


\title{
MEASUREMENT OF THE STRANGE-ANTISTRANGE ASYMMETRY AT NLO IN QCD FROM NUTEV DIMUON DATA
}

\author{
by \\ DAVID ALEXANDER MASON
}

\section{A DISSERTATION}

Presented to the Department of Physics and the Graduate School of the University of Oregon in partial fulfillment of the requirements for the degree of Doctor of Philosophy

March 2006 
"Measurement of the Strange-Antistrange Asymmetry at NLO in QCD from NuTeV Dimuon Data," a dissertation prepared by David Alexander Mason in partial fulfillment of the requirements for the Doctor of Philosophy degree in the Department of Physics. This dissertation has been approved and accepted by:

Professor Raymond E. Frey, Chair of the Examining Committee

Date

Committee in charge: $\quad$ Professor Raymond E. Frey, Chair

Professor Gregory D. Bothun

Professor James E. Brau

Professor Eugene D. Humphreys

Professor Davison E. Soper

Accepted by:

Dean of the Graduate School 
An Abstract of the Dissertation of David Alexander Mason for the degree of Doctor of Philosophy in the Department of Physics $\quad$ to be taken $\quad$ March 2006 Title: MEASUREMENT OF THE STRANGE-ANTISTRANGE ASYMMETRY AT NLO IN QCD FROM NUTEV DIMUON DATA

Approved:

Professor Raymond E. Frey

A measurement of the asymmetry between the strange and antistrange quark distributions, from a next to leading order QCD analysis of dimuon events measured by the $\mathrm{NuTeV}$ experiment at Fermilab is presented. Neutrino charged current events with two muons in the final state provide a direct means for studying charm production and measuring the strange sea. NuTeV's sign selected beam allows independent measurement of the strange and antistrange seas. An improved measurement of the neutrino and antineutrino forward dimuon cross section tables, using the complete charged current event sample for normalization is performed. These tables are then analyzed at NLO to measure the strange and antistrange seas. Detector acceptance is modeled using an NLO charm cross section differential in all variables required. The strange quark distribution is found to have an integrated momentum weighted asymmetry of $+0.00196 \pm 0.00046$ (stat) \pm 0.00045 (syst) \pm 0.00128 (external). The charm mass is found to be $1.41 \pm 0.10$ (stat) \pm 0.08 (syst) \pm 0.12 (external) $\mathrm{GeV}$. 


\section{CURRICULUM VITA}

NAME OF AUTHOR: David Alexander Mason

PLACE OF BIRTH: North Bend, Oregon

DATE OF BIRTH: September 18, 1970

GRADUATE AND UNDERGRADUATE SCHOOLS ATTENDED:

University of Oregon

DEGREES AWARDED:

Doctor of Philosophy in Physics, 2006, University of Oregon

Bachelor of Science, 1993, University of Oregon

AREAS OF SPECIAL INTEREST:

High Energy Physics 


\section{ACKNOWLEDGEMENTS}

It is impossible to complete something like this without relying heavily on the help and support of others. It is also impossible to give proper credit to all who have contributed to this work, either directly within the realm of physics, or indirectly via the support of friendship and family. But we will give it a shot.

I should start by thanking my committee, headed by my advisor, Ray Frey. Most of what I know about how to do science comes from Ray, and I cannot think of a better teacher. Ray's editing ability is amazing, and that this document makes any sense at all is in a large part thanks to his efforts. (He did not edit that last sentence)

I've learned from Greg Bothun to always go where the data leads, as well as many colorful metaphors. I've found Jim Brau's enthusiasm for the field, such that he probably spends more time in the air than on the ground in trying to keep it going, to be an inspiration. The only place I ever learned about things like heat transfer was in Gene Humphrey's geophysics class many years ago. The introduction of this thesis was essentially written for him and for Greg. There is more to Dave Soper than the Anthony Perkins facade lets on. He'll often surprise you with a really slick idea. It was his idea for example, to slightly re-scale the gluon sea in this analysis to maintain momentum conservation.

Panagiotis Spentzouris deserves to be included along with the committee members in this analysis. As the leader of the Dimuon analysis group, he has guided this analysis from its beginning to completion, always trying to make the 
time as he kept the other couple dozen balls in the air. I undoubtedly owe him many beers!

I have been very lucky in working with the people at the University of Oregon and with my collaborators on the $\mathrm{NuTeV}$ experiment. It is not often you can be involved with groups like this long enough to get to know everybody, and actually still like everyone! Both groups of people have always been very friendly and have always been willing to help. It will be hard to leave Eugene, and I'm not looking forward to the day when there are no more long distance phone meetings to FNAL.

I have also been very fortunate to have my family's strong support as I've pursued my dream of becoming a scientist. Whether it be through my dad's example in his insatiable appetite for books, or my mom letting me eat dinner in front of the TV so I could watch Carl Sagan when I was a kid, those are all pieces of this work.

While working on this project I was lucky enough to meet, fall in love with, and have Reiko Yamachika become my wife. I am forever grateful for her patience while waiting for me to finish, and her support throughout. With Reiko came a whole new family, and I am also thankful to Masahiko, Emiko and the Togai clan out East for supporting this poor guy who never seemed to be able to get out of school. And now Maiko is following a similar path. Hopefully this work serves as an inspiration, rather than reason to run away screaming!

Finally, while writing this document I came to meet a little guy named Naru. He doesn't really understand what all this paper is for (it doesn't taste very good), or why his daddy has such dark circles under his eyes, but now he can stick his tongue out and smile. And that's just fine with me! 


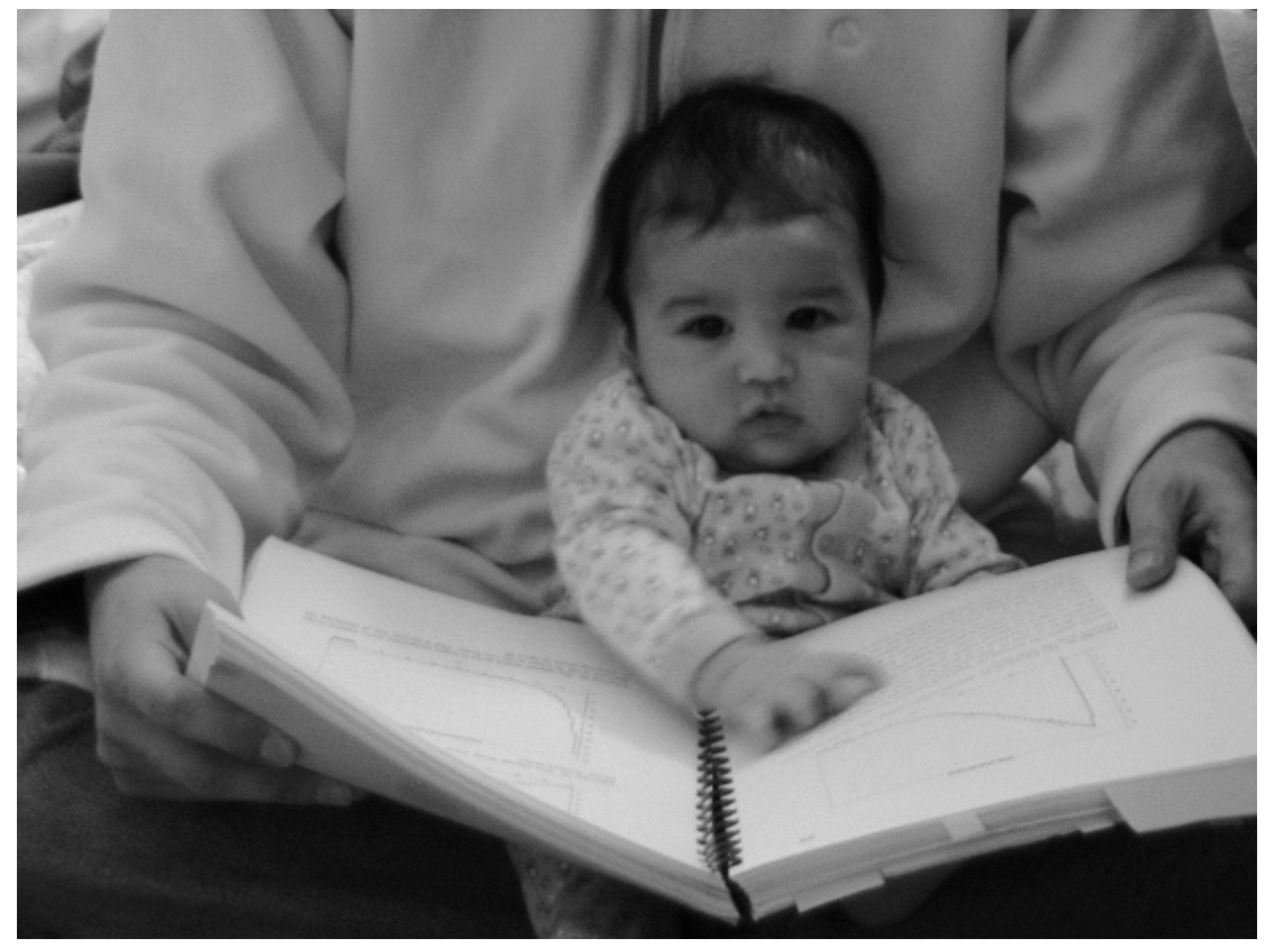

\section{For Narimasa Alexander Mason}

...without whose keen eye for detail and countless hours of editing, this document would not have been possible. 
viii

\section{TABLE OF CONTENTS}

Chapter Page

I. INTRODUCTION ........................... 1

1.1. A Personal Perspective on the History of Particle Physics . . . 3

1.2. The Life and Times of the Oppositely Signed Dimuon . . . . . 21

1.3. What to Expect in this Thesis . . . . . . . . . . . . . 44

II. SOME THEORY . . . . . . . . . . . . . . . . . 45

2.1. The Standard Model . . . . . . . . . . . . . . . 45

2.2. Charged Current Deep Inelastic Scattering . . . . . . . . . . . 49

2.3. Scaling Violation and $R_{L}$. . . . . . . . . . . 75

2.4. LO Charm Production . . . . . . . . . . . . 78

2.5. NLO Charm Production . . . . . . . . . . . . 96

III. THE NUTEV EXPERIMENT AND DATA SAMPLE . . . . . . . . . 121

3.1. Overview ..................... 121

3.2. The Neutrino Beam . . . . . . . . . . . . . . 122

3.3. The NuTeV Detector . . . . . . . . . . . . . . . . 137

3.4. The Calibration Beam . . . . . . . . . . . . . . . 152

3.5. Event Triggering and Data Acquisition . . . . . . . . . 154

3.6. The NuTeV Cruncher: e815_analysis . . . . . . . . . . . . 162

3.7. Event Reconstruction . . . . . . . . . . . . . . . 162

3.8. Data . . . . . . . . . . . . . . 166

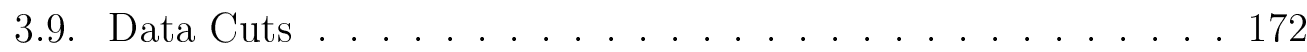

3.10. Detector Calibration . . . . . . . . . . . 177

IV. MONTE CARLO SIMULATION AND THE FORWARD DIMUON

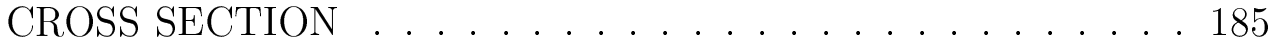

4.1. The NuTeV Monte Carlo . . . . . . . . . . . . . . . . . 191

4.2. MC Energy and Flux Corrections . . . . . . . . . . . . . 233

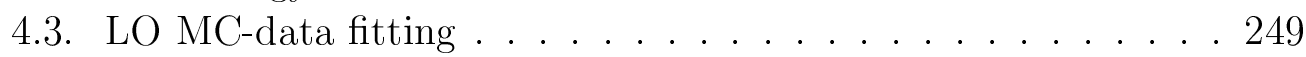

4.4. LO MC-data comparisons . . . . . . . . . . . . . 252

4.5. Forward Dimuon Cross Section Table . . . . . . . . . . 270

V. NLO FITS AND PHENOMENOLOGY . . . . . . . . . . . . . 300

5.1. NLO Enhancements to Numonte Dimuon Model . . . . . . . . 302 
5.2. NLO acceptance. . . . . . . . . . . . . . . 307

5.3. NLO inclusive charm cross section model . . . . . . . . . . . . 320

5.4. NLO table fits . . . . . . . . . . . . . . . . 336

5.5. NLO MC-data comparisons . . . . . . . . . . . . . . 355

5.6. NLO table re-extraction . . . . . . . . . . . . . . . . . . . . . . . . . . . . . . . . . . .

5.7. Discussion . . . . . . . . . . . . . . . . 375

5.8. Some Closing Remarks . . . . . . . . . . . 383

\section{APPENDIX}

A. THE NUTEV BEAM TOROID . . . . . . . . . . . . . 388

A.1. Introduction . . . . . . . . . . . . . . . . . . . . . . . . 388

A.2. Description ....................... 390

A.3. Some toroid physics and droop compensation . . . . . . . . 393

B. NUTEV SOFTWARE SETTINGS . . . . . . . . . . . . 399

C. RELEVANT CODE SNIPPETS . . . . . . . . . . . . 400

C.1. Irrelevant Code Chunks \& Snippets . . . . . . . . . . . . . 407

D. DIMUON CROSS SECTION TABLE VALUES . . . . . . . . . . 408

E. THE NUTEV COLLABORATION . . . . . . . . . . . . . 414

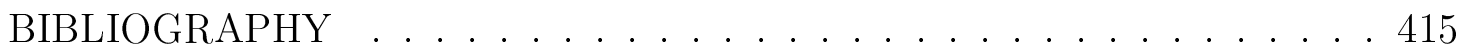




\section{LIST OF FIGURES}

Figure Page

1. Some examples of Feynman diagrams . . . . . . . . . . . . 7

2. The first version of the Reines-Cowan neutrino experiment . . . . . 11

3. Diagram of the Columbia-Brookhaven $\nu_{\mu}$ discovery experiment . . . 14

4. Columbia-Brookhaven "vertex event" ............. 15

5. FNAL neutrino beamline and HPWF experiment . . . . . . . . 22

6. A dimuon event from the HPWF experiment . . . . . . . . . . . 23

7. A dimuon event from the Caltech-Fermilab experiment . . . . . . 25

8. HPWFOR dimuon rates vs energy . . . . . . . . . . . 27

9. The CDHS detector . . . . . . . . . . . . . . 29

10. Cartoon of a drift chamber .................. 31

11. The CDHS tetramuon event .................. 32

12. Fanciful, and more realistic explanations of the tetramuon event . . . 33

13. The E-616 detector, with a dimuon event faintly seen in the crosssectional view at top .................. 35

14. E-744 strange and antistrange sea extractions . . . . . . . . . 36

15. The E-531 detector . . . . . . . . . . . . . . . 37

16. The CHARMII detector . . . . . . . . . . . . 38

17. The CHORUS detector . . . . . . . . . . . . . . 40

18. The NOMAD detector .................. . . 41

19. Cartoon of charged current . . . . . . . . . . . . 50

20. A Feynman diagram of CC DIS . . . . . . . . . . . . . . 54 
21. $\sigma_{C C}^{\nu}$ vs $E_{\nu}$ from FNAL-E-0001 and FNAL-E-0815 . . . . . . . . . 61

22. Possible helicity configurations for $\nu$ and $\bar{\nu}$ scattering . . . . . . . . 65

23. $\mathrm{CCFR}(\nu-N) F_{2}$ vs $\operatorname{EMC}(\mu-N) F_{2} \times \frac{18}{5}$ vs x ....... 70

24. Cartoon of $F_{2} \ldots \ldots \ldots \ldots \ldots \ldots$. . . . . . . . . . . . . . . . .

25. Callan-Gross violation vs $\mathrm{x}$ at different $Q^{2} \ldots \ldots$. . . . . . . 77

26. Charged current charm production. $\nu$ production at left, $\bar{\nu}$ at right. . 79

27. The hadronic vertex ................ 83

28. $\frac{\xi}{x}$ plotted vs $x$ and $Q \ldots \ldots \ldots \ldots$

29. $\frac{\xi_{\text {full }}}{\xi}$ vs $Q$ for both low and high x values . . . . . . . . 87

30. $D_{p}(x)$ for charm and bottom quarks . . . . . . . . . . . . 92

31. $D(x)$ from Peterson and Collins-Spiller parameterizations . . . . . . 93

32. A Feynman diagram of a neutrino induced dimuon event. . . . . . . . 95

33. Quark-quark interaction in QCD .............. 98

34. Some diagrams which are combined in charged particle scattering . . 99

35. Some diagrams which are combined in quark scattering . . . . . . . 102

36. The running of $\alpha_{s}\left(Q^{2}\right) \ldots \ldots \ldots \ldots$. . . . . . . . . . . . . . . .

37. Feynman diagrams included in NLO charm cross section calculation . 109

38. NLO charm cross section components versus $x \ldots \ldots$. . . . . 111

39. NLO charmed hadron cross section versus $z \ldots \ldots$. . . . . . 112

40. Cartoon explanation of why we have a negative bump at high z . . 113

41. Transverse momentum of LO and NLO diagrams . . . . . . . . . 114

42. Cross section vs rapidity and $z$ for fixed $x$ and $Q^{2}$. . . . . . . 116

43. Where NuTeV's neutrinos came from . . . . . . . . . . . . 123 
44. Diagram of fixed target beams . . . . . . . . . . . . . 125

45. TeVatron magnet ramp and proton occupancy versus time . . . . . 126

46. Timing of pings and slow spill . . . . . . . . . . . . . 127

47. The shape of the NuTeV pings . . . . . . . . . . . . . 128

48. Diagram of $\mathrm{NuTeV}$ beam target, beam optics and magnets . . . . . . 129

49. Saturation of the neutrino beamline SEM . . . . . . . . . . . . . 132

50. The NuTeV target "cave" under construction in 1995 . . . . . . . . 133

51. The SSQT . . . . . . . . . . . . . . . . . 134

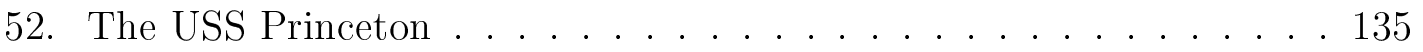

53. The NuTeV neutrino and antineutrino flux . . . . . . . . . . 136

54. NuTeV protons on target . . . . . . . . . . . . 137

55. The traditional NuTeV detector graphic . . . . . . . . . . 138

56. Photo of side of $\mathrm{NuTeV}$ detector . . . . . . . . . . . . . 139

57. Photo and diagram of calorimeter sandwich . . . . . . . . . . 140

58. A photograph of a disassembled scintillator counter . . . . . . . . . 141

59. A NuTeV scintillator counter . . . . . . . . . . . . . . . 142

60. Average response of a calorimeter scintillator . . . . . . . . . . . . 144

61. A NuTeV drift chamber ................... . . 145

62. A photo of the $\mathrm{NuTeV}$ toroid . . . . . . . . . . . . . . 148

63. Toroid coil and drift chamber layout . . . . . . . . . . . . . 149

64. Face-on view of the toroid and 4 coils . . . . . . . . . . . . . 149

65. Effect of burnt out coil on spectrometer toroid field . . . . . . . . . 150

66. The $\mathrm{NuTeV}$ veto wall . . . . . . . . . . . . . . 151 
67. The NuTeV Decay Channel helium bags . . . . . . . . . . . . 151

68. The NuTeV calibration beamline . . . . . . . . . . . . 153

69. The $\mathrm{NuTeV}$ calibration beam in relation to the NuTeV detector. . . . 154

70. A diagram of the phototube electronics. . . . . . . . . . . 156

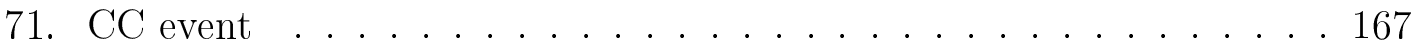

72. $\mathrm{NC}$ event . . . . . . . . . . . . . . . . . 168

73. Cosmic Ray . . . . . . . . . . . . . . . . 169

74. A classic dimuon event . . . . . . . . . . . . . . 170

75. A trigger 11 event . . . . . . . . . . . . . . 171

76. A bad trigger 11 event . . . . . . . . . . . . . . 172

77. Calibration Beam Electron event . . . . . . . . . . . 177

78. Calibration Beam Hadron event . . . . . . . . . . . . . 179

79. The hadron calibration . . . . . . . . . . . . . . 180

80. Trigger 6 event . . . . . . . . . . . . . . . 181

81. Calibration Beam Muon .................. 182

82. Calorimeter muon energy loss . . . . . . . . . . . . . 183

83. Calibration Beam and MC simulated $12.5 \mathrm{GeV}$ muons . . . . . . . . 184

84. Measured $E \Phi(E)$ vs $E$ in $\mathrm{GeV}$ for neutrino and antineutrino beams.

The $\mathrm{x}$ axis is in GeV. . . . . . . . . . . . . 195

85. Measured vertex distributions for $\nu$ (left) and $\bar{\nu}$ (right) beams . . . 196

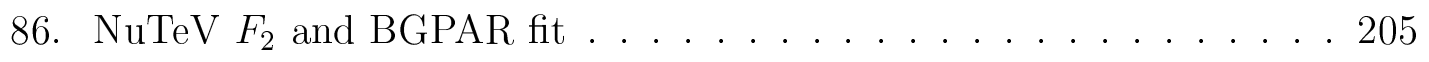

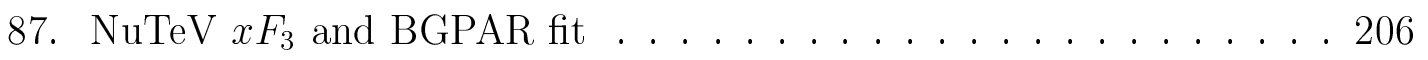

88. QED radiative correction diagrams . . . . . . . . . . . 208

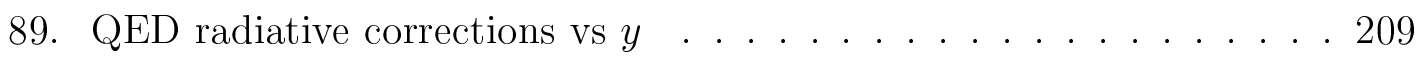


90. The NA14/2 $p_{\perp}^{2}$ data . . . . . . . . . . . . . 213

91. Energy stolen from D by neutrino . . . . . . . . . . . . 215

92. Decay muon energy vs energy of parent charmed meson . . . . . . . . 215

93. Charmed meson angle relative to $\mathrm{W}$ momentum vector . . . . . . . . 216

94. Decay muon angle relative to charmed meson momentum vector . . . 216

95. $\pi$-K parameterization vs $E_{\mu 2} \ldots \ldots \ldots \ldots$

96. wtcc from typical dimuon $\mathrm{MC}$ runs . . . . . . . . . . . . 224

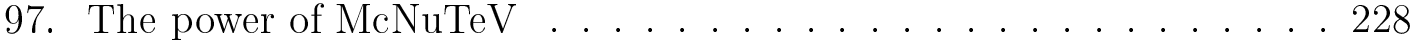

98. $E_{\pi^{0}}$ energy fraction . . . . . . . . . . . . . . 231

99. Average DC efficiencies . . . . . . . . . . . . . . . . . 234

100. Average DC efficiencies vs run . . . . . . . . . . . . . . . 234

101. The $E_{\mu-F F} \mathrm{MC}$ energy correction . . . . . . . . . . . 236

102. Hadronic correction mean parameterizations . . . . . . . . . 238

103. Hadronic correction width parameterizations . . . . . . . . 238

104. Neutrino and antineutrino mode flux corrections . . . . . . . . . . . . 240

105. Inclusive $E_{\mu 1}$ distribution . . . . . . . . . . . . . 242

106. Inclusive $E_{\text {Had }}$ distribution . . . . . . . . . . . . . . . . 243

107. Inclusive $E_{v i s}$ distribution . . . . . . . . . . . . . . . 244

108. Inclusive VERTX distribution . . . . . . . . . . . . . . . . 245

109. Inclusive VERTY distribution . . . . . . . . . . . . . . . . 246

110. Inclusive $\theta_{\mu 1}$ distribution . . . . . . . . . . . . . . . 247

111. Inclusive $E_{\mu-F F}$ distribution . . . . . . . . . . . . . 248

112. LO BGPAR fit output . . . . . . . . . . . . . 253 
113. Dimuon total reconstructed neutrino energy . . . . . . . . . 255

114. Dimuon hadronic shower energy . . . . . . . . . . . . . . 256

115. Dimuon primary muon energy . . . . . . . . . . . . . . 257

116. Dimuon $E_{\mu F F 1}$ distribution . . . . . . . . . . . 258

117. Dimuon $\theta_{\mu 1}$ distribution . . . . . . . . . . . . . . . 259

118. Dimuon charm decay muon energy . . . . . . . . . . . . . 260

119. Dimuon $E_{\mu F F 2}$ distribution . . . . . . . . . . 261

120. Dimuon $\theta_{\mu 2}$ distribution . . . . . . . . . . . . 262

121. Dimuon $\theta_{\mu 12}$ distribution . . . . . . . . . . . . 263

122. Dimuon $x_{v i s}$ distribution . . . . . . . . . . . 264

123. Dimuon $y_{v i s}$ distribution . . . . . . . . . . . . 265

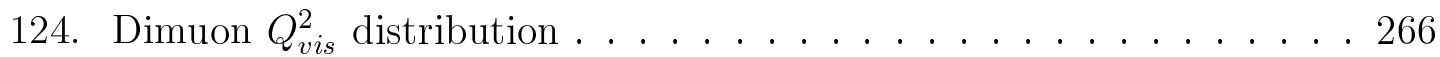

125. Dimuon $z_{v i s}$ distribution . . . . . . . . . . . . 267

126. Dimuon $W_{v i s}^{2}$ distribution $\ldots \ldots \ldots \ldots . \ldots \ldots$

127. Dimuon $m_{12-v i s}$ distribution . . . . . . . . . . . . . . 269

128. Extracted $\nu$ mode dimuon cross section table . . . . . . . . . . . 277

129. Extracted $\bar{\nu}$ mode dimuon cross section table . . . . . . . . . . . . 278

130. Table effective DOF, $\nu$ mode . . . . . . . . . . . . . . . 279

131. Table effective DOF, $\bar{\nu}$ mode . . . . . . . . . . . . . . . . . 280

132. Table unfolding correction, $\nu$ mode . . . . . . . . . . . . 281

133. Table unfolding correction, $\bar{\nu}$ mode . . . . . . . . . . . . . 282

134. $E_{\mu 2}>5 \mathrm{GeV}$ acceptance, $\nu$ mode . . . . . . . . . . 283

135. $E_{\mu 2}>5 \mathrm{GeV}$ acceptance, $\bar{\nu}$ mode . . . . . . . . . . . . 284 
136. Fake data pull study $m_{c} \ldots \ldots$. . . . . . . . . . . 286

137. Fake data pull study $\epsilon$. . . . . . . . . . . . . . . 286

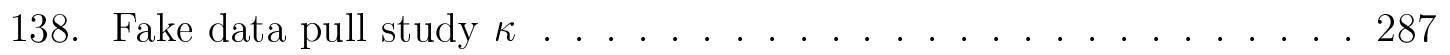

139. Fake data pull study $\bar{\kappa} \ldots$. . . . . . . . . . . . . 287

140. Fake data pull study $\alpha$. . . . . . . . . . . . . . . 288

141. Fake data pull study $\bar{\alpha} \ldots$. . . . . . . . . . . . . . 288

142. Fake data $B_{c}$ pull study . . . . . . . . . . . . . . . . . 289

143. Average DOF vs coverage . . . . . . . . . . . . . . . . 290

144. Average re-fitted $m_{c}$ vs coverage fraction . . . . . . . . . . . 291

145. Average re-fitted $\kappa$ vs coverage fraction . . . . . . . . . . . . 292

146. Average re-fitted $\bar{\kappa}$ vs coverage fraction . . . . . . . . . . . . . . 292

147. Average re-fitted $\alpha$ vs coverage fraction . . . . . . . . . . . 293

148. Average re-fitted $\bar{\alpha}$ vs coverage fraction . . . . . . . . . . . . . 293

149. $\nu$ table extracted based on CTEQ5 pdf set and BGPAR . . . . . . . 294

150. $\bar{\nu}$ table extracted based on CTEQ5 pdf set and BGPAR . . . . . . . 295

151. Numode Dimuon Cross Section with Systematics. Points are shown multiplied by $\frac{\pi}{G_{F}^{2} M E_{\nu}} \ldots \ldots \ldots$. . . . . . . . . . . 298

152. Nubarmode Dimuon Cross Section with Systematics. Points are shown

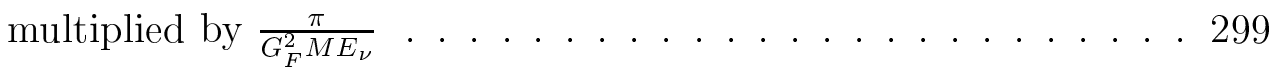

153. How rapidity is thrown . . . . . . . . . . . . . . . . . 304

154. MC precision vs rapidity binning scheme . . . . . . . . . . . 305

155. Cross section and acceptance for $\nu$ table point $3 \ldots . . . . . . .309$

156. Cross section and acceptance for $\nu$ table point 43 . . . . . . . . 310

157. $z$ acceptance for high and low $E_{\nu}$ table bins . . . . . . . . . . 312 
158. $z$ acceptance for hard and soft $\epsilon \ldots \ldots$. . . . . . . . . . 313

159. rapidity acceptance for high and low $E_{\nu}$ table bins . . . . . . . . . . 315

160. rapidity acceptance for high, low and medium $m_{c} \ldots \ldots$. . . . . 316

161. NLO acceptance corrections vs $\epsilon \ldots \ldots$. . . . . . . . . . 317

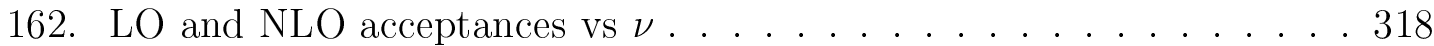

163. LO and NLO acceptances vs $Q^{2} \ldots \ldots$. . . . . . . . . 319

164. World $B_{c}$ measurements . . . . . . . . . . . . 321

165. Cartoon of nuclear effects . . . . . . . . . . . . . . 324

166. Nuclear valence correction . . . . . . . . . . . . . . 327

167. Nuclear sea quark correction . . . . . . . . . . . . . . . . . 328

168. Nuclear gluon correction . . . . . . . . . . . . . . . 328

169. deFlorian et al nuclear correction vs Hirai et al. . . . . . . . . . . . . 335

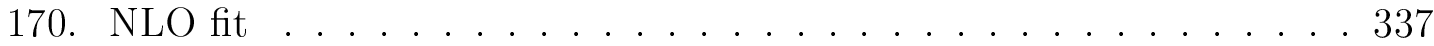

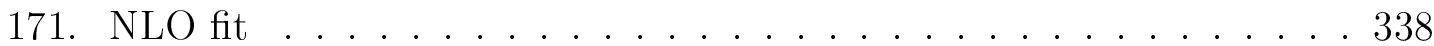

172. Data/MC zvis comparisons for $\epsilon=0.3,0.6$, and 0.9 . . . . . . . . 339

173. $\chi^{2}$ vs epsilon for $z_{v i s}$ and data fitter . . . . . . . . . . . . . 340

174. $\Delta S^{-}$vs $m_{c}$ and $\epsilon \ldots \ldots \ldots \ldots$. . . . . . . . . . . . . . . . . . . .

175. The variation of a typical acceptance point vs QCD scale . . . . . . 342

176. $\kappa$ - $\alpha$ QCD evolution fit $x s(x)$ and $x \bar{s}(x)$ vs $\mathrm{x} \ldots \ldots 347$

177. $\kappa$ - $\alpha$ QCD evolution fit $x s^{+}(x)$ vs $\mathrm{x} \ldots \ldots . \ldots . \ldots . \ldots 347$

178. $\kappa$ - $\alpha$ QCD evolution fit $x s^{-}(x)$ vs $\mathrm{x} \ldots \ldots . \ldots . \ldots . \ldots$

179. CTEQ parameterization $x s^{-}(x)$ vs $\mathrm{x} \ldots \ldots \ldots \ldots 2$

180. Comparison of $\nu$ dimuon charm mass measurements . . . . . . . . . 355 
181. NLO Dimuon total reconstructed neutrino energy . . . . . . . . . 356

182. NLO Dimuon reconstructed hadronic energy . . . . . . . . . . . 357

183. NLO Dimuon primary muon energy . . . . . . . . . . . . . . 358

184. NLO Dimuon $E_{\mu F F 1}$ distribution . . . . . . . . . . . . 359

185. NLO Dimuon $\theta_{\mu 1} \ldots \ldots \ldots \ldots$. . . . . . . . . . . . . . . . . . . . . . .

186. NLO Dimuon charm decay muon energy . . . . . . . . . . . . 361

187. NLO Dimuon $E_{\mu F F 2}$ distribution . . . . . . . . . . 362

188. NLO Dimuon $\theta_{\mu 2}$ distribution . . . . . . . . . . . . 363

189. NLO Dimuon $\theta_{\mu 12}$ distribution . . . . . . . . . . . . . . 364

190. NLO Dimuon $x_{v i s}$ distribution . . . . . . . . . . . 365

191. NLO Dimuon $y_{v i s}$ distribution . . . . . . . . . . . . . 366

192. NLO Dimuon $Q_{v i s}^{2}$ distribution . . . . . . . . . . . . 367

193. NLO Dimuon $z_{v i s}$ distribution . . . . . . . . . . . . . . 368

194. NLO Dimuon $W_{v i s}^{2}$ distribution . . . . . . . . . . . . 369

195. NLO Dimuon $m_{12-v i s}$ distribution . . . . . . . . . . . . . 370

196. NLO table vs LO table . . . . . . . . . . . . . . . . . 372

197. NLO table vs LO table . . . . . . . . . . . . . . . 373

198. Re-extracted $x s^{-}(x)$ with original fit . . . . . . . . . . . 374

199. Comparing $x s^{+}$to global fits . . . . . . . . . . . 376

200. $\eta_{s}$ vs time . . . . . . . . . . . . . . . 377

201. Comparison of 3 strange parameterizations . . . . . . . . . . . 379

202. Theoretical predictions for $s(x)-\bar{s}(x) \ldots \ldots$. . . . . . . 381

203. Theoretical predictions for $x s(x)-x \bar{s}(x) \ldots \ldots . \ldots . . . \ldots 382$ 
204. $S^{-}$and $\chi^{2}$ versus crossing point . . . . . . . . . . . . 384

205. $x s^{-}$versus $x$ for $x_{0}$ of $0.01,0.05$, and $0.15 \ldots \ldots . \ldots . \ldots$

206. $x s^{-}(x)$ vs $x$ at $Q^{2}=16 G e V^{2} \ldots \ldots . \ldots . \ldots . \ldots 38$

207. NuTeV's beam toroid before installation in the primary beam . . . . 389

208. A cartoon illustrating a beam toroid . . . . . . . . . . . . 390

209. A schematic diagram of NuTeV's beam toroid . . . . . . . . . . 391

210. A schematic of the use of the $\mathrm{NuTeV}$ toroid . . . . . . . . . . . 392

211. A simple equivalent circuit of the beam toroid and beam . . . . . . 393

212. Input and output current to $\mathrm{NuTeV}$ toroid . . . . . . . . . . . . . 394

213. The integral of the toroid output showing decay from signal droop . . 395

214. Circuit diagram of droop compensator circuit . . . . . . . . . . 396

215. Non-droop compensated and compensated test pulse waveforms . . . 397

216. Non-droop compensated and compensated ping waveforms . . . . . 398 


\section{LIST OF TABLES}

Table

Page

1. Dimuon cross section ratios measured by the HPWFOR collaboration 28

2. The CCFR neutrino experiment dynasty with dimuons reported . . . 34

3. Summary of neutrino experiments measuring dimuon events . . . . 43

4. The four known forces and their mediators. . . . . . . . . . . . 45

5. The six leptons .......................... 47

6. The six quarks . . . . . . . . . . . . . . . 49

7. Hadrons referred to in this thesis .............. 50

8. PMT signal summary . . . . . . . . . . . . . . . 155

9. Number of events from each trigger (non-exclusive) . . . . . . . . 160

10. Cuts applied to inclusive and dimuon events . . . . . . . . . . . . 174

11. Parameter values from NuTeV structure function BGPAR fit . . . . . 202

12. MC dimuon kinematics variable construction . . . . . . . . . . . 212

13. Charm semileptonic decay modes modeled in numonte . . . . . . . . 214

14. Vertex $\pi-K$ background model parameters . . . . . . . . . . . . 219

15. Shower $\pi-K$ background model parameters . . . . . . . . . . . . . 221

16. McNuTeV GEANT particle energy cut values . . . . . . . . . . 228

17. Legend for DC Efficiency plots (figures 99 and 100) . . . . . . . 233

18. Parameters for McNuTeV Hadronic Energy Correction . . . . . . . . 237

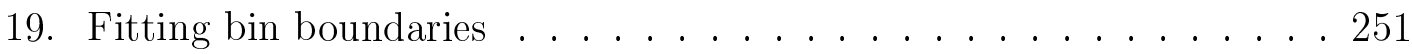

20. BGPAR fit parameters and the itemized effects of systematics . . . . 297 
21. $\kappa \alpha$ approximate evolution fit results . . . . . . . . . . . . 344

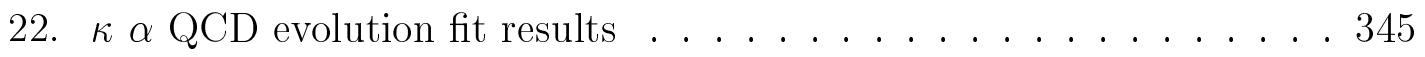

23. $\kappa \alpha$ QCD evolution fit systematics . . . . . . . . . . . 349

24. Sum rule satisfying fit parameters . . . . . . . . . . . . 350

25. Contributions to the error in $S^{-}$and $\eta_{s}$ for CTEQ fit . . . . . . . 351

26. $\kappa \alpha m_{c}$ fit systematics . . . . . . . . . . . . . 353

27. $\kappa \alpha m_{c}$ fit systematics . . . . . . . . . . . . . 354

28. Revised $\nu$ cross dimuon section for $E_{\nu} \sim 88.3 \mathrm{GeV} \ldots \ldots . . . . . .408$

29. Revised $\nu$ cross dimuon section for $E_{\nu} \sim 174.3 \mathrm{GeV}$. . . . . . . . 409

30. Revised $\nu$ cross dimuon section for $E_{\nu} \sim 247.0 \mathrm{GeV}$. . . . . . . . 410

31. Revised $\bar{\nu}$ cross dimuon section for $E_{\nu} \sim 77.9 \mathrm{GeV} \ldots \ldots . . . . . .411$

32. Revised $\bar{\nu}$ cross dimuon section for $E_{\nu} \sim 143.7 \mathrm{GeV}$. . . . . . . . . 412

33. Revised $\bar{\nu}$ cross dimuon section for $E_{\nu} \sim 226.8 \mathrm{GeV}$. . . . . . . . 413 


\section{CHAPTER I}

\section{INTRODUCTION}

This document is the description of the analysis of data taken in a particle physics experiment [1]. Particle physics is the study of what sorts of things, at the most fundamental level, everything in the universe is constructed from and how these things interact with each other. This study is pursued predominantly by throwing tiny pieces of the universe at each other, recording what happens, and using that information in constructing models to predict what might happen the next time. In constructing these models we hope that we can gain some knowledge about why the universe is the way it is and possibly even how it got that way.

In this particular experiment very elusive particles called neutrinos, and their antiparticle, antineutrinos, are used to illuminate the particles which make up protons and neutrons, which in turn make up us. Neutrinos are used to do this because they are able to "taste" the different flavors of these component particles, called quarks. Looking at things as small as the things that protons and neutrons are made out of requires a beam of very high energy particles. The higher energy the particles are that illuminate an object, the smaller the objects that can be resolved. It is also possible see things that happen within a very short space of time.

A person staring at a desk for an extended period of time may find it to be a dull, static, solid object. Zoom in however, and one finds it is constructed out of 
atoms, bound together, but still vibrating and jostling around. The atoms themselves are constructed out of smaller objects: A cloud of electrons surrounding a small nucleus. This nucleus is also constructed of component particles, called protons and neutrons. Zooming in still further, the protons and neutrons themselves also have substructure. Inside, quarks, antiquarks, and the particles that stick them together, called gluons, are moving around, interacting, being created and destroyed. How much of it can be observed all depends on how close one wants to look.

The NuTeV experiment looked at the protons and neutrons (collectively called "nucleons") in an iron target by measuring what happened when neutrinos or antineutrinos were crashed into it. The neutrinos were energetic enough to break apart the nucleons in the target. In some of these events, the pieces left over contained a charm quark.

There are 6 "flavors" of quarks, called "up", "down", "strange" "charm", "bottom", and "top". The up and down quarks are the most common kinds of quarks that are found inside nucleons, but strange quarks are light enough that they are also found fairly easily. When a neutrino hits a strange quark, it will most often produce a charm quark. Particles containing charm quarks can decay in ways that are easy to see in a detector, so looking for and these decays are a good way of measuring the characteristics of strange quarks inside nucleons.

When antineutrinos interact to produce an anticharm quark, it is most likely the result of an interaction with an antistrange quark in the nucleon. The NuTeV experiment was able to tell, with great precision whether neutrinos or antineutrinos interacted in its detector. This means $\mathrm{NuTeV}$ was able to measure, better than 
anyone else so far, whether there is any difference between the strange quarks and antistrange quarks in nucleons. It is this measurement and analysis which will be addressed in the pages which follow.

\subsection{A Personal Perspective on the History of Particle Physics}

Particle physics as a science can perhaps trace its origins back to human prehistory, where ancient humans would try to determine the nature of dark, unknown places by throwing rocks at them. Ancient physicists would gain understanding of the nature of such places through observing the effects of interactions between the thrown rocks and the place's contents. A clicking noise would mean something hard was present, a "plunk" noise would possibly mean there was water there, and so on. Repeating these measurements allowed them to build a picture, a model, of the mysterious place under study. Unfortunately all too frequently those early experiments were followed by the paleo-experimentalists becoming lunch.

It is perhaps for this reason that our understanding of what constitutes matter throughout the following millennia was dominated by superstition and supposition. Only in the last few centuries, and especially in the last hundred-odd years have physicists picked the rocks back up again and probed at nature's dark places through experimental and analytical means.

This transition from superstition-based models to a more systematic picture proceeded in fits and starts throughout the past few thousand years. One can assign a beginning at early cultures' flirtations with mathematical descriptions of planetary motion and geometry, with the Greek Philosophers works appearing

most prominently in Western accounts of history. We then see the beginning of 
the science of physics with the work of Galileo \& Newton, and an acceleration of experimental physics in the late 17th century and throughout the 1800's.

The birth of particle physics can probably best be placed at J.J. Thomson's 1897 discovery that cathode rays were not "rays", but better described by particles with a mass much less than that of a hydrogen atom [2]. These particles were electrons. This was followed by Ernest Rutherford's discovery of the atomic nucleus, and hence proton in 1911 [3], and James Chadwick's discovery of the neutron in 1932 [4]. The modern picture of the atom took shape, with electrons orbiting a nucleus made up of protons and neutrons.

During the same period several theoretical advances established the foundations of the models used to describe particle physics today, not the least of which was Einstein's theory of special relativity in 1905[5]. Relativity is still used with great success in describing the kinematics of particles in particle physics experiments 100 years later. In the late 1920's came the development quantum mechanics, culminating in Dirac's merger of quantum theory and relativity in 1928 $[6,7]$. Dirac's theory had an interesting feature though. It required that particles have both positive and negative energy states. These negative energy states meant there had to be particles just like electrons, but with a positive charge.

This prediction of "antimatter" was confirmed in an elegant cloud chamber experiment by Carl Anderson in 1933 [8]. A cloud chamber is a tank of gas super saturated, in this case with alcohol. As a charged particle passes through it tiny beads of alcohol condense together along the particle's path allowing one to see and photograph its track. In the Anderson experiment, a 6 millimeter lead plate was placed in the middle of such a chamber which in turn was placed in a magnetic 
field. The combination of the plate and magnetic field allowed measurement of the energy and mass of cosmic rays passing through the chamber, and the difference in curvature of the tracks above and below the plate determined the direction the particles were moving, and hence charge. Anderson observed 15 tracks of particles with masses much less than that of a proton, but were nevertheless positively charged. These particles were the antiparticle partner to the electron, which he called the "positron".

At about this same time theorists were confronted with a dilemma in trying to understand nuclear beta decay. In nuclear beta decay, the nucleus decays emitting a "beta particle", named as such before it was determined that it was actually an electron. The problem confronting the theorists, however, was that detailed accounting of the energies of the decay products did not add up properly. Energy did not seem to be conserved. In a letter to the participants of a conference in Tubingen, Germany in 1930 [9], Wolfgang Pauli suggested that the reason energy didn't seem to be conserved was that there might be another particle involved that wasn't seen. That particle would have to be neutral so as not to leave an ionizing trail visible by experiments, "not larger than 0.01 proton mass," with "something like 10 times the penetrating capacity of a $\gamma$ ray." Pauli referred to this particle as a "neutron." Two years later Enrico Fermi [10] incorporated Pauli's mysterious particle into his theory of beta decay, renaming it the "neutrino." The energy balancing problem was solved, but physicists were left with an invented particle that was to remain unobserved for another 20 years.

In 1937 Neddermeyer and Anderson [11] discovered a deeply penetrating particle with their cloud chamber, which they called the "meson", since it had 
a mass between that of electrons and protons. Though not realized at first, this particle was the muon, the first of an entire new generation of particles. For the next decade particle physics took to the back burner while those who studied it plied their talents toward winning World War II.

One of these combatants was Richard Feynman, who while working for the Manhattan Project, organized a room full of human operated mechanical calculators into a sort of parallel processor to simulate what would happen during the atomic explosion [12] . This was possibly the world's first Monte-Carlo simulator. After the war, Feynman returned to thinking about more peaceful electrons and photons, completing his development of quantum electrodynamics, or QED [13]. QED is the first (and most) successful quantum field theory - a theory so successful that it now agrees with experiment to eight decimal places. Julian Schwinger and Shinichiro Tomonaga both developed equivalent QED field theories around that time, but Feynman's description has left a lasting mark on particle physics with his visual method of accounting for the terms in the series expansions used in calculations. These diagrams are now called "Feynman Diagrams," and are ubiquitous in modern particle physics.

Four examples of Feynman diagrams are shown in figure 1. In the diagrams, time flows from left to right, the lines with arrows indicate fermions like electrons and neutrinos, wavy lines bosons like photons, and curly lines gluons, carriers of the strong force. If an arrow in the fermion line is in the same direction as time (to the right) it is a particle, if going the opposite direction, it is an antiparticle. The diagram in 1-a shows an electron and positron annihilating into a virtual photon, which then emits another electron-positron pair. This process is called 


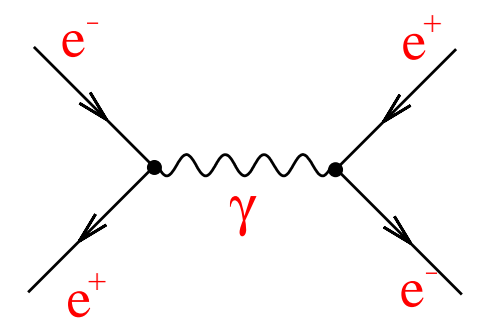

(a)

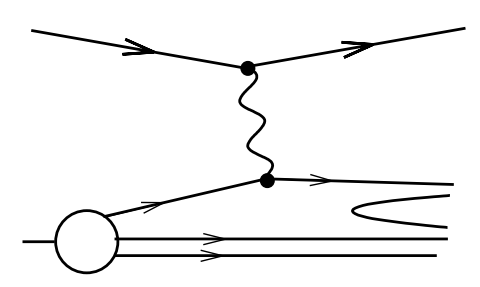

(c)

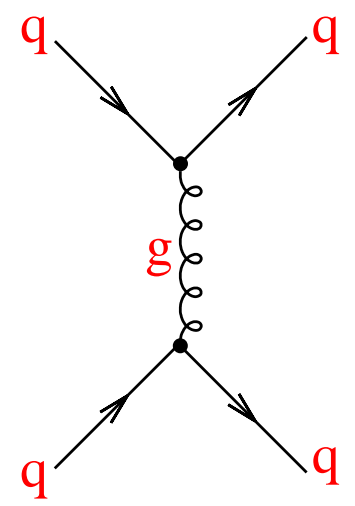

(b)

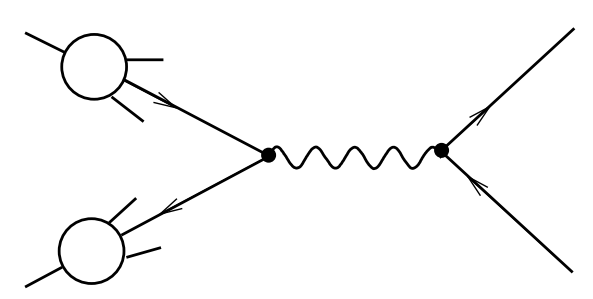

(d)

FIGURE 1. Some examples of Feynman diagrams. (a) is Bhahba scattering, (b) is quark scattering, (c) is deep inelastic lepton-nucleon scattering, and (d) decorates the floor of Willamette Hall (proton-antiproton annihilation and lepton production)

"Bhahba scattering." "Virtual" means that the photon isn't observable, and in fact, in order to conserve momentum in this case would need to carry mass. Real photons are massless. Weird behavior like this is perfectly acceptable in field theory. Diagram 1-b is an example of a quark interacting with another quark via the strong interaction. The loopy line between the two quarks is a gluon, which mediates the strong interaction. Diagrams 1-c and d are more complicated, with c being very relevant to this thesis. Diagram c represents deep inelastic scattering. Since the particles aren't labeled it could represent either charged lepton scattering, 
with the fermion line on the top representing an electron or muon, and the wavy boson a photon. The round ball with 3 lines emanating from it would be a nucleon, a proton or a neutron. The photon interacts with one of the 3 constituent fermions (a quark), knocking it out of the nucleon. The elliptical line at the end indicates fragmentation, the process by which the never seen bare quarks form observable particles. In this thesis we will be working with "charged current neutrino deep inelastic scattering", which the same diagram would represent if the incoming fermion line were a neutrino, which then exchanges a $\mathrm{W}$ boson with the wavy line, converting to a muon in the outgoing fermion line. The vertex where the (virtual) W boson and neutrino/muon lines meet is called the "leptonic vertex", and where the $\mathrm{W}$ and the quarks meet is the "hadronic vertex". This diagram will be discussed in more detail in the next chapter. The final diagram in the figure decorates the floor of Willamette Hall. It represents lepton pair production in proton-antiproton scattering. The two round balls indicate a proton and antiproton, which collide and a constituent quark and antiquark annihilate into a virtual boson. The virtual boson then emits a lepton pair on the right.

One of Feynman's brothers-in-arms at the Manhattan Project was Fred Reines, who continued working in Los Alamos' nuclear program after the war. In 1949 Reines was joined by Clyde Cowan, who had received the Bronze Star for his work on radar in Britain during the war. In 1951 Reines and Cowan began working on the world's first neutrino experiment, to try and directly observe the neutrino, which they called "Project Poltergeist". Neutrinos are very difficult to detect, since they only feel the weak nuclear force. ${ }^{1}$ Either a lot of neutrinos need

\footnotetext{
${ }^{1}$ Technically, since they're now known to have mass, they also feel gravity, but since both the mass and the strength of the gravitational interaction is so small, gravity can be ignored here
} 
to pass through a detector or a detector must be very massive in order to catch one of them. Reines and Cowan constructed a detector, nicknamed "El Monstro", because at 10 cubic feet, it was by far the largest detector ever conceived at the time [14]. El Monstro consisted of a large tank filled with liquid scintillator, surrounded by 90 evenly spaced phototubes. Scintillators are materials that give out small amounts of light as charged particles pass through them, and the total amount of light given off is proportional to the energy deposited. Phototubes were used to detect the light, and hence the particles interacting inside the tank. The detector was designed to detect the reverse reaction from nuclear beta decay: According to Fermi's beta decay theory neutrons decay following the process:

$$
n \rightarrow p+e^{-}+\nu
$$

(We now know the $\nu$ in the above equation is actually $\bar{\nu}_{e}$, an antineutrino, but that was the theory at the time) The reaction Reines and Cowan were looking for (according to Fermi theory) was the reverse of this:

$$
\nu+p \rightarrow n+e^{+}
$$

El Monstro, now renamed "Herr Auge" or German for "Mr. Eye", was to detect the signal from the positron, the " $e^{+"}$, emitted in the detector. The positron would produce photons in the scintillator as it travelled through it, then annihilate with an electron, producing gamma rays. These gamma rays would then be detected by some of the phototubes lining the walls of the detector.

With the detection method established, one then needs a source of particles 
to detect. Neutrinos were originally conceived to explain beta decay, so one might think a sample of beta decaying nuclei would be a good source. Unfortunately, because the cross section for neutrino interactions is so small, an incredibly dangerous quantity of this material would be required, with radiation levels of tens to hundreds of Curies, in order for any interactions to be seen. Another good source would have been a nuclear reactor, however it would be difficult to shield the detector properly from the flux of neutrons and gamma rays.

Both Reines and Cowan knew from their experience with nuclear tests that a huge pulse of neutrinos is produced during the initial explosion, but only for a short time. This large pulse would be easily distinguishable from any background positrons, if it could be detected. The experiment they originally conceived was to take advantage of this large neutrino pulse [15]. They planned to dig a 50 meter vertical shaft about 40 meters from ground zero of a 20 kiloton nuclear bomb (figure 2). The top half of that shaft they would seal to shield from the blast, and the bottom half of the shaft would be put under vacuum. At the top of the evacuated shaft they would attach their detector, "Herr Auge". When the bomb detonated, a trigger system would release the detector, which would drop for about 2 seconds, until encountering a pile of feathers and foam rubber at the bottom of the shaft. While the detector was in free fall, everything around it would be shaking like mad by the shock wave from the nuclear blast. So in essence what they were trying to do, in less than two seconds, is detect elusive particles using a free falling, 75 gallon tank full toluene, benzene, and of all sorts of noxious chemicals, directly underneath a detonating atomic bomb. 


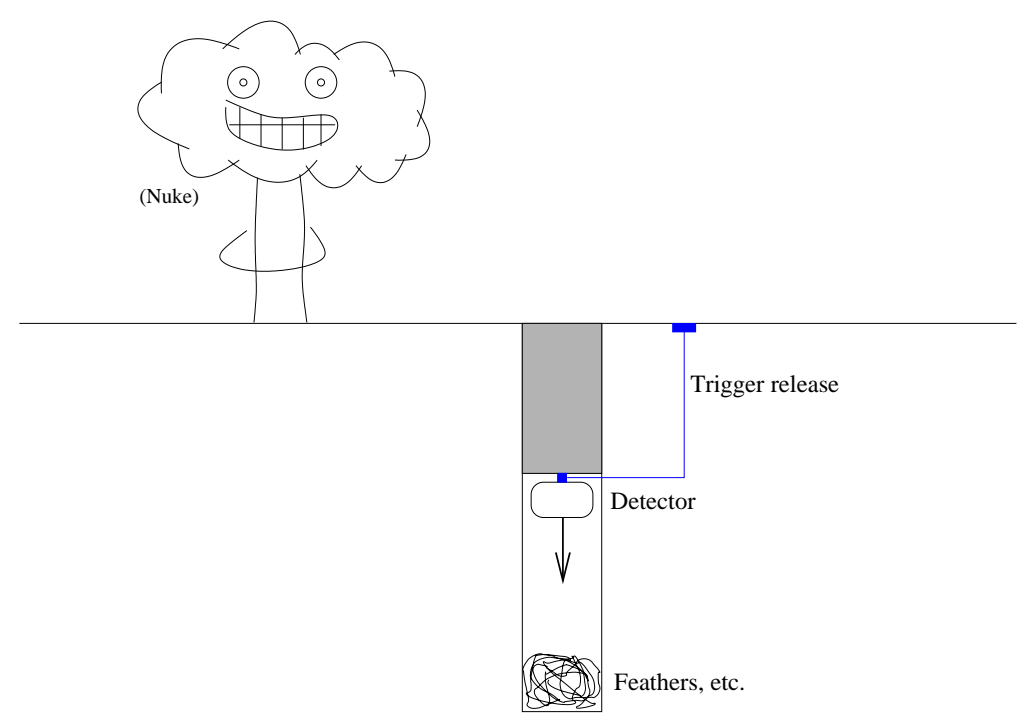

FIGURE 2. The first version of the Reines-Cowan neutrino experiment

As they began digging their shaft at the Nevada test site, and fitting phototubes into Herr Auge, the head of physics division at Los Alamos suggested that they perhaps re-think whether it was feasible to do their experiment at a nice, quiet, nuclear reactor. After some more thought, they hit upon the solution: They would look for a double pulse in their detector, one from the positron, and one from the neutron. The neutrons, being uncharged particles wouldn't leave a signal as they bounced around inside the scintillator, however if captured by an atomic nucleus, detectable gamma rays would be emitted. A very good neutron absorber is cadmium, which they dissolved into their tank to increase the probability of neutron capture. The signal from the positron and the gamma rays released in neutron capture were expected to occur a few microseconds apart, an easily distinguishable signal. With that problem behind them, the group shifted gears, the head of physics division probably breathed a sigh of relief, and Reines and Cowan reconfigured the experiment to run at one of the Hanford nuclear reactors. In 1953, 
they reported their first results [16]: The background was 5 times higher than the expected rate, but the difference in count rate between when the reactor was on and off agreed with the predicted rate for neutrinos. This was close to discovery, but confirmation was needed. After checking their shielding, re-testing their detector underground, they found that the background was in fact due to cosmic rays. They went back to Los Alamos, designed a new detector that was sensitive to the direction of the neutrinos to reduce the cosmic ray background, and confirmed the neutrino signal in a second experiment at the Savannah River plant in South Carolina[17]. After almost 30 years, the neutrino was finally a real particle. They sent Pauli a telegram right away, which was forwarded to him at a conference he was attending at CERN. He interrupted the meeting to read it to the rest of the participants, then polished off a case of champagne with some friends later that evening. Pauli's reply to the telegram was somewhat more restrained: "Thanks for the message. Everything comes to him who knows how to wait." Following their discovery, Reines and Cowan sought somewhat of an encore [18]:

Having Detected a neutrino associated with nuclear beta decay, we puzzled as to whether the neutral particle from $(\pi \mu)$ decay was the same as the neutrino from nuclear beta decay. ...Cowan and I proposed to go to an accelerator and test the identity. The reaction we got from Los Alamos was difficult to understand. The advice went something as follows "You two fellows have had enough fun. Why don't you go back to work."

In their case they were doubting that the neutral particle emitted in the transition from what was supposed to be a "strongly" interacting particle $(\pi)$ to a "weakly" interacting particle $(\mu)$ was the same as was emitted in weak nuclear beta decay. 
A more compelling case came from the absence of an observed $\mu \rightarrow e \gamma$ decay. The lack of this decay implied that neutrinos associated with muon decay were different from those in nuclear beta decay [19].

To answer the question whether those neutrinos were the same or not, Melvin Schwartz of Columbia University led an unprecedentedly large collaboration of 7 physicists in an experiment at Brookhaven's new Alternating Gradient Synchrotron (AGS) accelerator in 1962 [20]. Figure 3 shows a diagram of the experiment. 15 GeV protons were peeled from the synchrotron beam and driven into a beryllium target, producing a shower of particles containing predominantly pions and kaons. Those particles were allowed to decay into muons and (as was yet to be determined) muon neutrinos. Pions decay into a muon and muon neutrino pair $99.99 \%$ of the time, while charged Kaons do so $63.4 \%$ of the time, and into pions $21 \%$ of the time. So essentially if one lets the $\pi-K$ beam coast for a while in vacuum, you will end up with a beam containing mostly muons and muon neutrinos. At the end of the decay region shielding is placed to absorb the muons, leaving a beam of neutrinos to interact with the detector. This technique for producing a neutrino beam has been used in every accelerator based neutrino experiment since, including $\mathrm{NuTeV}$.

Their detector was the largest that had been constructed so far, a 10 ton aluminum spark chamber. A spark chamber is a series of metal planes, alternating between those that are at ground and those for which high voltage is applied when the detector is triggered. In between the plates is some kind of inert gas, like neon or argon. Scintillator counters were placed in between each block of 10 planes to detect whether a particle had passed through the spark chamber. When that happened the voltage was applied on the spark chamber, and the gas would break 


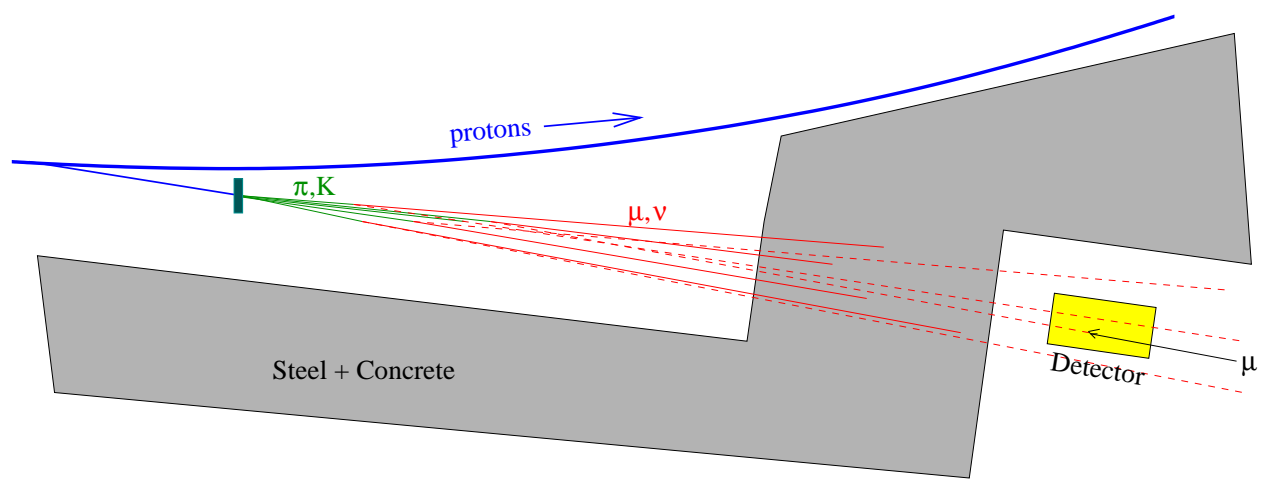

FIGURE 3. Diagram of the Columbia-Brookhaven $\nu_{\mu}$ discovery experiment

down along the particle's ionized path. The result is a series of sparks illuminating the tracks of particles in the detector. Melvin Schwartz recalled from their one page proposal [21]:

A curious item labeled "Health Physics and General Safety Requirements" mentions only that "spark chambers require 7 kilovolts to fire." That was the sum total of our concern for health physics and general safety! By the way, we certainly found the right problem. I almost got killed by a 7 kilovolt shock when Leon walked in and turned on the chamber once while I was in the back. So that was the one safety problem that we specified.

This "Leon" character was Leon Lederman, who would go on to be a Nobel Prize winner, discoverer of the bottom quark, and the second director of Fermilab.

The Columbia group rigged cameras around the detector to take photographs of any tracks produced by the neutrino beam. In those photographs they found 34 events with tracks that started in the detector, then trailed off out the back: muons. After checking that the tracks pointed back to the target (they did), that shielding the pions at the target reduced the event rate as expected (it did), and that changing the shielding near the detector didn’t affect the event rate (it didn`t), they were convinced that these muons were the result of neutrino interactions in 


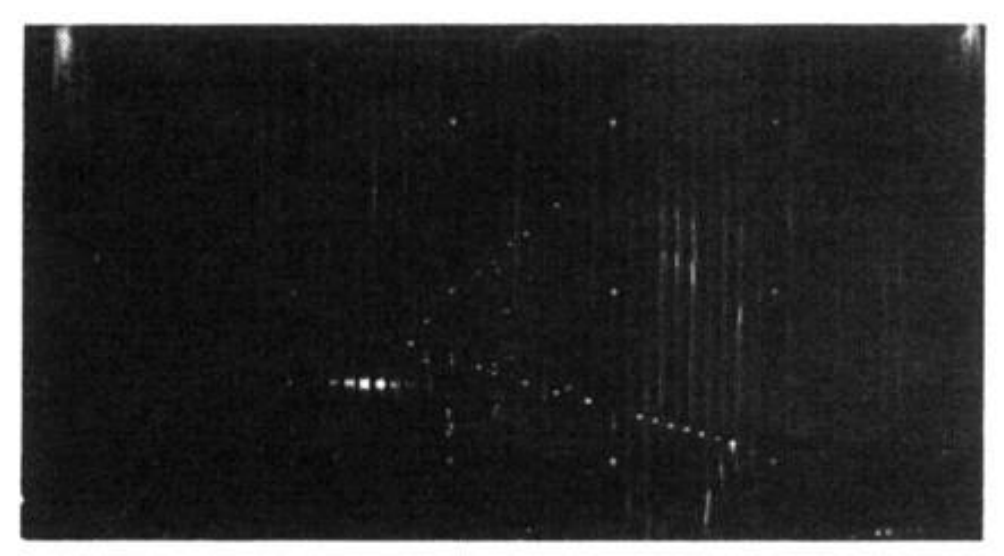

FIGURE 4. A "vertex event" from the Columbia-Brookhaven $\nu_{\mu}$ experiment. The two muon tracks form a sideways "V" opening to the right.

the detector. Those neutrinos were behaving differently from those in reactor experiments, which would produce electrons. These new neutrinos were the muon neutrino, the discovery of which completed the second family of leptons. They also saw a few events with more than one track, including an event which produced a pair of muons, shown in figure 4. In the figure, a photograph of the sparks in the spark chamber, one can just make out the spark chamber planes, with a faint " $<$ " shape. The bright smudge just to the left of the vertex of the " $<$ " is a reflection off the edges of the spark chambers.

The existence of the $\nu_{\mu}$ began to give some credence to the idea that weak interactions were not a point interaction, as supposed in Fermi theory, but were mediated by some kind of intermediate boson. This idea gained more acceptance up to the early 70's as the theoretical foundations of what was to become the Standard Model of electroweak interactions were becoming established. In 1957 Julian Schwinger took a stab at unifying the electromagnetic interaction and the weak interaction by introducing two vector bosons in addition to the photon [22]. 
This was taken further by Sheldon Glashow (and independently by Abdus Salam and J.C. Ward [23]) in 1960, who realized a neutral vector boson needed to be added to the theory [24]. But acceptance of these theories was problematic, in part because they could not explain why the weak bosons, which needed to have large masses in order for the weak interaction to maintain its observed short distance scale behavior, should be massive, while the photon was not. At least not without unleashing a flood of massless massless scalar bosons called "Goldstone Bosons" [25]. In 1967, Steven Weinberg developed a model for leptons which solved these problems [26], utilizing the "Higgs mechanism" [27] to both remove the Goldstone boson problem and explain the mass differences between the photon and weak bosons. In 1964 Peter Higgs showed that the addition of a scalar field which couples to what would otherwise be massless vector bosons, can break that symmetry, giving mass to the weak bosons, while leaving the photon massless. Weinberg's theory still had a sticky problem that had plagued its predecessors - it wasn't renormalizable. The theories had unphysical divergences in them for which, unlike Feynman's QED, there were no known fixes. In fact at that time field theory was mistrusted to such a level that physicists in the Soviet Union and Berkeley were actually forbidden to study or work on it [28].

About this time Murray Gell-Mann [29], and George Zweig [30] had independently proposed models explaining that the symmetries seen in the dozens of new particles discovered in the 50's came about because they were in fact composite particles. Zweig called the subcomponents "aces", and described them as real particles which came in pairs to construct the mesons, and "treys" for the baryons, 
like protons and neutrons. Gell-Mann however believed them to be merely theoretical constructs, which he called "quarks":

It is fun to speculate about the way quarks would behave if they were particles of finite mass (instead of purely mathematical entities as they would be in the limit of infinite mass).

A search for stable quarks of charge $-\frac{1}{3}$ or $+\frac{2}{3}$ and/or stable di-quarks of charge $-\frac{2}{3}$ or $+\frac{1}{3}$ or $+\frac{4}{3}$ at the highest energy accelerators would help to reassure us of the non-existence of real quarks.

Three quarks were all that was required to construct all the known mesons and baryons at the time: up, down and strange. Quite a few searches reminiscent of Millikan's oil drop experiment [31] were launched to try and measure fractional charges, yet no evidence was found. In fact after 40 years there is still no evidence for single free quarks. We will learn why in the next chapter.

The late 60's were a fairly dismal time for particle theory. The models that were proposed had nasty infinities in them that had to be ignored, the quarks that were predicted didn't seem to exist, and neither did the weak neutral current that was required to exist if the electromagnetic and weak interactions were to be unified. This non-observation of the weak neutral current was probably the main sticking point. There was no reason in the theory why, for example, the "neutral current" charged Kaon decay: $K^{+} \rightarrow \pi^{+}+\nu+\bar{\nu}$ should not occur. The charged current decay: $K^{+} \rightarrow \pi^{0}+\mu^{+}+\bar{\nu}$ was observed, but the neutral current decay was excluded to a level of $10^{-5}$ relative to it. Then in 1970 Glashow, Iliopoulos, and Maiani proposed a radical fix: There was a fourth quark, which they called charm [32]. Adding this new quark made two families of quark pairs ("doublets") mirror the already known pair of lepton doublets. It also added another set of diagrams, or 
pieces to the calculation, for the charged kaon decay rate which almost completely canceled out the neutral current. This gave the needed suppression to make the prediction for charged kaon decay agree with observations. So in the early 1970's then, if electroweak theory was to be believed, experimentalists were left with 2 things to hunt for: The weak neutral current, and the charm quark.

In 1971 the world's largest bubble chamber, "Gargamelle" had been constructed and began taking data at CERN [33]. A bubble chamber is somewhat like the cloud chamber used to study cosmic rays in the 1930's. Bubble chambers are essentially large tanks filled with some kind of liquid, at a temperature above its boiling point, but held at pressure so that it does not boil. When the detector is triggered, the pressure is released, and small bubbles form along the trajectories of charged particles. At the same time as the pressure is released, cameras take photographs of the tracks so that they may be "scanned" or studied to determine the physics that happened inside the detector. The Gargamelle bubble chamber was 4.8 meters long and 1.9 meters in diameter, filled with 20 tons of freon. It was based at the $25 \mathrm{GeV}$ CERN "Proton Synchrotron", producing a 1-2 GeV neutrino horn beam. To make the neutrino beam, as was the case at Brookhaven, protons struck a target, producing a secondary beam of mostly pions and kaons. The secondary beam was then focused using a horn shaped (hence "horn beam") toroidal coil placed coaxially with the beam, so that the neutrino intensity at the detector (again behind shielding to remove the muons) would be greater. Gargamelle's ten item priority list was topped of with a search for the $W^{ \pm}$bosons, then studying nucleon structure, etc. Way down towards the bottom of the list, number 8, was to look for neutral currents. 
This relative lack of interest in neutral currents changed almost immediately when Gerard 't Hooft proved that theories like Weinberg's were renormalizable [34]. With the theory now able to make finite predictions, people started paying attention. The Gargamelle group started poring through their photographs, trying to find evidence for neutral currents. Because Weinberg's paper was restricted to the case of lepton scattering, they began searching for the reaction: $\bar{\nu}_{\mu}+e^{-} \rightarrow$ $\bar{\nu}_{\mu}+e^{-}$. Charles Baltay and Donald Perkins estimated that out of 1.4 million photographs they should find between 5 and 30 of these events depending on the mixing between the $Z^{0}$ and $W$. They found 3 [35]. Neutral currents were finally observed, another of the pieces for acceptance of the Standard Model was in place.

Neutral currents could have been discovered at SLAC. In 1970, Melvin Schwartz, having recovered from his shocking discovery of $\nu_{\mu}$, was running a neutrino experiment there under the guise of trying to observe something he called "strange light":

The experiment began running in September 1970, and we saw a number of events within a few weeks. Most events were standard chargedcurrent neutrino interactions producing a muon. There were, however, three events that had no visible muon but rather one or more strongly interacting particles. I remember showing these events at the Amsterdam Conference in the summer of 1971 and having them called "melons". In retrospect, they were undoubtedly neutral-current events, but there were just too few of them to conclude anything.

Evidently were it not for the SLAC Program Advisory Committee forcing the experiment to relocate 4 times further from the target than they would have preferred, thereby reducing the sensitivity by a factor of 16, we could today be talking about measurements of the "melon width at LEP", " $\alpha_{s}$ at the melon" or advocating a "melon factory at the ILC". But alas, it was not to be. 
With the publication of Gargamelle's observations in 1973, Glashow and Iliopoulos were quite confident that the charm quark would then be found. Glashow made a wager at a conference on hadron spectroscopy [36]:

I would bet on charm's existence and discovery. There are just three possibilities:

1. Charm is not found and I eat my hat.

2. Charm is found by hadron spectroscopists, and we celebrate.

3. Charm is found by outlanders and you eat your hats.

In the summer of 1974, having already won several bottles of wine from betting on the discovery of neutral currents, Iliopoulos made a more financially risky bet to the audience at a conference in London [37]:

I am ready to bet now a whole case [of wine] that the entire next conference will be dominated by the discovery of charmed particles

That fall two groups of Glashow's outlanders were starting to see weird peaks in their data. One at SLAC, was studying electron and positron collisions at the SPEAR collider, the other from MIT was looking for electron and positron pairs from resonances produced by protons smashing into a target at Brookhaven. The SLAC-LBL group was investigating the rise in the ratio of the number of events which produced hadrons, to the number that produced muon pairs. For three quarks, this ratio was expected to be:

$$
R\left(E_{c m}\right)=3 \sum_{m_{i}<E_{c m}} Q_{i}^{2}
$$

or the sum of the squared charges of all the quarks with masses less than the center of mass squared, multiplied by the number of colors, 3. If only the up, down, 
and strange quarks existed, $\mathrm{R}$ was expected to stay flat at 2. An experiment at Cambridge that year had reported seeing it rise as high as 4 or 5 . At the same conference Iliopoulos made his "case of wine" bet (and probably one of the reasons why he was confident enough to do it), Burton Richter presented the SLAC-LBL [38] data verifying the Cambridge data, showing an apparent rise from 2 all the way to 5 . That fall while going over this rise more carefully they began seeing a peak at about 3.1 GeV. At the same time, the MIT group, led by Samuel Ting, a very precise, demanding physicist, were seeing peaks in their data. Ting had two independent groups analyzing the data who were forbidden to talk to each other until presenting results at group meetings [39]. Both groups saw their peaks at 3.1 GeV. In November of 1974, both SLAC and MIT published their results, Ting calling the new particle the "J", and the SLAC-LBL group calling it the $\psi$. The peak was a bound state of a charm and anticharm quark, now called the $J / \psi$. Two years later, at a spectroscopy conference in Mexico, candy sombreros were passed out to be consumed by the participants, as charm had been discovered by "outlanders". At least as of 1994, Iliopoulos had yet to receive his case of wine.

\subsection{The Life and Times of the Oppositely Signed Dimuon}

While the foundations of the Standard Model were being solidified with the discovery of neutral currents and charm, foundations were being poured in the corn fields 50 miles east of Chicago. Fermilab, then called The National Accelerator Laboratory, or "NAL", was built to be a central accelerator complex where users could come and perform their experiments. The labs at SLAC, Brookhaven, Berkeley, etc. had become somewhat like feudal kingdoms, where the directors 


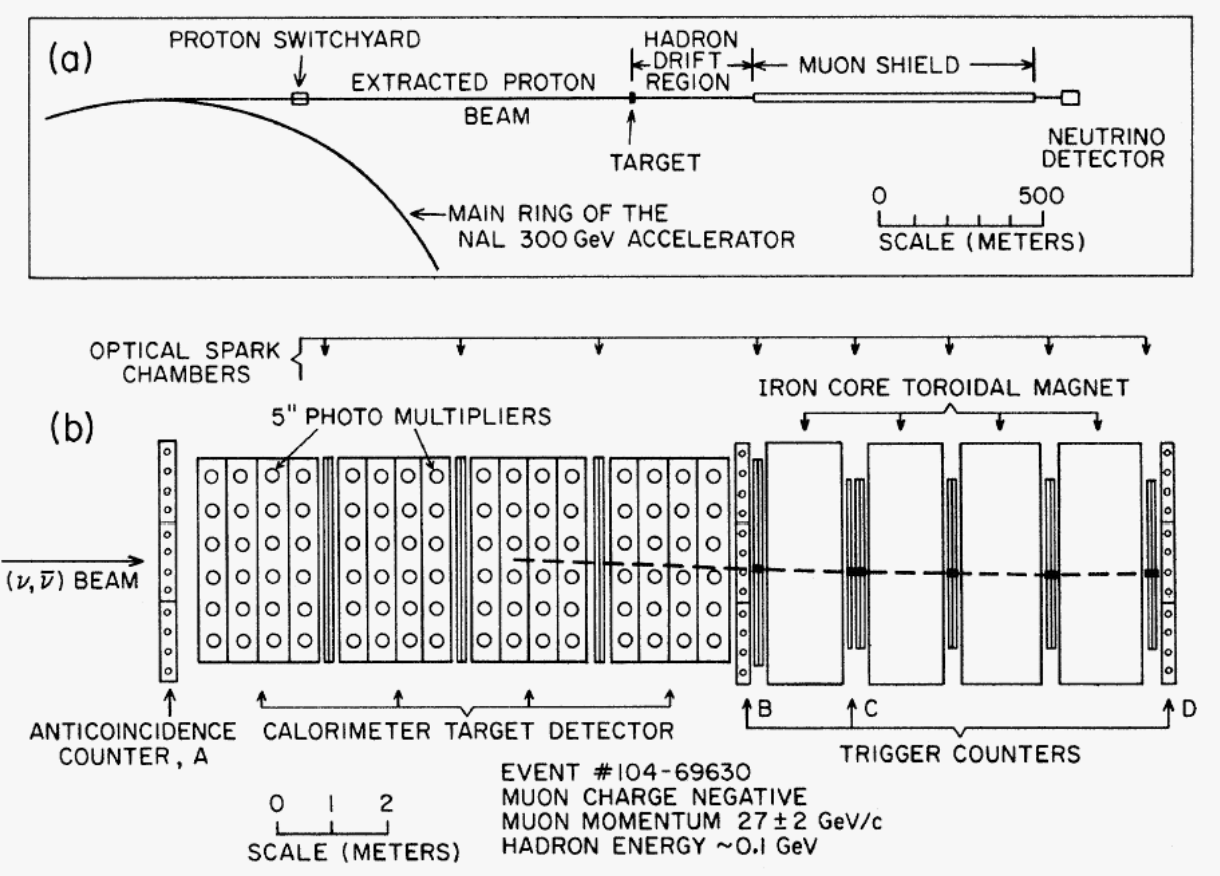

FIGURE 5. The FNAL neutrino beamline (top) and HPWF experiment (bottom)

of each accelerator lab had total control over what experiments were done. In 16th century Japan, the first Shogun required the feudal lords from each region to spend some number of months in Edo, the new capital of Japan. In that way revolts would be less likely, as some member of the clan's family was always under the watchful eyes of the shogun. In a similar way, Fermilab was to be a "user's paradise" where physicists could come and perform their experiments in any of three experimental areas, one for mesons, one for protons, and a neutrino area at the end of a mile long decay pipe and shielding berm. A 15 foot diameter bubble chamber was placed at the end of the neutrino beamline, and experimental halls placed ahead of it. FNAL-E-0001, a neutrino experiment run by Harvard, Pennsylvania, Wisconsin, and Fermilab was assembled there. 


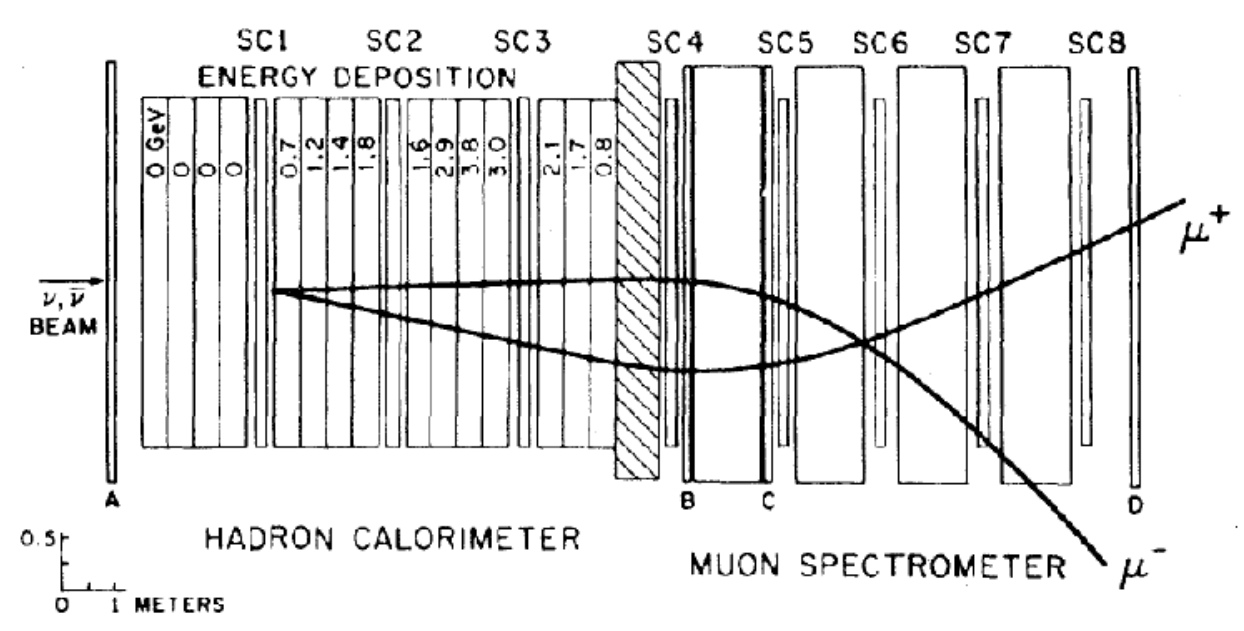

FIGURE 6. A dimuon event from the HPWF experiment

The top half of figure 5 shows the neutrino beamline [40], and the bottom shows the layout of the HPWF detector. The HPWF experiment was the first neutrino experiment to use a calorimeter as both a target and detector, followed by a highly magnetized muon spectrometer [41, 42]. The calorimeter was a stacked array of liquid scintillator detectors with 4 spark chambers in between for particle tracking. The muon spectrometer consisted of 4 magnetic coils with spark chambers sandwiched in between. The calorimeter was designed to measure the characteristics of the hadron shower, while the muon spectrometer would measure the momentum any muons produced in charged current interactions by their deflection in the magnetic field. The neutrino beam was produced in a similar way as in the Brookhaven experiment ten years earlier, though with a much higher 300 GeV proton beam energy.

Charged current events were identified by a muon in the muon spectrometer, and neutral current events were identified by a hadron shower in the calorimeter with no muon in the spectrometer. The HPWF experiment started data taking in 
early 1974. They presented some initial results at the same conference Iliopoulos bet his case of wine, including an observation of a charged current event with two muons in the final state instead of the usual one. Although not known at the time, the second muon was likely the result of the decay of a charm quark produced in the neutrino interaction [43].

After the Gargamelle discovery of the neutral current, the HPWF experiment announced a confirmation of their signal. (although there was a brief period of confusion when the signal went away due to a misconfiguration of their detector [33].) As they continued running, they eventually measured 14 more "dimuon" events. Figure 6 shows a diagram of the HPWF detector and one of the dimuon events $[44,45]$. The events were consistent with a new particle with a mass somewhere between 1 and $4 \mathrm{GeV}$. It was not clear at the time however, whether this particle was a charm quark, or some kind of neutral heavy lepton. In the intervening time between the proposal of charm quarks and the discovery of the neutral current by Gargamelle, Howard Georgi and Sheldon Glashow had come up with an alternative model for electroweak unification that involved introducing a neutral heavy lepton instead of a neutral current [46]. In order to distinguish between charm and this other proposed mechanism for creating dimuons, HPWF would have needed to be able to determine which muons came from the leptonic vertex, and which came from the hadronic vertex. The detector did not have the resolution required to do so.

A second electronic neutrino detector was built by the Caltech-Fermilab group, shown in figure 7 [47]. Like the HPWF detector it had an iron and scintillator based calorimeter, followed by a magnetized muon spectrometer. The 


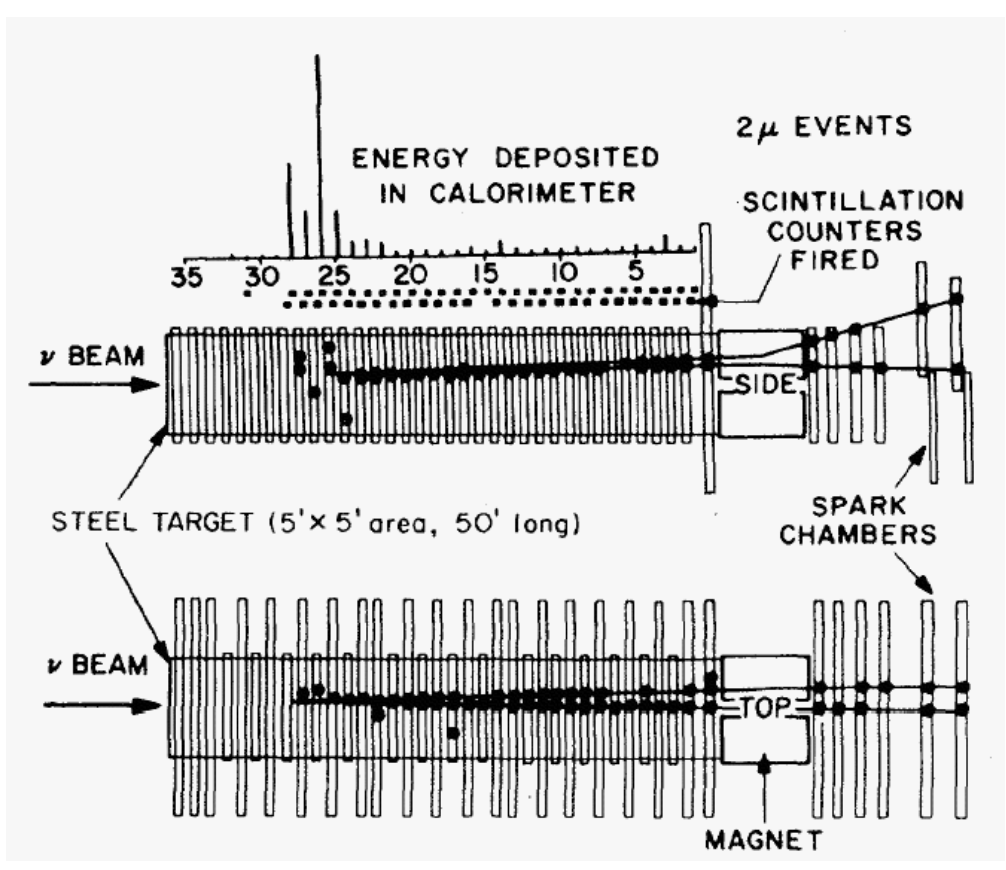

FIGURE 7. A dimuon event from the Caltech-Fermilab experiment

spectrometer was a bit different from HPWF in that the magnet and spectrometer were not interleaved, but separate elements. A toroidal magnet immediately followed the calorimeter, which was then followed by a series of spark chambers to measure the displacement of the muon's tracks by the magnetic field. The CaltechFermilab group was the first ancestor of a long line of experiments extending to the experiment discussed in this thesis, $\mathrm{NuTeV}$. One could loosely say that the lineology goes something like: Caltech-Fermilab begat CFR, begat CFNRR, begat CCFRR, begat CCFR, begat NuTeV. This dynasty of neutrino experiments took data over the course of 20 years with more or less the same detector configuration - scintillator calorimeter followed by magnetized muon spectrometer.

The Caltech-Fermilab group was able to confirm the HPWF dimuon signal with their observation of 8 dimuons in 1976. They too however were unable 
to distinguish whether these events were emanating from the leptonic vertex or the hadronic vertex. The identification of these events with charm came with a more detailed examination of the interactions at Brookhaven and Fermilab. The Brookhaven bubble chamber experiment used a 7 foot bubble chamber at the Brookhaven AGS accelerator. They recorded 100 neutrino interactions, and one was consistent with the production of a charmed baryon. The Caltech-Brookhaven group, using the Fermilab 15 foot bubble chamber, also observed 64 events in a peak centered at $1850 \mathrm{GeV}$ in the mass spectrum of neutrino events which produced a $K^{0} \pi^{+} \pi^{-}$[48]. This was perfectly consistent with both what was seen at SLAC in 1976 [49], HPWF and the Caltech-Fermilab experiments. Dimuons were produced from the production of charm quarks.

The HPWF (now HPWFOR with the addition of Ohio State and Rutgers) collaboration had substantially upgraded their detector, adding an iron target section with spark chambers for particle tracking in the calorimeter, and an 8 meter wide, high angle muon spectrometer to record the momentum of lower energy muons [50]. They also modified their beamline to sign select the pions and kaons in the secondary beam, allowing them to effectively select neutrino or antineutrino beams illuminating their detector. Using this detector, called NEULAND, they observed 199 dimuon events from neutrino scattering, and 44 dimuon events from antineutrino scattering [51]. With their wide angle spectrometer they were also able to make a first observation of 6 trimuon events - events with 3 muons in the final state. These events can be produced in several ways, most likely being the decay of a pion or kaon in the hadronic shower accompanying a dimuon, but also 

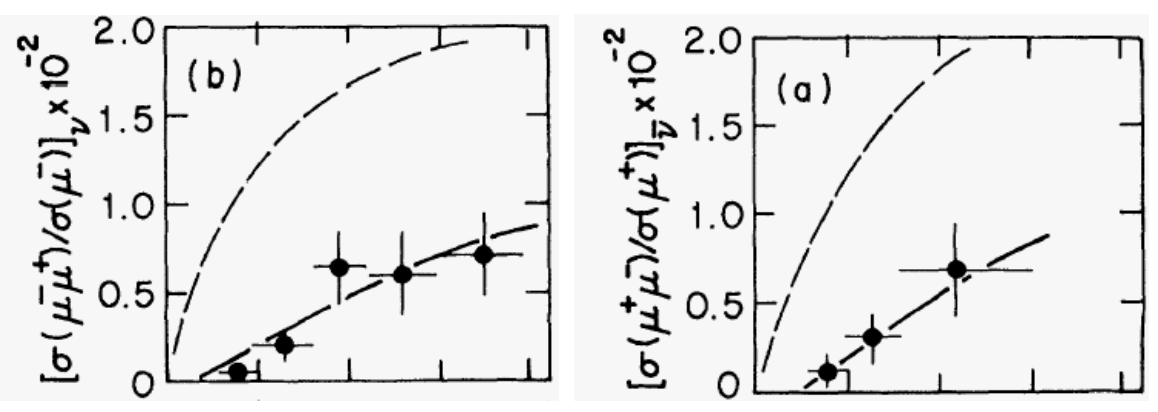

FIGURE 8. The measured ratio of dimuon events to all charged current events vs energy. Left is for the neutrino beam and right is for the antineutrino beam. The $\mathrm{x}$ axis in both cases ranges from 0 to $200 \mathrm{GeV}$

from production of a $J / \psi$, or a bottom quark. Bottom quarks were discovered by a Fermilab experiment led by Leon Lederman later that year [52].

The HPWFOR collaboration was the first to attempt a measurement of the strange quark content of nucleons. It had been observed that charmed particles preferentially decayed to strange particles. It stood to reason then that significant charm production from scattering off nucleons implied that they had a significant strange component. They looked at the struck quark fractional momentum (Bjorken $\mathrm{x}$, which will be discussed in more detail in the next chapter) as measured from dimuon production in both neutrino and antineutrino data, and found that antineutrinos had a softer momentum spectrum than neutrino data. This was consistent with the picture that the antineutrinos were scattering from the low momentum quark "sea". The ratio of the total dimuon cross section to the total charged current cross section is a good "back of the envelope" way of estimating the size of the strange sea. The HPWFOR collaboration measured these ratios for both neutrino and antineutrino generated dimuons. Their dependence on incident neutrino energy is shown in figure 8 (the $x$ axis scale ranges from 0 to $200 \mathrm{GeV}$ ). The threshold behavior due to the charm mass is clearly visible in 
how the points increase, then flatten out as a function of increasing energy. They also measured ratios of dimuon events with neutrino energy above $80 \mathrm{GeV}$ (i.e. safely above the threshold for making charm) to inclusive charged current events for a measurement of the overall size of the strange sea relative to the nonstrange quarks. The ratios are shown in table 1 . Since its now known that $90 \%$ of charm production in antineutrino mode originates from scattering off the antistrange sea, the top number is a reasonably good estimate of the overall size of the strange and antistrange seas. It is in good agreement with measurements we have today.

TABLE 1. Dimuon cross section ratios measured by the HPWFOR collaboration

\begin{tabular}{|c|c|}
\hline Ratio & Value \\
\hline $\bar{S} \equiv \frac{\sigma_{\bar{\nu}}\left(\mu^{+} \mu^{-}\right)}{\sigma_{\bar{\nu}}\left(\mu^{+}\right)}$ & $0.076 \pm 0.027$ \\
$\frac{S}{D} \equiv \frac{\sigma_{\nu}\left(\mu^{-} \mu^{+}\right)}{\sigma_{\nu}\left(\mu^{-}\right)}$ & $0.099 \pm 0.035$ \\
\hline
\end{tabular}

Dimuon production at this time was not the exclusive domain of Fermilab. A few months after the HPWF dimuon discovery results were published, Gargamelle published a charm candidate event where the charmed particle decays semileptonically with a positron [53], lending further support that charm was being generated. As the $400 \mathrm{GeV}$ CERN SPS became available in 1977, several detectors came online to take advantage of it. Gargamelle was moved from the $25 \mathrm{GeV}$ CERN PS to the new SPS, and began taking data right away. They saw 117 dimuon events, and were able to determine that there was missing energy in the charm decay, implying that a neutrino is also produced [54]. Unfortunately Gargamelle developed an irreparable leak in 1978, bringing an end to a very successful run [55]. 


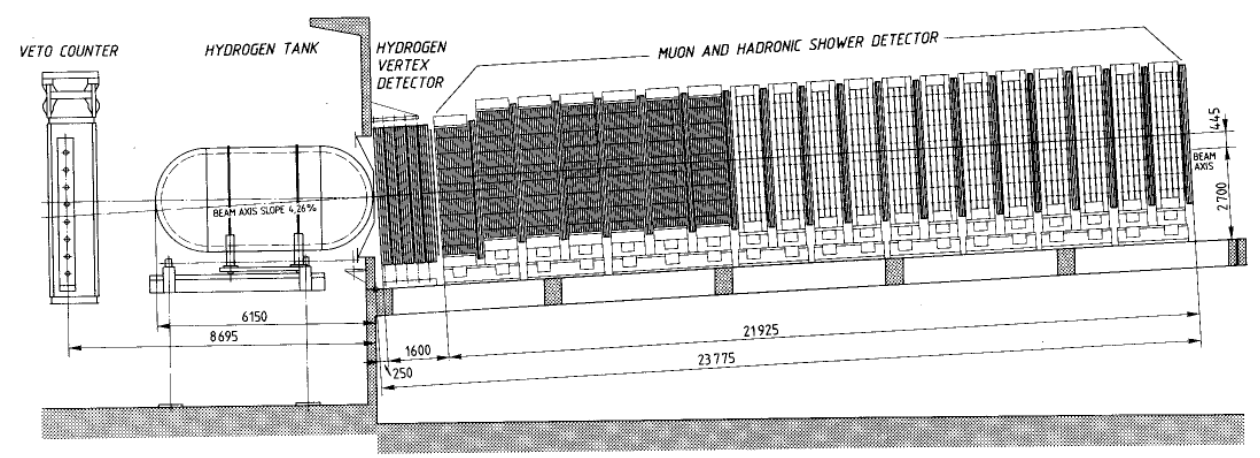

FIGURE 9. The CDHS detector

A new electronic detector also began operation at CERN in 1977 by the CERN, Dortmund, Heidelberg, Saclay (CDHS) Collaboration [56]. This detector was designed somewhat differently than the experiments at Fermilab in that there was no segregation between the muon spectrometer and the calorimeter. Magnet sections were placed throughout the length of the detector so that its acceptance for muons would be increased. Instead of spark chambers it used hexagonal drift chambers, with the wires in different planes either vertical or $\pm 60^{\circ}$ from vertical $[57]$.

A drift chamber is essentially an array of parallel wires under high voltage, placed inside a gap between a pair of separated grounded plates (figure 10). The gap separating the wires and the ground planes is filled with a gas, like argon, helium, etc. When a charged particle passes through the gas, it creates a trail of ions. Those ions are accelerated towards the nearest wires generating an electric signal. That signal is used to measure the position of the particle track in the direction perpendicular to the direction of the wires. A scintillator detector is usually used with drift chambers to provide a starting time, so that the time it takes for the ions to drift to the wires can be used to give a more precise measurement 
of the position. In the CDHS detector, (as well as others, such as the $\mathrm{NuTeV}$ detector) the scintillators then provide a dual function, the size of the pulses are proportional to the energy deposited, and the timing is used to start the clock for measuring the drift times in the drift chambers. Drift chambers are usually used in pairs to measure both dimensions of the charged particle track's position. There is an ambiguity with the simple drift chamber shown in figure 10 though: It is not always possible to tell which side of a wire the track went through. This can lead to multiple solutions when trying to reconstruct the particle's track. The ambiguity can be resolved if there are chambers placed to measure in more than two directions. One way this is done is to place chambers rotated at some angle to resolve the ambiguity. CDHS solved this problem by having hexagonally shaped chambers rotated at $60^{\circ}$ angles from each other, so that track positions could be measured along 3 axes.

The CDHS experiment ran through the early 80's recording an as yet unmatched sample of 10,381 neutrino induced dimuons, and 3,513 antineutrino induced dimuons [58]. Their neutrino energies ranged up to $300 \mathrm{GeV}$, sign selected in a limited way using a horn beam. CDHS used two methods of producing its neutrino beam. The first was a narrow band beam which momentum selected the secondary particles within a narrow range, thus producing a neutrino beam with a predictable energy depending on where the interaction took place in the detector. This had the advantage of making kinematic reconstruction easier, since the incoming momentum of the neutrino was more or less known, but the disadvantage of a much lower neutrino rate. CDHS saw only 660 neutrino and 257 antineutrino induced dimuon events with this narrow band beam. The other beam used was 


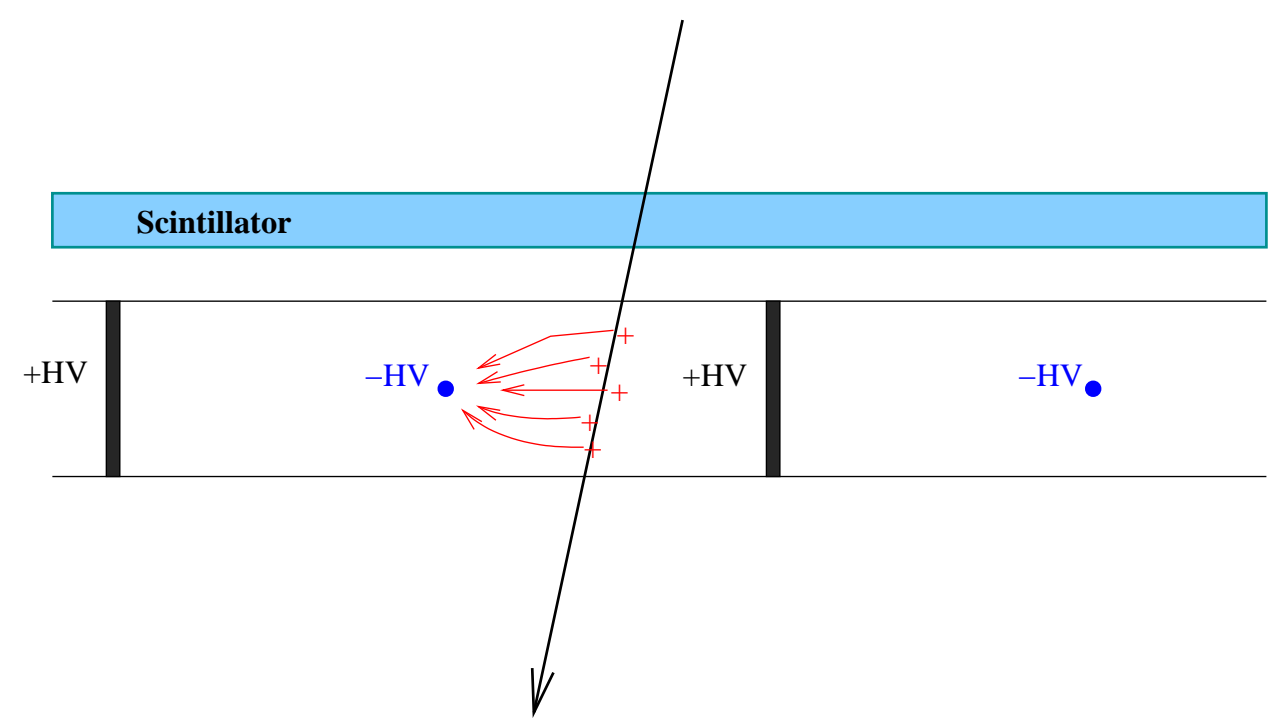

FIGURE 10. Cartoon of a drift chamber in action. A charged particle (arrow) passes through the chamber and produces ions, which are then accelerated towards the wires. The scintillator shown would be used to set the start time for measuring the ion drift time

the "wide band" beam, which used a horn to focus the secondaries toward the target. This sacrificed quality for quantity, as there was no momentum selection. Both beams were sign selected, though the wide band antineutrino beam had a very high $(>10 \%)$ neutrino contamination.

CDHS used the narrow band events and 2000 of their wide band events to debug their reconstruction software. With their large dimuon sample they were able to determine that the strange quarks carry about half (52 $\pm 9 \%$ ) of the momentum of up or down quarks in the quark sea. They were able to fit the Bjorken $x$ distribution of the antineutrino dimuons with the same shape as was determined by their total charged current sample for highly inelastic events (those events would also likely have produced charm, just not necessarily charm that decayed semileptonically). They then used the shape from that distribution 

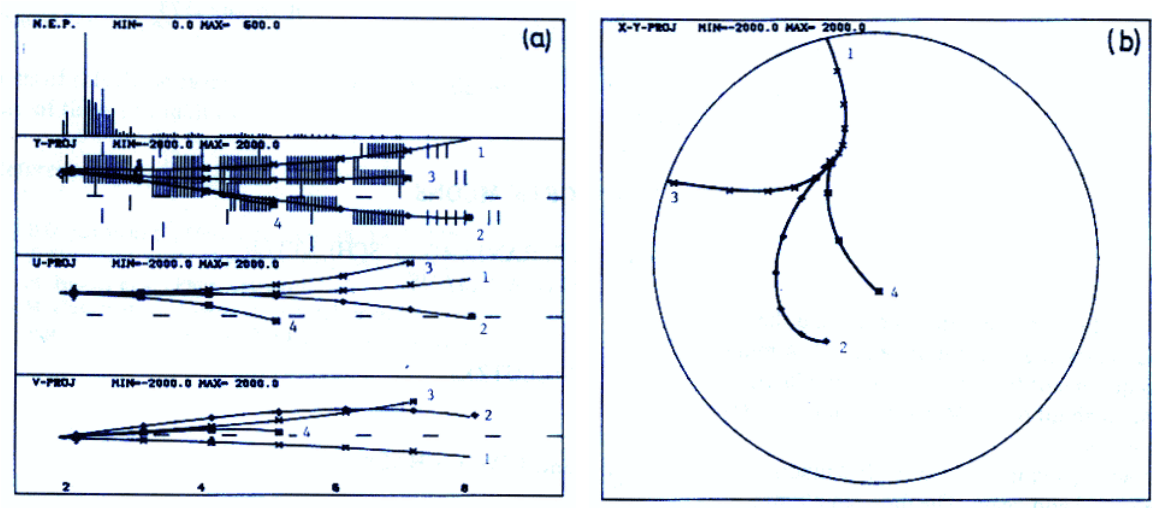

FIGURE 11. The CDHS tetramuon event, longitudinal and cross sectional views

to determine that about $48 \%$ of the events in neutrino mode came from scattering off the strange sea (they assumed the strange and antistrange seas were the same). CDHS's high statistics and good angular acceptance also allowed 76 trimuon events and a single tetramuon (4 muon) event to be recorded.

The trimuon events were explained as mostly originating from the generation of a pair of charm quarks instead of the usual single charm quark, with a less than $10 \%$ chance that they could be due to the production and decay of a bottom quark. The tetramuon event contained two positive antimuons and two negative muons [59]. A diagram of the event is shown in figure 11, looking from the sides of the detector on the left, then looking at the event from the beam axis on the right. It is interesting to see the explanations given in the original 1978 paper for what this event could be, shown in figure 12. The left half of the figure is probably somewhat optimistic given that the total energy of the event, $91.4 \pm 7.3 \mathrm{GeV}$, is about half of the now known mass of the top quark, $\sim 173 \mathrm{GeV}$. No tetramuon events have been reported in any of the neutrino experiments performed since CDHS. 

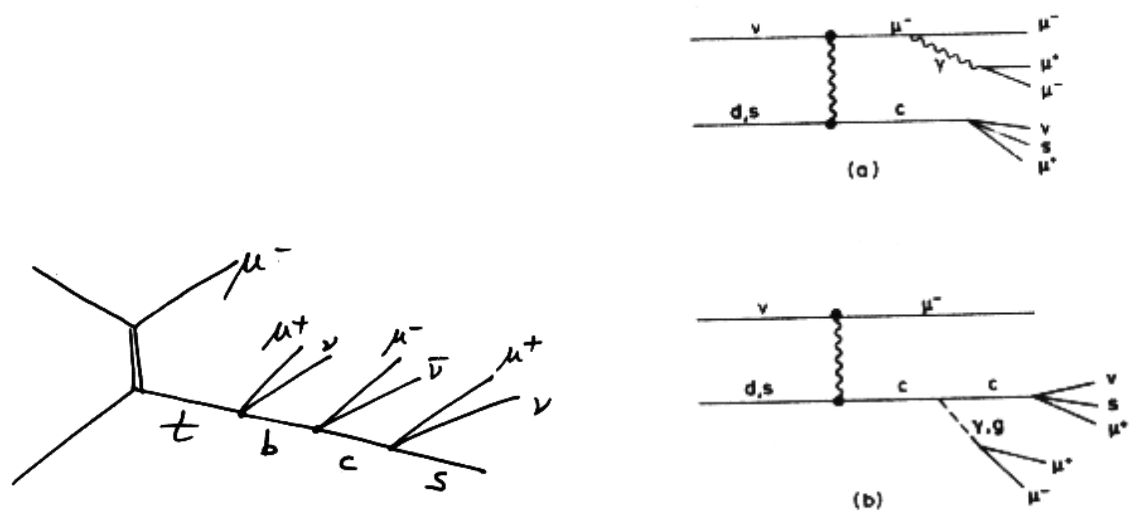

(b)

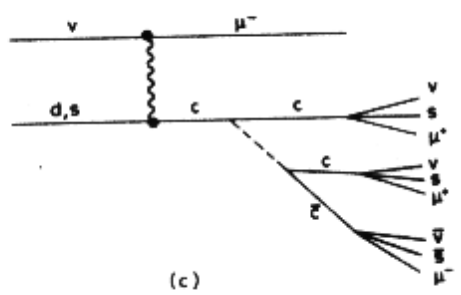

FIGURE 12. Fanciful, and more realistic explanations of the tetramuon event

Another detector took data at about the same time as CDHS, run by the CHARM collaboration (for CERN, HAmburg, Rome, Moscow, not the quark flavor). The CHARM detector was situated directly behind the CDHS detector. It had a magnetized calorimeter constructed out of 78, 2 inch thick marble slabs held in a magnetized iron frame. Unlike CDHS, CHARM had a magnetized muon spectrometer at the downstream end of its detector as well. Although CHARM's main purpose was to look for neutrino oscillations, during its run it recorded 495 $\nu$ induced dimuons, and $285 \bar{\nu}$ induced dimuons. Their properties were consistent with past measurements.

While neutrino physics at CERN took a short hiatus for the construction of the LEP $e^{+} e^{-}$collider program, the CCFR family of experiments at Fermilab 


\begin{tabular}{|ccccc|}
\hline FNAL-E-XXX & Name & Proposed & Run End & $\mu^{+} \mu^{-}$Reported \\
\hline E-021 & Caltech-Fermilab & 1970 & 1975 & 8 \\
E-356 & CFR & 1974 & 1979 & $\downarrow$ \\
E-482 & CFNRR & 1976 & 1978 & 100 \\
E-616 & CCFRR & 1979 & 1980 & $\downarrow$ \\
E-701 & CCFR & 1981 & 1982 & $852 \nu, 68 \bar{\nu}$ \\
E-744 & CCFR & 1983 & 1985 & $2159 \nu, 610 \bar{\nu}$ \\
E-770 & CCFR & 1985 & 1988 & $2568 \nu, 702 \bar{\nu}$ \\
E-815 & NuTeV & 1990 & 1997 & $5139 \nu, 1380 \bar{\nu}$ \\
\hline
\end{tabular}

TABLE 2. The CCFR neutrino experiment dynasty with dimuons reported

was quite active studying deep inelastic neutrino scattering. These experiments provided measurements of electroweak parameters, nucleon structure, and of course charm production via dimuon events. Table 2 summarizes the experiments and the number of opposite sign dimuons measured in each. The "l" in some of the dimuon entries indicates that experiment's data was combined with the later experiment.

The detector used for the bulk of these measurements is essentially the same detector used for the $\mathrm{NuTeV}$ experiment, discussed in much more detail in chapter 3. The original detector of the Caltech-Fermilab group had changed quite a bit by the E-616 CCFRR experiment. The layout for the E-616 detector is shown in figure 13 [60]. The separate magnet and muon spectrometer had by this time been replaced by the more integrated spectrometer with spark chambers in between the toroidal magnet sections. The calorimeter was a repeated sandwich of iron plates, with liquid scintillators for measuring the energy of any hadronic showers in the event, and spark chambers for locating the tracks of particles produced in the interaction. The figure shows an example of a dimuon event in the detector. Although aesthetically pretty, spark chambers are somewhat "high maintenance" 


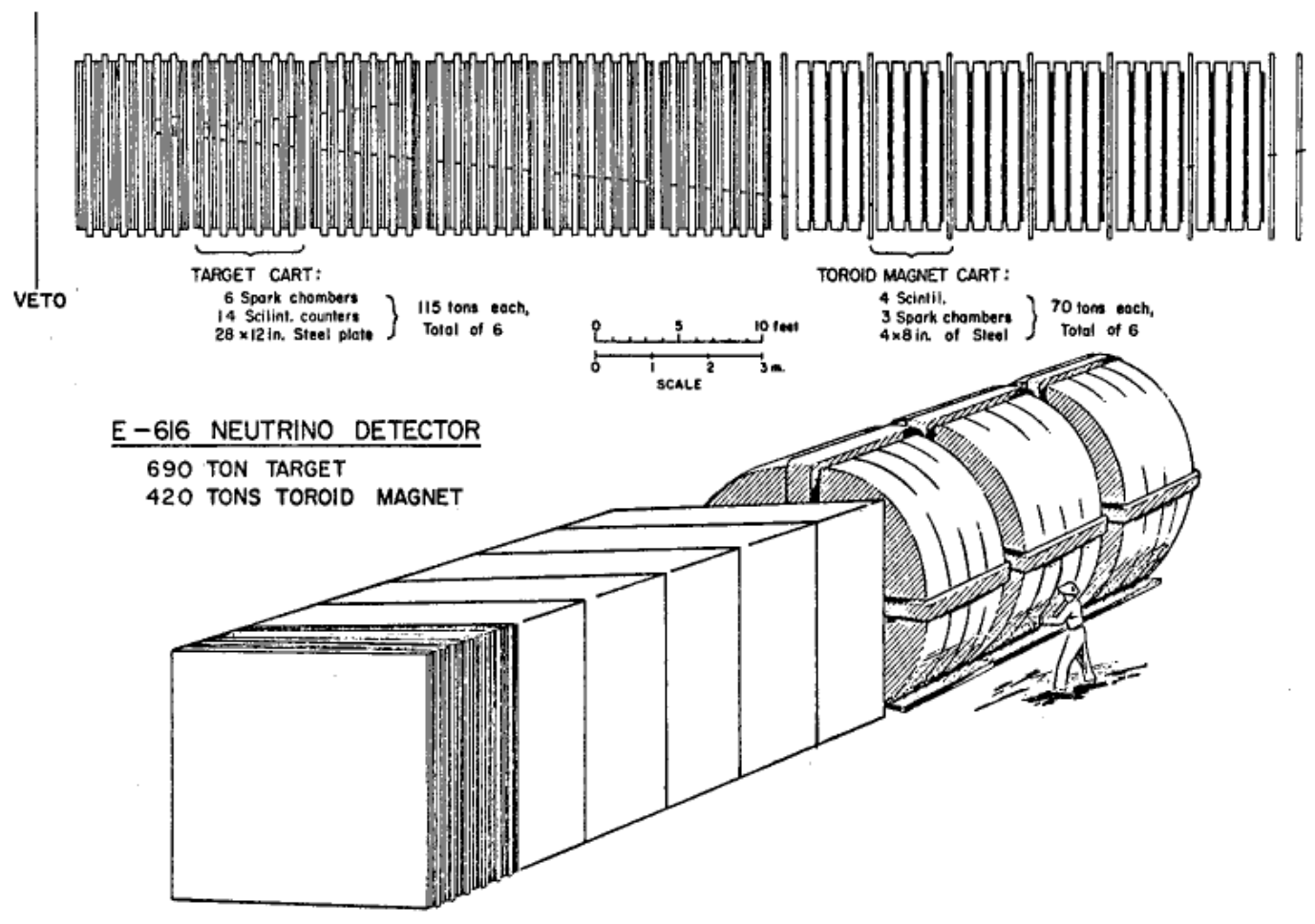

FIGURE 13. The E-616 detector, with a dimuon event faintly seen in the crosssectional view at top

way of recording the characteristics of an event. Like bubble chambers they are primarily a visual means of recording events, and reconstruction of event kinematics requires a substantial amount of human effort. Their spatial resolution is also not very good, which limits their accuracy. After the E-616 and 701 experimental runs, the CCFR group replaced their spark chambers with drift chambers, which could be digitally read out with greater precision.

In the E-744 data analysis CCFR extracted the strange and antistrange quark distributions independently for the first time [61]. Their measured $x s(x)$ and $x \bar{s}(x)$ distributions plotted as a function of Bjorken $x$ are shown in figure 14. They found that the strange and antistrange seas are consistent within errors, however the 

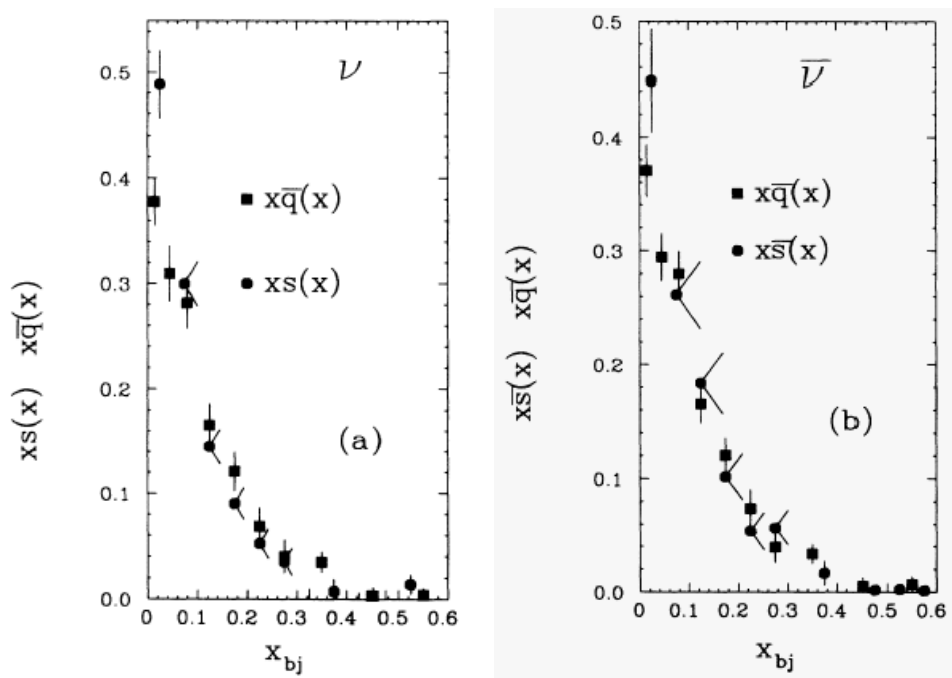

FIGURE 14. E-744 strange (left) and antistrange (right) sea extractions. Both are plotted with the total $(x \bar{q}(x))$ sea distributions, scaled to the same size for comparison.

strange sea appeared to be a touch larger at low $x$ than the antistrange sea. This was attributed to a possible deficiency in the charm mass threshold behavior in the cross section model they used.

After the continuation of data taking in the E-770 Run, two more analyses were performed on dimuon events: one based on the simple quark model that had been used in all past measurements [62], and another utilizing some new calculations that included, to first order, some of the contributions from gluons in the charm production cross section (more on that next chapter) [63]. Both measurements found that the strange and antistrange seas were consistent within errors, however the uncertainty due to the mixed neutrino and antineutrino beams makes those errors large.

CCFR was not the only game at Fermilab in terms of neutrino scattering. Another neutrino experiment, E-531, was run from 1979-1980 to try and see if $\nu_{\mu}$ 's 

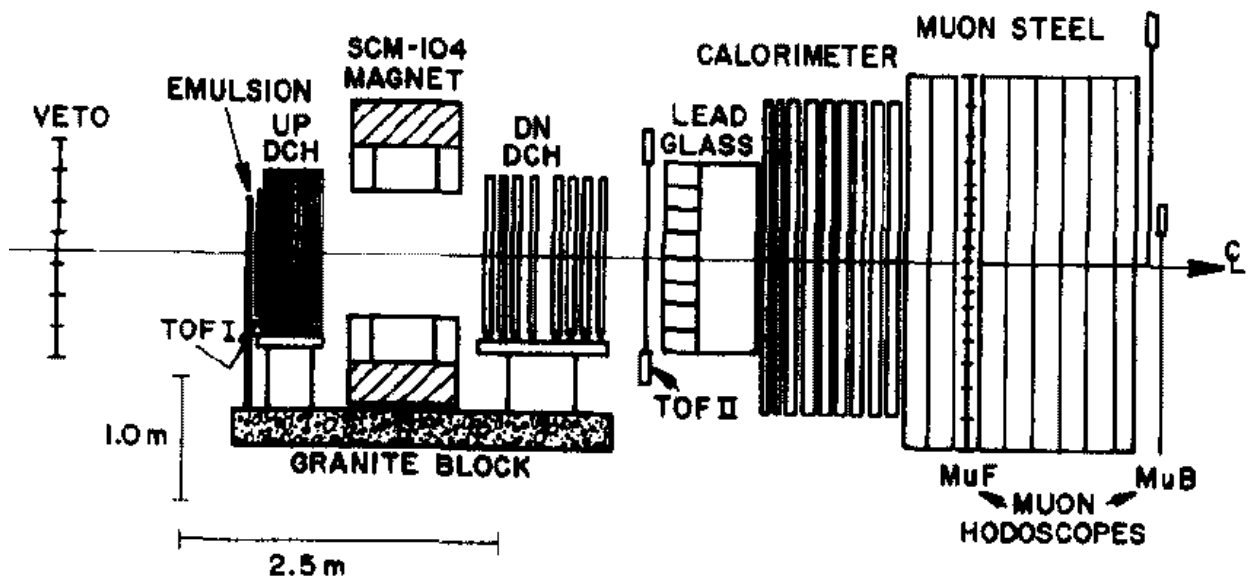

FIGURE 15. The E-531 detector

oscillate into $\nu_{\tau}$. The general idea was to put a detector in a muon neutrino beam, and watch for the production of tau's rather than muons. In order to do this a detector with very good spatial resolution was required, as any prospective tau would decay after travelling a microscopic distance in the detector. E-531 did this by placing plates of nuclear emulsion - essentially thick photographic plates, at the front of the detector as a target. When an interaction happened in the emulsion the tracks would be preserved in it, allowing measurements of the event vertex to microscopic accuracy [64]. Figure 15 shows a layout of the detector. Behind the emulsion layer they had a drift chamber based tracking detector to record when events happened in the emulsion, and provide a general estimate where to look to find the more detailed vertex from of the interaction in the emulsion itself. After the run, the nuclear emulsions were pulled out of the detector, developed and scanned, and computers matched up the drift chamber tracks with scanned dots in the emulsions. E-531 did not see any evidence of muon neutrinos changing into tau neutrinos, however they were able to provide measurements of the production and 


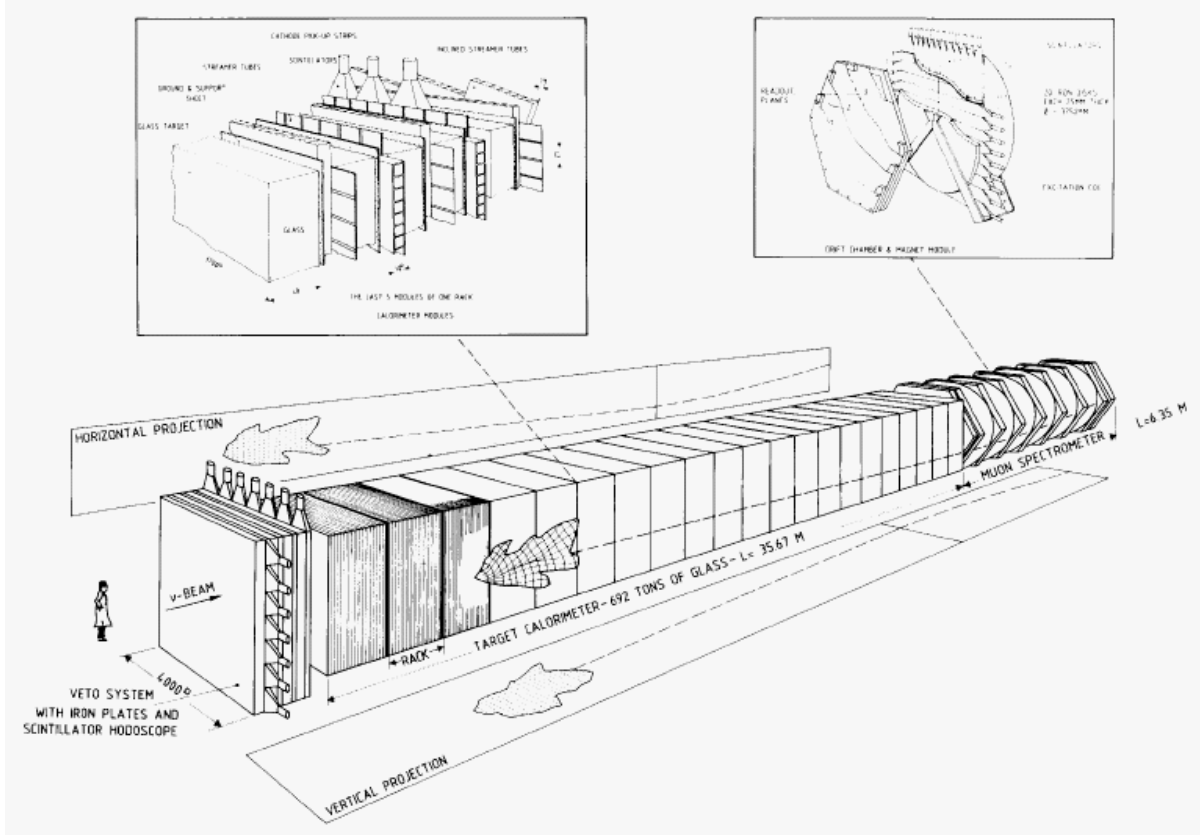

FIGURE 16. The CHARM II detector, with close-ups of the components and a charged current event superposed

decay characteristics of 122 charmed particles created in their emulsion [65]. Some of their measurements are still the only data available for neutrino production of charmed mesons.

During the late 80's and 90's three neutrino scattering experiments were executed at CERN to study electroweak and neutrino oscillation physics: CHARM II [66], NOMAD [67], and CHORUS[68]. CHARM II was designed to study electroweak interactions between muon neutrinos and electrons. To do this they employed a low density target so that they could more easily distinguish between scattering with electrons and hadrons by the shape of the resulting particle shower. The light target allowed the shower to propagate further, making it easier to use the width of the shower, narrow for electrons, and wide for hadrons, to determine whether the event was from electron or nuclear (hadron) scattering. The detec- 
tor is shown in figure 16. They used an array of 2 inch thick glass plates as a low mass target, held together in a magnetized frame like the original CHARM detector. Following each glass plate they had scintillators and an array of 352, $1 \mathrm{~cm}$ wide streamer tubes for particle tracking. The streamer tubes were sort of like small tubular spark chambers, shaped like coaxial cables with gas as an insulator. When a charged particle passed through the scintillators, a high voltage $(4.3 \mathrm{kV})$ pulse would be applied to the outside of the tube, which would create streamers, like sparks, along any tracks through the tube. The sparks would create a pulse on the inside wire that could be measured, giving a position measurement. Like drift chambers, arrays of streamer tubes measure position in one direction and must be used in (at least) pairs to get both directions. Like its predecessor CHARM, CHARM II also had a muon spectrometer following the calorimeter based on 6 toroidal magnets with drift chambers for tracking in between. CHARM II recorded $4111 \nu_{\mu}$ and $871 \bar{\nu}_{\mu}$ induced dimuon events, and measured the size and shape of the strange and (assumed equal) antistrange seas.

The CHORUS experiment, shown in figure 17, was designed to look for $\nu_{\mu} \rightarrow \nu_{\tau}$ oscillations like the Fermilab E531 experiment. Also like E531, CHORUS employed nuclear emulsions to try to resolve any tau vertices in their target. Their detector design, was similar to E531, however instead of using drift chambers to track particles emanating from the emulsion detectors, CHORUS employed scintillating fiber detectors. Scintillating fibers are just "wires" of flexible scintillator. In the CHORUS case they were pasted together into ribbons, then attached to a CCD. If a particle interacted in one of the fibers, the resulting light traveled along its length, and was converted to an electronic signal by one of the pixels in the 


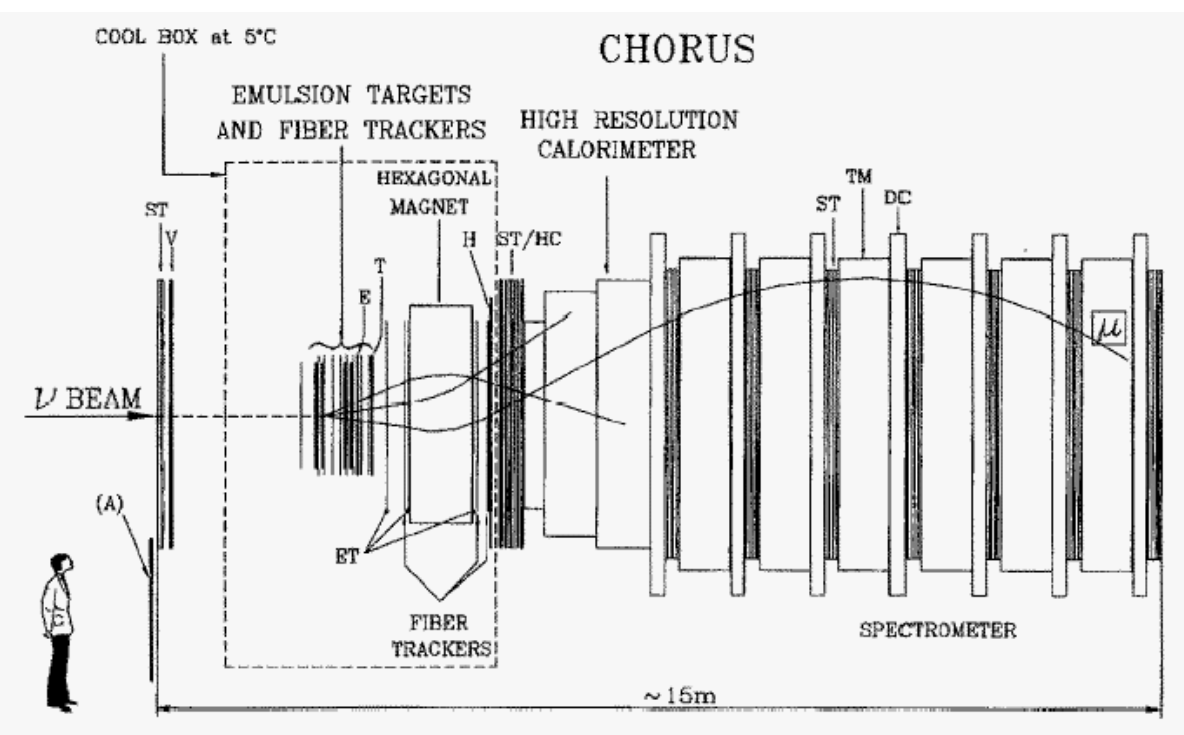

FIGURE 17. The CHORUS detector

CCD. The fibers used by the CHORUS experiment were $0.5 \mathrm{~mm}$ wide, providing very good position resolution for matching tracks up with hits in the emulsions. Following the fiber detectors was a scintillator based calorimeter, and magnetized muon spectrometer using drift chambers and streamer tube arrays for tracking. CHORUS is expected to significantly improve on the charm production and decay measurements of E531, however gargantuan task of scanning the emulsions is still underway.

The NOMAD detector ran at the same time as CHORUS, in the same neutrino beam, and was also designed for a $\nu_{\mu} \rightarrow \nu_{\tau}$ oscillation search. The detector is shown in figure 18. The target was at the very front of the detector, constructed from iron and arrays of $18.5 \mathrm{~cm}$ wide scintillator strips. The bulk of the detector was placed inside a 0.4 Tesla dipole magnet, with drift chambers forming the "active target". These were followed by an array of TRD, or transition radiation 


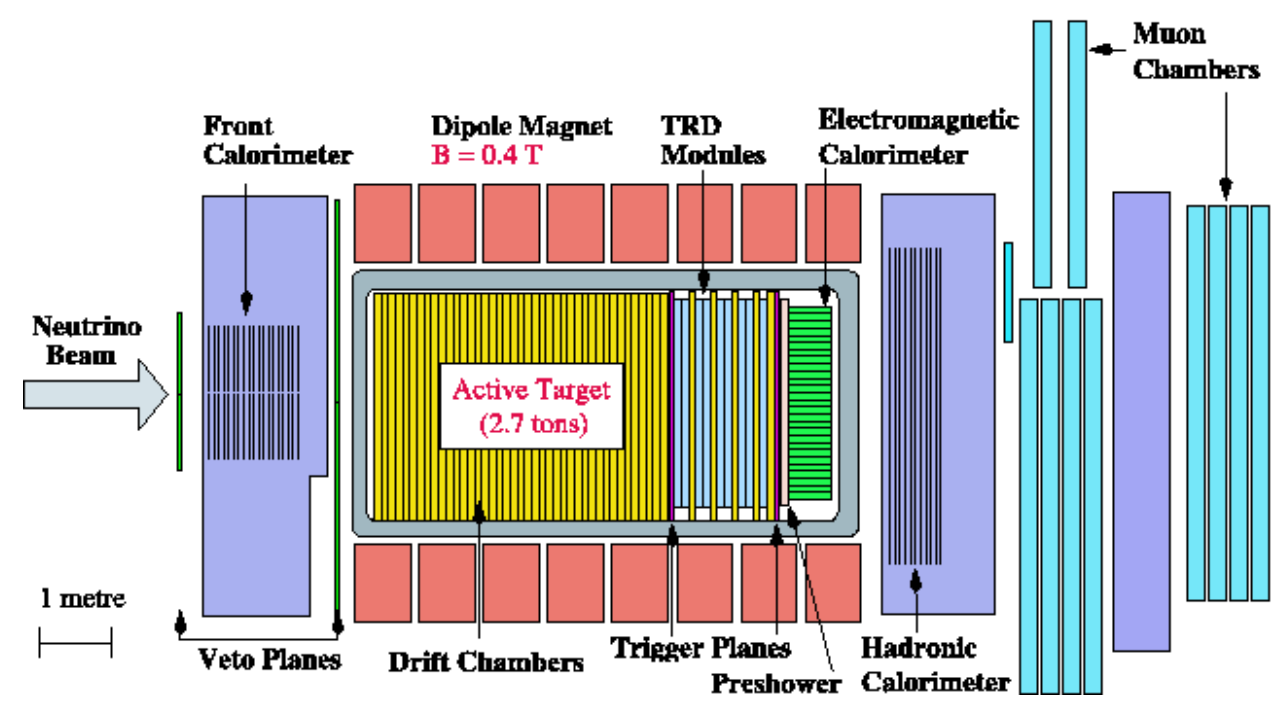

FIGURE 18. The NOMAD detector

detector modules. A transition radiation detector consists of two different materials, each with different dielectric constants. When a relativistic charged particle crosses the boundary between the two dielectrics the sudden change in the electric field causes detectable photons to be emitted. The number of photons emitted is proportional to the relativistic boost of the particle that travels across the dielectric boundary, so that TRD's coupled with another calorimeter can be used to identify the particles travelling through the detector. Following the TRD's in the NOMAD detector was a stacked array of lead glass blocks as an electromagnetic calorimeter. Lead glass is used as a Cerenkov radiation detector. When a charged particle exceeds the speed of light in a medium, it emits light that can be detected by a photomultiplier. Following the magnetized active target region is an array of iron plates and $18 \mathrm{~cm}$ wide scintillator paddles for measuring the energy of neutral hadrons. Those hadrons would interact in the high density iron, and energy 
measured in the scintillators. Following that is a toroidal magnet and set of drift chambers to measure the momentum of any muons resulting from the interaction. NOMAD (as well as CHORUS) ran with a 95\% pure neutrino beam, and measured the overall level of the strange sea with a sample of 2829 dimuon events[69]. Like many of their predecessors without a pure neutrino beam, they relied on a transverse momentum dependent algorithm to determine which muon came from the weak vertex and which from the charm decay. Using this algorithm they determined that 2714 events were from neutrino scattering, and 115 were from antineutrino scattering.

Table 1.2 summarizes neutrino experiments that have measured opposite signed dimuon events. The early measurements concentrated on establishing that opposite sign dimuons were in fact due to charm production, and gave first looks at the size of the nucleon strange component. As the intensities of neutrino beams increased, so did the number of dimuons seen in neutrino detectors. Most of the experiments listed used neutrino and antineutrino beams with relatively high levels of impurity, so that either symmetry between the strange and antistrange seas needed to be assumed, or semi-accurate algorithms based on the event kinematics needed to be used to try to determine whether a dimuon originated from a neutrino or antineutrino event. With high statistics samples of both better than $99 \%$ pure neutrino and antineutrino beams $\mathrm{NuTeV}$ has been able to use the charge of the observed muons to determine which originated from the weak vertex, and which originated from the charmed particle decay. This allows more precise determination of event kinematics. Further, a priori knowledge whether the event originated by neutrino or antineutrino scattering allows independent determination of the sizes 


\begin{tabular}{|c|c|c|c|c|c|}
\hline Experiment & Date & Dimuons & SS Beam & Strange & Ref's \\
\hline Gargamelle & $72-75$ & 117 & no & level & {$[54]$} \\
\hline HPWF & '74-'75 & 14 & no & no & {$[44,45]$} \\
\hline $\begin{array}{l}\text { Caltech- } \\
\text { Fermilab }\end{array}$ & '74-'75 & 8 & no & level & {$[47]$} \\
\hline HPWFOR & '76-'78 & $\begin{array}{c}199 \nu \\
44 \bar{\nu}\end{array}$ & $\operatorname{both}^{a}$ & level & {$[51]$} \\
\hline CFNRR & '77-'78 & 100 & no & no & {$[70]$} \\
\hline CDHS & '77-'83 & $\begin{array}{l}10381 \nu \\
3513 \bar{\nu}\end{array}$ & $\begin{array}{l}\sim 99 \% \nu \\
\sim 80 \% \bar{\nu}\end{array}$ & $\mathrm{LO}, s=\bar{s}$ & [58] \\
\hline CCFRR & '80-'82 & $\begin{array}{c}852 \nu \\
68 \bar{\nu}\end{array}$ & yes & level & {$[71]$} \\
\hline CHARM & '82-'83 & $\begin{array}{l}495 \nu \\
285 \bar{\nu}\end{array}$ & $\begin{array}{c}\sim 91 \% \nu \\
\sim 96 \% \bar{\nu}^{b}\end{array}$ & $s=\bar{s}, p_{\perp}$ & {$[72]$} \\
\hline CCFR & '85-'88 & $\begin{array}{l}5048 \nu \\
1063 \bar{\nu}\end{array}$ & no & $\begin{array}{c}\mathrm{LO}, \sim \mathrm{NLO} \\
s=\bar{s}, s \neq \bar{s}, p_{\perp}\end{array}$ & {$[61,62,63]$} \\
\hline FMMF & 1988 & 393 & no & level & {$[73]$} \\
\hline CHARM II & '86-'91 & $\begin{array}{l}4111 \nu \\
871 \bar{\nu}\end{array}$ & $\begin{array}{l}92.7 \% \nu \\
86.4 \% \bar{\nu}\end{array}$ & $\mathrm{LO}, s=\bar{s}$ & {$[74]$} \\
\hline $\mathrm{NuTeV}$ & '96-'97 & $\begin{array}{l}5139 \nu \\
1380 \bar{\nu}\end{array}$ & $\begin{array}{l}99.9 \% \nu \\
99.7 \% \bar{\nu}\end{array}$ & $\begin{array}{c}\mathrm{LO}, \mathrm{NLO} \\
s=\bar{s}, s \neq \bar{s}\end{array}$ & {$[75]$} \\
\hline CHORUS & '94-'97 & $\begin{array}{c}10718 \nu \\
420 \bar{\nu}\end{array}$ & $94 \% \nu$ & level,$s=\bar{s}$ & {$[76]$} \\
\hline NOMAD & '94-'98 & $\begin{array}{c}2714 \nu^{c} \\
115 \bar{\nu}\end{array}$ & $94 \% \nu$ & LO, level & {$[69]$} \\
\hline
\end{tabular}

TABLE 3. Summary of neutrino experiments measuring dimuon events. For each experiment, the date it took data, number of dimuons recorded, whether or not a sign selected beam was used, what strange sea measurements were performed (if any), and references to their dimuon analysis are listed. In the "Strange" column, "level" indicates only the overall level was measured, LO or NLO indicates the order in $\alpha_{s}$ of the measurement," $s=\bar{s}$ " or " $s \neq \bar{s}$ " whether or not the strange and antistrange seas were assumed equal, and " $p_{\perp}$ ", indicates that a kinematics based algorithm was used to determine the muons from the leptonic or hadronic vertices.

${ }^{a}$ HPWFOR took data with both a "Quad Triplet" mixed $\nu / \bar{\nu}$ beam, and a sign selected beam

${ }^{b} \mathrm{CHARM}$ cut $\bar{\nu}$ dimuons which had $E_{\mu-\text { charm }}>2 E_{\mu-\text { eptonic }}$ to increase purity

${ }^{c}$ Results with $\nu$ statistics around 13,600 events are expected soon [77] 
and shapes of the strange and antistrange seas, so that they need not be assumed identical. This data then provides the best opportunity so far to study whether there is a difference between the strange and antistrange seas.

\subsection{What to Expect in this Thesis}

This thesis is organized in five chapters: The first you have just read. The second attempts to provide some necessary theoretical background for understanding neutrino deep inelastic scattering, structure functions, charm production and QCD, with further details scattered throughout subsequent chapters as necessary. The following chapter covers the $\mathrm{NuTeV}$ experiment, beamline, detector and electronics, along with some of the details of data taking. The fourth chapter describes the $\mathrm{NuTeV}$ Monte Carlo software models and the extraction of the forward dimuon cross section used in the NLO analysis, discussed in detail in chapter 5. A series of appendices immediately follows, and then the bibliography. At the very end there is a blank page.. 


\section{CHAPTER II}

\section{SOME THEORY}

\subsection{The Standard Model}

"The Standard Model" is the name particle physicists have given to the theory which to date has successfully described the fundamental components of matter and their interactions with each other. In it, everything in the universe is either constructed from or interacts by means of particles. These particles come in two varieties, fermions and bosons. To be overly simplistic, one could say fermions are what everything is made of, and the bosons are what holds them together.

\begin{tabular}{|l|c|c|c|}
\hline Force & Relative Strength & Mediator & Mediator Mass \\
\hline Strong & 1 & gluon & 0 \\
Electromagnetic & $10^{-2}$ & photon & 0 \\
Weak & $10^{-5}$ & $W^{+}, W^{-}$ & $80.4 \mathrm{GeV}$ \\
Gravity & $10^{-42}$ & $Z^{0}$ & $91.2 \mathrm{GeV}$ \\
\hline
\end{tabular}

TABLE 4. The four known forces and their mediators.

Table 2.1 summarizes the four known forces in nature, and their associated bosons which mediate those forces [78]. The forces are classified by their strength at distance scales around that of an atomic nucleus. The strongest force is the aptly named "strong force" which binds together the particles that make up atomic nuclei. It is mediated by a massless particle called the gluon. The behavior of gluons will be discussed in more detail a bit later, when we talk about Quantum Chro- 
modynamics, the theory of the strong interaction. The next strongest force is the familiar electromagnetic force, mediated by the massless photon. The electromagnetic force is the union of electricity and magnetism, which in turn has been unified with the "weak force" in the Standard Model. The weak force is mediated by the exchange of either charged $W$ bosons, or the uncharged $Z^{0}$ boson. $W$ exchange is referred to as a "charged current" weak interaction, and $Z^{0}$ exchange is referred to as a "neutral current" weak interaction. All fermions interact via weak interactions, but only those with charge are able to interact via the electromagnetic interaction. The weak force is not the weakest force however, at subatomic distances gravity is by far the weakest of the four. Gravity affects all the different kinds of particles, but its affect upon them is minuscule compared to the other forces at the distances investigated in accelerator experiments.

This perhaps seems backwards. Why is it in everyday life gravity seems to be the strongest of the forces, governing even planetary and galactic motion? Why isn't gravity on the top of the list? Part of the reason is that both the electromagnetic and strong interactions are in some sense too strong for their own good, finding ways to cancel out their effects by balancing out their respective charges very quickly. A person and a chair both contain a tremendous number of electric charges, but because the charges are for the most part paired up into stable, neutral atoms, people generally do not become permanently bound to their chairs when they sit down, and end up only fighting the force of gravity when they stand back up again. By the same token the color charges in atomic nuclei also cancel each other out, with the strong interaction being so strong, that if one tries 
separating quarks from the proton or particle they are bound in, it turns out to be much easier to create particles out of the vacuum in order to cancel out the resulting bare color charges.

With the strong and electromagnetic forces more or less out of the picture, we are left with the the weak interaction and gravity. The weak interaction does not have "charges" that may cancel in the sense of the previous two forces. Being higher on the list one would then expect it to be stronger than gravity. It is, but because the bosons which mediate it have large masses the range of the weak force becomes short, constrained more or less the distance of an atomic nucleus. Adding a mass to the particle which mediates a force modifies the familiar $\frac{1}{r^{2}}$ dependence we see in the electromagnetic force with an $e^{-r / a}$ factor, where $a$ becomes smaller the larger the mass of the boson is. So that more or less leaves gravity as little guy left over after the teams have been picked.

\begin{tabular}{|c|c|c|}
\hline Lepton & Charge $(e)$ & Mass \\
\hline $\mathrm{e}$ & -1 & $0.511 \mathrm{MeV}$ \\
$\nu_{e}$ & 0 & $<3 \mathrm{eV}$ \\
\hline$\mu$ & -1 & $105.7 \mathrm{MeV}$ \\
$\nu_{\mu}$ & 0 & $<0.17 \mathrm{MeV}$ \\
\hline$\tau$ & -1 & $1777 \mathrm{MeV}$ \\
$\nu_{\tau}$ & 0 & $<18.2 \mathrm{MeV}$ \\
\hline
\end{tabular}

TABLE 5. The six leptons

The other half of the way we've divided up the universe, the fermions, come in two kinds: quarks and leptons. The quarks feel the strong interaction, the leptons do not. There are six known types of each, divided into 3 "generations". The leptons are summarized in table 5. Each of the three generations of lepton consist of a charged lepton and neutrino pair. Each pair, or "doublet", shares the 
same characteristic called "lepton number", which is conserved in all interactions. Of the charged leptons, only the electron is a stable particle. Both the muon and tau decay, with the muon lifetime around 2 microseconds, and the tau lifetime (an incredibly short) $3 \times 10^{-13}$ seconds.

The neutrinos are somewhat different beasties than their charged partners. They are uncharged leptons, so therefore only feel the weak interaction. Because of this they interact only very rarely with matter. So rarely in fact that on average, a $35 \mathrm{GeV}$ neutrino can travel through 1000 Earths before interacting [79]! Within the Standard Model the neutrinos are stable massless particles. Experimentally it is unknown whether the $\mu$ and $\tau$ neutrinos decay like their charged partners, and all direct measurements of neutrino mass to date have yielded results consistent with zero. There is now compelling evidence however that transitions between the neutrino flavors do occur[80, 81, 82], which imply that the neutrino masses, though small, are not exactly zero. These transitions are consistent with the idea that the neutrino mass eigenstates and flavor eigenstates are different, causing the flavors to mix. This mixing leads to oscillatory behavior between neutrino flavors. Depending on when you look, a neutrino produced as a $\nu_{e}$ can behave like a $\nu_{\mu}$, then again like a $\nu_{e}$ later. The extent to which this is true and the details of neutrino oscillations are currently a very active field of study, which we will make no attempt to summarize here. In this thesis we are primarily interested in neutrinos as sophisticated hammers rather than neutrinos as objects of study.

The other group of fermions are the quarks, summarized in table 6. Quarks are the particles which make up hadrons, like protons, neutrons, and pions. One can approximately say that protons are constructed from two up quarks and a 


\begin{tabular}{|c|c|c|}
\hline Quark & Charge $(e)$ & Mass \\
\hline up & $+2 / 3$ & $1.5-4 \mathrm{MeV}$ \\
\hline down & $-1 / 3$ & $4-8 \mathrm{MeV}$ \\
\hline strange & $-1 / 3$ & $80-130 \mathrm{MeV}$ \\
\hline charm & $+2 / 3$ & $1150-1350 \mathrm{MeV}$ \\
\hline bottom & $-1 / 3$ & $4100-4400 \mathrm{MeV}$ \\
\hline top & $+2 / 3$ & $173000 \mathrm{MeV}$ \\
\hline
\end{tabular}

TABLE 6. The six quarks

down quark, and a neutron is constructed from two down quarks and an up quark. Nobody has ever observed a quark by itself. Because of the nature of the strong interaction which binds them together, quarks will always be bound to other quarks. There are two kinds of hadrons that the quarks form: baryons and mesons. Baryons are bound states of 3 quarks or 3 antiquarks, and mesons are bound states of a quark-antiquark pair. Quarks are electrically charged, but also carry a "color" charge. Unlike the two charge polarities in electromagnetism, The strong charge has three different polarities, called color in analogy with the primary colors. To make a baryon, three quarks, one of each color, bind together to become "color neutral". For a meson a color and its anti-color bind together to become color neutral. Hadrons that will be referred to in this thesis and their quark makeup are summarized in table 7. Mesons and Baryons are grouped together in the top and bottom halves of the table respectively.

\subsection{Charged Current Deep Inelastic Scattering}

Neutrinos are excellent tools for illuminating the structure within protons and neutrons. As far as we know neutrinos are without structure themselves, and act like point projectiles. Since they are uncharged and do not feel the strong in- 


\begin{tabular}{|c|c|c|c|}
\hline Hadron & Mass & Mean Lifetime & Quark Makeup \\
\hline$\pi^{+}, \pi^{-}$ & $0.140 \mathrm{GeV}$ & $2.603 \times 10^{-8} \mathrm{~s}$ & $u \bar{d}, d \bar{u}$ \\
$\pi^{0}$ & $0.135 \mathrm{GeV}$ & $8.4 \times 10^{-17} \mathrm{~s}$ & $(u \bar{u}-d \bar{d}) / \sqrt{2}$ \\
$K^{+}, K^{-}$ & $0.494 \mathrm{GeV}$ & $1.234 \times 10^{-8} \mathrm{~s}$ & $u \bar{s}, \bar{u} s$ \\
$K^{0}, \bar{K}^{0}$ & $0.498 \mathrm{GeV}$ & $0.893 \times 10^{-10} \mathrm{~s}(\mathrm{short})$ & $s \bar{d}, \bar{s} d$ \\
$D^{+}, D^{-}$ & $1.869 \mathrm{GeV}$ & $1.057 \times 10^{-12} \mathrm{~s}$ & $c \bar{d}, \bar{c} d$ \\
$D^{0}, \bar{D}^{0}$ & $1.865 \mathrm{GeV}$ & $0.415 \times 10^{-12} \mathrm{~s}$ & $c \bar{u}, \bar{c} d$ \\
$D_{s}^{+}, D_{s}^{-}$ & $1.968 \mathrm{GeV}$ & $0.467 \times 10^{-12} \mathrm{~s}$ & $c \bar{s}, \bar{c} s$ \\
\hline proton & $0.938 \mathrm{GeV}$ & stable & $u u d$ \\
neutron & $0.940 \mathrm{GeV}$ & $887 \mathrm{~s}$ & $d d u$ \\
$\Lambda_{c}$ & $2.284 \mathrm{GeV}$ & $0.206 \times 10^{-12} \mathrm{~s}$ & $u d c$ \\
\hline
\end{tabular}

TABLE 7. Hadrons referred to in this thesis

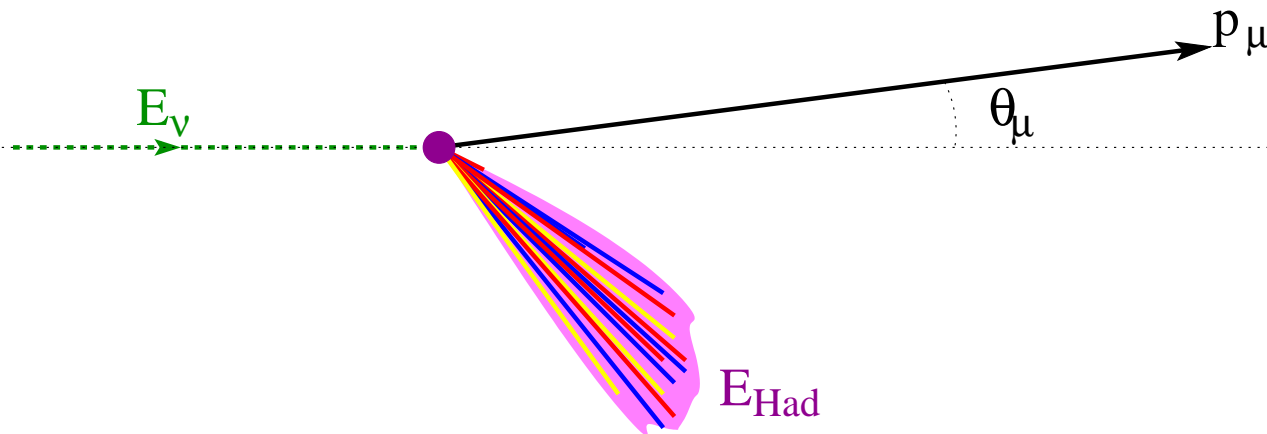

FIGURE 19. Cartoon of charged current event as observed in detector, with measured quantities shown. An unobserved neutrino with energy $E_{\nu}$ comes in from the right, and interacts with a nucleon, producing a muon with momentum $p_{\mu}$ at an angle of $\theta_{\mu}$, and a hadronic shower with energy $E_{H a d}$

teraction, they only interact weakly with the protons and neutrons in a prospective target. As we will see, this can allows the structure of the particles struck by the neutrinos to be studied in a unique way. 


\subsubsection{Charged Current Deep Inelastic Scattering Kinematics}

A charged current interaction in the $\mathrm{NuTeV}$ detector looks somewhat like the cartoon shown in figure 19. In the figure, an unobserved high energy neutrino interacts inelastically with one of the stationary protons or neutrons in the detector, breaking it apart, then converting to an outgoing muon. When a charged current event like this occurs in the $\mathrm{NuTeV}$ detector, two kinematic quantities are measured. They are the momentum vector of the outgoing muon $\left(p_{\mu}, \theta_{\mu}, \phi_{\mu}\right)$, and the energy deposited in the detector by the hadron shower $\left(E_{H A D}\right)$.

We will want to understand the cross section for these events, but before doing that we need to define some useful kinematic variables. The neutrino (or antineutrino) enters along the detector axis ( $z$ axis) carrying 4-momentum $\mathbf{k}$ and interacts inelastically with a hadron sitting at rest (4-momentum $\mathbf{p})$ in the target. 4-vectors for the initial state would be (note that we are following the particle physics convention where $c=\hbar=1$ ):

$$
\begin{aligned}
& \mathbf{k}=\left(E_{\nu}, 0,0, E_{\nu}\right) \\
& \mathbf{p}=(M, 0,0,0)
\end{aligned}
$$

Here $M$ is the mass of the struck hadron and $E_{\nu}$ is the energy of the incoming neutrino. $E_{\nu}$ ideally can be reconstructed from measured quantities:

$$
E_{\nu}=\sqrt{p_{\mu}^{2}+m_{\mu}^{2}}+E_{H A D}
$$


The square of the center of mass energy, $s$, would be:

$$
\begin{aligned}
s & =(\mathbf{k}+\mathbf{p})^{2} \\
& =2 M E_{\nu}+M^{2}
\end{aligned}
$$

We will give the outgoing muon 4 -momentum $\mathbf{k}^{\prime}$. In the lab frame, this is:

$$
\mathbf{k}^{\prime}=\left(E_{\mu}, p_{\mu} \sin \theta_{\mu} \cos \phi_{\mu}, p_{\mu} \sin \theta_{\mu} \sin \phi_{\mu}, p_{\mu} \cos \theta_{\mu}\right)
$$

The momentum transferred to the hadronic system would be $\mathbf{q}=\mathbf{k}-\mathbf{k}^{\prime}$. The momentum transfer squared, $q^{2}$, is then:

$$
\begin{aligned}
q^{2} & =\left(\mathbf{k}-\mathbf{k}^{\prime}\right)^{2} \\
& =\left(E_{\nu}-E_{\mu},-p_{\mu} \sin \theta_{\mu} \cos \phi_{\mu},-p_{\mu} \sin \theta_{\mu} \sin \phi_{\mu}, E_{\nu}-p_{\mu} \cos \theta_{\mu}\right)^{2} \\
& =2 E_{\nu}\left(p_{\mu} \cos \theta_{\mu}-E_{\mu}\right)+m_{\mu}^{2}
\end{aligned}
$$

This is often used as $Q^{2}=-q^{2}$ :

$$
Q^{2}=2 E_{\nu}\left(E_{\mu}-p_{\mu} \cos \theta_{\mu}\right)-m_{\mu}^{2}
$$

At high $E_{\nu}$, as is the case with $\mathrm{NuTeV}$, the $m_{\mu}^{2}$ term can be safely neglected. Doing so:

$$
\begin{aligned}
Q^{2} & =2 E_{\nu}\left(E_{\mu}-E_{\mu} \cos \theta_{\mu}\right) \\
& =4 E_{\nu} E_{\mu} \sin ^{2} \frac{\theta_{\mu}}{2}
\end{aligned}
$$


One could further simplify this with the small angle approximation:

$$
Q^{2} \simeq E_{\nu} E_{\mu} \theta_{\mu}^{2}
$$

The energy transferred to the hadronic system, $\nu$ is:

$$
\begin{aligned}
\nu & =\frac{\mathbf{p} \cdot \mathbf{q}}{M} \\
& =E_{\nu}-E_{\mu} \\
& =E_{H A D}
\end{aligned}
$$

The invariant mass of the hadronic system squared, $W^{2}$, is:

$$
\begin{aligned}
W^{2} & =(\mathbf{p}+\mathbf{q})^{2} \\
& =M^{2}+2 M\left(E_{\nu}-E_{\mu}\right)+q^{2} \\
& =M^{2}+2 M \nu-Q^{2}
\end{aligned}
$$

For a heavy particle, like any of the charmed mesons, to be produced in the hadronic shower, $W^{2}$ would need to be greater than the mass squared of that particle.

The inelasticity, $y$ is defined as:

$$
\begin{aligned}
y & =\frac{\mathbf{p} \cdot \mathbf{q}}{\mathbf{p} \cdot \mathbf{k}} \\
& =\frac{M \nu}{M E_{\nu}} \\
& =\frac{E_{H A D}}{E \nu}
\end{aligned}
$$




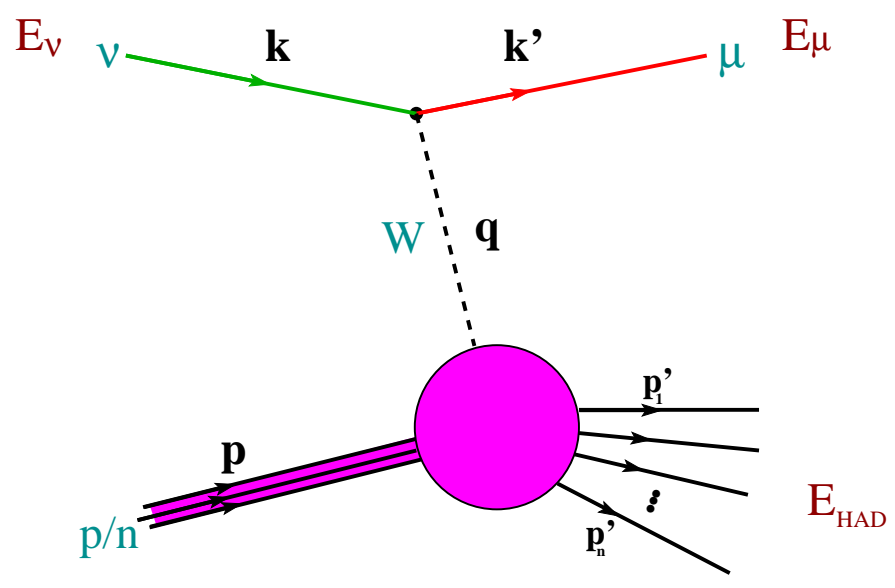

FIGURE 20. A Feynman diagram of CC DIS

An event with $y=0$ would pass no energy to the hadronic system and therefore be elastic, an event with $y=1$ would pass all the neutrino's energy to the hadronic system, so would be completely inelastic.

\subsubsection{DIS Cross Section and Structure Functions}

In this thesis we are investigating events which were the result of inelastic charged current interactions of muon neutrinos with protons and neutrons. In charged current neutrino scattering, the incoming neutrino interacts with the nucleon via a W boson (which is charged, hence "charged current"). A diagram of a charged current interaction is shown in figure 20. The incoming neutrino with four-momentum $\mathbf{k}$ exchanges a $\mathrm{W}$ with momentum $\mathbf{q}$ in interacting with the proton or neutron. This inelastic interaction breaks the nucleon into $\mathrm{n}$ particles labeled $\mathbf{p}_{\mathbf{1}}-\mathbf{p}_{\mathbf{n}}$ and, conserving charge, converts the muon neutrino into an outgoing muon with momentum $\mathbf{k}^{\prime}$. 
We want to understand the cross section of these events $[83,84,85]$. It is usual to calculate a cross section by starting with Fermi's Golden Rule:

$$
d \sigma=\frac{|\mathcal{M}|^{2}}{\Phi} d \Gamma
$$

Here $\mathcal{M}$ is the invariant scattering amplitude, $\Phi$ is the flux of the incident particles, and $d \Gamma$ is a differential slice of final state phase space per incident particle. The flux factor $\Phi$ is given by:

$$
\Phi=2 \triangle\left(s, m_{\nu}^{2}, M^{2}\right)
$$

Here the "triangle" function $\triangle(a, b, c)$ is given by:

$$
\triangle(a, b, c) \equiv \sqrt{a^{2}+b^{2}+c^{2}-2(a \cdot b+a \cdot c+b \cdot c)}
$$

In the rest frame, and following the very good approximation that $m_{\nu}$ is zero:

$$
\Phi=4 M E_{\nu}
$$

The final state phase space is the product of all possible kinematic configurations of the final state particles, namely the muon and particles in the hadron shower:

$$
d \Gamma=S \cdot \underbrace{\frac{d^{3} \mathbf{k}^{\prime}}{(2 \pi)^{3} 2 E_{\mu}}}_{\mu} \underbrace{\sum_{n} \prod_{j=1}^{n} \frac{d^{3} \mathbf{p}_{\mathbf{j}}^{\prime}}{(2 \pi)^{3} 2 E_{j}^{\prime}}}_{\text {hadron shower }} \underbrace{(2 \pi)^{4} \delta^{4}\left(\mathbf{k}+\mathbf{p}-\mathbf{k}^{\prime}-\sum_{i=1}^{n} \mathbf{p}_{\mathbf{i}}^{\prime}\right)}_{\text {momentum conservation }}
$$

Each of the components that make up $d \Gamma$ are bracketed in equation 2.30. There is a differential factor for the outgoing muon and all combinations of outgoing 
particles in the hadron shower, a delta function to enforce momentum conservation, and a statistical factor $S$. $S$ includes a combinatorial factor of $1 / j$ ! for each group of $j$ identical particles in the final state.

The last and most interesting component of the cross section is $|\mathcal{M}|^{2}$. This is the invariant scattering amplitude, and it is here where the physics of the CC interaction lies. At the most fundamental level, the interaction can be factored into a weak vertex, propagator for the $\mathrm{W}$ boson, and a hadronic vertex. The matrix element $\mathcal{M}$ is then:

$$
\mathcal{M}=\underbrace{\sqrt{2} G_{F} \bar{\mu}\left(k^{\prime}\right) \gamma^{\alpha}\left(1-\gamma^{5}\right) \nu(k)}_{E W \text { vertex }} \underbrace{\frac{1}{1+\frac{Q^{2}}{M_{W}^{2}}}}_{\text {propagator }} \underbrace{\left\langle X\left|J_{\alpha}^{W e a k}\right| p, \sigma\right\rangle}_{\text {hadronic vertex }}
$$

where $\nu$ and $\bar{\mu}$ are spinors representing the incoming neutrino and outgoing muon respectively, the $\gamma^{\alpha}\left(1-\gamma_{5}\right)$ is the $\mathrm{V}$-A weak interaction, where $\gamma^{\alpha}$ is one of the usual Dirac gamma matrices:

$$
\begin{aligned}
& \gamma^{0}=\left[\begin{array}{cc}
1 & 0 \\
0 & -1
\end{array}\right] \\
& \gamma^{i}=\left[\begin{array}{cc}
0 & \sigma^{i} \\
-\sigma^{i} & 0
\end{array}\right]
\end{aligned}
$$

where the $i$ in the $\gamma^{i}$ gamma matrices ranges from 1 to 3 , and the $\sigma^{1-3}$ are the Pauli spin matrices. $\gamma^{i}$ is the product of the four gamma matrices:

$$
\gamma^{5}=i \gamma^{0} \gamma^{1} \gamma^{2} \gamma^{3}=\left[\begin{array}{ll}
0 & 1 \\
1 & 0
\end{array}\right]
$$


The weak current, $J_{\alpha}^{\text {Weak }}$ transitions the initial nucleon state $|p, \sigma\rangle$ to the final hadronic state $\langle X|$. The superscript and subscript $\alpha$ 's indicate a sum over all four possible values, 0-3.

The cross section is proportional to the square of square of $\mathcal{M}$, which can be written:

$$
|\mathcal{M}|^{2}=\frac{2 G_{F}^{2}}{1+\frac{Q^{2}}{M_{W}^{2}}} L_{\alpha \beta} W^{\alpha \beta}
$$

where $L_{\alpha \beta} W^{\alpha \beta}$ are tensors describing the leptonic and hadronic vertices respectively. Because the neutrino and muon are point particles, the leptonic tensor is easily written down:

$$
\begin{aligned}
L_{\alpha \beta} & =\left[\bar{\nu}(k) \gamma_{\alpha}\left(1-\gamma_{5}\right) \mu\left(k^{\prime}\right)\right]\left[\bar{\mu}\left(k^{\prime}\right) \gamma_{\beta}\left(1-\gamma_{5}\right) \nu\left(k^{\prime}\right)\right] \\
& =8\left[k_{\alpha} k_{\beta}^{\prime}+k_{\beta} k_{\alpha}^{\prime}-\left(k \cdot k^{\prime}-m_{\mu}^{2}\right) g_{\alpha \beta} \mp i \epsilon_{\alpha \beta \gamma \delta} k^{\gamma} k^{\prime \delta}\right]
\end{aligned}
$$

where $\epsilon_{\alpha \beta \gamma \delta}$ is the totally antisymmetric tensor. It is +1 for $\epsilon_{0123}$ and all even permutations, -1 for all odd permutations, and zero if any of the indices are the same. The ₹ in equation 2.37 is subtraction for neutrino-nucleon scattering, addition for antineutrino-nucleon scattering.

We don't at this point want to make any assumptions about the structure of the hadronic vertex. The most general form for the hadronic tensor assuming only Lorentz invariance is:

$$
\begin{array}{r}
W^{\alpha \beta}=-g^{\alpha \beta} W_{1}+\frac{p^{\alpha} p^{\beta}}{M^{2}} W_{2}-\frac{i \epsilon^{\alpha \beta \gamma \delta} p_{\gamma} q_{\delta}}{2 M^{2}} W_{3}+\frac{q^{\alpha} q^{\beta}}{M^{2}} W_{4} \\
+\frac{p^{\alpha} q^{\beta}+p^{\beta} q^{\alpha}}{M^{2}} W_{5}+\frac{i\left(p^{\alpha} q^{\beta}-p^{\beta} q^{\alpha}\right)}{2 M^{2}} W_{6}
\end{array}
$$


Here the W's are Lorentz scalars dependent on $Q^{2}$ and $\nu$. They are called structure functions and describe the structure of the target that the neutrino or antineutrino is interacting with. Several of these structure functions can be eliminated upon evaluating $L_{\alpha \beta} W^{\alpha \beta}$ :

- The $W_{6}$ term of $L_{\alpha \beta} W^{\alpha \beta}$ becomes: $\epsilon_{\alpha \beta \gamma \delta} k^{\gamma} k^{\prime \delta} \frac{\left(p^{\alpha} q^{\beta}-p^{\beta} q^{\alpha}\right)}{2 M^{2}} W_{6}$. This will be zero, since $q=k-k^{\prime}$

- Both the $W_{4}$ and $W_{5}$ terms will be proportional to the mass of the outgoing muon, so can be safely neglected at NuTeV's energies.

This leaves $W_{1-3}$ which when contracted with $L_{\alpha \beta}$ gives the following differential cross section[86]:

$$
\begin{array}{r}
\frac{d \sigma^{\nu(\bar{\nu})}}{d Q^{2} d \nu}=\frac{G_{F}^{2}}{2 \pi M\left(1+\frac{Q^{2}}{M_{W}^{2}}\right)} \frac{E_{\mu}}{E_{\nu}}\left[W_{2}^{\nu(\bar{\nu})} \cos ^{2} \frac{\theta^{*}}{2}+2 W_{1}^{\nu(\bar{\nu})} \sin ^{2} \frac{\theta^{*}}{2}\right. \\
\left. \pm\left(\frac{E_{\nu}+E_{\mu}}{M}\right) W_{3}^{\nu(\bar{\nu})} \sin ^{2} \frac{\theta^{*}}{2}\right]
\end{array}
$$

Here $Q^{2}$ and $\nu$ are as defined in equations 2.10 and 2.18 respectively, $\theta^{*}$ is the CM muon scattering angle, $E_{\nu}$ and $E_{\mu}$ are the incoming muon-neutrino and outgoing muon energies. $G_{F}$ is the Fermi constant, the coupling constant for the weak interaction, where $G_{F} /(\hbar c)^{3}=1.166 \times 10^{5} \mathrm{GeV}^{-2}[87]$. The \pm in equation 2.39 is + for neutrino scattering, and - for antineutrino scattering. The cross section and structure functions are also different depending on whether neutrinos or antineutrinos interacted with the nucleon. This is indicated with the $\nu(\bar{\nu})$ superscripts.

In charged lepton $\left(e\right.$ or $\mu$ ) scattering, the $W_{3}$ term would disappear, as it comes about from the interference between the vector and axial vector weak cur- 
rents. Since parity is conserved in electromagnetic interactions, which dominate in deep inelastic electron and muon scattering, this term would vanish.

In 1969, Bjorken suggested that the structure functions should depend on a single "scaling variable" at very large values of $Q^{2}$ and $\nu$ [86]. This removed the explicit dependence on $Q^{2}$, which was equivalent to saying the scattering occurs from free point particles. As $Q^{2}$ increases, the boson in the interaction is able to resolve smaller and smaller distance scales. The cross section's dependence on $Q^{2}$ would indicate that substructure existed to be resolved. A free point particle, however would have the same cross section no matter how close one looked, no matter the $Q^{2}$. For free point particles then:

$$
\begin{aligned}
2 W_{1}^{\text {point }} & =\frac{Q^{2}}{2 M^{2}} \delta\left(\nu-\frac{Q^{2}}{2 M}\right) \\
W_{2}^{\text {point }} & =\delta\left(\nu-\frac{Q^{2}}{2 M}\right) \\
W_{3}^{\text {point }} & =\delta\left(\nu-\frac{Q^{2}}{2 M}\right)
\end{aligned}
$$

These can be re-arranged to:

$$
\begin{aligned}
2 M W_{1}^{\text {point }} & =\frac{Q^{2}}{2 M \nu} \delta\left(1-\frac{Q^{2}}{2 M \nu}\right) \\
\nu W_{2}^{\text {point }} & =\delta\left(1-\frac{Q^{2}}{2 M \nu}\right) \\
\nu W_{3}^{\text {point }} & =\delta\left(1-\frac{Q^{2}}{2 M \nu}\right)
\end{aligned}
$$


In scattering off Dirac point particles, the structure functions become merely functions of a single variable proportional to the ratio of $Q^{2}$ and $\nu$. This scaling variable is defined as:

$$
\begin{aligned}
x & =\frac{Q^{2}}{2 \mathbf{p} \cdot \mathbf{q}} \\
& =\frac{Q^{2}}{2 M \nu}
\end{aligned}
$$

In the limit where $Q^{2}$ and $\nu$ are large (with large being $>1 G e V$ ) the cross section, 2.39, can be re-expressed with the following substitutions:

$$
\begin{aligned}
M W_{1}^{\nu(\bar{\nu})}\left(Q^{2}, \nu\right) & \rightarrow F_{1}^{\nu(\bar{\nu})}(x) \\
\nu W_{2}^{\nu(\bar{\nu})}\left(Q^{2}, \nu\right) & \rightarrow F_{2}^{\nu(\bar{\nu})}(x) \\
\nu W_{3}^{\nu(\bar{\nu})}\left(Q^{2}, \nu\right) & \rightarrow F_{3}^{\nu(\bar{\nu})}(x) \\
\cos \theta^{*} & \rightarrow 1-2 y
\end{aligned}
$$

Giving:

$$
\begin{array}{r}
\frac{d \sigma^{\nu(\bar{\nu})}}{d x d y}=\frac{G_{F}^{2} M E_{\nu}}{\pi}\left[\left(1-y-\frac{M x y}{2 E_{\nu}}\right) F_{2}^{\nu(\bar{\nu})}(x)+\frac{y^{2}}{2}\left[2 x F_{1}^{\nu(\bar{\nu})}(x)\right]\right. \\
\left. \pm y\left(1-\frac{y}{2}\right)\left[x F_{3}^{\nu(\bar{\nu})}(x)\right]\right]
\end{array}
$$

Here for the moment we're also dropping the propagator $\left(1 /\left[1+\frac{Q^{2}}{M_{W}^{2}}\right]\right)$ term, a reasonably good approximation for fixed target experiments like $\mathrm{NuTeV}$, where $Q^{2}$ 


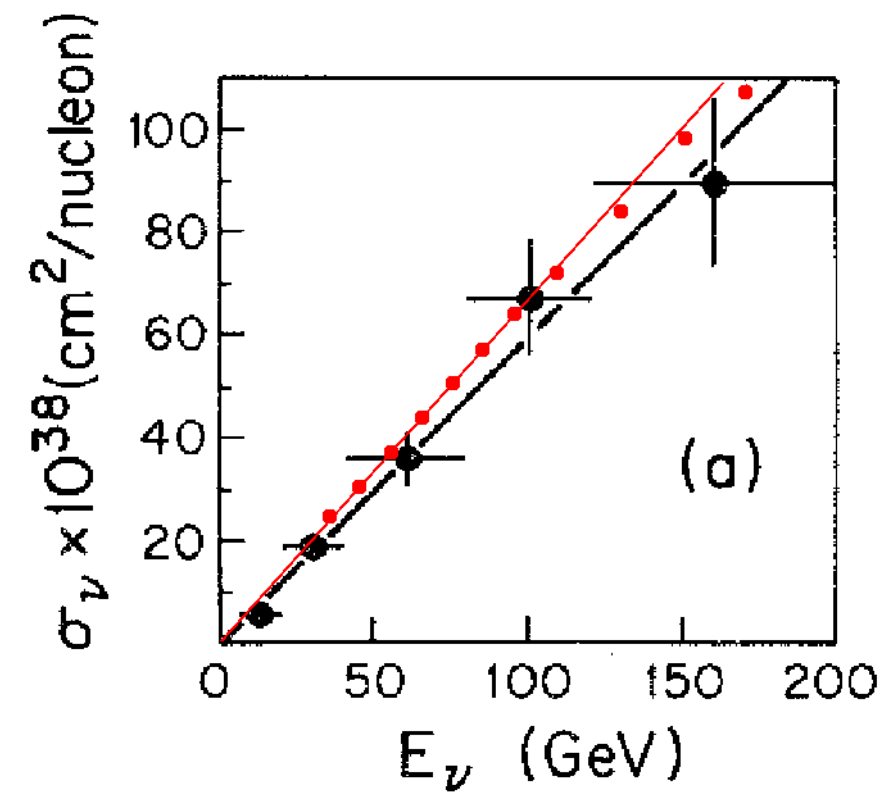

FIGURE 21. $\sigma_{C C}^{\nu}$ vs $E_{\nu}$ from FNAL-E-0001 and FNAL-E-0815. The red points and line are from E-815, superposed on top of the original FNAL-E-0001 plot. The errors on the E-815 data are smaller than the point size.

rarely tops $100 \mathrm{GeV}^{2}$, let alone $M_{W}^{2}$. We can also safely drop the $\frac{M x y}{2 E_{\nu}}$ term ahead of $F_{2}^{\nu(\bar{\nu})}(x)$. The total cross section is found by integrating over $x$ and $y$ :

$$
\begin{aligned}
\sigma= & \frac{G_{F}^{2} M E_{\nu}}{\pi} \int_{0}^{1} d x \int_{0}^{1} d y\left[(1-y) F_{2}^{\nu(\bar{\nu})}(x)+\frac{y^{2}}{2}\left[2 x F_{1}^{\nu(\bar{\nu})}(x)\right]\right. \\
& \left. \pm y\left(1-\frac{y}{2}\right)\left[x F_{3}^{\nu(\bar{\nu})}(x)\right]\right] \\
= & \frac{G_{F}^{2} M E_{\nu}}{\pi} \int_{0}^{1} d x\left[\frac{1}{2} F_{2}^{\nu(\bar{\nu})}(x)+\frac{1}{6}\left(2 x F_{1}^{\nu(\bar{\nu})}(x)\right) \pm \frac{1}{3}\left(x F_{3}^{\nu(\bar{\nu})}(x)\right)\right]
\end{aligned}
$$

This leads to a prediction: The total neutrino cross section is proportional to the energy of the incident neutrino. This was confirmed by the Gargamelle Bubble chamber at CERN in 1972[88], and FNAL Experiment 0001 in 1973[40]. A plot of FNAL E-0001's measured total neutrino cross section[89] as a function of energy 
is shown in figure 21. NuTeV's total neutrino cross section measurement is superposed on the original figure (red points, NuTeV's errors are smaller than the size of the points). Scaling is still valid after 30 years!

\subsubsection{The Quark Parton Model}

Following Bjorken's lead, e-p deep inelastic scattering experiments at SLAC looked for and found scaling behavior in the structure functions in the late 60's and early 70's [90, 91]. Upon seeing some of the first SLAC results, Richard Feynman adapted a model of hadron structure he was developing for hadronhadron interactions to the electron-proton case. In Feynman's model, a proton was made up of point like "partons", each carrying a fraction of the proton's momentum, $x[92,93,94]$. The model utilized the "infinite momentum" reference frame, where the proton was boosted to near infinite momentum relative to the incident particle. The advantage of this reference frame is that the partons are slowed down through time dilation to such an extent as to prevent interactions between them. They then can be treated as free particles, and one can begin to consider probing them without necessarily requiring detailed understanding of the forces binding them together. The infinite momentum frame becomes reasonable to use at energies greater than $1 \mathrm{GeV}$, where the scaling behavior was seen in the SLAC experiments.

Although with the confirmation of scaling it became clear that nucleons had substructure, at that time it was unclear what the nature of Feynman's partons really were. The quark model[29, 30, 95] had been in circulation for several years, and very successfully gave order to the zoo of hadrons and mesons discovered in the 
50 's and 60's, but was still viewed with some degree of skepticism. The quark model constructs a proton out of 3 spin $1 / 2$ particles called quarks, seemingly violating the Fermi exclusion principle. To avoid this problem a new quantum number, color $[96,97,98]$ was invented. In a proton, each of the 3 quarks would carry a different value of the 3 possible color quantum numbers, allowing them to bind together in the same nucleon. Only "color neutral" particles can exist, hadrons, where the 3 primary colors would cancel to become "color neutral", and mesons, where a color would match up with its "anti-color" to become color neutral. There also was (and continues to be) no experimental evidence for unbound free quarks. To explain this, the concept of quark confinement, that the binding between bound quarks actually increases one tries to them apart, was introduced. If one imagines slowly trying to pull a pair of quarks apart, the potential energy increases linearly. It eventually increases to such a level that it becomes more energetically advantageous to pull quark-antiquark pairs out of the vacuum, which then combine together with the original quarks into mesons. Leonard Susskind describes some of the early theoretical work on quark confinement in the context of quark-antiquark production in electron positron collisions[99]:

Feynman's theory of the process was very simple. He would put his hands in front of his face, almost touching, Then he would quickly draw them apart. While doing this he would make a sound something like "Brrrrrrrrrp." I interpreted this to mean that each outgoing quark would radiate a pair, which would almost keep up with their parents. Then one of the newly produced quarks would mate with its parent, producing a meson..."

Quark confinement may have explained why unbound quarks were not seen, but this seemed to contradict the scaling behavior seen experimentally. The concept of asymptotic freedom $[100,101]$ solved this problem by showing that the 
strong coupling effectively disappears at short timescales, as in the case of the infinite momentum reference frame, which allows the quarks to behave like free particles within the nucleon. The stage was then set. In 1974 the charmed $J / \psi$ meson, predicted to exist by the quark parton model, was jointly discovered by groups at Brookhaven and SLAC [102, 103]. This confirmation brought general acceptance of the quark parton model as a description of nucleon structure.

How then are partons, at this point assumed to be quarks, distributed inside the nucleon? To answer this we start by looking at elastic scattering of point spin $1 / 2$ particles. Elastic scattering of point particles depends only on the center of mass energy and the total angular momentum of the system. In the case of elastic neutrino and antineutrino scattering off spin $1 / 2$ point particles, there are two possibilities for the total angular momentum: 0, and 1. Neutrinos are always left handed, and antineutrinos are always right handed. The $\gamma^{\mu}\left(1-\gamma^{5}\right)$ operator in charged current weak interactions (i.e. in eq. 2.31) projects out left handed particles, or right handed antiparticles. This is the V-A, "Vector minus Axial vector", structure of the weak interaction, where $\gamma^{\mu}$ corresponds to a vector coupling, and $\gamma^{\mu} \gamma^{5}$ corresponds to an axial vector coupling. This means the weak interaction violates parity: If you reflect a left handed quark in a mirror, making a right handed quark, it can no longer interact via the weak interaction. This is different from the electromagnetic interaction which is a vector $\left(\gamma^{\mu}\right)$ coupling and conserves parity. The V-A structure for weak interactions gives the 4 helicity possibilities shown in figure 22 . 

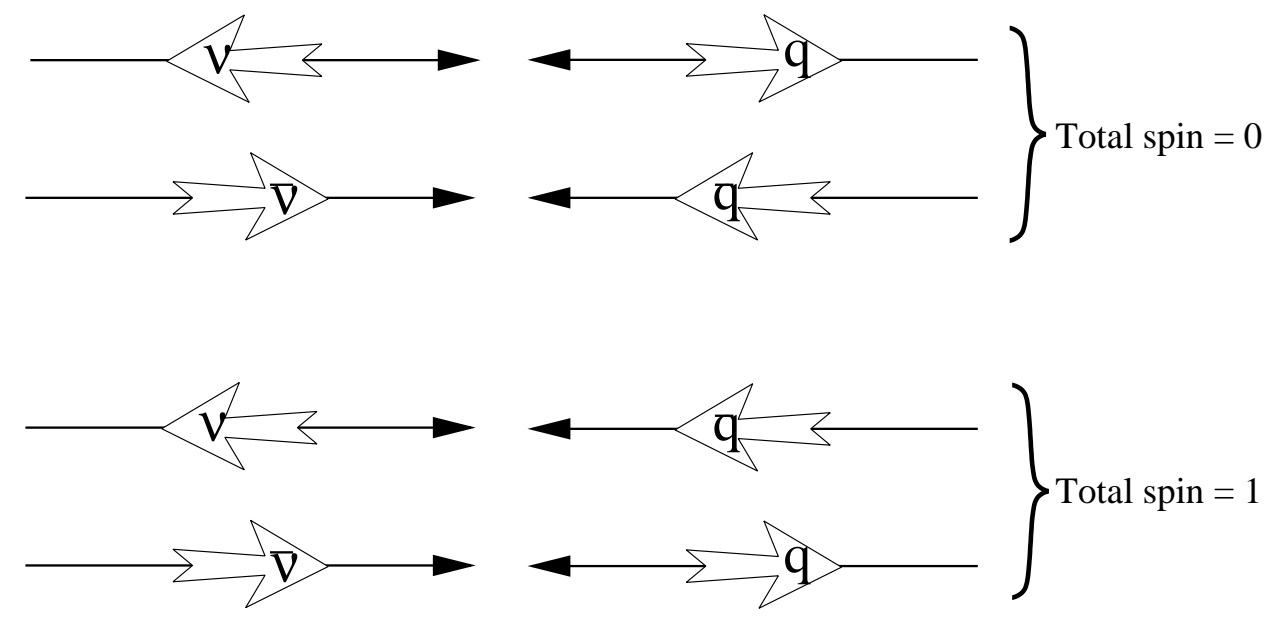

FIGURE 22. Possible helicity configurations for $\nu$ and $\bar{\nu}$ scattering

The total cross sections for each of these cases are, for total spin zero:

$$
\frac{d \sigma^{\nu q}}{d \cos \theta^{*}}=\frac{d \sigma^{\overline{\nu q}}}{d \cos \theta^{*}}=\frac{G_{F}^{2} M E}{\pi\left(1+Q^{2} / M_{W}^{2}\right)^{2}}
$$

and for total spin 1:

$$
\frac{d \sigma^{\nu \bar{q}}}{d \cos \theta^{*}}=\frac{d \sigma^{\bar{\nu} q}}{d \cos \theta^{*}}=\frac{G_{F}^{2} M E}{\pi\left(1+Q^{2} / M_{W}^{2}\right)^{2}}\left(\frac{1+\cos \theta^{*}}{2}\right)^{2}
$$

We can apply the substitution in eq. 2.51 to eq. 2.56:

$$
\frac{d \sigma^{\nu \bar{q}}}{d y}=\frac{d \sigma^{\bar{\nu} q}}{d y}=\frac{G_{F}^{2} M E}{\pi\left(1+Q^{2} / M_{W}^{2}\right)^{2}}(1-y)^{2}
$$

The above equations give the cross section for neutrino interacting with a generic spin $1 / 2$ particle, however we want to scatter off quarks. To do so we introduce the idea of a parton distribution function. A parton distribution function $q_{i}(x)$ is defined in the simple quark parton model to be the probability that a 
struck quark of flavor $i$ has the momentum fraction $x$ of a hadron's momentum. The momentum distribution of the struck quark is given by $x q_{i}(x)$. Following what we found in the generic case then, the differential cross section for neutrino quark scattering would be:

$$
\frac{d \sigma^{\nu q_{i}}}{d x d y}=\frac{G_{F}^{2} M E_{\nu}}{\pi\left(1+Q^{2} / M_{W}^{2}\right)^{2}} x q_{i}(x)
$$

Similarly for neutrino scattering off antiquarks:

$$
\frac{d \sigma^{\nu \overline{q i}}}{d x d y}=\frac{G_{F}^{2} M E_{\nu}}{\pi\left(1+Q^{2} / M_{W}^{2}\right)^{2}}(1-y)^{2} x \bar{q}_{i}(x)
$$

Making the total cross section for neutrino scattering:

$$
\frac{d \sigma^{\nu N}}{d x d y}=\frac{G_{F}^{2} M E_{\nu} x}{\pi\left(1+Q^{2} / M_{W}^{2}\right)^{2}} \sum_{i}\left[q_{i}(x)+(1-y)^{2} \bar{q}_{i}(x)\right]
$$

Here the $q_{i}$ 's would be defined as pdfs for that particular nucleon (typically a proton). Antineutrino scattering follows in an analogous way:

$$
\frac{d \sigma^{\nu N}}{d x d y}=\frac{G_{F}^{2} M E_{\nu} x}{\pi\left(1+Q^{2} / M_{W}^{2}\right)^{2}} \sum_{i}\left[(1-y)^{2} q_{i}(x)+\bar{q}_{i}(x)\right]
$$

We can use the $y$ dependence of these two expressions to determine the quark composition of the structure functions in equation 2.52. In doing so we find:

$$
\begin{aligned}
2 x F_{1}^{\nu(\bar{\nu}) N} & =2 \sum_{i}\left[x q_{i}^{\nu(\bar{\nu}) N}(x)+x \bar{q}_{i}^{\nu(\bar{\nu}) N}(x)\right] \\
F_{2}^{\nu(\bar{\nu}) N} & =2 \sum_{i}\left[x q_{i}^{\nu(\bar{\nu}) N}(x)+x \bar{q}_{i}^{\nu(\bar{\nu}) N}(x)\right] \\
x F_{3}^{\nu(\bar{\nu}) N} & =2 \sum_{i}\left[x q_{i}^{\nu(\bar{\nu}) N}(x)-x \bar{q}_{i}^{\nu(\bar{\nu}) N}(x)\right]
\end{aligned}
$$


One can immediately see in the simple quark model, where the scattering is exclusively off free spin 1/2 partons, the Callan-Gross [104] relation:

$$
2 x F_{1}=F_{2}
$$

This relation was confirmed in early experiments at SLAC[105], demonstrating that scattering was in fact occurring off spin 1/2 quarks. The Callan-Gross relation is slightly violated however: Interactions between the quarks can give them transverse momentum, which causes them to act like spin-0 particles. This violation of scaling is one of the pieces of evidence that lends support to quantum chromodynamics, the very successful theory of strong interactions.

Charged current weak interactions change the flavor of the struck quark. Charge conservation then constrains which quarks contribute to the structure functions for neutrino and antineutrino scattering. To conserve charge at the quark vertex, neutrinos may only scatter from $d, s, \bar{u}$, and $\bar{c}$ quarks, and antiquarks may only scatter from $u, c, \bar{d}$, and $\bar{s}$ quarks. The structure functions for neutrino-proton scattering therefore become:

$$
\begin{aligned}
F_{2}^{\nu p}(x) & =2 x[d(x)+s(x)+\bar{u}(x)+\bar{c}(x)] \\
x F_{3}^{\nu p}(x) & =2 x[d(x)+s(x)-\bar{u}(x)-\bar{c}(x)]
\end{aligned}
$$

For antineutrinos:

$$
\begin{aligned}
F_{2}^{\bar{\nu} p}(x) & =2 x[u(x)+c(x)+\bar{d}(x)+\bar{s}(x)] \\
x F_{3}^{\bar{\nu} p}(x) & =2 x[u(x)+c(x)-\bar{d}(x)-\bar{s}(x)]
\end{aligned}
$$


The neutron structure functions are found in an analogous way, we apply strong isospin invariance which demands:

$$
\begin{aligned}
& d^{n}(x)=u^{p}(x) \\
& u^{n}(x)=d^{p}(x) \\
& \bar{d}^{n}(x)=\bar{u}^{p}(x) \\
& \bar{u}^{n}(x)=\bar{d}^{p}(x)
\end{aligned}
$$

To find the parton content of structure functions for a nuclear target $(\mathrm{N})$ we will assume that the nucleus in question is isoscalar, that there is an equal number of protons and neutrons. This is not unreasonable for most nuclear targets, including iron, the main component of NuTeV's target. Assuming an isoscalar target, and using the symmetries in eq's (2.70-2.73) we obtain the structure functions for a nuclear target:

$$
\begin{aligned}
F_{2}^{\nu N}(x) & =x[u(x)+d(x)+\bar{u}(x)+\bar{d}(x)+2 s(x)+2 \bar{c}(x)] \\
x F_{3}^{\nu N}(x) & =x[u(x)+d(x)-\bar{u}(x)-\bar{d}(x)+2 s(x)-2 \bar{c}(x)]
\end{aligned}
$$

Here all the parton distributions above are those as defined for a proton. For antineutrino scattering:

$$
\begin{aligned}
F_{2}^{\bar{\nu} N}(x) & =x[u(x)+d(x)+\bar{u}(x)+\bar{d}(x)+2 \bar{s}(x)+2 c(x)] \\
x F_{3}^{\bar{\nu} N}(x) & =x[u(x)+d(x)-\bar{u}(x)-\bar{d}(x)-2 \bar{s}(x)+2 c(x)]
\end{aligned}
$$


Structure functions in charged lepton scattering are derived in a similar way, however as mentioned earlier $x F_{3}$ vanishes because the electromagnetic interaction, which dominates in charged lepton scattering, conserves parity. The electromagnetic interaction is also sensitive to the quark's charge rather than flavor, so the structure functions are modified by factors of the quark charges squared. $F_{2}$ for an isoscalar target in charged lepton scattering is:

$$
F_{2}^{l N}=\frac{5}{18} x[u(x)+\bar{u}(x)+d(x)+\bar{d}(x)]+\frac{1}{9} x(s(x)+\bar{s}(x))+\frac{4}{9} x(c(x)+\bar{c}(x))
$$

Here the $\frac{5}{18}$ comes about from the average between proton and neutron structure functions in assembling the nuclear structure function. In comparing the neutrino and lepton structure functions, one can find an interesting relationship:

$$
F_{2}^{l N}=\frac{5}{18} F_{2}^{\nu N}-\frac{1}{3} x s(x)+\frac{1}{3} x c(x)
$$

The strange and charm contributions to $F_{2}$ are small, so:

$$
F_{2}^{l N} \simeq \frac{5}{18} F_{2}^{\nu N}
$$

This approximate $\frac{5}{18}$ factor provides a simple, but more importantly testable, connection between charged lepton and neutrino structure function measurements. Figure 23 compares $F_{2}$ for iron at similar $Q^{2}$ values from a muon-nucleon scattering experiment performed by the EMC collaboration [106], with that from a neutrinonucleon scattering experiment performed by the CCFR collaboration [107], scaling the EMC data by $\frac{18}{5}$. The agreement is not perfect, but reasonably close. This ap- 


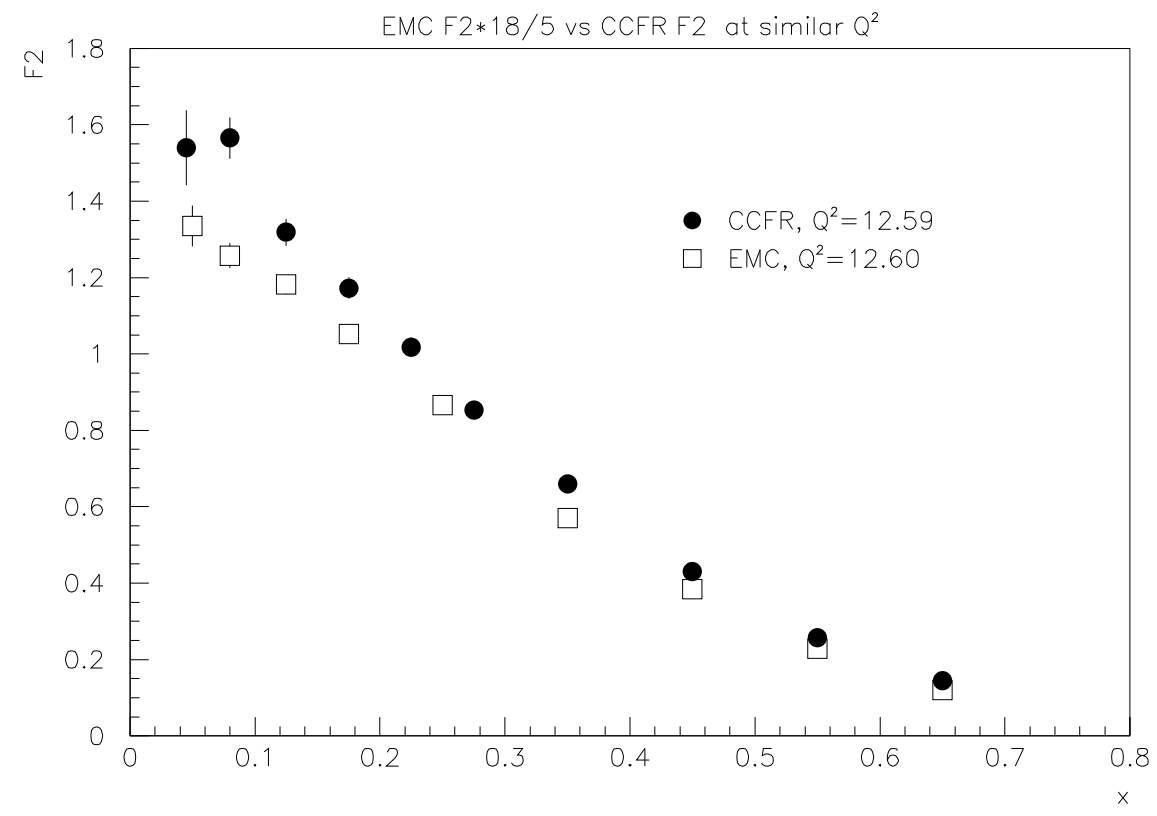

FIGURE 23. $\operatorname{CCFR}(\nu-N) F_{2}$ vs $\operatorname{EMC}(\mu-N) F_{2} \times \frac{18}{5}$ vs x

proximate $\frac{5}{18}$ relationship between charged lepton and neutrino structure functions is one of the important pieces of evidence which points to spin $1 / 2$, fractionally charged quarks as nucleon constituents.

The shape of $F_{2}$ also tells us something about the constituents of the nucleon [108]. Figure 24 shows what $F_{2}$ might look like with increasingly sophisticated models of nucleon structure. On the top is the simplest case, where the nucleon is constructed of 3 noninteracting quarks of equal mass. $F_{2}$ would then be a delta function at $x=\frac{1}{3}$. If we were to introduce some means for the quarks to interact with each other, the quarks would then be able to re-distribute momentum between themselves, which would cause this delta function to be smeared in $\mathrm{x}$ as shown in the center of figure 24. This picture still does not match the measurements shown 

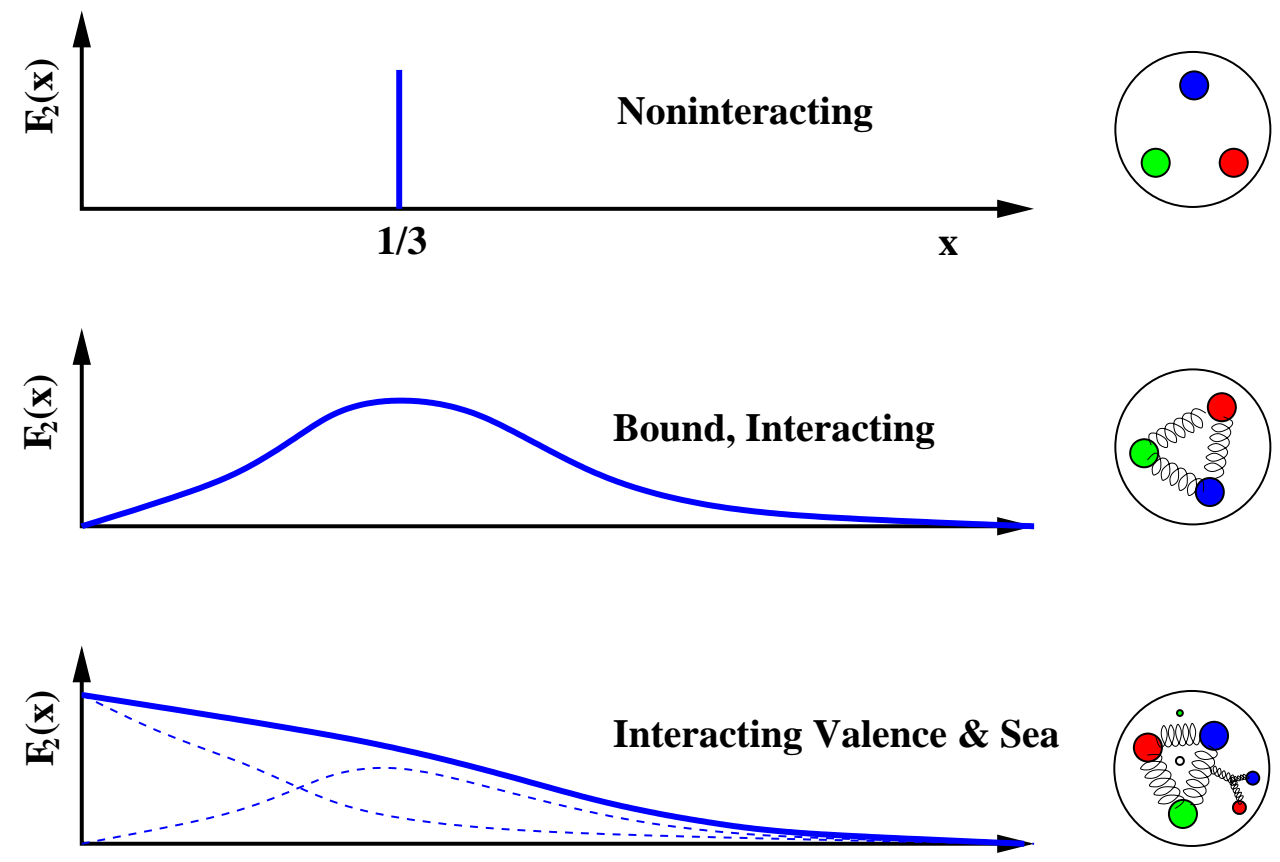

FIGURE 24. Cartoon of $F_{2}$. The expected $F_{2}$ for noninteracting (top), bound valence quarks (middle), and with sea contribution (bottom)

in figure 23 however. We must finally introduce a "sea" of low momentum quarks spontaneously generated and destroyed within the nucleon as shown on the bottom of figure 24. This implies that there are two groups of quarks in the nucleon: More or less permanent "valence" quarks, which provide the nucleon's physical characteristics like charge and spin, and a constantly fluctuating, low momentum component called the "sea". The pdfs can reformulated to reflect this:

$$
\begin{aligned}
u & =u_{v}+u_{s} \\
d & =d_{v}+d_{s} \\
u_{s} & =\bar{u}_{s} \\
d_{s} & =\bar{d}_{s}
\end{aligned}
$$


Here the " $v$ " subscript refers to the valence component, and the "s" subscript refers to the sea component. Protons and neutrons do not have net strange or charm quantum numbers, so there is no strange or charm valence distribution. Applying these to the structure functions:

$$
\begin{array}{r}
F_{2}^{\nu N}(x)=x\left[u_{v}(x)+d_{v}(x)+u_{s}(x)+d_{s}(x)+\bar{u}_{s}(x)+\bar{d}_{s}(x)\right. \\
+2 s(x)+2 \bar{c}(x)] \\
x F_{3}^{\nu N}(x)=x\left[u_{v}(x)+d_{v}(x)+u_{s}(x)+d_{s}(x)-\bar{u}_{s}(x)-\bar{d}_{s}(x)\right. \\
+2 s(x)-2 \bar{c}(x)]
\end{array}
$$

If for the moment we assume quark and antiquark seas are equal for each flavor:

$$
\begin{aligned}
F_{2}^{\nu N}(x) & =x\left(u_{v}(x)+d_{v}(x)+2\left[u_{s}(x)+d_{s}(x)+s(x)+c(x)\right]\right) \\
x F_{3}^{\nu N}(x) & =x\left[u_{v}(x)+d_{v}(x)+2 s(x)-2 c(x)\right]
\end{aligned}
$$

For antineutrinos:

$$
\begin{aligned}
F_{2}^{\bar{\nu} N}(x) & =x\left(u_{v}(x)+d_{v}(x)+2\left[u_{s}(x)+d_{s}(x)+s(x)+c(x)\right]\right) \\
x F_{3}^{\bar{\nu} N}(x) & =x\left[u_{v}(x)+d_{v}(x)-2 s(x)+2 c(x)\right]
\end{aligned}
$$

In this case:

$$
\begin{aligned}
F_{2}^{\nu N}(x) & =F_{2}^{\overline{\nu N}}(x) \\
& =x[V(x)+S(x)]
\end{aligned}
$$


Here $V(x)$ would be the total valence distribution and the $S(x)$ would be the total sea distribution. If one averages $x F_{3}^{\nu N}(x)$ and $x F_{3}^{\overline{\nu N}}(x)$ :

$$
\begin{aligned}
x F_{3}^{N}(x) & =\frac{x F_{3}^{\nu N}(x)+x F_{3}^{\bar{\nu} N}(x)}{2} \\
& =x\left[u_{v}(x)+d_{v}(x)\right] \\
& =x V(x)
\end{aligned}
$$

The averaged $F_{3}$ is the total valence quark distribution. The parton distribution functions are the probability of finding a particular quark at a momentum fraction $x$. A proton is composed of two up quarks and one down quark, so when integrating over all $\mathrm{x}$ :

$$
\begin{aligned}
& \int_{0}^{1} u_{v}(x) d x=2 \\
& \int_{0}^{1} d_{v}(x) d x=1
\end{aligned}
$$

The integral of $F_{3}$ should then be:

$$
\int_{0}^{1} F_{3}^{N}(x) d x=3
$$

This is called the Gross Llewellyn-Smith[109] (GLS) sum rule. The GLS sum rule can be determined from measured structure functions, and the values obtained are close but not quite 3. The current world average for the GLS sum rule is $2.64 \pm 0.06$ [85]. It is now known that there are corrections dependent on the strong coupling $\alpha_{s}$ which pull the value obtained by the quark parton model down by approximately 0.34 [94]. These corrections make determining the GLS sum rule 
a useful tool in measuring $\alpha_{s} . F_{3}(x)$ is measured, parameterized, and integrated, and the required QCD corrections are used to determine the value of $\alpha_{s}[110]$. We will discuss $\alpha_{s}$ further in the next section.

The difference in $F_{2}$ between proton and neutron scattering provides another useful, measurable, sum rule. Following the example of equation 2.66 for the structure function of the proton and using isospin symmetry to convert it for the neutron:

$$
F_{2}^{\nu n}(x)=2 x[u(x)+s(x)+\bar{d}(x)+\bar{c}(x)]
$$

Subtracting the two and applying equations 2.83 and 2.84:

$$
\begin{aligned}
F_{2}^{\nu n}(x)-F_{2}^{\nu p}(x) & =2 x[u(x)+\bar{d}(x)-d(x)-\bar{u}(x)] \\
& =2 x\left[u_{v}(x)-d_{v}(x)\right]
\end{aligned}
$$

Integrating this and dividing by $2 x$ :

$$
\int_{0}^{1} \frac{F_{2}^{\nu n}-F_{2}^{\nu p}}{2 x} d x=1
$$

This is called the Adler sum rule [111], and has been measured in neutrino scattering in hydrogen for $F_{2}^{\nu p}$ and deuterium for $F_{2}^{\nu n}$ to obtain a value of $1.01 \pm 0.20$ $[112]$.

An analogous sum rule for charged lepton scattering is the Gottfried sum rule:

$$
\int_{0}^{1} \frac{1}{x}\left[F_{2}^{l n}(x)-F_{2}^{l n}(x)\right] d x=\int_{0}^{1}[u(x)-d(x)]\left[\left(\frac{2}{3}\right)^{2}-\left(\frac{1}{3}\right)^{2}\right]=\frac{1}{3}
$$


Here the $\left[\left(\frac{2}{3}\right)^{2}-\left(\frac{1}{3}\right)^{2}\right]$ factor comes from the up and down quark charges. When this was measured in muon scattering however, the measured value was $0.240 \pm$ 0.016 [113], quite a bit different from the expected $\frac{1}{3}$. The difference between measurement and the expectation was explained if the up and down seas, assumed to be the same in the derivation of the Gottfried sum rule, were in fact different. This is the first instance where sea distributions assumed to be the same by symmetry arguments in fact turned out to be different.

A final sum rule we want to consider is the integral of $F_{2}$ in neutrino scattering. $F_{2}$ is essentially the momentum weighted sum of all the quark flavors in the nucleon. If the quarks carry all of the nucleon's momentum, the integral of $F_{2}$ should be 1 . If we look again at figure 23 more closely however, we can see that even by eye the integral of $F_{2}$ is not 1 , but more like 0.5 . This means that something other than the quarks carries half the nucleon's momentum. The missing momentum would have to be carried by uncharged particles, or they would contribute to the charged lepton scattering structure functions. The particles in question must also not directly experience weak interactions, or they would contribute to the neutrino scattering structure functions. These "missing" particles are gluons, the carriers of the strong force.

\section{$\underline{2.3}$ Scaling Violation and $R_{L}$}

One of the fundamental predictions of scaling is that the structure functions should be independent of $Q^{2}$. This is only approximately true. One of the ways this scaling violation manifests itself is by breaking the Callan Gross relation (eq. 2.65). The derivation of the Callan Gross relation assumed that the spin $\frac{1}{2}$ quarks 
were noninteracting. With roughly half of the nucleon momentum taken up by the carriers of the strong force however, its quite clear that they are interacting. These interactions can impart angular momentum to the quarks, making them behave as if they were spin zero particles.

We want to "patch up" the structure functions to take this effect into account. Scaling violation in the Callan Gross relation is typically expressed in terms of a "longitudinal structure function", $R_{L} . R_{L}$ is defined as the ratio of the longitudinal cross section to the transverse cross section:

$$
\begin{aligned}
R_{L}\left(x, Q^{2}\right) & =\frac{\sigma_{L}}{\sigma_{T}} \\
& =\frac{\left[1+\frac{4 M^{2} x^{2}}{Q^{2}}\right] F_{2}\left(x, Q^{2}\right)-2 x F_{1}\left(x, Q^{2}\right)}{2 x F_{1}\left(x, Q^{2}\right)}
\end{aligned}
$$

Here the fact that the structure functions are no longer independent of $Q^{2}$ is indicated in the structure functions' dependence on $x$ and $Q^{2}$. "Longitudinal" and "transverse" refer to the polarization of the interacting virtual W boson in the infinite momentum frame. A longitudinally polarized $\mathrm{W}$ boson has $m_{s}=0$ (scalar), and transversely polarized $\mathrm{W}$ has $m_{s}=+1$ or $m_{s}=-1$ (right or left handed polarization). Longitudinally polarized bosons are not able to interact with spin $1 / 2$ partons and conserve helicity. This is the case in the simple quark parton model where $R_{L}$ approaches zero and the Callan Gross relation applies. Conversely if the boson were transversely polarized, it would not be able to interact with a spin zero parton and conserve helicity. If the target were exclusively made from spin zero partons, $R_{L}$ would then be infinite. Figure 25 shows the level to which the Callan Gross relation is violated for several values of $Q^{2}$, utilizing a 


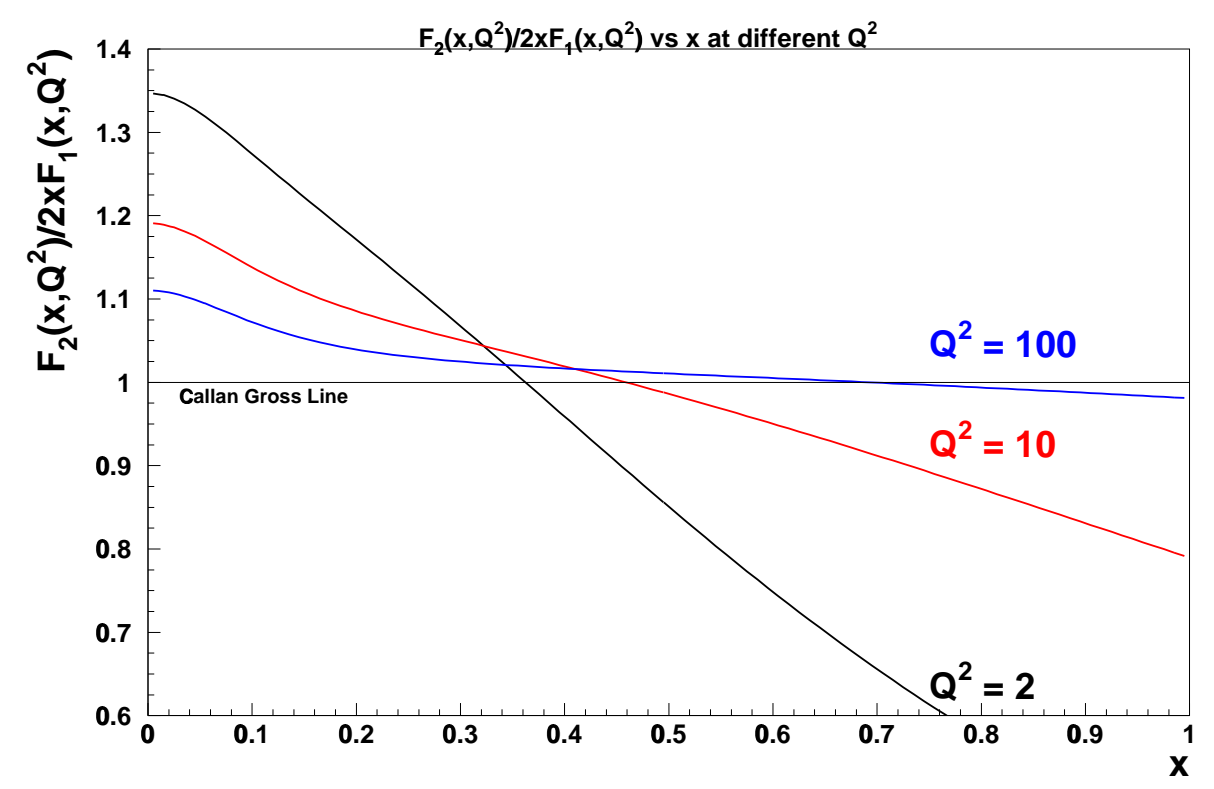

FIGURE 25. Callan-Gross violation vs x at different $Q^{2}$. As $Q^{2}$ increases, The Callan-Gross assumption becomes more accurate

parameterization of $R_{L}$ from fits to charged lepton scattering data [114]. The line at 1 indicates perfect agreement between $F_{2}$ and $2 x F_{1}$. At small $Q^{2}$ the structure function ratio deviates considerably from 1 , but at higher $Q^{2}$ the ratio approaches unity. This is asymptotic freedom at work. At short distances (high $Q^{2}$ ) quarks act like free spin 1/2 particles, but for longer distances they are able to interact, and the Callan Gross relation is increasingly violated at lower and lower $Q^{2}$. One can re-express the Callan-Gross relation including $R_{L}$ :

$$
2 x F_{1}\left(x, Q^{2}\right)=\frac{1+\frac{4 M^{2} x^{2}}{Q^{2}}}{1+R_{L}\left(x, Q^{2}\right)} F_{2}\left(x, Q^{2}\right)
$$

which approaches equation 2.65 as $R_{L}$ goes to zero (also implying large $Q^{2}$ so that $\left(\frac{2 M x}{Q^{2}}\right)^{2} \rightarrow 0$ as well) 
Using 2.106, the total cross section in equation 2.52 can be reformulated in terms of $F_{2}, x F_{3}$, and $R_{L}$ :

$$
\begin{array}{r}
\frac{d \sigma^{\nu(\bar{\nu})}}{d x d y}=\frac{G_{F}^{2} M E}{\pi\left(1+\frac{Q^{2}}{M_{W}^{2}}\right)^{2}}\left[\left(1-y-\frac{M x y}{2 E_{\nu}}+\frac{y^{2}}{2}\left[\frac{1+\frac{4 M^{2} x^{2}}{Q^{2}}}{1+R_{L}\left(x, Q^{2}\right)}\right]\right) \times\right. \\
\left.F_{2}^{\nu(\bar{\nu})}\left(x, Q^{2}\right) \pm y\left(1-\frac{y}{2}\right) x F_{3}^{\nu(\bar{\nu})}\left(x, Q^{2}\right)\right]
\end{array}
$$

Alternatively, one can invert equation 2.106, and express the cross section in terms of $2 x F_{1}, x F_{3}$, and $R_{L}$ (it all depends on what you want to measure):

$$
\begin{aligned}
\frac{d \sigma^{\nu(\bar{\nu})}}{d x d y}=\frac{G_{F}^{2} M E}{\pi\left(1+\frac{Q^{2}}{M_{W}^{2}}\right)^{2}}( & {\left[\left(1-y-\frac{M x y}{2 E_{\nu}}\right) \frac{1+R_{L}\left(x, Q^{2}\right)}{1+\frac{4 M^{2} x^{2}}{Q^{2}}}+\frac{y^{2}}{2}\right] \times } \\
& \left.2 x F_{1}^{\nu(\bar{\nu})}\left(x, Q^{2}\right) \pm y\left(1-\frac{y}{2}\right) x F_{3}^{\nu(\bar{\nu})}\left(x, Q^{2}\right)\right)
\end{aligned}
$$

Reformulating the cross section like this allows one to maintain the intuitiveness of the simple quark parton model definitions of the structure functions while incorporating the effects of the interactions between them in the form of $R_{L}$. We will use both these equations in different ways, with the first being used to describe the inclusive $\mathrm{CC}$ cross section. In both cases $R_{L}$ is usually taken from external lepton scattering data.

\subsection{LO Charm Production}

One can learn a considerable amount of information about nucleon structure from the inclusive cross section discussed in the previous few sections, however additional information can be obtained through study of noninclusive cross sections, where a particular final state is selected. A useful noninclusive cross section, 

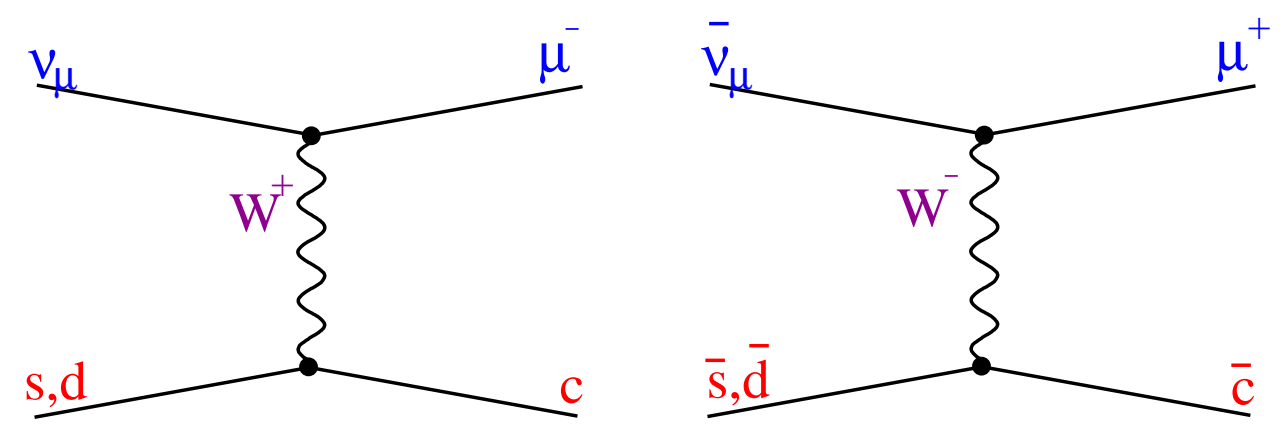

FIGURE 26. Charged current charm production. $\nu$ production at left, $\bar{\nu}$ at right.

especially since it is a primary subject of this dissertation, is the charged current charm production cross section.

Figure 26 displays the Born level Feynman diagrams for charged current neutrino charm production. As in figure 20, the muon neutrino (on the right, analogous antineutrino scattering is shown on the left) interacts with a quark in the nucleon via a $\mathrm{W}$ boson, converting into a muon. In the final state, in particular we want to select a charm quark. In order for a charm quark to be produced and for charge to be conserved at the hadronic vertex, the quarks "struck" by the $\mathrm{W}$ can only be a strange or down quark (or bottom, but the distribution is very small, so can be ignored). In antineutrino scattering, an anti-charm quark is produced, so the only nucleon constituents that may participate are the anti-down and antistrange quarks. Examining the properties of charged current events which produce a charm quark in the final state then gives a direct way of examining the strange and down nucleon distributions for neutrino scattering, and anti-strange and antidown distributions in anti-neutrino scattering.

charm quarks are not produced in equal probability from neutrino scattering off down or strange quarks. When we refer to a "down", "strange", or "bottom" quark, we are referring specifically to that flavor's mass eigenstate. In the Standard 
Model however, the quark mass eigenstates are not the same as the eigenstates of the weak interaction. We could ignore this fact in inclusive scattering because we did not care what kind of quarks were produced in the final state, and the translation between the mass and weak eigenstates is unitary. In the noninclusive case however, we will need to be able to "convert" between the two bases. To do this a rotation matrix called the "CKM" matrix, after Cabibbo, Kobayashi and Maskawa $[115,116]$ is used. The conventional way of defining the CKM matrix is as follows:

$$
\left(\begin{array}{c}
d^{\prime} \\
s^{\prime} \\
b^{\prime}
\end{array}\right)=\left(\begin{array}{ccc}
V_{u d} & V_{u s} & V_{u b} \\
V_{c d} & V_{c s} & V_{c b} \\
V_{t d} & V_{t s} & V_{t b}
\end{array}\right)\left(\begin{array}{l}
d \\
s \\
b
\end{array}\right)
$$

The current values of the $V_{x x}$ matrix elements with their $90 \%$ confidence level errors[78]:

$$
\left(\begin{array}{ccc}
0.9745 \pm 0.0006 & 0.224 \pm 0.003 & 0.0037 \pm 0.0008 \\
\mathbf{0 . 2 2 4} \pm \mathbf{0 . 0 0 3} & \mathbf{0 . 9 7 3 7} \pm \mathbf{0 . 0 0 0 7} & \mathbf{0 . 0 4 1 5} \pm \mathbf{0 . 0 0 2 5} \\
0.0094 \pm 0.0046 & 0.040 \pm 0.003 & 0.9991 \pm 0.0001
\end{array}\right)
$$

Note that this is close to being a diagonal matrix. The elements involved in charm production are emphasized in bold. The amplitude for $d \rightarrow c$ production is proportional to $V_{c d}$, the amplitude for $s \rightarrow c$ is proportional to $V_{c s}$ and $b \rightarrow$ $c$ is proportional to $V_{c b}$. Probabilities go as the amplitude squared, so all else being equal, this means that a charm quark production from strange quarks is $\left|V_{c s}\right|^{2} /\left|V_{c d}\right|^{2}$, or approximately 19 times more likely than from down quarks. Charm production from strange is approximately 550 times more likely than from bottom 
quarks. This preference for charm quarks to be produced from strange quarks in neutrino deep inelastic scattering makes the events where they are produced a unique sample in which to study the strange (and antistrange) components of the nucleon.

The cross section for charged current charm production can be extracted from the total cross section. A first step in determining the charm cross section is pulling out the parts of the structure functions that produce charm. Starting with $2 x F_{1}$ for the proton:

$$
\begin{gathered}
2 x F_{1}^{\nu p}\left(x, Q^{2}\right)=2 x\left[d\left(x, Q^{2}\right)+s\left(x, Q^{2}\right)+\bar{u}\left(x, Q^{2}\right)+\bar{c}\left(x, Q^{2}\right)\right] \\
=2 x\left[\left(\left|V_{u d}\right|^{2}+\left|V_{c d}\right|^{2}+\left|V_{t d}\right|^{2}\right) d\left(x, Q^{2}\right)+\right. \\
\\
\left(\left|V_{u s}\right|^{2}+\left|V_{c s}\right|^{2}+\left|V_{t s}\right|^{2}\right) s\left(x, Q^{2}\right)+ \\
\left(\left|V_{u d}\right|^{2}+\left|V_{u s}\right|^{2}+\left|V_{u b}\right|^{2}\right) \bar{u}\left(x, Q^{2}\right)+ \\
\left.\left(\left|V_{c d}\right|^{2}+\left|V_{c s}\right|^{2}+\left|V_{c b}\right|^{2}\right) \bar{c}\left(x, Q^{2}\right)\right]
\end{gathered}
$$

Here we've expanded out the different CKM matrix terms for each parton type. We then cross out any of the pieces that do not produce charm:

$$
\begin{aligned}
2 x F_{1}^{\nu p-\text { charm }}\left(x, Q^{2}\right)= & 2 x[ \\
& \left(\left|V_{u d}\right|^{2}+\left|V_{c d}\right|^{2}+\left|V_{c d}\right|^{2}\right) d\left(x, Q^{2}+\left|V_{t s}\right|^{2}\right) s\left(x, Q^{2}\right)+ \\
& \left(\left|V_{u d}\right|^{2}+\left|V_{u s}\right|^{2}+\left|V_{u b}\right|^{2}\right) \bar{u}\left(x, Q^{2}\right)+ \\
& \left.\left(\left|V_{c d}\right|^{2}+\left|V_{c s}\right|^{2}+\left|V_{c b}\right|^{2}\right) \bar{c}\left(x, Q^{2}\right)\right] \\
= & 2 x\left[\left|V_{c d}\right|^{2} d\left(x, Q^{2}\right)+\left|V_{c s}\right|^{2} s\left(x, Q^{2}\right)\right]
\end{aligned}
$$


$x F_{3}^{\nu p-c h a r m}\left(x, Q^{2}\right)$ is the same in neutrino mode:

$$
2 x F_{1}^{\nu p-c h a r m}\left(x, Q^{2}\right)=x F_{3}^{\nu p-c h a r m}\left(x, Q^{2}\right)=2 x\left[\left|V_{c d}\right|^{2} d\left(x, Q^{2}\right)+\left|V_{c s}\right|^{2} s\left(x, Q^{2}\right)\right]
$$

However for antineutrinos the sign on $x F_{3}^{\overline{\nu p} p \text { charm }}\left(x, Q^{2}\right)$ will flip, since the anticharm quark is produced off the down or strange sea quarks, which are negative in $x F_{3}^{\overline{\nu p}}\left(x, Q^{2}\right)$ (see equation 2.77).

\subsubsection{Slow Rescaling}

In the kinematics discussion at the beginning of this chapter, we assumed the masses of the partons involved in DIS were negligible. Charm quarks, however do not have a negligible mass at the energies relevant for the $\mathrm{NuTeV}$ experiment. The $J / \Psi$ meson, a bound state of a charm and anti-charm quark, for example has a mass of about $3.1 \mathrm{GeV}$, and the $D^{+}$meson, a bound state between a charm quark and a d quark, has a mass around $1.9 \mathrm{GeV}$. It is clear that some kind of threshold should exist that governs whether it is kinematically possible to produce a heavy charm quark in an interaction. As mentioned on page $53, W^{2}$ should at least be as large as the mass of the particles produced for energy to be conserved. A threshold is applied to the cross section in the form of a step, or theta function. The theta function is a construct similar to a delta function, such that $\Theta(>0)$ is 1 , and $\Theta(<0)$ zero. For bare charm quark production the cross section should be multiplied by $\Theta\left(W^{2}-m_{c}^{2}\right)$. This is referred to as "fast rescaling" [117]. (We will modify this threshold slightly a bit later).

To see how scaling is affected by the final state quark mass we start with the 4 -vectors of the interacting boson and quark, illustrated in figure 27 . In the 


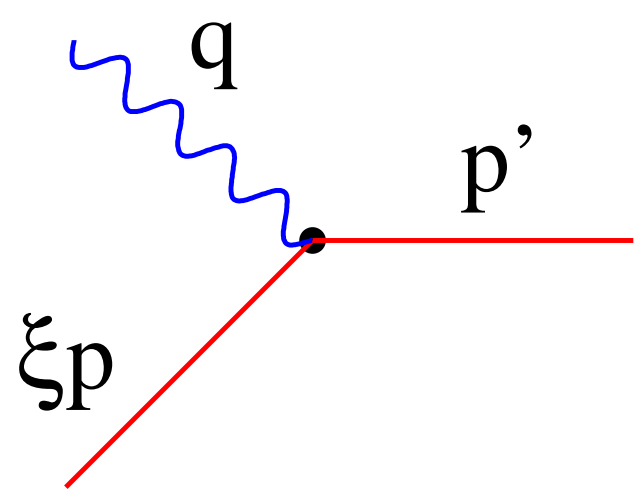

FIGURE 27. The hadronic vertex. A W boson with momentum $q$ interacts with a parton with momentum $\xi p$, producing a charm quark with momentum $p^{\prime}$.

figure, a $\mathrm{W}$ boson having momentum $q$, interacts with a quark having momentum $\xi p$, producing in this case a charm quark with momentum $p^{\prime}$. The variable $\xi$ is a more general parton scaling variable, analogous to Bjorken $x$, but instead includes a "rescaling" factor to account for the production of a massive quark like charm. This is referred to as "slow rescaling" $[118,119,120]$. Finding $\xi$ begins with the squared center of mass energy :

$$
\begin{aligned}
s^{\prime} & =(q+\xi p)^{2} \\
& =q^{2}+\xi q \cdot p+\xi^{2} p^{2}
\end{aligned}
$$

Which in the lab frame (see equations $2.4,2.5,2.15,2.18$ ) is:

$$
s^{\prime}=-Q^{2}+2 \xi M \nu+\xi^{2} M^{2}
$$


$s^{\prime}$ is invariant, and in the $\mathrm{W}$ - partonic center of mass frame would need to be greater than the mass squared of the quark produced:

$$
\begin{aligned}
\xi^{2} M^{2}+2 \xi M \nu-Q^{2} & =s^{\prime} \\
& =m_{c}^{2} \\
2 \xi M \nu-Q^{2} & \simeq m_{c}^{2} \\
\xi \frac{Q^{2}}{x}-Q^{2} & \simeq m_{c}^{2} \\
\frac{\xi}{x}-1 & \simeq \frac{m_{c}^{2}}{Q^{2}} \\
\xi & \simeq x\left(1+\frac{m_{c}^{2}}{Q^{2}}\right)
\end{aligned}
$$

Here we've neglected the $\xi^{2} M^{2}$ term. Like Bjorken $x, \xi$ is constrained between 0 and 1 , so $\xi^{2} M^{2}$ is typically small compared to the other terms, especially at low $x$ and high $Q^{2}$. If in addition $Q^{2}$ were sufficiently high that the charm mass can be considered small, $\xi$ reduces to the usual Bjorken $x$.

If one were to not neglect the $\xi^{2} M^{2}$ term, and include the initial quark mass as well, the expression for $\xi$ would be [121]:

$$
\xi_{f u l l}=\frac{Q^{2}-m_{i}^{2}+m_{c}^{2}+\Delta\left(-Q^{2}, m_{s}^{2}, m_{c}^{2}\right)}{2 Q^{2}\left(\frac{1}{2 x}+\sqrt{\frac{1}{4 x^{2}}+\frac{M^{2}}{Q^{2}}}\right)}
$$

Here $m_{i}$ refers to the initial quark mass, either that of a $d$ or an $s$ quark, and the $\Delta(\ldots)$ is the usual triangle function, defined in equation 2.28. The struck quark 
masses are small, around $6 \mathrm{MeV}$ for down quarks, and $100 \mathrm{MeV}$ for strange quarks, and can be safely neglected. In doing so one finds:

$$
\xi_{f u l l} \simeq x\left(1+\frac{m_{c}^{2}}{Q^{2}}\right)\left(1-\frac{x^{2} M^{2}}{Q^{2}}\right)
$$

Which reduces to equation 2.124 if we neglect the typically small $\frac{x^{2} M^{2}}{Q^{2}}$ term. The behavior of the slow rescaling variable with the different approximations is shown in figures 28 and 29. The left plot in figure 28 plots the $\frac{\xi}{x}$ ratio as a function of $x$ for a $Q^{2}$ value of $2 \mathrm{GeV}^{2}$. at this $Q^{2}$, and at high values of $x$, the values of $\xi_{\text {full }}$ with and without the initial mass term begin to deviate substantially from the simple expression in equation 2.124. This deviation gets smaller as $Q^{2}$ increases, shown in the right hand side of the figure, where the ratio is plotted for a fixed $x$ value of 0.2 versus $Q$. Figure 29 shows the effect of the initial quark mass by looking at the ratio of equations 2.125 and 2.124 at both low (left, $x=0.2$ ) and high (right, $x=0.8$ ) values of $x$, plotted vs $Q$. The left plot is more relevant for investigating the strange sea, and shows neglecting the strange quark mass makes only a sub $1 \%$ level mistake. This difference is smaller at lower values of $\xi$. 

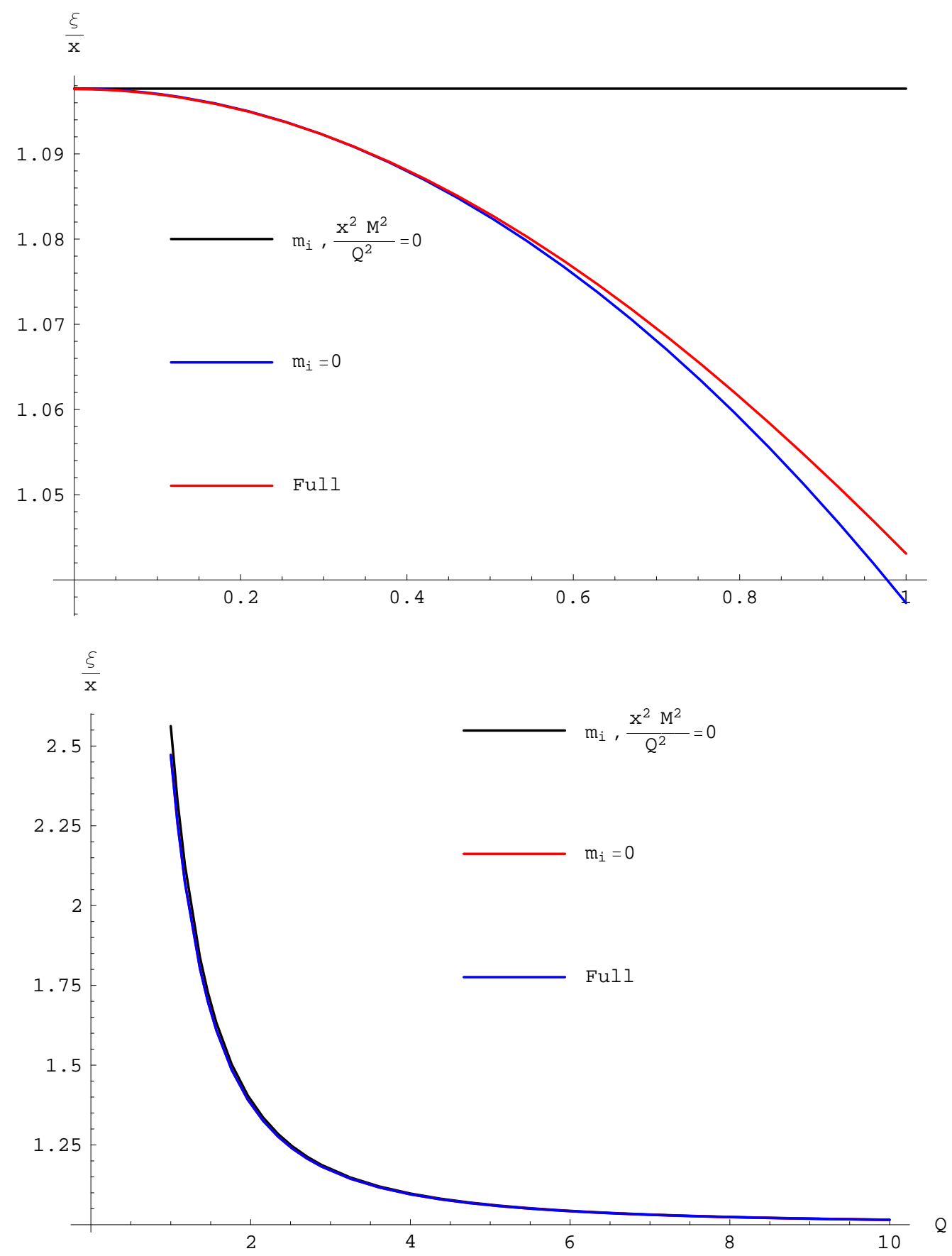

FIGURE 28. $\frac{\xi}{x}$ plotted vs $x$ and $Q$. The red curve shows the full expression, eq. 2.125 , blue ignoring the initial quark mass, and black also ignoring the $\frac{x^{2} M^{2}}{Q^{2}}$ term 


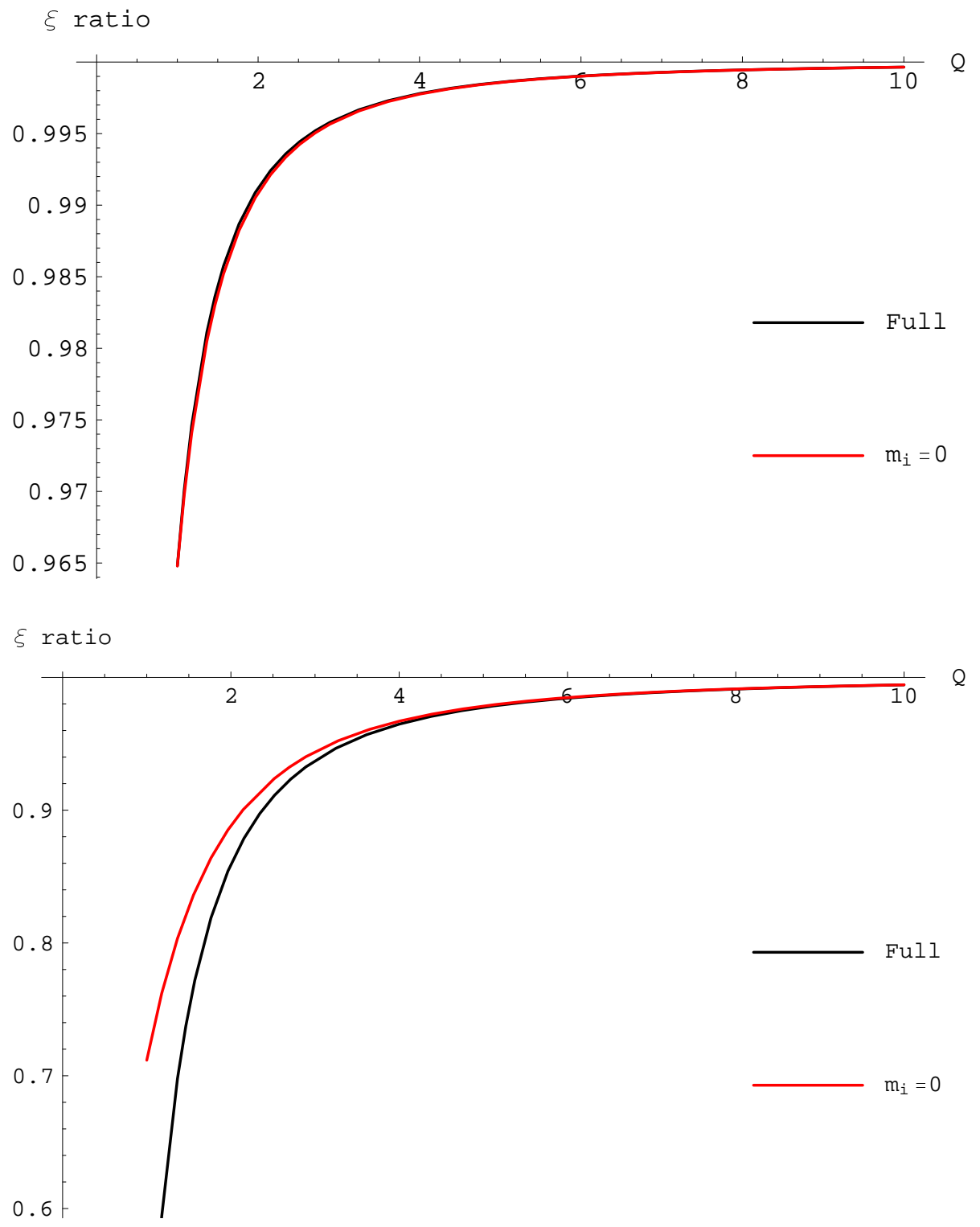

FIGURE 29. $\frac{\xi_{\text {full }}}{\xi}$ vs $Q$ for both low and high x values. The black curves are eq. 2.125 , red ignores the initial quark mass. Left plot is for $x=0.2$, right is for $x=0.8$ 


\subsubsection{The Charm Cross Section}

We now have the tools we need to construct the total charm cross section. Starting with equation 2.108 and introducing slow-rescaling:

$$
\begin{aligned}
& \frac{d \sigma_{\text {charm }}^{\nu p}}{d \xi d y}=\frac{G_{F}^{2} M E}{\pi\left(1+\frac{Q^{2}}{M_{W}^{2}}\right)^{2}}\left[\left(1-y-\frac{M x y}{2 E}\right) F_{2}^{\nu p-\text { charm }}\left(\xi, Q^{2}\right)+\right. \\
& \frac{y^{2}}{2} \frac{2 x}{2 \xi} 2 \xi F_{1}^{\nu p-c h a r m}\left(\xi, Q^{2}\right)+ \\
& \left.y\left(1-\frac{y}{2}\right) \frac{x}{\xi} \xi F_{3}^{\nu p-c h a r m}\left(\xi, Q^{2}\right)\right]
\end{aligned}
$$

We substitute out $F_{2}^{\nu p-c h a r m}\left(\xi, Q^{2}\right)$ using a slow rescaled version of equation 2.106:

$$
\begin{array}{r}
\frac{d \sigma_{\text {charm }}^{\nu p}}{d \xi d y}=\frac{G_{F}^{2} M E}{\pi\left(1+\frac{Q^{2}}{M_{W}^{2}}\right)^{2}}\left[\left(1-y-\frac{M x y}{2 E}\right)\left(\frac{1+R_{L}\left(\xi, Q^{2}\right)}{1+\frac{4 M^{2} \xi^{2}}{Q^{2}}}\right) 2 \xi F_{1}^{\nu p-c h a r m}\left(\xi, Q^{2}\right)+\right. \\
\left.\frac{x y^{2}}{2 \xi} 2 \xi F_{1}^{\nu p-c h a r m}\left(\xi, Q^{2}\right)+\left(\frac{x y}{\xi}-\frac{x y^{2}}{2 \xi}\right) \xi F_{3}^{\nu p-c h a r m}\left(\xi, Q^{2}\right)\right]
\end{array}
$$

Here we've assumed $R_{L}$ has the same behavior for charm production as for the inclusive cross section. Gathering together terms and substituting in equation 2.115:

$$
\begin{array}{r}
\frac{d \sigma_{c h a r m}^{\nu p}}{d \xi d y}=\frac{2 G_{F}^{2} M E \xi}{\pi\left(1+\frac{Q^{2}}{M_{W}^{2}}\right)^{2}}\left[\left(1-y-\frac{M x y}{2 E}\right)\left(\frac{1+R_{L}\left(\xi, Q^{2}\right)}{1+\frac{4 M^{2} \xi^{2}}{Q^{2}}}\right)+\frac{x y}{\xi}\right] \times \\
\left(\left|V_{c d}\right|^{2} d\left(\xi, Q^{2}\right)+\left|V_{c s}\right|^{2} s\left(\xi, Q^{2}\right)\right)
\end{array}
$$


Applying isospin symmetry for the neutron cross section and adding it in to construct the cross section for an isoscalar nucleus:

$$
\begin{aligned}
\frac{d \sigma_{c h a r m}^{\nu N}}{d \xi d y}= & \frac{G_{F}^{2} M E \xi}{\pi\left(1+\frac{Q^{2}}{M_{W}^{2}}\right)^{2}}\left[\left(1-y-\frac{M x y}{2 E}\right)\left(\frac{1+R_{L}\left(\xi, Q^{2}\right)}{1+\frac{4 M^{2} \xi^{2}}{Q^{2}}}\right)+\frac{x y}{\xi}\right] \times \\
\left(\left|V_{c d}\right|^{2}\left[d\left(\xi, Q^{2}\right)+u\left(\xi, Q^{2}\right)\right]+2\left|V_{c s}\right|^{2} s\left(\xi, Q^{2}\right)\right) & \\
= & \frac{G_{F}^{2} M E \xi}{\pi\left(1+\frac{Q^{2}}{M_{W}^{2}}\right)^{2}}\left[\left(1-y-\frac{M x y}{2 E}\right)\left(\frac{1+R_{L}\left(\xi, Q^{2}\right)}{1+\frac{4 M^{2} \xi^{2}}{Q^{2}}}\right)+\frac{x y}{\xi}\right] \times \\
& \left(\left|V_{c d}\right|^{2}\left[d_{v}\left(\xi, Q^{2}\right)+u_{v}\left(\xi, Q^{2}\right)+d_{s}\left(\xi, Q^{2}\right)+u_{s}\left(\xi, Q^{2}\right)\right]+\right. \\
& \left.2\left|V_{c s}\right|^{2} s\left(\xi, Q^{2}\right)\right)
\end{aligned}
$$

Here the valence and sea quark distributions are explicitly shown to emphasize that charm production can occur off the valence quarks in neutrino scattering. (Indeed, approximately half of neutrino induced charm is from the valence quarks). The $u$ quark parton distributions do not indicate charm production from up quarks, but that the down distribution in the neutron is the same as the up distribution in the proton. As before all parton distributions are defined in terms of the proton. In antineutrino scattering only the seas are involved:

$$
\begin{aligned}
\frac{d \sigma_{\text {charm }}^{\overline{\nu N}}}{d \xi d y}= & \frac{G_{F}^{2} M E \xi}{\pi\left(1+\frac{Q^{2}}{M_{W}^{2}}\right)^{2}}\left[\left(1-y-\frac{M x y}{2 E}\right)\left(\frac{1+R_{L}\left(\xi, Q^{2}\right)}{1+\frac{4 M^{2} \xi^{2}}{Q^{2}}}\right)+\frac{x y}{\xi}\right] \times \\
& \left(\left|V_{c d}\right|^{2}\left[\bar{d}_{s}\left(\xi, Q^{2}\right)+\bar{u}_{s}\left(\xi, Q^{2}\right)\right]+2\left|V_{c s}\right|^{2} \bar{s}\left(\xi, Q^{2}\right)\right)
\end{aligned}
$$


In antineutrino charm production, scattering occurs predominantly off the antistrange quarks, since the valence d distributions are not involved, and scattering off the $\bar{d}$ sea is suppressed by the $\left|V_{c d}\right|^{2}$ factor.

\subsubsection{From Charm to Observables}

What has been calculated above are cross sections for bare charm quark production. Quarks are extremely modest creatures, and desperately require to be associated with other quarks in order to cover up their nakedness. The process of acquiring, producing, and attaching to one or more quarks to produce a color neutral hadron is called "hadronization", or "fragmentation". If we did not care what kind of final state hadron was produced, the cross section for inclusive charmed hadron production would be equal to the cross section for charm quark production. Generally however, some kind of energy cut needs to be applied to candidate charm events distinguish them from unwanted background events. The fragmentation process is energy dependent, and that energy dependence will need to be included in the cross section.

In the simple quark parton picture we are currently following, fragmentation is applied to the cross section by multiplying a parameterization of the probability a charmed hadron keeps a ratio $z$ of the parent quark's momentum, called a "fragmentation function". This more or less empirical function accounts for the 
momentum lost to the charm quark by "grabbing and dragging along" the quarks needed to become an observable hadron. Including the fragmentation function, the LO cross section becomes:

$$
\begin{aligned}
& \frac{d \sigma_{D}^{\nu(\bar{\nu}) N}}{d \xi d y d z}=\frac{d \sigma_{\text {charm }}^{\nu(\bar{\nu}) N} D(z)}{d \xi d y} D \\
& \frac{d \sigma_{D}^{\nu(\bar{\nu}) N}}{d \xi d y}=\int_{0}^{1} \frac{d \sigma_{\text {charm }}^{\nu(\bar{\nu}) N}}{d \xi d y} D(z) d z
\end{aligned}
$$

The " $D$ " subscript indicates a charmed hadron rather than bare charm quark. $\mathrm{D}(\mathrm{z})$ is the "fragmentation function", and $z$ is defined to be the ratio of the initial quark's momentum carried by the observable hadron. it is Lorentz invariant, and reduces to the ratio of the charmed hadron's energy over the energy transferred to the hadronic system in the lab frame:

$$
\begin{aligned}
z & \equiv \frac{p_{D} \cdot p}{q \cdot p} \\
& =\frac{M p_{D-l a b}}{M \nu} \\
& \sim \frac{E_{D-l a b}}{\nu}
\end{aligned}
$$

where $p$ is the nucleon momentum, and $q$ is the momentum carried by the $\mathrm{W}$ boson. $z$ ranges from zero to 1 , and the distribution $D(z)$ will have a shape peaking closer to 1 (keeping all of the parent quark's momentum) the larger the mass of the parent quark. 


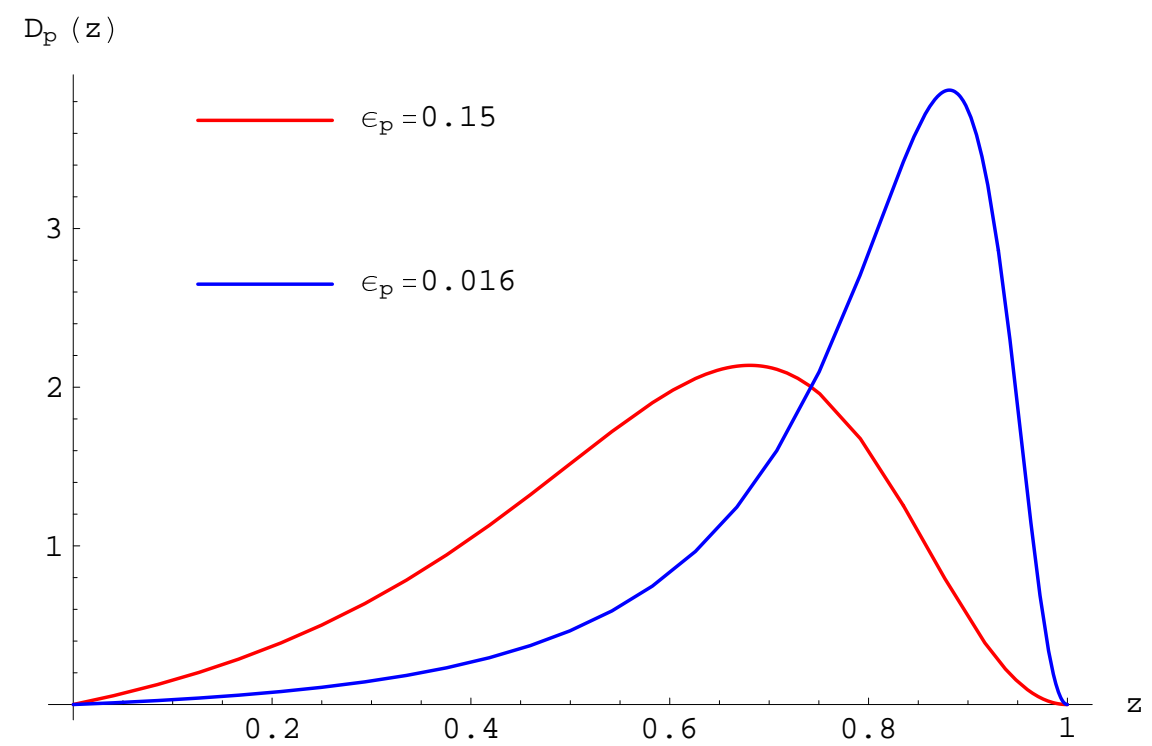

FIGURE 30. $D_{p}(x)$ for charm and bottom quarks. The distribution for charm is softer (red) than bottom (blue).

Many parameterizations for $D(z)$ exist, the two most used by neutrino scattering experiments are the Peterson [122], and Collins-Spiller [123] parameterizations. The Peterson function is:

$$
D_{p}(z)=\frac{N}{z\left(1-\frac{1}{z}-\frac{\epsilon_{p}}{1-z}\right)^{2}}
$$

Here $\mathrm{N}$ is a normalization factor, defined such that $\int_{0}^{1} D_{p}(z) d z=1$. $\epsilon_{p}$ defines the shape of the function, with a value of zero corresponding to the hadron keeping all of the initial quark's momentum. $\epsilon_{p}$ can be related to the heavy quark's mass:

$$
\epsilon_{p} \sim \frac{m_{q}^{2}}{m_{Q}^{2}}
$$




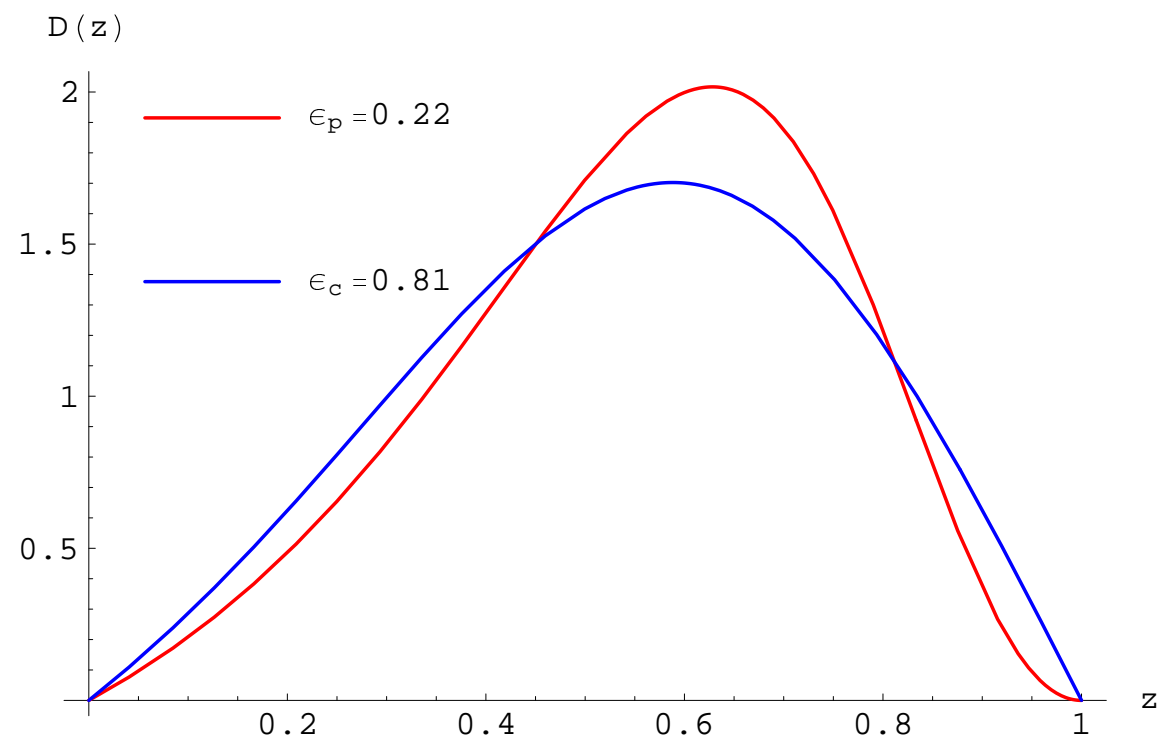

FIGURE 31. $D(x)$ from Peterson and Collins-Spiller parameterizations. Peterson is in red, Collins-Spiller in blue. Both are from fits to CCFR data.

Here $m_{Q}$ corresponds to the heavy quark (i.e. charm) and $m_{q}$ the light quark bound to it. Figure 30 shows the Peterson fragmentation function with epsilons corresponding to what might be expected for a charm quark $\left(\epsilon_{p} \simeq 0.15\right)$ and for a bottom quark $\left(\epsilon_{p} \simeq 0.016\right)$. The heavier bottom quark peaks closer to 1 .

The Collins-Spiller fragmentation function was proposed because the asymptotic behavior of the Peterson function as $z \rightarrow 1$ supposedly did not satisfy requirements imposed on it by dimension counting rules. Collins \& Spiller reported that the Peterson function behaved like $D_{p}(z) \sim(1-z)^{2}$, however dimension counting arguments suggested instead it should behave like $D_{p}(z) \sim(1-z)$ as $z \rightarrow 1$. In fact the Peterson function should satisfy the dimension counting arguments, with 
$D_{p}(z) \sim \frac{(1-z)^{2}}{z}$. The CCFR and $\mathrm{NuTeV}$ collaborations however have come to use the Collins-Spiller parameterization because it tends to provide a better fit to their data $[124,75]$. The Collins-Spiller parameterization is:

$$
D_{c}(z)=\frac{N\left(\frac{1-z}{z}+\frac{2-z}{1-z} \epsilon_{c}\right)(1+z)^{2}}{\left(1-\frac{1}{z}-\frac{\epsilon_{c}}{1-z}\right)^{2}}
$$

As in the Peterson function, $N$ is a normalization factor enforcing $\int_{0}^{1} D_{c}(z) d z=1$, and $\epsilon_{c}$ controls the "hardness" of the fragmentation function, with a smaller $\epsilon_{c}$ also corresponding to the hadron keeping more of the parent quark's mass. $\epsilon_{c}$ is similar to $\epsilon_{p}$ in that it is also inversely proportional to the heavy quark mass squared, but instead of being a ratio to the light quark mass squared, it is defined to be:

$$
\epsilon_{c} \equiv \frac{\left\langle k_{T}^{2}\right\rangle}{m_{Q}^{2}}
$$

Here $\left\langle k_{T}^{2}\right\rangle$ would correspond to an average squared transverse momentum, or the "size" of the hadron in momentum space. Figure 31 compares the Peterson and Collins-Spiller functions for charm with $\epsilon_{p}$ and $\epsilon_{c}$ from CCFR's measurements.

We have until this point neglected "fast rescaling". As mentioned at the beginning of the previous subsection, we need to apply thresholds on the cross section which require there be sufficient energy available for a charmed hadron to be produced. This condition is imposed as a multiplicative theta function: $\Theta\left(W^{2}-m_{D}^{2}\right)$, where again the " $D$ " in $m_{D}$ is a generic label for whatever hadron is produced.

All the cross section pieces are then in place to calculate the probability of producing an observable charmed hadron. Unfortunately, large, coarsely grained 


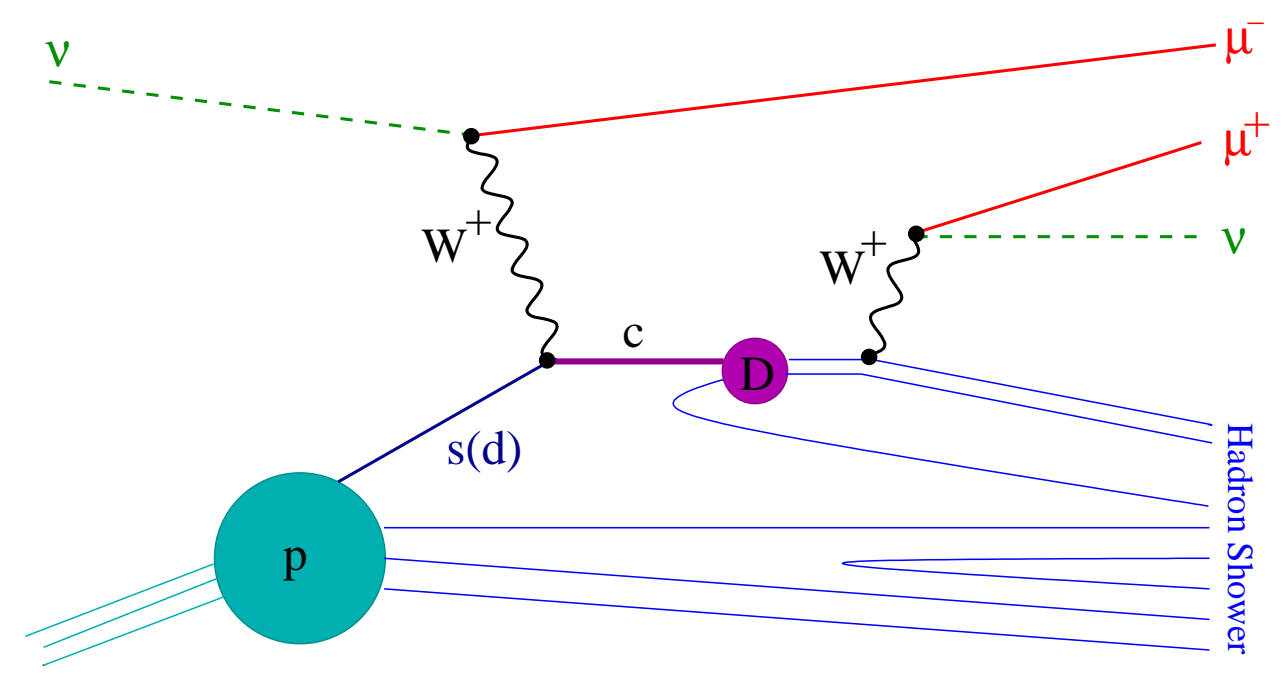

FIGURE 32. A Feynman diagram of a neutrino induced dimuon event.

detectors such as that employed by $\mathrm{NuTeV}$ are unable to distinguish between charmed hadrons and any of the other non-charmed particles produced in the hadron shower. Approximately ten percent of the time however, charmed hadrons decay semi-leptonically such that a muon is produced. Muons travel large distances through detectors, so provide a very clear signal by which one can distinguish charm produced events from charged current events as a whole. This second muon will have a charge opposite from the muon produced at the electroweak vertex. These events are called "dimuon" events, an example of a neutrino dimuon event is shown in figure 32 .

The dimuon cross section is the charmed hadron cross section multiplied by the semileptonic branching ratio for that hadron. Because we cannot distinguish what type of charmed hadron is produced however, we must use an averaged semileptonic branching ratio, averaged over all produced charmed hadrons. This average is found by convolving the neutrino energy spectrum, measured hadronic 
production fractions, and the individual semileptonic decays for those hadrons together:

$$
B_{c}=\sum_{i} \int P(E) f_{i}(E) B_{\mu-i} d E
$$

Here the sum is over all charmed hadron types $\left(D^{0}, D^{+}, D_{s}, \Lambda_{c}\right), P(E)$ is the incident neutrino energy distribution, $f_{i}(E)$ is the energy dependent production fraction for each hadron, and $B_{\mu-i}$ is the semi-muonic branching ratio for each hadron. In practice, for most charmed hadrons $B_{\mu-i}$ is known very poorly, and equivalence between muon and electron production is invoked so that $B_{e-i}$ may used instead. Even the $B_{e-i}$ ratios are not known so well however, and often it is more advantageous to include $B_{c}$ as a parameter when performing fits to data.

With the addition of $B_{c}$, our charmed hadron cross section becomes a dimuon cross section:

$$
\frac{d \sigma_{D}^{\nu(\bar{\nu}) N}}{d \xi d y d z}=\frac{d \sigma_{\text {charm }}^{\nu(\bar{v}) N}}{d \xi d y} D(z) B_{c}
$$

As no measurements indicate to the contrary (with emphasis on "no measurements"), and no reasons from the theory to assume otherwise, it is assumed that both fragmentation and $B_{c}$ are the same for neutrino and antineutrino scattering.

\subsection{NLO Charm Production}

In calculating cross sections in the previous two sections, we relied on on the intuitive, but slightly naive simple quark parton model (albeit enhanced with an empirical $R_{L}$ term). Using it, it is possible to describe $\mathrm{NuTeV}$ data quite well, however measurements of quark masses and parton distributions really only have 
meaning within that scheme. Because the value of $R_{L}$ is nonzero for example, we know that the force carriers play an important role in nucleon cross sections. We also know from integrating $F_{2}$ that half the nucleon momentum is carried by gluons. When measuring the parton distributions in the simple quark parton model however we do not fully account for the presence of gluons, and so the quark distributions extracted really are a sort of amalgam of quarks and gluon related effects. We have gone as far as we can with this simple model, to understand better what is going on inside the nucleon we will need to take on a more complete model.

\subsubsection{Quantum Chromodynamics}

Quantum Chromodynamics, or QCD, is the theory of the strong interaction, which binds quarks together. In QCD, quarks carry one of 3 possible charges, referred to as color charges in analogy with the 3 primary colors. The three colors, or a color and its anti-color combined (baryons and mesons respectively) produce a color singlet state is no longer able to interact directly via the strong interaction. The strong interaction occurs between colored objects by means of the exchange of a gluon. Gluons are massless, spin-1 bosons which themselves carry color, allowing them to interact with other gluons. Gluons can carry one of nine possible color combinations, 8 of which interact. In terms of group theory, the strong interaction follows $\mathrm{SU}(3)$ symmetry, where the $\mathrm{SU}(3)$ means $3 \otimes \overline{3}=8 \oplus 1$, or that 3 colors combined with 3 anti-colors gives you 8 color states, +1 color singlet. The 8 interacting quark color combinations are: $r \bar{g}, r \bar{b}, g \bar{b}, g \bar{r}, b \bar{r}, b \bar{g}, \frac{1}{\sqrt{2}}(r \bar{r}-b \bar{b})$, and $\frac{1}{\sqrt{6}}(r \bar{r}+b \bar{b}-g \bar{g})$. The noninteracting color singlet gluon is: $\frac{1}{\sqrt{3}}(r \bar{r}+b \bar{b}+2 g \bar{g})$. 


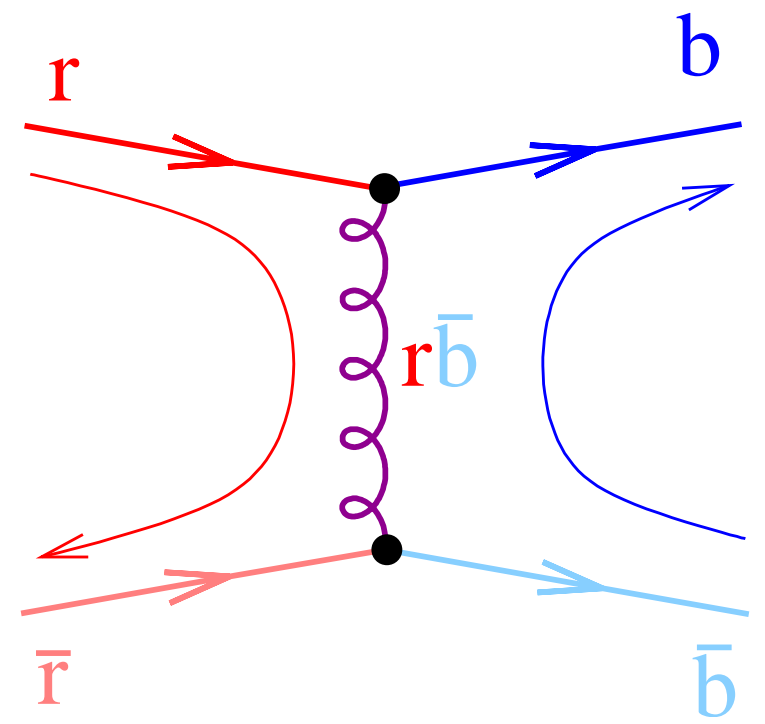

FIGURE 33. Quark-quark interaction in QCD. Colors in the figure show the colors of the quarks, curved arrows to the side show how color flows in the interaction.

Figure 33 shows a diagram of a quark interacting with another quark via the strong interaction, with arrows indicating how color flows in the interaction.

QCD is a field theory, and as such is defined by a Lagrangian density:

$$
\mathcal{L}_{Q C D}=\sum_{f} \bar{\psi}_{f}^{i}\left(i \gamma_{\mu} D^{\mu}-m_{f}\right) \psi_{f}^{j}-\frac{1}{4} F_{a}^{\mu \nu} F_{\mu \nu}^{a}
$$

Here the sum is over all quark fields, $D^{\mu}$ is the covariant derivative, $m_{f}$ quark masses, and $F_{a}^{\mu \nu}$ the field strength tensor. On the surface, the QCD Lagrangian is very similar to the QED Lagrangian:

$$
\mathcal{L}_{Q E D}=\sum_{f} \bar{\psi}_{f}\left(i \gamma_{\mu} D^{\mu}-m_{f}\right) \psi_{f}-\frac{1}{4} F^{\mu \nu} F_{\mu \nu}
$$




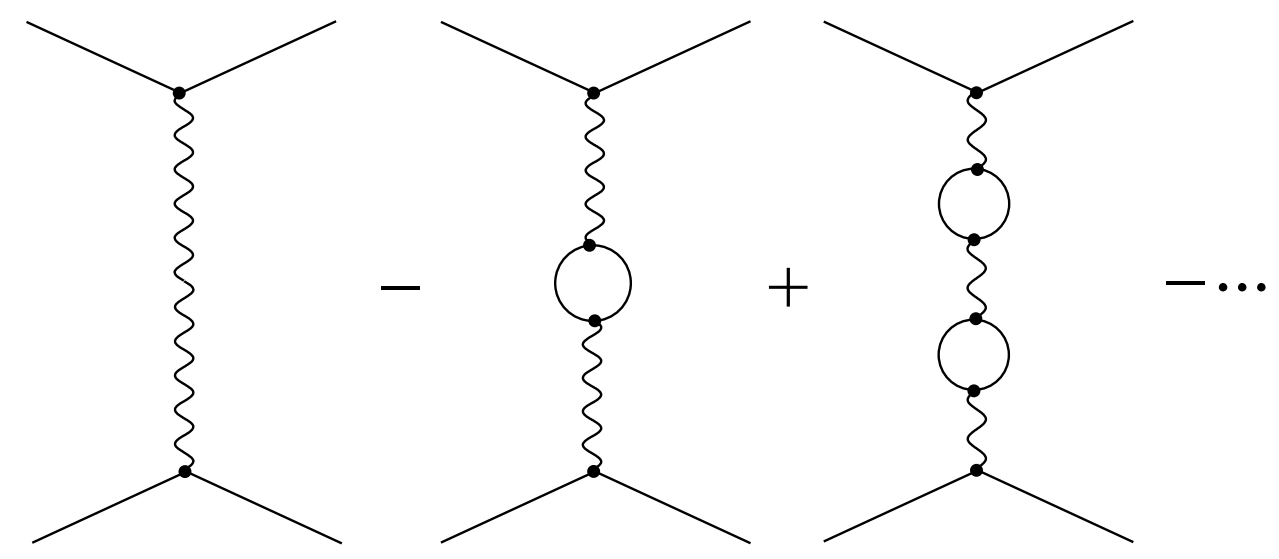

FIGURE 34. Some diagrams which are combined in charged particle scattering

However an important difference lies in the field strength tensors:

$$
\begin{array}{ll}
F^{\mu \nu}=\partial^{\mu} A^{\nu}-\partial^{\nu} A^{\mu} & (Q E D) \\
F_{a}^{\mu \nu}=\partial^{\mu} A_{a}^{\nu}-\partial^{\nu} A_{a}^{\mu}+g_{s} f^{a b c} A_{b}^{\mu} A_{c}^{\nu} & (Q C D)
\end{array}
$$

The last term in the QCD field strength tensor indicates that unlike photons, the gluons are able to interact with each other. This self interaction gives QCD profoundly different behavior than electromagnetic interactions, but it is this difference that makes QCD so successful at describing both quark confinement and asymptotic freedom.

To get a better idea how this difference between QCD and QED works, we will need to briefly discuss loop diagrams and renormalization. We will start by looking at $\mathrm{QED}[108,125]$. In calculating the amplitude for a process in a field theory like QED, all possible ways that the electrons may interact must be included in the calculation. Feynman diagrams are used as tools to represent the terms in this expansion. For example figure 34 shows some of the Feynman diagrams that 
must be summed to calculate the measured cross section. The simplest case is the exchange of a single photon (left). It is however also completely possible for the photon to briefly split into an electron-positron pair which then recombine into a photon again (middle). This can happen however many times (right). There is a bit of a problem with the middle and rightmost diagrams though, in order to calculate the amplitude corresponding to those diagrams, one must integrate over the momentum of the internal loops. There is no restriction on the momentum inside those loops, so that integral must range from zero to infinity. The correction due to the middle diagram is proportional to:

$$
I\left(q^{2}\right)=\frac{\alpha_{0}^{2}}{3 \pi} \int_{m_{e}^{2}}^{\infty} \frac{d p^{2}}{p^{2}}-\frac{2 \alpha_{0}}{\pi} \int_{0}^{1} z(1-z) \log \left(1-\frac{q^{2} z(1-z)}{m_{e}^{2}}\right) d z
$$

Here we are deliberately labeling $\alpha$ with a subscript zero to indicate that the value applied at the vertex of each diagram in the series is different than the one which is measured (which would be resulting from the sum of all these diagrams). The problem with $I\left(q^{2}\right)$ comes from the fact that it is logarithmically divergent. This and all subsequent loop diagrams are infinite. There is a way to get around this however, via a process called "renormalization". In renormalization the infinity in the upper limit of the $\int_{m_{e}^{2}}^{\infty} \frac{d p^{2}}{p^{2}}$ integral is temporarily replaced with a finite cutoff M. $\quad \alpha$ is then redefined to suck up the logarithmic dependence on this cutoff. That "renormalized" $\alpha$ is the one we measure. This seems fundamentally weird on the surface, however some level of reassurance should come from the fact that whatever mathematical tomfoolery is going on here allows physical quantities to be calculated, and, in the case of QED, predictions can be made that agree with experimental measurements to 8 decimal places. 
A consequence of renormalization is that the electromagnetic coupling, $\alpha$ becomes dependent on $Q^{2}$, and a reference energy scale $\mu$ which was added to remove the dependence on $M$. This dependence goes like:

$$
\alpha\left(Q^{2}\right)=\frac{\alpha\left(\mu^{2}\right)}{1-\frac{1}{\pi} \alpha\left(\mu^{2}\right) \log \left(\frac{Q^{2}}{\mu^{2}}\right)}
$$

including the contribution from one loop. $\alpha\left(Q^{2}\right)$ is referred to as a "running coupling constant", because of its dependence in $Q^{2}$. In QED, the coupling increases with increasing $Q^{2}$. Increasing $Q^{2}$ is equivalent to looking at smaller and smaller distance scales. At low $Q^{2}$ the effect of the loop diagrams, called vacuum polarization, is strongest. This can be envisioned as being like a cloud of virtual electron-positron pairs surrounding the electron being probed in an interaction. Like a dielectric, this cloud "screens" the bare electron charge at large distance scales. As we probe deeper, penetrating this cloud at higher and higher $Q^{2}$, the effective coupling increases. We would then be seeing more of the bare electron charge.

Something here should also be said about this "reference scale" $\mu$. Its presence is perhaps a bit worrisome. It may seem as if we have introduced an arbitrary free variable. The rules of the game however are that any observable quantity must be independent of the reference scale. The scale merely selects how important higher order loops are in the calculation. Calculating the Feynman diagrams to all orders is impossible to do, and the scale choice is then limited to something near the $Q^{2}$ of the problem under study. If a calculation is performed only up to certain order, and there is a strong dependence on this $\mu$ scale, then that is an indication that higher order diagrams need to be included in the calculation [126]. 


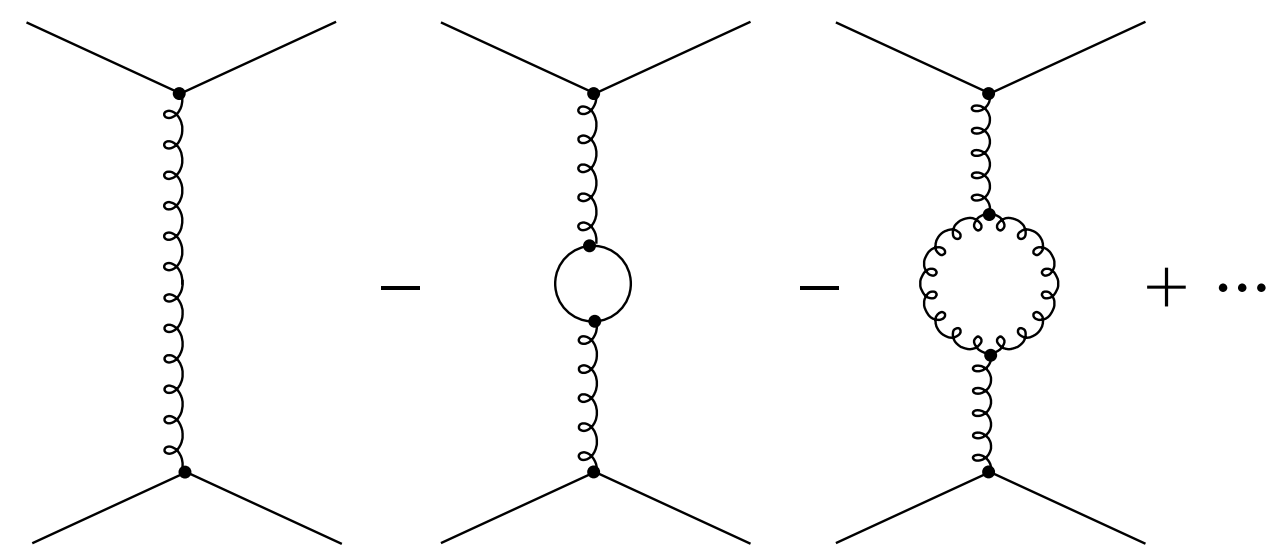

FIGURE 35. Some diagrams which are combined in quark scattering

Examining the scale dependence of an observable is a way of examining the level of certainty that that order of calculation may have on the observable.

The purpose for this whole discussion of QED is intended to illuminate some of the important characteristics of QCD. Recall from equation 2.147 that the difference between QCD and QED is the presence of the $g_{s} f^{a b c} A_{b}^{\mu} A_{c}^{\nu}$ term, the selfcoupling of the gluons. This self-coupling changes the sorts of diagrams that must be summed from what we've seen in QED. Figure 35 shows some of the diagrams summed to calculate quark scattering in QCD. Like QED, we have the simple exchange of a gluon (left), then also like QED, we can have gluons splitting into quark-antiquark pairs and recombining (middle). Were these diagrams all there was, we would have a theory which behaved exactly like QED, with charge screening, etc. There is the diagram on the right however. The effect of the gluon-gluon coupling is opposite from the vacuum polarization diagram - it anti-screens the bare charge. As one looks at larger and larger distance scales, more and more 
gluon loops appear, which effectively magnifies the charge. This gives the observed behavior of quark confinement. As we look at smaller and smaller distance scales, at higher and higher $Q^{2}$, the coupling grows small. This is asymptotic freedom.

Including diagrams with up to one loop the QCD running coupling is given by:

$$
\alpha_{s}\left(Q^{2}\right)=\frac{\alpha_{s}\left(\mu^{2}\right)}{1+\frac{\alpha_{s}\left(\mu^{2}\right)}{12 \pi}\left(33-2 n_{f}\right) \log \left(Q^{2} / \mu^{2}\right)}
$$

where the $n_{f}$ refers to the number of flavors participating in the interaction. More than 16 would be needed to make the coupling behave like QED, up to the energies we have been able to reach, there are at most 6 .

The running coupling expression is typically redefined in terms of a scale which sets the boundary between QCD's "confinement" behavior, and its "asymptotic freedom" behavior:

$$
\Lambda_{Q C D}^{2}=\mu^{2} \exp \left(\frac{-12 \pi}{\left(33-2 n_{f}\right) \alpha_{s}\left(\mu^{2}\right)}\right)
$$

making the running coupling $\alpha_{s}$ :

$$
\alpha_{s}\left(Q^{2}\right)=\frac{12 \pi}{\left(33-2 n_{f}\right) \log \left(\frac{Q^{2}}{\Lambda_{Q C D}^{2}}\right)}
$$

The value of $\Lambda_{Q C D}$ defines the behavior of QCD, and can be obtained from measuring $\alpha_{s}\left(Q^{2}\right)$ at different energies. Figure 36 shows the decrease in $\alpha_{s}\left(Q^{2}\right)$ as a function of energy [87]. The current world average for $\alpha_{s}$ at $Z^{0}$ mass is $0.1187 \pm 0.002$, and $\Lambda_{Q C D}=217_{-23}^{+25} \mathrm{MeV}$. 


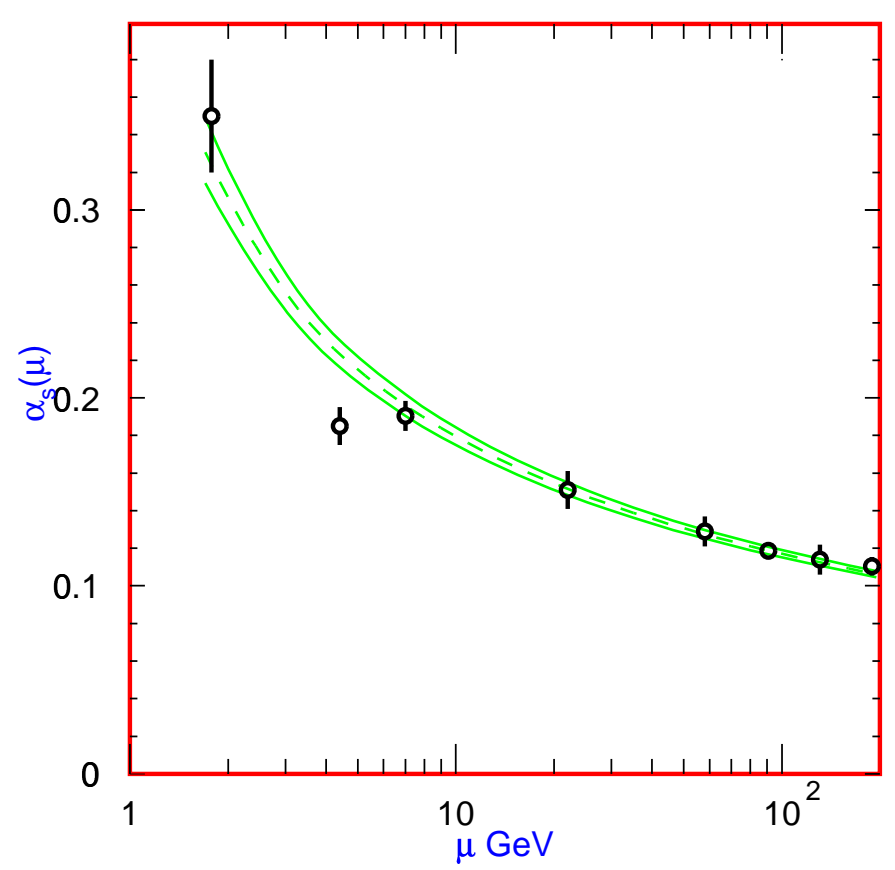

FIGURE 36. The running of $\alpha_{s}\left(Q^{2}\right)$ with increasing energy scale. $\left(\sqrt{Q^{2}}\right.$ labeled as $\mu$ in figure) Data points are experimental measurements.

\subsubsection{Factorization and Cross Sections}

Having gone over some of the features of QCD, we need to work our way back to the problem at hand, using QCD to calculate the cross section for charm production. This may be done using perturbation theory for $Q^{2}$ safely above $\Lambda_{Q C D}$ - i.e. above around $1 \mathrm{GeV}^{2}$. The cross section in terms of the structure functions described in, for example, equation 2.52 is still completely correct in QCD, however the construction of the structure functions themselves must be generalized [94]:

$$
F_{1}^{(V h)}\left(x, Q^{2}\right)=\sum_{i=q, \bar{q}, G} \int_{0}^{1} \frac{d \xi}{\xi} H_{1}^{V i}\left(\frac{x}{\xi}, \frac{Q^{2}}{\mu^{2}}, \frac{\mu_{f}^{2}}{\mu^{2}}, \alpha_{s}\left(\mu^{2}\right)\right) \phi_{i, h}\left(\xi, \mu_{f}, \mu^{2}\right)
$$




$$
\begin{aligned}
& F_{2}^{(V h)}\left(x, Q^{2}\right)=\sum_{i=q, \bar{q}, G} \int_{0}^{1} d \xi H_{2}^{V i}\left(\frac{x}{\xi}, \frac{Q^{2}}{\mu^{2}}, \frac{\mu_{f}^{2}}{\mu^{2}}, \alpha_{s}\left(\mu^{2}\right)\right) \phi_{i, h}\left(\xi, \mu_{f}, \mu^{2}\right) \\
& F_{3}^{(V h)}\left(x, Q^{2}\right)=\sum_{i=q, \bar{q}, G} \int_{0}^{1} \frac{d \xi}{\xi} H_{3}^{V i}\left(\frac{x}{\xi}, \frac{Q^{2}}{\mu^{2}}, \frac{\mu_{f}^{2}}{\mu^{2}}, \alpha_{s}\left(\mu^{2}\right)\right) \phi_{i, h}\left(\xi, \mu_{f}, \mu^{2}\right)
\end{aligned}
$$

These structure function definitions utilize the factorization theorem [127]: that the long distance dependence of the cross section may be factorized apart from the short distance dependence.

This makes sense intuitively, things which are happening quickly can do so without the slow parts of the interaction knowing what's going on. In that case short and long distance processes should be incoherent, shouldn't mix, so there are therefore no cross terms which would prevent the slow and fast processes from factorizing. In the structure functions above, the short distance pieces are contained in the hard scattering terms: $H_{n}^{V i}\left(\frac{x}{\xi}, \frac{Q^{2}}{\mu^{2}}, \frac{\mu_{f}^{2}}{\mu^{2}}, \alpha_{s}\left(\mu^{2}\right)\right)$, and are calculable using perturbation theory. The $\phi_{i, h}\left(\xi, \mu_{f}, \mu^{2}\right)$ are parton distribution functions, and include all the "non-perturbative" long distance scale pieces of the cross section. The parton distributions are not calculable in perturbation theory, so must be determined from experimental measurements. The stream of labels and scales included in equations 2.153-2.155 are meant to highlight various features of the structure functions and pieces thereof. The superscript "V" for each structure function indicates that they are different depending on what sort of vector boson is interacting - the structure functions for photon scattering will be different from those for $\mathrm{W}$ or $\mathrm{Z}$ scattering. The "h" superscript indicates that the structure functions are different for whatever hadron is struck - i.e. a proton or neutron. The sum is over all quark and anti-quark flavors is as in the simple parton model case, however now the gluon is also included ("G"). The hard scattering term $H_{n}^{V i}()$ 
depends on $x$ and $Q^{2}$, and of course $\alpha_{s}$. Both the hard scattering term and the associated parton distribution functions are dependent on the renormalization scale $\mu$ as well as an additional "factorization" scale, $\mu_{F}$. The factorization scale controls the cutoff between what is considered to be a short distance scale contribution, and included in $H_{n}^{V i}()$, and what would be a long distance scale contribution, and included in with the parton distribution functions. Often $\mu$ and $\mu_{F}$ are set to be equal to keep things simple.

The parton distribution functions, despite the necessity of determining them from experimental measurements, are themselves not observables. The convolutions in equations 2.153-2.155 tie the parton distribution functions together with the hard scattering terms to produce the observable structure functions. Experiments are performed to measure the structure functions, and parton distribution functions are extracted using hard scattering terms calculated at a desired order of $\alpha_{s}$. The resulting parton distribution functions (pdfs) are then only usable when convolved with hard scattering terms calculated at that order. The hard scattering terms may be for different processes, but must always be calculated at the same order in $\alpha_{s}$ and with the same assumptions about the factorization scale $\mu_{f}$.

Different ways of dividing the structure functions into the hard scattering and pdfs are referred to as different "factorization schemes". At lowest order in $\alpha_{s}$, the pdfs are delta functions in $(1-\xi)$, independent of scheme, and we recover the structure function definitions of the simple parton model used earlier. At higher orders though, there are several factorization scheme choices one can make. Two of the most commonly used schemes are the DIS [128] and $\overline{M S}[129]$ schemes. The DIS scheme tries to preserve the definition of $F_{2}$ to be the same at all orders of $\alpha_{s}$, 
but does so at the expense of making $2 x F_{1}$ and $x F_{3}$ more complicated. The $\overline{M S}$ scheme defines the pdfs in terms of the creation and annihilation operators for the partons in question. The makes $\overline{M S}$ much simpler to perform calculations in, and it is therefore more often used. The two schemes are equivalent, and should yield the same answer up to what one hopes are small higher order corrections. The calculations used in this thesis all follow the $\overline{M S}$ scheme.

The usefulness of the pdfs as measured in a particular scheme and at a particular order in $\alpha_{s}$ is not limited to the $Q^{2}$ at which they were measured. Parton distribution functions measured at any $Q^{2}$ may be evolved to any other $Q^{2}$ by solving what are called the DGLAP (after Dokshitzer [130], Gribov and Lipatov [131] and Altarelli and Parisi [132]) equations:

$$
\mu^{2} \frac{d}{d \mu^{2}} \phi_{i, h}\left(x, \mu^{2}\right)=\sum_{j=q, \bar{q}, G} \int_{x}^{1} \frac{d \xi}{\xi} P_{i j}\left(\frac{x}{\xi}, \alpha_{s}\left(\mu^{2}\right)\right) \phi_{j, h}\left(\xi, \mu^{2}\right)
$$

where $\mu_{F}$ has been set equal to $\mu$. These are a series (the subscript $i$ in $\phi_{i, h}$ on the left hand side stands for gluons, or any active quark or antiquark flavor) of coupled integro-differential equations which govern the $\mu$ dependence of the parton distribution functions. The $P_{i j}$ 's are the QCD splitting functions, and represent the probability of producing parton type $i$ from type $j$. They are calculated perturbatively to the particular order of $\alpha_{s}$ one is working in.

Pdfs can be be determined from measurements at a particular $Q^{2}$, then the DGLAP equations are solved numerically to find the value of the pdfs at any desired $Q^{2}$. Typically they are described by parameterized functions at a particular scale $Q_{0}$, then evolved and compared to experimental measurements at various $Q^{2}$ via 
the appropriate cross section calculations. The parameters for these functions are determined by the best fit to thousands of data points from dozens of experiments.

\subsubsection{The NLO Charm Cross Section}

The charm cross section is calculated in a similar way as in the simple parton model $[133,134,135]$. For simplicity, we will define $\mathcal{F}_{i}^{\text {charm }}$ structure functions as: $\mathcal{F}_{1}^{\text {charm }} \equiv F_{1}^{\text {charm }}, \mathcal{F}_{2}^{\text {charm }} \equiv \frac{1}{2 \xi} F_{2}^{\text {charm }}, \mathcal{F}_{3}^{\text {charm }} \equiv \frac{1}{2} F_{3}^{\text {charm }}$. We may then define the

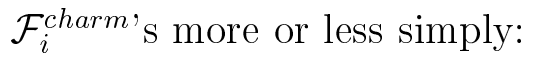

$$
\mathcal{F}_{i}^{\text {charm }}=q\left(\xi, \mu^{2}\right)+\frac{\alpha_{s}\left(\mu^{2}\right)}{2 \pi} \int_{\xi}^{1} \frac{d \xi^{\prime}}{\xi^{\prime}}\left[H_{i}^{q}\left(\xi^{\prime}, \mu^{2}\right) q\left(\frac{\xi}{\xi^{\prime}}, \mu^{2}\right)+H_{i}^{g}\left(\xi^{\prime}, \mu^{2}\right) g\left(\frac{\xi}{\xi^{\prime}}, \mu^{2}\right)\right]
$$

Here $\xi$ is the usual slow rescaling variable, and $q(), g()$ are pdfs for the quarks and gluons respectively. The quark distribution is similar to what was done in the simple parton model case, and for neutrino scattering off an isoscalar nucleus would be:

$$
\begin{aligned}
q\left(\xi, \mu^{2}\right)= & \left|V_{c d}\right|^{2}\left[d_{v}\left(\xi, \mu^{2}\right)+u_{v}\left(\xi, \mu^{2}\right)+d_{s}\left(\xi, \mu^{2}\right)+u_{s}\left(\xi, \mu^{2}\right)\right] \\
& +2\left|V_{c s}\right|^{2} s\left(\xi, \mu^{2}\right)
\end{aligned}
$$

And for antineutrinos:

$$
\bar{q}\left(\xi, \mu^{2}\right)=\left|V_{c d}\right|^{2}\left[d_{s}\left(\xi, \mu^{2}\right)+u_{s}\left(\xi, \mu^{2}\right)\right]+2\left|V_{c s}\right|^{2} \bar{s}\left(\xi, \mu^{2}\right)
$$

Here as usual we've assumed both isoscalar invariance and that the up and down seas are the same as their anti-partner seas. The hard scattering terms, $H_{i}^{q}$ and 


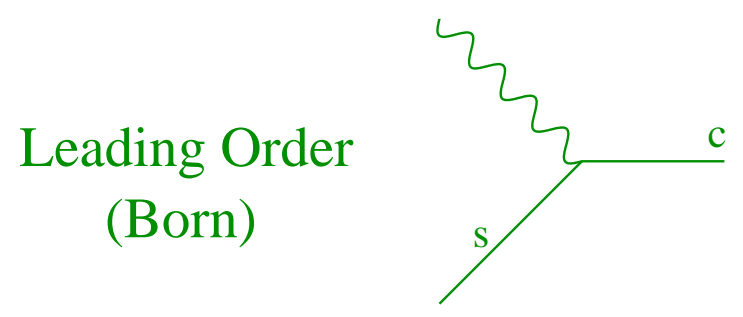

Next To Leading Order (gluon initiated)

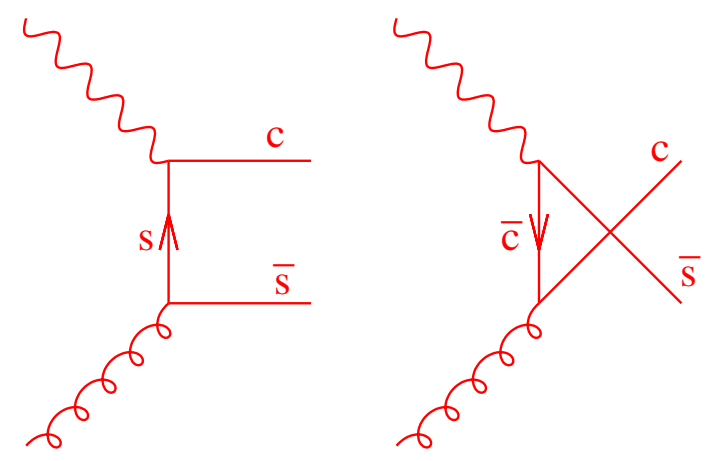

\section{Next To Leading Order}
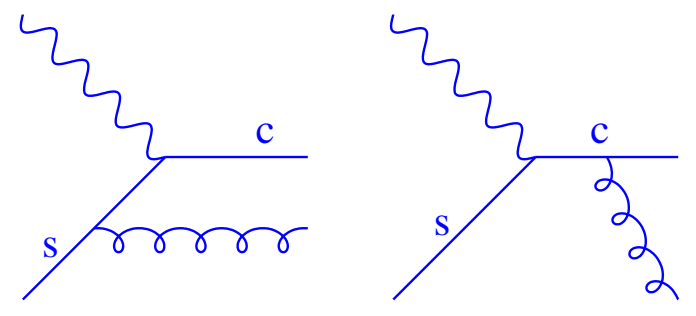

\section{(quark initiated)}

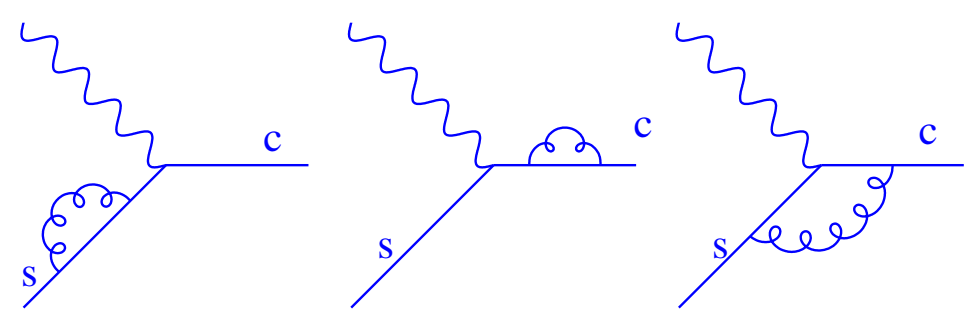

FIGURE 37. Feynman diagrams included in NLO charm cross section calculation 
$H_{i}^{g}$ are calculable in perturbation theory, and represent the first order QCD contributions initiated off quarks or gluons respectively. The Feynman diagrams in figure 37 represent those which are included in the charm cross section calculation. The topmost diagram labeled "Leading Order" is the Born term, $q\left(\xi, \mu^{2}\right)$ in equation 2.157. In this case "leading order", or "LO" means no gluon vertices, or the case we considered in section 2.4. Although the same Feynman diagram represents both the LO charm cross section and the Born term in the NLO cross section, they are not equal, by virtue of the fact that the pdfs in the two cases are not equal. The two sets of diagrams classified as "Next To Leading Order" represent the terms in the structure functions with one gluon vertex, or first order in $\alpha_{s}$. The "gluon initiated" diagrams are those included in the $H_{i}^{g}\left(\xi^{\prime}, \mu^{2}\right)$ hard scattering term, and the bottom "quark initiated" diagrams are those included in the $H_{i}^{q}\left(\xi^{\prime}, \mu^{2}\right)$ hard scattering term. Figure 38 shows the components of the NLO charm cross section as a function of $x$, along with the simple parton model cross section (labelled "LO"). Note the difference between the Born curve in green, and the LO term in purple. This is essentially the difference between the quark pdfs at LO and at NLO. Also note that the total NLO cross section and the LO cross section are very similar. This is because those total cross sections were what was fit to data to determine the pdfs. If those fits were at all correct, one would expect the total cross sections to be fairly similar!

We now have the bare charm cross section at NLO, and again we must clothe this poor charm quark to form an observable charmed hadron. Fragmentation beyond LO however is not as simple as it was in the simple parton model case. In 


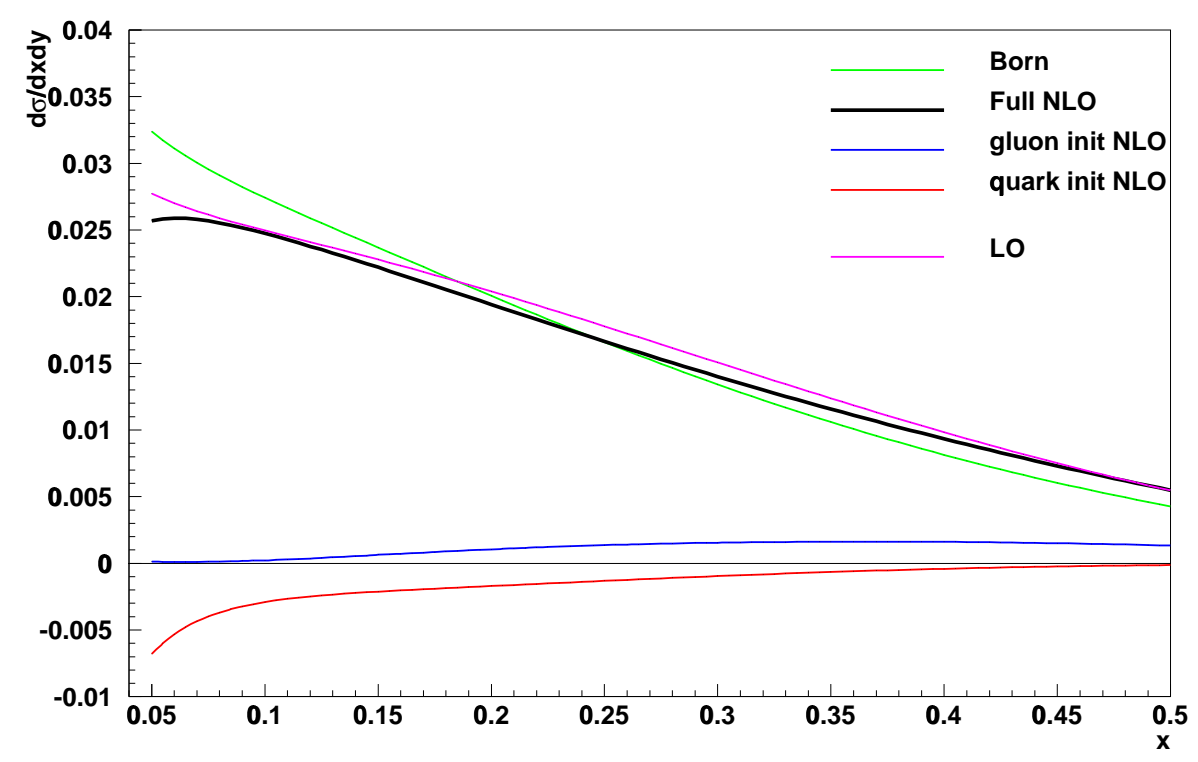

FIGURE 38. NLO charm cross section components versus $x$

the NLO case we have:

$$
\begin{array}{r}
\mathcal{F}_{i}^{D}=q\left(\xi, \mu^{2}\right) D(z)+\frac{\alpha_{s}\left(\mu^{2}\right)}{2 \pi} \int_{\xi}^{1} \frac{d \xi^{\prime}}{\xi^{\prime}} \int_{z}^{1} \frac{d \zeta}{\zeta}\left[H_{i}^{q}\left(\xi^{\prime}, \mu^{2}, \zeta\right) q\left(\frac{\xi}{\xi^{\prime}}, \mu^{2}\right) D\left(\frac{z}{\zeta}\right)+\right. \\
\left.H_{i}^{g}\left(\xi^{\prime}, \mu^{2}, \zeta\right) g\left(\frac{\xi}{\xi^{\prime}}, \mu^{2}\right) D\left(\frac{z}{\zeta}\right)\right]
\end{array}
$$

Here we now have to convolve the fragmentation function with the hard scattering terms in the cross section over all possible virtual charmed quark momenta, represented by $\zeta \equiv \frac{p_{c} \cdot p}{q \cdot p}$. Note that at LO, without the $\alpha_{s}$ terms we return to the multiplicative application of the fragmentation function as we had done earlier (equation 2.133).

Figure 39 shows the differential NLO charm cross section $\frac{d \sigma}{d x d y d z}$ plotted as a function of $z$. Included in the plot are the Born, gluon initiated NLO, and 


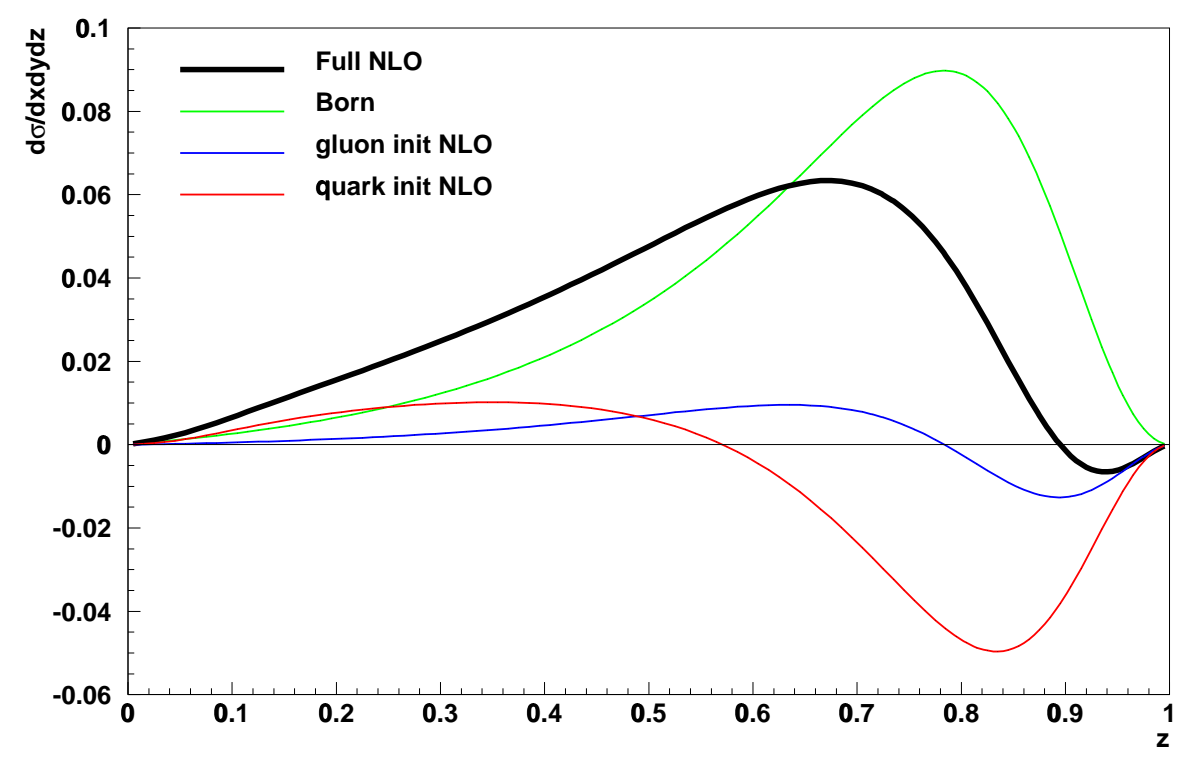

FIGURE 39. NLO charmed hadron cross section versus $z$

quark initiated NLO components of the cross section. As one might expect the $z$ dependence of the Born piece is exactly as it is at LO, but the $z$ dependence of the two NLO pieces have somewhat different behavior. Most disconcerting is the negative dip they have at high z. Figure 40 attempts to show how this comes about in terms of the LO and gluon initiated NLO diagrams. On the top of the figure we have the Born level diagram labelled "LO". Next to it we have the $\zeta$ distribution for that diagram. At LO, the charm quark is produced collinearly with the interacting W-boson in the W-parton CMS frame, so the $\zeta$ distribution is a delta function at the maximum value. When this is convolved with the fragmentation function $D\left(\frac{z}{\zeta}\right)$, the usual distribution as in figure 31 results. Similar goes for the "SUB" term, which also is collinear with the incoming $\mathrm{W}$, but as one might suspect from the name, is negative. Finally we have the gluon initiated term, for which the charm 

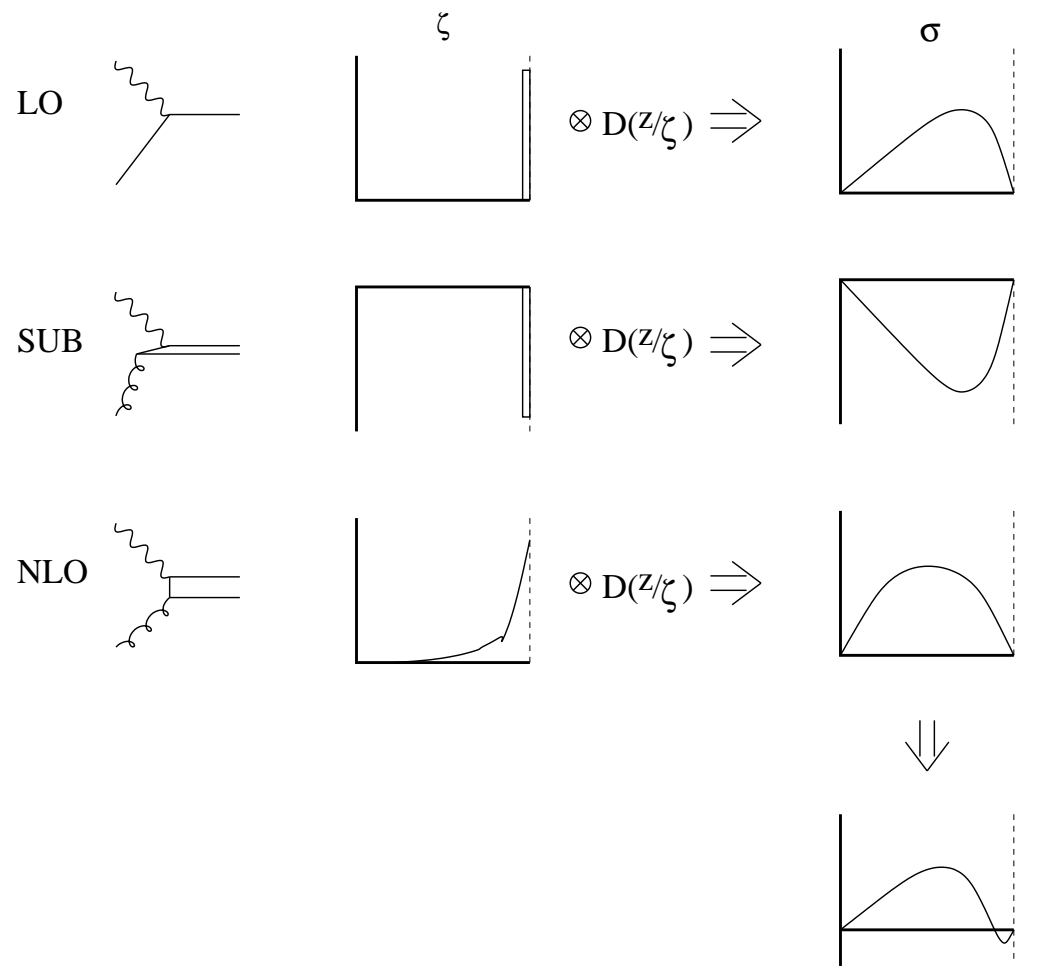

FIGURE 40. Cartoon explanation of why we have a negative bump at high z

is not necessarily collinear with the W. This makes the $\zeta$ distribution, although still peaked at the collinear end, now have a finite width. The effect of this is to produce a $D(z)$ distribution after the convolution that is peaked at lower $z$ than the LO and SUB cases. The three pieces are added together, producing the $z$ distribution shown at the bottom, which results in an undershoot from the three pieces having $z$ distributions that don't exactly line up.

This negative undershoot can cause trouble when generating Monte Carlo. Often what is done is a random value of $z$ is thrown, and the cross section as a function of $z$, proportional to the probability of obtaining that value of $z$, is used to determine whether that throw should be kept or not. The negative dip would lead to a negative probability for $z$ 's in that range however. The integral over $z$ of the 

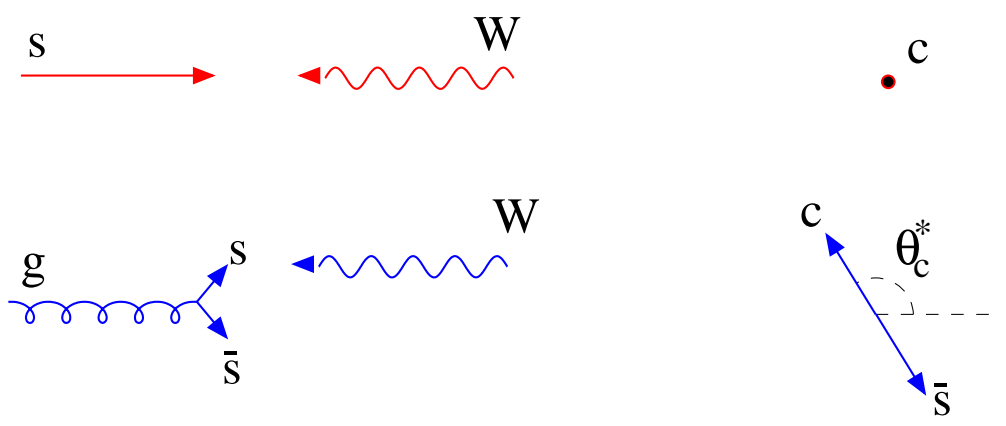

FIGURE 41. Transverse momentum of LO and NLO diagrams

cross section does properly lead to a cancellation of the collinear divergence that the subtraction term removes, so the solution to this problem is to evaluate the cross section in piecewise integrated bins with a width such that negative weights occur. As the Monte-Carlo needs to model the acceptance for a particular event, as long as those bins are small enough that the bins are insignificant compared to the resolution of the detector, we are saved.

The transverse momentum due to the diagrams with gluons in them are of concern with respect to event acceptance. Figure 41 shows how the transverse momentum is different in the LO and NLO cases in the center of mass frame between the $\mathrm{W}$ boson and the struck parton. At LO, there are only two bodies interacting, the struck quark and boson. That results in a charm quark at rest in that frame (though called collinear with the $\mathrm{W}$ because of the boost to get to that frame). A gluon-W interaction is shown in the NLO case. There a gluon splits into a quark-antiquark pair, one of which interacts with the $\mathrm{W}$ boson. The splitting between the quark and antiquark pair imparts transverse momentum to the charm quark in the CMS frame. Whether or not the decay products from this charm quark are accepted due to applied energy cuts in the actual measurement depends 
on how much transverse momentum there is. Transverse momentum is in some sense "lost" when the system is boosted to the lab frame, so that more transverse momentum means less probability of an event passing energy cuts. The collinear case, with zero transverse momentum, has the best chance of being accepted.

A quantity related to transverse momentum is rapidity:

$$
\eta_{c}=\frac{1}{2} \log \frac{E+p_{\|}}{E-p_{\|}}
$$

Here $p_{\|}$refers to the particle momentum in the same direction as the frame in which its evaluated. The symbol $\eta_{c}$ is used rather than the usual $y$ for rapidity to avoid confusion with the inelasticity. Rapidity is a useful quantity to work with as it transforms well between different reference frames. The log also tends to smooth distributions, making it easier to work with in a Monte-Carlo (though the log of a singularity is still a singularity!).

Event acceptance depends on both fragmentation $z$ and on the $\eta_{c}$ of the charm quark. Producing an NLO cross section differential in both of these variables (and Bjorken $x$, and $y$ ) however leads to an over-constrained problem, leaving singularities in the resulting $d \sigma / d x d y d z d \eta_{c}$ cross section. This difficulty is solved in a similar way as the negative dip problem in the fragmentation distribution [136].

Both $z$ and $\eta_{c}$ are integrated within bins large enough to bulldoze away any singularities, but small enough so as not to be visible within detector resolution. Figure 42 shows an example of the cross section at fixed neutrino energy, $x$ and $y$, plotted as a function of rapidity and fragmentation $z$. Along the back wall, at most negative (negative thanks to an unfortunate $\mathrm{z}$ axis direction choice early on in the calculation) rapidity is the collinear limit, where $p_{\perp}$ is zero. There we can 


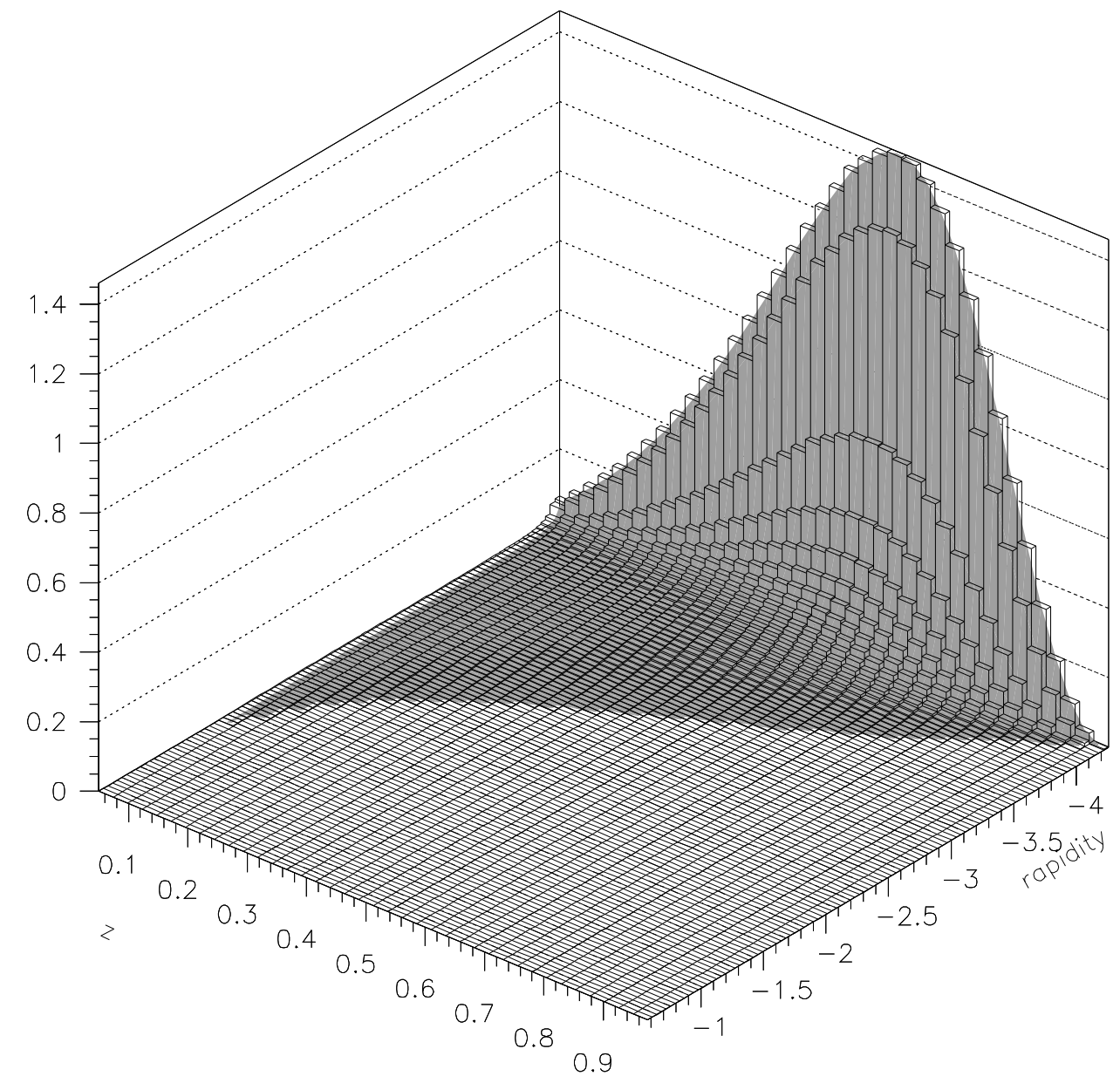

FIGURE 42. Cross section vs rapidity and $z$ for fixed $x$ and $Q^{2}$. 
see the familiar shape of the fragmentation function vs $z$. The leading order case would only have this function along the back wall, with zero extent away from collinear rapidity. Here in the NLO case, as we pull away from the back wall, we are increasing the transverse momentum component, the cross section gets smaller quickly, but also prefers smaller values of $z$. We will see more of the effects of this when we discuss event acceptance in a later chapter.

\subsubsection{Some Words About CCFR... .}

About 10 years prior to this analysis, the CCFR collaboration performed the first NLO QCD analysis of $\nu$ and $\bar{\nu}$ charm production $[63,135]$. At that time NLO QCD calculations for this process were in their infancy, and as a result several approximations had to be taken in the CCFR analysis. As the work documented here has benefited greatly from the iterative discussions and work on both the experimental and theoretical sides since then [135], it is appropriate to discuss the approach taken in the CCFR analysis, and compare it to what is done now.

The CCFR analysis was based on the newly-published ACOT [134] calculation, where it was argued that the Born diagram and gluon initiated NLO diagrams were a sufficient approximation for the full NLO charm production cross section. The NLO quark initiated diagram, being multiplied by a factor of $\alpha_{s} \sim 0.1$ was assumed to be be small compared to the NLO gluon initiated diagram. In the gluon case however, the large size of the gluon pdf would effectively cancel that factor.

The diagrams included in the CCFR calculation were then the top two rows in figure 37, with an additional "subtraction" term that removed the collinear 
singularity in the gluon initiated diagram. (We did not go into the technical details of the calculation, but the calculation we use has this subtraction term as well, as well as two more in the quark initiated diagrams. A singularity occurs in the gluon initiated diagram when the charm quark is collinear with the $\mathrm{W}$ boson in the W-parton CMS frame. The subtraction term removes this singularity, as well as the double counting between that case and the Born diagram - which always is collinear with the $\mathrm{W}$ boson.).

Fragmentation was handled as it is at LO, as a multiplicative term to the total cross section. The CCFR analysis used re-weighted semiinclusive $\left(\frac{d \sigma}{d x d y}\right)$ MonteCarlo generated with a LO cross section [62], and therefore did not have the $p_{\perp}$ dependence that an NLO model would have. To correct for the effects of $p_{\perp}$ at NLO, an acceptance correction was applied to the gluon-initiated term in the NLO cross section:

$$
W_{W+N \rightarrow c+X}=\underbrace{f_{q}^{N} \otimes \omega^{0}}_{\text {born }}-\underbrace{f_{g}^{N} \otimes f_{g}^{q} \otimes \omega^{0}}_{\text {subtraction }}+\underbrace{\mathcal{A} f_{g}^{N} \otimes \omega^{1}}_{\text {gluon } L O}
$$

Here the f's are the pdfs, $\omega$ 's are hard scattering terms, and the $\otimes$ indicates the convolution over $\xi^{\prime}$. $\mathcal{A}$ is the acceptance factor applied to the gluon initiated diagrams in the cross section. A constant value of 0.6 was used, which was the average acceptance calculated from fixed points ranging from $x$ values of $\sim 0$ to 0.15 and $Q^{2}$ from 1 to $5000 \mathrm{GeV}^{2}[124]$. 
CCFR also described the strange sea in terms of external level and shape parameters applied to already evolved sea pdfs. In the CCFR case:

$$
x s\left(x, \mu^{2}\right)=A_{s}(\kappa)(1-x)^{\alpha}\left[\frac{x \bar{u}\left(x, \mu^{2}\right)+x \bar{d}\left(x, \mu^{2}\right)}{2}\right]
$$

The factor $A_{s}$ is a normalization factor which forced the level of the total sea quark distributions be unchanged with shifts in the size of the strange sea. Technically, applying the $(1-x)^{\alpha}$ factor to $x$ dependent solutions to a set of integro-differential equations (eq. 2.156) is an approximation, resulting in an effective re-definition of what the fitted parameters mean. One strictly would not be able to use the fitted $\kappa$ and $\alpha$ parameters as fitted in the case of equation 2.163 , and apply them to a properly evolved sea, defined at a single $Q_{0}$ scale.

The following is a summary of the improvements in the NLO charm model since the CCFR analysis, now applied in this $\mathrm{NuTeV}$ analysis:

- All diagrams in figure 37 are now included in the cross section calculation.

- Fragmentation is now properly convolved with the NLO terms (equation 2.160)

- Charm $p_{\perp}$ dependence is now directly included in the cross section

- Pdf definitions are all applied at an initial $Q_{0}^{2}$ scale, then evolved using numerically solved DGLAP evolution equations 
Add these model improvements to the large antineutrino sample, and very pure sign selected beams, and we should be able to have some useful things to say about the strange and antistrange seas. We'll start seeing how to do that in the next chapter. 


\section{CHAPTER III}

\section{THE NUTEV EXPERIMENT AND DATA SAMPLE}

\section{$\underline{3.1 \text { Overview }}$}

A very specialized environment is needed to examine the physics outlined in the previous chapter. There are two parts to the construction of this environment. To study neutrino scattering, neutrinos are of course required, and that is provided in the form of a particle beam of neutrinos. To examine the incredibly small timescales involved in deep inelastic scattering the neutrinos need to be very energetic, 10's to 100's of GeV. How the neutrinos were produced and controlled in the beam for the $\mathrm{NuTeV}$ experiment is explained in the beamline section of this chapter.

The second part of this specialized environment is the target that the neutrinos interact with, and detector to record that interaction. As neutrinos only interact with matter via the aptly named weak interaction, they do not do so very often. This means a neutrino experiment requires either a lot of neutrinos, or a lot of material in the target. NuTeV did both. The target material must also be instrumented adequately so the interaction can be recorded with enough information so as to decipher the physics processes which occurred. The detector section below describes the $\mathrm{NuTeV}$ neutrino detector, which also served as the target for the neutrino interactions. 


\subsection{The Neutrino Beam}

The beam of neutrinos which interacted with the $\mathrm{NuTeV}$ detector began life as a beam of $800 \mathrm{GeV}$ protons in the TeVatron. Figure 43 shows schematically the path these protons took from their small bottle of hydrogen gas to their untimely demise in the $\mathrm{NuTeV}$ beryllium oxide target. The gas was ionized inside the Cockroft-Walton accelerator. Despite what one might expect, the electrons were not stripped from the hydrogen atoms right away, but instead ionized to have a negative charge. A DC potential then accelerated the $H^{-}$ions to an energy of 750 keV. From the Cockroft-Walton, the ions were inserted into a 500 foot long linear accelerator, which accelerated them to an energy of $400 \mathrm{MeV}$. The electrons were then stripped from the hydrogen ions, making them bare protons. The protons were then inserted into a the "Booster" synchrotron accelerator, which accelerated them to an energy of $8 \mathrm{GeV}$.

When NuTeV ran in the 1996-97 fixed target run, the protons were then inserted from the booster into the "main ring". The aging main ring was the original high energy accelerator at Fermilab, and was replaced by the "main injector" after the fixed target run. The main ring accelerated the protons to $150 \mathrm{GeV}$, and then handed the protons off to the TeVatron. The TeVatron remains, at least of this writing, the world's highest energy particle accelerator. During the NuTeV run, the TeVatron accelerated protons to $800 \mathrm{GeV}$. Since then the performance of the accelerator has improved considerably, and it is now able to achieve energies in the upper $900 \mathrm{GeV}$ range.

Once the proton energy was ramped up to $800 \mathrm{GeV}$ in the TeVatron, protons were extracted out and directed into the neutrino beamline. The neutrino beamline 


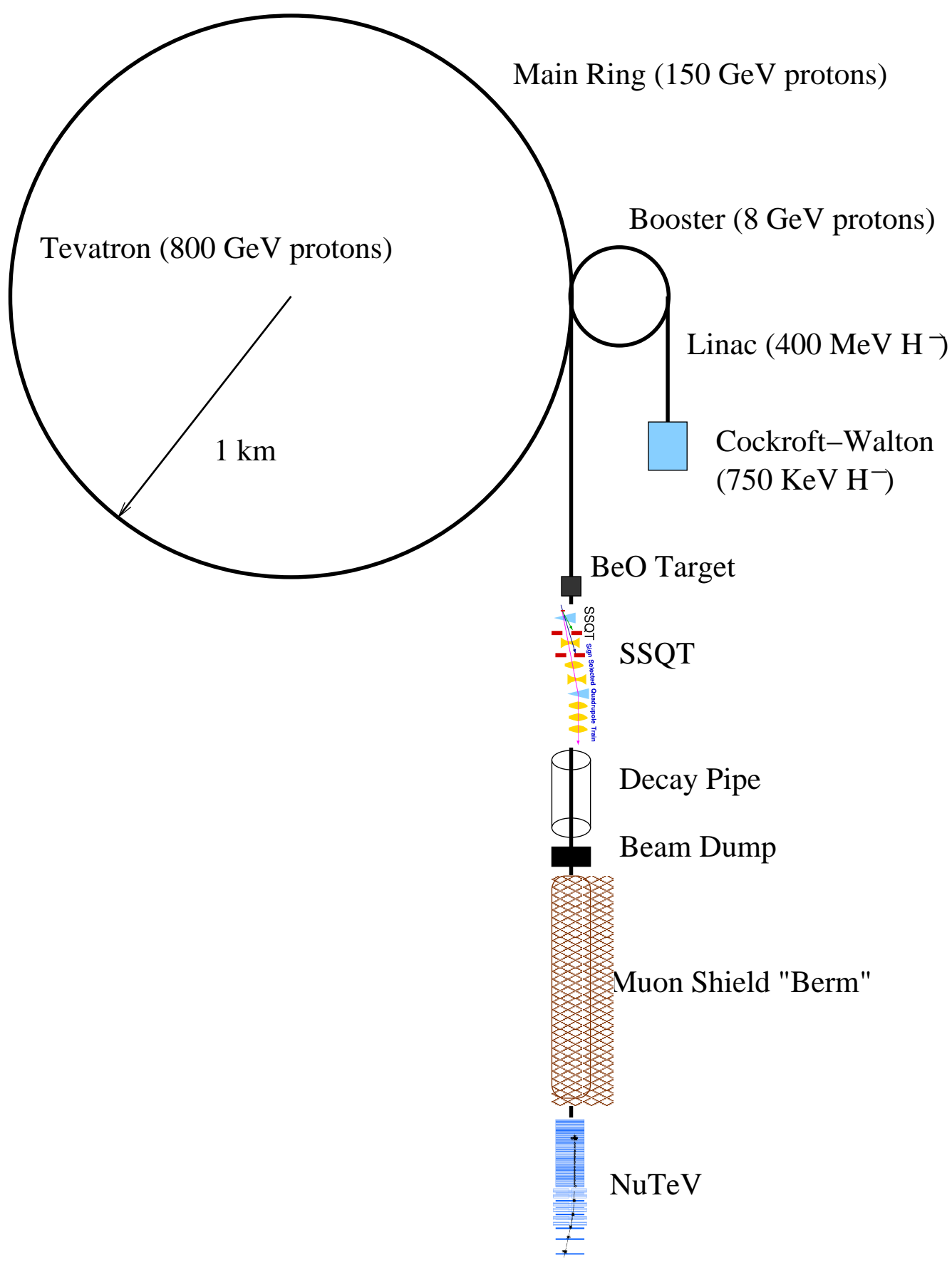

FIGURE 43. Where NuTeV's neutrinos came from, the path particles took from $H^{-}$ions in the Cockroft-Walton, to protons in the TeVatron, to neutrinos en route to the $\mathrm{NuTeV}$ detector. 
at Fermilab was one of 3 beam lines constructed for fixed target experiments. Its layout is shown in figure 44, with protons originating from the switchyard at the bottom of the figure. The neutrino beam cuts through the center of the figure, reaching the $\mathrm{NuTeV}$ detector towards the top. The $\mathrm{NuTeV}$ test beam is also highlighted just to the right of the neutrino beamline. The KTeV experiment also shared the neutrino beamline, located towards the lower left of the figure.

The $\mathrm{NuTeV}$ detector was necessarily very large, and this large size brought with it the disadvantage of having cosmic rays interact within the detector volume frequently. Under certain circumstances cosmic rays could be mistaken for real neutrino interactions. In order to reduce the background due to these cosmic rays, the protons were not extracted from the TeVatron ring continuously, but in five short bursts called "pings". The pings concentrated the beam into a short time period, minimizing the chance of a cosmic ray interacting in the detector at the same time.

Figure 45 shows a sketch of the TeVatron magnet current and an accompanying plot of the number of protons in the beam over time. The TeVatron ran in one minute cycles, during each of which the TeVatron was filled, ramped up to energy, then dumped to the fixed target experiments. The proton occupancy plot shows the TeVatron initially full, then dumping beam in steps during the five pings (called "fast spill"), then slowly dribbling out beam at the end in "slow spill". Slow spill was used for the $\mathrm{NuTeV}$ calibration beam, as well as the other fixed target experiments running at the same time. Figure 46 shows a schematic of the neutrino beamline proton intensity, with the timing of the pings and slow spill. Figure 47 shows the shape of individual pings both before and after they were widened 


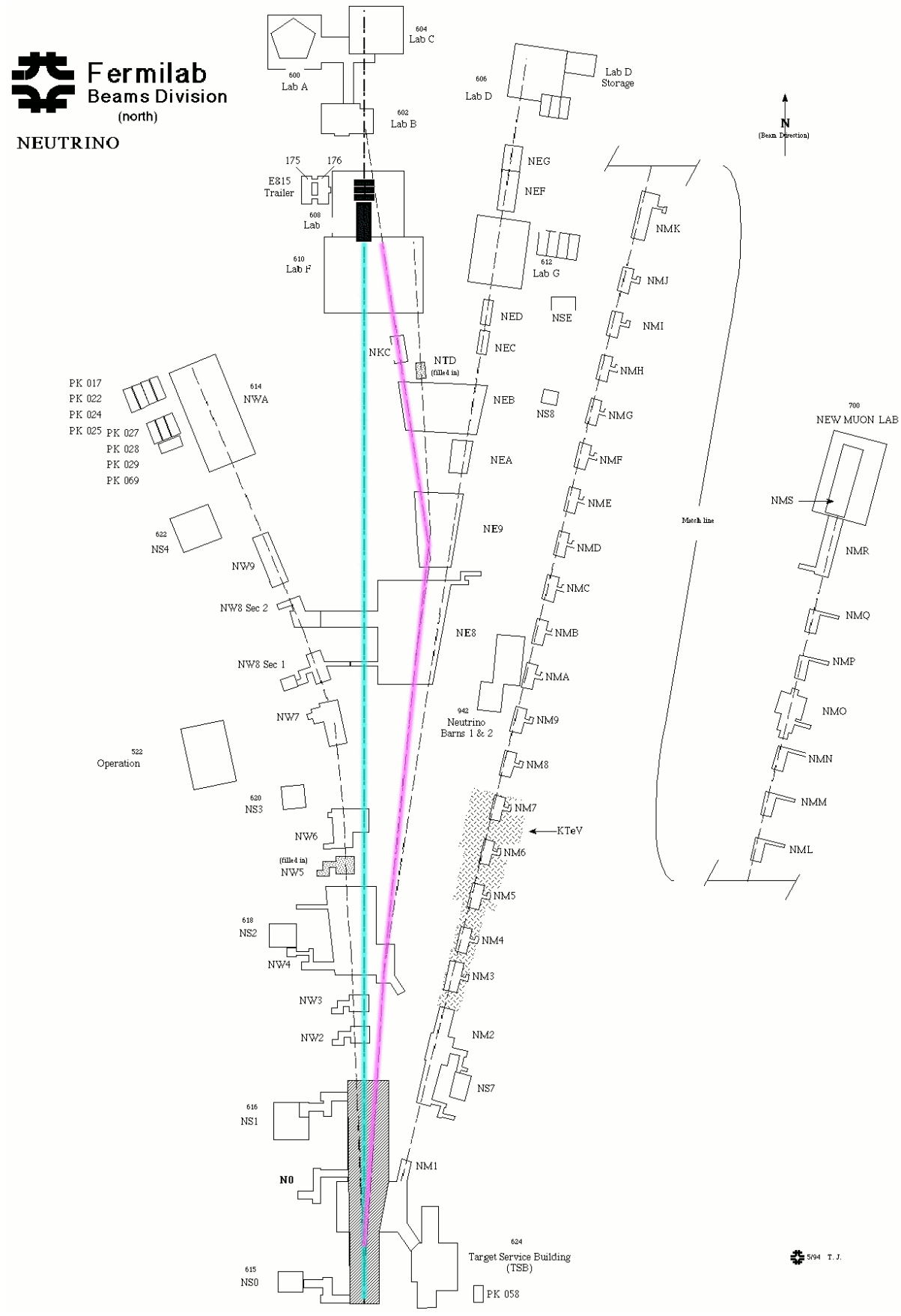

FIGURE 44. Diagram of fixed target beams. The neutrino beam is highlighted in blue, and the calibration beamline is highlighted in pink. The $\mathrm{NuTeV}$ detector is shown in black in the LabE/F enclosures. 


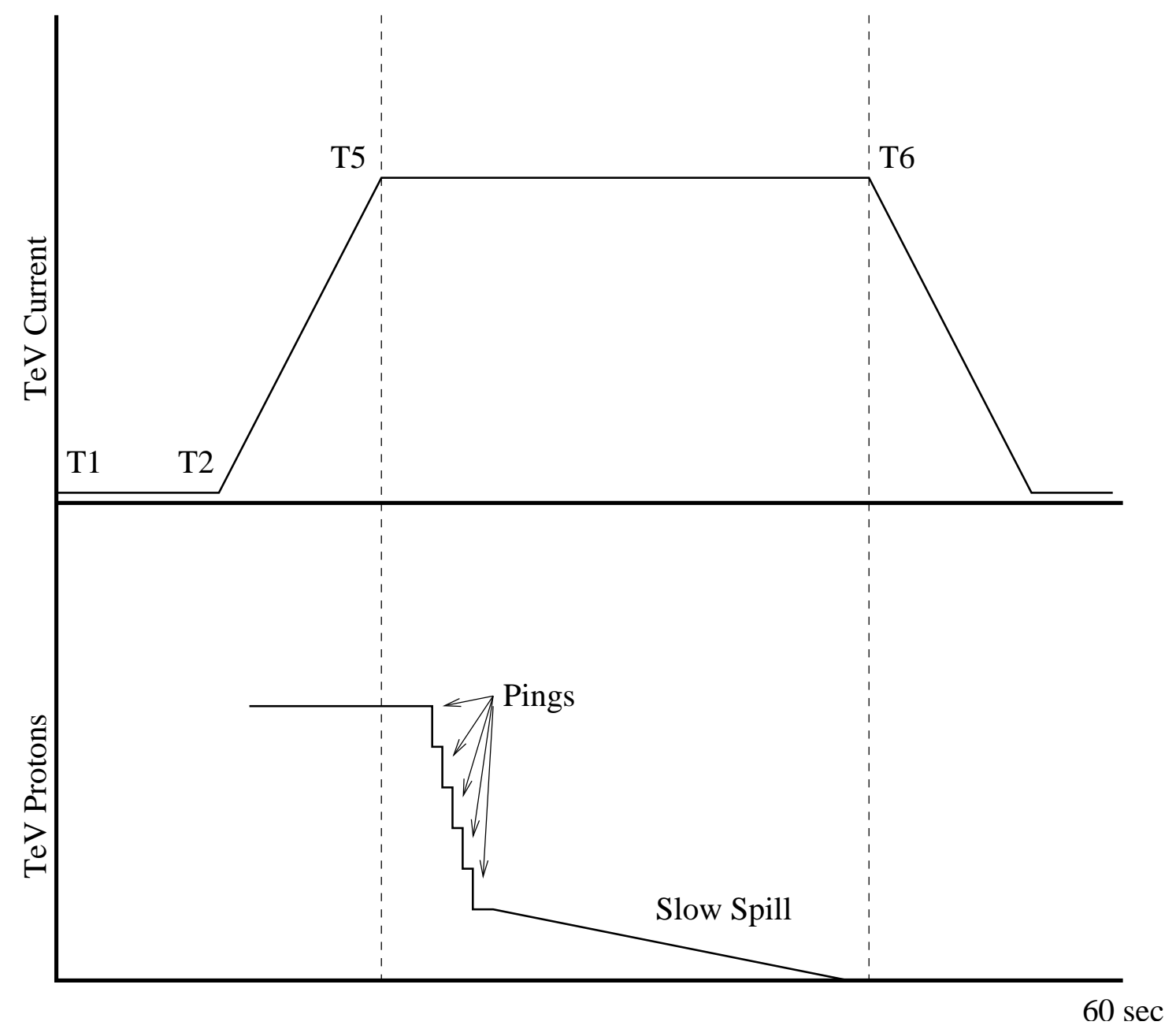

FIGURE 45. TeVatron magnet ramp and proton occupancy versus time

during the early part of the run in 1997. The more Gaussian shaped pings sent too high intensity beam to the detector at their peak, causing readout electronics to saturate due to multiple interactions per ping. The pings were widened to spread the beam out over a bit more time and reduce detector downtime by about $66 \%$ $[137]$.

With beam extracted from the TeVatron, most of it was directed down the neutrino beamline towards the $\mathrm{NuTeV}$ target. A schematic of the neutrino beam- 


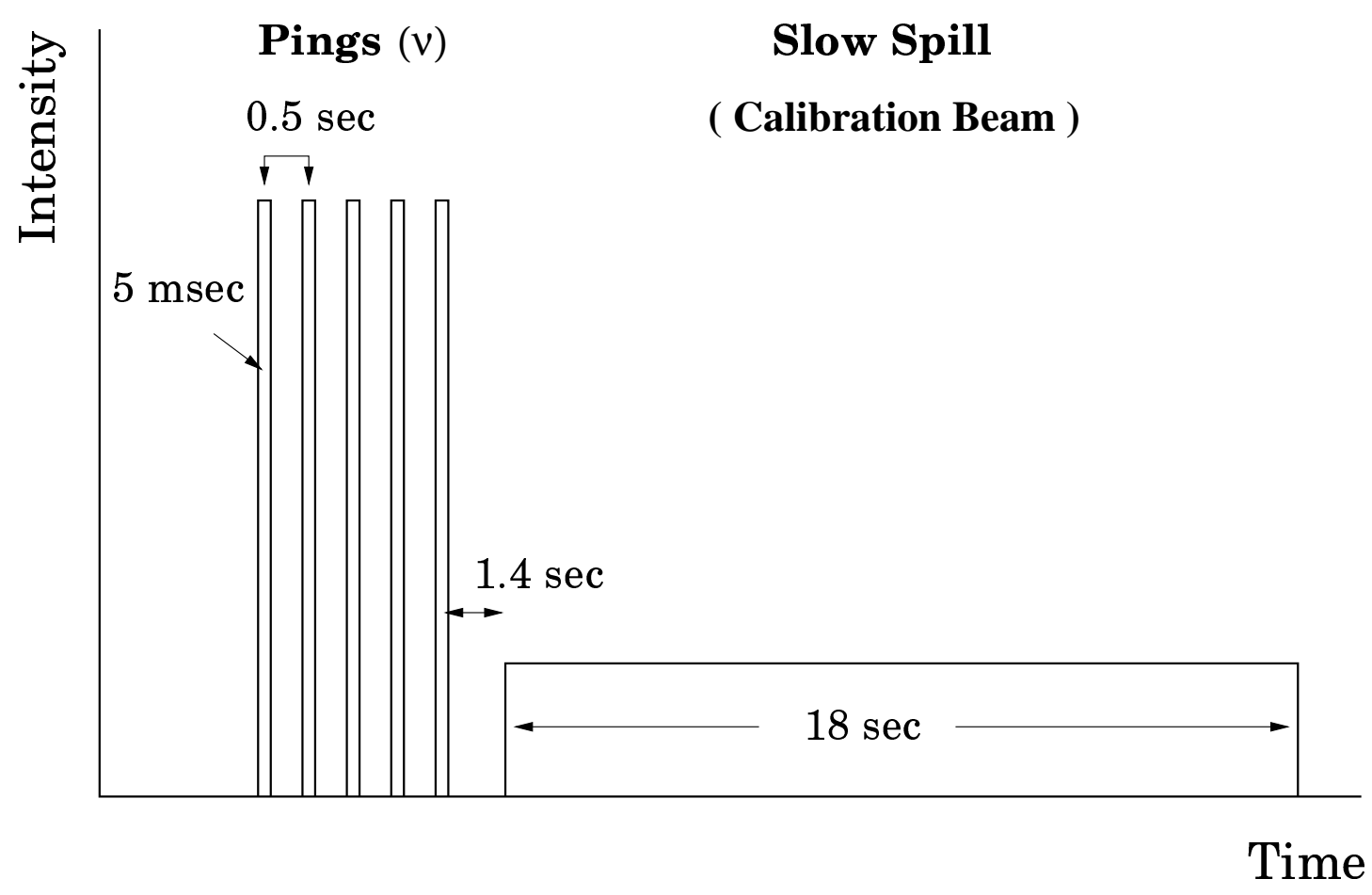

FIGURE 46. Timing of pings and slow spill

line is shown in figure 48. The schematic shows both beam monitoring devices and magnets. In the figures, triangles are dipole magnets, which changed the direction of the beam, convex or concave lens shapes are quadrupole magnets, which focused or defocused the beam. Quadrupole magnets focus a charged particle beam along one axis and defocus it along the other. Whether a quadrupole is shown as a convex or concave lens is determined by whether it defocused or focused along the beam $\mathrm{x}$ axis.

Several of the devices used to measure the characteristics of the beam are also shown in figure 48. The circles with a pair of "(" shaped lines in them are beam position monitors (BPMs), essentially capacitors aligned with the beam axis passing between the plates. The difference in the induced charge between the 


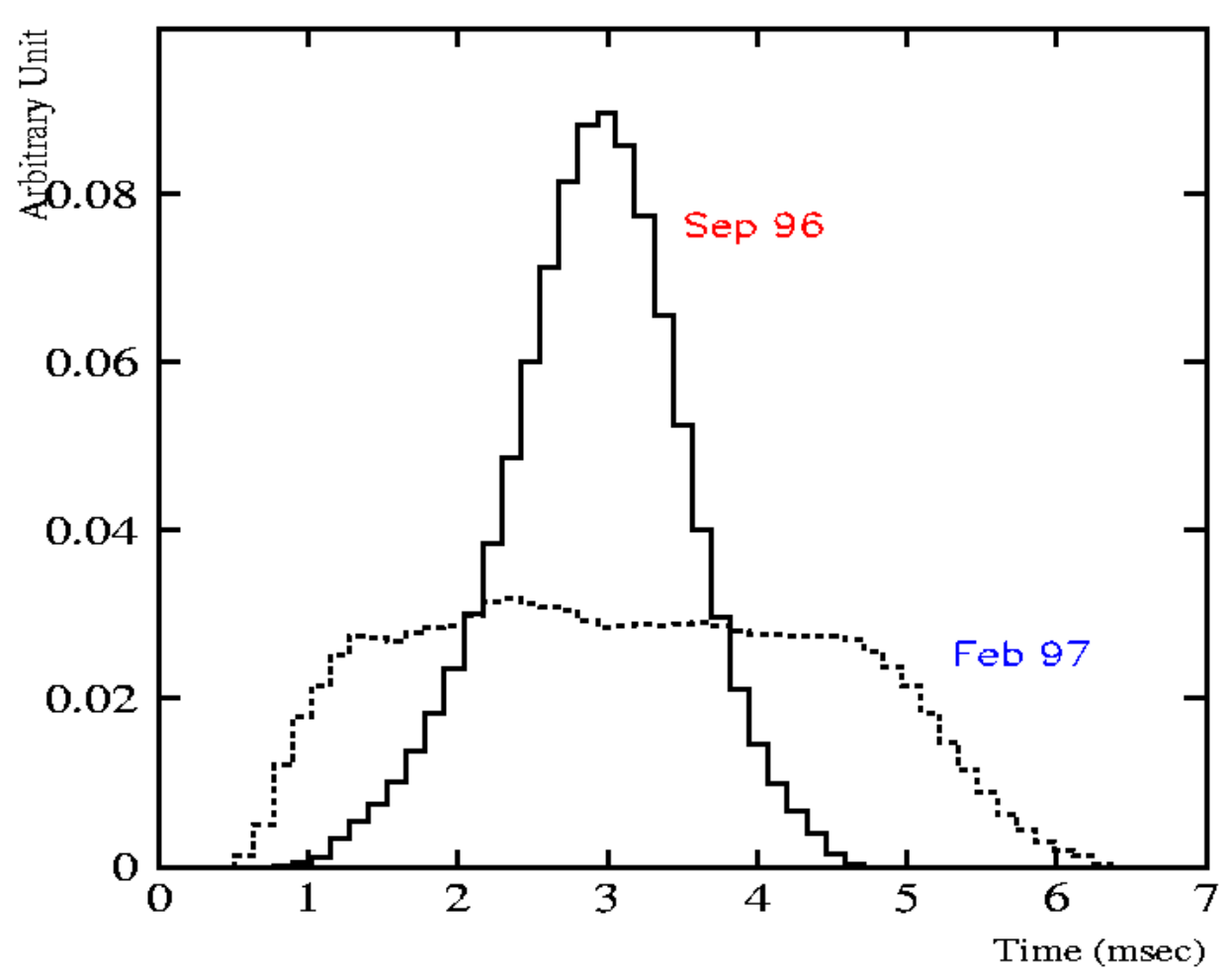

FIGURE 47. The shape of the NuTeV pings, as measured by the beam toroid, both before and after widening in early 1997

two plates would determine the beam position, and their sum the relative beam intensity. BPMs are typically used in pairs, each of the two measuring the position in the $\mathrm{x}$ or $\mathrm{y}$ direction. The four highlighted BPMs were devices constructed by the University of Oregon machine shop.

The squares with lined circles in the middle of them represent "SWICs", or "Segmented Wire Ionization Chambers". These measured the cross sectional intensity profile of the proton beam along the $\mathrm{x}$ or $\mathrm{y}$ axis. SWICs consist of a series of wires placed within a gas chamber held at a high voltage relative to the body of the device. As the beam passes through the gas, it ionizes it, and a charge is induced in the wires, allowing measurement of the beam profile. The presence 
From Switchyard
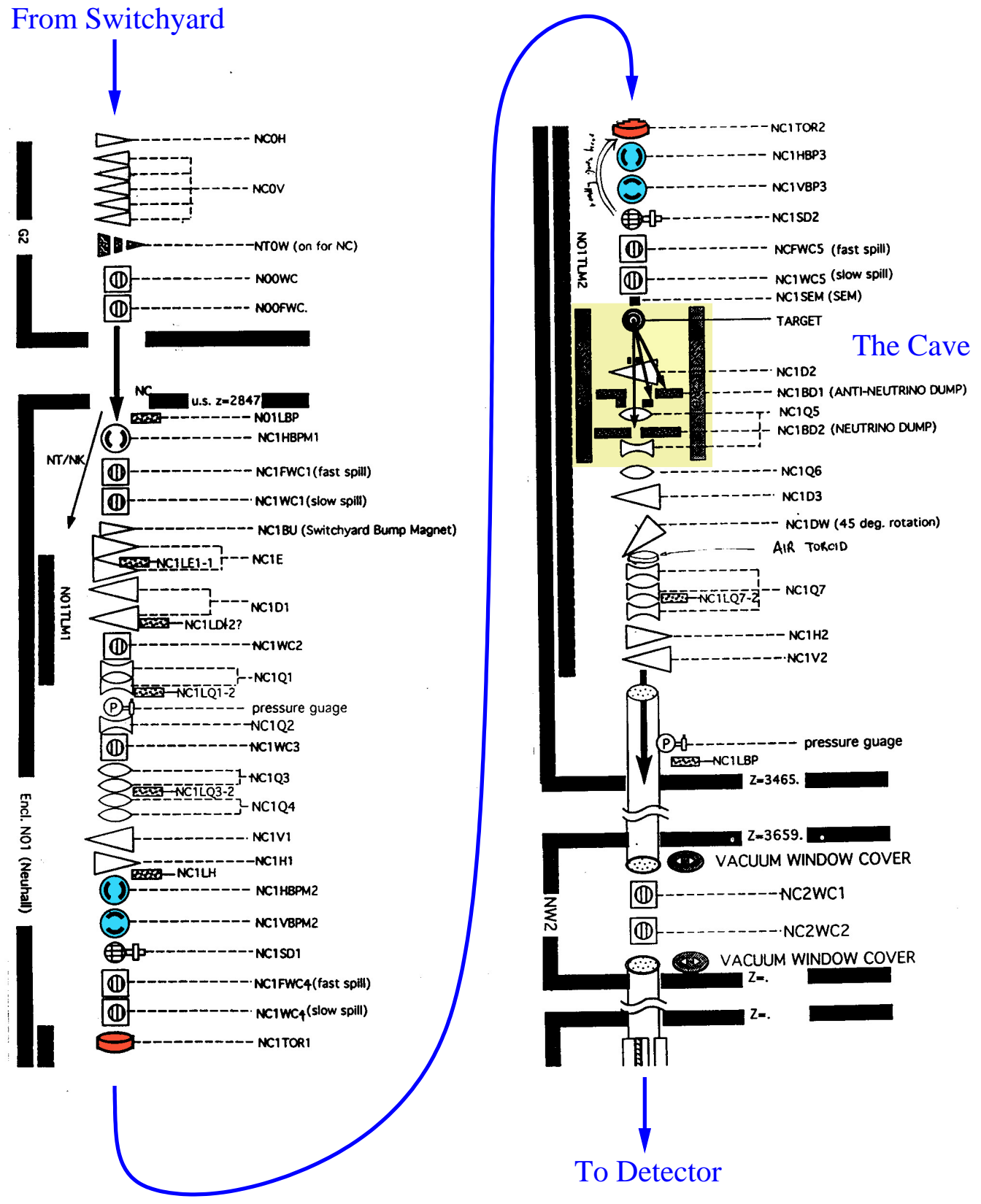

To Detector

FIGURE 48. Diagram of $\mathrm{NuTeV}$ beam target, beam optics and magnets 
of the gas both enables the measurement, but also causes smearing in the profile measurement due to dispersion within the gas.

The hatched circles with cross-like shapes attached to them are the "SEEDs", or "Secondary Emission Electron Detectors" [138]. These detectors were used to measure the beam profile more directly, and were used as a means of calibrating the SWICs. The SEEDs were related to the SWICs, except there was no gas inside to disperse the charge. In their case the charge was induced directly on the wires themselves. This allowed finer measurement of the beam profile, but did so at the expense of the wires. To reduce damage on the wires from the beam, the SEEDs were dipped in and out of the beam periodically throughout the run.

The highlighted pill-like shapes at the bottom of the left and top of the right half of figure 48 are beam toroids, discussed in some detail in appendix A. These devices were toroidal coils placed coaxial with the beam to measure the beam current. They were used as a "beam gate" for the $\mathrm{NuTeV}$ experiment, as well as to calibrate the intensity measurements of the other beam monitoring devices, as the beam current can be determined from first principles. There were three toroids placed in the beamline, two (one active, one spare) in front of the target to measure the intensity of the proton beam, and one placed in the secondary beam. The secondary beam toroid was called the "air toroid", because unlike the two in the primary beam it was not placed under vacuum. The air toroid was a relic from an old experiment, and was both too large and too leaky to be trusted under the beampipe vacuum. It was placed coaxially with a ceramic section of the beam pipe in order to measure the intensity of the secondary beam. The air toroid however had numerous problems. The halo from the secondary beam interacted 
with both the air in between the air toroid and the ceramic pipe, and the readout electronics, mounted on the exterior casing of the device itself. This led to rather confusing behavior. The secondary beam would flip sign depending if the beam was placed in neutrino or in antineutrino mode. The signal in the air toroid, in principle based on the secondary beam current, was then also expected to flip sign. Instead, the air toroid measured the signal of the electrons being stripped off the air between it and the beamline, producing a signal, though somehow related to the beam intensity, which was always the same sign. As if this were not enough, the beam halo interacting with the electronics caused the amplifiers to arbitrarily shift gain settings, causing strange jumps in the output signal. Because of these features, the air toroid was never used, and it is only mentioned here as a kind of warning to anyone who may have the bright idea to try such a thing in the future.

Just ahead of the NuTeV target in figure 48, is a tiny rectangle, representing a "SEM", or "Secondary Emission Monitor". The SEM was a cylinder coaxial with the beam, with foils placed in the beam. As the beam passed through the foils, it would knock electrons off them and the resulting charge was a measurement of the beam intensity. The NuTeV pings however sent a large pulse $\left(\sim 10^{13}\right.$ protons $)$ through the foils in a short time. There are only so many electrons available to be knocked off the foil, and as a result the SEM intensity measurement saturated during the higher intensity pings. Figure 49 shows the intensity measurement from the primary beam toroid vs that from the SEM. Saturation is clearly seen beginning at ping intensities greater than about $1.5 \times 10^{12}$ protons.

The purpose of the beam was to deliver neutrinos and antineutrinos to the $\mathrm{NuTeV}$ detector. The first step in this was to drive the proton beam into a target, 


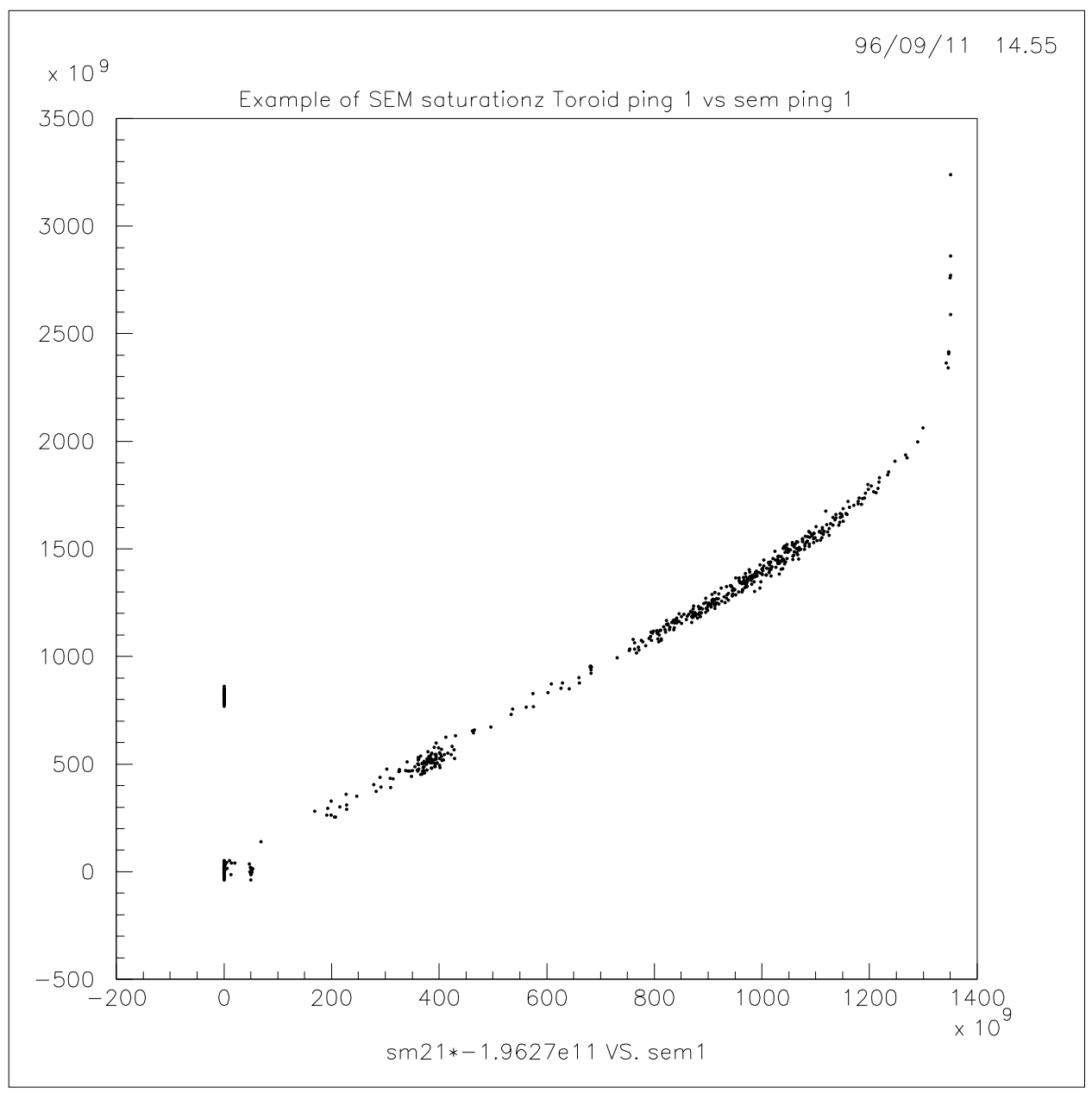

FIGURE 49. Saturation of the neutrino beamline SEM. The $\mathrm{x}$ axis is the SEM intensity measurement, the $\mathrm{y}$ axis is the measurement from the primary beam toroid. We see saturation beginning to occur roughly above $1.75 \times 10^{12}$ protons. 


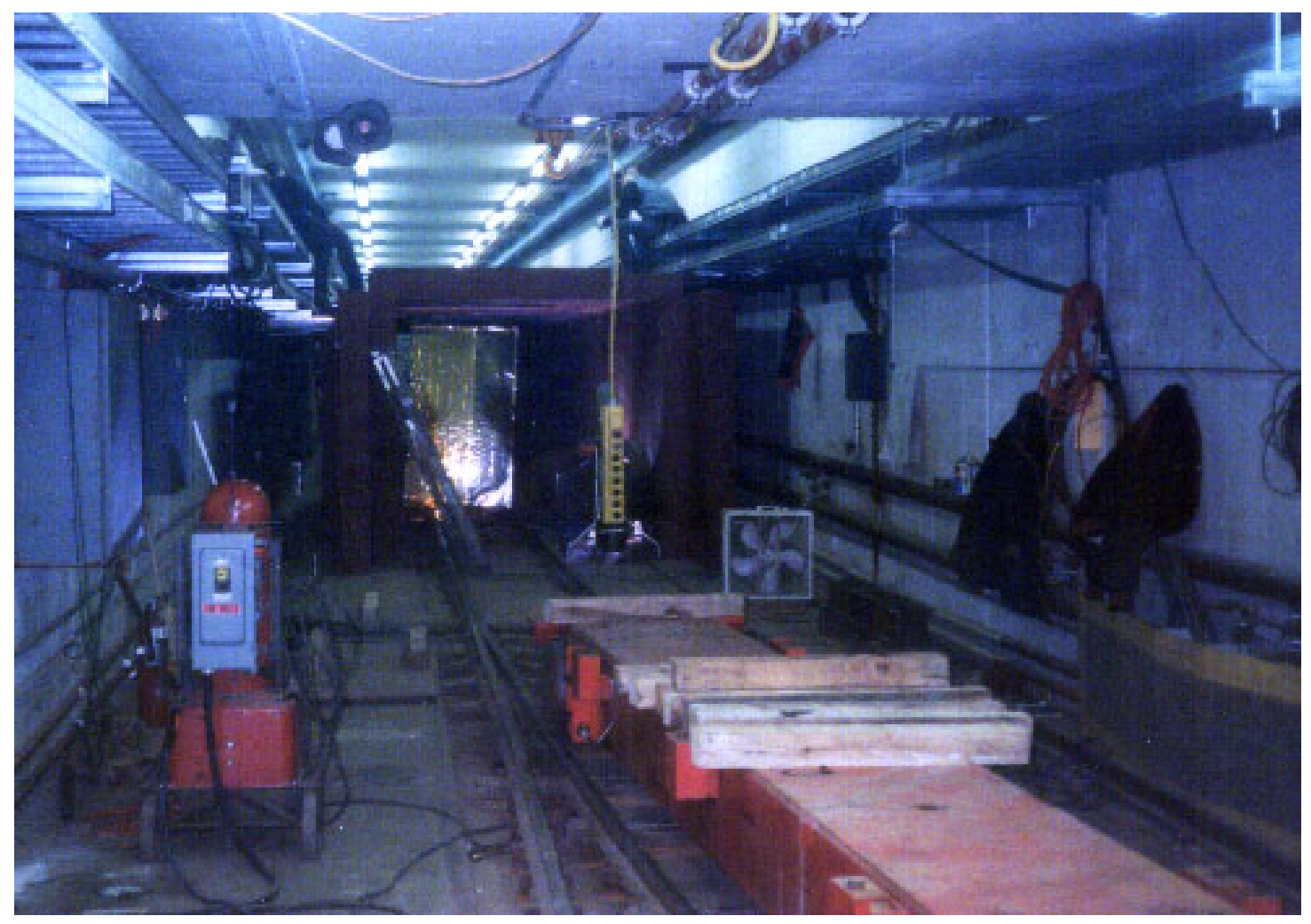

FIGURE 50. The NuTeV target "cave" under construction in 1995. The beam magnets were placed on carts which were rolled into place via the train tracks.

resulting in a beam of all sorts of hadrons, whose decay products included the neutrinos and antineutrinos $\mathrm{NuTeV}$ detects. As a result of the large number of protons striking this target it became very radioactive. Because of this, in addition to the concrete enclosure that housed the entire the beamline, the target region was enshrouded in a steel "cave", highlighted in figure 48. Figure 50 shows a photograph of the construction of the cave during the summer of 1995, prior to NuTeV running. This cave prevented radiation from reaching the outside of the beam enclosure.

Immediately after the target, and also housed inside the cave, was the main improvement NuTeV had over its predecessors: the "SSQT" or "Sign Selected 


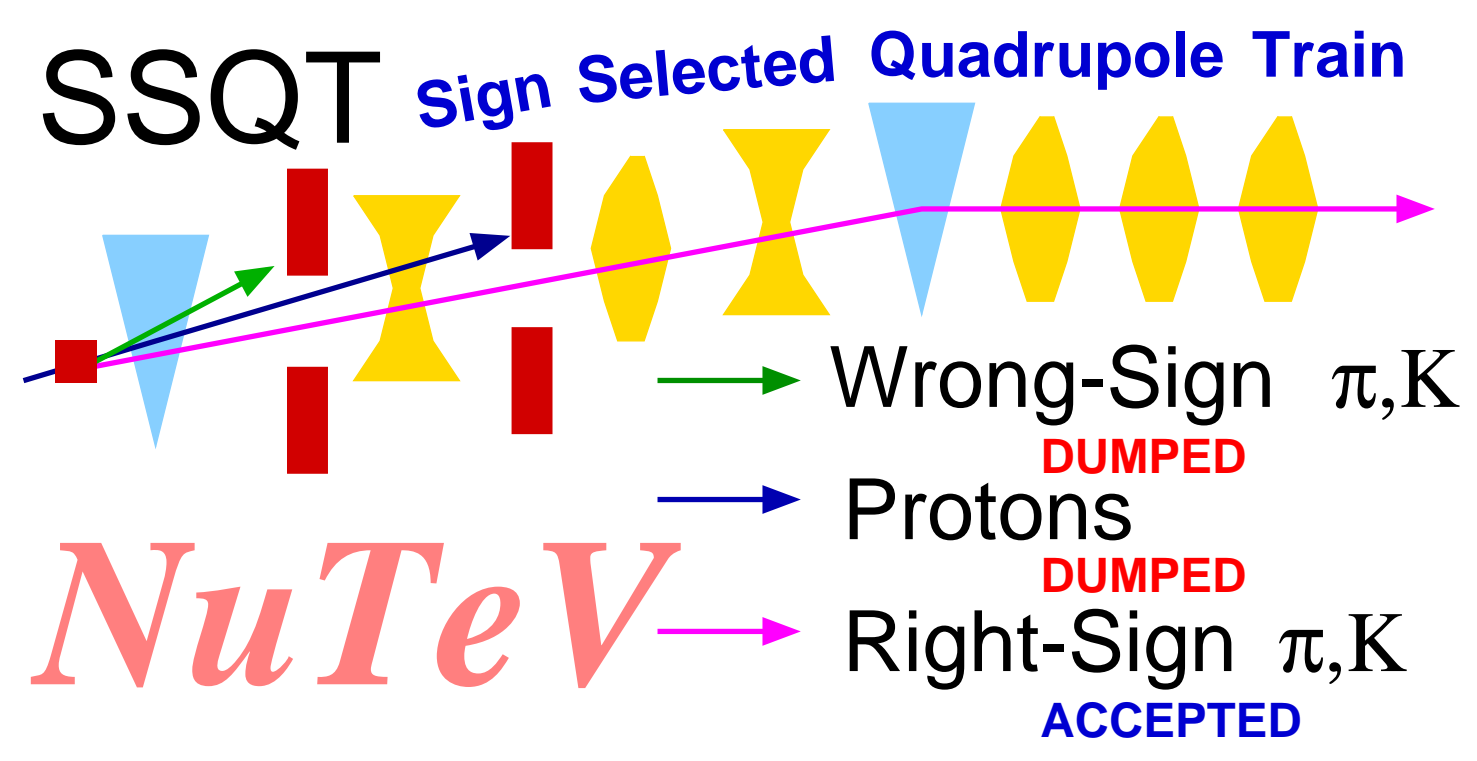

FIGURE 51. The Sign Selected Quadrupole Train. Correct sign secondaries are bent and focused toward the detector, while wrong signs and neutral particles are driven into a beam dump.

Quadrupole Train" [139]. Shown in figure 51, the SSQT included a dipole magnet which steered one sign of secondary particles into a beam dump, and the opposite sign in the direction of the $\mathrm{NuTeV}$ detector. Neutral particles, unaffected by the dipole, as well as protons and higher mass particles, were driven into a second dump. The net effect of this "sign selection" in the secondary beam, was that $\mathrm{NuTeV}$ had the ability to choose whether highly pure $\nu_{\mu}$ 's or $\bar{\nu}_{\mu}$ beams would be directed toward the detector.

After sign selection, the neutrino beam was formed much the same way as was the beam for the Columbia-Brookhaven experiment that discovered the $\nu_{\mu}$ in the early 60's (page 13). The correct sign secondaries were steered and focused towards the NuTeV detector, then left to coast down a "decay pipe". There, the beam particles, mostly pions and kaons, were given a chance to decay, producing a beam of muons and muon neutrinos (or their anti-partners, depending on the 


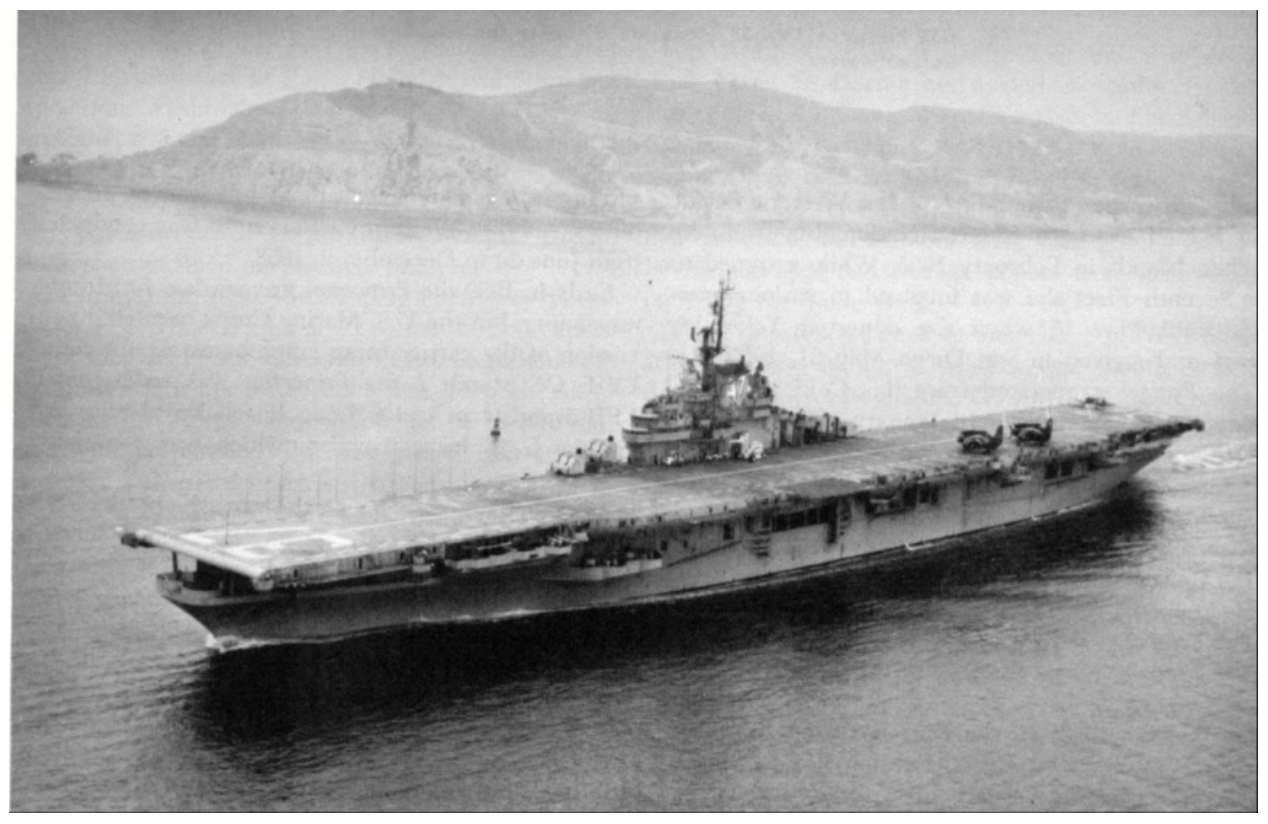

FIGURE 52. The USS Princeton circa 1947. U.S. Navy photo

SSQT polarity). At the end of the decay pipe was a long length of shielding. Its purpose in immediate terms was to stop any remaining pions and kaons, but also to remove the muons from the decay beam. This was achieved by about a half mile of concrete and steel. The steel used in this long shielding berm, was actually the steel plating from the aircraft carrier USS Princeton, shown in figure 52. Incidentally, ribs from the Princeton are also what the "Broken Symmetry" sculpture at the north entrance to Fermilab is constructed from.

The long berm of steel, dirt, and concrete filtered out almost all of the muons, resulting in a neutrino beam which passed through Lab E. Interactions of the neutrinos with material in the berm also created a background sample of muons called "straight through muons". This more or less uniform background of predominantly straight muons provided a useful tool for aligning and calibrating elements in the $\mathrm{NuTeV}$ detector. 

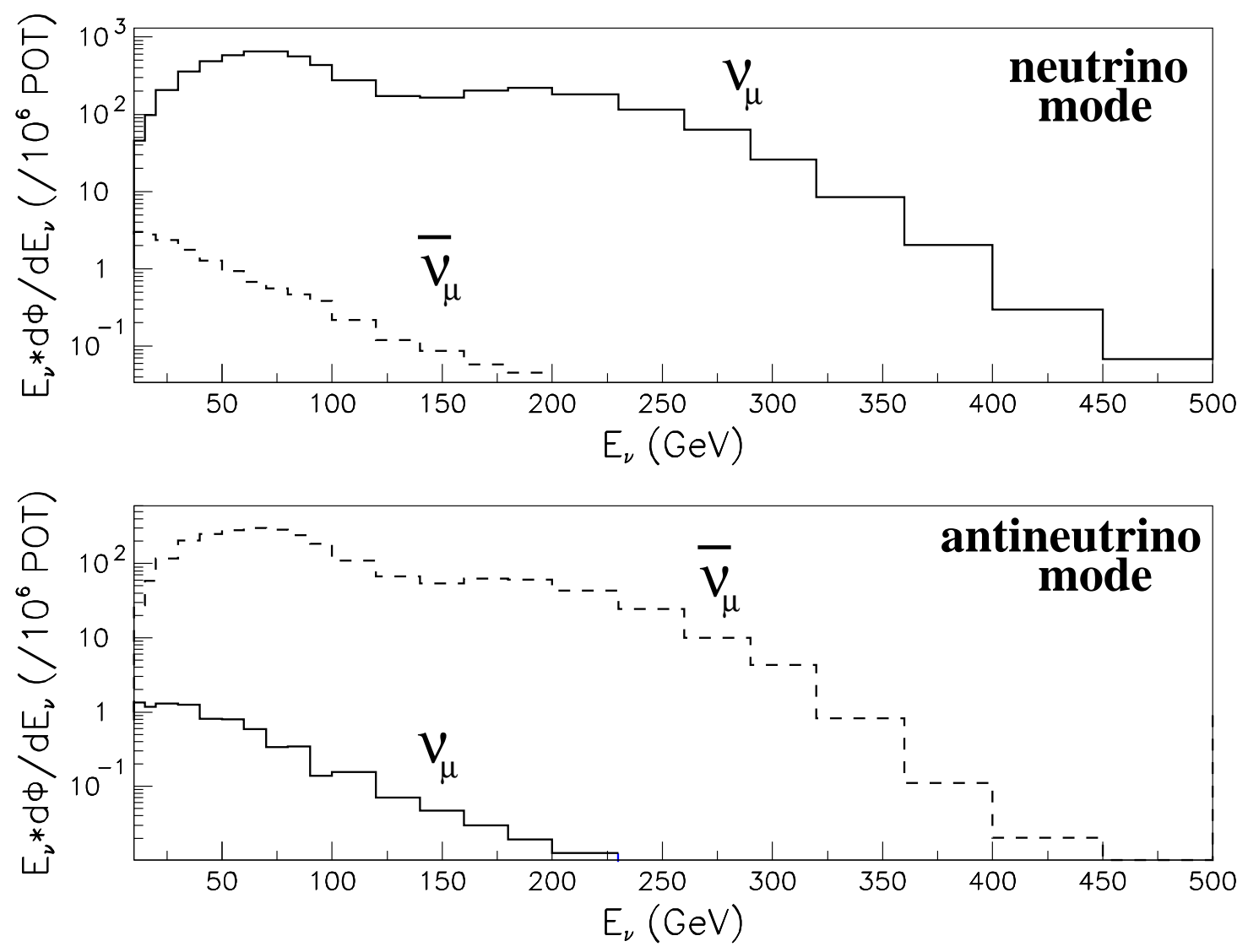

FIGURE 53. The NuTeV neutrino and antineutrino flux. Both plots show the small impurity due to the wrong sign backgrounds.

Figure 53 shows the flux of neutrinos or antineutrino in the beam reaching the $\mathrm{NuTeV}$ detector, plotted versus energy. There are two peaks in the energy distribution, the lower originating from pions in the decay beam, the higher energy peak from the Kaons in the beam. The figure also shows the "wrong sign" backgrounds in each polarity. The purity of the neutrino beam was 99.9\%, and was $99.7 \%$ for the antineutrino beam.

By the end of data taking, NuTeV took $1.43 \times 10^{18}$ protons on target in neutrino running, and $1.72 \times 10^{18}$ protons in antineutrino mode. Figure 54 shows the integrated protons on target as a function of time from the $\mathrm{NuTeV}$ data logs. 


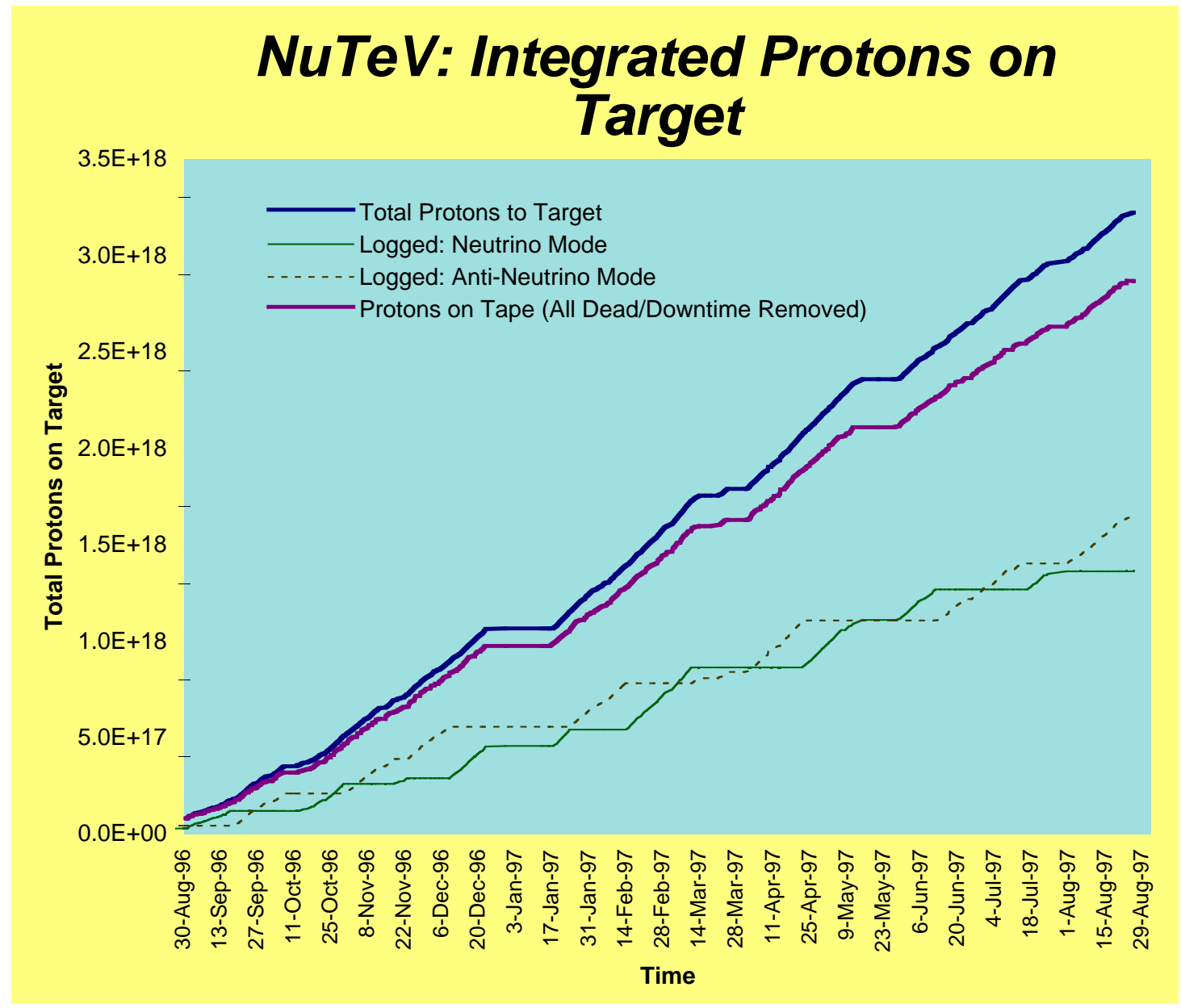

FIGURE 54. NuTeV protons on target

\subsection{The NuTeV Detector}

The $\mathrm{NuTeV}$ detector sat approximately half a mile from the primary target. The detector itself served as both the target and means of detecting the neutrinos and antineutrinos directed towards it. Neutrinos only interact with matter via the weak interaction, so the detector needed to include a large amount of material to maximize the probability of a neutrino interacting within it. This material took the form of the almost 700 tons of steel composing the target calorimeter. The 


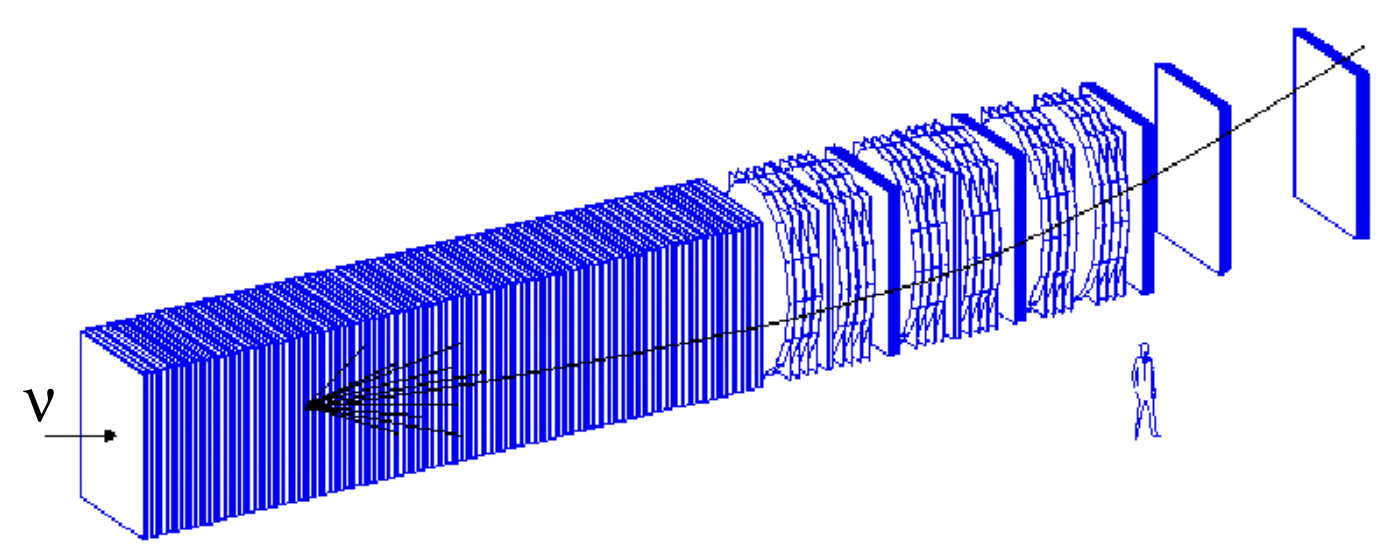

FIGURE 55. The traditional NuTeV detector graphic, showing a cartoon of a charged current event.

traditional cartoon of the $\mathrm{NuTeV}$ detector is shown in figure 55, with a cartoon of a charged current event depicted within it.

The NuTeV detector had two parts, the frontmost of which was the target calorimeter [140]. This part of the detector served two purposes: It was the target in which the neutrinos interacted, and it measured the energy and position of the hadron shower produced by the interaction. Hadron showers produced in the calorimeter would likely be contained within it, however any muons that would be produced could require as much as 10-20 times the length of the calorimeter to be completely contained within. Muons penetrate long distances in materials, because for the most part they only lose energy through ionization. (As opposed to electrons, which, because of their much smaller mass, makes them $\sim 40,000$ times more likely to lose energy through bremsstrahlung, drastically shortening their range.) This penetrating behavior allows muons to present very clean and clear 


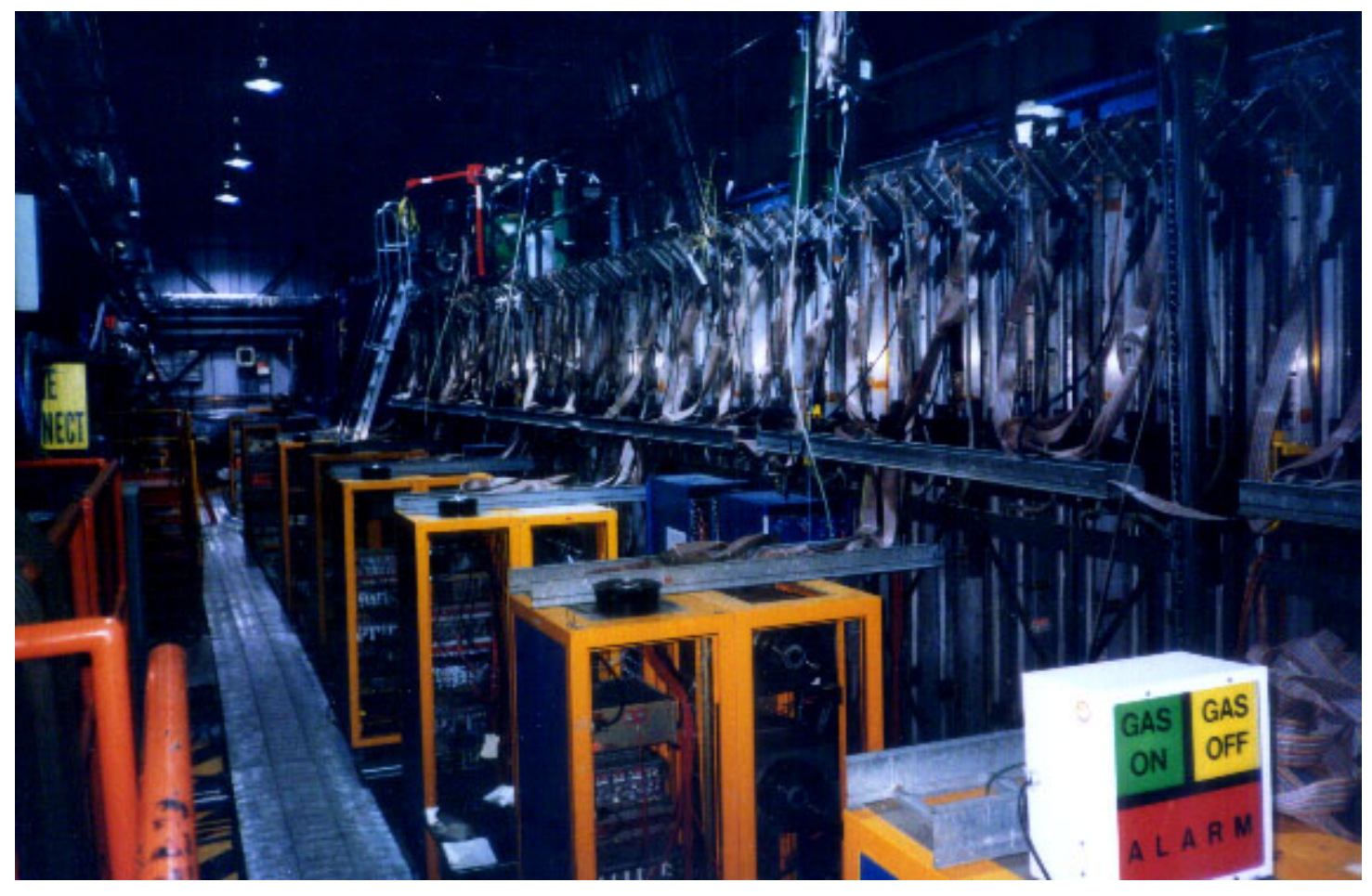

FIGURE 56. Photo of side of NuTeV detector

signatures in the detector, but it also makes it difficult to contain them for energy measurements. Rather than extend the calorimeter into Warrenville, a magnetized muon spectrometer was placed at the rear of the detector. The muon spectrometer consisted of three toroidal magnets with drift chambers interspersed between them. The momentum and sign of the muons could be measured by the direction and amount that they were bent by the magnetic field. Figure 56 shows a photograph of a side view the $\mathrm{NuTeV}$ detector. In the foreground is the calorimeter with power supply and data acquisition racks below. Behind the ladder in back was the toroid spectrometer. 

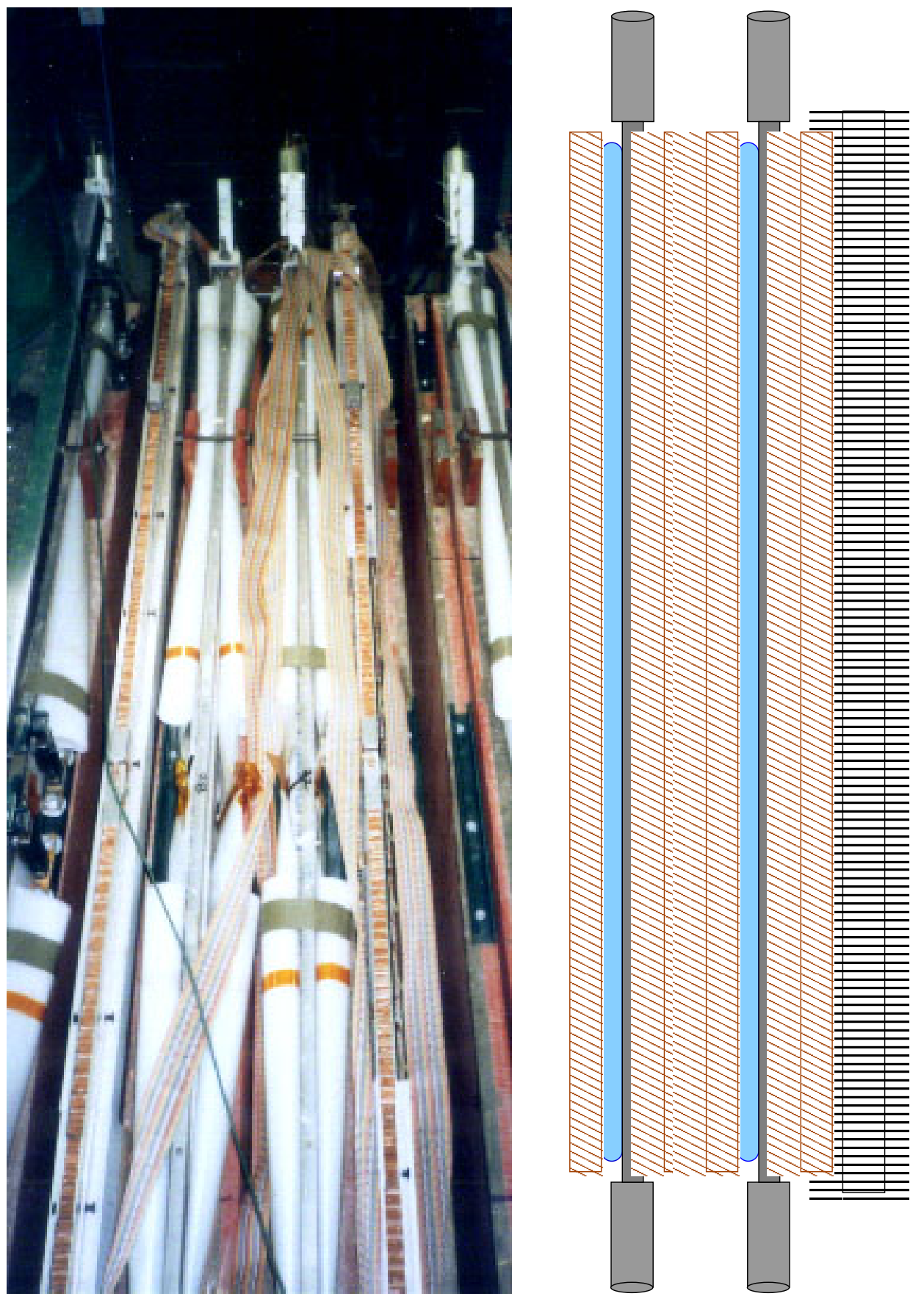

FIGURE 57. Photo of detector counter-DC sandwich. The diagram shows two scintillator modules followed by one drift chamber. A scintillator module consists of a scintillator, sandwiched between a pair of water bags, which are sandwiched between two two-inch steel plates. Photomultipliers are shown on the ends of the scintillators. 


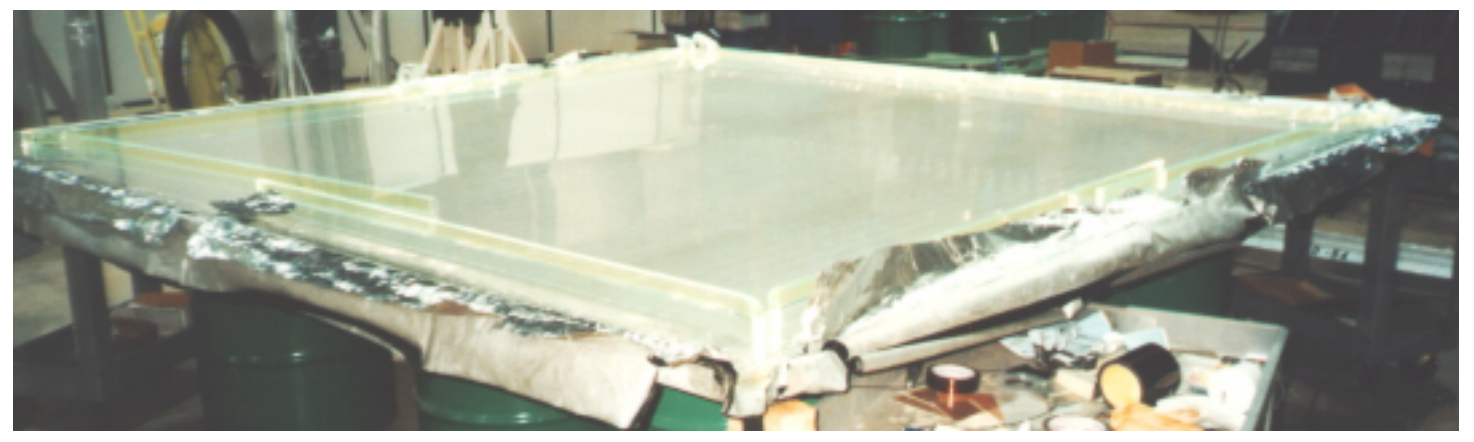

FIGURE 58. A photograph of a disassembled scintillator counter, with waveshifter bars laying on top.

\subsubsection{The Target Calorimeter}

The target calorimeter served as both the target for the neutrino interactions, and the means by which the event location and hadronic shower energy was measured. It was approximately 60 feet long, with a $10 \times 10$ foot cross section. Its construction was fairly simple, based on a repeated array of 42 scintillator/drift chamber modules, shown in figure 57. Each module included a pair of liquid scintillator counters, followed by a drift chamber (both $\mathrm{x}$ and y planes). The scintillators consisted of a lucite framework filled with Bicron 517L liquid scintillator oil, and were used both to measure deposited energy and for event timing. To balance the pressure from the scintillator oil, each scintillator was supported by a pair of water bags, which were in turn supported by 2 inch thick steel plates. Figure 58 shows a photo of a disassembled scintillator, and figure 59 shows the structure of a scintillator in more detail. The full length of the calorimeter contained about 28 feet of steel, corresponding to 522 radiation lengths.

The scintillator counters were essentially large, flat boxes held together by transparent lucite ribs along their length. Because the lucite ribs were not active 


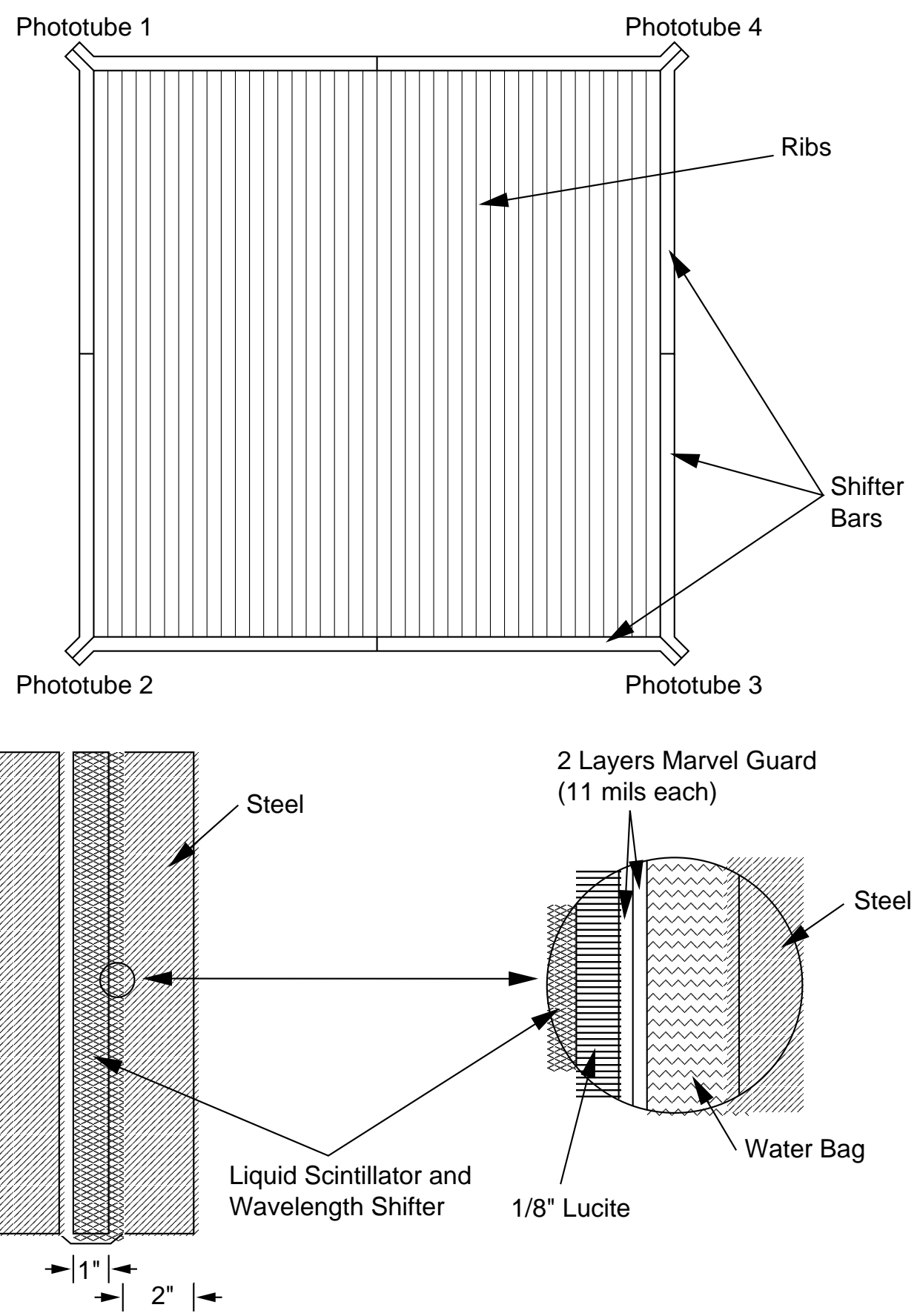

FIGURE 59. A NuTeV scintillator counter 
themselves, the scintillators were offset from each other slightly so that the ribs would not line up along the neutrino beam axis. The scintillator had two active fluors, the first of which radiate ultraviolet light when excited by the passage of a charged particle [141]. The UV light would then be picked up by a second fluor, which would radiate blue light. Blue light has a longer attenuation length than UV light in the scintillator material. The surface of the lucite box was covered with reflective material and made light tight. Photons which reached the outer edge of the scintillator were picked up by a set of "wave shifter bars", plastic bars doped with yet another fluor, that emitted green light when excited by the blue photons. This green light was picked up more efficiently by the four phototubes mounted on the corners of the scintillators. Ten stage Hamamatsu 2154 phototubes were used, with green-extended photocathodes. The cathodes were held at a voltage of about 1400 volts, and were about $20 \%$ efficient at converting a photon into a photoelectron. Each photoelectron was multiplied to about a million electrons within the photomultiplier tube.

Straight through muons were used to determine the efficiency of the calorimeter scintillators. As minimum ionizing particles, the muons deposited a roughly constant amount of energy along their path as they pass through the calorimeter. Figure 60 shows the average response of a scintillator as a function of where these muons passed through it. You can see the peaks near the corners, where the photomultipliers are mounted, and the minimum at the middle of the scintillator. The signal from a minimum ionizing muon track in the center of a scintillator corresponds to about 30 photoelectrons. Nearer to the phototubes that signal is approximately doubled. 


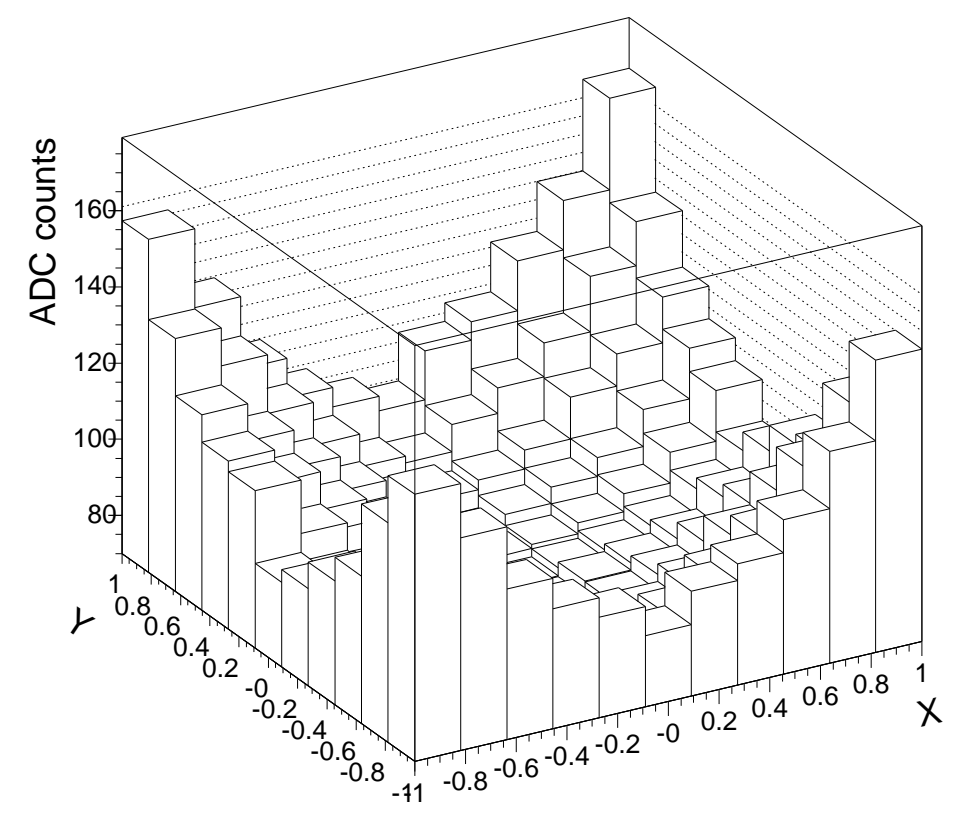

FIGURE 60. Average response of a calorimeter scintillator to the passage of muons as a function of track position

A drift chamber was placed after each pair of scintillators in the calorimeter to measure the position of charged particle tracks [142]. Each drift chamber included two planes, one for a position measurement in $\mathrm{x}$, the other in $\mathrm{y}$. Each plane was divided into 24 parallel 5 inch wide cells, filled with an even mixture of argon and ethane gas. A cross section of one of the cells is shown in figure 61. The cell walls are made from copper-clad G10, with 19 evenly spaced cathode strips. The I-beams at the borders of each cell are held at -4500 volts, and supply voltage to the strips. The strip voltage is stepped down incrementally via voltage dividers to maintain a uniform electric field inside the cell.

When a charged particle passed through a cell, it left behind a trail of ions and electrons in the gas. The electrons drifted (hence the name "drift chamber") 


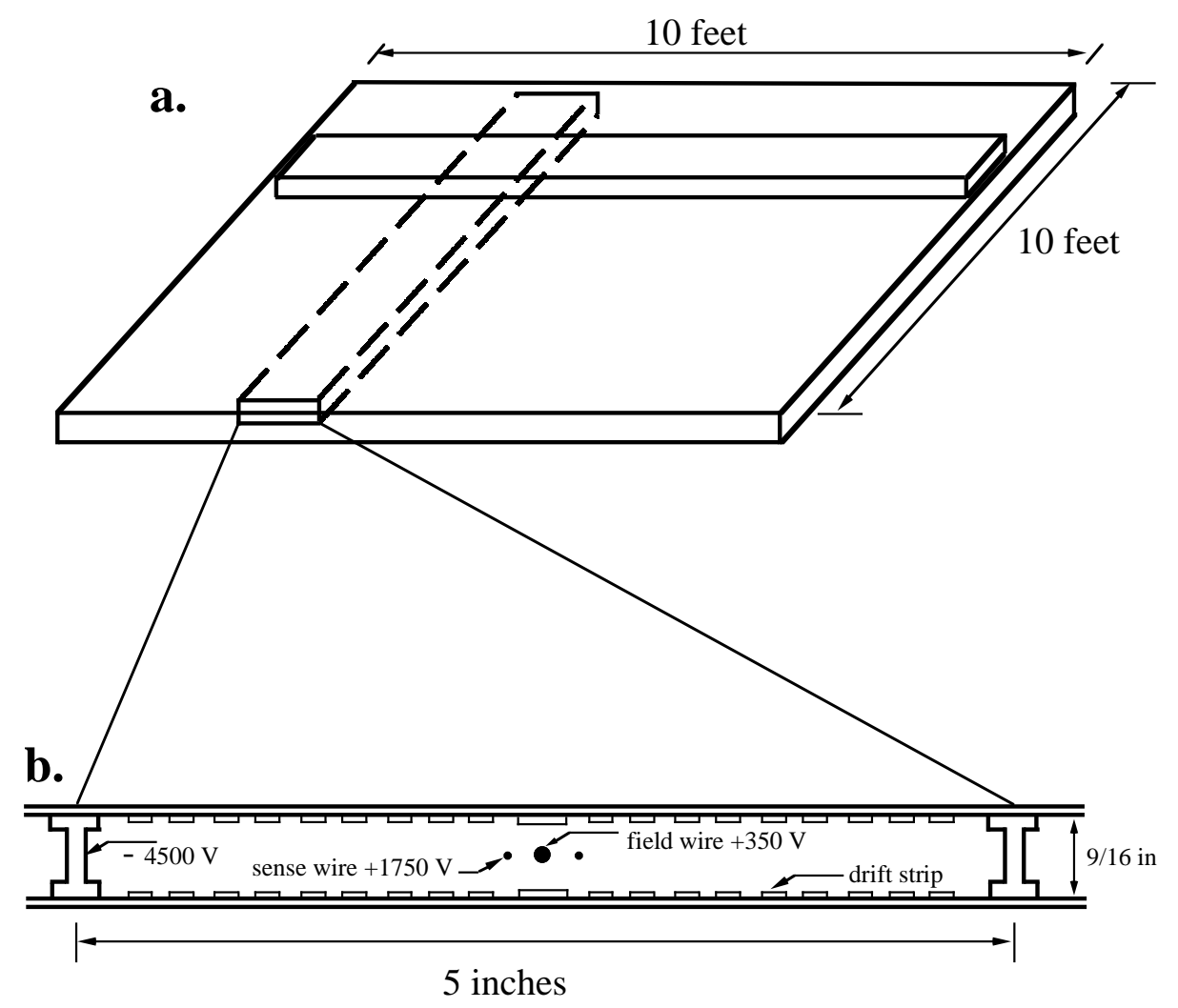

FIGURE 61. A NuTeV drift chamber

at an approximately constant velocity of $52.4 \mu \mathrm{m} / \mathrm{ns}$. The time that the ions took to induce a signal on the wire was measured and used to determine the position of where the particle went through the cell. The start time was defined by the scintillators upstream. (The scintillators are fast devices, with very fine time resolution measured in nanoseconds, while drift chamber velocities are such that the drift times are measured in hundreds of nanoseconds.)

Once the electrons got close to one of the sense wires, the rapidly increasing electric field there accelerated them and caused them to initiate a cascade of elec- 
tron showers. This amplified the total charge from the track, making the signal easier to measure. In the calorimeter drift chambers, there are two sense wires, one on each side of the field shaping wire. This was so it was possible to tell the difference between a track that passed on the left or right of the cell.

The electric signals from the sense wires were amplified and discriminated, producing an ECL logic pulse. This logic pulse, along with a pulse from the scintillators ahead of the drift chamber was fed into a TDC ("time to digital converter") to determine the electron drift time and therefore position of the drift chamber "hit".

\subsubsection{The Toroid Spectrometer}

After the calorimeter came the toroid muon spectrometer, shown if figure 62. This was a series of three toroidal magnets with drift chambers in between for position measurements. The layout of the back end of the $\mathrm{NuTeV}$ detector is shown in figure 63. After each toroid coil was placed an array of single wire drift chambers, followed by an additional array of drift chambers at the very back of the detector (called the "blue cart").

The toroid drift chambers were slightly different from the chambers in the calorimeter. Instead of three wires, they had only one sense wire in the middle held at +1900 volts. This necessitated the presence of a pair of drift chambers (called "u-v" chambers) tilted at a 5 degree angle so that the ambiguity between hits on the left or right side of a cell could be resolved by following fitted muon tracks. 
Each of the three toroid magnets were divided into eight 8 inch thick toroid "washers". The washers had an outer radius of 70 inches, with a 5 inch hole in the center. A cross sectional view, from the neutrino beam's perspective is shown in figure 64. Each of the washers were split forming top and bottom halves, with space in the middle to allow a Hall probe to be inserted to measure the magnetic field. There were four copper coils at about 90 degree intervals wrapped around the iron washers. Current applied to the coils magnetized the washers to provide a 1.5 Tesla magnetic field. This field gave about a $2.4 \mathrm{GeV}$ transverse kick to muons which passed through all 3 coils. The toroid polarity could be flipped so that muons from neutrino interactions or anti-muons from anti-neutrino interactions would be focused in the spectrometer.

Early in the run, one of the coils on the left side of the second toroid (from the neutrino's perspective) developed a short, and had to be disconnected. The change in the magnetic field as a result is shown in figure 65. The figure shows the ratio of the toroid field with only 3 coils vs the full 4 -coil field. The greatest effect was in the region of the burnt-out coil, on the left of the figure. This map was incorporated into the $\mathrm{NuTeV}$ analysis software when determining the energy of muons displaced in the toroid.

\subsubsection{Other Detector Features}

A large detector like the one discussed above is very sensitive to cosmic rays. Some of these events, especially those at low angles on the horizon can masquerade as neutrino events, and set off NuTeV's triggers. NuTeV built a large "veto wall" in front of the detector consisting of a large steel plate and a series of scintillators 


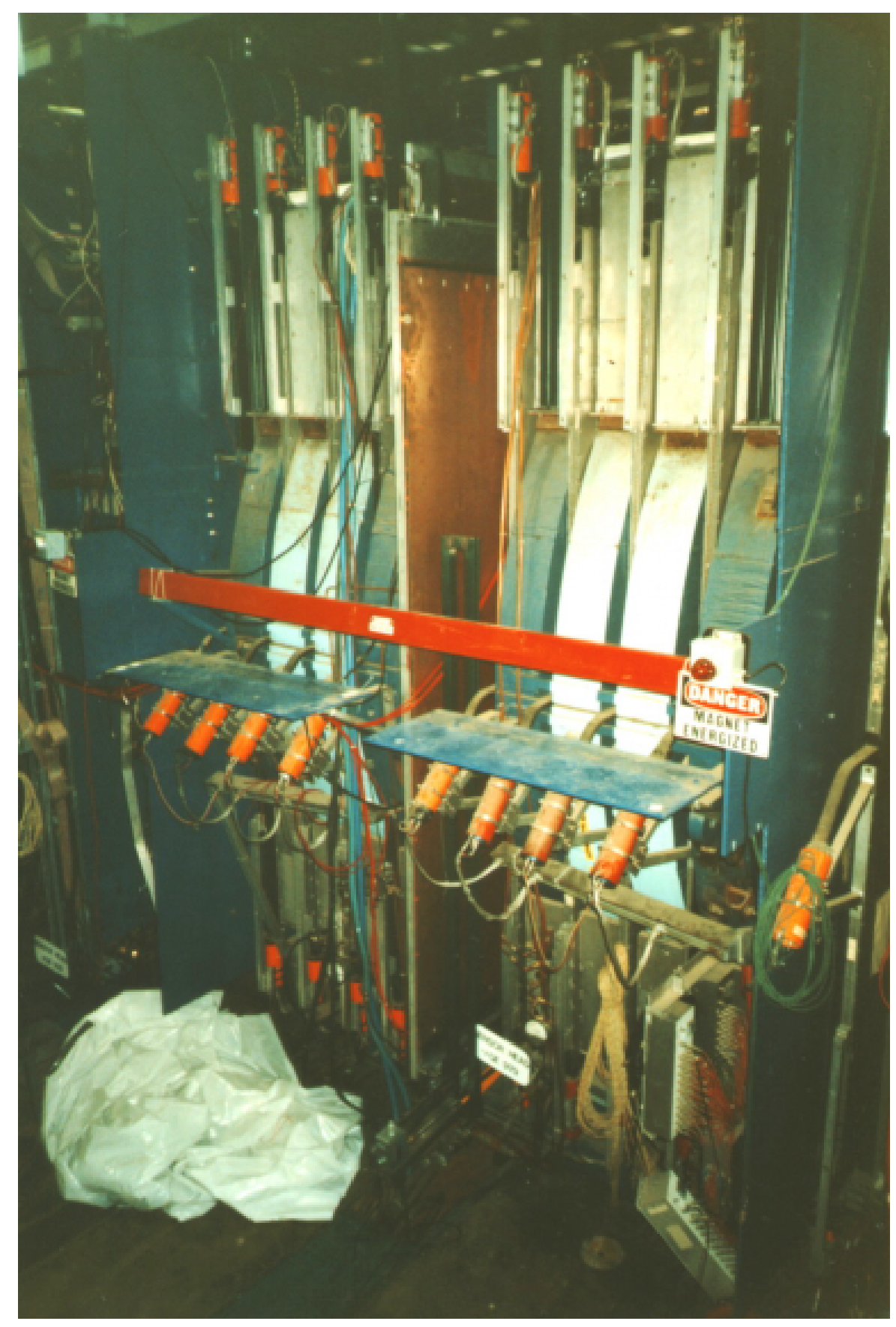

FIGURE 62. A photo of the NuTeV toroid 


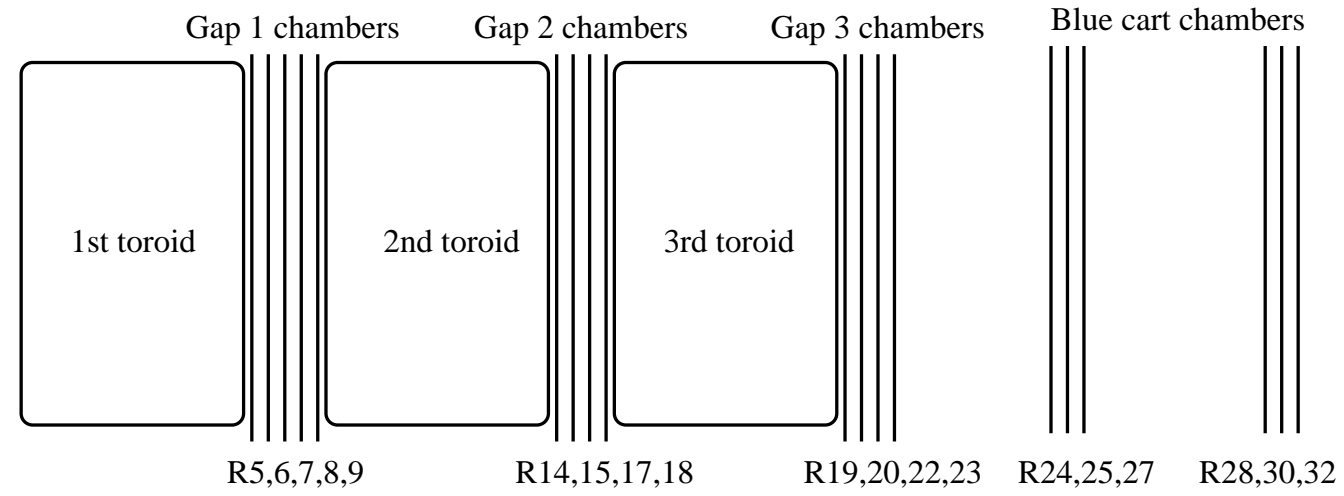

FIGURE 63. The layout of the toroid coils (large marshmallow shapes) and the drift chambers between and behind them

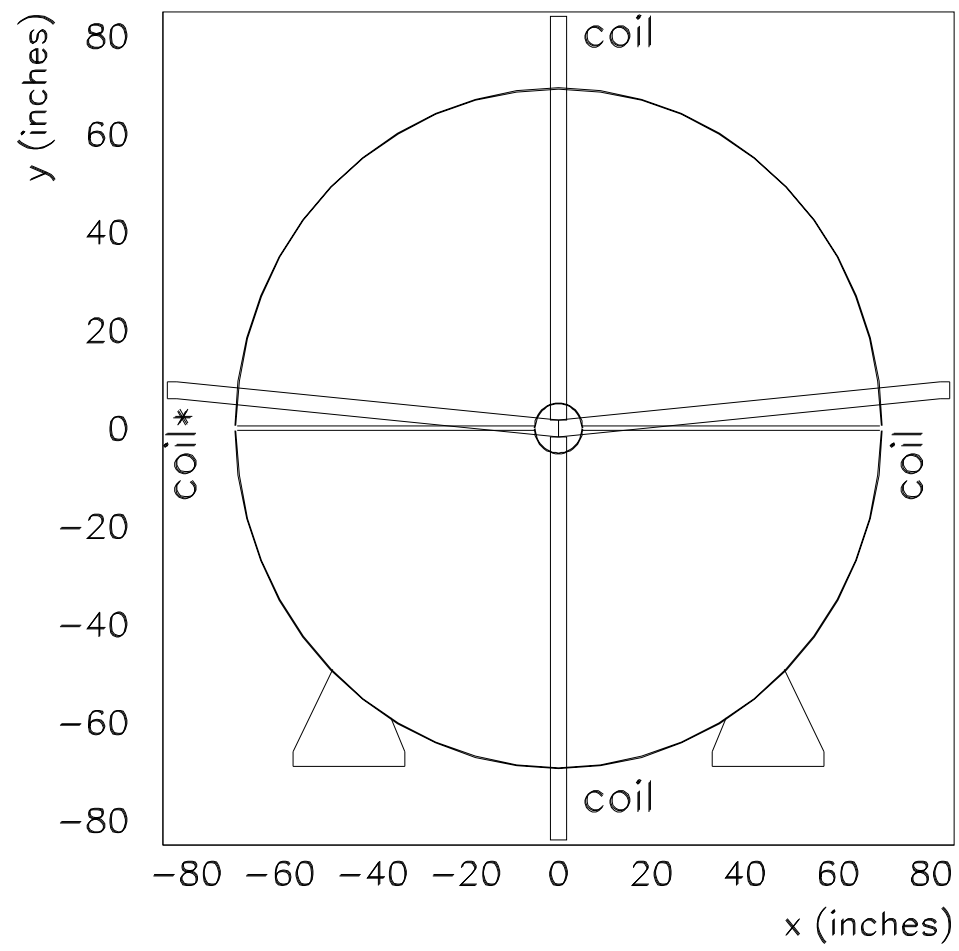

FIGURE 64. Face-on view of the toroid and 4 coils. The coils were loops around the steel washers, passing through the center hole, around the back and back forward. 


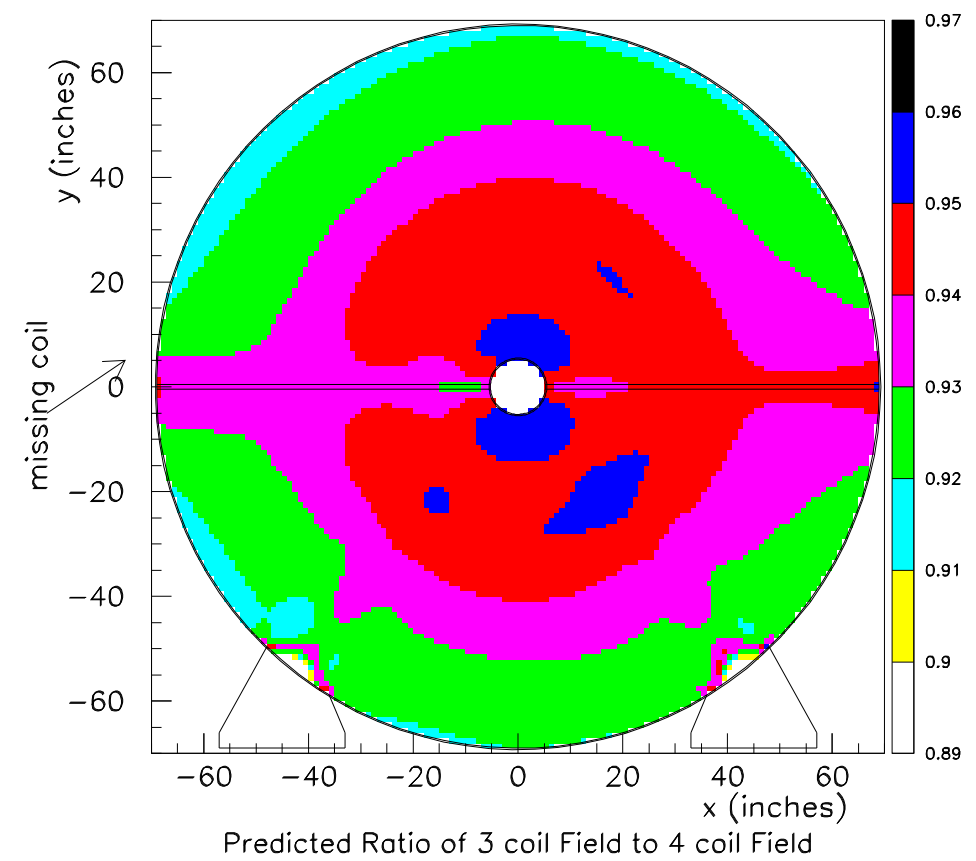

FIGURE 65. Results of an ANSYS simulation of the effect of the burnt out coil on the magnetic field of the toroid spectrometer. The coil on the left side of the figure was burned out, where the ratio with the 4 coil field deviates greatest from 1.

to detect these events [143]. The purpose of the large steel plate was to filter out low energy "beam halo" particles, and the scintillators would flag events which originated ahead of the detector. A hole was left in the middle so that the NuTeV calibration beam could still reach the detector unimpeded. Figure 66 shows the $\mathrm{NuTeV}$ veto wall in its final painted form. The fact that University of Oregon colors were chosen is merely a happy coincidence.

A "decay channel" was placed ahead of the NuTeV detector to try and observe the decay of proposed neutral heavy leptons, should they exist [144]. These particles were expected to behave like neutrinos, but with a large mass. If they 




FIGURE 66. The NuTeV veto wall

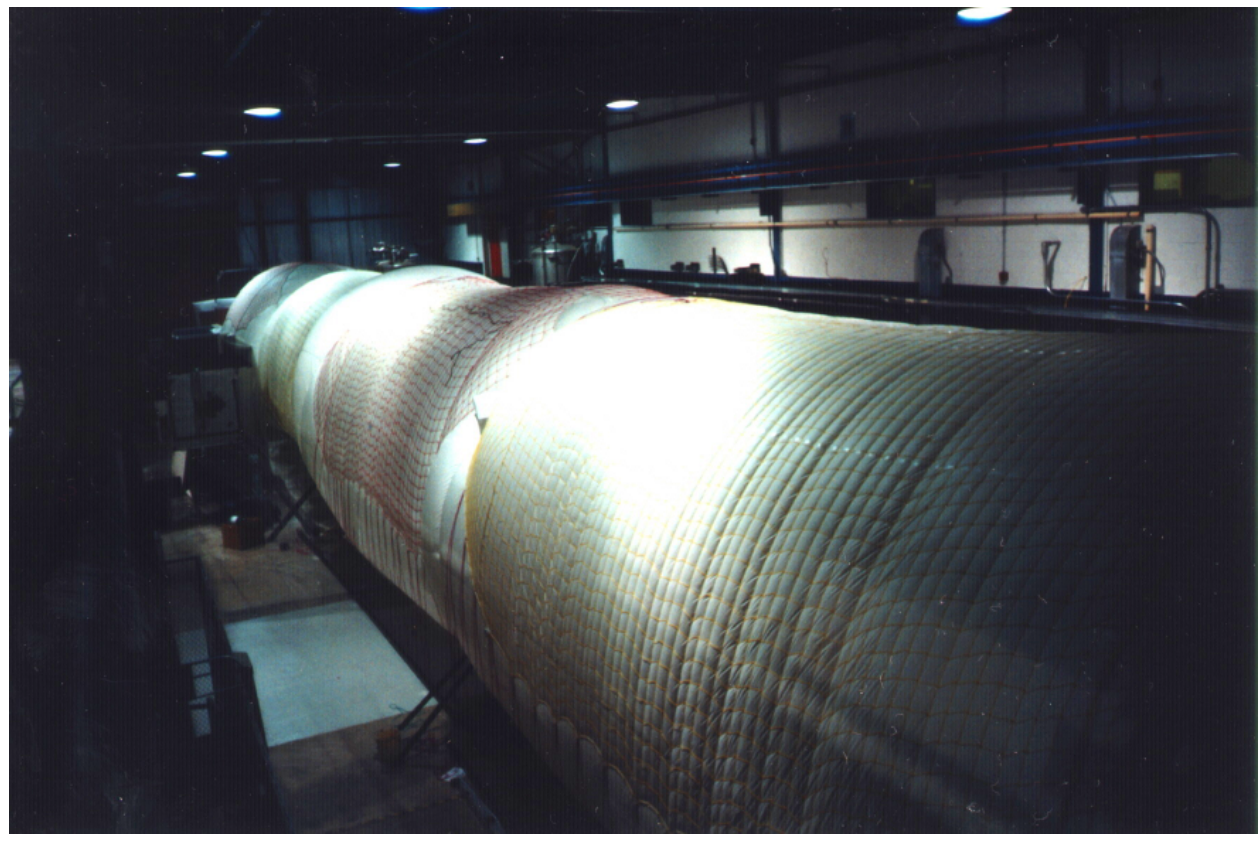

FIGURE 67. The NuTeV Decay Channel helium bags. The NuTeV detector is beyond the edge of the photo to the lower right. 
were produced at some point at the primary beam target upstream, their decay might have been observable in the $\mathrm{NuTeV}$ detector. A series of helium balloons, with drift chambers in between, was placed in the space ahead of the NuTeV detector to provide a low density region in which to observe such a decay. The purpose of the low density region was to minimize the background from normal neutrino scattering events. One would then look for a pair of tracks originating from a vertex located inside one of the helium bags, indicating the possibility that one of these particles decayed within it. A photograph of the helium bags, looking away from the NuTeV detector, toward the neutrino beam is shown in figure 67 .

\subsection{The Calibration Beam}

Two fundamental improvements set $\mathrm{NuTeV}$ apart from its predecessor, CCFR. The first was the sign selected beam, discussed already above. The other improvement was NuTeV's continuous calibration beam. The separate beamline used for the calibration beam is highlighted in pink in figure 44 on page 125 . A schematic of the beamline itself is shown in figure 68. Protons struck an aluminum target during slow spill, after the fast spill neutrino pings. The resulting secondary particles were focused by the quadrupoles immediately following the beam dump, then passed through a series of collimators and dipoles which selected for particle type and momentum. The calibration beam had the ability to project electrons, muons, or hadrons over a wide range of energies to the $\mathrm{NuTeV}$ detector. Once the particles were selected, they passed through a spectrometer to measure their energy, shown

in figure 69. This energy measurement was compared to the measurement by the $\mathrm{NuTeV}$ detector to determine how accurate the $\mathrm{NuTeV}$ detector was. 


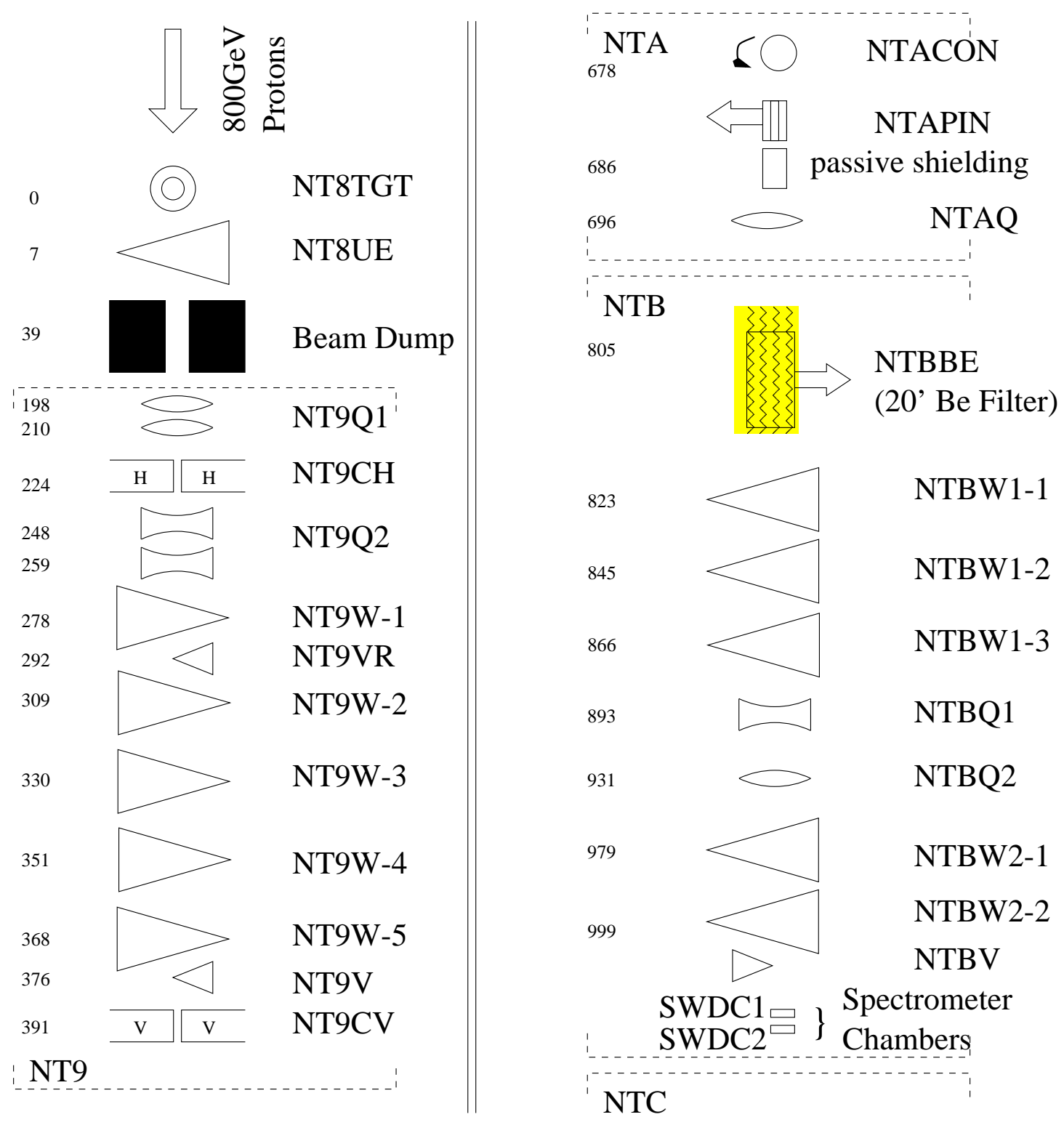

FIGURE 68. The NuTeV calibration beamline 


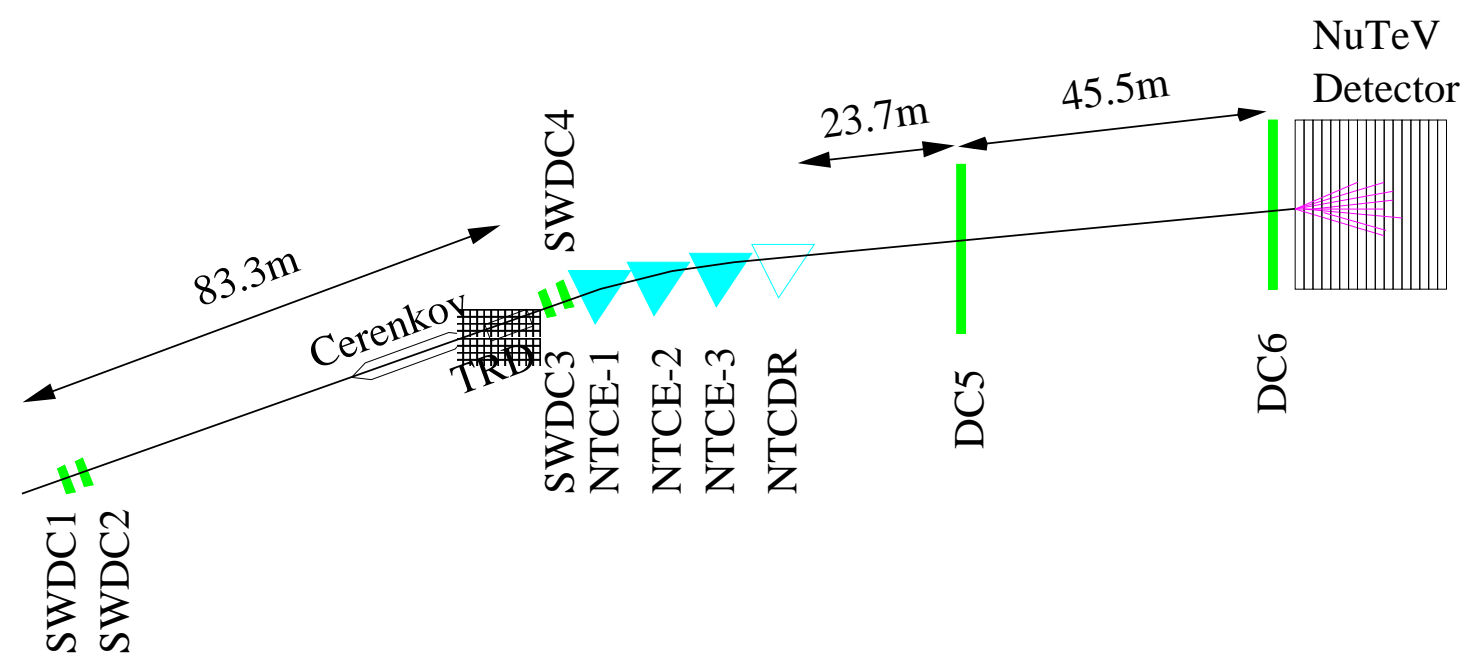

FIGURE 69. The NuTeV calibration beam in relation to the $\mathrm{NuTeV}$ detector.

\subsection{Event Triggering and Data Acquisition}

Any particle physics experiment requires some number of conditions to be met before it makes sense for its measurements to be recorded. Usually digitization of those measurements requires some finite amount of time, during which the detector is effectively blind. In this case its best to only begin the digitization process when its likely something worth digitizing has happened. One typically assembles a collection of fast devices with which to make some preliminary measurements of what is happening in the detector, and use them to tell the data acquisition system to start recording after certain conditions are met. This is called "triggering" the detector, and the devices and accompanying logic would be called the "trigger".

\subsubsection{Phototube Permutations}

Scintillators and phototubes can provide fast signals which are often well suited to trigger a detector. The scintillators in the $\mathrm{NuTeV}$ calorimeter, coupled 


\begin{tabular}{|c|c|c|c|}
\hline Name & Source & Amplification & MIP \\
\hline LOW & 1 PMT & $\times 1$ & 2 \\
COMBINATION LOW & $\sum 4$ counter PMTs & $\times 1$ & 8 \\
HIGH & COMBINATION LOW & $\times 10$ & 80 \\
SUPERLOW & $\sum 8$ PMTs & $\div 10$ & 0.2 \\
\hline
\end{tabular}

TABLE 8. Summary of the types of signals combined from the calorimeter PMTs. The "MIP" column indicates the number of ADC counts for a minimum ionizing muon.

with more in the veto wall and upstream were essential components in the NuTeV trigger. The sorts of events that were sought in the $\mathrm{NuTeV}$ experiment however included particles which either deposited large (hadron showers) or small (muon tracks) amounts of energy in the scintillators. Different combinations of phototube signals were therefore assembled to expand the dynamic range of the calorimeter.

Figure 70 shows a schematic of what happens to the signals from each of the four phototubes on a counter. Four variations of the phototube signals were used for triggering and energy measurement: LOW, COMBINATION LOW, HIGH, and SUPERLOW. The simplest of the four was the LOW, which was merely the unamplified signal from a single phototube. There were $84 \times 4=336$ LOWs. The COMBINATION LOW (C.L.) was the sum of the signals from all 4 phototubes in a counter. There were 84 C.L.'s. The HIGHs took the same phototube sums as the C.L.'s and amplified them by 10. The SUPERLOWs were the digitized sums of 8 phototube signals, with each phototube separated by 10 counters. This sum was then attenuated by a factor of 10 .

Table 8 summarizes the 4 signal types, and the average number of ADC counts that would be seen from the passage of a minimum ionizing particle, like a muon. The amplified HIGH channels were used to measure the energy deposited by muons. A typical hadron shower however could deposit as much as $100 \mathrm{GeV}$ in 


\section{Counter 1 readout}

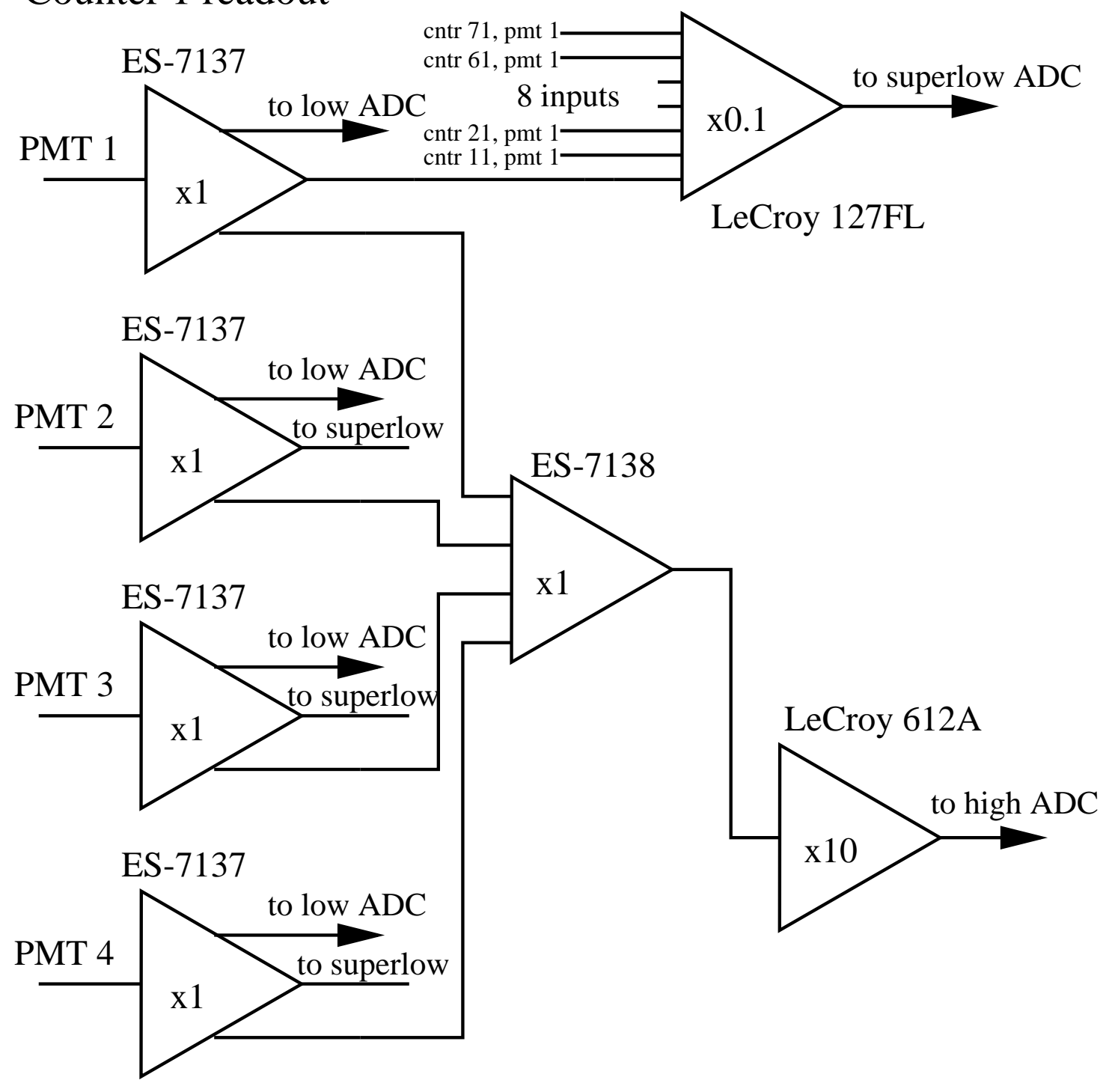

FIGURE 70. A diagram of the phototube electronics. 
a single counter, saturating the HIGHs. The LOWs were usually used to measure the hadronic shower energy, though occasionally for large showers that occurred near one of the phototubes, the SUPERLOWs were required.

Different combinations of the C.L. signals were assembled into four types of logic events. These "bits" were combined to construct the dozen or so different triggers used by the $\mathrm{NuTeV}$ experiment. The simplest of the bits was the Sbit, taken from a C.L. signal which was amplified by a factor of 100, then passed through a discriminator with a $150 \mathrm{mV}$ threshold (corresponding to about a quarter MIP). The Sbits were intended to flag whether at least one muon passed through a particular counter. Similar to the Sbits, Tbits took the C.L. signal through a higher $450 \mathrm{mV}$ threshold. The Tbits were designed to distinguish between a single muon track and a hadron shower.

The two remaining bits were designed to flag the presence of hadronic showers in the calorimeter. The Nbit took the sum of eight consecutive C.L. signals without amplification, and ran it into a discriminator with a $55 \mathrm{mV}$ threshold. These sums of 8 counters progressed through the calorimeter such that in the middle of the detector each counter would contribute to eight Nbits. The other shower indication bit was the NCbit. This looked at sets of four consecutive counters, requiring that the Tbits fired in at least two, and the NBIT including those counters plus the four upstream also fired. The NCbits were intended flag a shower with some longitudinal extent in the calorimeter. 


\subsubsection{Event Triggers}

In addition to forming the logical components of each of the triggers, the different bits were also recorded by the $\mathrm{NuTeV}$ data acquisition system for trigger efficiency and diagnostic purposes. Twelve different trigger events were defined by different bit combinations added to external information such as timing events from the TeVatron, scintillators placed upstream of the detector and in the Veto wall, and the beam gate discussed in appendix I. The triggers were non-exclusive, so that any particular event usually had more than one of the triggers flagged. The different triggers were as follows:

Trigger 1 This was the charged current trigger. All the data events in this thesis (inclusive charged current and dimuon) fired trigger 1. This was designed to flag charged current events which originated in the calorimeter, and had a muon pass through the toroid. One of two conditions needed to be met for this trigger to fire: 1.) hits in at least two of the last four counters, and hits in two toroid gaps. 2.) hits in at least two of the last four counters, hits from at least two counters upstream, and hits in just the first toroid gap.

Trigger 2 This was the neutral current trigger, and also the most active of the neutrino event triggers. This looked only for hadronic showers, so was set off if any of the NCbits fired.

Trigger 3 This was the calorimeter exit trigger. It looked for short charged current events that were not able to reach the toroid, either because the muon ranged out in, or escaped from, the calorimeter. It required that sixteen of the Sbits (not necessarily consecutive) fired in the calorimeter. 
Trigger 4 This was actually a redundant charged current trigger, used to test trigger 1 efficiency. It used different logic devices than trigger 1, and sets of counters slightly upstream of the trigger 1 requirements. It also used a slightly stricter gap requirement than trigger 1 such that every trigger 4 should also be a trigger 1 .

Trigger 5 This was the calibration beam trigger, flagged during slow spill and when a pair of scintillation counters upstream of the detector in the calibration beam line detected a particle en route to the detector. Since this was for detector calibration, no requirement from the calorimeter or toroid was made.

Trigger 6 This was the straight-through muon trigger, which was designed to flag muons produced from charged current events in the berm upstream. These events typically produced muons which traveled the length of the detector. The trigger required a hit in each target cart, and hits in the same quadrant of each of the toroid gaps. The toroid quadrant requirement selected for muons which were of higher energy, and therefore left relatively straight tracks through the detector.

Trigger 8 The cosmic ray trigger. This was designed to look for cosmic ray muons which traversed a significant portion of the detector. It required at least 40 semi-consecutive counters and hits in the first toroid gap.

Trigger 9 This was the neutral heavy lepton trigger, designed to trigger when tracks reached the front end of the calorimeter. 


\begin{tabular}{|ccc|}
\hline Trigger & $\nu$ events & $\bar{\nu}$ events \\
\hline 1 & $2,574,783$ & 914,886 \\
2 & $5,445,024$ & $2,804,981$ \\
3 & $4,306,217$ & $3,065,412$ \\
5 & \multicolumn{2}{c}{$\sim 17,000,000$} \\
6 & $1,712,591$ & 735,222 \\
9 & $1,334,675$ & $1,616,637$ \\
10 & 189,855 & 183,794 \\
11 & 785,040 & 153,461 \\
\hline
\end{tabular}

TABLE 9. Number of events from each trigger (non-exclusive)

Trigger 10 This was the in-spill pedestal trigger, set up to trigger the detector randomly during the spill so as not to be correlated with neutrino beam. This activated the data acquisition electronics to measure their pedestals, or zero values when no events should be present.

Trigger 11 This is the toroid shower pedestal trigger. It was designed to fire when events showered catastrophically in the toroid. The intent was to trigger the detector on events where something happened in the toroid, but nothing happened in the calorimeter, so that pedestals in the calorimeter while the beam was active could be measured. It was later found that regular charged current events with larger than usual energy loss in the toroid were also able to fire this trigger. This led to trouble for the first Dimuon analysis, discussed in 3.8.2 starting on page 169 .

Trigger 12 This was a pedestal trigger which sampled while the beam was inactive. This was set to fire about 10 times right after the beginning of each 60 second TeVatron cycle. 
Table 9 shows the number of events associated with each trigger during the $\mathrm{NuTeV}$ run. In addition to the triggers three "gates" defined when the data acquisition system was allowed to accept triggers and record data to tape. These were:

Fast Gate This corresponded to fast spill, or during the pings. It was defined by the TeVatron "T11" ping times and the presence of beam indicated by the beam gate.

Slow Gate This indicated that slow spill was in progress, and was defined by timing signals from the TeVatron.

Cosmic Ray Gate This was active when the beam was not, for 5 seconds each spill.

This was used to measure the background due to cosmic ray events.

When the gates were active, and a trigger fired, the electronic signals from the various components of the detector were converted to digital form, and recorded. There were generally five sources of digital information from the detector: 1) ADCs, Analog to Digital Converters from the phototubes, 2) TDCs, Time to Digital Converters for the drift chambers, 3) Trigger and phototube bits, 4) Beam monitoring data for magnet settings and EPICURE beam monitoring device data [145], and 5) Slow monitoring data, like temperatures and high voltage settings.

NuTeV used 11 bit LeCroy 4300 FERA ADCs to digitize the various signals produced by the calorimeter phototubes. These were read out by means of a CAMAC bus system. The TDCs were custom made boards placed in racks in the Lab E detector hall. They had a 4 nanosecond time resolution, and were able to store 32 events in their buffers for each spill. The buffers were read out by the 
DAQ system at the end of each spill. The VME system wrote the data in the form of several ZEBRA [146] banks. Different branches of the data stream would be written in different banks: Beam monitoring data, CAMAC ADC and scaler data, TDC readouts, slow monitoring data, and calibration beam monitoring and chamber data. The ZEBRA banks were written to exabyte tapes, typically at a rate of about two 5 gigabyte tapes per day. Approximately 600 of these data tapes were written over the course of $\mathrm{NuTeV}$ running.

\subsection{The NuTeV Cruncher: e815_analysis}

The $\mathrm{NuTeV}$ data (and GEANT based McNuTeV detector simulation which we'll discuss in the next chapter) are "crunched" into a usable form by a package aptly named "e815_analysis" ${ }^{1}$ E815_analysis reads in the ZEBRA banks written to tape by the $\mathrm{NuTeV}$ DAQ, unpacks them, reconstructs measured energies and positions, and calculates physical parameters for each event. The output of e815_analysis is usually an HBOOK file containing histograms and ntuples, or a DST which is further processed by PHDST, the DST reader and physics analysis package. In this analysis e815_analysis has been used to generate ntuples which were processed with the fitting software described in the next chapter.

\section{$\underline{3.7 \text { Event Reconstruction }}$}

As the ZEBRA banks are unpacked, the data within is used to determine what was measured in each component of the detector. The TDC bank gets

\footnotetext{
${ }^{1}$ E815_analysis can claim descent from a smaller program: "CNFCRAP", which stood for "Completely Non-Fancy Cosmic Ray Analysis Package". CNFCRAP was renamed e815_analysis once NuTeV began taking neutrino data, because "CNFNUAP" did not have sufficient pizazz.
} 
transformed into an array of drift chamber hit locations and times and the ADC bank becomes an array of phototube pulse height information. These data are used to calculate the characteristics of the particles which interacted in the detector. The following is a listing of the variables used in this analysis and how they are obtained:

RUN,EVENT The event and run number are recorded in the header of each event. They are useful for example, to flag blocks of data that was taken when something may have been wrong with the detector or beam.

LTRIGB This is a logical array containing the trigger bits for an event. Since this is an analysis of charged current events, $\operatorname{LTRIGB}(1)$ is required to be set.

TORPOL Toroid polarity, either +1 or -1 , and is found from a small database based on the run log.

PLACE This is intended to indicate in which counter the event started. PLACE is defined to be the most upstream of the upstream pair of counters with more than 4 MIPs of energy deposited in them.

SHEND Short for "shower end", although not used in any cuts, it is used in the definition of the shower energy. SHEND is defined to be the first counter downstream of PLACE followed by three consecutive counters with less than four MIPs of energy deposited in them.

VERTZ This is the physical position of the center of the PLACE counter

VERTX,VERTY These are the $\mathrm{x}$ (horizontal) and $\mathrm{y}$ (vertical) locations of the event vertex. These are defined by following the tracks back to their most 
upstream hits, rejecting those out of time and not ending near PLACE ("not near PLACE" is defined to be 4 chambers). The event vertex positions are then the weighted average positions of the track endpoints one chamber upstream of PLACE. The weights are taken from the two counters adjacent to each chamber.

TIMMU1,TIMMU2 These are the Sbit times associated with the drift chamber hits of muon track 1 and 2. The "zero" time for a track is near 236 TDC counts.

CHARG1,CHARG2 These are the reconstructed charges of muons 1 and 2. They are based on the direction of curvature of the track in the muon spectrometer. If there is more than one muon track reconstructed, muon 1 is defined to be the highest energy muon with a charge one would expect from the primary charged current interaction (in neutrino mode this would be -1, antineutrino mode +1 ). Muon 2 is then the muon with opposite charge (or if the muon is the same charge, its the one with lower energy. A muon must pass through the toroid for the charge to be determined. If a dimuon event only has one muon pass through the spectrometer its charge is used to determine whether its muon 1 (primary) or 2 (charm decay). The other muon is the other muon, and is assumed to have opposite charge.

EMU1,EMU2 These are the reconstructed muon energies. There are two pieces that contribute to the muon energy. If the track reached the muon spectrometer, the momentum calculated from the curvature of the fitted track is placed in the variable EMUFF1 (or 2). The portion of the muon energy 
within the calorimeter is calculated in different ways depending if there is one or more tracks. If there is more than one track, the energy is calculated based on the muon track length using range tables from reference [147]. In the case of one track, the counter pulse heights are used to directly measure the energy back to SHEND. From there, parameterization of the average energy loss for a minimum ionizing particle is used to calculate the energy lost within the hadronic shower.

EMUFF1,EMUFF2 The energies of the muon at the front face of the steel in the muon spectrometer. See above.

XFF,YFF These are the extrapolated $\mathrm{x}$ and $\mathrm{y}$ positions of the track at the front face of the muon spectrometer.

STEEL This is a number from 0 to 1 of the fraction of the toroid steel that a muon track passes through. The fitted track is followed and this number is calculated based on its shape.

THETAVR,PHIVR These are the calculated polar coordinates of each muon track at the event vertex

GAP1,2,3 These are flags based on toroid drift chamber hits indicating whether the track passed through each of the three toroid gaps. The code setting these flags is listed in the appendices on page 402

EHCC3 EHCC3 is the transverse position corrected hadronic energy deposited in the scintillator counters from PLACE to SHEND-5, with the muon contribution subtracted out. 
These variables are used first in event selection, and then to construct the physics parameters used in this analysis.

\section{$\underline{3.8 \text { Data }}$}

The NuTeV data was taken over the period of 1 year, from August of 1996 through August of 1997 . During that time approximately $3.15 \times 10^{18}$ protons were taken on the NuTeV target, producing 2,574,783 $\nu$ and 914,886 $\bar{\nu}$ trigger 1 events.

Figure 71 shows a good example of a trigger 1 charged current event from the $\mathrm{NuTeV}$ event display (see for example figure 19 on page 50, and figure 20 on page 54). In the figure, the neutrino beam enters from the left, and interacts, producing a hadronic shower (black blob of X's) and muon track (sequence of black X's trailing off to the right). Each " $\mathrm{X}$ " signifies a hit in one of the drift chambers. Two views of the detector are shown, the y view shows the detector view as if one were standing on its east side, the $\mathrm{x}$ view shows the detector as if one were hovering over the top, looking down. The series of blue lines indicate the layers of scintillators and drift chambers, and the three toroid coils, with the drift chambers in between and behind can be seen in the rear half of the detector. The red lines above the y view are the scintillator pulse heights from the highs. One can see the spike from the hadron shower, then the near constant stretch of pulse heights as the muon travels through the detector. The green dots below the pulse heights indicate which Sbits fired for that event. On the very top of the display, in addition to the run and event information are the triggers which fired for that event, and to the right, estimates of the hadron and muon energies. Figure 72 


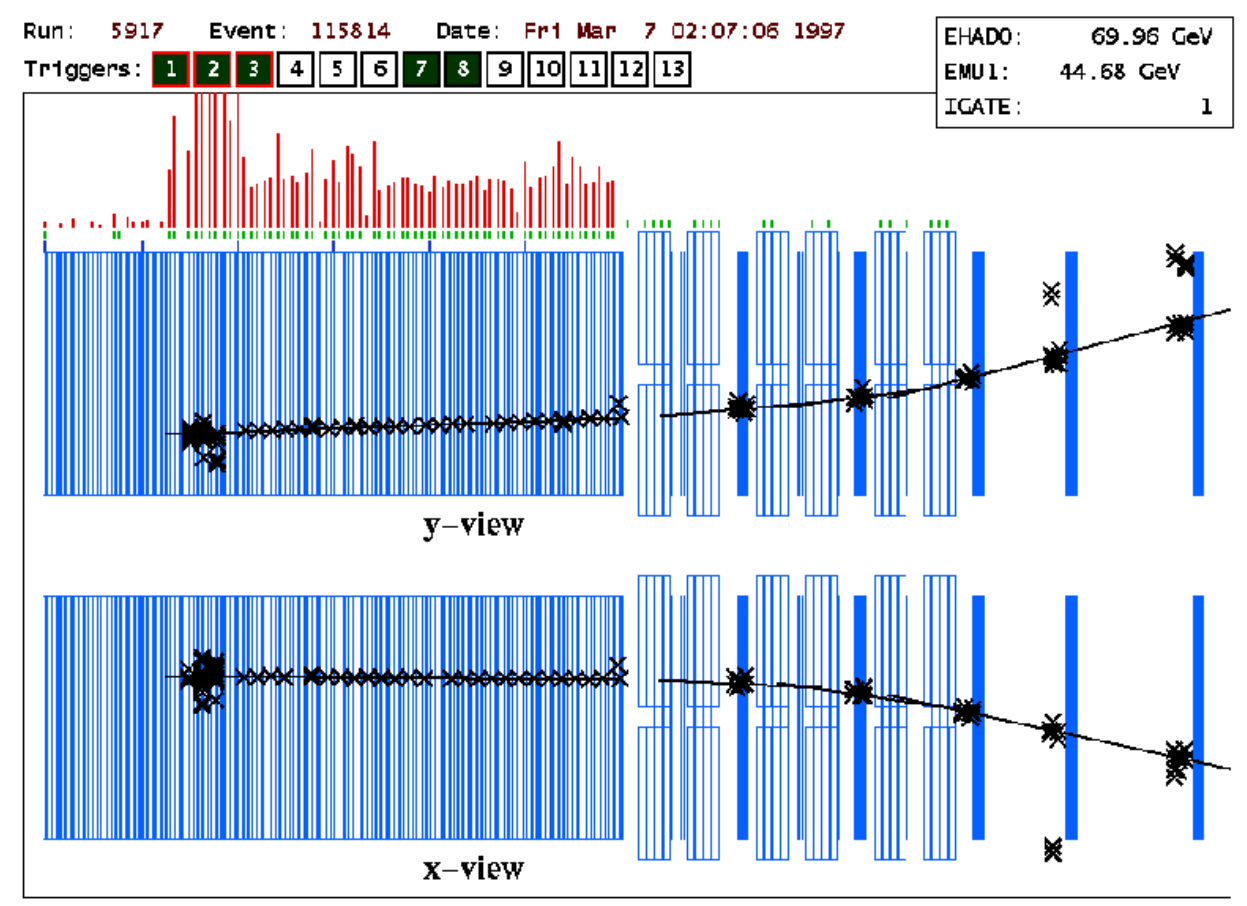

FIGURE 71. A charged current event in the $\mathrm{NuTeV}$ event display

shows another event display with a neutral current event. In this case there is only a hadron shower, and no muon. Figure 73 shows an example of a cosmic ray that had interacted in the detector.

\subsubsection{The dimuon split}

Immediately after data taking, it was determined that the full sample of trigger 1 events was too unwieldy to use in the dimuon analysis. The compressed ZEBRA data files consume approximately 75 Gigabytes of disk space, which was rather large at a time when a 9 Gigabyte disk was the norm. To simplify analysis, a subsample was stripped out of the full trigger 1 sample. This subsample, the "dimuon split", was chosen to include likely dimuon events, but be done with 


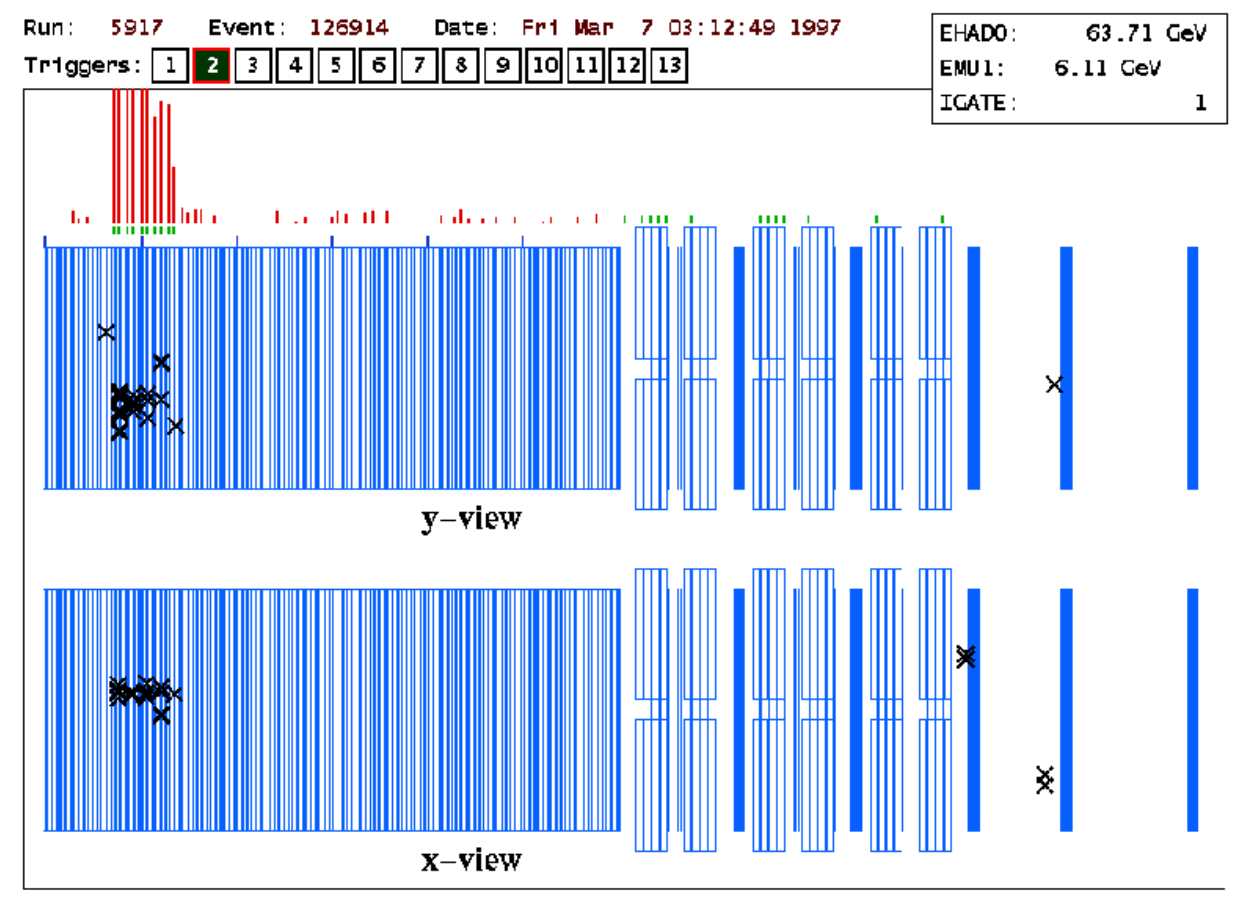

FIGURE 72. A neutral current event from the $\mathrm{NuTeV}$ event display

cuts loose enough so that legitimate dimuon events wouldn't be removed from the sample. The conditions imposed on events which were passed to the dimuon split were:

$-10 \leq \mathrm{PLACE} \leq 82$

$--60 " \leq$ VERTX,VERTY $\leq 60 "$

- At least 1 track be present in both views in the calorimeter chambers

- 10 counters must have $>1.3$ MIPs, allowing for a 2 counter gap.

This brought the trigger 1 sample down to a more manageable 193,033 $\nu$ and $51,032 \bar{\nu}$ dimuon split events. If one now applies the analysis cuts below to both the split sample and the full sample as a check, indeed no usable events were lost 


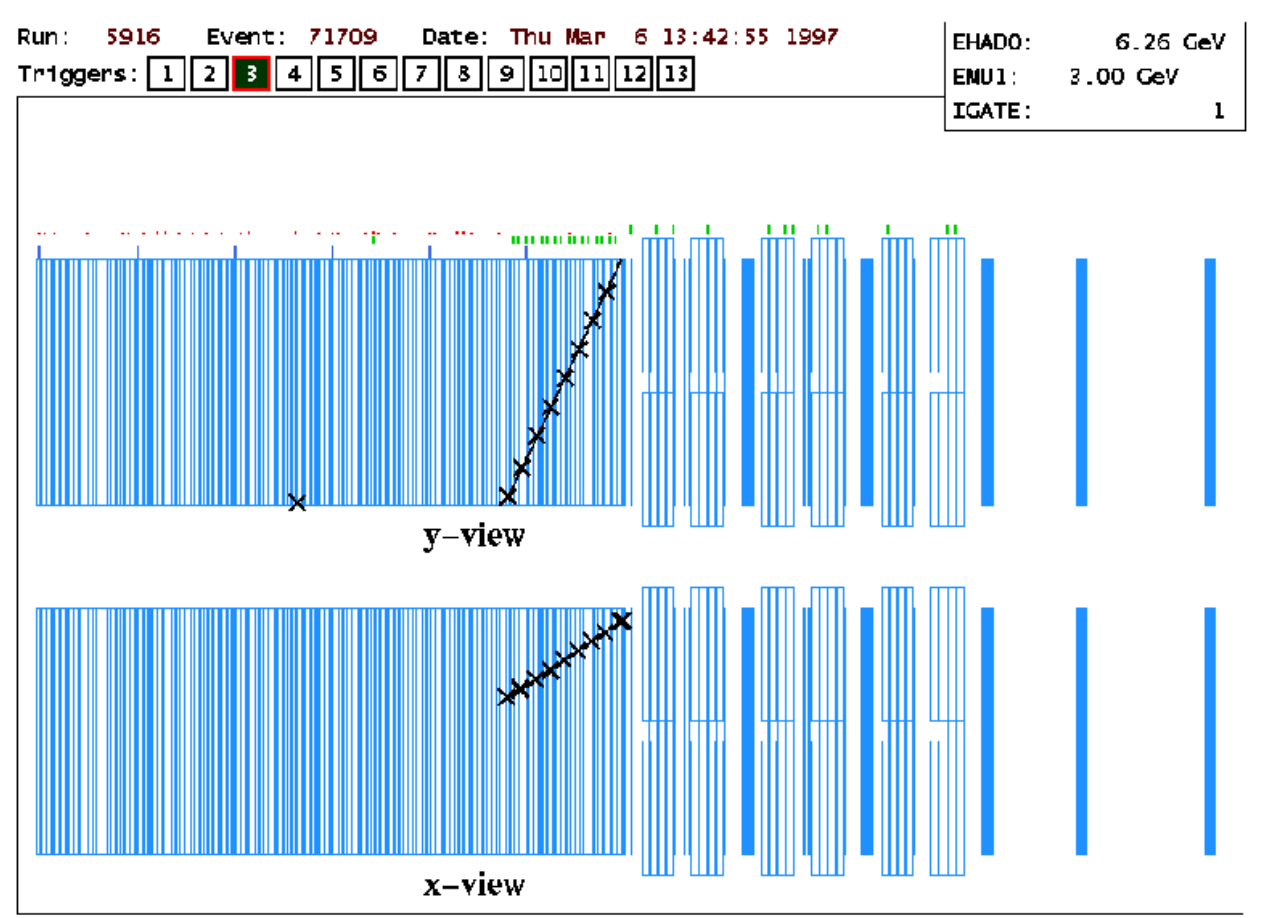

FIGURE 73. A cosmic ray background event

in the dimuon split sample. An example of a dimuon event is shown in figure 74 . Both muons are seen tracking back from the hadron shower through to the back of the spectrometer. The muon from the leptonic vertex was focused (the more straight of the two muons), and the muon from the charm decay, of opposite sign, was ejected from the spectrometer.

\subsubsection{The $5 \%$ split}

Because of the dimuon event sample's relatively low statistics ( $1 \%$ of inclusive charged current events), the full trigger 1 sample was not needed to normalize the dimuon sample with sufficient precision. A "5\% split" of all trigger 1's was extracted so that a normalization sample could be readily available on disk. Splitting 


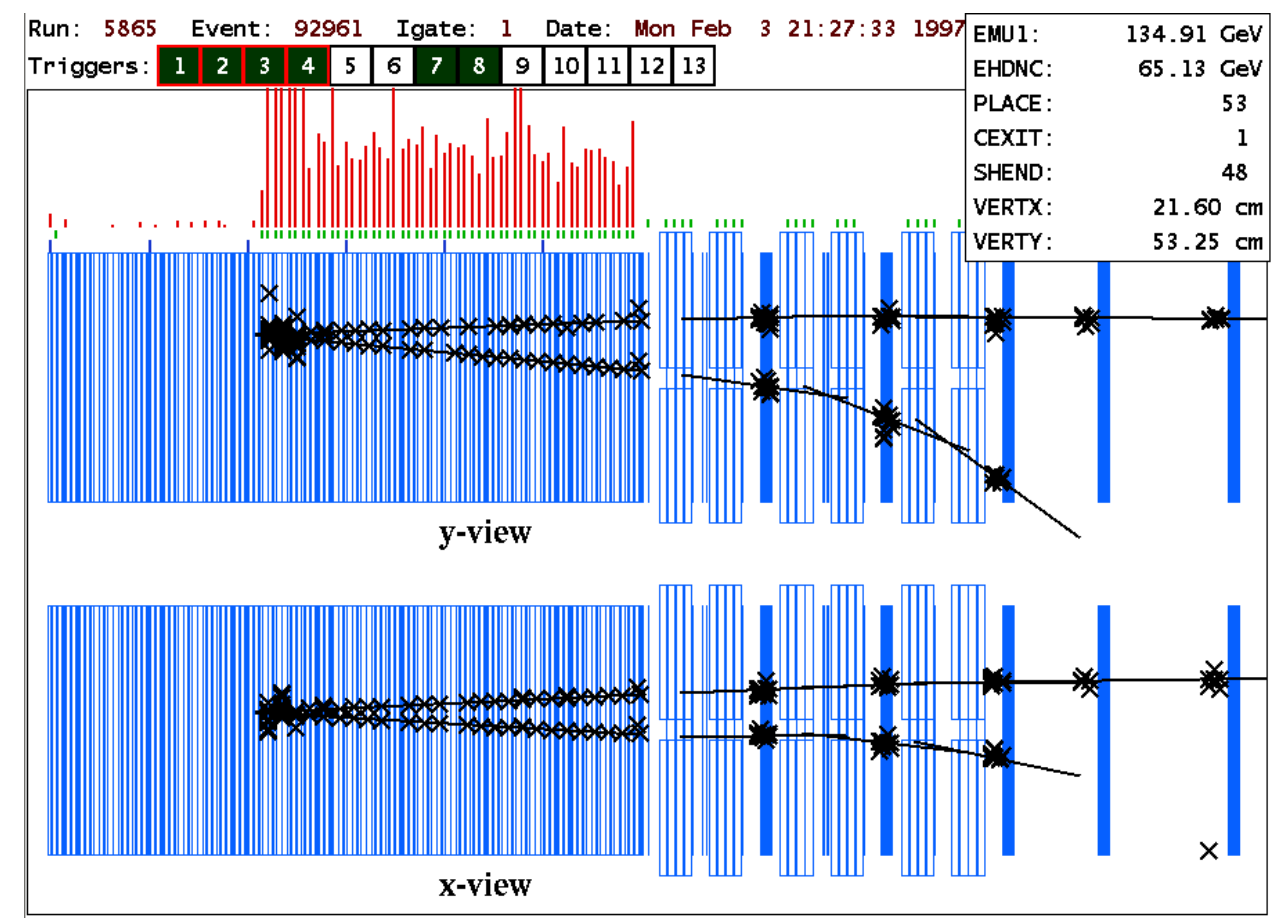

FIGURE 74. A textbook example of a dimuon event.

out this sample was simple, every 20th event was written out in the split stream. Because pedestal events were still needed to set the zero levels of the electronics, however, all pedestal triggers were passed through to the split stream as well, but without the prescale. This led to a problem however, discovered only recently.

Trigger 11 was intended to flag events where a neutrino interaction happened in the toroid spectrometer. These events were called "toroid pedestal" events, because the calorimeter would in principle be empty, and pedestals could be measured under "beam on" conditions. The event displayed in figure 75 is an example of such an event. It turned out however that legitimate trigger 1's with perhaps more muon energy than usual would also fire trigger 11. Since trigger 11 events were also passed into the $5 \%$ split without a prescale, the $5 \%$ split became a $5 \%$ trigger 


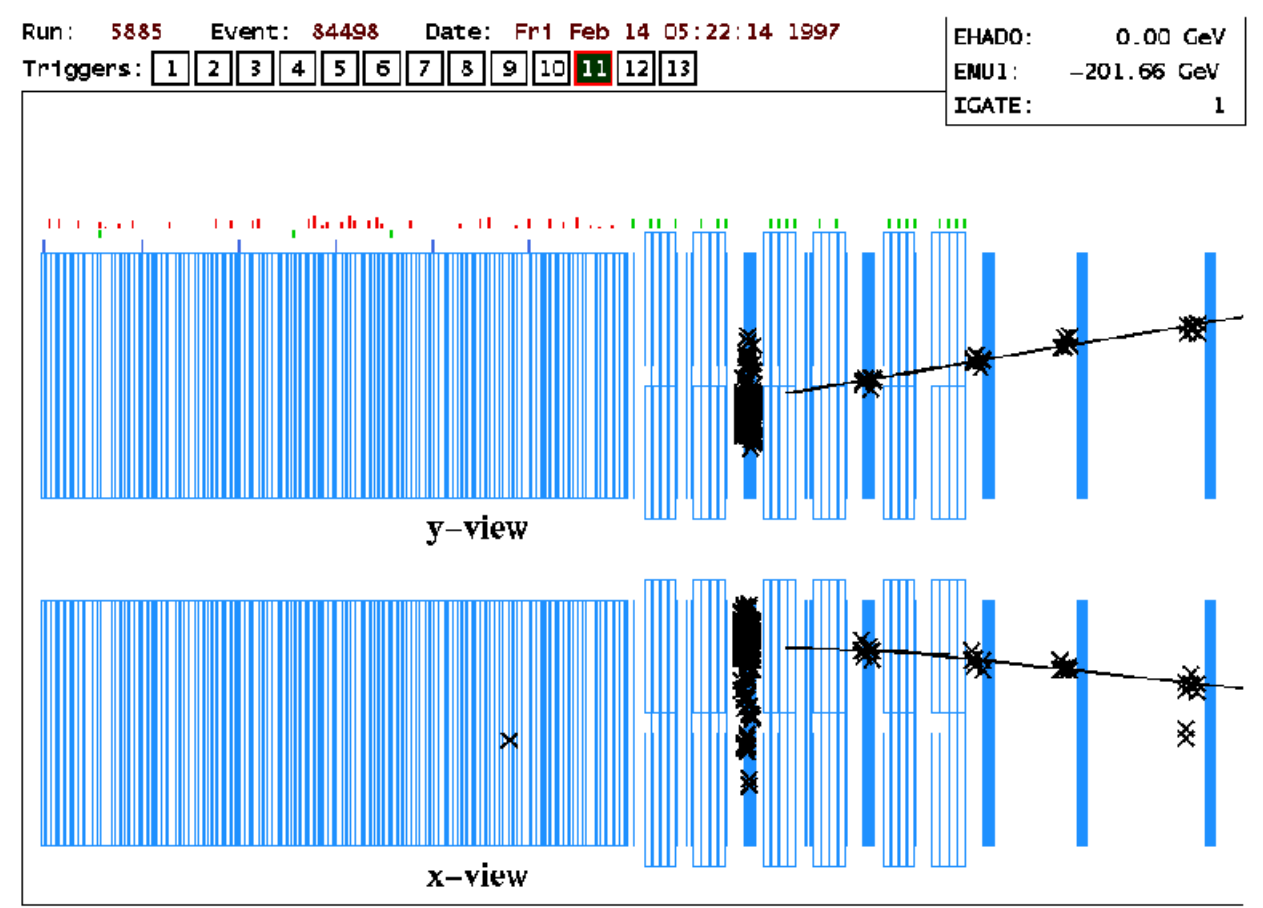

FIGURE 75. A proper trigger 11 event

$1+100 \%$ split of trigger 1's which also fired trigger 11. Figure 76 shows one of these perfectly legitimate trigger 1's which also set off trigger 11.

This trigger 11 event excess in the $5 \%$ split affected the normalization in the previous NuTeV dimuon analysis. Because firing trigger 11 tended to happen for larger muon energies, the effect was $y$ (inelasticity) dependent, so that not only was the overall normalization affected, but also the relative normalization between neutrino and antineutrino dimuon events. This normalization change affects both the strange sea measurement as well as the measurement of the difference relative to the antistrange sea. In this analysis the full sample is used to eliminate the trigger 11 problem, and the forward dimuon cross section will be re-extracted, described in detail in the next chapter. 


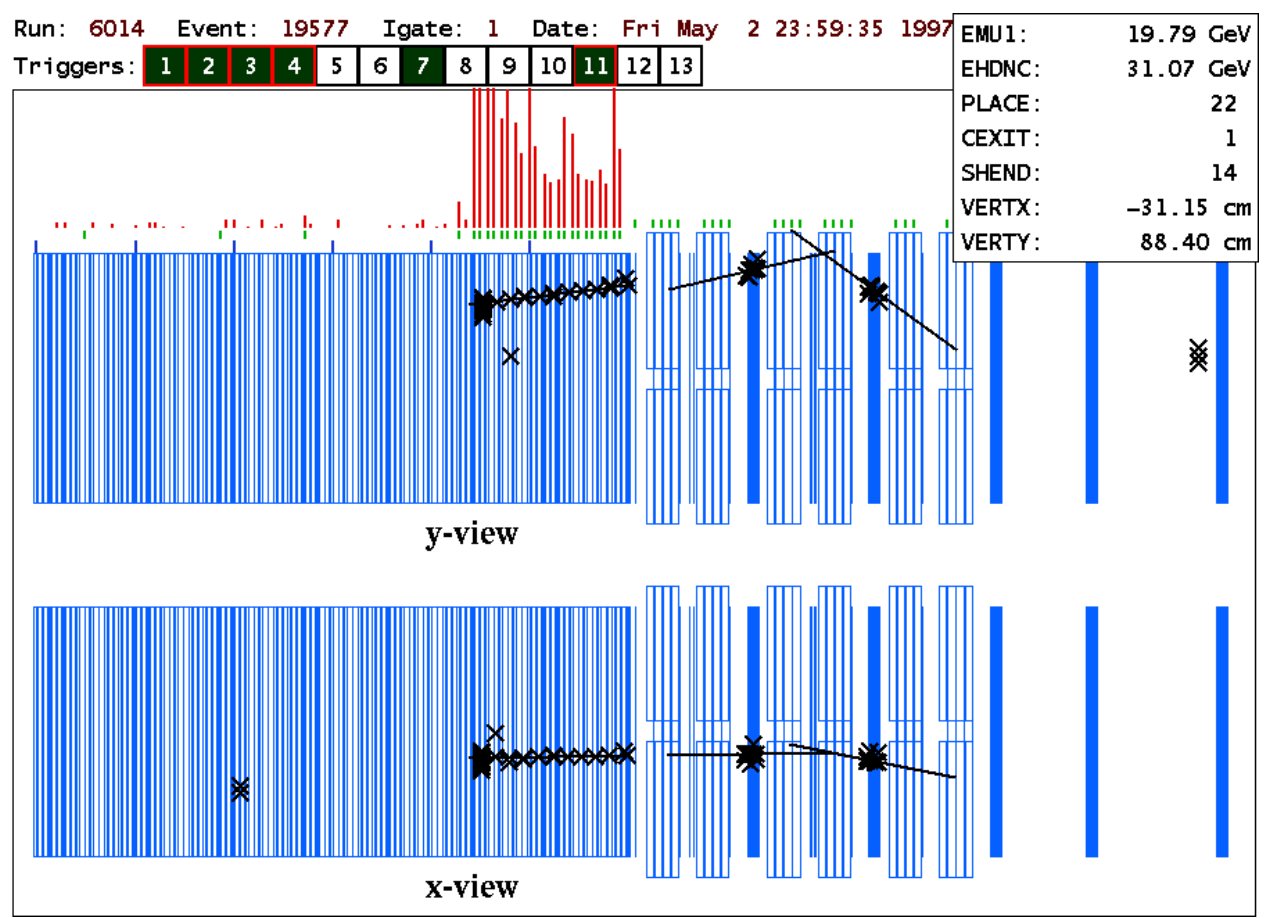

FIGURE 76. An example of a reasonable CC event masquerading as a trigger 11 event.

\subsection{Data Cuts}

The detector's ability to reconstruct events and the validity of calibrations and MC models require limits, or "cuts" be applied on many of a candidate events parameters. The two classes of data and $\mathrm{MC}$ events (inclusive and dimuon) require different sets of cuts, with the inclusive cuts being a subset of the dimuon cuts. Dimuons are further classified by how many of the muons were reconstructed in the muon spectrometer, indicated by whether their charge was reconstructed or not. Table 10 lists the cuts applied to each class of event. These same cuts were applied to both data and McNuTeV Monte-Carlo. 
The philosophy guiding the cuts displayed in table 10 can be expressed in two statements:

1. For the dimuon sample to be properly normalized to the inclusive sample, all events should be a subset of the inclusive sample.

2. All events must have at least one muon reconstructed in the toroid. This is so charge may be used to identify which muon is the primary muon and which is the muon from charm decay.

It turns out however that rule $\# 1$ is bent in the dimuon case to increase statistics. Some of the energy that would otherwise be in the hadronic shower is taken away by the charm decay muon. This would effectively shorten the hadronic shower on average [148], so that a slightly longer fiducial region can be defined for dimuon events. This increases dimuon statistics by about $7 \%$. Increasing the dimuon fiducial region does not affect dimuon normalization as long as the same fiducial region is selected in the Monte Carlo, and it is.

\subsubsection{Description of cuts}

trigger 1 Trigger 1 is the charged current trigger, so all inclusive and dimuon events must have set off trigger 1

badruns This removes runs and/or event blocks where known hardware failures rendered the data useless. Code is included in appendix C.0.6. 


\begin{tabular}{|c|c|c|c|}
\hline Cut & Inclusive & \multicolumn{2}{|c|}{ Dimuon } \\
\hline & & $\begin{array}{c}\text { charg } 1 \neq 0 \text {.and } \\
\text { charg } 2 \neq 0\end{array}$ & $\operatorname{charg} 2=0$ \\
\hline trigger 1 & $\sqrt{ }$ & $\sqrt{ }$ & $\sqrt{ }$ \\
\hline badruns & $\sqrt{ }$ & $\sqrt{ }$ & $\sqrt{ }$ \\
\hline $15 \leq$ place $\leq 81$ & $\sqrt{ }$ & $\sqrt{ }$ & $\sqrt{ }$ \\
\hline $50 "$ box & $\sqrt{ }$ & $\sqrt{ }$ & $\sqrt{ }$ \\
\hline $60 "$ radius & $\sqrt{ }$ & $\sqrt{ }$ & $\sqrt{ }$ \\
\hline $\mid$ timmu1-236| $\leq 9$ & $\sqrt{ }$ & $\sqrt{ }$ & $\sqrt{ }$ \\
\hline emu $1 \geq 5 \mathrm{GeV}$ & $\sqrt{ }$ & $\sqrt{ }$ & $\sqrt{ }$ \\
\hline $10 \leq$ ehcc $3 \leq 300 \mathrm{GeV}$ & $\sqrt{ }$ & $\sqrt{ }$ & $\sqrt{ }$ \\
\hline $20 \leq$ enuvis $\leq 400 \mathrm{GeV}$ & $\sqrt{ }$ & $\sqrt{ }$ & $\sqrt{ }$ \\
\hline $20 \leq$ place $\leq 80$ & $\sqrt{ }$ & & \\
\hline charg1 = torpol & $\sqrt{ }$ & $\sqrt{ }$ & $\sqrt{ }$ \\
\hline $60 " \mu 1 \mathrm{FF}$ radius & $\sqrt{ }$ & $\sqrt{ }$ & $\sqrt{ }$ \\
\hline$\mu 180 \%$ steel & $\sqrt{ }$ & $\sqrt{ }$ & $\sqrt{ }$ \\
\hline$\mu 1$ gap cut & $\sqrt{ }$ & $\sqrt{ }$ & $\sqrt{ }$ \\
\hline $5 \leq$ emuff $1 \leq 400 \mathrm{GeV}$ & $\sqrt{ }$ & $\sqrt{ }$ & $\sqrt{ }$ \\
\hline phivr $1 \leq 2 \pi$ & $\sqrt{ }$ & $\sqrt{ }$ & $\sqrt{ }$ \\
\hline emu $2 \geq 5 \mathrm{GeV}$ & & $\sqrt{ }$ & $\sqrt{ }$ \\
\hline $\mid$ timmu2-236| $\leq 9$ & & $\sqrt{ }$ & $\sqrt{ }$ \\
\hline charges opposite & & $\sqrt{ }$ & \\
\hline $60 " \mu 2 \mathrm{FF}$ radius & & $\sqrt{ }$ & \\
\hline$\mu 280 \%$ steel & & $\sqrt{ }$ & \\
\hline phivr $2 \leq 2 \pi$ & & $\sqrt{ }$ & \\
\hline
\end{tabular}

TABLE 10. Cuts applied to the inclusive event samples, and for both toroid analyzed and calorimeter range-out/exit muons 
$15 \leq$ place $\leq 81$ This ensures the event happened 3 counters downstream of the front counter (counter 84) in the calorimeter, and that the event happened far enough upstream of the toroid that the hadron shower is contained within the calorimeter.

50 " box Requires both vertx and verty be between $-50 "$ and +50 ". This also is to ensure hadron shower containment within the calorimeter.

60 " radius This is the transverse radius cut on vertx and verty. This rounds off the corners on the 50" box. It removes events which would tend to produce muons passing outside the good field region in the muon spectrometer, or miss it entirely.

$\mid$ timmu1-236 $\mid \leq 9$ This is the timing cut on the primary muon (a similar cut is used for the secondary muon from the charm decay). This is to remove "double" events, where a second event may have happened, or was in the process of happening while data was being read out. The units of this cut are in ADC counts, with each ADC count corresponding to 4 nanoseconds.

$80 \%$ steel psteel1 $/ 2$, defined in appendix C.0.5 must be greater than or equal to 0.8 , indicating that $80 \%$ of the muon's path in the muon spectrometer was in the steel.

emu1 $\geq 5 \mathrm{GeV}$ Both muons must be energetic enough to extend beyond the hadronic shower. This cut is somewhat redundant with the requirements on the muon track in the toroid. 
$10 \leq e h c c 3 \leq 300 \mathrm{GeV}$ The hadronic shower calibration is very poor below an energy of $10 \mathrm{GeV}$. The upper limit is applied to remove grossly misreconstructed hadronic energies.

$20 \leq$ enuvis $\leq 400 \mathrm{GeV}$ This is the energy range that the neutrino flux is valid.

charg1 $=$ torpol The primary muon must have the proper sign. This cut is also applied for the dimuons, even though it is possible for the charm decay muon to have been reconstructed well. Those events however would tend to either be dimuons from wrong sign events, or from low $y$ events not present in the charged current sample.

60" FF radius A 60" radius cut on the position where the muon tracks cross the plane of the muon spectrometer front face. This ensures the muon enters where the spectrometer magnetic field is well understood.

gap cuts The requirement that at least one view of the muon track be reconstructed and pass through at least two consecutive gaps in the muon spectrometer. The routine gapper.F (appendix C.0.7) takes the encoded bits and applies the cut. This guarantees a good muon track fit, and good muon momentum measurement.

$5 \leq$ emuff $1 \leq 400$ This is to ensure that the muon have sufficient energy to pass through the entire toroid.

phivr $1 \leq 2 \pi$ This removes grossly misreconstructed events. 


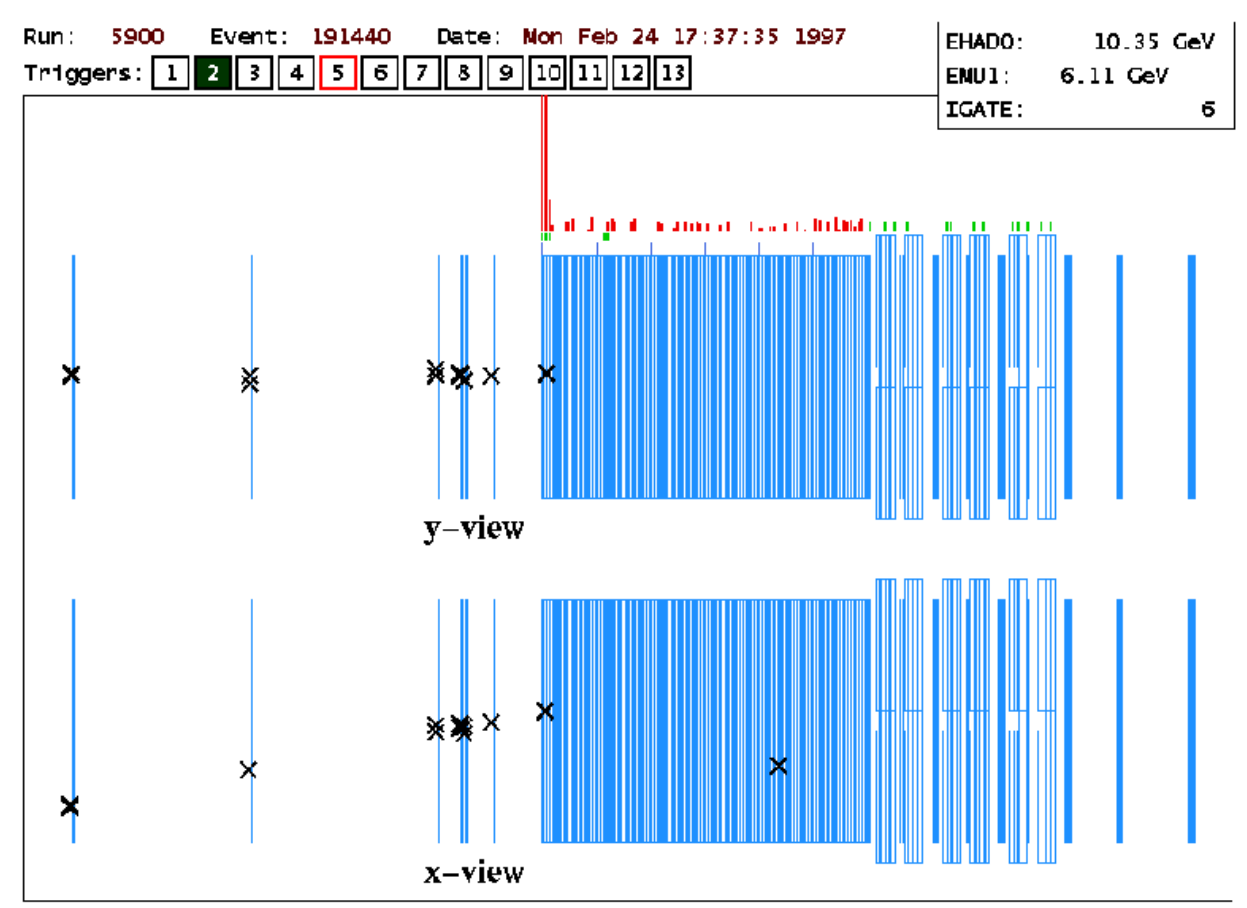

FIGURE 77. A calibration beam electron event

\section{$\underline{3.10 \text { Detector Calibration }}$}

The detector was calibrated throughout data running by muon, electron, and hadron beams. The electron beam was used to study the difference between short ranged electron-generated showers versus those generated by hadrons. This information was primarily used in the $\nu_{\mu} \rightarrow \nu_{e}$ oscillation search [149], and in the $\sin \left(\theta_{W}\right)$ analysis [150] for background studies. A calibration electron event is shown in figure 77. Electrons are thousands of times more likely than muons to lose energy through bremsstrahlung, making their range very short in the calorimeter. In the event shown in the figure, the electron only reached the 3rd counter before being totally absorbed. 
Calibration Beam hadrons, mostly pions were used to measure the scintillator phototube response to hadronic showers in the calorimeter. A typical calibration hadron event is shown in figure 78. Hadrons with energies ranging from 5 to 190 $\mathrm{GeV}$ were momentum analyzed upstream in the calibration spectrometer, before striking the front of the $\mathrm{NuTeV}$ detector. An example of calibration beam hadron event is shown in figure 78 . Figure 79 shows the hadron energy response as a function of the momentum measured by the calibration beam spectrometer. The energy resolution of the calorimeter was found to be:

$$
\frac{\sigma}{E_{H A D}}=\frac{0.86 \pm 0.01}{\sqrt{E_{H A D}}}+0.022 \pm 0.001
$$

For a typical $50 \mathrm{GeV}$ hadron shower this would correspond to an energy resolution of about $14 \%$.

Both calibration beam and straight-through muons (figures 81 and 80 respectively) were used to calibrate the phototubes in the calorimeter. The position dependence shown in figure 60 on page 144 was obtained by measuring the phototube response as a function of straight through muon track position. These "muon maps" were obtained for each of the 84 scintillator counters in the calorimeter, and were used correct out the position dependence of the pulse height determined energies. These muons were also used to propagate the hadron calibrations measured at the front of the detector back through the rest of the detector.

The calibration beam muons were also used to study detector pulls and smearing in the calorimeter and toroid. Figure 82 shows the measured energy loss in the calorimeter for calibration beam muons, as a function of total energy. Muons of energy ranging from 12.5 to $190 \mathrm{GeV}$ were used to study energy response, 


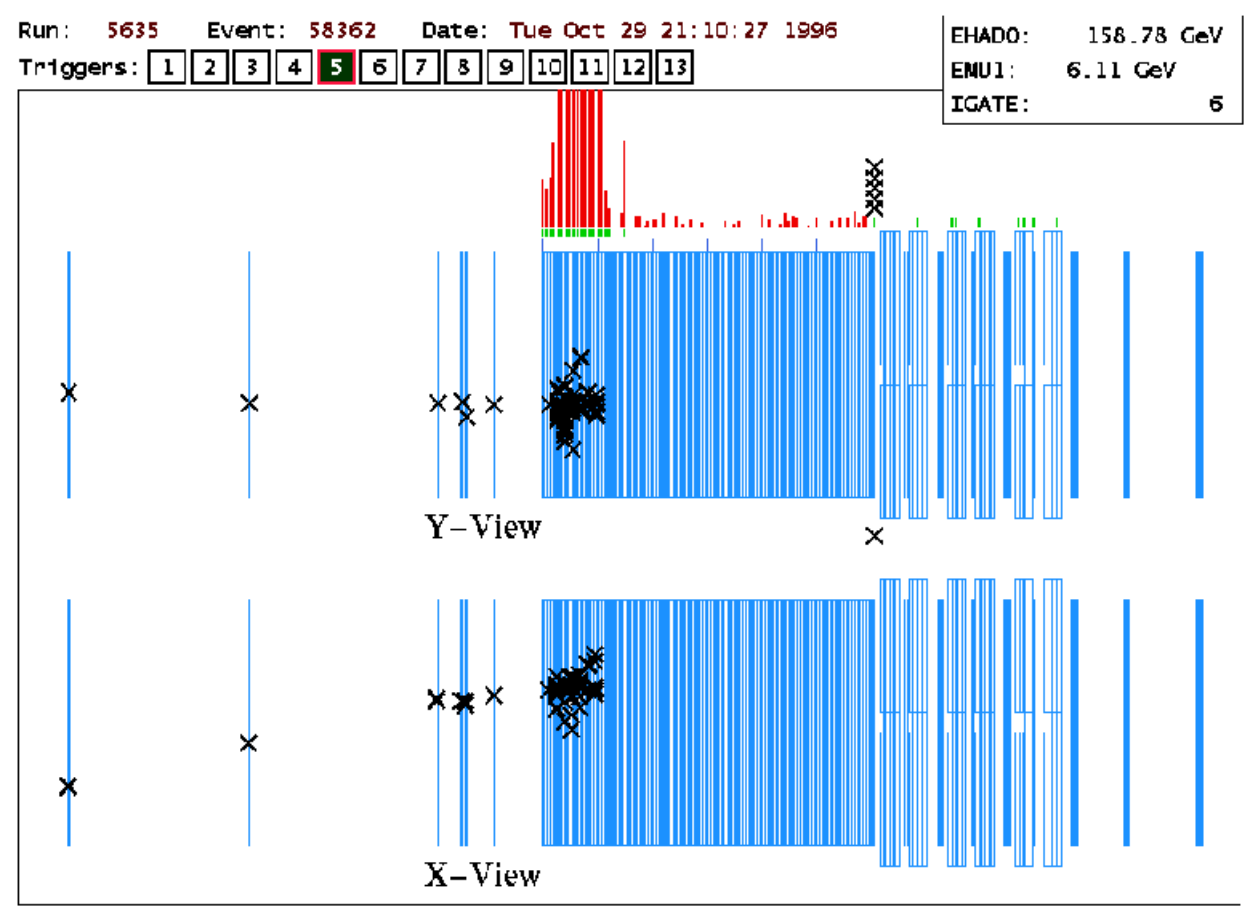

FIGURE 78. A calibration beam hadron event

and 50 and $100 \mathrm{GeV}$ muons were scanned in a grid pattern over the spectrometer magnets to calibrate the energy response to better than $1 \%$. The momentum resolution $\frac{\Delta p}{p}$ was found to be $11 \%$.

\subsubsection{Dimuon range-out study}

The calibration muons were also used to check the muon range method of determining energies for dimuons which ranged out in the detector. As mentioned above, the energy of single muon tracks is determined by counter pulse heights, but when a second muon is present, this is impossible, since one cannot tell which fraction of a counter pulse height corresponds to which muon. The best that can be done then is to use the range of the muon, the distance it travels, as a measurement 


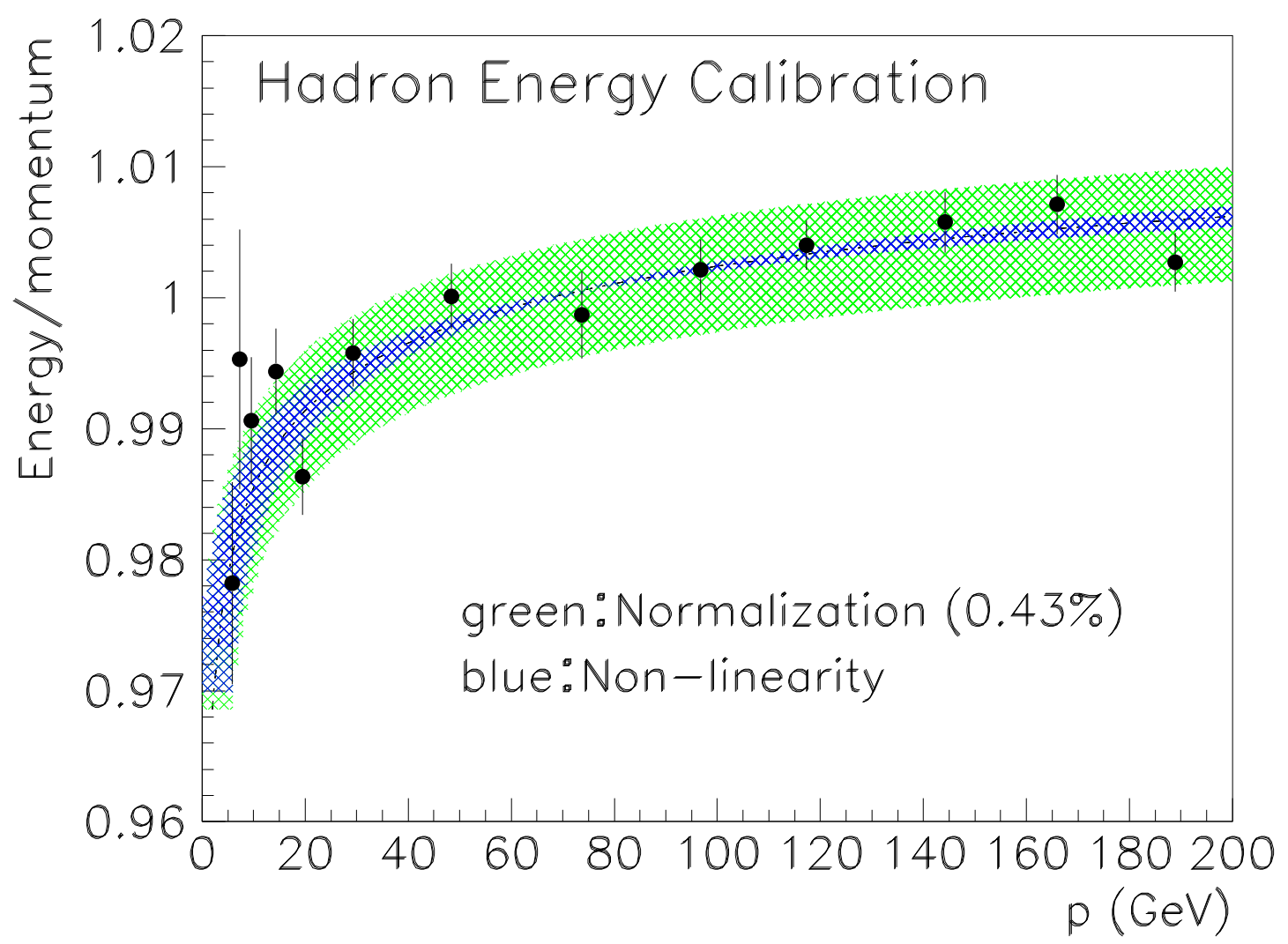

FIGURE 79. The hadron calibration. The green shaded region is the error on the overall hadronic energy scale, $0.43 \%$.

of the muon's energy. Calibration beam muons with energies low enough that they "range out", or stop inside the calorimeter can be used to check the range method of determining energy loss. From figure 82, it can be seen that the energy for such muons need to be less than about $14 \mathrm{GeV}$.

Only one set of calibration muon runs, those with energy $12.5 \mathrm{GeV}$ barely satisfied this condition. The energy of these muons as measured by the calibration beam spectrometer was compared to the energy obtained from the muon range in the detector. E815_analysis was modified to force it to use the range algorithm even when one muon was present. Figure 83 shows the results of a comparison 


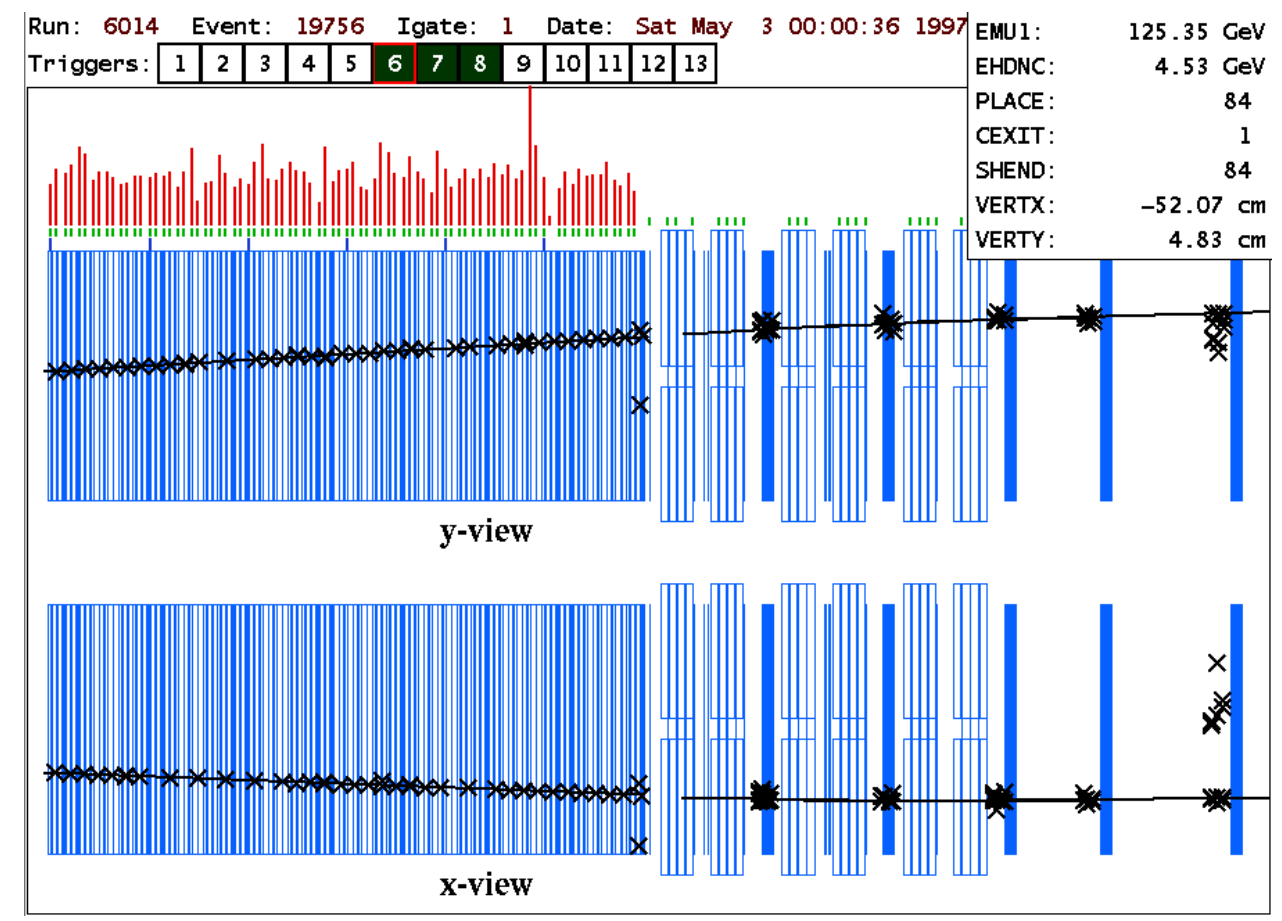

FIGURE 80. A "straight through muon" event

between the calibration beam data and Monte-Carlo. The calibration beam data has a $2 \%$ shift relative to the MC. This difference is taken as a systematic error in the range out muon energy calibration in this analysis. 


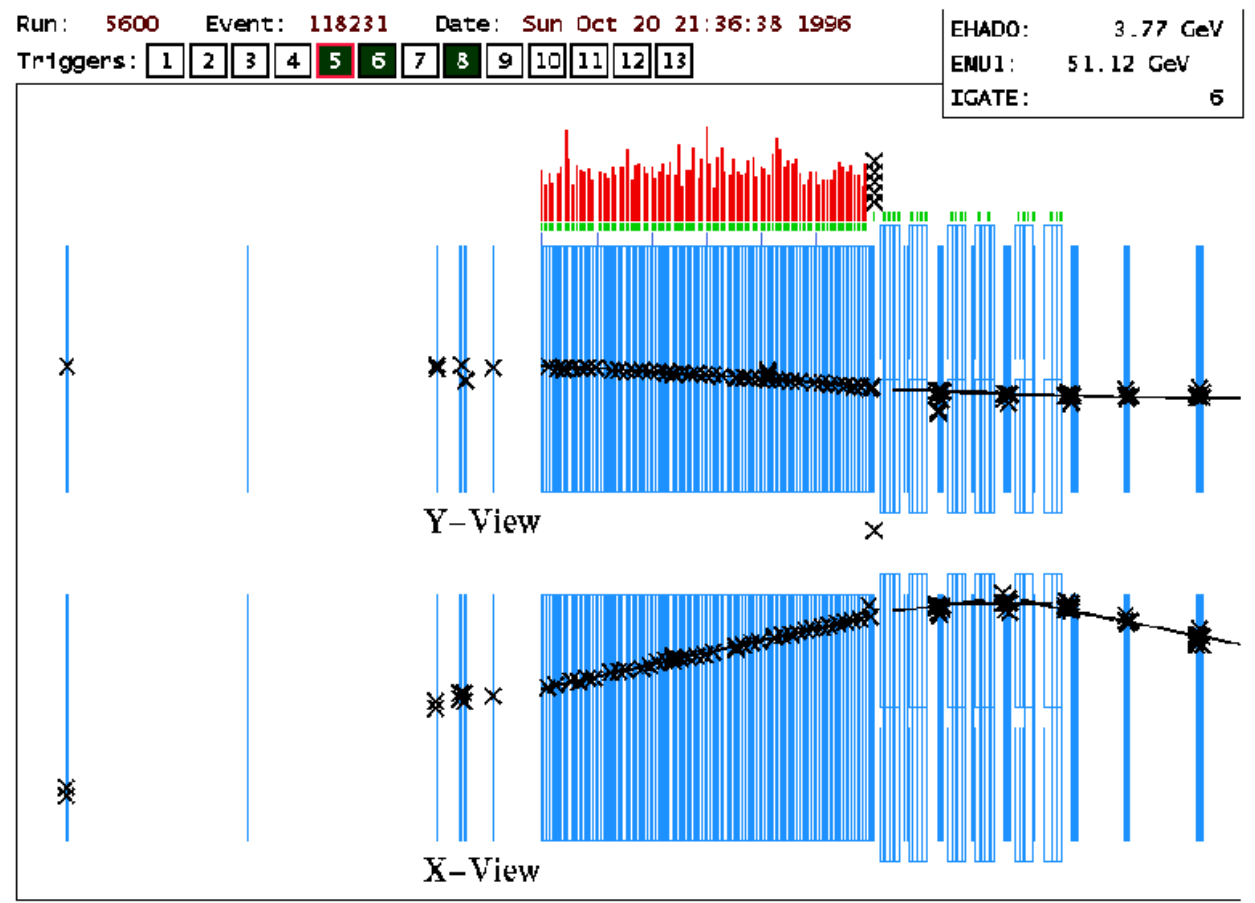

FIGURE 81. A calibration beam muon event 

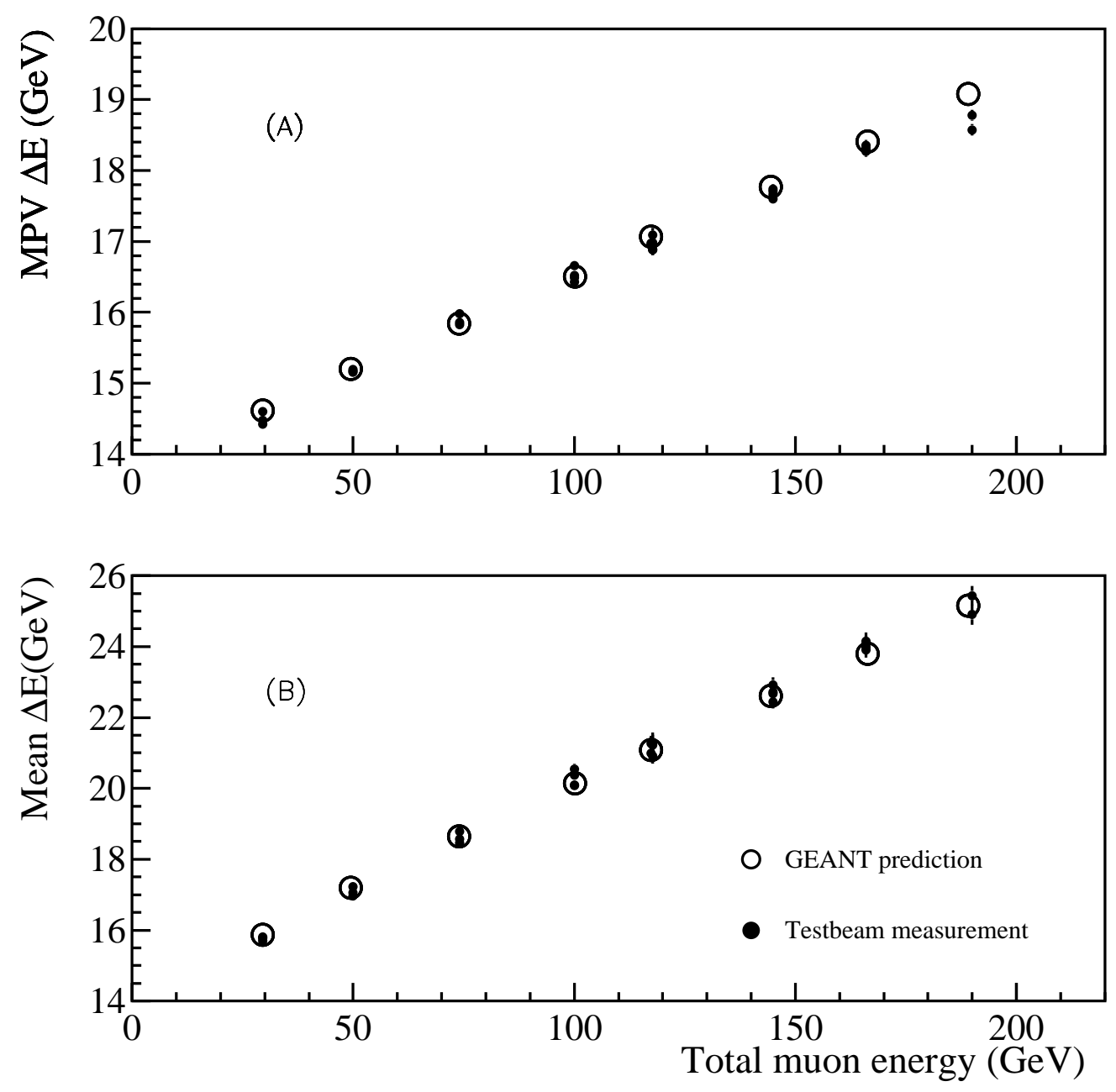

FIGURE 82. The most probable (top) and mean value (bottom) of muon energy loss in the calorimeter based on pulse heights. 

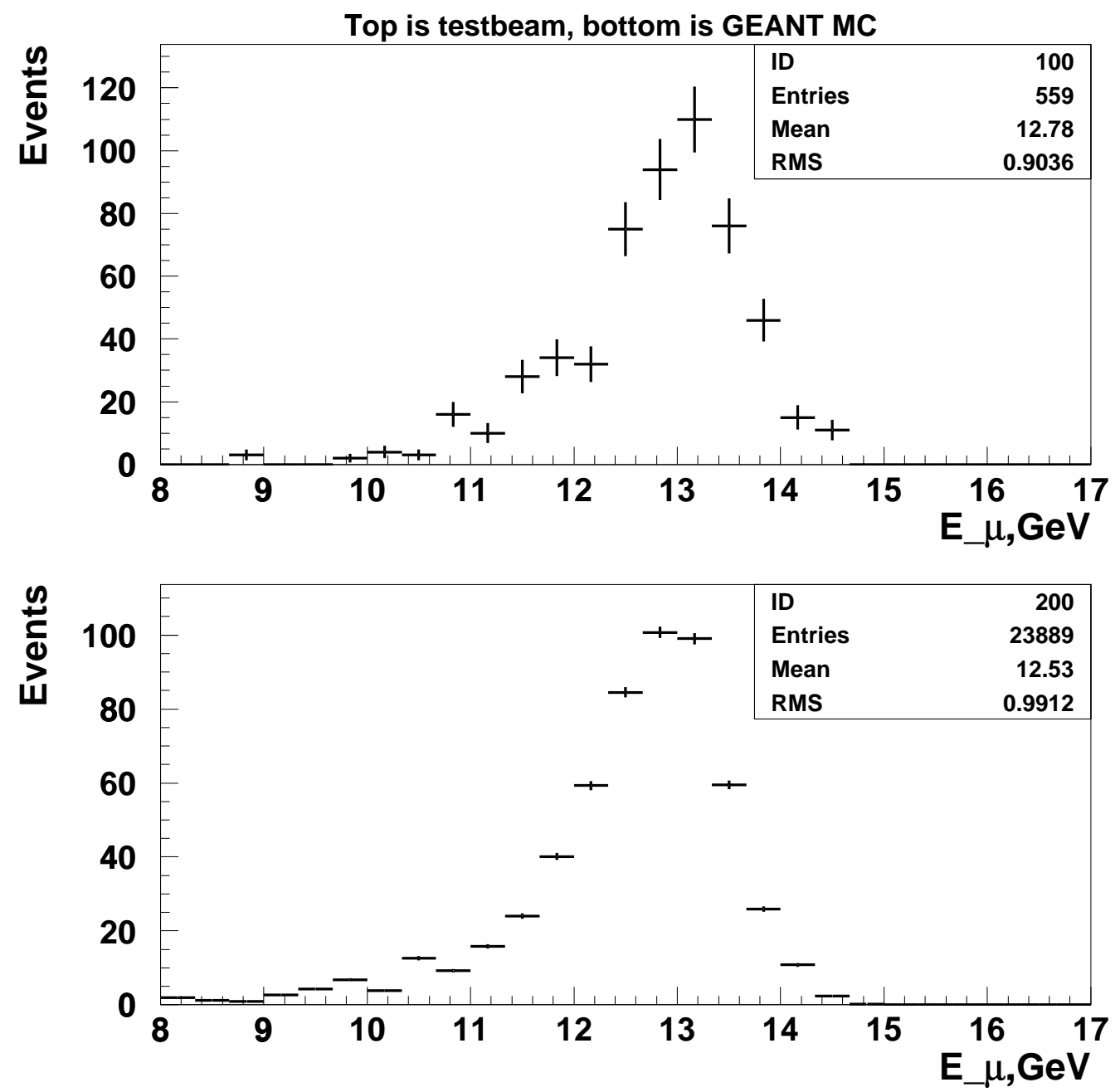

FIGURE 83. Reconstructed muon energies for $12.5 \mathrm{GeV}$ calibration beam muons (top) and Monte-Carlo simulated muons (bottom). The mean energies show a $2 \%$ shift. 


\section{CHAPTER IV}

\section{MONTE CARLO SIMULATION AND THE FORWARD DIMUON CROSS SECTION}

Extracting physics from data taken in a particle physics experiment can be a complicated business. The data that is taken is typically a collection of events for which some finite number of attributes, like kinematics, timing, location, etc. are recorded. Those attributes are recorded by a detection system whose ability to measure them is limited. These limitations can be due to the geometry of the detector, noise and inefficiencies in electronics and detector components, particles like neutrinos that escape undetected, the list goes on and on. The effects of an experiment's limitations can have a complicated effect on what is measured versus the physics that produced the events in the first place.

The way particle physicists usually take these complicated effects into account is by trying to create a simulation of the physics and detection environment which reproduces what is observed in the experiment. Fundamentally the simulation of an experiment requires the convolution of two elements:

$$
[\text { physics }] \otimes[\text { experimental effects }] \simeq[\text { data }]
$$

The [physics] piece typically includes as variables the quantities that are to be measured, such as particle masses, coupling constants, etc. Those quantities would be varied to provide the best agreement to the data, which when found becomes 
the measurement of those quantities. The [experimental effects] piece includes the limited resolution, noise, and efficiency effects due to the detection environment. Any uncertainties due to data statistics and in physical constants or corrections applied in the simulation translate into uncertainties in the measurement.

The probabilistic nature of the processes like those studied in particle physics suggest that an analogous approach be taken in simulating those processes. The convolution of the probability that a particular event occurs with all the experiment related corrections and efficiencies is usually impossible to describe in a sensible analytical expression. The numerical technique of Monte Carlo integration is therefore used to evaluate the convolution between physics and experiment.

In a Monte Carlo simulation, event characteristics are generated ("thrown") according to probability distributions describing the distribution of kinematic quantities and types of incoming particles, the cross sections for generating the process being measured (and for generating background events) as well as inefficiencies and changes in the detection environment. There are two equivalent ways that this is done, the first, "darts Monte Carlo", follows the rejection method of generating random numbers. In the simulation a prospective set of event characteristics are thrown, usually with a uniform, flat distribution, and a random number is compared to the probability of those characteristics occurring. If the the probability is greater than the thrown random number, the event is kept, if not it is thrown out and a new event is thrown. The second method is "weighted Monte Carlo", where all events are kept, however a weight is applied based on the probability of each event occurring. Due to the organic evolution of the Monte Carlo (MC) 
packages throughout the CCFR family of experiments up to the current experiment, $\mathrm{NuTeV}$, both methods are used in software for this analysis. This is not necessarily a bad thing.

A large collection of MC events are generated so that they may be processed in the same way as the real data. In the case of this analysis, events were generated with a full detector simulation such and recorded in even the same file format as the actual data. This way the same analysis software could be used for both data and $\mathrm{MC}$ processing, reducing the probability of mistakes. (Or at minimum mistakes due to processing would be more likely to cancel.)

A very inefficient way of determining the measurement quantities embedded in the data would be to re-run MC samples with different values to find the parameter values that provide the best fit. A better way is to assume (as is usually the case) that the [experimental effects] piece of the convolution is decoupled from the [physics] piece, and re-weight a single MC sample. When re-weighting MC, a large sample is generated and processed as would the data. Any changes in the input parameters under study are applied to the sample by multiplying the weight of each event by the ratio of probabilities from the initial parameters which were used to generate it and the new ones. This can actually be done for either darts MC or weighted MC, (where usually each event in the darts MC has an initial weight of 1), however because less likely regions of phase space are often underpopulated in darts generated MC, biases dependent on the initial generation parameters can be introduced. It is therefore often safer to re-weight weighted MC. Even with weighted $\mathrm{MC}$ however, if the variables being determined somehow affect kinematic quantities that are subject to analysis cuts, (which strictly speaking means the 
factorization of [physics] $\otimes$ [experimental effects] would not be not perfect), one must also be careful that the values used to generate the initial MC sample do not bias the result.

In order to determine the physics parameters which best fit the data, both processed MC and data events are assigned to bins in relevant kinematic variables, and then an extreme value of some kind of quality of fit parameter is found such that the fit quality between the two is maximized. In the limit that Gaussian statistics may be applied, this quality of fit parameter can be the $\chi^{2}$, which would be minimized:

$$
\chi^{2}=\sum_{i} \frac{\left(N_{i}^{M C}-N_{i}^{D A T A}\right)^{2}}{\left(\sigma_{i}^{M C}\right)^{2}+\left(\sigma_{i}^{D A T A}\right)^{2}}
$$

Here the sum is over all bins, $N_{i}^{D A T A}$ is the number of data events in each bin $i$, $N_{i}^{M C}$ is the number of MC events (or normalized sum of weights) in each bin, and the $\sigma_{i}^{M C}$ and $\sigma_{i}^{D A T A}$ are the uncertainties in each bin for MC and data respectively. A search is performed in parameter space to find the minimum $\chi^{2}$. Frequently a software package such as MINUIT [151] (the case for this analysis) is used to search for the minimum, and calculate the statistical errors from the fit. In the case of a $\chi^{2}$ fit those errors are defined to be where the $\chi^{2}$ surface is 1 unit from the minimum.

A more general technique for determining the best values of physics parameters is the "Maximum Likelihood Method". A function, called a "likelihood function" is defined to be the product of probabilities that the data in each bin came from the parent distribution described by the Monte Carlo (or vice versa) in that bin:

$$
\mathcal{L}=\prod_{i} P_{i}
$$


Where $P_{i}$ is the probability bin $i$ has the number of data events it has with respect to the probability distribution defined by the MC. The probability distributions in this case need not be Gaussian (however if they were, this would be equivalent to the $\chi^{2}$ method above). The game then is to try and maximize this quantity. $\mathcal{L}$ can be a very large number, so typically the quantity that is maximized is $\log \mathcal{L}$. If a minimization software package like MINUIT is used then $-\log \mathcal{L}$ is minimized.

For cases where the number of events in bins are small, Poisson statistics is more appropriate than the Gaussian approximation. In this case the probability that a bin $i$ has a number $N_{i}^{M C} \mathrm{MC}$ events and $N_{i}^{D A T A}$ data events is:

$$
P_{i}=\frac{\left(N_{i}^{M C}\right)^{N_{i}^{D A T A}}}{N_{i}^{D A T A} !} e^{-N_{i}^{M C}}
$$

The likelihood function is then:

$$
\begin{aligned}
\mathcal{L} & =\prod_{i} \frac{\left(N_{i}^{M C}\right)^{N_{i}^{D A T A}}}{N_{i}^{D A T A} !} e^{-N_{i}^{M C}} \\
\log \mathcal{L} & =\sum_{i} \log \left[\frac{\left(N_{i}^{M C}\right)^{N_{i}^{D A T A}}}{N_{i}^{D A T A} !} e^{-N_{i}^{M C}}\right] \\
& =\sum_{i}\left(N_{i}^{D A T A} \log N_{i}^{M C}-\underline{\log \left(N_{i}^{D A T A} !\right)}-N_{i}^{M C}\right)
\end{aligned}
$$

Here the $\log \left(N_{i}^{D A T A}\right.$ !) term has been crossed out, because in finding the minimum only differences in $\log \mathcal{L}$ are of interest. Since the data never changes, that term is just a constant that can be removed. This leaves:

$$
-\log \mathcal{L}=\sum_{i}\left(N_{i}^{M C}-N_{i}^{D A T A} \log N_{i}^{M C}\right)
$$

as the expression that is minimized when fitting to the data. 
The general procedure then is to run a lot of $\mathrm{MC}$ events, process them like the data, assign a set of kinematics bins for each and populate them, then begin an minimization process where weights in the MC are re-weighted as a function of the parameters under study until a minimum value for the log likelihood is found. For this analysis this will only be a first step.

This is an NLO analysis of dimuon events (the first complete NLO analysis of dimuon events). The differential cross section code which describes neutrino charm production at NLO requires a two dimensional integral (c.f. equation 2.160) which is slow to calculate. In the fitting procedure above, several tens of thousands of MC events must be re-weighted for each prospective set of parameters being determined. On a typical processor currently available, a single round of re-weighting takes on the order of 24 hours to complete. A well behaved fit typically takes several hundred rounds before converging to a believable minimum. Therefore performing a direct fit with the NLO cross section can take several months if not a year or more to perform. This does not include the additional fits required to determine systematic uncertainties, etc.

Instead in this analysis, NLO fits to an extracted cross section table for dimuons are performed. These NLO fits may be accomplished in several hours time. This cross section is extracted by first performing fits to data at LO, and extracting the resulting dimuon cross section in a model independent way [75]. The NLO model is then fit to this cross section data, and then MC using that model and the fitted parameters is generated. The MC is compared to data to verify a good fit. As a final check, the cross section table will be re-extracted from the NLO MC, and that the table was indeed model independent will be verified. The 
remainder of this chapter will document the LO dimuon model and the extraction of the cross section table. The following chapter then will discuss the NLO fits to the table, and resulting physics.

\subsection{The NuTeV Monte Carlo}

$\mathrm{NuTeV}$ has assembled two Monte Carlo packages to describe its data. One is a "fast" MC, called "numonte", originally descended from MC code used by CCFR and its predecessors. Numonte contains all the primary physics of the interaction, as well as an extensive set of parameterized smearing functions used to simulate the effects of the detector environment on the interactions under study. In this analysis only the physics generation part of this software package is used.

The second $\mathrm{MC}$ package, called "McNuTeV", takes the generated particles and kinematics as an input and simulates their behavior in a GEANT [152] based simulation of the full detector. This simulation software tracks the particles through the detector, simulating energy loss, multiple scattering, etc, as well as electronic noise and inefficiencies in detector elements. Events generated in numonte are transferred to $\mathrm{McNuTeV}$ via an ASCII based interface called "McPipe". Each event which is simulated is written in the same data format as the raw data was taken in, then processed the same way. In this case, following the convolution expression in equation 4.165, [numonte $] \otimes[\mathrm{McNuTeV}] \simeq$ data $]$.

\subsubsection{Numonte \& Flux}

The dimuon analysis requires two MC samples for both neutrino and antineutrino modes. One sample simulates the full inclusive charged current data 
sample, and is used for normalization, and the other the dimuon MC. Numonte was originally designed to generate the inclusive sample as a "darts" MC, and the dimuon mode was added later to enable more efficient analysis of dimuon events (rather than the equivalent, but tedious, running about 100 times more inclusive MC than the desired number of dimuon events, and pulling out the dimuons from that sample). The dimuon MC is weighted MC. Discussion for now will be limited to the total cross section and inclusive event generation, then what modifications have been made to include the dimuon cross section.

The probability of a particular interaction happening in the detector is proportional to how many neutrinos are passing through it multiplied by the cross section for producing that interaction:

$$
P\left(E_{\nu}, V_{x}, V_{y}, x, y\right) \propto \Phi\left(E_{\nu}, V_{x}, V_{y}\right) \frac{d \sigma\left(E_{\nu}, x, y\right)}{d x d y}
$$

Where $V_{x}$, and $V_{y}$ are the vertex positions transverse to the beam axis, $E_{\nu}, x$ and $y$ are as defined in chapter 3 . In numonte, neutrino energies and vertex positions are thrown according to the measured flux, then Bjorken $x$ and $y$ are thrown against the cross section. Subsequent observable kinematics, like the momentum vector of the outgoing muon(s), energy of the hadron shower, etc are calculated from these variables.

The flux is measured from data using what is called the "fixed $\nu$ " method [153]. This uses the sample of charged current events with low hadronic energy to come as close to directly measuring the neutrino flux as possible. Charged current events with low hadronic energy are those that transfer the least momentum to the hadronic system, so are those for which the outgoing muon carries the greatest 
percentage of the original neutrino's momentum. A further advantage of this method is it uses a largely independent sample from that which is used to measure the cross section, as the hadronic energy of this sample is mostly below the cut applied for the cross section analysis. A disadvantage of this method is that it is only capable of measuring the energy dependence of the flux and not the overall level. That must be determined by the normalization of the total cross section $(\sigma)$ relative to external measurements. Additionally, the very small $\nu_{e}$ component to the flux cannot be determined with this method (at least not in the NuTeV detector), and must be found by beam simulations. Fortunately in charged current analyses the $\nu_{e}$ component to the flux is not a factor thanks to the requirement that at least one muon be reconstructed.

The fixed $\nu$ method comes about in the following way - starting with equation 2.107, which is repeated here so the reader won't have to flip through a million pages:

$$
\begin{array}{r}
\frac{d \sigma^{\nu(\bar{\nu})}}{d x d y}=\frac{G_{F}^{2} M E}{\pi\left(1+\frac{Q^{2}}{M_{W}^{2}}\right)^{2}}\left[\left(1-y-\frac{M x y}{2 E}+\frac{y^{2}}{2}\left[\frac{1+\frac{4 M^{2} x^{2}}{Q^{2}}}{1+R_{L}}\right]\right) \times\right. \\
\left.F_{2}^{\nu(\bar{\nu})} \pm y\left(1-\frac{y}{2}\right) x F_{3}^{\nu(\bar{\nu})}\right]
\end{array}
$$

Where again the \pm is "+" for neutrinos, and "-" for antineutrinos. These events are low $\nu$, which means low $Q^{2}$, which means the propagator $\left(1+\frac{Q^{2}}{M_{W}^{2}}\right)^{2}$ term may be safely dropped, since it will be nearly 1 . Substituting $\nu / E$ in for $y$ :

$$
\begin{array}{r}
\frac{E d \sigma^{\nu(\bar{\nu})}}{d x d \nu}=\frac{G_{F}^{2} M E}{\pi}\left[\left(1-\frac{\nu}{E}-\frac{M x \nu}{2 E^{2}}+\frac{\nu^{2}}{2 E^{2}}\left[\frac{1+\frac{2 M x}{\nu}}{1+R_{L}}\right]\right) \times\right. \\
\left.F_{2}^{\nu(\bar{\nu})} \pm \frac{\nu}{E}\left(1-\frac{\nu}{2 E}\right) x F_{3}^{\nu(\bar{\nu})}\right]
\end{array}
$$


Cancelling the duplicated factor of $\mathrm{E}$ on both sides and shuffling terms a bit:

$$
\frac{d \sigma^{\nu(\bar{\nu})}}{d x d \nu}=\frac{G_{F}^{2} M}{\pi}\left[F_{2}-\frac{\nu}{E}\left(F_{2} \mp x F_{3}\right)+\frac{\nu^{2}}{2 E^{2}}\left(\left(\frac{1+\frac{2 M x}{\nu}}{1+R_{L}}-\frac{M x}{\nu}\right) F_{2} \mp x F_{3}\right)\right]
$$

With terms now organized by factors of $\frac{\nu}{E}$. For convenience define:

$$
1+\mathcal{R} \equiv \frac{1+\frac{2 M x}{\nu}}{1+R_{L}}-\frac{M x}{\nu}
$$

We can then re-write things as:

$$
\frac{d \sigma^{\nu(\bar{\nu})}}{d x d \nu}=\frac{G_{F}^{2} M}{\pi}\left[F_{2}-\frac{\nu}{E}\left(F_{2} \mp x F_{3}\right)+\frac{\nu^{2}}{2 E^{2}}\left(F_{2} \mp x F_{3}+\mathcal{R} F_{2}\right)\right]
$$

Integrating over $x$ then gives:

$$
\frac{d \sigma^{\nu(\bar{\nu})}}{d \nu}=\frac{G_{F}^{2} M}{\pi}[\underbrace{\int F_{2}}_{A}-\frac{\nu}{E}(\underbrace{\left(\int F_{2} \mp \int x F_{3}\right)}_{B}-\frac{\nu^{2}}{2 E^{2}} \underbrace{\left(\int F_{2} \pm \int x F_{3}-\int \mathcal{R} F_{2}\right)}_{C}]
$$

Where all the integrals are over $x$, ranging from 0 to 1 . The bracketed " $A$ ", " $B$ ", " $C$ "'s are approximately independent of $\nu$. Multiplying in the flux, $\Phi(E)$, allows this to be expressed in terms of the number of events:

$$
\frac{d N(E)}{d \nu}=\Phi(E)\left(A+\frac{\nu}{E} B-\frac{\nu^{2}}{2 E^{2}} C\right)
$$

For small $\nu$, the flux is then approximately proportional to the derivative of the number of events with respect to $\nu$, however there is an energy dependence from the full expression in parentheses above. 


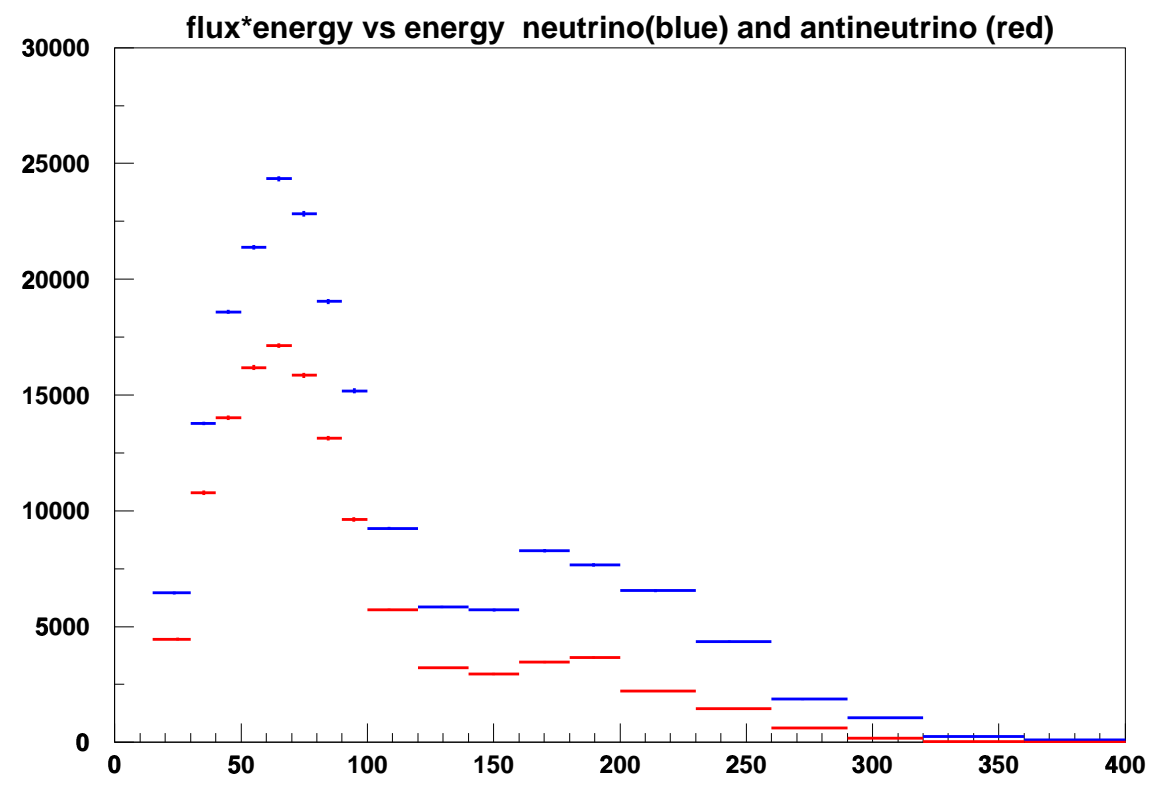

FIGURE 84. Measured $E \Phi(E)$ vs $E$ in $\mathrm{GeV}$ for neutrino and antineutrino beams. The $\mathrm{x}$ axis is in $\mathrm{GeV}$.

This is traditionally re-arranged in terms of $\frac{B}{A}$ :

$$
\frac{d N(E)}{d \nu}=\Phi(E) A\left[1+\frac{\nu}{E}\left(\frac{B}{A}\right)-\frac{\nu^{2}}{2 E^{2}}\left(\frac{B}{A}-\frac{\int F_{2} \mathcal{R}}{\int F_{2}}\right)\right]
$$

To obtain the flux this is inverted:

$$
\Phi(E) \propto \int_{0}^{\nu_{0}} \frac{\frac{d N(E)}{d \nu}}{1+\frac{\nu}{E}\left\langle\frac{B}{A}\right\rangle-\frac{\nu^{2}}{2 E^{2}}\left(\left\langle\frac{B}{A}\right\rangle-\frac{\int F_{2} \mathcal{R}}{\int F_{2}}\right)} d \nu
$$

where the integral is up to a value of $\nu_{0}$, which is small to minimize correlation with the data sample, but large enough such that there are adequate statistics for a believable determination of the flux. $\frac{d N(E)}{d \nu}$ is determined by subdividing each neutrino energy $(E)$ bin in $\nu$ bins. $\left\langle\frac{B}{A}\right\rangle$ is the average value of $\frac{B}{A}$, corrected for scaling violations within the $\nu=0 \rightarrow \nu_{0}$ range. 

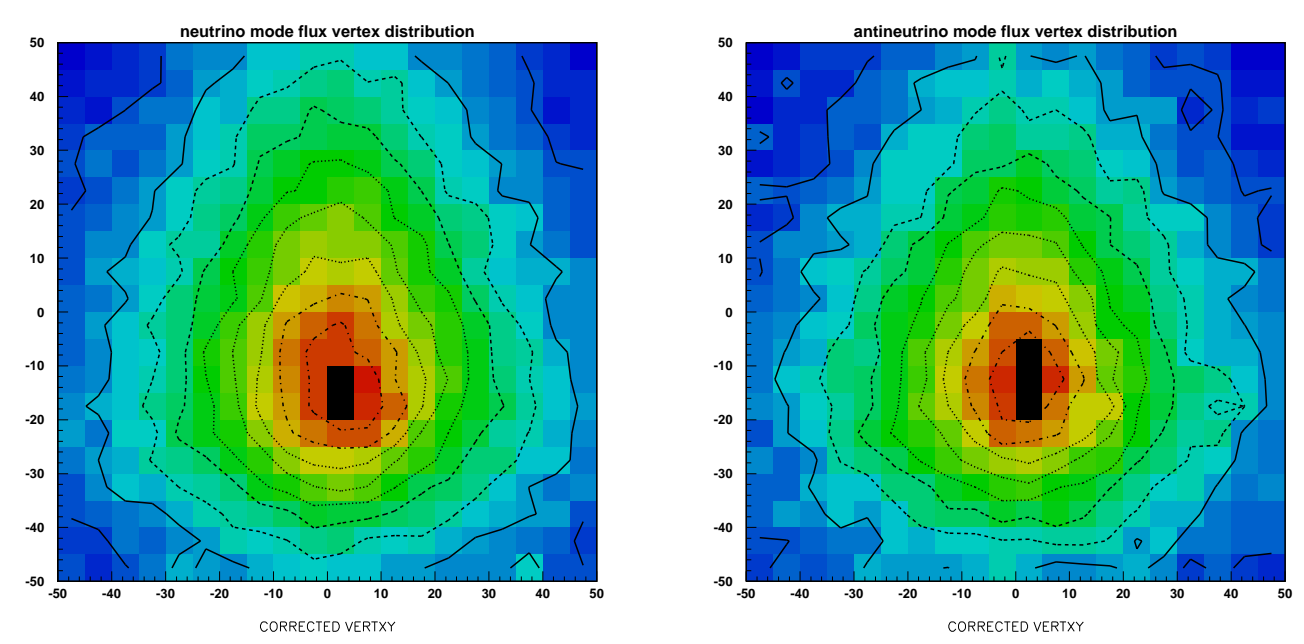

FIGURE 85. Measured vertex distributions for $\nu$ (left) and $\bar{\nu}$ (right) beams

The flux used in this analysis was determined for the $\mathrm{NuTeV}$ cross section measurement [154], with $\nu_{0}$ cut of $20 \mathrm{GeV}$. It is measured in 23 neutrino energy bins, ranging from $20 \mathrm{GeV}$ to $550 \mathrm{GeV}$. The bins have widths such that approximately the same number of events are included in each. Figure 84 shows $E \Phi(E)$ plotted vs $E$ in $\mathrm{GeV}$ for both neutrino (in blue) and neutrino (red) beams. (Its a bit more intuitive to look at plots of $E \Phi(E)$ rather than just $\Phi(E)$, as the cross section is proportional to $E$. Multiplying the flux by $E$ then makes it look more like what one sees when looking at the data.) The peak due to the $\pi$ component of the secondary beam can be seen around $70 \mathrm{GeV}$, and the kaon peak around 175 $\mathrm{GeV}$. The plot has been adjusted for the differing bin widths. Figure 85 shows the energy averaged vertex distributions of both beams (left is neutrino, right is antineutrino) as seen at the front face of the detector. (It is not a mammogram.) 


\subsubsection{Numonte \& BGPAR}

In numonte, the neutrino energy and event vertex are thrown according to these measured flux distributions. This gives half of equation 4.173. To get the other half, Bjorken $x$ and the inelasticity $y$ are thrown against the total cross section $\frac{d \sigma}{d x d y}[155]$. This is essentially the total cross section from equation 2.107. A parameterization of the $\mathrm{NuTeV}$ cross section measurement from neutrino and antineutrino scattering in the $\mathrm{NuTeV}$ detector steel is used. The structure functions are parameterized by an extension of the Buras-Gaemers ("BGPAR") parton distribution function parameterization [156]. The original 9 parameter Buras-Gaemers description has been extended to a more general 20 parameters described below $[142,157]$.

In the $\mathrm{NuTeV}$ enhanced BG parameterization, the up and down valence distributions are described by:

$$
\begin{aligned}
& x u_{v}\left(x, Q_{0}^{2}\right)=u_{v}^{t o t}\left[x^{E_{1}}(1-x)^{E_{2}}+A V_{2} x^{E_{3}}(1-x)^{E_{4}}\right] \\
& x d_{v}\left(x, Q_{0}^{2}\right)=d_{v}^{t o t} x u_{v}\left(x, Q_{0}^{2}\right)(1-x)
\end{aligned}
$$

Where the $E_{1-4}$ exponents and $A V_{2}$ describe the $\mathrm{u}$ valence distribution, and the softer $\mathrm{d}$ valence distribution is tied to that by a fixed $(1-x)$ factor. Evolution is through a simple logarithmic function of $Q^{2}$. The parameterization does not provide a perfect description of the parton distributions at all values of $Q^{2}$, however the fits using this model describe $\mathrm{NuTeV}$ data quite well within the limits of its $x$ and $Q^{2}$ range. Evolution in $Q^{2}$ is accomplished by modifying the $E_{n}$ 's in the 
following way:

$$
\begin{aligned}
& E_{1}=E_{10}+s E_{11} \\
& E_{1}=E_{20}+s E_{21} \\
& E_{1}=E_{30}+s E_{31} \\
& E_{1}=E_{40}+s E_{41}
\end{aligned}
$$

Where $s$ describes the evolution:

$$
s \equiv \log \left[\frac{\log \left(\frac{Q^{2}}{A_{0}^{2}}\right)}{\log \left(\frac{Q_{0}^{2}}{A_{0}^{2}}\right)}\right]
$$

$Q_{0}^{2}$ is set to $12.6 \mathrm{GeV}^{2}$, close to the average value of $Q^{2}$ used for the fit, but actually chosen somewhat by tradition for the CCFR family of experiments. (It was the mean $Q^{2}$ for E616/E701.) $A_{0}^{2}$ sets the evolution scale in an analogous way to $\Lambda_{Q C D}$, discussed in chapter 2 on page 103 .

In equations 4.182 and $4.183, u_{v}^{\text {tot }}$ and $d_{v}^{\text {tot }}$ describe the overall size of the valence distributions and are found by an application of the GLS sum rule (e.g. see equation 2.98 on page 73$)$ :

$$
\begin{aligned}
\int_{0}^{1} x F_{3}\left(x, Q^{2}\right) d x & =3\left(1-\frac{\alpha_{s}\left(Q^{2}\right)}{\pi}\right) \\
& =3\left(1-\frac{A_{1}}{\log \left(\frac{Q^{2}}{A_{0}^{2}}\right)}-\frac{A_{2}}{\left[\log \left(\frac{Q^{2}}{A_{0}^{2}}\right)\right]^{2}}\right)
\end{aligned}
$$


$A_{1}$ and $A_{2}$ are valence normalization factors. Breaking this up in terms of $u_{v}$ and $d_{v}:$

$$
\begin{aligned}
\int_{0}^{1} x u_{v}\left(x, Q^{2}\right) d x & =2\left(1-\frac{A_{1}}{\log \left(\frac{Q^{2}}{A_{0}^{2}}\right)}-\frac{A_{2}}{\left[\log \left(\frac{Q^{2}}{A_{0}^{2}}\right)\right]^{2}}\right) \\
\int_{0}^{1} x d_{v}\left(x, Q^{2}\right) d x & =\left(1-\frac{A_{1}}{\log \left(\frac{Q^{2}}{A_{0}^{2}}\right)}-\frac{A_{2}}{\left[\log \left(\frac{Q^{2}}{A_{0}^{2}}\right)\right]^{2}}\right)
\end{aligned}
$$

There is also a constraint from the quark charges:

$$
\begin{aligned}
\left(+\frac{2}{3}\right) \int_{0}^{1} x d_{v}\left(x, Q^{2}\right) d x+\left(-\frac{1}{3}\right) & \int_{0}^{1} x u_{v}\left(x, Q^{2}\right) d x= \\
& 1-\frac{A_{1}}{\log \left(\frac{Q^{2}}{A_{0}^{2}}\right)}-\frac{A_{2}}{\left[\log \left(\frac{Q^{2}}{A_{0}^{2}}\right)\right]^{2}}
\end{aligned}
$$

Where equations 4.188 - 4.190 set the overall levels of the valence distributions.

The quark sea is described by:

$$
\begin{aligned}
x \bar{u}(x)=x \bar{d}(x) & =\frac{1}{2(\kappa+2)} x S(x) \\
& =\frac{1}{2(\kappa+2)}\left[A S(1-x)^{E S}+A S_{2}(1-x)^{E S_{2}}\right]
\end{aligned}
$$

where the parameter $\kappa$ controls the relative normalization between the strange quark sea and nonstrange quark sea. $A S, A S_{2}, E S, E S_{2}$ are parameters determined as follows:

$$
\begin{aligned}
& A S_{2}=A S_{20}+A S_{21} \log Q^{2} \\
& E S_{2}=E S_{20}+E S_{21} \log Q^{2}
\end{aligned}
$$


Where $A S_{20}, A S_{21}, E S_{20}$, and $E S_{21}$ are free parameters determined by the fit to the total inclusive CC sample. $E S$ and $A S$ are:

$$
\begin{aligned}
E S & =\frac{S Q_{2}-\frac{A S_{2}}{E S_{2}+1}}{S Q_{3}-\frac{A S_{2}}{\left(E S_{2}+1\right)\left(E S_{2}+2\right)}}-2 \\
A S & =(E S+1)\left(\frac{S Q_{2}-A S_{2}}{E S_{2}+1}\right)
\end{aligned}
$$

$S Q_{2}$ and $S Q_{3}$ are first and second moments from the sea distribution with $S Q_{n}$ defined as follows:

$$
S Q_{n}=\int_{0}^{1} x^{n-2} x S\left(x, Q^{2}\right) d x
$$

where $x S\left(x, Q^{2}\right)$ is as in eq. 4.192. This may seem circular, however these moments are determined at leading order QCD. Assuming a 4-flavor (at typical $\mathrm{NuTeV} Q^{2}$ values, up, down, strange, charm are really all one need to worry about) and $\mathrm{SU}(3)$ gauge theory (i.e. QCD), the first and second moments are specified by:

$$
\begin{aligned}
S Q_{2} & =\frac{3}{4} D_{22}+\frac{1}{4} D_{12} \\
S Q_{3} & =\frac{3}{4} D_{23}+\frac{1}{4} D_{13}
\end{aligned}
$$

The $D_{x y}$ 's are:

$$
\begin{aligned}
D_{12}= & S_{2} e^{-0.427 s} \\
D_{13}= & S_{3} e^{-0.667 s} \\
D_{22}= & {\left[(1-0.429)\left(S_{2}+V_{82}\right)-0.429 G_{2}\right] e^{-0.747 s} } \\
& +\left[0.429\left(S_{2}+V_{82}\right)+0.429 G_{2}\right]-V_{82} e^{-0.427 s}
\end{aligned}
$$




$$
\begin{aligned}
D_{23}= & {\left[(1-0.925)\left(S_{3}+V_{83}\right)-0.288 G_{3}\right] e^{-1.386 s} } \\
& +\left[0.925\left(S_{3}+V_{83}\right)+0.288 G_{3}\right] e^{-0.609 s}-V_{83} e^{-0.667 s}
\end{aligned}
$$

Where the numerical values in equations 4.200-4.204 are from table 1 in reference [156], $s$ is as defined by equation $4.185, S_{2}$, and $S_{3}$ are free parameters determined from the fit, and $V_{82}$ and $V_{83}$ are:

$$
\begin{aligned}
V_{82}= & u_{v}^{\text {tot }}\left[\beta\left(E_{1}+1, E_{2}+1\right)+A V_{2} \beta\left(E_{3}+1, E_{4}+1\right)\right]+ \\
& d_{v}^{\text {tot }}\left[\beta\left(E_{1}+1, E_{2}+2\right)+A V_{2} \beta\left(E_{3}+1, E_{4}+2\right)\right] \\
V_{83}= & u_{v}^{\text {tot }}\left[\beta\left(E_{1}+2, E_{2}+1\right)+A V_{2} \beta\left(E_{3}+2, E_{4}+1\right)\right]+ \\
& d_{v}^{\text {tot }}\left[\beta\left(E_{1}+2, E_{2}+2\right)+A V_{2} \beta\left(E_{3}+2, E_{4}+2\right)\right]
\end{aligned}
$$

These are evaluated at $Q=Q_{0} \cdot \beta(m, n)$ is the Euler beta function:

$$
\beta(m, n)=\int_{0}^{1} x^{m-1}(1-x)^{n-1} d x
$$

The $G_{2}$, and $G_{3}$ in equations 4.200-4.204 are the second and third moments of the gluon distribution, with $G_{2}$ constrained by the momentum sum rule:

$$
G_{2}+\int_{0}^{1} \frac{1+R_{L}\left(x, Q_{0}^{2}\right)}{1+\frac{4 M^{2} x^{2}}{Q_{0}^{2}}} x\left[u_{v}\left(x, Q_{0}^{2}\right)+d_{v}\left(x, Q_{0}^{2}\right)+S\left(x, Q_{0}^{2}\right)\right] d x=1
$$

and $G_{3}$ is left free as a fit parameter. 


\begin{tabular}{|cll|}
\hline Parameter & Value & Error \\
\hline$A_{0}$ & 0.583 & 0.017 \\
$A_{1}$ & 0.295 & 0.013 \\
$A_{2}$ & 0.17 & 0.03 \\
$E_{10}$ & 0.5333 & 0.0025 \\
$E_{11}$ & -0.028 & 0.011 \\
$E_{20}$ & 2.61 & 0.015 \\
$E_{21}$ & 1.31 & 0.045 \\
$A V_{2}$ & 637.0 & 75.0 \\
$E_{30}$ & 4.56 & 0.14 \\
$E_{40}$ & 12.5 & 0.35 \\
$A S_{20}$ & 1.06 & 0.11 \\
$A S_{21}$ & 1.76 & 0.25 \\
$E S_{20}$ & 185.0 & 20.0 \\
$E S_{21}$ & 8.4 & 8.0 \\
$S_{2}$ & 0.1625 & 0.0013 \\
$S_{3}$ & 0.0159 & 0.0004 \\
$G_{3}$ & 0.031 & 0.003 \\
\hline
\end{tabular}

TABLE 11. Parameter values from NuTeV structure function BGPAR fit [154]

The strange component to the sea distribution is described by:

$$
\begin{aligned}
x s(x)=x \bar{s}(x) & =\frac{\kappa}{2(\kappa+2)} x S S\left(x, Q^{2}\right) \\
& =\frac{\kappa}{2(\kappa+2)} \frac{A S}{E S+1}(E S+\alpha+1)(1+x)^{E S+\alpha} \\
& =\kappa \frac{x \bar{u}(x)+x \bar{d}(x)}{2}(1-x)^{\alpha}
\end{aligned}
$$

$\kappa$ describes the relative normalization of the strange to the nonstrange seas. The exponent $\alpha$ affects the relative shape between the strange and nonstrange seas. The BG parameterization assumes that the strange and antistrange seas are equal. In order to fit for the strange and antistrange seas separately within the BG model, 
the neutrino and antineutrino data are taken as separate data sets tied together with a common charm mass, semileptonic branching ratio and fragmentation shape parameter $\epsilon$.

The up and down seas are assumed the same in the original BGPAR prescription, which has been shown not to be the case experimentally. A parameterization of the measured asymmetry between the $\bar{u}$ and $\bar{d}$ distributions by FNAL E866 $[158,159]$ is used to correct for this difference in the form of the ratio $\bar{d} / \bar{u}$ :

$$
f(\bar{d} / \bar{u})=\frac{1}{\operatorname{MAX}\left(0.1,1-x\left(2.7-0.14 \log \left(Q^{2}\right)-1.9 x\right)\right)}
$$

Where the MAX() function caps the ratio at 10\%. The modified up and down seas $\left(\bar{u}^{\prime}\right.$ and $\left.\bar{d}^{\prime}\right)$ are constrained so that their total is unchanged:

$$
\begin{aligned}
\bar{u}^{\prime} & =\bar{u} \frac{\bar{u}+\bar{d}}{\bar{u}+\bar{d} \cdot f(\bar{d} / \bar{u})} \\
\bar{d}^{\prime} & =\bar{d} \frac{\bar{u}+\bar{d}}{\bar{u}+\bar{d} \cdot f(\bar{d} / \bar{u})} f(\bar{d} / \bar{u})
\end{aligned}
$$

The up and down valence distributions are also corrected:

$$
\begin{aligned}
u_{v}^{\prime} & =\frac{u_{v}}{1+\delta(d / u) \frac{u_{v}}{u_{v}+d_{v}}} \\
d_{v}^{\prime} & =\frac{d_{v}+u_{v} \delta(d / u)}{1+\delta(d / u) \frac{u_{v}}{u_{v}+d_{v}}}
\end{aligned}
$$

where $\delta(d / u)$ is parameterized as:

$$
\delta(d / u)=0.12079-1.3303 x+4.9829 x^{2}-8.4465 x^{3}+5.7324 x^{4}
$$


Strictly speaking, in LO QCD, the Callan Gross relation (c.f. page 67) should hold. The NuTeV MC however includes an empirical correction to incorporate the effects of gluons in the cross section. This correction is in the form of a parametrized longitudinal structure function as described in section 2.3. The parameterization is based on a fit to SLAC electron deep inelastic scattering data [114] and is given as follows:

$$
R_{L}\left(x, Q^{2}\right)=\frac{0.0635}{\log \left(\frac{Q^{2}}{0.04}\right)} \Theta\left(x, Q^{2}\right)+\frac{0.5747}{Q^{2}}-\frac{0.3534}{Q^{4}+0.04}
$$

where $\Theta\left(x, Q^{2}\right)$ is:

$$
\Theta\left(x, Q^{2}\right)=1+12\left(\frac{Q^{2}}{1+Q^{2}}\right)\left(\frac{0.125^{2}}{x^{2}+0.125^{2}}\right)
$$

The degree of violation of the Callan Gross relation is shown in figure 25 on page 77.

Corrections for higher order effects also take the form of a "higher twist" correction to the total cross section. Higher twist effects are due to interactions between the quark struck in the interaction and the other "spectator quarks" also present in the nucleon. These effects are nonperturbative, and are suppressed by a factor of $\left(\frac{1}{Q^{2}}\right)^{\frac{\tau}{2}-1}$. The $\tau$ in the exponent is the "twist", named as such because of its relation to the order of spin ("twist" kind of like "spin") operators included in the operator product expansion formulation of deep inelastic scattering [160, 161]. The lowest meaningful value of twist for QCD operators is 2, and terms of greater than leading order in QCD all have twists of 4 or higher. Those pieces have a greater effect on the cross section at low $Q^{2}$, and at high $x$. The higher twist 


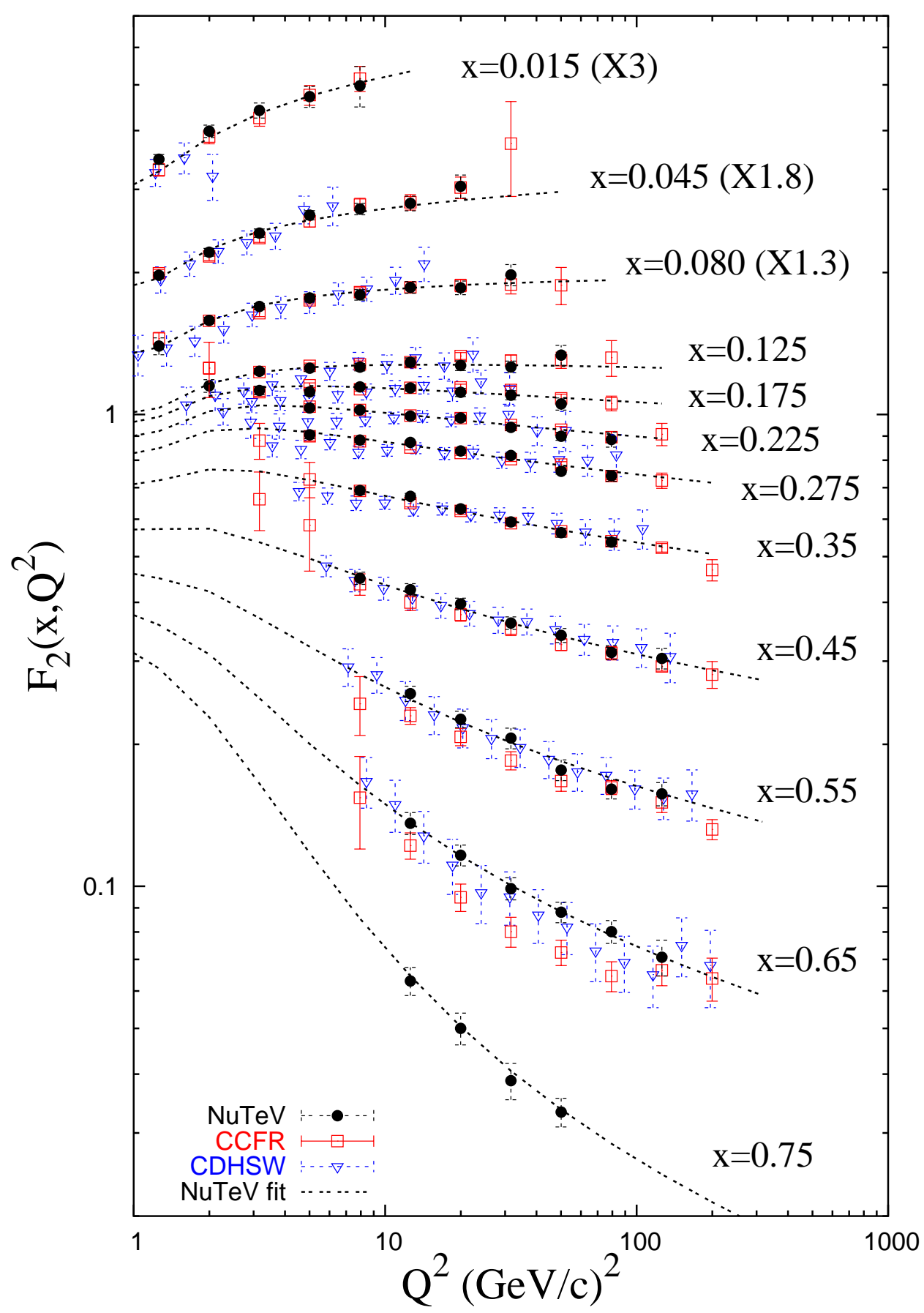

FIGURE 86. The NuTeV $F_{2}$ measurement with BGPAR fit superposed. Only the black points were used in the BGPAR parameterization. The points from CCFR and CDHSW are included for comparison purposes only. (plot courtesy Martin Tzanov) 


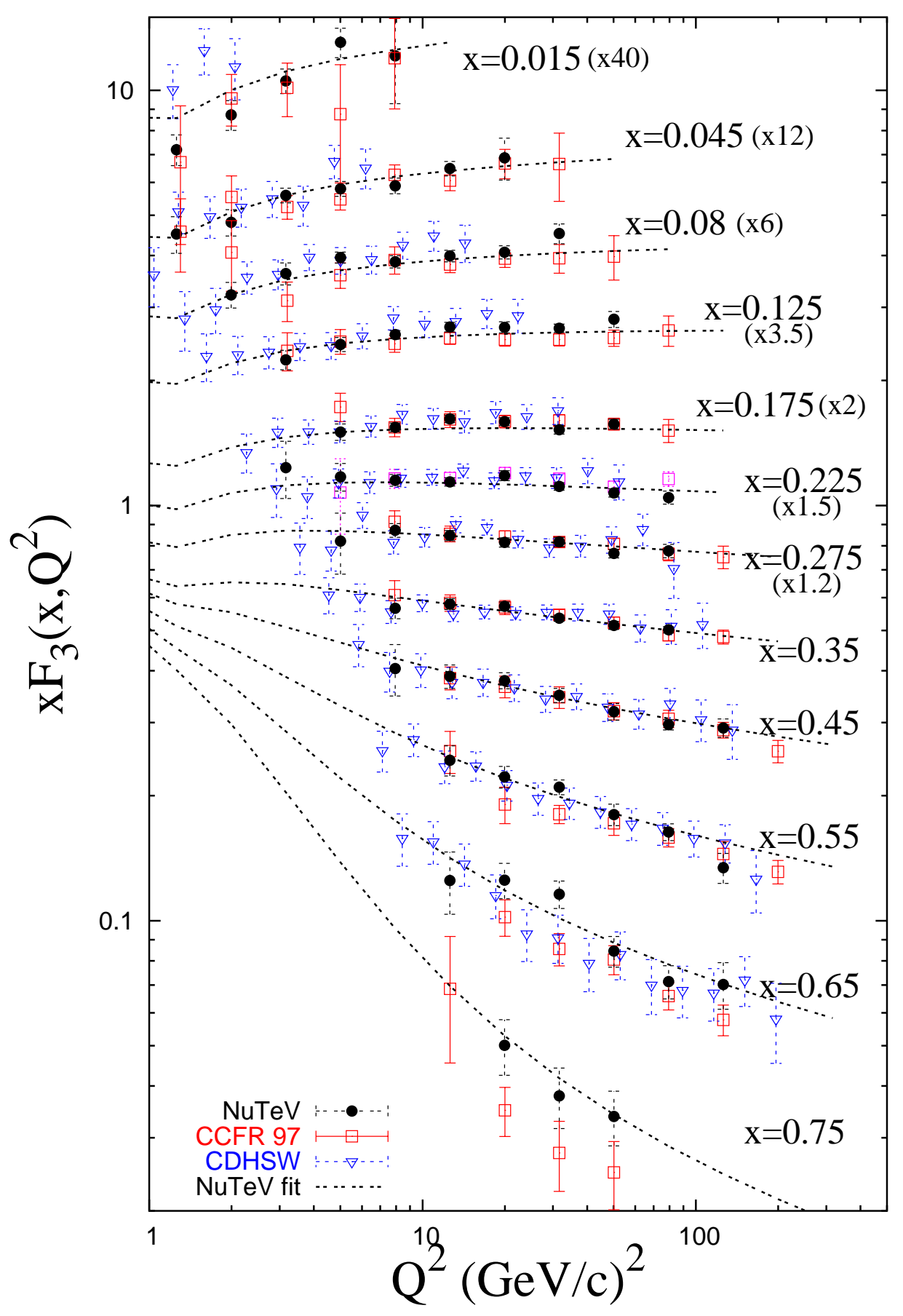

FIGURE 87. The NuTeV $x F_{3}$ measurement with BGPAR fit superposed. Only the black points were used in the BGPAR parameterization. The points from CCFR and CDHSW are included for comparison purposes only. (plot courtesy Martin Tzanov) 
effects are modeled by employing a parameterization which rescales $x$ in a similar way to slow rescaling in charm production. The following parameterization is used to correct for higher twist effects[162]:

$$
x \rightarrow \xi_{H T}=x \frac{Q^{2}+B_{H T}}{Q^{2}+A_{H T} x}
$$

with the parameters:

$$
\begin{aligned}
& A_{H T}=1.187 \pm 0.035 \\
& B_{H T}=0.33 \pm 0.02
\end{aligned}
$$

resulting from a fit to charged lepton DIS data. Utilizing all of these corrections, the flux and BGPAR pdfs were simultaneously fit to the NuTeV measured structure functions $F_{2}$, and $x F_{3}$. The ability of the BGPAR model to describe the $\mathrm{NuTeV}$ structure function data is shown in figures 86 and 87 . The BGPAR parameterization is based on only the $\mathrm{NuTeV}$ data shown in the figures.

The charged current cross section is also corrected for electromagnetic radiative effects to 1 loop in QED, including the Feynman diagrams shown in figure 88. The effect of the electromagnetic radiation on the final state is typically to increase the measured hadronic energy over that of the bare process. For example, when the muon radiates a photon, as in the top left of the figure, its momentum will be lessened, and that photon will initiate an electromagnetic shower which in the $\mathrm{NuTeV}$ detector will be added into the energy of the hadronic shower. The radiative corrections are those of Bardin [163], evaluated in a table binned in Bjorken $x$ and the inelasticity $y$, which are then interpolated. They are calculated and 

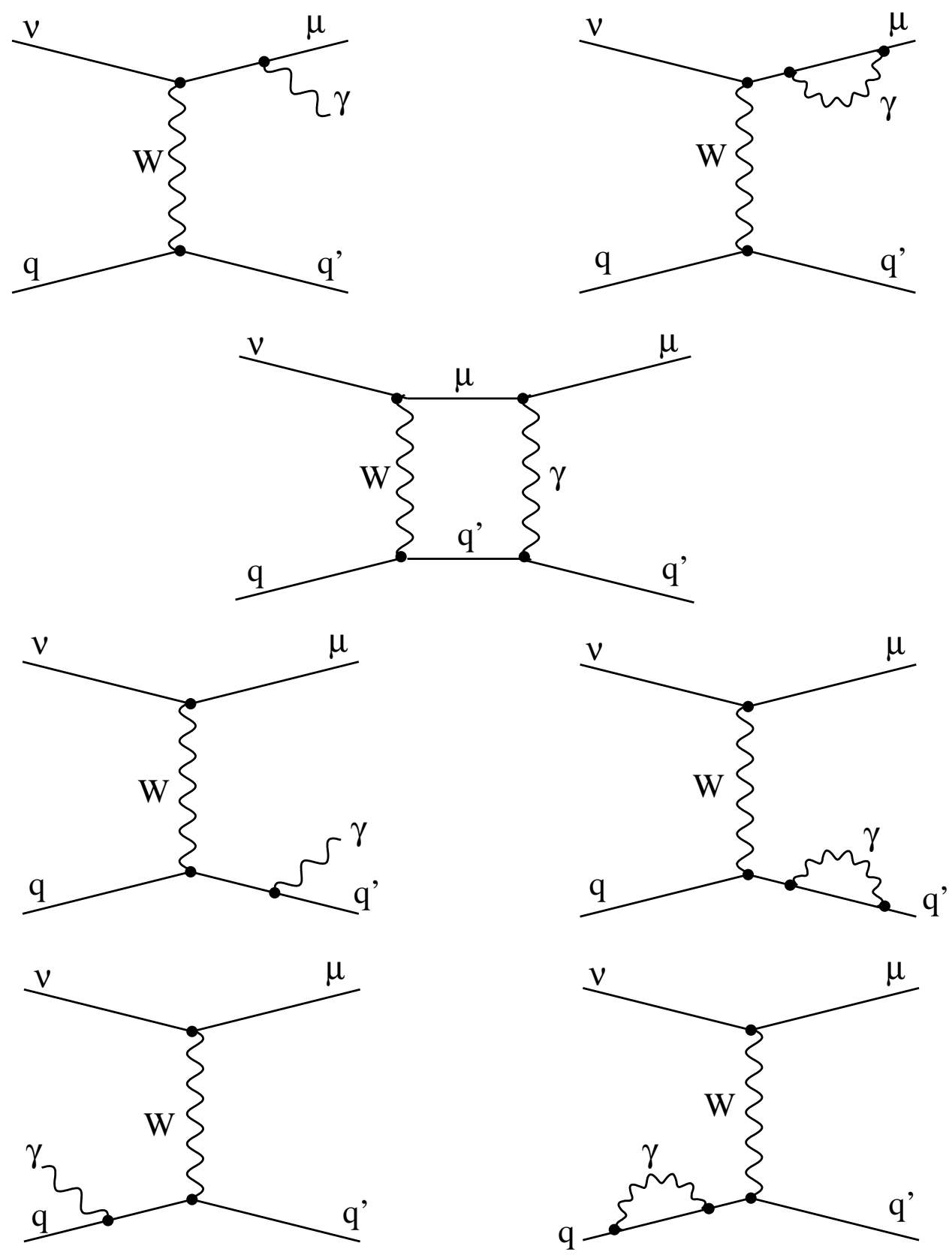

FIGURE 88. Feynman diagrams of both initial and final state QED radiative corrections 

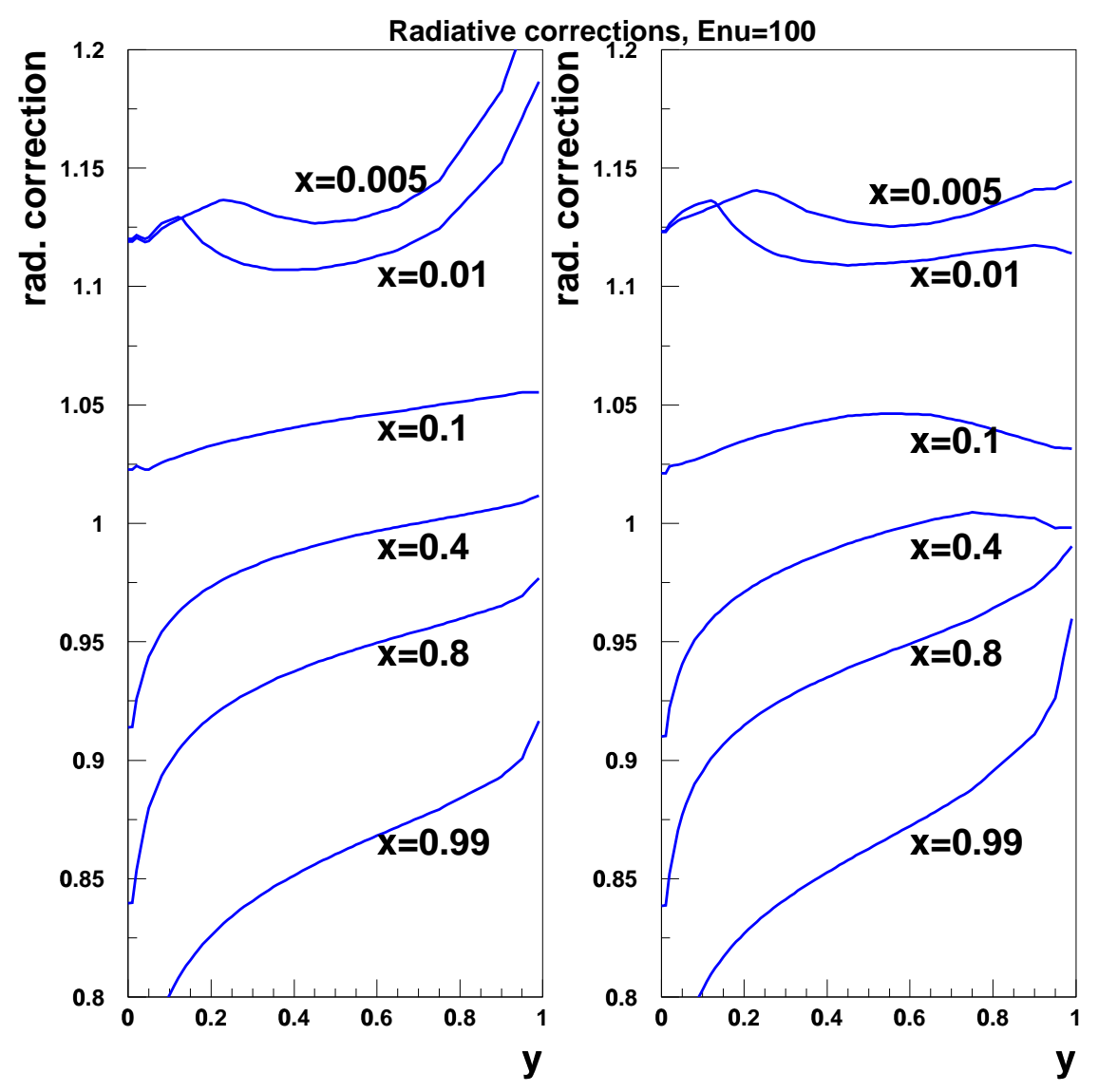

FIGURE 89. Total CC QED radiative corrections for neutrino (left) and antineutrino (right) cross sections, plotted for $E_{\nu}=100 \mathrm{GeV}$.

applied as multiplicative factors to the uncorrected "Born" cross section:

$$
\frac{d \sigma}{d x d y}=\left[\frac{\frac{d \sigma_{1-\text { loop }}}{d x d y}}{\frac{d \sigma_{\text {Born }}}{d x d y}}\right] \cdot \frac{d \sigma_{\text {Born }}}{d x d y}
$$

Where the bracketed piece is the applied correction. Figure 89 shows the magnitude of the QED radiative corrections as a function of $y$ for different values of $x$. 


\subsubsection{Inclusive dimuon production}

Dimuon events are a subset of the total charged current cross section, with the distinguishing characteristic of having an additional muon of opposite charge to the primary muon from the weak vertex. When generating inclusive MC, a throw is performed against the ratio of the dimuon cross section to the total cross section. If passed, roughly $1 \%$ of the time, and energy thresholds for producing a charmed meson are also passed, a dimuon event is generated. In inclusive charged current event generation (darts MC) the dimuon event replaces the "single muon" charged current event that would have otherwise been generated and is given a weight of 1 .

Table 12 outlines the construction of kinematic variables in generating a LO dimuon event. The neutrino energy and event vertex in the detector are determined from the flux, and $x$ and $y$ are determined from the total cross section. The momentum vector for the muon originating from the weak vertex is calculated from these quantities (with a flat $\phi_{\mu}$ distribution assumed), and the total momentum available for the hadronic system is then determined from momentum balancing. For inclusive charged current events, this is the end of the story, and the hadronic shower is generated with that momentum. If charm is generated however, kinematics are generated in the hadronic CMS frame, with a boost of $\gamma=E_{\text {had }} / \sqrt{W^{2}}$. The type of charmed particle generated is selected by throwing against the following production fractions [164]: $29 \%$ for charged D's, $64 \%$ neutral D's, and $7 \% D_{s}$ mesons. $\Lambda_{c}$ production is not simulated. The charmed meson is assigned a 
momentum of $P_{D}=z \cdot P_{D \max }$, where $P_{D \max }$ is $\sqrt{W^{2} / 4-M_{D}^{2}}$. (Here and in table 12 , the subscript " $D$ " is a used as a generic label for any of the charmed mesons). $z$ results from a throw against the fragmentation function (e.g. equation 2.140 on page 94$)$.

The charmed meson is assumed to remain collinear with the $\mathrm{W}$ boson in the LO model. Additional transverse momentum that would originate from higher order diagrams and nonperturbative effects is added based on external measurements, as the NuTeV detector is unable to fully resolve the details of the hadronic vertex. The amount of " $p_{\perp}$ smearing" imparted to the charmed particle relative to the $\mathrm{W}$ boson direction is determined by looking at the transverse momentum between the pair of charmed mesons produced in $c \bar{c}$ events in fixed target protonproton scattering. The momentum perpendicular to the axis defined by their center of momentum vector can be measured, and used to set the magnitude of this $p_{\perp}$ smearing. The LEBC-EHS experiment at CERN [165] measured the transverse momentum spectrum of $29 c \bar{c}$ events produced in $360 \mathrm{GeV}$ fixed target protonproton scattering. Their measured distribution of $p_{\perp}^{2}$ was found to fit a function of the form:

$$
\frac{d \sigma}{d p_{\perp}^{2}} \propto e^{-a p_{\perp}^{2}}
$$

with $a=1.1 \pm 0.3(\mathrm{GeV} / \mathrm{c})^{2}$. This function and measured smearing was used by the CCFR experiment in their dimuon analyses. The NA14/2 experiment at CERN also measured the $p_{\perp}^{2}$ distribution from more than 1000 measured $c \bar{c}$ events from $40-160 \mathrm{GeV}$ proton scattering [166]. Their $p_{\perp}^{2}$ distribution and a fit of the same form is shown in figure 90 . The fit gives a width $a=0.98 \pm 0.06$, completely consistent with the value used in past analyses, but with better precision. 


\begin{tabular}{|c|c|c|}
\hline McKinem.F & $\begin{array}{l}\text { - } E_{\nu} \text {, vertex thrown against (anti)neutrino flux } \\
\text { - } \mathrm{x}, \mathrm{y} \text { from throwing darts against } \frac{d \sigma}{d x d y} \\
\text { - } Q^{2}=2 * M_{p} * E_{\nu} * x * y \\
\text { - } E_{\mu}=E_{\nu} *(1-y) \\
\text { - } E_{h a d}=E_{\nu} * y \\
\text { - } \theta_{\mu}=E_{\mu 1}-M_{p} * x * y-m_{\mu}^{2} /\left(2 * E_{\nu}\right) \\
\text { - } \phi_{\mu} \text { thrown flat }(0-2 \pi)\end{array}$ & \\
\hline chrm_gen.F & $\begin{array}{l}\text { - } \text { Meson type thrown according to ref. [164] } \\
\text { - } W^{2}=M_{p}^{2}+2 * M_{p} * E_{\nu} * y *(1-x) \\
\text { - } P_{\mu}=\sqrt{E_{\mu}^{2}-m_{\mu}^{2}} \\
\text { - } P_{h a d}=\sqrt{E_{h a d}^{2}-W^{2}} \\
\text { - } P_{\mu x}=P_{\mu} * \sin \theta_{\mu} * \cos \phi_{\mu} \\
\text { - } P_{\mu y}=P_{\mu} * \sin \theta_{\mu} * \sin \phi_{\mu} \\
\text { - } P_{\mu z}=\sqrt{\left(P_{\mu}^{2}-P_{\mu x}^{2}-P_{\mu y}^{2}\right)} \\
\text { - } P_{h a d x}=-P_{m u x} \\
\text { - } P_{h a d y}=-P_{m u y} \\
\text { - } P_{h a d z}=\sqrt{P_{h a d}^{2}-P_{h a d x}^{2}-P_{h a d y}^{2}}\end{array}$ & 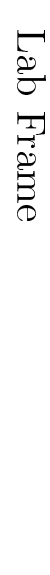 \\
\hline qfrag_ccfr.F & $\begin{array}{l}\text { - } P_{D \max }^{2}=W^{2} / 4-M_{D}^{2} \\
\text { - } \text { throw z against fragmentation function } \\
\text { - } P_{D}=z * P_{D \max } \\
\text { - } E_{D}=\sqrt{P_{D}^{2}-M_{D}^{2}} \\
\text { - Additional } p_{T} \text { smearing applied to } \mathrm{D} \\
\text { - boost } P_{D} \text { to the lab frame, } \gamma=E_{\text {had }} / \sqrt{W^{2}}\end{array}$ & 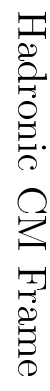 \\
\hline chrm_dk.F & $\begin{array}{l}\text { - Use } 3 \text { body decay to break D into observables } \\
\text { (muon }+ \text { hadron }+ \text { invisible neutrino })\end{array}$ & 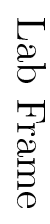 \\
\hline
\end{tabular}

TABLE 12. Definitions of dimuon kinematics as used in analysis and MC codes 


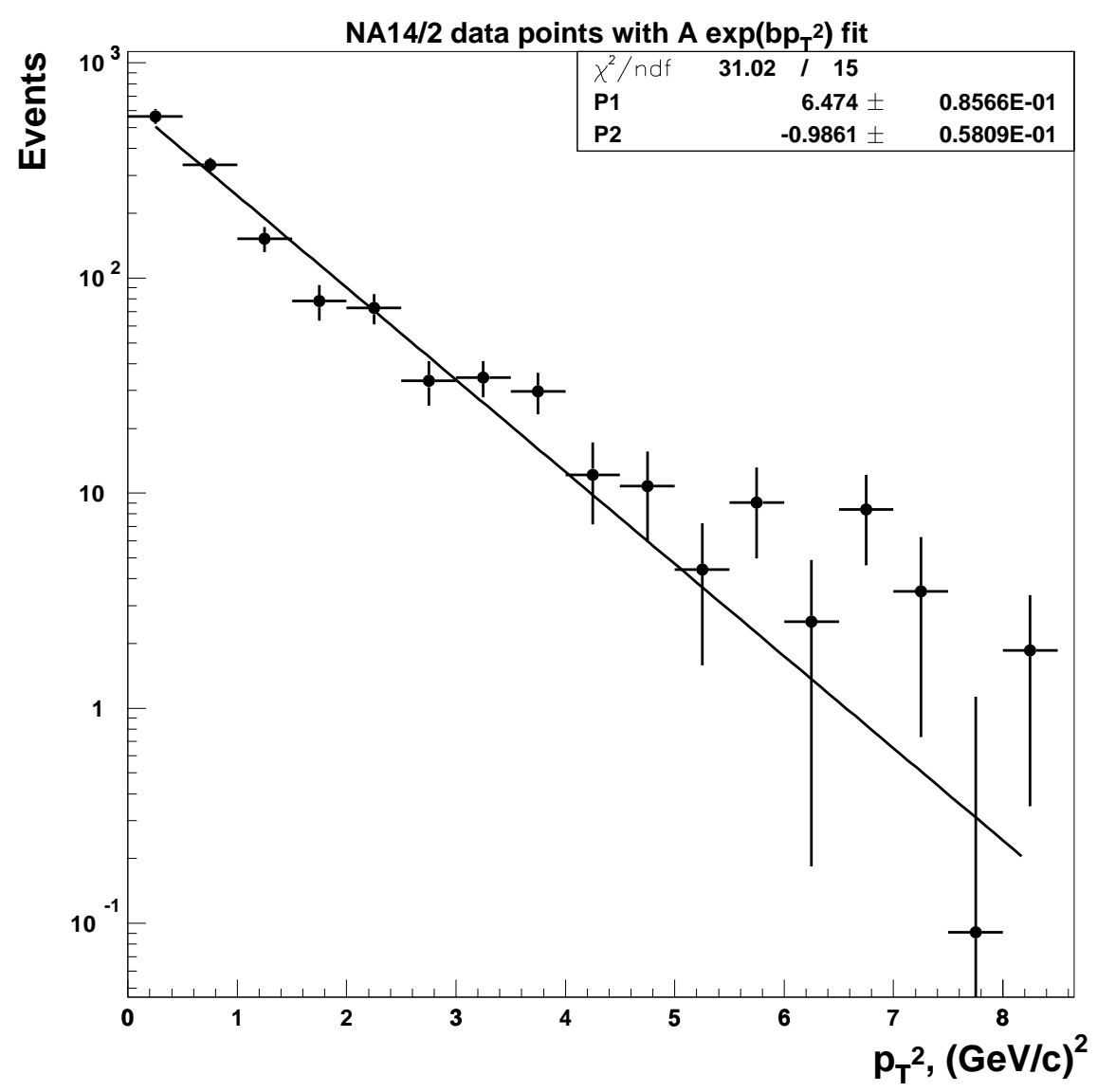

FIGURE 90. The NA14/2 $p_{\perp}^{2}$ distribution and superposed $e^{-a p_{\perp}^{2}}$ fit

A throw against this $p_{\perp}$ smearing function is performed and the charmed meson's momentum vector is rotated (with absolute magnitude unchanged) relative to the $\mathrm{W}$ boson axis such that the final vector has the thrown transverse momentum. The $\phi$ distribution is assumed to be uniform.

With the kinematics of the charmed meson completely determined in the hadronic center of mass frame, the meson is then boosted back to the lab frame where it is semileptonically decayed according to PDG branching fractions [78]. 


\begin{tabular}{|l|c|c|}
\hline Mode & 1997 PDG [167] & 2005 PDG [78] \\
\hline$D \rightarrow K_{0} \mu \nu$ & $0.067 \pm 0.008$ & $0.091 \pm 0.009$ \\
$D \rightarrow(K \pi)_{s} \mu \nu$ & $0.032 \pm 0.004$ & $0.038 \pm 0.005$ \\
$D \rightarrow \pi^{0} \mu \nu$ & $0.0057 \pm 0.0022$ & $0.0038 \pm 0.0019$ \\
$D \rightarrow K_{0}^{*} \mu \nu$ & $0.032 \pm 0.003$ & $0.035 \pm 0.003$ \\
$D \rightarrow \rho_{0} \mu \nu$ & $0.003 \pm 0.007$ & $0.003 \pm 0.008$ \\
\hline$D^{0} \rightarrow K \mu \nu$ & $0.0321 \pm 0.0018$ & $0.0320 \pm 0.0017$ \\
$D^{0} \rightarrow\left(K_{0} \pi\right) \mu \nu$ & $0.016 \pm 0.009$ & $0.018 \pm 0.008$ \\
$D^{0} \rightarrow \pi \mu \nu$ & $0.0037 \pm 0.0006$ & $0.0024 \pm 0.0004$ \\
$D^{0} \rightarrow K^{*} \mu \nu$ & $0.0134 \pm 0.0022$ & $0.0131 \pm 0.0017$ \\
\hline$D_{s} \rightarrow \eta \mu \nu$ & $0.025 \pm 0.007$ & $0.025 \pm 0.007$ \\
$D_{s} \rightarrow \eta^{\prime} \mu \nu$ & $0.0088 \pm 0.0034$ & $0.0089 \pm 0.0033$ \\
$D_{s} \rightarrow \phi \mu \nu$ & $0.020 \pm 0.005$ & $0.020 \pm 0.005$ \\
$D_{s} \rightarrow \mu \nu$ & $0.004 \pm 0.002$ & $0.005 \pm 0.002$ \\
\hline
\end{tabular}

TABLE 13. Charm semileptonic decay modes modeled in numonte. In neutrino mode the muon charge is positive, negative in antineutrino mode.

The type of charmed meson, $D^{+}, D^{0}$, or $D_{s}$ is determined by throwing against measured production fractions. At that time a throw is also performed against the semileptonic branching fractions for that particular charmed meson, shown in table 13. The kinematics are thrown assuming the charmed meson broke up via a standard weak decay [168], resulting in the momentum vectors of the remaining uncharmed meson, a neutrino, and a muon. The muon then is the second muon in the dimuon event, the neutrino slips away undetected, carrying some amount of the original D meson's energy with it (figure 91), and the remnant meson becomes a part of the hadronic shower. The average energy of the decay muon versus that of the parent charmed meson is shown in figure 92. The angular spread of the charmed meson momentum relative to the $\mathrm{W}$ boson momentum vector is shown in figure 93, and the amount that the weak decay of the charmed meson scatters the observable final state decay muon is shown in figure 94 . 


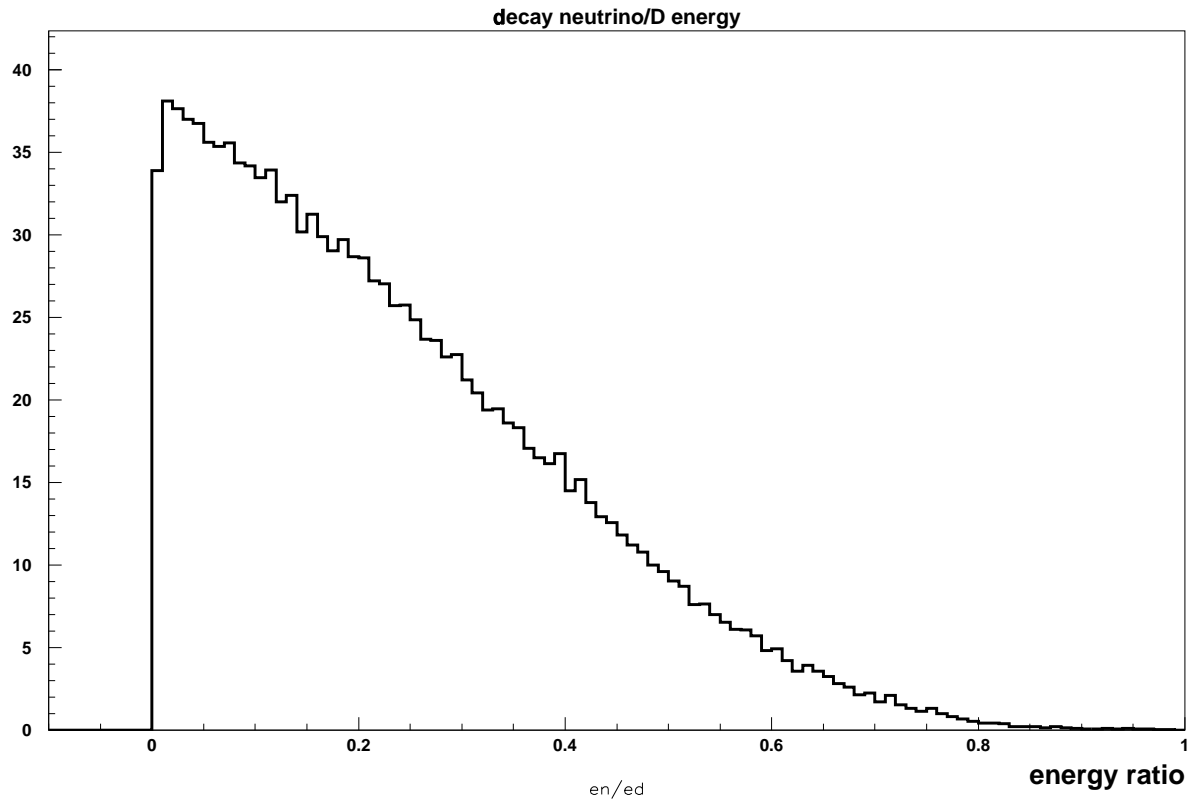

FIGURE 91. The fraction of the charmed meson's energy lost via the undetected neutrino from the weak decay

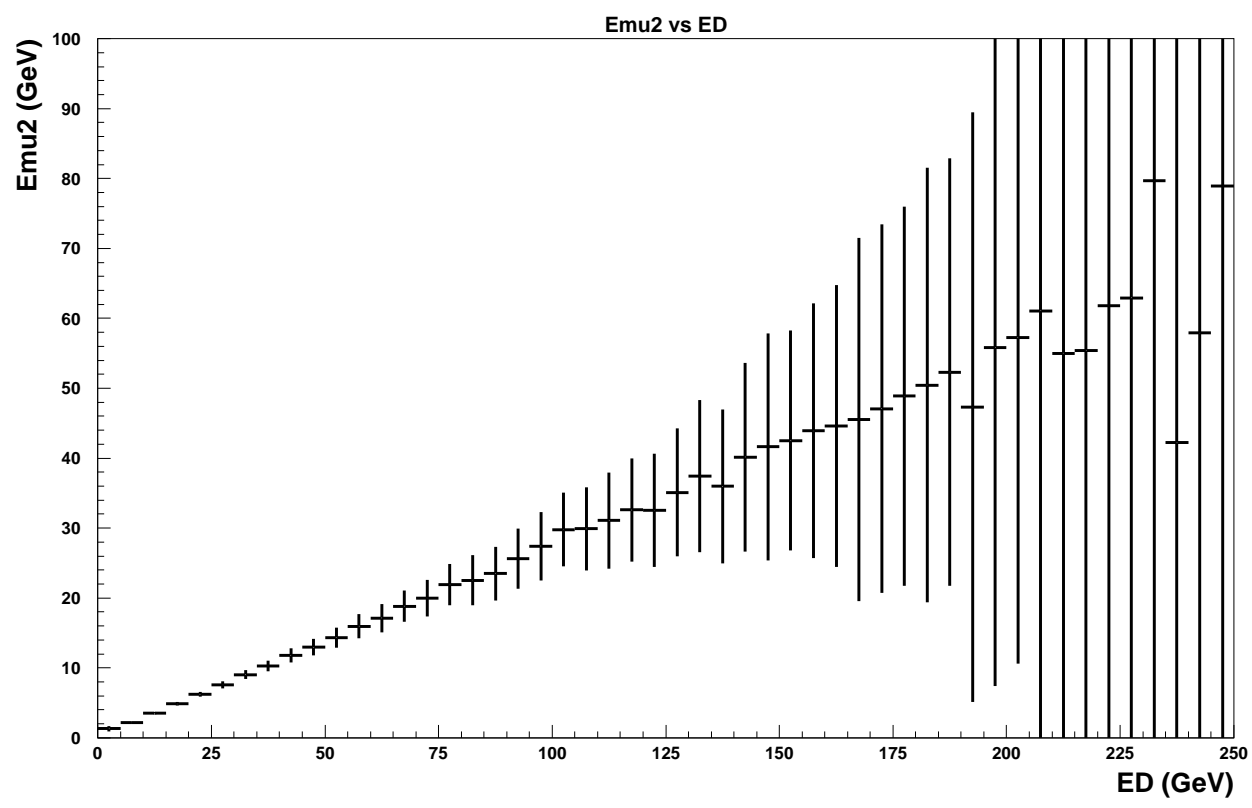

FIGURE 92. The average decay muon energy as a function of the energy of the parent charmed meson 


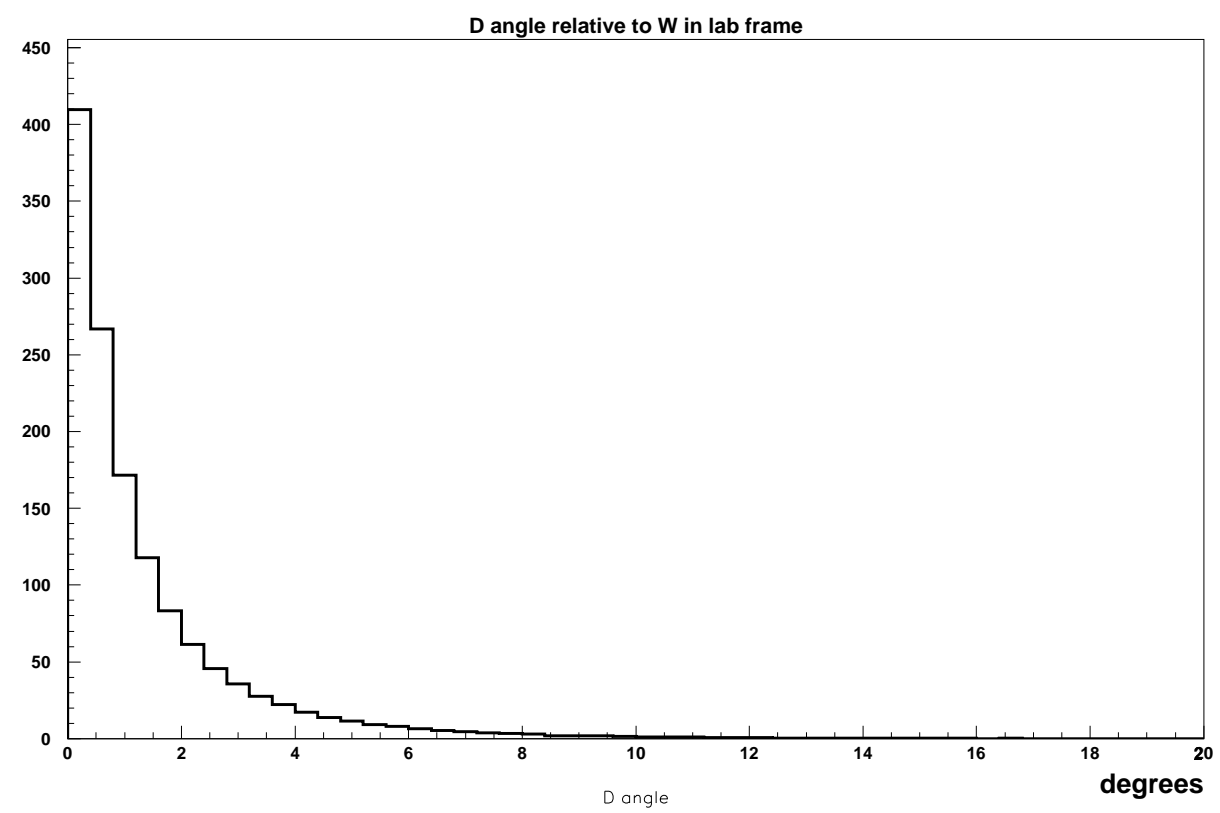

FIGURE 93. The angular spread of the D meson momentum vector relative to the direction of the $\mathrm{W}$ boson in the Lab frame. This is another way of displaying the $p_{\perp}$ smearing shown in figure 90

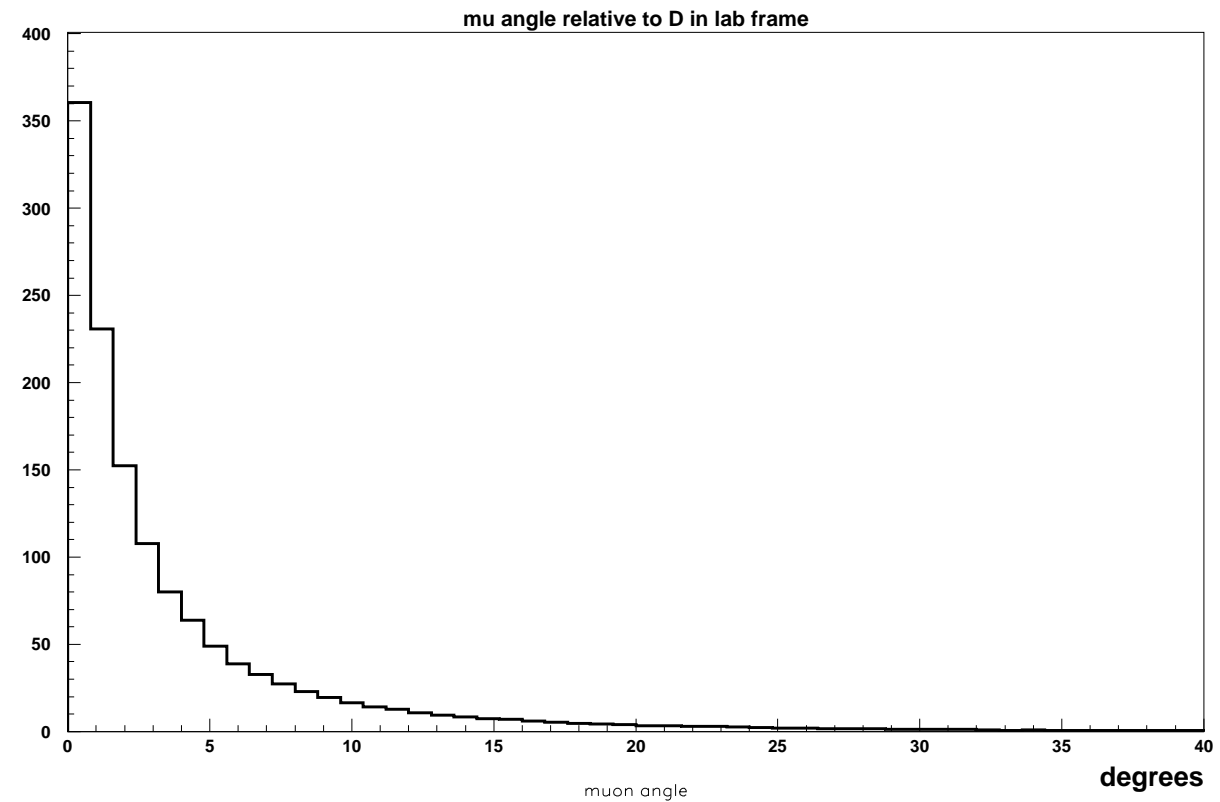

FIGURE 94. The angular spread of the charm decay muon relative to the direction of the charmed meson in the Lab frame 


\subsection{4 $\pi-K$ background}

Dimuons can be produced from two sources in numonte. The first, is the semileptonic decay of a charmed particle, the events targeted in this analysis. The second is a background to this desired process, and occurs when a pion or kaon in a typical charged current hadronic shower decays semi-muonically before being destroyed through interactions within the target. These events occur predominantly with lower muon energy, and are one of the reasons why a cut on the energy of the charm decay muon must be applied. Knowing the characteristics of this background is important in order to measure the size of the charm generated dimuon component correctly. The model used has two parts, one from the decay of a primary meson at the hadronic vertex, the other from the decay of secondary pions or kaons generated within the hadronic shower. We model both pieces of the background from empirical parameterizations based on simulation in the vertex case, and measurements in the shower case [169, 170].

The $\pi-K$ decay background parameterization from vertex pions and kaons is based on simulating the hadron shower using the LUND [171] Monte-Carlo coupled with Particle Data Group predictions for the semileptonic decay of each particle type in the hadronic shower. The parameterization used, a function of the hadronic energy, decayed muon energy and Bjorken $x$, is as follows:

$$
\frac{d P_{\mathrm{V}-\mathrm{pikdk}}}{d E_{\mu 2}}=\left(\frac{A}{E_{\mu 2}}\right)^{B}\left(1-\frac{E_{\mu 2}}{E_{H A D}}\right)^{2} e^{-C_{\frac{E_{\mu 2}}{E_{H A D}}}}
$$


The parameters $A, B$, and $C$ are Bjorken $x$ dependent expansions:

$$
\begin{aligned}
& A=A_{0}+A_{1} x+A_{2} x^{2} \\
& B=B_{0}+B_{1} x+B_{2} x^{2} \\
& C=C_{0}+C_{1} x+C_{2} x^{2}
\end{aligned}
$$

where $x$ is reset to 0.025 if $x<0.025$ and to 0.65 if $x>0.65$. The $A_{i}, B_{i}$, and $C_{i}$ 's themselves are expansions in multiples of $\log \left(E_{H A D} / 30\right)$ :

$$
\begin{aligned}
& A_{i}=a_{i 0}+a_{i 1} \log \left(\frac{E_{H A D}}{30}\right)+a_{i 2} \log ^{2}\left(\frac{E_{H A D}}{30}\right) \\
& B_{i}=b_{i 0}+b_{i 1} \log \left(\frac{E_{H A D}}{30}\right)+b_{i 2} \log ^{2}\left(\frac{E_{H A D}}{30}\right) \\
& C_{i}=c_{i 0}+c_{i 1} \log \left(\frac{E_{H A D}}{30}\right)+c_{i 2} \log ^{2}\left(\frac{E_{H A D}}{30}\right)
\end{aligned}
$$

where the $a_{i j}, b_{i j}, c_{i j}$ are parameters resulting from the CCFR fit to the LUND results, shown in table 14 .

To model the $\pi-K$ background originating from the decay of mesons in the hadronic shower, a parameterization of the measured rates for $E_{\mu 2}>4 \mathrm{GeV}$ muons from CCFR hadron test beam data is used:

$$
\frac{d P_{\mathrm{S}-\mathrm{pikdk}}}{d E}=\left(\frac{A_{1}}{E_{H A D}}\right)^{A_{2}}\left(1-\frac{E_{\mu 2}}{E_{H A D}}\right)^{2} e^{-\frac{E_{\mu 2}}{E_{H A D}} A_{3}}\left(A_{4}+A_{5} x\right)^{A_{6}}
$$

The parameters $A_{i}$ are determined from an expansion in hadronic energy:

$$
A_{i}=a_{i 1}+a_{i 2} \log \left(\frac{E_{H A D}}{30}\right)+a_{i 3} \log ^{2}\left(\frac{E_{H A D}}{30}\right)
$$




\begin{tabular}{|c|ccc|ccc|}
\hline & \multicolumn{3}{|c|}{ neutrino } & \multicolumn{3}{c|}{ antineutrino } \\
\hline \hline$A_{i}$ & $a_{i 0}$ & $a_{i 1}$ & $a_{i 2}$ & $a_{i 0}$ & $a_{i 1}$ & $a_{i 2}$ \\
\hline$A_{0}$ & 10.063 & -1.5887 & 0.11658 & 10.171 & -1.7365 & 0.14722 \\
$A_{1}$ & -3.3512 & 11.4168 & 0.0 & 3.4393 & -0.60418 & -0.19621 \\
$A_{2}$ & 1.1309 & -0.74166 & 0.0 & -0.36881 & -0.43144 & 0.0 \\
\hline$B_{i}$ & $b_{i 0}$ & $b_{i 1}$ & $b_{i 2}$ & $b_{i 0}$ & $b_{i 1}$ & $b_{i 2}$ \\
\hline$B_{0}$ & 1.4709 & 0.11446 & -0.011556 & 1.4438 & 0.11772 & -0.00963 \\
$B_{1}$ & 0.3 & 0.0 & 0.0 & 0.3 & 0.0 & 0.0 \\
$B_{2}$ & -0.3 & 0.0 & 0.0 & 0.0 & 0.0 & 0.0 \\
\hline$C_{i}$ & $c_{i 0}$ & $c_{i 1}$ & $c_{i 2}$ & $c_{i 0}$ & $c_{i 1}$ & $c_{i 2}$ \\
\hline$C_{1}$ & 3.8336 & 0.58488 & -0.17086 & 3.4293 & 0.60418 & -0.19621 \\
$C_{2}$ & -1.4510 & 0.32336 & 0.0 & -1.3544 & 0.34998 & 0.0 \\
$C_{3}$ & 0.0 & 0.0 & 0.0 & 0.0 & 0.0 & 0.0 \\
\hline
\end{tabular}

TABLE 14. Parameters used for vertex $\pi-K$ model

The values for the $a_{i j}$ parameters used to model the shower generated $\pi-K$ background are listed in table 15 . The average probability of making a $\pi-K$ generated dimuon compared to a charm produced dimuon within the BGPAR model as a function of decay muon energy is shown in figure 95. The $\pi-K$ background becomes a significant component of dimuon events at low $E_{\mu 2}$, eventually dominating at extremely low $E_{\mu 2}$. The $\pi-K$ background for neutrino scattering is larger relative to antineutrino scattering due to the large contribution of the valence quark distributions. The valence distributions are Cabibbo suppressed in charm production, which is why the charm curve is not enhanced to the same degree as the $\pi-K$ curve in neutrino mode.

\subsubsection{Noninclusive dimuon production MC}

Dimuon events, whether originating from charm or from the decay of pions and kaons, only make up about $1 \%$ of the total charged current event sample. When 

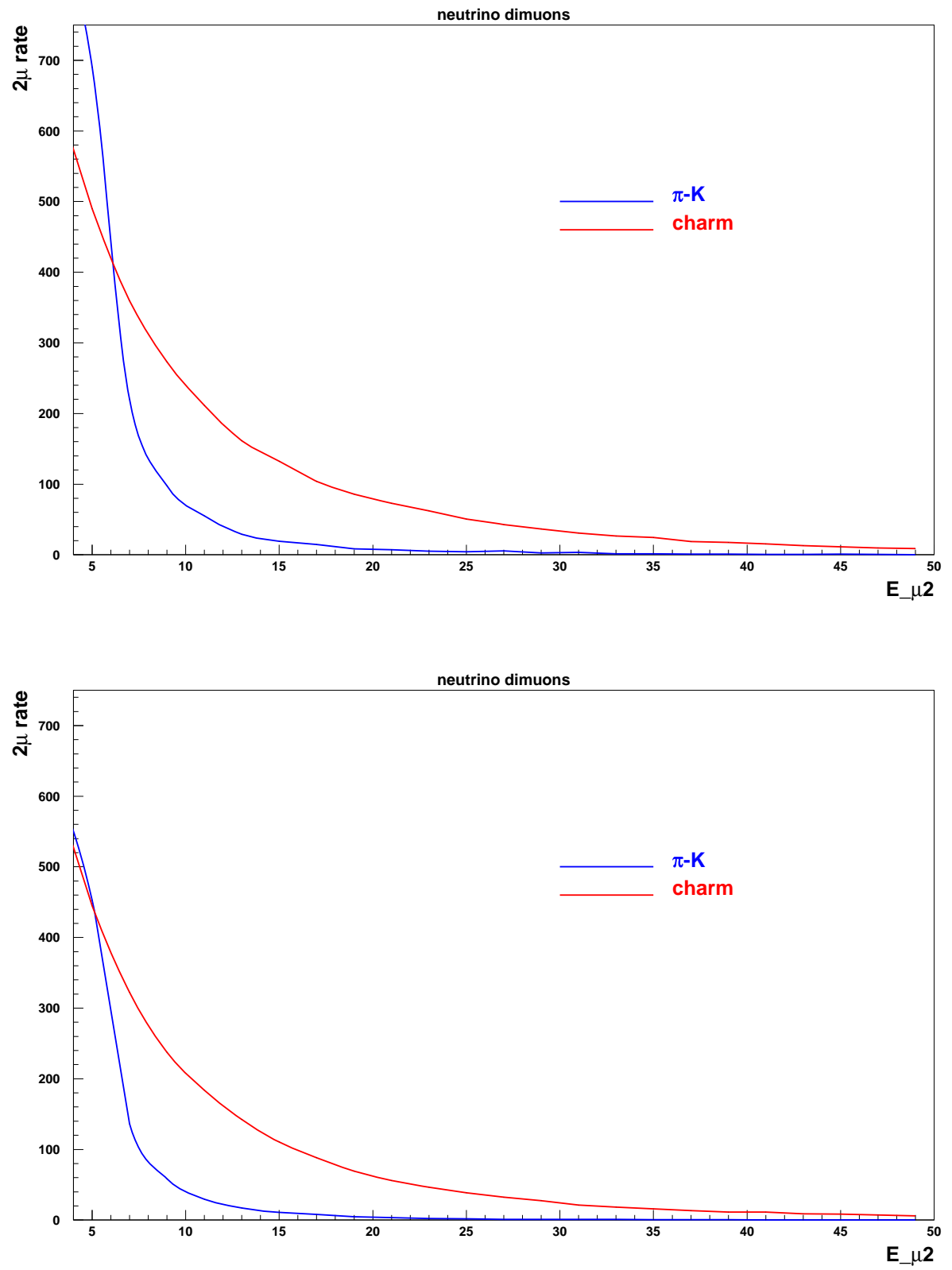

FIGURE 95. $\pi$-K parameterization in $\nu$ (top) and $\bar{\nu}$ (bottom) modes vs $E_{\mu 2}$. The $\mathrm{x}$ axis in both plots is in $\mathrm{GeV}$ 


\begin{tabular}{|c|ccc|}
\hline & $a_{i 1}$ & $a_{i 2}$ & $a_{i 3}$ \\
\hline$A_{1}$ & 1.3604 & -0.09049 & 0.0 \\
$A_{2}$ & 3.7356 & -0.24801 & 0.0 \\
$A_{3}$ & 3.4547 & -0.75553 & 0.0 \\
$A_{4}$ & 5.5783 & 1.8321 & 0.3805 \\
$A_{5}$ & -0.40785 & -0.10043 & 0.0 \\
$A_{6}$ & 2.95 & -0.02936 & 0.0 \\
\hline
\end{tabular}

TABLE 15. Parameters used for shower generated $\pi-K$ model

generating Monte Carlo events to compare to dimuon data, it is usually much more efficient to generate dimuons without the extra $99 \%$ overhead. Numonte also provides the ability to exclusively generate dimuon events as weighted MC as opposed to sporadically during inclusive event generation.

In this case, event generation proceeds as it would for inclusive charged current event generation, with $E_{\nu}$, and vertex thrown against the flux, and $x$ and $y$ thrown against the total CC cross section. For each event that passes the required energy thresholds to produce a charmed meson (or in the case of $\pi-K$ decay, pion or Kaon), dimuon event kinematics are generated, and the event is assigned a weight:

$$
w t c c=\frac{w t_{2 \mu}}{w t_{t o t C C}}
$$

Here $w t_{2 \mu}$ represents the probability of producing a dimuon event, and $w t_{t o t C C}$ is proportional to the inclusive cross section. For $\pi-K$ decay events $w t_{2 \mu}$ comes from the $\frac{d P_{\text {pikdk }}}{d E_{\mu 2}}$ parameterizations above. For LO charm $w t_{2 \mu}$ is:

$$
w t_{2 \mu}=\frac{d \sigma_{c h a r m}}{d \xi d y} B_{c}
$$


where $B_{c}$ is the averaged charm semi-muonic branching ratio, described in section 2.4.3, on page 90. The fragmentation function is not included in the weight. Instead $z$ is thrown against the fragmentation function $D(z)$. The charm cross section is essentially that of equations 2.131 and 2.132 on page 89, but without assuming isoscalar symmetry. For neutrino mode then:

$$
\begin{aligned}
\frac{d \sigma_{\text {charm }}^{\nu N}}{d \xi d y}= & \frac{\xi}{\left(1+\frac{Q^{2}}{M_{W}^{2}}\right)^{2}}\left[\left(1-y-\frac{M x y}{2 E}\right)\left(\frac{1+R_{L}\left(\xi, Q^{2}\right)}{1+\frac{4 M^{2} \xi^{2}}{Q^{2}}}\right)+\frac{x y}{\xi}\right] \times \\
& {\left[\begin{array}{l}
\left|V_{c d}\right|^{2} \frac{Z\left(d_{v}\left(\xi, Q^{2}\right)+d_{s}\left(\xi, Q^{2}\right)\right)+N\left(u_{v}\left(\xi, Q^{2}\right)+u_{s}\left(\xi, Q^{2}\right)\right)}{A}+ \\
\left.2\left|V_{c s}\right|^{2} s\left(\xi, Q^{2}\right)\right]
\end{array}\right.}
\end{aligned}
$$

here two modifications have been made: The factor of $\frac{G_{F}^{2} M E}{\pi}$ has been removed. The normalization convention within numonte is that the cross sections are multiplied by a factor of $\frac{\pi}{G_{F}^{2} M E}$. Also the relative (nonequal) number of neutrons and protons in the $\mathrm{NuTeV}$ target is taken into account by the factors of $Z$ (atomic number), $A$ (atomic mass), and $N=A-Z$. The strange quark distribution is assumed to be the same in protons and neutrons. The values used for $Z, A$, and $N$ are based on averages measured in samples taken from the steel plates in the NuTeV detector:

$$
\begin{aligned}
& Z=23.403 \\
& N=26.215 \\
& A=49.618
\end{aligned}
$$


Antineutrino mode is similar, but without the valence component:

$$
\begin{aligned}
\frac{d \sigma_{c h a r m}^{\nu N}}{d \xi d y}=\frac{\xi}{\left(1+\frac{Q^{2}}{M_{W}^{2}}\right)^{2}}\left[\left(1-y-\frac{M x y}{2 E}\right)\left(\frac{1+R_{L}\left(\xi, Q^{2}\right)}{1+\frac{4 M^{2} \xi^{2}}{Q^{2}}}\right)+\frac{x y}{\xi}\right] \times \\
{\left[\left|V_{c d}\right|^{2} \frac{Z d_{s}\left(\xi, Q^{2}\right)+N u_{s}\left(\xi, Q^{2}\right)}{A}+2\left|V_{c s}\right|^{2} s\left(\xi, Q^{2}\right)\right] }
\end{aligned}
$$

Figure 96 shows the $w t c c$ distributions for typical numonte runs in both neutrino and antineutrino modes. At high $x$, wtcc converges to:

$$
\begin{aligned}
\text { wtcc } \stackrel{\text { high x }}{\longrightarrow}\left|V_{c d}\right|^{2} B_{c} \frac{\xi\left(u_{v}+d_{v}\right)}{x\left(u_{v}+d_{v}\right)} \\
\sim\left|V_{c d}\right|^{2} B_{c} \\
\sim \mathcal{O}(0.005)
\end{aligned}
$$

since the seas largely disappear at high $x$. The bottom plot of figure 96 shows the average $x$ dependence of $w t c c$, and the neutrino curve converges towards a constant value near $\left|V_{c d}\right|^{2} B_{c}$ at high $x$. Charm production in antineutrino mode does not have a valence component, making wtcc approach zero at high $x$ in antineutrino mode. Rather than just being a peculiarity of the Monte Carlo, the behavior of neutrino wtcc at high $x$ demonstrates that in fits, that region in phase space is what constrains $V_{c d}$ and $B_{c}$ (or if an external $V_{c d}$ is assumed, just $B_{c}$ ).

The NuTeV dimuon data is described quite well with the leading order BGPAR model outlined above. The BGPAR model may be thought of as a physics motivated empirical parameterization of the $\mathrm{NuTeV}$ data. In analyses where only a good description of the $\mathrm{NuTeV}$ data, such as the cross section extraction described later in this chapter, an accurate parameterization of the cross section, no 

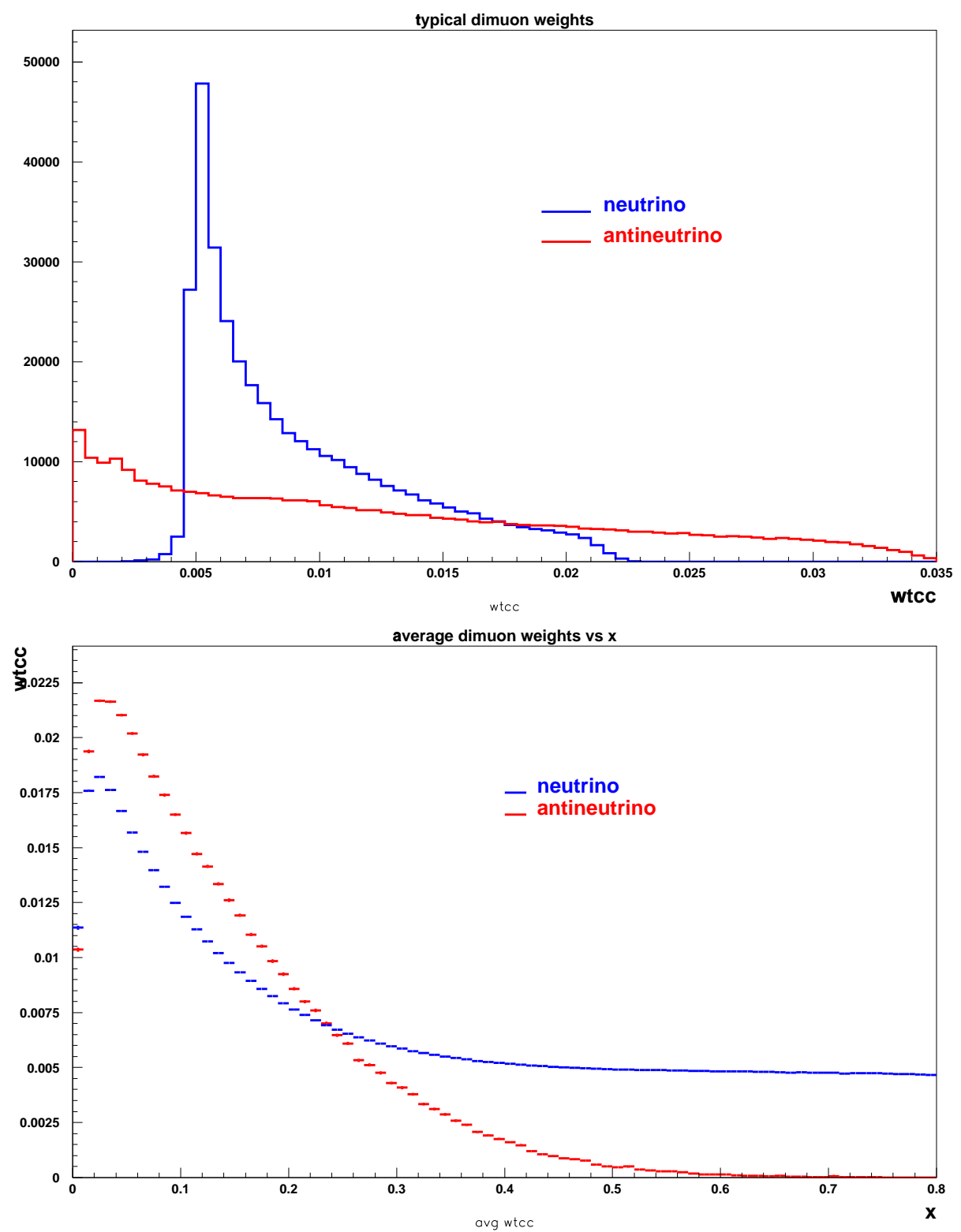

FIGURE 96. The values of wtcc for typical neutrino and antineutrino MC runs. The bottom plot is the dependence of $w t c c$ on $x$. One sees neutrino mode converging towards $\left|V_{C D}\right|^{2} B_{c}$ at high $\mathrm{x}$, while antineutrino mode goes to zero. 
matter what the internal structure might be, is all that is necessary. The BGPAR model however is really only expected to be useful in the phase space region of the NuTeV data, where the model parameters are determined. For example, the $Q^{2}$ evolution of the parton distribution functions is approximate. The BGPAR model is also a leading order model, with only a minimal description of the substantial gluon contribution to nucleon structure. In order to interpret the dimuon data in a way that may be better applied to other experimental situations, a more general NLO QCD model must be used to describe the data. This is described in the next chapter.

\subsection{6 $\mathrm{McNuTeV}$}

Generating the kinematics and calculating the cross sections of Monte Carlo events is of course of fundamental importance when trying to reproduce the data, however it is useless without a good simulation of the effects due to the detector environment on each event. There are two ways detector related smearing can be imparted to numonte-generated Monte Carlo. The first is through numonte itself, which includes an extensive library of smearing routines based on data taken with NuTeV's calibration beam. The measurement of dimuon events however is very dependent on the ability of the NuTeV analysis software's ability to reconstruct the second muon track, so a more detailed simulation of what happens in the detector in each event is needed. For this reason the second method of imparting detector effects to events was created, called McNuTeV.

McNuTeV is a GEANT [172] based "hit level" simulation of the NuTeV detector. GEANT is a library of routines used to model the passage of particles 
through the different materials a detector might be constructed out of. Using the GEANT library, one virtually builds the detector within the simulation by defining volumes of the different materials in the code. One then tells it to propagate whatever particles one desires (and often as important, those one doesn't) through the detector volumes, simulating interactions along the way. These interactions include processes like multiple scattering, bremsstrahlung, pair production, etc. The particle interactions used in $\mathrm{McNuTeV}$ are best listed by going through the entries in the card file used to control it:

MULS 2 Turns on multiple Coulomb scattering using Moliere theory

HADR 1 Turns on hadron interactions.

BREM 1 Bremsstrahlung scattering,

ANNI 1 Electron-positron annihilation

PAIR 1 Pair production

$D C A Y 1$ Allows particle decays

PHOT 1 Photoelectric effect

COMP 1 Compton scattering

RAYL 1 Rayleigh scattering

MUNU 1 Nuclear interactions 
LOSS 1 Energy loss with restricted energy loss fluctuations

\section{$D R A Y 1$ Delta ray production}

\section{PFIS 1 Photon fission}

In all of these flags the "1" (and in the case of "MULS" the 2) used as an argument indicates that any generated secondary particles will in turn also be propagated through the detector. To prevent this geometrically increasing collection of particles from severely bogging down the computer simulating them, minimum energy thresholds are set to tell it when it can stop. At that point the particle is no longer propagated through the detector, and the leftover energy is then added to the total. These energy thresholds should be set low enough so that the final track lengths and observables are not affected, but high enough so that GEANT doesn't spend an undue amount of time propagating millions of particles with energy too low to affect the results. The cuts used in this analysis are shown in table 16. The entire configuration file for $\mathrm{McNuTeV}$ is listed in appendix B.0.1.

Although McNuTeV has a plethora of physics processes to apply to the particles moving through the simulated $\mathrm{NuTeV}$ detector, it does not have any of the particle physics machinery to generate NuTeV events. This lends some flexibility, for example when simulating calibration beam events, one essentially just needs to tell McNuTeV something like "go make a muon with this energy there, and then do it 10000 times". An example of McNuTeV's flexibility is shown in figure 97, where McNuTeV was instructed to place $225 \mathrm{GeV}$ hadron showers at the top of the detector, a $7 \mathrm{GeV}$ shower in the middle, with a $4 \mathrm{GeV}$ muon at the bottom. Shown is the y view of a $\mathrm{NuTeV}$ event display after the particles were propagated 


\begin{tabular}{|l|c|}
\hline Cut & Value \\
\hline gammas & $1 \times 10^{-4} \mathrm{GeV}$ \\
electrons & $1 \times 10^{-4} \mathrm{GeV}$ \\
charged hadrons & $1 \times 10^{-4} \mathrm{GeV}$ \\
neutral hadrons & $1 \times 10^{-4} \mathrm{GeV}$ \\
muons & $1 \times 10^{-3} \mathrm{GeV}$ \\
electron bremsstrahlung & $1 \times 10^{-4} \mathrm{GeV}$ \\
electron delta rays & $1 \times 10^{-4} \mathrm{GeV}$ \\
muon delta rays & $1 \times 10^{-4} \mathrm{GeV}$ \\
muon produced $e^{+} e^{-}$pairs & $1 \times 10^{-4} \mathrm{GeV}$ \\
time of flight cut & $0.00204 \mathrm{GeV}$ \\
\hline
\end{tabular}

TABLE 16. McNuTeV GEANT particle energy cut values

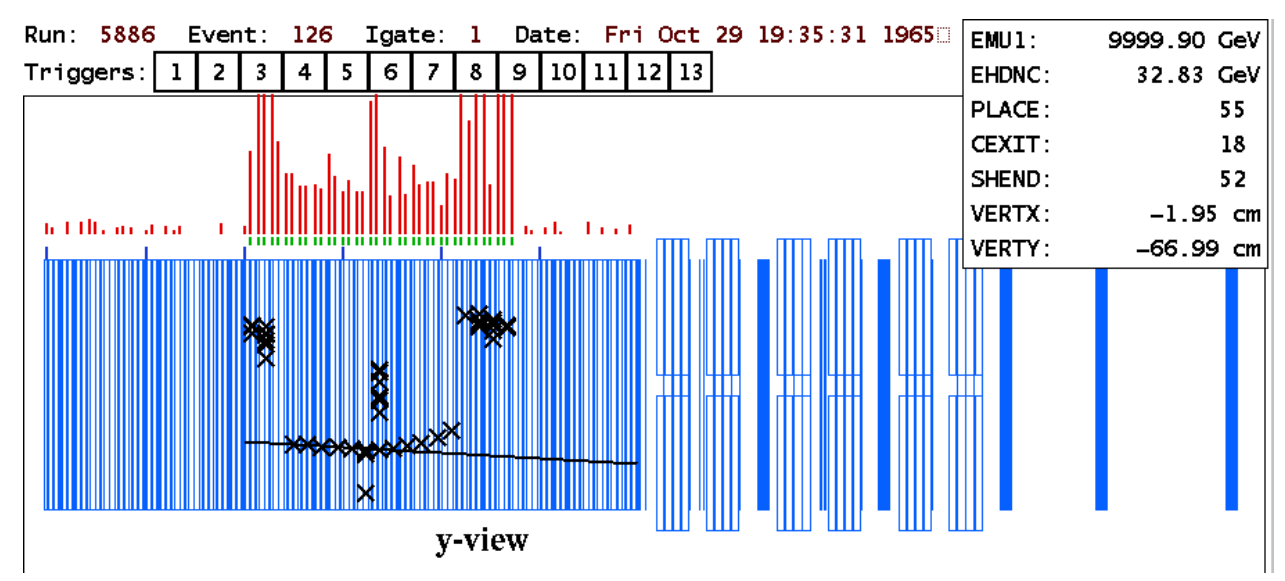

FIGURE 97. An MC event demonstrating the power and flexibility of McNuTeV.

through the detector, and the resulting data file processed and displayed via the $\mathrm{NuTeV}$ event display. The effects of multiple scattering are evident in the sarcastic upward-going displacement of the $4 \mathrm{GeV}$ muon. This functionality may be activated in McNuTeV by including the flag "GUKINE 37" in the card file. The implementation of "GUKINE 37" events instigated the hardwired subtraction of 40 years from the timestamp in $\mathrm{McNuTeV}$ data files to prevent confusion with real data. 
Manually instructing $\mathrm{McNuTeV}$ to place various particles in the detector may be useful at times, but in order to simulate real data, this process must be automated, and tied into the physics generator in numonte. The connection between detector simulation and physics simulation is achieved through an ASCII interface called "McPipe". As events are generated in numonte, the kinematics of final state particles, as well as event weights and variables necessary for physics analysis are written to a sequential ASCII McPipe file. McNuTeV then reads in this file, and propagates the events through the detector. The outputted data files from $\mathrm{McNuTeV}$ are in a format which is identical to the real data files, however an extra data bank (called the "truth bank") is added which includes the original kinematic variables and unsmeared position and momentum values of the particles involved in the interaction. The weight and physics variables brought in via the McPipe file are written to the truth bank as well.

In addition to physical smearing effects as particles traverse the $\mathrm{NuTeV}$ detector, McNuTeV also includes the digitization and inefficiency effects of the electronics recording the events. When a particle passes through the volume of a drift chamber or scintillator in the simulation, the code calculates the signal that should have been read out via the electronics, applying relevant corrections for phototube efficiencies, rounding errors from ADCs, and so on. These signals are dependent on when the event happened over the course of data taking, due to hardware failures, temperature variations or drifts in the electronics. A perl script called McWrapper is usually used to run $\mathrm{McNuTeV}$ in such a way that the events are distributed within different runs as they are in the real data. McWrapper is called for either neutrino or antineutrino modes for the desired total number of events. It then 
calculates the number of events to be simulated for each $\mathrm{NuTeV}$ run number, and calls separate McNuTeV jobs for each event block. For each run McNuTeV uses the measured characteristics of the electronics and detector based on monitoring performed throughout the duration of the experiment. The outputted MC data files are then placed in a single "McWrapOut" directory, which can then be combined into a single file to be analyzed in the same way as the data.

\subsection{7 $3-\pi$ hadronic shower model}

Although the event kinematics are determined in numonte, exactly what goes on within the hadronic shower is not. Numonte generates a momentum vector for the hadronic shower as a whole, but does not specify what particles are produced within it (except of course, in the case of charm production). This is fundamentally because the $\mathrm{NuTeV}$ detector is unable to resolve these particles individually, and records the shower as a stream of drift chamber hits with an associated deposition of energy in the calorimeter. In order to simulate a hadronic shower in $\mathrm{McNuTeV}$, 3 pions, a $\pi^{0}, \pi^{+}$, and $\pi^{-}$, are created with randomly distributed momenta such that the total is that of the total hadronic shower vector passed from numonte.

Although this 3 pion model balances the total charge of the hadronic shower, the ratio of energy lost due to hadronic interactions to electromagnetic interactions is not necessarily correct. The weights of each event are adjusted so that the energy lost by the $\pi^{0}$ component matches that of a LUND [171, 148] simulation of hadronic showers. 


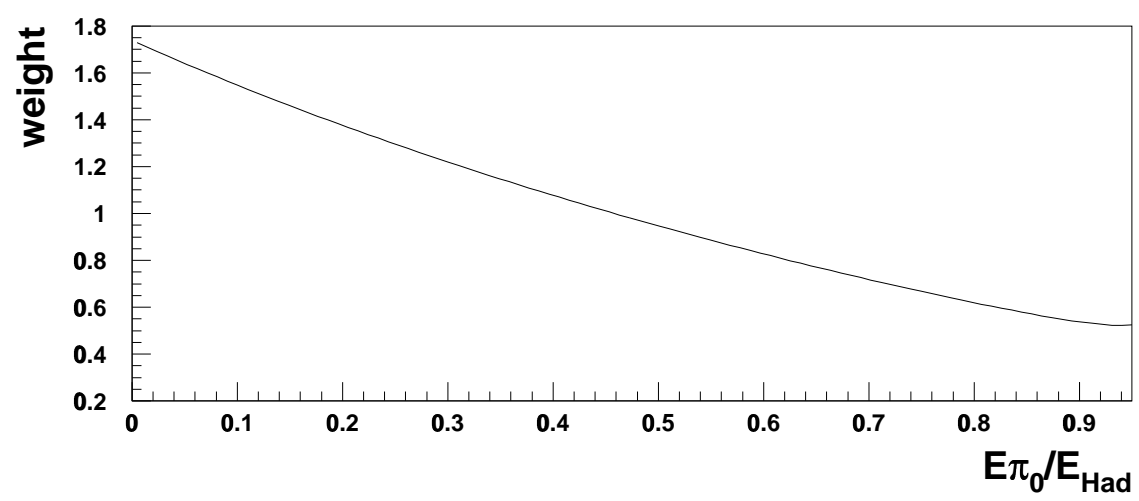

FIGURE 98. The correction rescaling the weights of events as a function of $\pi^{0}$ energy fraction

The probability an $\mathrm{MC}$ event has a particular $\frac{E_{\pi^{0}}}{E_{H A D}}$ ratio was found to fit the following curve:

$$
\mathcal{P}_{M C}\left(\frac{E_{\pi^{0}}}{E_{H A D}}\right)=34862-16998 \frac{E_{\pi^{0}}}{E_{H A D}}-18244\left(\frac{E_{\pi^{0}}}{E_{H A D}}\right)^{2}
$$

This probability from the LUND simulation was found to fit:

$$
\mathcal{P}_{L U N D}\left(\frac{E_{\pi^{0}}}{E_{H A D}}\right)=48423-79213 \frac{E_{\pi^{0}}}{E_{H A D}}+30774\left(\frac{E_{\pi^{0}}}{E_{H A D}}\right)^{2}
$$

The weight of each MC event multiplied by their normalized ratio:

$$
w t c c^{\prime}=w t c c \cdot \frac{\mathcal{P}_{L U N D}\left(\frac{E_{\pi} 0}{E_{H A D}}\right)}{\mathcal{P}_{M C}\left(\frac{E_{\pi^{0}}}{E_{H A D}}\right)}
$$

Figure 98 shows the magnitude of this correction, as a function of $\frac{E_{\pi^{0}}}{E_{H A D}}$. 


\subsubsection{Drift Chamber Efficiency}

The drift chambers as modeled in the $\mathrm{McNuTeV}$ Monte Carlo are 100 percent efficient. In order for this $\mathrm{MC}$ to better represent the slightly inefficient drift chambers in the data, drift chamber hits are removed according to the measured drift chamber cell efficiencies on a run by run basis.

These efficiencies were measured using the tracks of muons created by neutrino interactions in the shielding berm upstream of Lab E. These events, called "straight through muons" or just "trigger 6's", are an independent data sample not directly used in any physics analyses. As they are essentially a constant background of straight tracks passing through the entire detector and are very easy to reconstruct, they are therefore very useful as a tool to monitor the detector for changes in the drift chamber behavior. The technique for measuring the efficiencies then is to track these muons through the detector, and tally up the locations of chamber cells which are missing along the length of the tracks. The numbers of these straight through muon events are high enough that this can be done on a run by run basis, providing an empirical record of when chamber cells are disabled, inefficient, or when chambers were removed from the detector during the run for repair. This information is then used in post-processing the NuTeV MonteCarlo to make it better match the data. When $\mathrm{McNuTeV}$ data is run through the processing software, e815_analysis, drift chamber hits are removed according to random throws against these measured efficiencies for each run.

Figures 99 and 100 represent these measured efficiencies. Table 17 explains the color schemes for the figures. (Note that the scale to the right of each figure is actually the "inefficiency", or 1-efficiency, chosen to emphasize the differences 
near 100\% efficiency. See table 17) Figure 99 shows the average efficiencies of the drift chambers throughout all of $\mathrm{NuTeV}$ data taking. The vertical axis is chamber cell number, ranging from 0 to 23. The horizontal axis is chamber number, where calorimeter chambers range from 0 to 43, with \#43 towards the front of the calorimeter, and 1 right before the muon spectrometer. Numbers above 43 are drift chambers in the muon spectrometer. Note that the most efficient drift chambers were placed in the spectrometer. The plots in figure 100 are the average inefficiency for each chamber as a whole (x axis), plotted by run number (y axis). Run numbers increase with time, so one can see how the efficiency of chambers changed as data taking progressed.

\begin{tabular}{|l|c|}
\hline \multicolumn{2}{|c|}{ Efficiency. Plot Color Code } \\
\hline \hline Color & approx efficiency range \\
\hline Black & $0-58 \%$ \\
\hline Blue & $88-82 \%$ \\
\hline Red & $93-96.8 \%$ \\
\hline Pink & $96.8-98.7 \%$ \\
\hline Green & $98.7-99.4 \%$ \\
\hline Blue & $99.4-99.7 \%$ \\
\hline Yellow & $99.7-100 \%$ \\
\hline White & (can also mean run was missing) \\
\hline
\end{tabular}

TABLE 17. Legend for DC Efficiency plots (figures 99 and 100)

\section{$\underline{4.2 \mathrm{MC} \text { Energy and Flux Corrections }}$}

The MC is not perfect, and several small corrections need to be applied to force muon and hadronic energy smearing to agree with measurements from the $\mathrm{NuTeV}$ calibration beam. Additionally, the neutrino flux was extracted with 

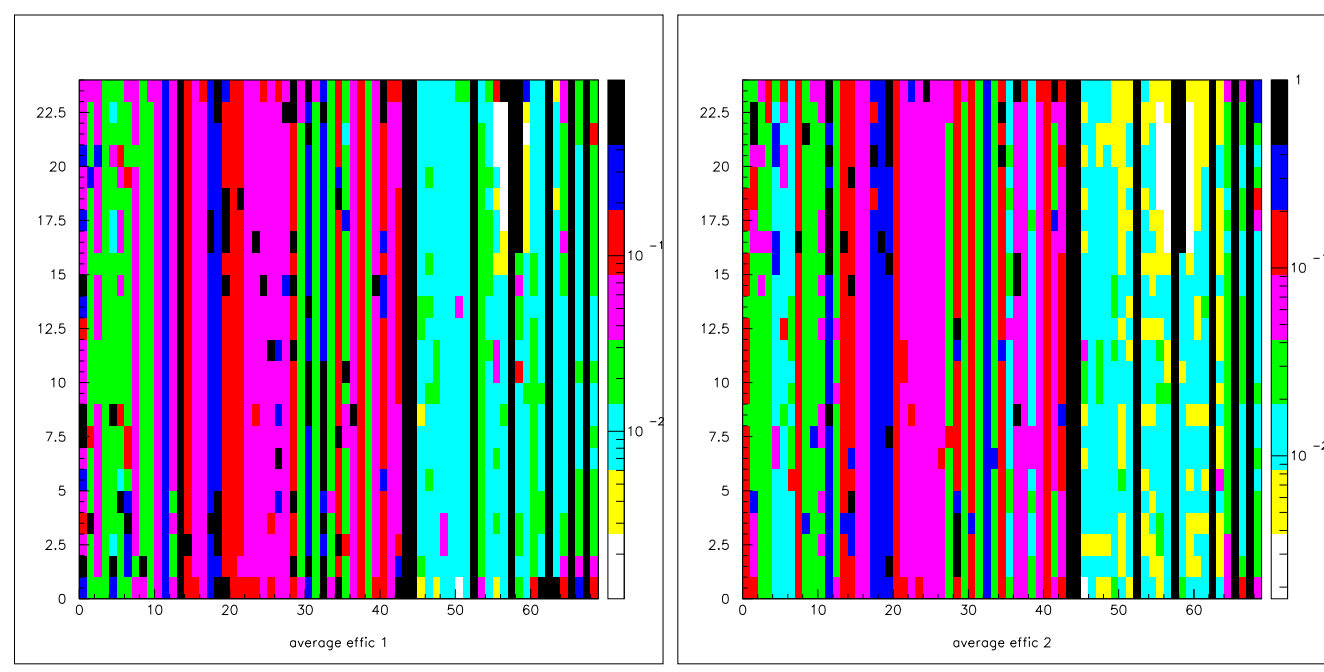

FIGURE 99. Average DC efficiency over all runs cell vs chamber (views 1,2)
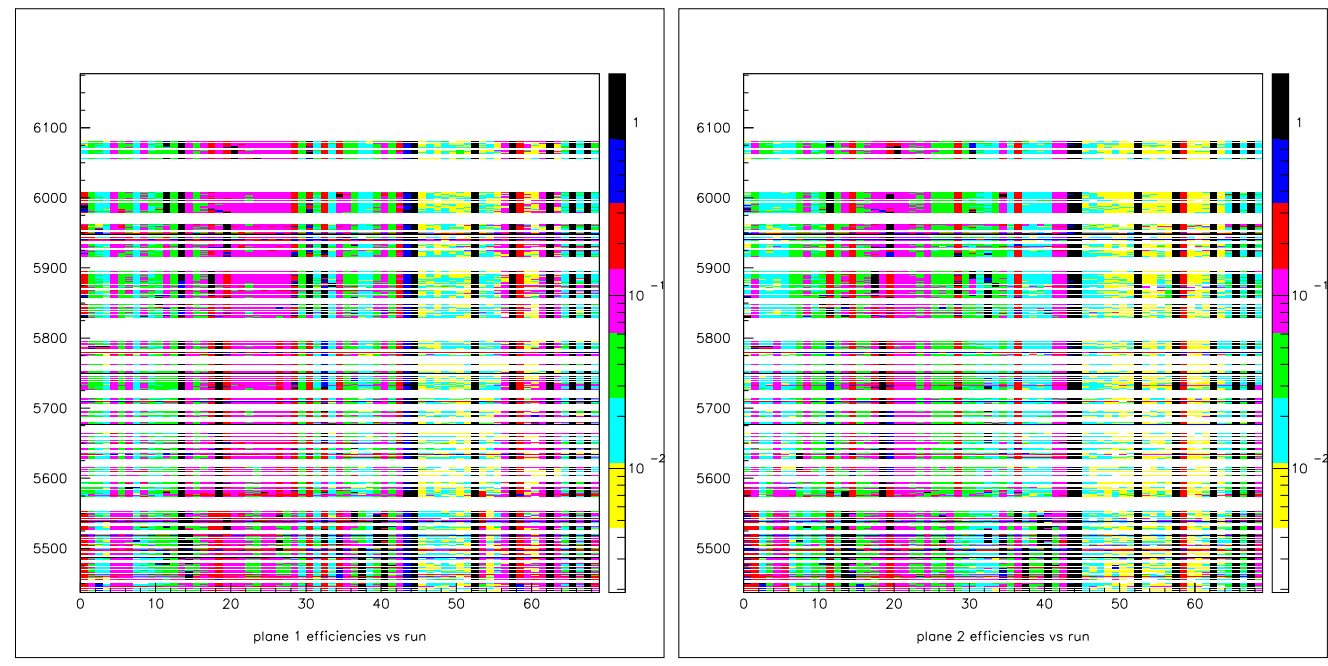

FIGURE 100. Average DC efficiency for each chamber vs run (views 1,2) 
a slightly different set of kinematic constraints than is required for the dimuon analyses (for example the flux was extracted with a harder cut on the primary muon and more restrictive gap cut), so the inclusive data and MC samples are used to empirically correct the flux.

\subsubsection{Muon energy correction}

The magnetized steel washers in the $\mathrm{McNuTeV}$ simulation of the muon spectrometer were defined slightly wider than was later found from direct measurements of the detector. The effect of this is to artificially increase the MC muon energy. There are three more or less equivalent ways one can remedy this situation:

1. Change the widths of the washers in the $\mathrm{McNuTeV}$ software to reflect the measured value.

2. Use the McNuTeV widths when calculating the muon energies in the reconstruction software.

3. Don't change anything in the software, and apply a muon energy correction post-reconstruction.

Of the three, the last was chosen, so that pre-existing $\mathrm{McNuTeV} \mathrm{MC}$ would not need to be regenerated, and to minimize confusion within the reconstruction software. The (at most) $2 \%$ level correction that is applied to $\mathrm{McNuTeV}$ muon energies is of the form:

$$
\mathcal{C}\left(E_{\mu-F F-g e n}\right)=\left.\frac{\left\langle E_{\mu-F F-\text { rec-correct-washer }}\right\rangle}{\left\langle E_{\mu-F F-\text { rec-wide-washer }}\right\rangle}\right|_{E_{\mu-F F-\text { gen }}}
$$




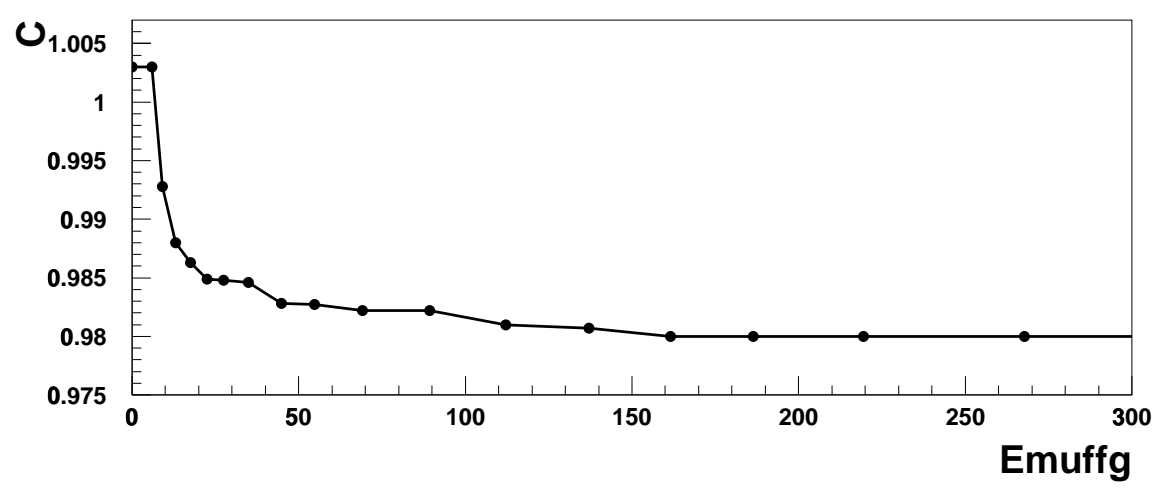

FIGURE 101. The correction applied to reconstructed $E_{\mu-F F-r e c}$ as a function of $E_{\mu-F F-g e n}$. The $\mathrm{x}$ axis scale is in $\mathrm{GeV}$.

where $\mathcal{C}$ is a function of the true muon energy within the spectrometer region, $E_{\mu-F F-g e n}$, and is applied only to the portion of the muon energy reconstructed by the muon spectrometer $\left(E_{\mu-F F-r e c}\right)$. The portion reconstructed within the calorimeter is subtracted from the total muon energy, then the remainder is scaled by $\mathcal{C}$, and the calorimeter part is added back in. Figure 101 shows the magnitude of this correction as a function of the true muon energy within the spectrometer.

\subsubsection{Hadronic energy corrections}

The hadronic shower smearing model in GEANT does not completely reproduce the energy smearing as measured from the $\mathrm{NuTeV}$ hadronic test beam data. A correction is applied in addition to the electromagnetic/hadronic energy correction mentioned above, to simultaneously shift the mean and width of the smeared hadronic energy produced by $\mathrm{McNuTeV}$ to match a parameterization of test beam measurements. 
Both the mean and width may be simultaneously adjusted through the following transformation:[148]:

$$
E_{r e c}^{\prime}=E_{g e n}\left[\frac{W_{T B}}{W_{M C}}\left(\frac{E_{r e c}}{E_{g e n}}-M_{M C}\right)+M_{T B}\right]
$$

where $E_{g e n}$ refers to the unsmeared energy, $E_{\text {rec }}$ is the raw smeared energy from $\mathrm{McNuTeV}$, and the W's and M's refer to the widths and means of the McNuTeV (MC) and parameterized calibration beam (TB) distributions.

The means and widths are dependent on generated hadronic energy, and are parameterized according to the following functions:

$$
\begin{aligned}
M & =A \frac{\log \left(B \cdot E_{g e n}\right)}{E_{g e n}+C}-D \\
W & =A \exp \left[B\left(E_{\text {gen }}+C\right)\right]+\frac{D}{E_{\text {gen }}}+F
\end{aligned}
$$

The parameters for each $\mathrm{TB}$ and $\mathrm{MC}$ curve are shown in table 18. Equation 4.245

\begin{tabular}{|l|c|c|c|c|c|}
\hline & $\mathrm{A}$ & $\mathrm{B}$ & $\mathrm{C}$ & $\mathrm{D}$ & $\mathrm{F}$ \\
\hline$M_{M C}$ & 8.538 & 0.03207 & 181.9 & -0.02805 & $\mathrm{~N} / \mathrm{A}$ \\
$M_{T B}$ & 2.592 & 0.0157 & 98.76 & -0.006912 & $\mathrm{~N} / \mathrm{A}$ \\
$W_{M C}$ & 0.1989 & -0.01176 & 70.05 & 0.9378 & 0.0793 \\
$W_{T B}$ & 0.09966 & -0.01075 & 18.13 & 1.248 & 0.04832 \\
\hline
\end{tabular}

TABLE 18. Parameters for McNuTeV Hadronic Energy Correction

is applied to the hadronic energies during event reconstruction, and limited so that for energies $E_{\text {gen }}<12 \mathrm{GeV}$ and $E_{\text {gen }}>200 \mathrm{GeV}$, the M's and W's are evaluated at 12 and $200 \mathrm{GeV}$ respectively. The functions described by the parameters in table 18 are shown in figures 102 and 103 


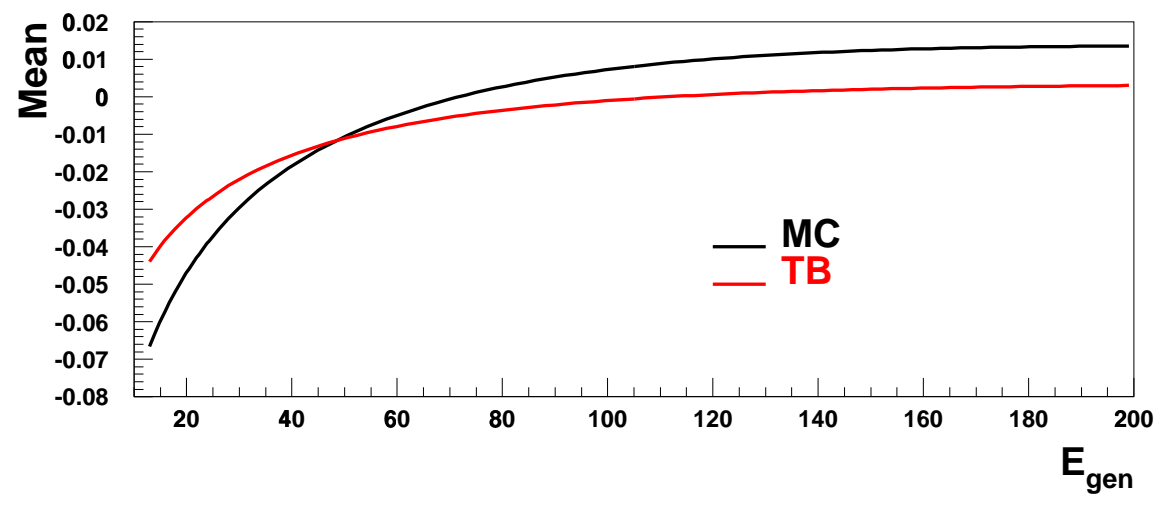

FIGURE 102. Parameterizations of the smearing function means for McNuTeV and test beam. The $\mathrm{x}$ axis scale is in $\mathrm{GeV}$

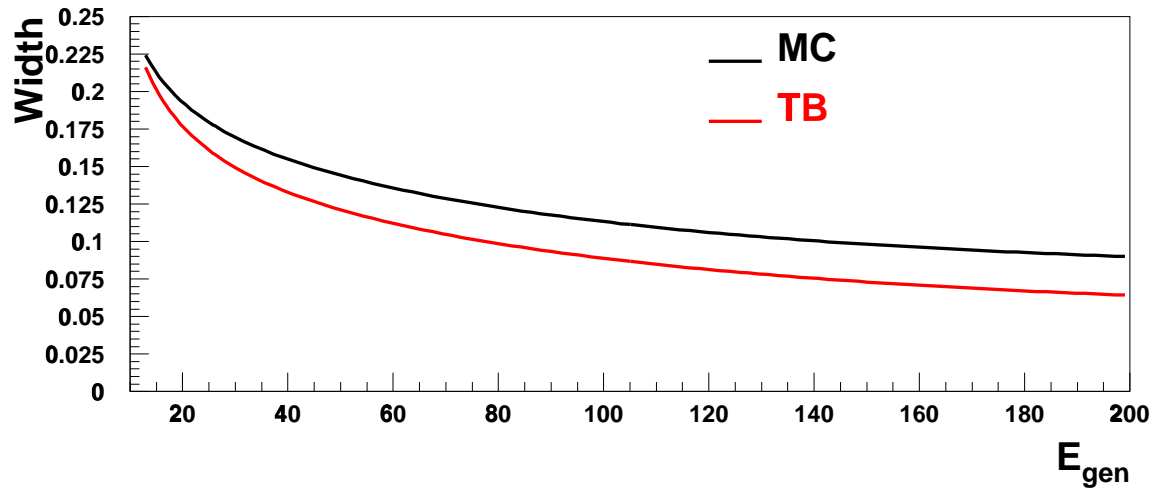

FIGURE 103. Parameterizations of the smearing function widths for $\mathrm{McNuTeV}$ and test beam. The $\mathrm{x}$ axis scale is in $\mathrm{GeV}$. 


\subsubsection{Flux Corrections}

The final correction to MC is the empirical adjustment to the flux to correct for the difference in kinematic cuts between this analysis and cuts used for its initial extraction.

This is found by binning data and MC events from the full inclusive sample in neutrino energy and vertex position. The correction is used to correct the flux for the dimuon sample. In each MC bin the weights are rescaled so that the overall shape matches the data. After applying the energy corrections and analysis cuts, $E_{\nu}$ is divided into the 23 bins shown in figure 84 on page 195. Since the flux is also vertex dependent, the transverse vertex position is also divided into a 10 by 10

grid along the $\mathrm{x}$ and $\mathrm{y}$ axes. Within each $\left(E_{\nu}\right.$, vertx, verty) bin, the ratio, $\frac{E_{\text {data }}}{E_{M C}}$ is calculated from the inclusive sample after energy corrections and cuts are applied, and used to rescale the dimuon MC flux. This function is interpolated linearly between the bins for each event's vertex position and energy. The correction as a function of reconstructed neutrino energy is shown in figure 104. 

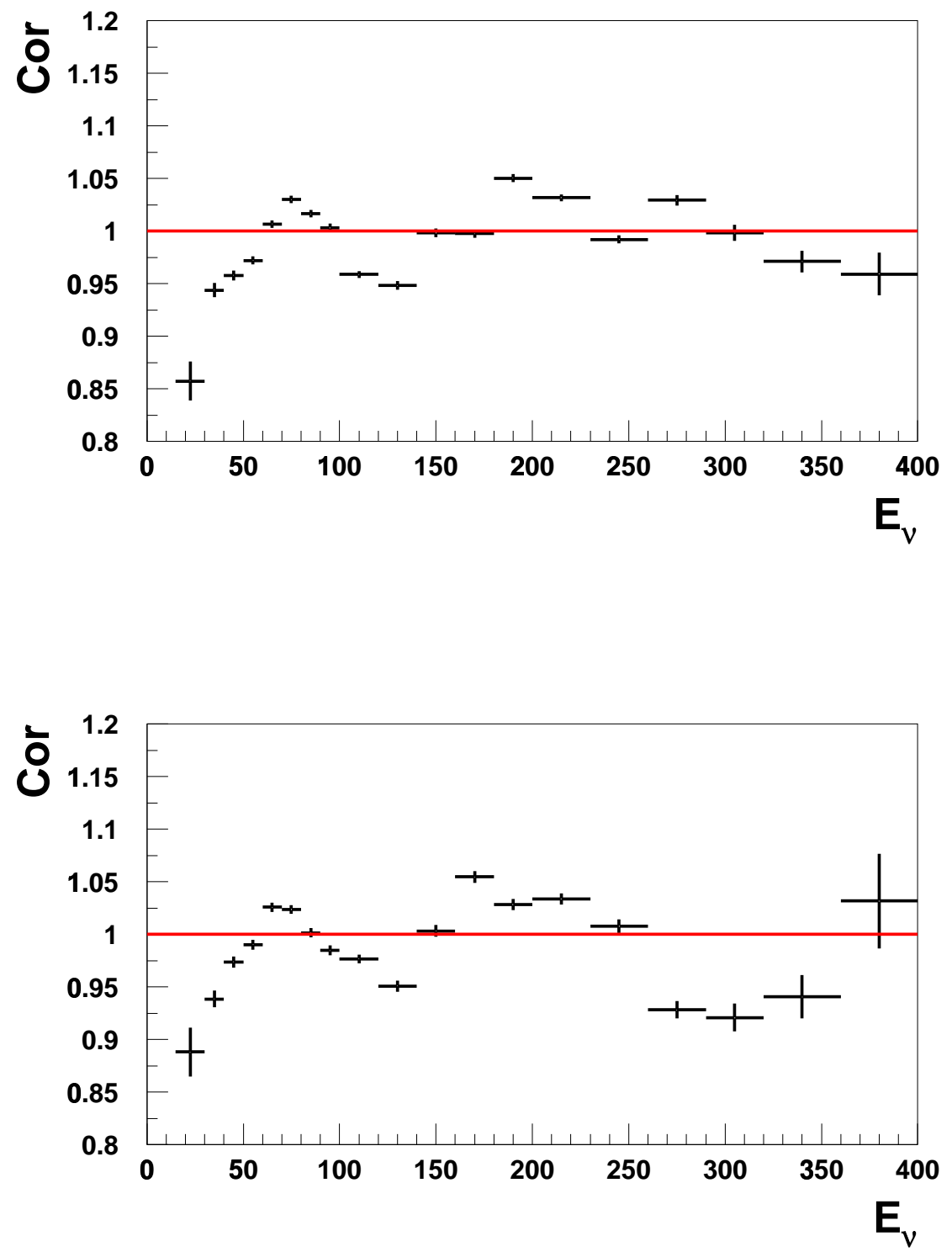

FIGURE 104. Neutrino (top) and antineutrino (bottom) flux corrections as a function of reconstructed neutrino energy, in $\mathrm{GeV}$. 


\subsubsection{Inclusive Data/MC Comparisons}

One million each of $\nu$ and $\bar{\nu}$ inclusive $\mathrm{CC}$ MC events were generated in numonte, processed through $\mathrm{McNuTeV}$, had the above energy corrections and cuts from table 10 applied, and compared to the total inclusive data sample. After cuts, $379304 \nu \mathrm{MC}$ events, and $399807 \bar{\nu} \mathrm{MC}$ events survived to be compared to 886004 $\nu$ and $255045 \bar{\nu}$ data events. The MC samples were normalized to the inclusive sample by scaling the number of MC events to be equal to data for each polarity.

Figures 105-111 compare the distributions of the observable variables between data and MC for both $\nu$ and $\bar{\nu}$. Each pair of plots compares the total distributions and displays a ratio plot $\left(\frac{d a t a}{M C}-1\right)$. The variables plotted are defined as follows:

$E_{\mu 1}$ : The energy of the muon from the weak vertex

$E_{H A D}$ : The reconstructed hadronic energy. The energies due to any muons present are subtracted out by assuming the energy loss for the muons as 1 MIP within the length of the shower.

$E_{v i s}:$ Total reconstructed neutrino energy, $E_{\mu}+E_{H A D}$. If a second muon is present $E_{\mu 2}$ is also added.

vertx, verty: The transverse $\mathrm{x}$ and $\mathrm{y}$ positions of the event vertex

$\theta_{\mu 1}$ : The opening angle relative to the beam axis of the muon from the weak vertex

$E_{\mu F F}$ : The muon spectrometer component of the primary muon energy

The yellow band in each plot indicates the magnitude of systematic uncertainties, described in detail in section 4.5.2. 

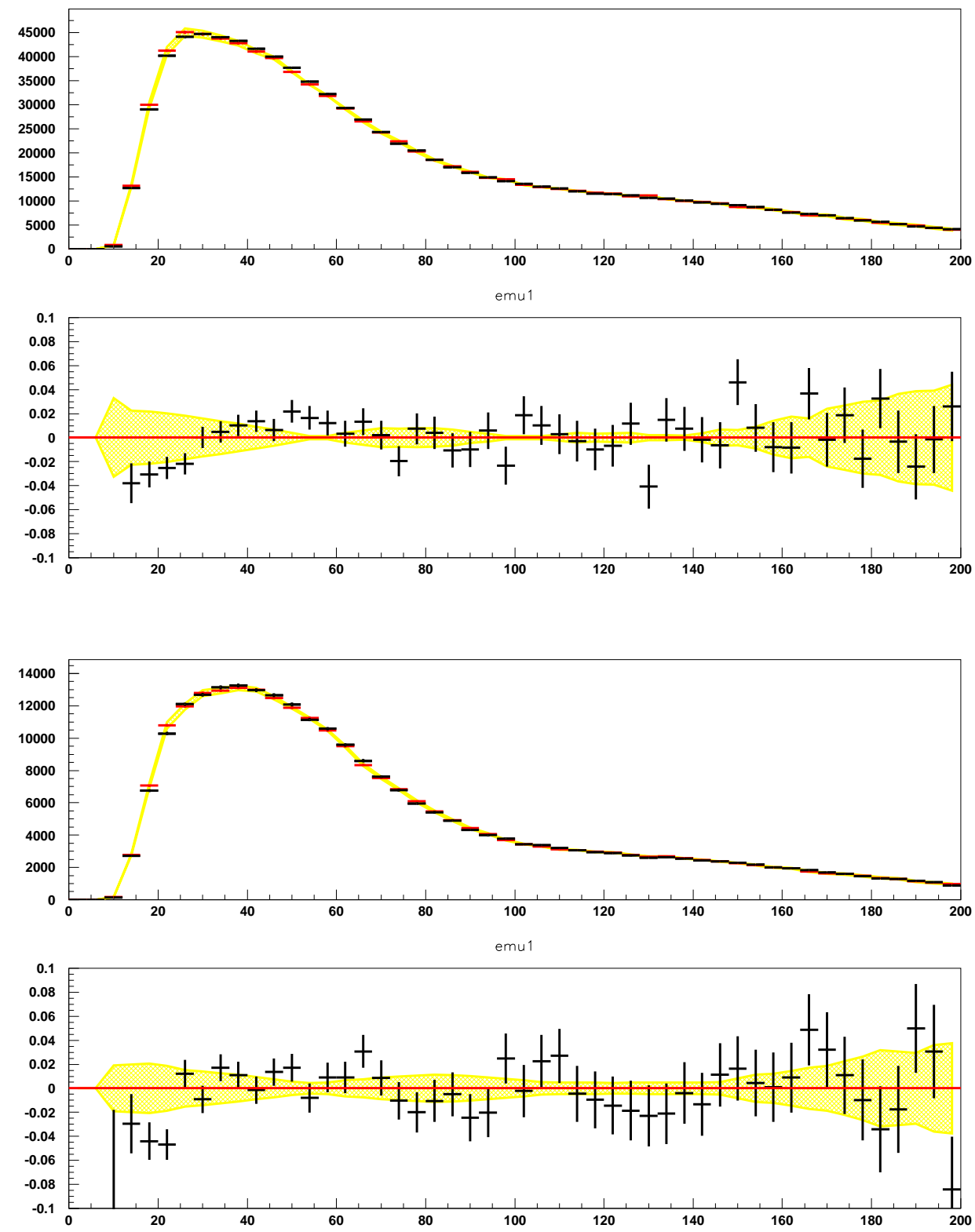

FIGURE 105. Comparisons of the reconstructed muon energy, $E_{\mu 1}$, in data vs MC for the inclusive data sample, for both neutrino (top) and antineutrino (bottom) modes. Plotted are both absolute comparisons, with data in black, and $\mathrm{MC}$ in red, above ratio plots, showing data/MC-1. The shaded regions are the systematic errors, and the $\mathrm{x}$ axis is in $\mathrm{GeV}$. 

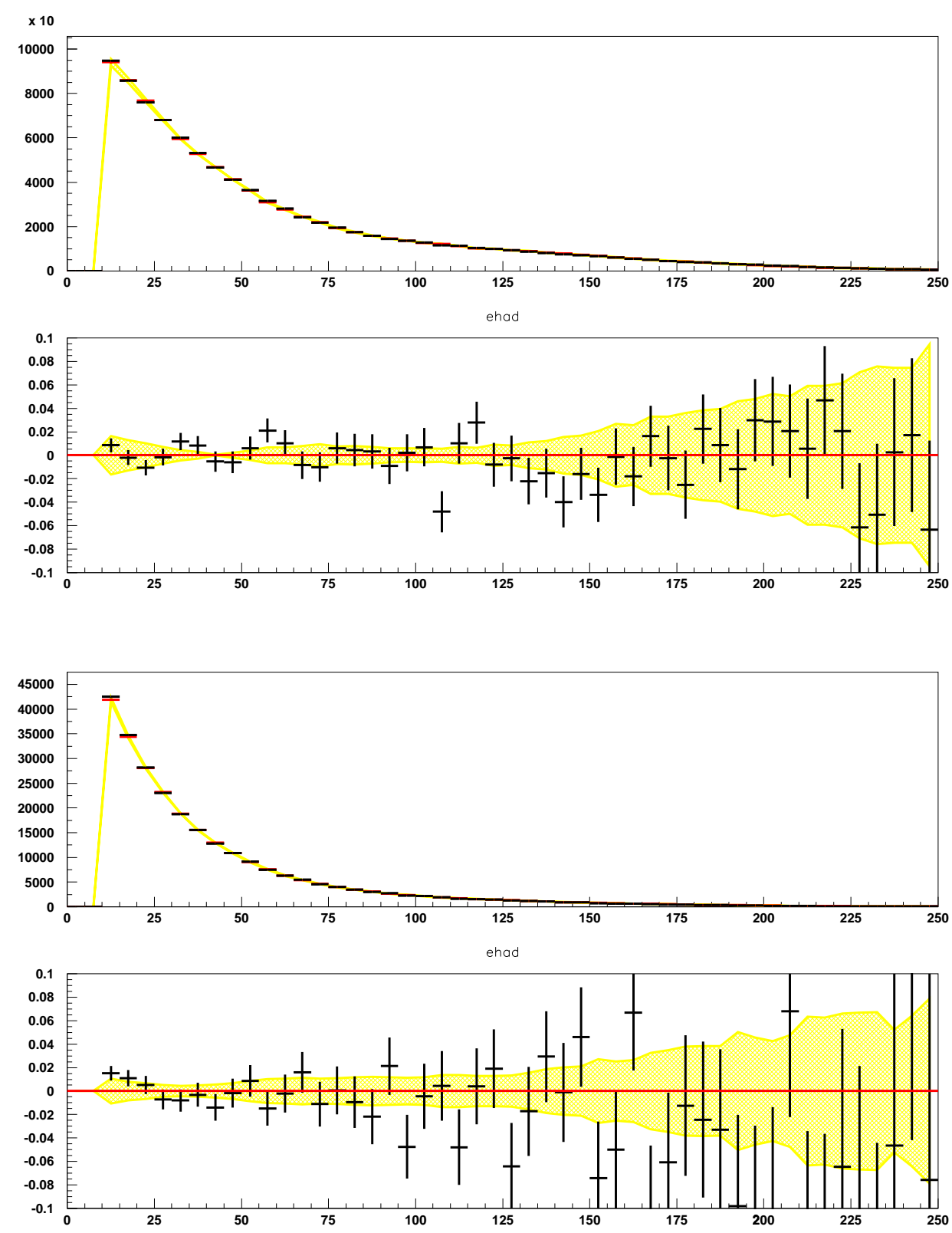

FIGURE 106. Comparisons of the reconstructed hadronic energy, $E_{H A D}$, in data vs MC for the inclusive data sample, for both neutrino (top) and antineutrino (bottom) modes. Plotted are both absolute comparisons, with data in black, and $\mathrm{MC}$ in red, above ratio plots, showing data/MC-1. The shaded regions are the systematic errors, and the $\mathrm{x}$ axis is in $\mathrm{GeV}$. 

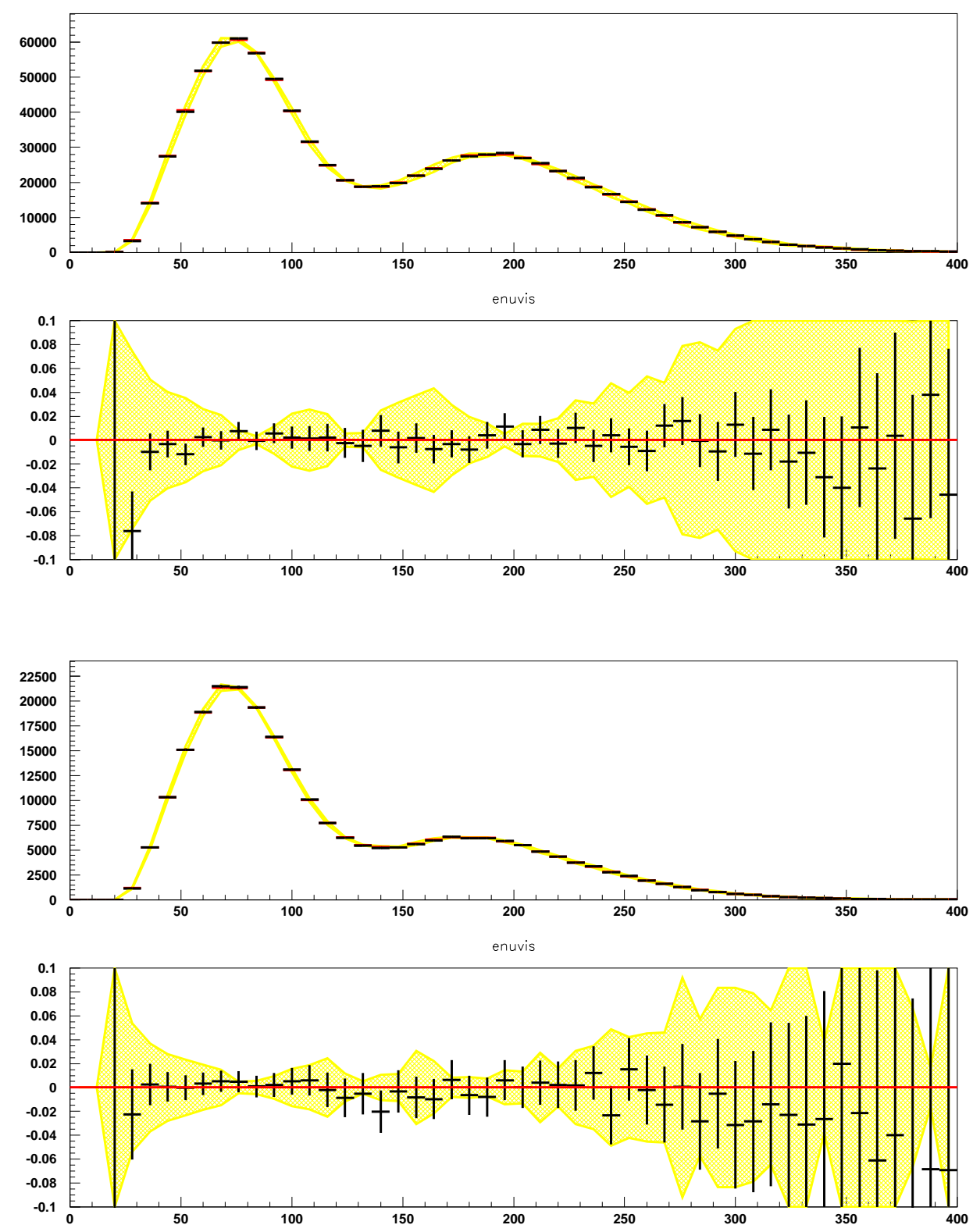

FIGURE 107. Comparisons of the total reconstructed neutrino energy, $E_{v i s}$, in data vs MC for the inclusive data sample, for both neutrino (top) and antineutrino (bottom) modes. Plotted are both absolute comparisons, with data in black, and $\mathrm{MC}$ in red, above ratio plots, showing data/MC-1. The shaded regions are the systematic errors, and the $\mathrm{x}$ axis is in $\mathrm{GeV}$. 

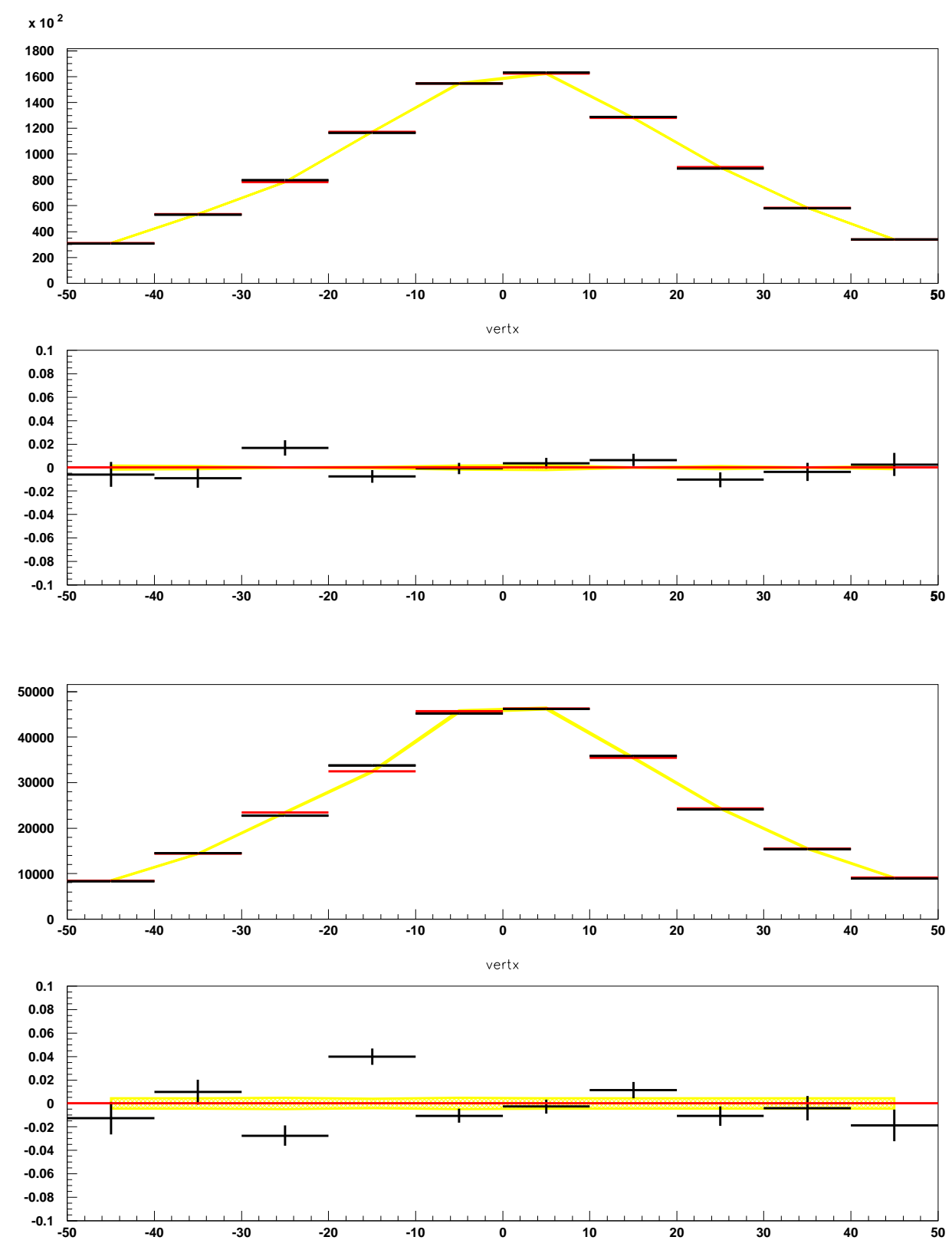

FIGURE 108. Comparisons of the reconstructed event vertex in the $\mathrm{x}$ direction, vertx, in data vs $\mathrm{MC}$ for the inclusive data sample, for both neutrino (top) and antineutrino (bottom) modes. Plotted are both absolute comparisons, with data in black, and MC in red, above ratio plots, showing data/MC-1. The shaded regions are the systematic errors, and the $\mathrm{x}$ axis is in inches. 

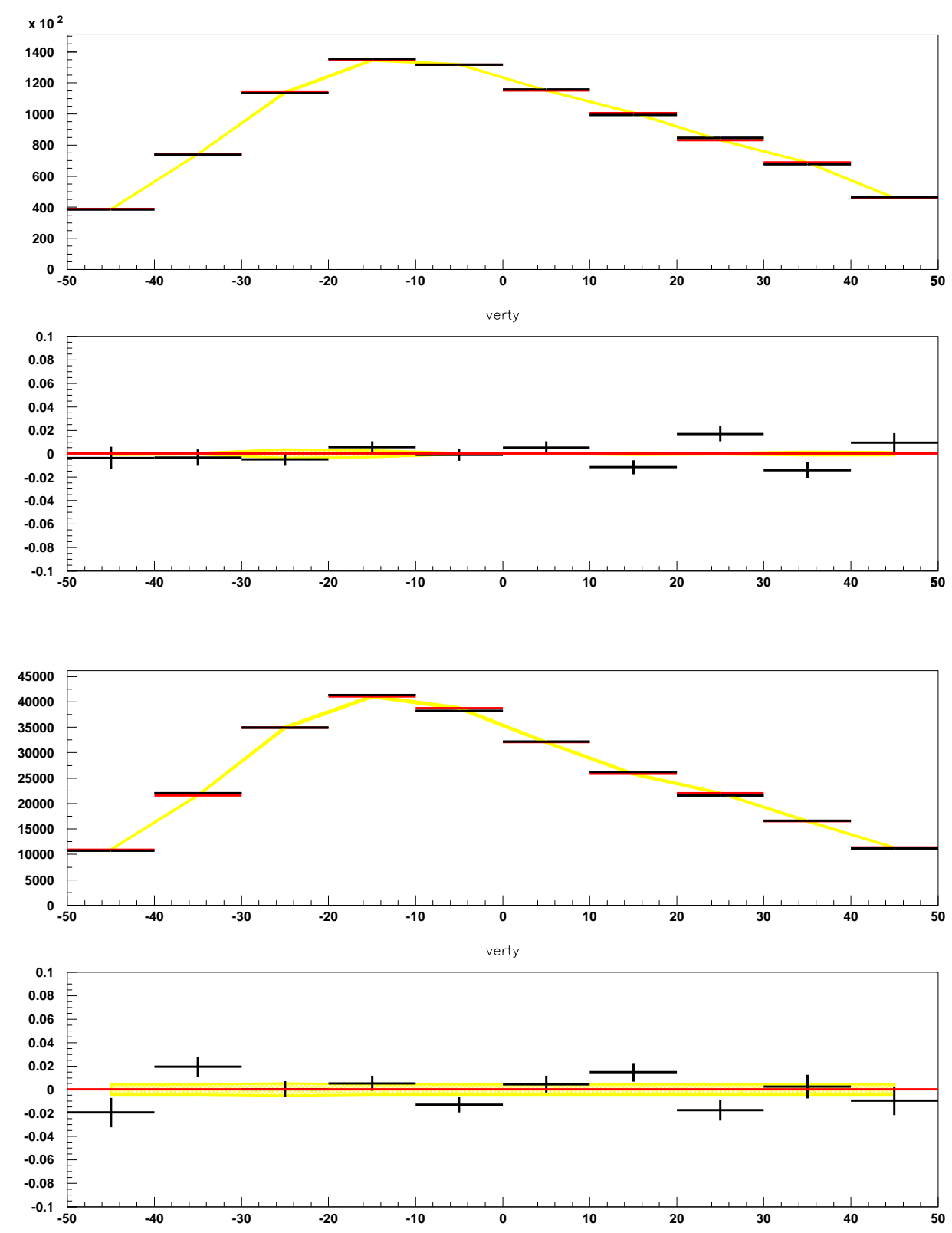

FIGURE 109. Comparisons of the reconstructed event vertex in the y direction, verty, in data vs $\mathrm{MC}$ for the inclusive data sample, for both neutrino (top) and antineutrino (bottom) modes. Plotted are both absolute comparisons, with data in black, and MC in red, above ratio plots, showing data/MC-1. The shaded regions are the systematic errors, and the $\mathrm{x}$ axis is in inches. 

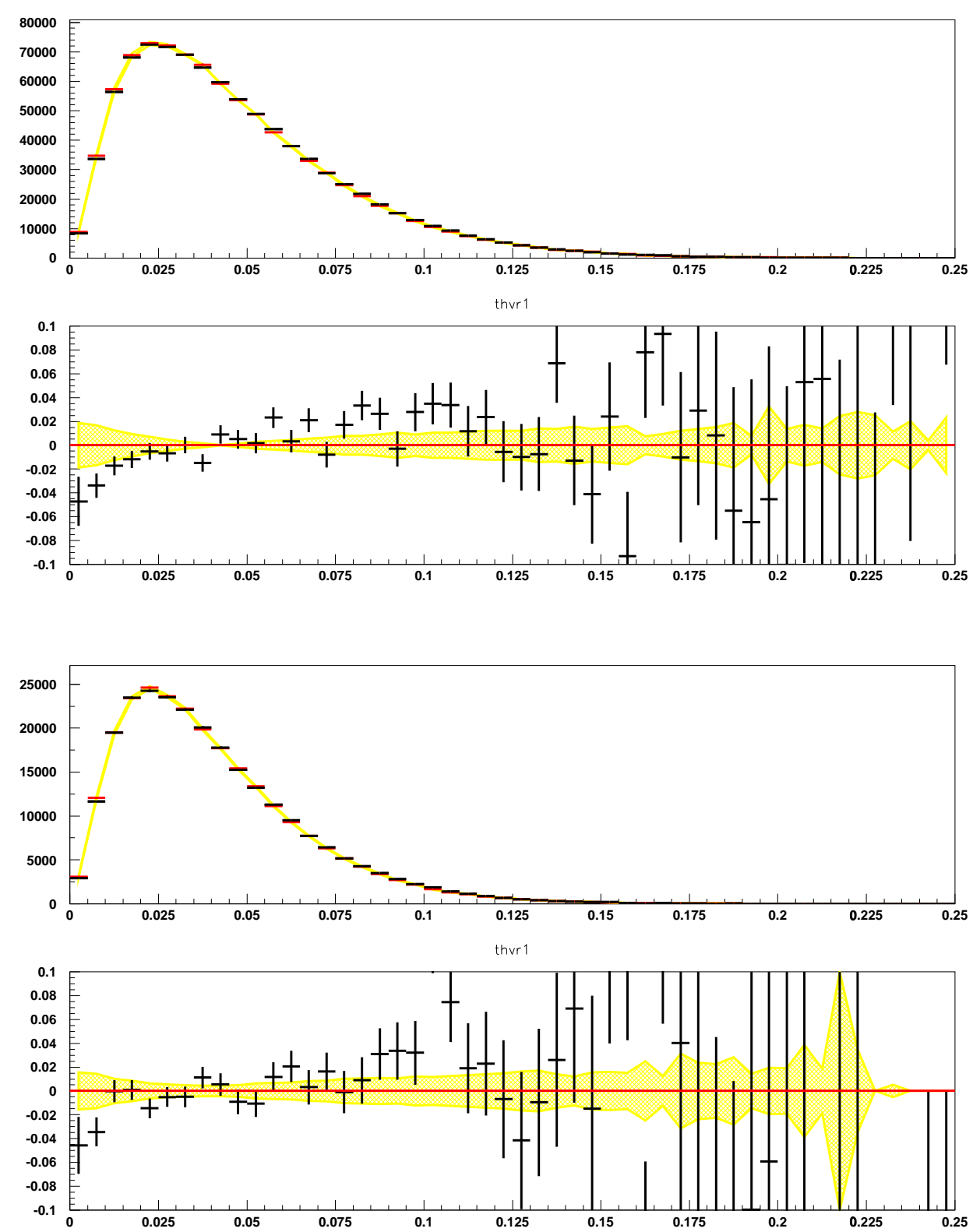

FIGURE 110. Comparisons of the reconstructed muon angle, $\theta_{\mu 1}$, in data vs MC for the inclusive data sample, for both neutrino (top) and antineutrino (bottom) modes. Plotted are both absolute comparisons, with data in black, and MC in red, above ratio plots, showing data/MC-1. The shaded regions are the systematic errors, and the $\mathrm{x}$ axis is in radians. 

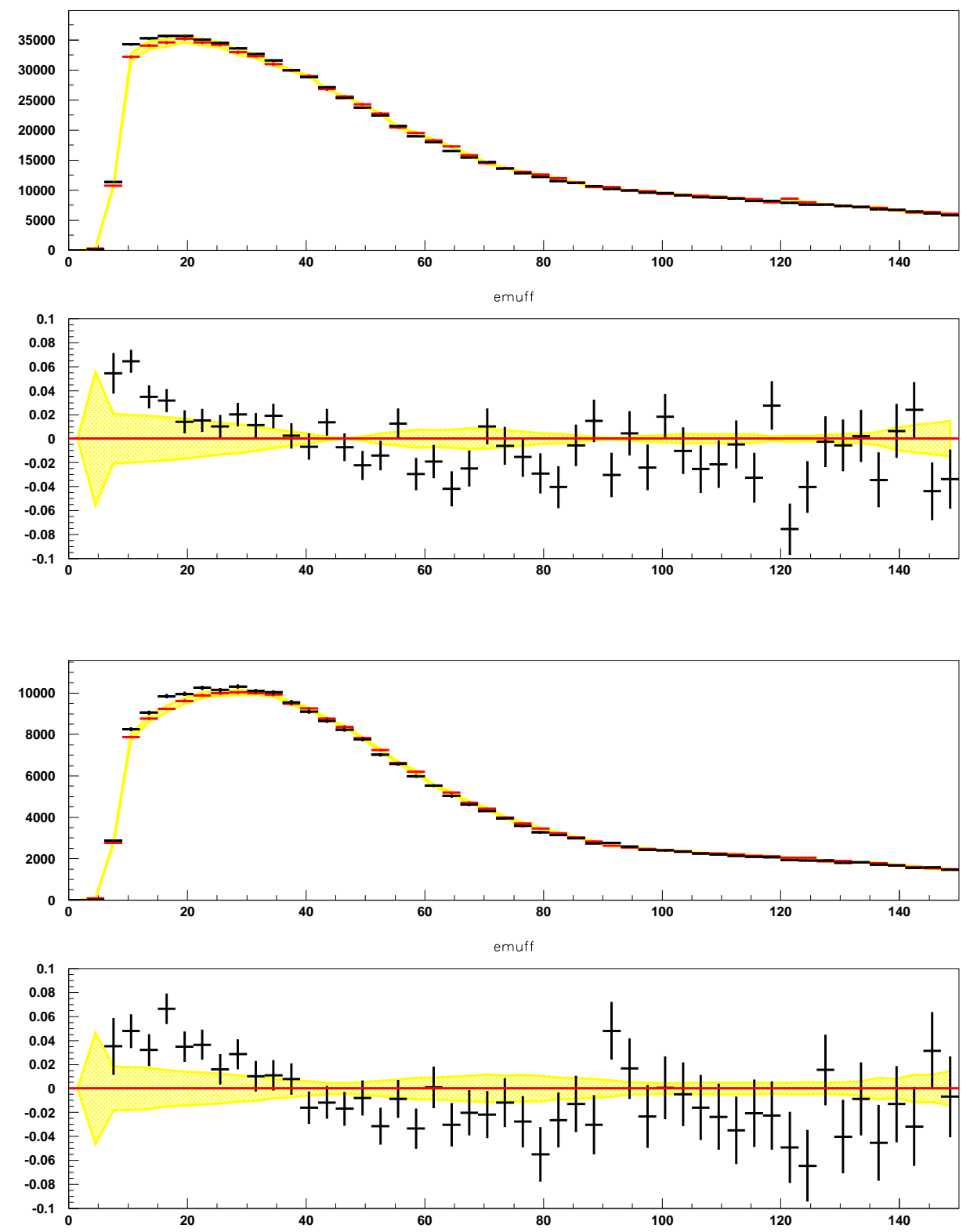

FIGURE 111. Comparisons of the reconstructed muon energy immediately after the calorimeter, $E_{\mu-F F}$, in data vs $\mathrm{MC}$ for the inclusive data sample, for both neutrino (top) and antineutrino (bottom) modes. Plotted are both absolute comparisons, with data in black, and MC in red, above ratio plots, showing data/MC-1. The shaded regions are the systematic errors, and the $\mathrm{x}$ axis is in $\mathrm{GeV}$. 


\subsection{LO MC-data fitting}

With acceptable reproduction of the data by the inclusive MC sample achieved, one may then work towards fitting the BGPAR based dimuon model to the dimuon data. The dimuon data and $\mathrm{MC}$ were processed using the same corrections as the inclusive sample, and dimuon cuts as outlined in table 10 on page 174 . The surviving events for each sample were then placed into a series of bins, whose populations were used to define a likelihood function via equation 4.172. The fit procedure, a discussed at the beginning of this chapter, was then a search for the set of physics parameters which produced the lowest value of $-\log (\mathcal{L})$, and therefore provided the best fit to data.

In the fit, The parameters allowed to vary were the charm mass $m_{c}$, strange sea via $\kappa, \alpha$ (see equation 4.210 ), the antistrange sea via $\bar{\kappa}, \bar{\alpha}$, Collins-Spiller fragmentation parameter $\epsilon$, and the semi-muonic branching ratio $B_{c}$. The strange and antistrange seas were not constrained relative to each other, so net strangeness was allowed to deviate from zero. Neutrino and antineutrino data were essentially treated as independent data sets tied together with the same $m_{c}, B_{c}$, and $\epsilon$.

To fit MC to data, one must first start with an initial sample of MC to reweight. This sample should be generated with parameters that do not bias the sample through elimination of events via threshold effects like slow rescaling. This means that the charm mass in this seed sample should be below where the final value is expected to be, and epsilon should be for a harder fragmentation function. On the other hand one does not want to be too far away from the eventual final values of the fit, so that the re-weighting corrections remain relatively small. The initial values for the dimuon $\mathrm{MC}$ sample were then chosen to be: $m c=0.9$, 
$\epsilon=0.81, B_{c}=0.092, \kappa=\bar{\kappa}=0.373, \alpha=\bar{\alpha}=2.5$. The initial values of the branching ratio and strange sea parameters are less important to the final outcome of the fit, so were chosen to match the LO CCFR values [62] used in the inclusive sample BGPAR fit. $1300196 \nu$ dimuon MC events and $819989 \bar{\nu}$ events were generated for the fit. After kinematic cuts were applied, these samples were whittled down to $224343 \nu$ events, and $130310 \bar{\nu}$ events.

After a dimuon MC sample is generated and processed, the next step in assembling the likelihood function is determining the normalization of the dimuon $\mathrm{MC}$ relative to the data. Unlike the inclusive sample, the dimuon MC sample is not normalized relative to the dimuon data, as the overall number of dimuon events is important in determining physics parameters like the size of the strange sea, semimuonic branching ratio and charm mass. To properly normalize the dimuon MC to the dimuon data, dimuon $\mathrm{MC}$ event weights must be rescaled by the following expression:

$$
w t c c_{2 \mu_{\_} M C}^{\prime}=w t c c_{2 \mu \_M C} \cdot N_{1 \mu} \cdot \frac{1}{N_{\text {dimu_MC_run }}} \cdot \frac{N_{\text {inclusive_MC_run }}}{N_{\text {inclusive_MC }}}
$$

where $N_{1 \mu}$ and $N_{\text {inclusive_MC }}$ are the total number of inclusive data and MC events after cuts, and $N_{d i m u \_M C \_r u n}$ and $N_{\text {inclusive_MC_run }}$ are the pre-cut numbers of Mc$\mathrm{NuTeV}$ generated $\mathrm{MC}$ events for the dimuon and inclusive samples respectively.

Each event in the normalized dimuon $\mathrm{MC}$ and the data are then assembled into a binned three dimensional grid in $E_{v i s}, x_{v i s}$ and $z_{v i s}$. Five bins in $E_{v i s}$, 4 bins in $z_{v i s}$, and 7 bins in $x_{v i s}$ were used in both neutrino and antineutrino modes. An alternate 4-dimensional scheme with additional binning in $y_{v i s}$ was tried, however because $z_{v i s}$ is dependent on $y_{v i s}$, this resulted in several of the 4-D 


\begin{tabular}{|c|c|c||c|c|c|}
\hline \multicolumn{3}{|c||}{$\nu$} & \multicolumn{3}{c|}{$\bar{\nu}$} \\
\hline$E_{\text {vis }}$ & $x_{\text {vis }}$ & $z_{\text {vis }}$ & $E_{\text {vis }}$ & $x_{\text {vis }}$ & $z_{\text {vis }}$ \\
\hline 34.83 & 0.00011 & 0.0188 & 31.70 & 0.00014 & 0.024 \\
82.44 & 0.027 & 0.088 & 74.63 & 0.019 & 0.106 \\
121.64 & 0.050 & 0.147 & 96.79 & 0.035 & 0.163 \\
164.92 & 0.080 & 0.217 & 134.60 & 0.056 & 0.232 \\
196.30 & 0.113 & 0.313 & 172.64 & 0.078 & 0.328 \\
233.01 & 0.156 & 0.870 & 207.08 & 0.107 & 0.808 \\
399.63 & 0.208 & & 393.31 & 0.149 & \\
& 0.305 & & & 0.218 & \\
& 0.973 & & & 0.991 & \\
\hline
\end{tabular}

TABLE 19. Boundaries on bins in LO fit

bins becoming unpopulated in data or MC, making it difficult for fits to converge. Different numbers of bins along each axis were also tried, with the above chosen to maximize the total number of bins while keeping a reasonable number of events in each bin.

The bin boundaries were determined along each of the $3 E_{v i s}, x_{v i s}$ and $z_{v i s}$ axes by sorting the data in that variable and dividing it into equal populations. The bin boundaries in the fit for both neutrino and antineutrino modes are shown in table 19. For each polarity, two arrays with this binning were maintained, one for data and one for MC. The data arrays were filled once at the beginning of a fit. When a data event was assigned to a particular bin, that bin in the data array is incremented by 1 .

The fit process would proceed with MINUIT searching for its best fit by varying the parameters to minimize $-\log \mathcal{L}$. With each prospective parameter set, all the MC events were re-weighted by the ratio of the new cross section to the old cross section, then the MC arrays were re-filled. Each MC event is placed in its 
appropriate bin, which was incremented by the rescaled weight for that iteration. After all the MC events were re-weighted and placed in their appropriate bins, $\mathcal{L}$ was calculated, and the cycle continued until a minimum was found. For a typical fit this process could take a few hundred iterations.

The results of this procedure are shown in figure 112. The fit achieved a $\chi^{2}$ of 111 for 106 degrees of freedom. Agreement between LO MC run with these fit parameters and data is good, and is shown in figures 113-127 in the next section.

\subsection{LO MC-data comparisons}

A minimum likelihood and good $\chi^{2}$ are not the only measure of the quality of fit between data and MC. The agreement between different kinematic and physics distributions should be checked as well. Figures 113-127 compare several kinematic distributions for data and MC run with the parameters resulting from the LO BGPAR fit. The variables plotted are the reconstructed versions of quantities defined in chapter 2.1, including the measured quantities: $E_{\mu 1}, E_{\mu 2}$, the reconstructed energies of the muon from the weak vertex $(\mu 1)$, and that from the charm vertex $(\mu 2), E_{H A D}$, the reconstructed hadronic energy, and $\theta_{1}$ and $\theta_{2}$, the reconstructed muon opening angles. Also included are several "visible" quantities derived from the reconstructed energies and angles:

$$
\begin{aligned}
E_{v i s}= & E_{H A D}+E_{\mu 1}+E_{\mu 2} \\
\theta_{\mu 12}= & \arccos \left[\cos \theta_{\mu 1} \cos \theta_{\mu 2}+\right. \\
& \left.\sin \theta_{\mu 1} \sin \theta_{\mu 2} \sin \left(\phi_{\mu 1}-\phi_{\mu 2}\right)\right] \\
Q_{v i s}^{2}= & 2 E_{v i s} E_{\mu 1}\left[1-\cos \left(\theta_{\mu 1}\right)\right]
\end{aligned}
$$


FIGURE 112. The output from the LO BGPAR fit using MINUIT. Listed are the 7 fit parameters, the estimated statistical errors, then below is the error matrix (elements including and below the diagonal), and the correlation matrix. 


$$
\begin{aligned}
x_{v i s}= & \frac{Q_{v i s}^{2}}{2 M E_{H A D}} \\
y_{v i s}= & \frac{E_{H A D}+E_{\mu 2}}{E_{\mu 1}} \\
z_{v i s}= & \frac{E_{\mu 2}}{E_{H A D}+E_{\mu 2}} \\
W_{v i s}^{2}= & 2 M E_{H A D}\left(1-x_{v i s}\right)+M^{2} \\
m_{12 v i s}^{2}= & \left(E_{\mu 1}+E_{\mu 2}\right)^{2}-\left(p_{\mu 1 x}+p_{\mu 2 x}\right)^{2}- \\
& \left(p_{\mu 1 y}+p_{\mu 2 y}\right)^{2}-\left(p_{\mu 1 z}+p_{\mu 2 z}\right)^{2}
\end{aligned}
$$

where $\phi_{\mu 1,2}$ is analogous to $\theta_{\mu 1,2}$, and $p_{\mu 1,2 x}, p_{\mu 1,2 y}, p_{\mu 1,2 z}$ are the vector components of each of the two muons $(1,2)$. Also plotted below are the variables $E_{\mu F F 1}$ and $E_{\mu F F 2}$, which are the muon energies as reconstructed in the muon spectrometer (i.e. without the portion deposited in the calorimeter). The plots show histograms of each variable in data and MC superimposed on each other. The errors on the histogram bin values represent the statistical errors of each bin, and a shaded band indicating the effect of various systematic uncertainties like energy calibrations, model uncertainties, etc. is also shown. A full listing of these systematics and their effects will be discussed in section 4.5.2. 

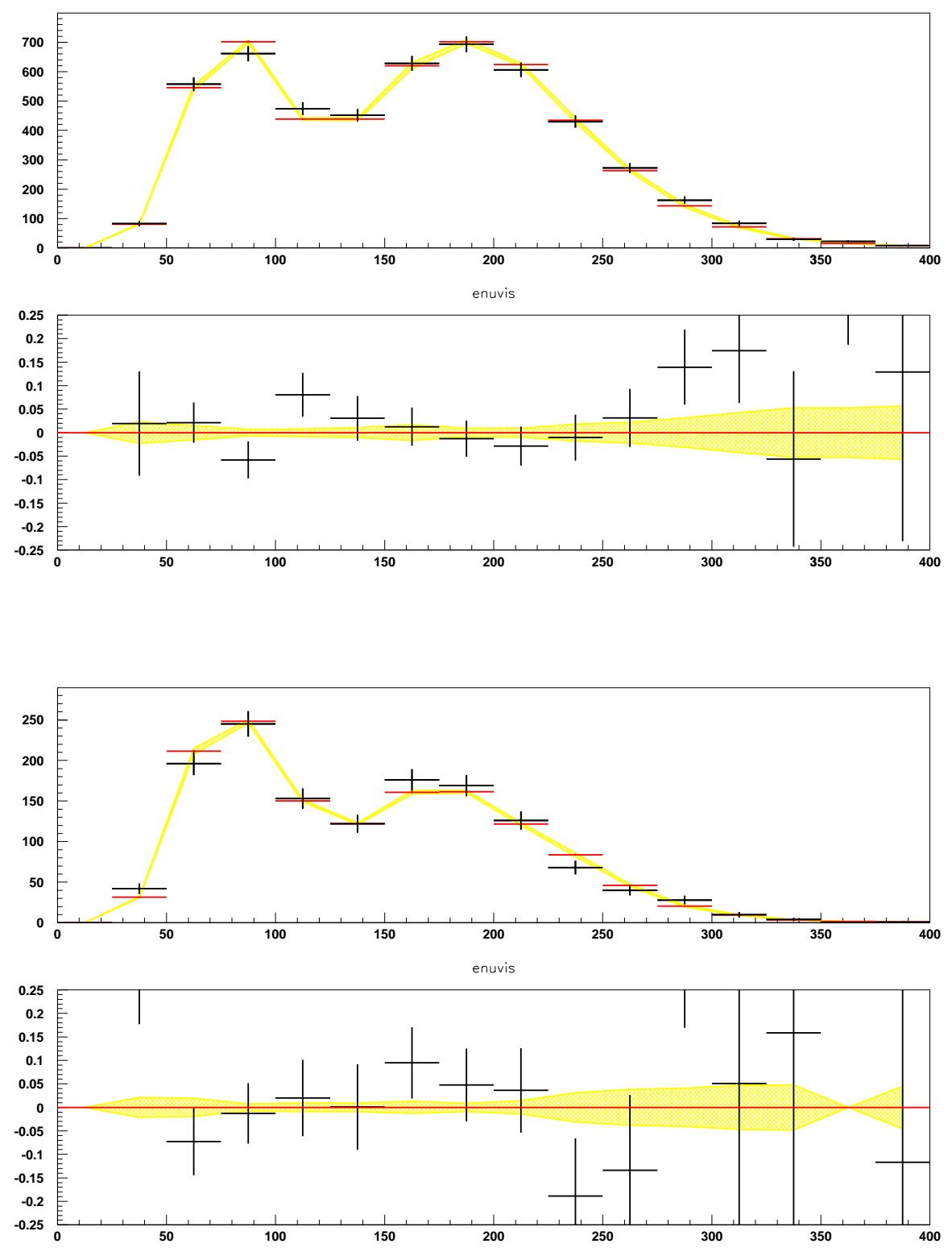

FIGURE 113. Total reconstructed neutrino energy, $E_{v i s}$, in data vs MC for the LO dimuon sample, for both neutrino (top) and antineutrino (bottom) modes. Plotted are both absolute comparisons, with data in black, and $\mathrm{MC}$ in red, above ratio plots, showing data/MC-1. The shaded regions are the systematic errors, and the $\mathrm{x}$ axis is in $\mathrm{GeV}$. 

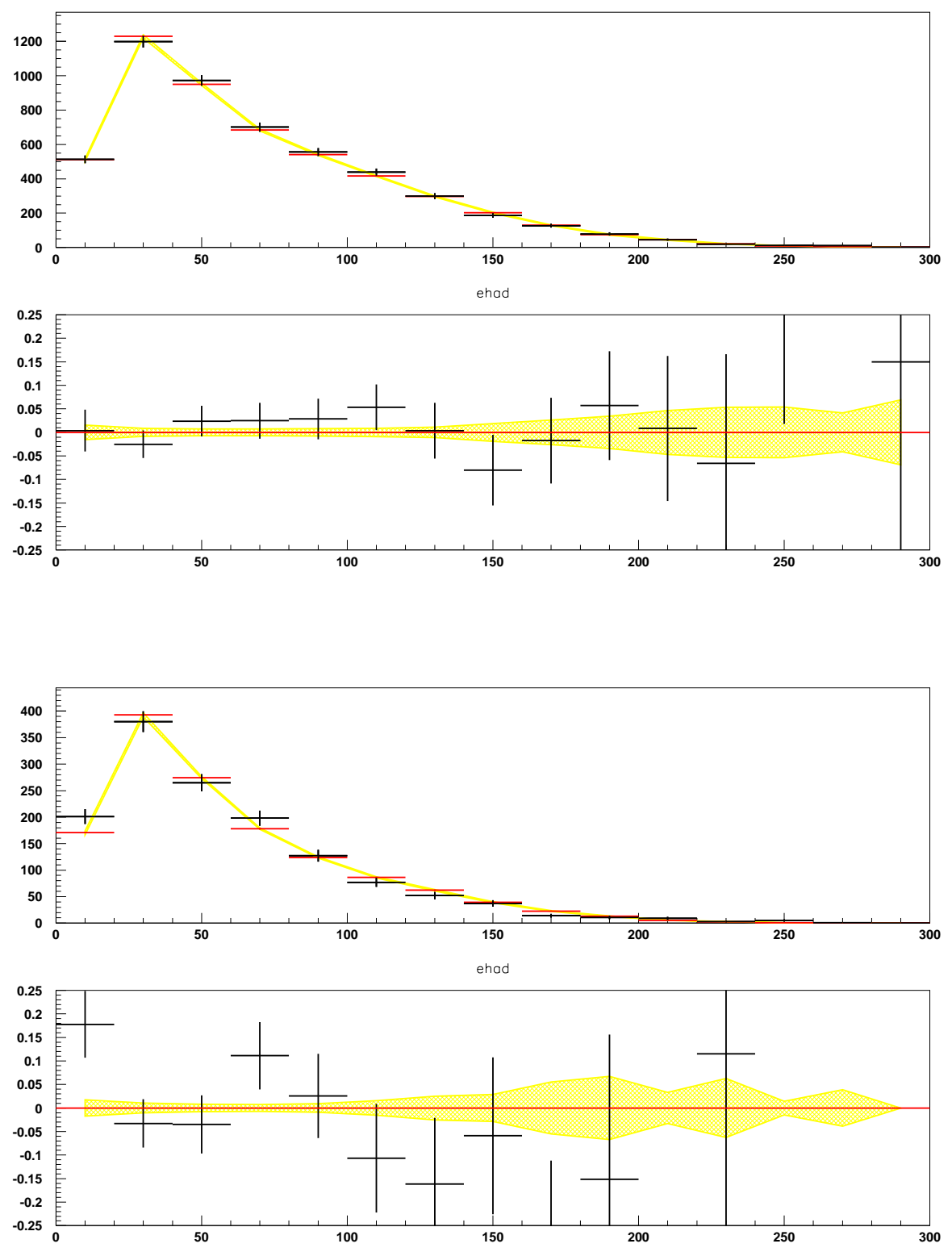

FIGURE 114. Reconstructed hadron shower energy, $E_{H A D}$ in data vs MC for the LO dimuon sample, for both neutrino (top) and antineutrino (bottom) modes. Plotted are both absolute comparisons, with data in black, and MC in red, above ratio plots, showing data/MC-1. The shaded regions are the systematic errors, and the $\mathrm{x}$ axis is in $\mathrm{GeV}$. 

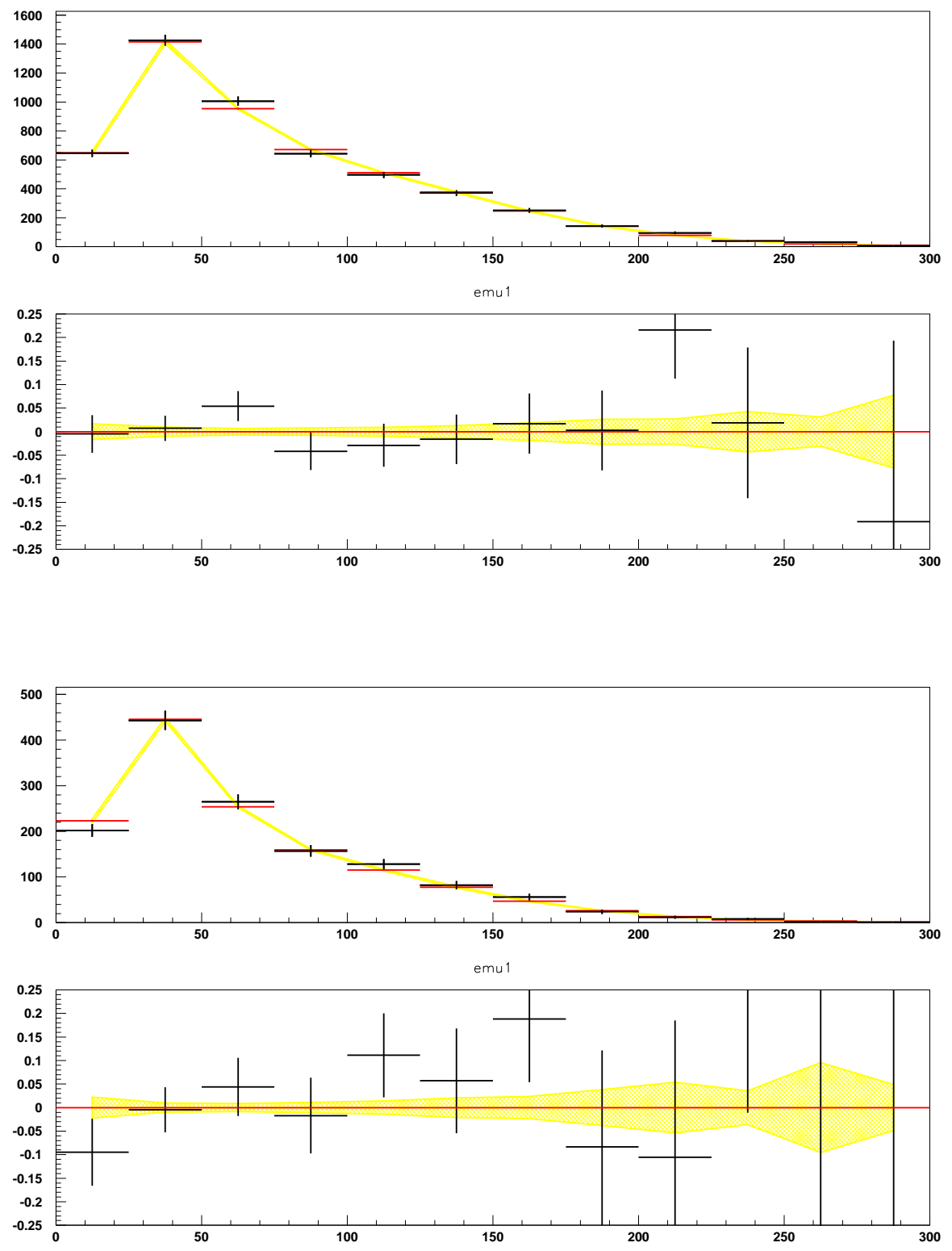

FIGURE 115. Reconstructed energy of the muon from the weak vertex, $E_{\mu 1}$, in data vs MC for the LO dimuon sample, for both neutrino (top) and antineutrino (bottom) modes. Plotted are both absolute comparisons, with data in black, and $\mathrm{MC}$ in red, above ratio plots, showing data/MC-1. The shaded regions are the systematic errors, and the $\mathrm{x}$ axis is in $\mathrm{GeV}$. 

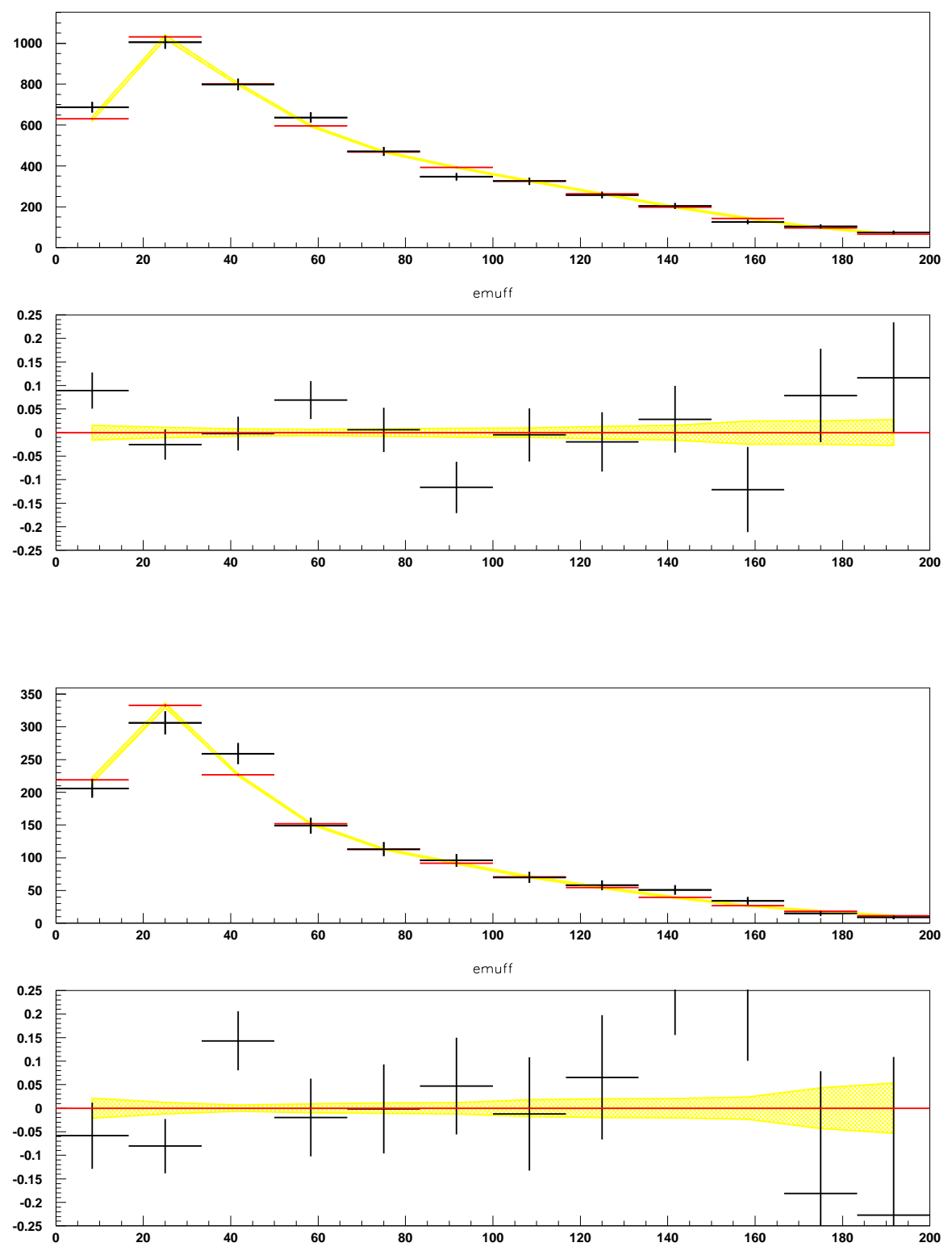

FIGURE 116. Reconstructed energy of the muon from the weak vertex immediately after the calorimeter, $E_{\mu F F 1}$, in data vs MC for the LO dimuon sample, for both neutrino (top) and antineutrino (bottom) modes. Plotted are both absolute comparisons, with data in black, and $\mathrm{MC}$ in red, above ratio plots, showing data/MC-1. The shaded regions are the systematic errors, and the $\mathrm{x}$ axis is in $\mathrm{GeV}$. 

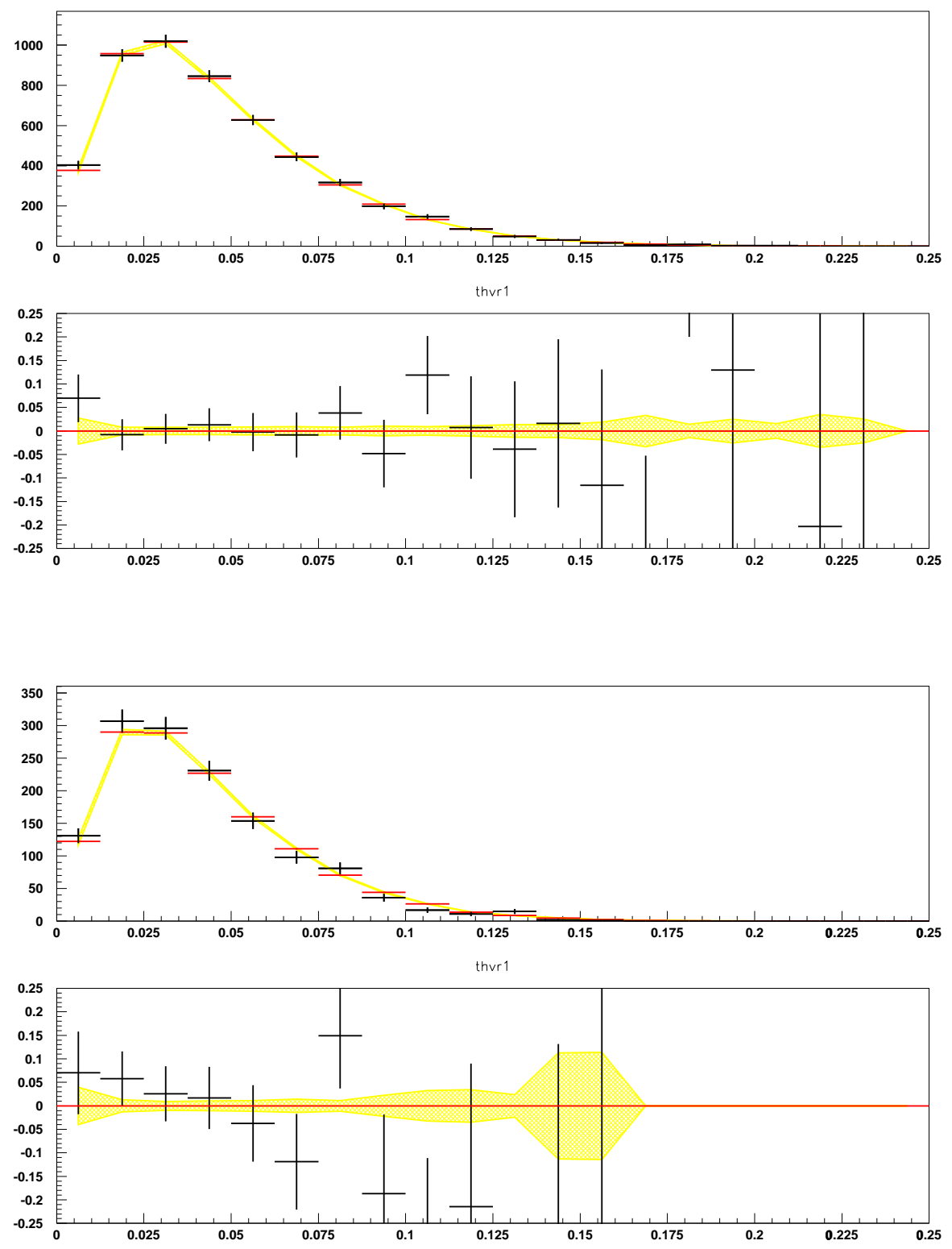

FIGURE 117. Reconstructed opening angle of the muon from the weak vertex, $\theta_{\mu 1}$, in data vs MC for the LO dimuon sample, for both neutrino (top) and antineutrino (bottom) modes. Plotted are both absolute comparisons, with data in black, and $\mathrm{MC}$ in red, above ratio plots, showing data/MC-1. The shaded regions are the systematic errors, and the $\mathrm{x}$ axis is in radians. 

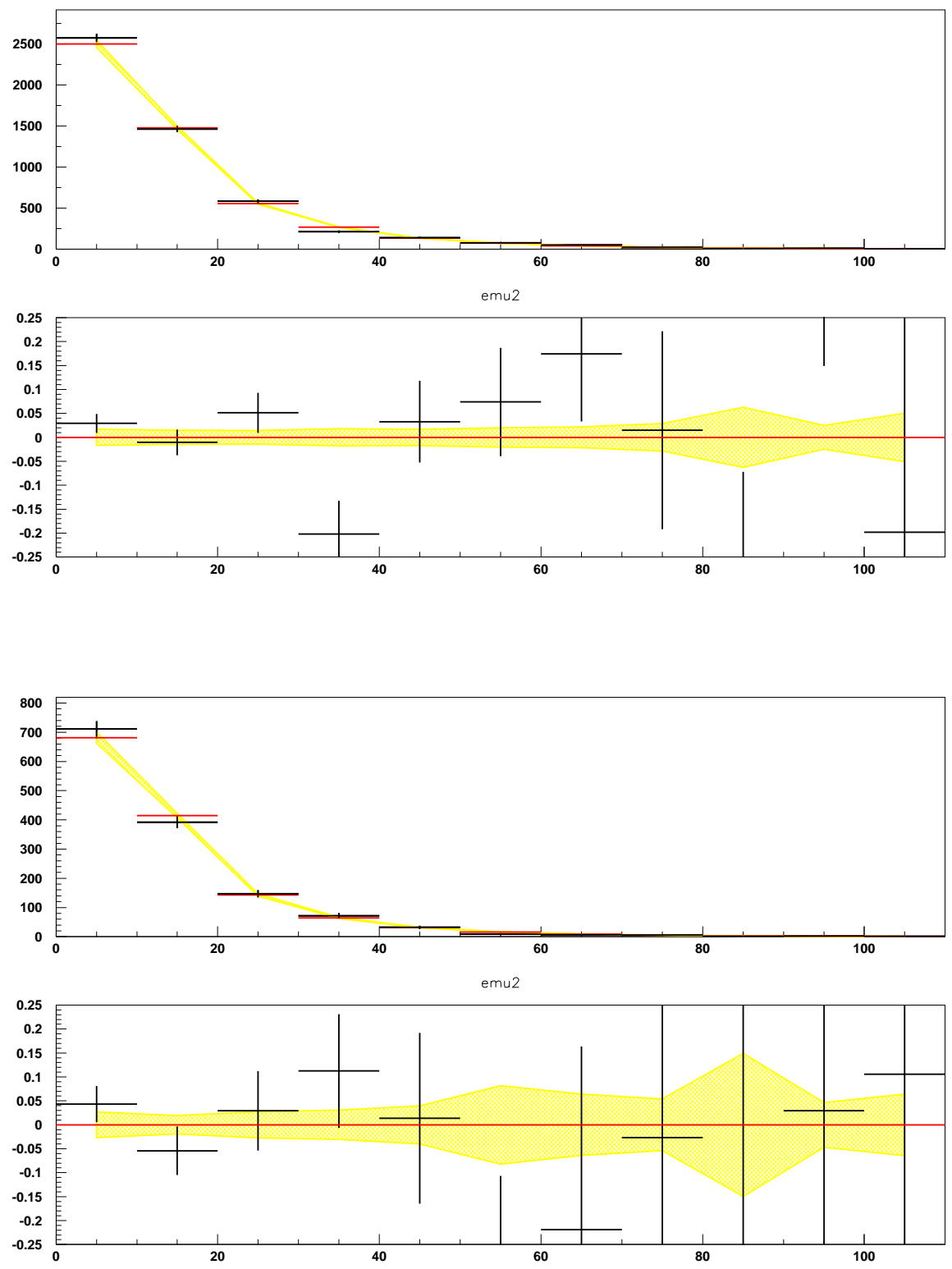

FIGURE 118. Reconstructed energy of the muon from the charm decay, $E_{\mu 2}$ in data vs MC for the LO dimuon sample, for both neutrino (top) and antineutrino (bottom) modes. Plotted are both absolute comparisons, with data in black, and $\mathrm{MC}$ in red, above ratio plots, showing data/MC-1. The shaded regions are the systematic errors, and the $\mathrm{x}$ axis is in $\mathrm{GeV}$. 

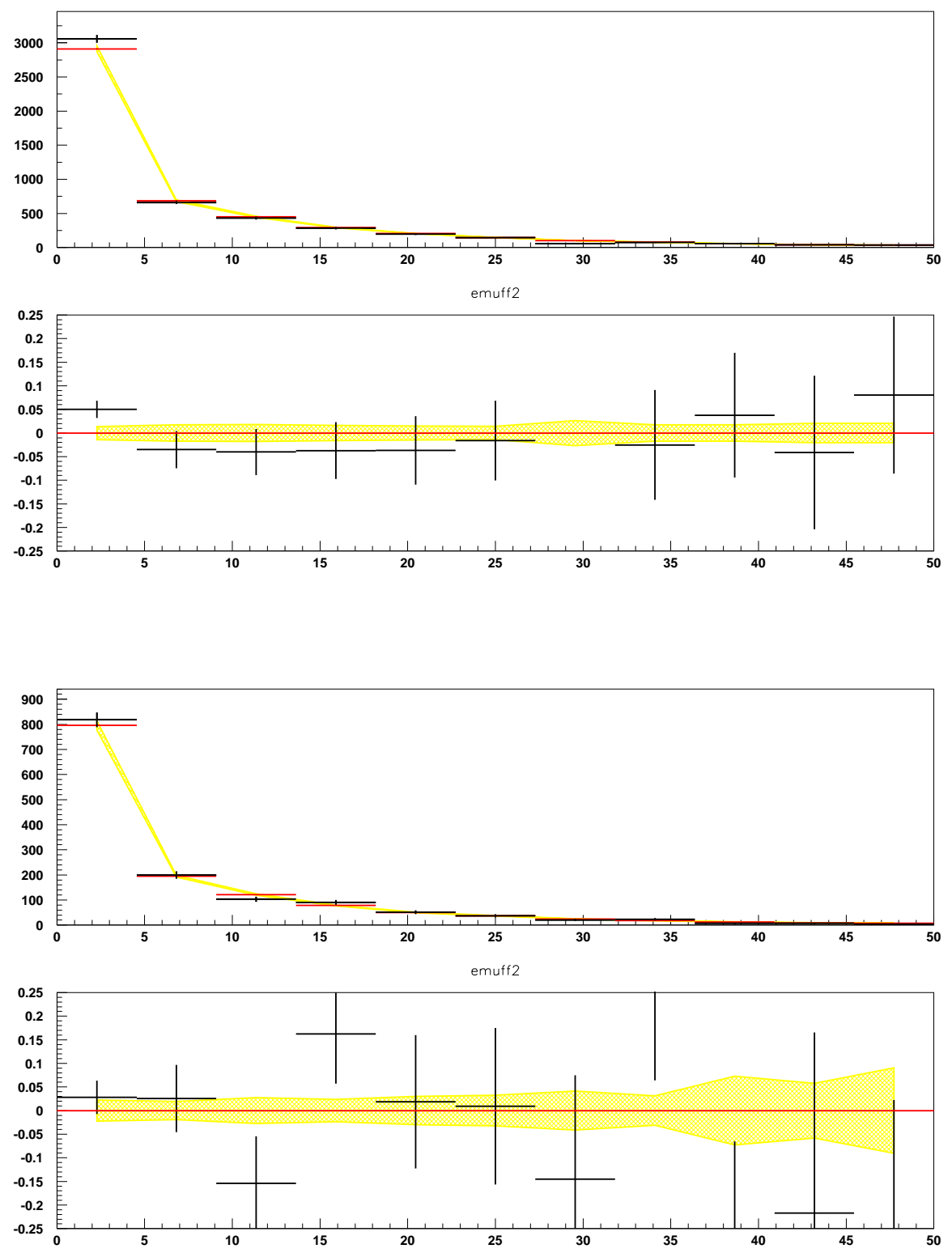

FIGURE 119. Reconstructed energy of the muon from the charm decay immediately after the calorimeter, $E_{\mu F F 2}$, in data vs MC for the LO dimuon sample, for both neutrino (top) and antineutrino (bottom) modes. Plotted are both absolute comparisons, with data in black, and $\mathrm{MC}$ in red, above ratio plots, showing data/MC-1. The shaded regions are the systematic errors, and the $\mathrm{x}$ axis is in $\mathrm{GeV}$. 

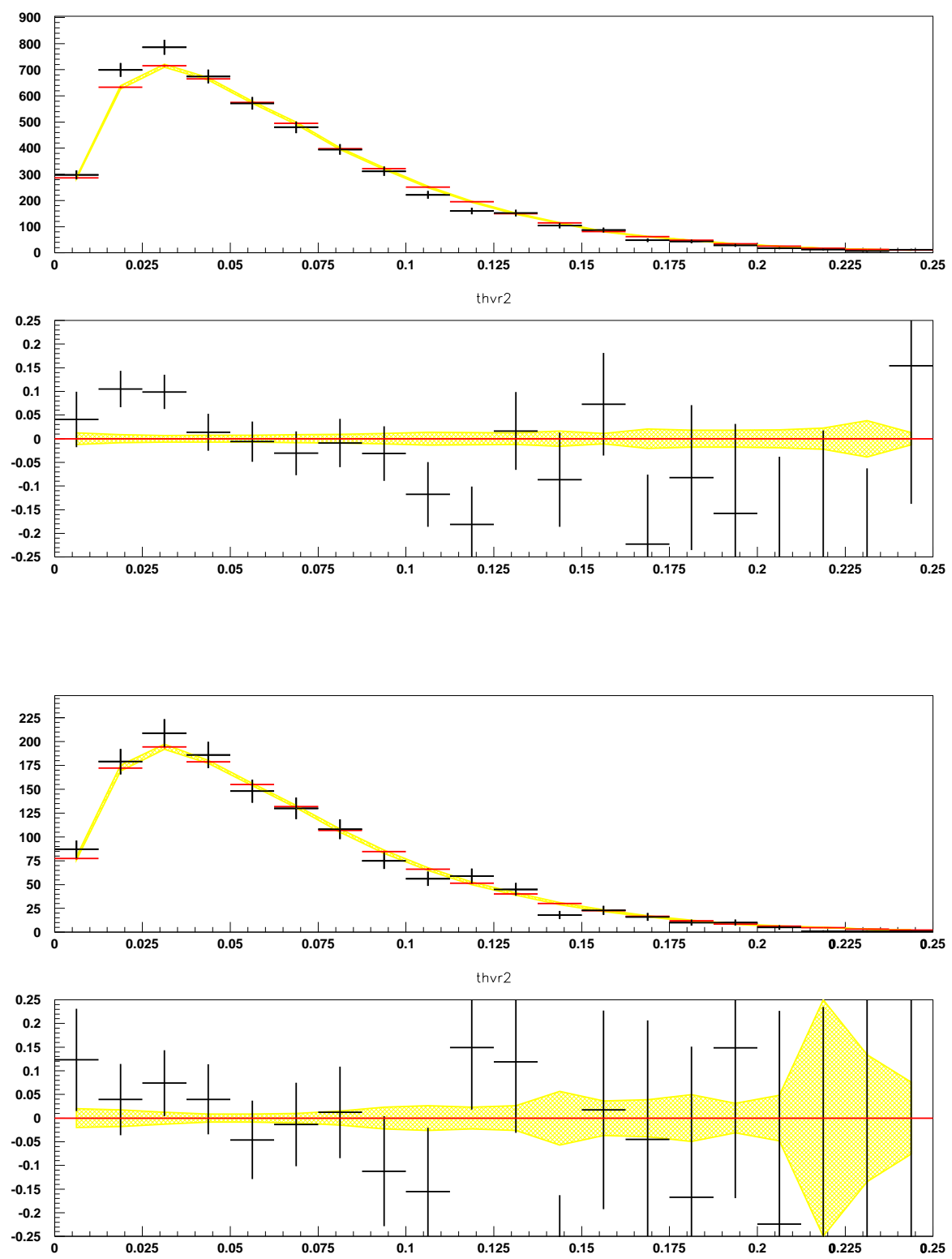

FIGURE 120. Reconstructed opening angle from the charm decay muon, in data vs MC for the LO dimuon sample, $\theta_{\mu 2}$, for both neutrino (top) and antineutrino (bottom) modes. Plotted are both absolute comparisons, with data in black, and $\mathrm{MC}$ in red, above ratio plots, showing data/MC-1. The shaded regions are the systematic errors, and the $\mathrm{x}$ axis is in radians. 

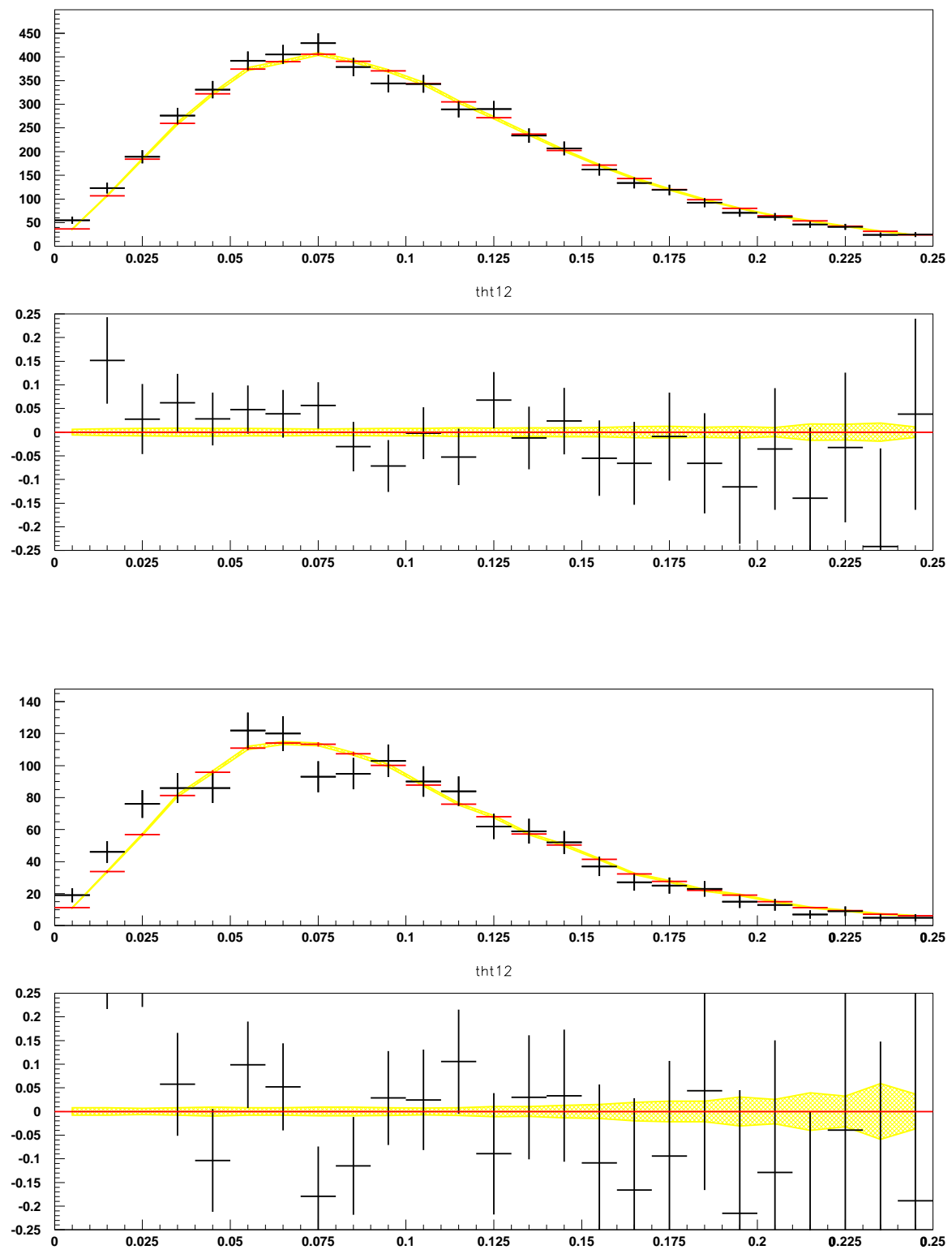

FIGURE 121. Reconstructed muon opening angle between the two muons, $\theta_{\mu 12}$, in data vs MC for the LO dimuon sample, for both neutrino (top) and antineutrino (bottom) modes. Plotted are both absolute comparisons, with data in black, and $\mathrm{MC}$ in red, above ratio plots, showing data/MC-1. The shaded regions are the systematic errors, and the $\mathrm{x}$ axis is in radians. 

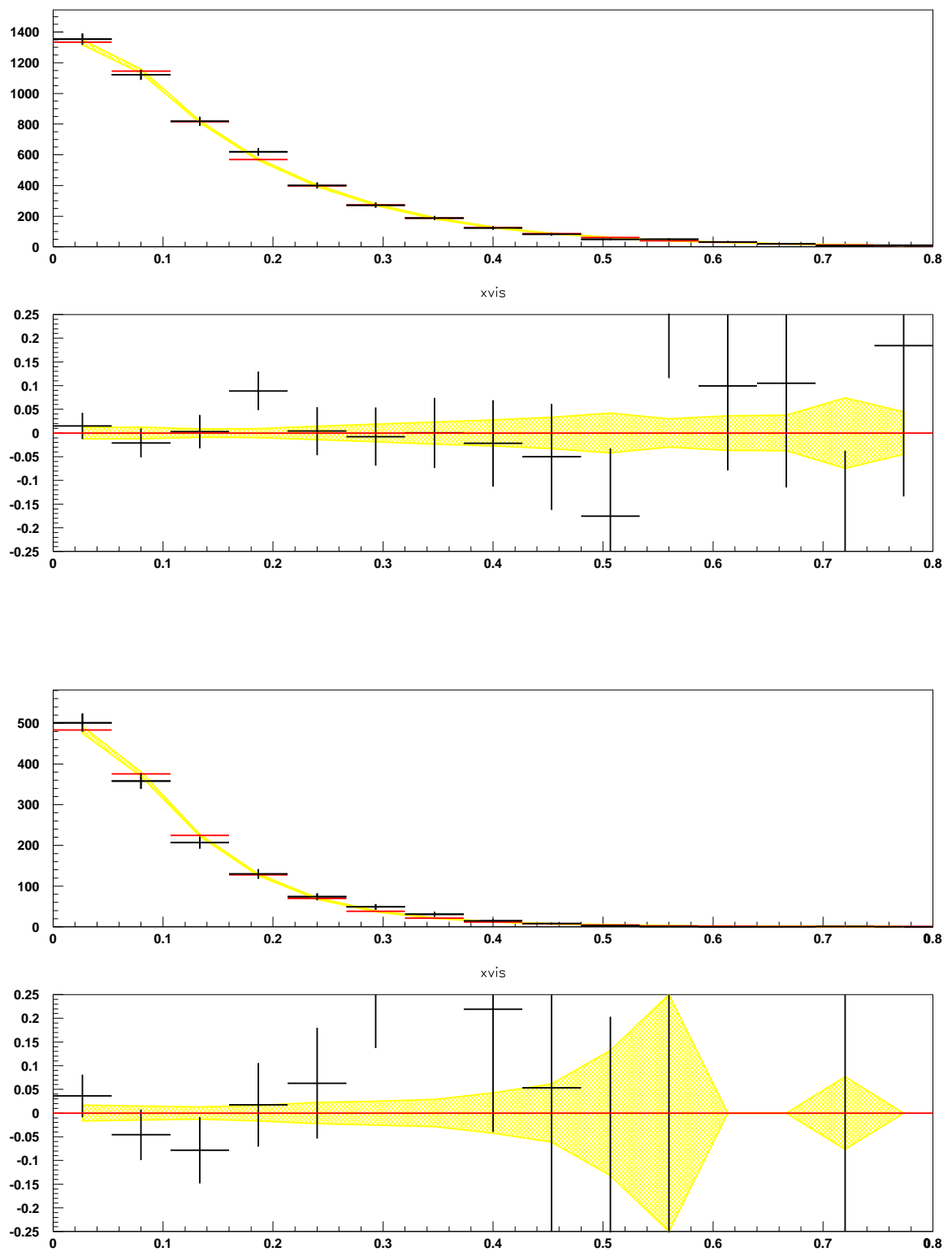

FIGURE 122. Reconstructed Bjorken $x, x_{v i s}$, in data vs MC for the LO dimuon sample, for both neutrino (top) and antineutrino (bottom) modes. Plotted are both absolute comparisons, with data in black, and MC in red, above ratio plots, showing data/MC-1. The shaded regions are the systematic errors. 

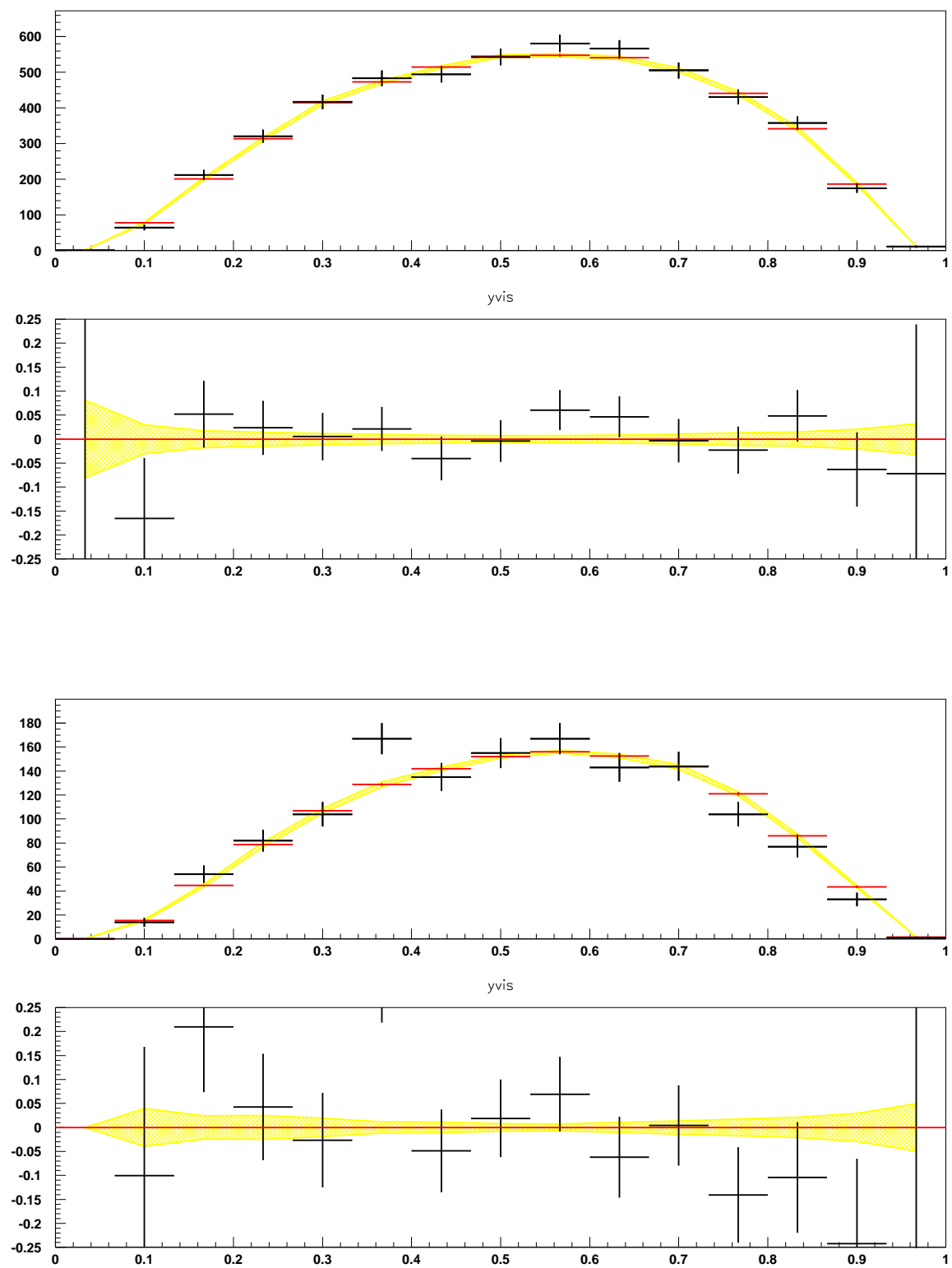

FIGURE 123. Reconstructed Inelasticity, $y, y_{v i s}$, in data vs MC for the LO dimuon sample, for both neutrino (top) and antineutrino (bottom) modes. Plotted are both absolute comparisons, with data in black, and MC in red, above ratio plots, showing data/MC-1. The shaded regions are the systematic errors. 

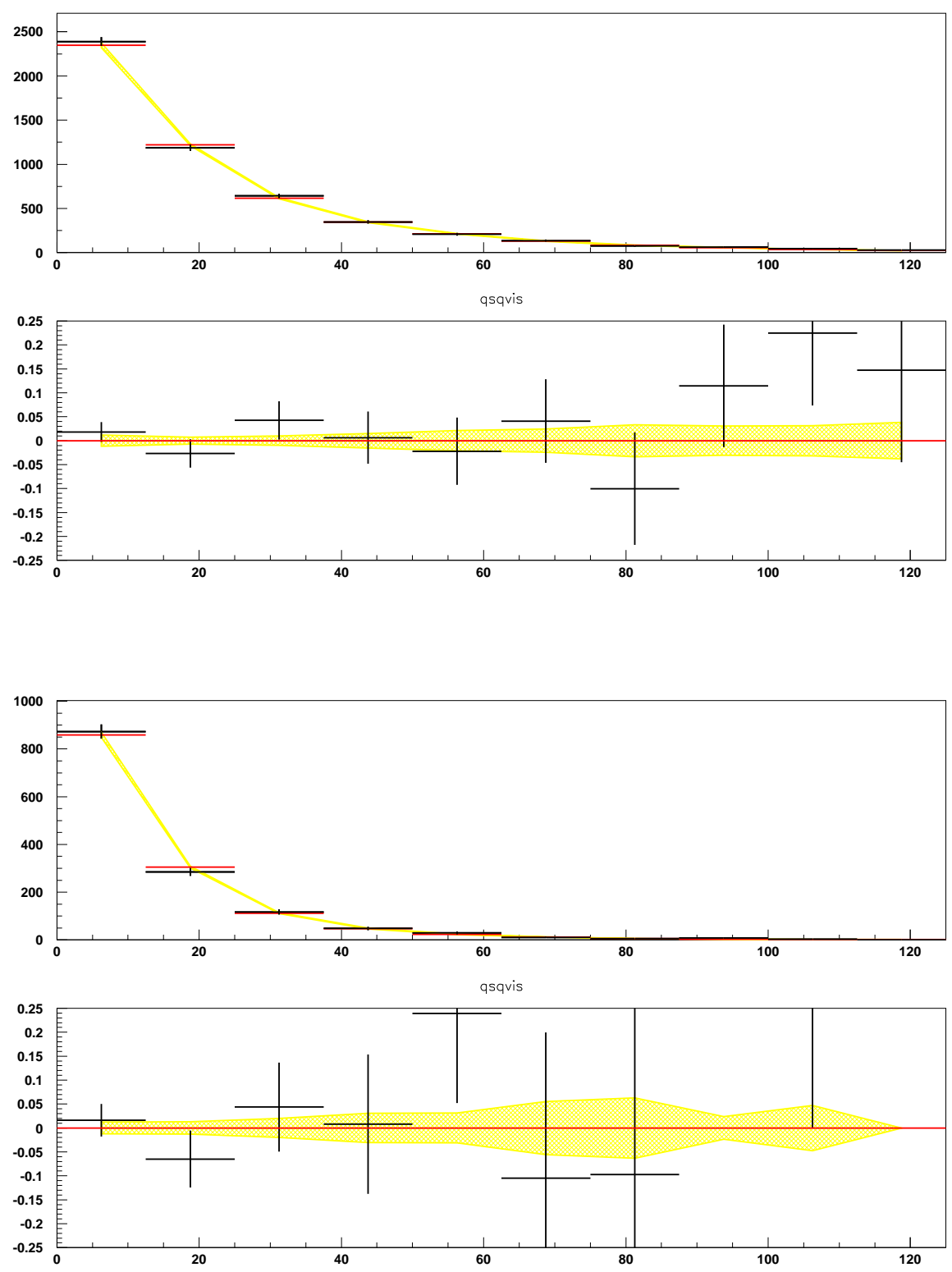

FIGURE 124. Reconstructed $Q^{2}, Q_{v i s}^{2}$,in data vs MC for the LO dimuon sample, for both neutrino (top) and antineutrino (bottom) modes. Plotted are both absolute comparisons, with data in black, and MC in red, above ratio plots, showing data/MC-1. The shaded regions are the systematic errors, and the $\mathrm{x}$ axis is in $\mathrm{GeV}^{2}$. 

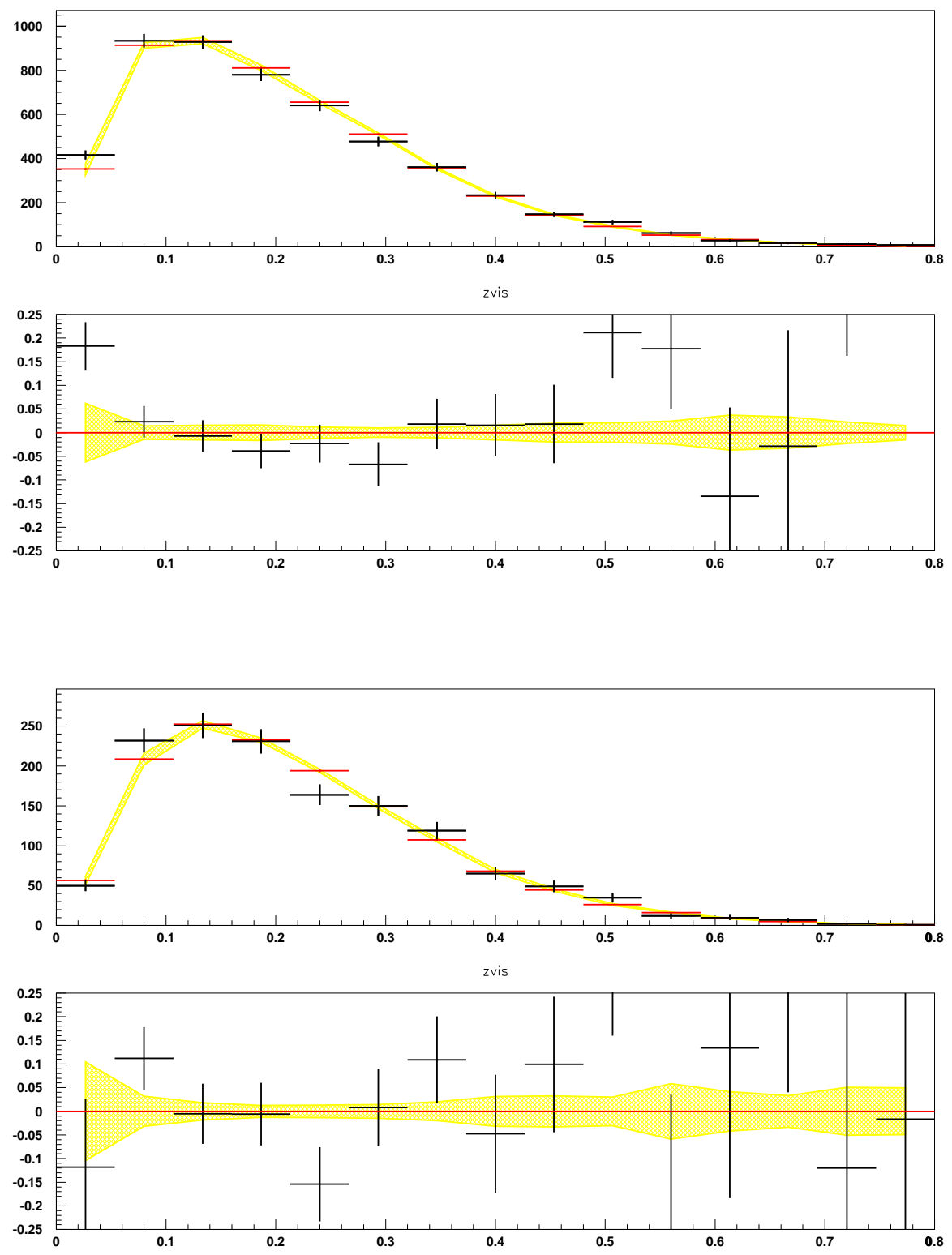

FIGURE 125. Reconstructed $z_{v i s}=\frac{E_{\mu 2}}{E_{\mu 2}+E_{H A D}}$, in data vs MC for the LO dimuon sample, for both neutrino (top) and antineutrino (bottom) modes. Plotted are both absolute comparisons, with data in black, and $\mathrm{MC}$ in red, above ratio plots, showing data/MC-1. The shaded regions are the systematic errors. 

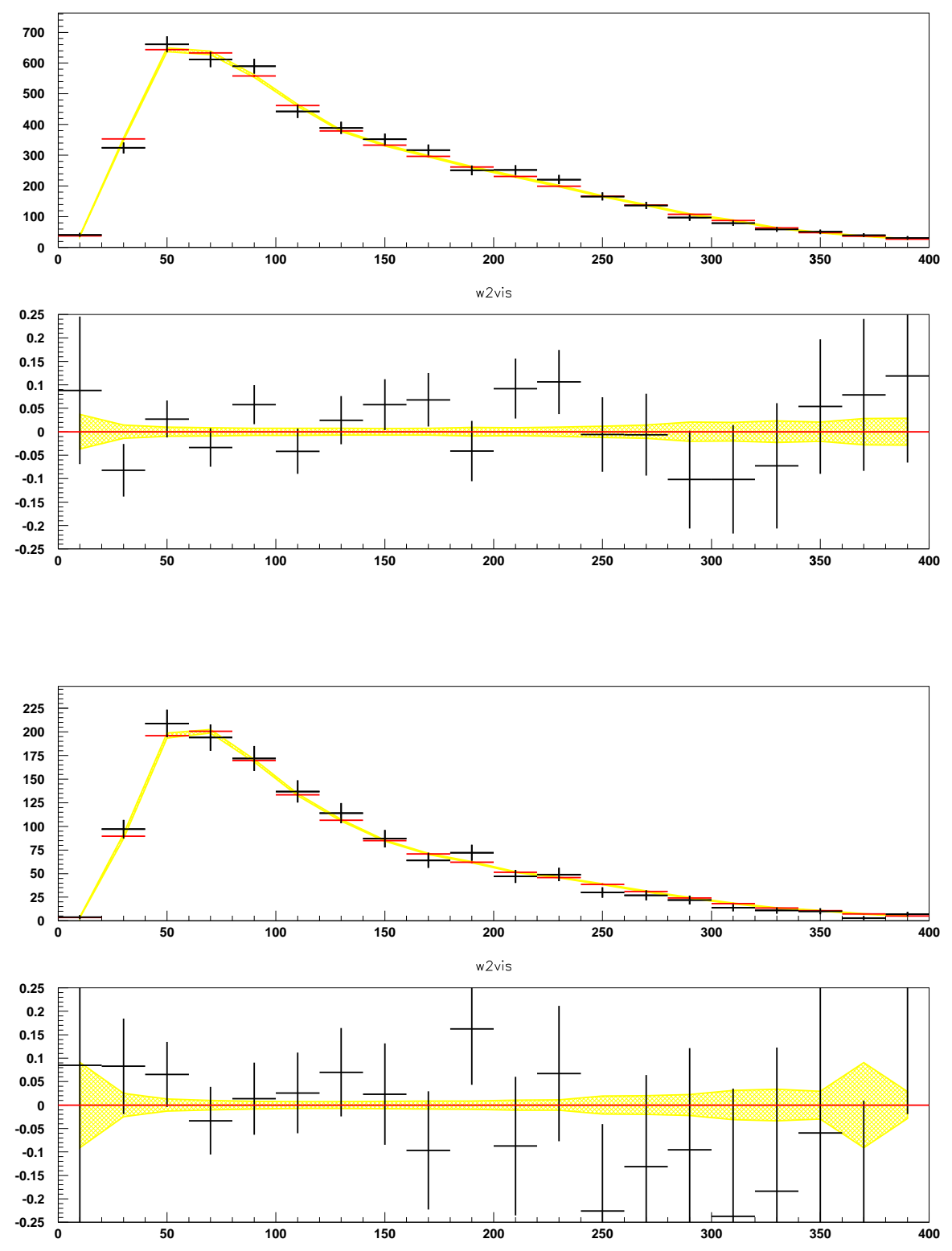

FIGURE 126. Reconstructed $W_{v i s}^{2}$, in data vs MC for the LO dimuon sample, for both neutrino (top) and antineutrino (bottom) modes. Plotted are both absolute comparisons, with data in black, and MC in red, above ratio plots, showing data/MC-1. The shaded regions are the systematic errors, and the $\mathrm{x}$ axis is in $\mathrm{GeV}^{2}$. 

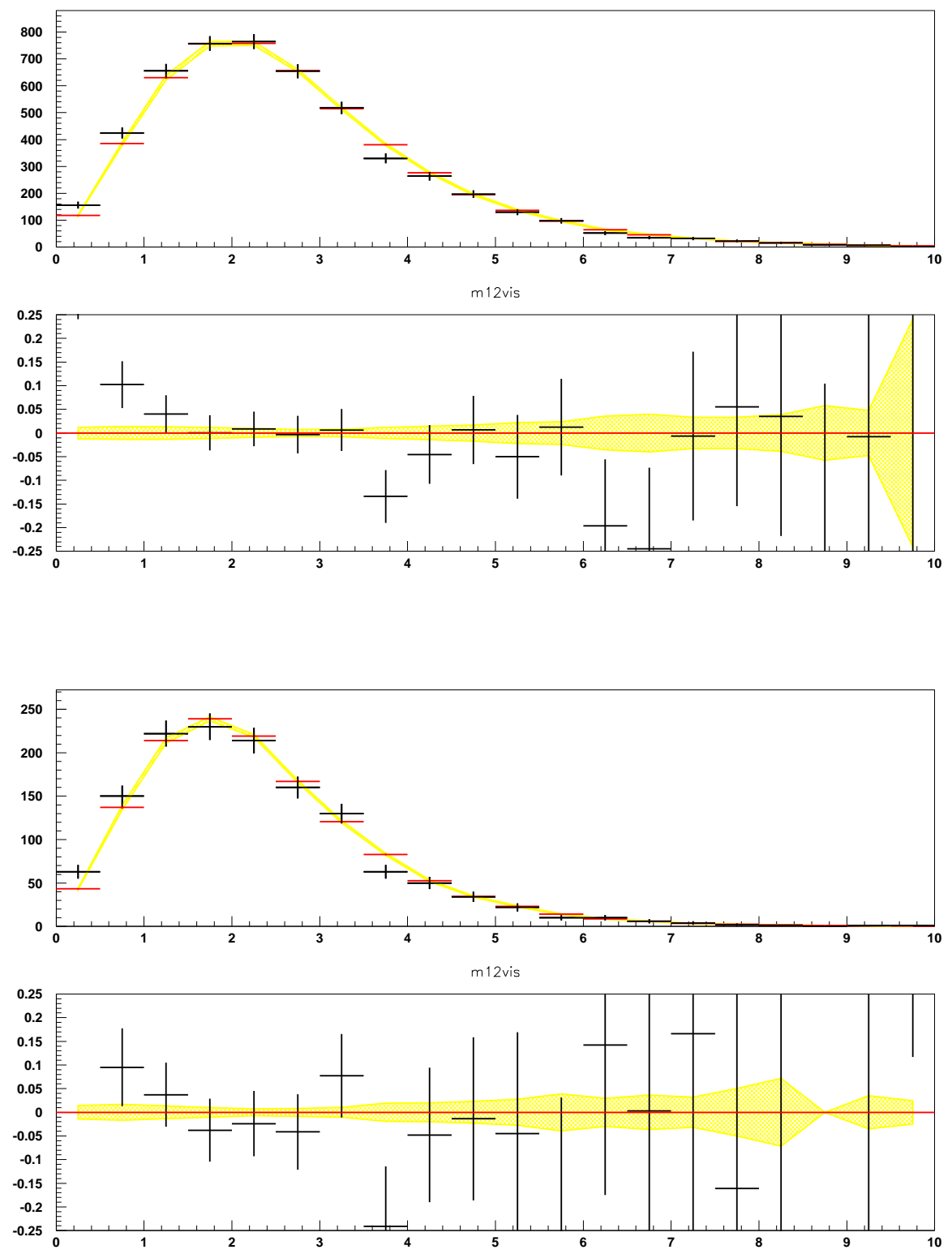

FIGURE 127. Reconstructed invariant mass between the two muons, $m_{12 v i s}$, in data vs MC for the LO dimuon sample, for both neutrino (top) and antineutrino (bottom) modes. Plotted are both absolute comparisons, with data in black, and $\mathrm{MC}$ in red, above ratio plots, showing data/MC-1. The shaded regions are the systematic errors, and the $\mathrm{x}$ axis is in $\mathrm{GeV}$. 


\subsection{Forward Dimuon Cross Section Table}

If the goal of this analysis were to extract parameters within the BGPAR model, we would be able to finish here now with some level of discussion of the physics implications of these results. Before $\mathrm{NuTeV}$, analyses of dimuon events

had indeed stopped here. Doing so however is perhaps not very useful outside of the context of neutrino deep inelastic scattering. For one thing the parameters obtained really only have meaning within this leading order BGPAR model. This means for example, that the strange and antistrange seas extracted here do not account for the substantial gluon component to the nucleon. The charm mass also was extracted with the kinematic assumptions of a leading order model. Anyone who wanted to try to use the BGPAR numbers to make predictions would need to use this BGPAR model, which may give entirely inappropriate results at the energy scale they are working at, or material they are working in.

Ideally this situation could be remedied by publishing the data, and allowing people to fit it with their favorite model themselves. This extreme is also dangerous, as it requires some knowledge of the experimental environment for such fits to be believable. A middle path though is to distill the data into a table of cross section values which have been corrected for experimental effects. This distillation must be done in such a was as to be independent of the model used to extract the cross section. The advantage of doing this is the data is presented in a way that makes it possible to fit a computationally expensive model to the extracted cross section, and detailed knowledge of the detector environment is not required. The disadvantage of doing this is that some of the flexibility afforded by fitting the raw data is lost in the process. 
What follows here is an extraction of the forward cross sections of neutrino and antineutrino induced dimuons, originally done in references [75, 148]. The prior analysis has been updated here, providing a model independent cross section upon which to base the NLO model analysis discussed in the next chapter.

Fundamental to extracting a model independent cross section from the $\mathrm{NuTeV}$ data is the understanding that neutrino experiments like $\mathrm{NuTeV}$ do not directly measure charm production. What is actually measured is the production of dimuon events, which result from the unobservable production of a charmed particle. The characteristics of charm production may be studied, but only through the window provided by a particular model of how the charmed quarks hadronize and decay semileptonically to make the observed dimuon events. To break up the [physics] $\otimes$ [experiment] expression in equation 4.165, it might look like:

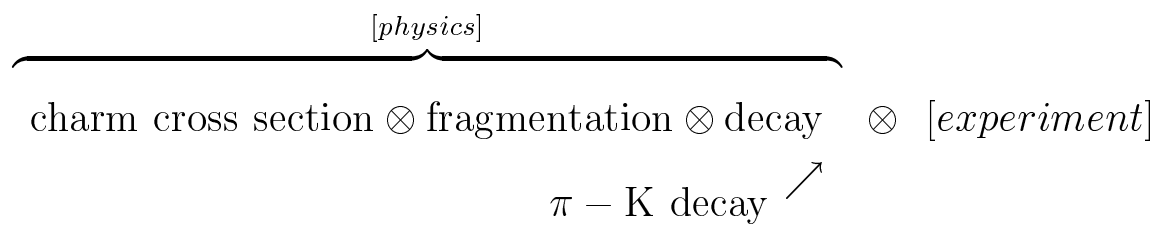

Where the background contribution due to $\pi-K$ decay has been inserted.

Using the LO parameters extracted in the previous section requires following the assumptions in the "fragmentation" and "decay" pieces in expression 4.257. Of these how the the fragmentation piece is applied is dependent on the order in $\alpha_{s}$ at which the charm cross section calculation is performed. So as not to subject potential users of the measured cross section to a particular set of model choices, and (more importantly) bias the NLO analysis which follows, the cross section of neutrino dimuon production, rather than the charm cross section is extracted. 
That is, 4.257 becomes:

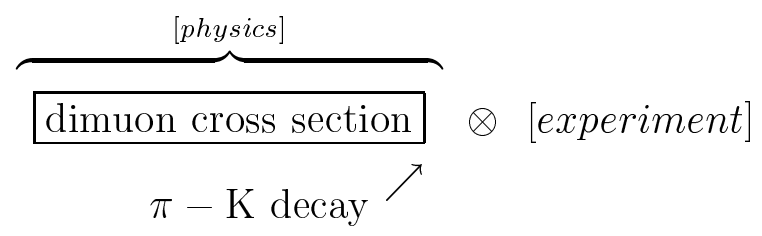

Here the model for producing dimuons is effectively assumed to be a black box. In doing this the only corrections necessary to apply are those due to detector effects ([experiment $]$ ) and the background from $\pi-K$ semileptonic decays. Both of these pieces are understood through external means: [experiment], or the smearing due to the detector environment, is known through extensive study of the $\mathrm{NuTeV}$ detector with its calibration beam. The kinematics of the particles from the calibration beam were measured independent from the detector, which allowed direct measurement of the biases and smearing in detector measurements. In other words, the calibration beam data provided a direct measurement of [experiment]. The $\pi-K$ decay model, outlined earlier in this chapter, is based on an external empirical measurement from semi-muonic decays of CCFR test beam hadrons and predictions from the LUND model.

In measuring the dimuon cross section, any model dependence then only occurs in those effects which cross the " $\otimes$ " boundary, by means of, for example, things like kinematic cuts. Assuming the dimuon cross section model is reasonable, any model dependence that leaks into the final measurement should then be a second order effect, happening whenever it affects whether an event passes or fails a cut. The model is ensured to be reasonable by requiring that a good fit to data be achieved. If the [experiment] piece is well understood through calibration beam studies, as well as the contribution due to $\pi-K$ decay, then a good fit 
to data guarantees the cross section model be reasonable, whether it be based on BGPAR, NLO QCD. The effect of cuts can also be limited by only using data where acceptance is large, so that the effect of cuts are relatively small (multiplying a small model difference by a small effect from cuts). An energy threshold on the muon from charm decay is therefore applied. A $5 \mathrm{GeV}$ cut is both large enough so that the muon track extends sufficiently beyond the hadron shower, while being small enough not to eliminate all the data. With the application of this cut, the cross section measurement becomes a "forward dimuon cross section". - the cross section of charm induced dimuon events such that the muon from charm decay has energy greater than $5 \mathrm{GeV}$.

Measuring the forward cross section and unfolding the experimental effects and $\pi-K$ background from it involves defining sets of bins in both "generated space", i.e. the unsmeared variables in the [physics] piece of 4.258, and in "visible space", the smeared version of those variables after the convolution with [experiment]. Bins in $x, y$, and $E_{\nu}$ in both visible and generated space are defined such that projections in each of the 3 axes contain an equal number of events. The cross section is computed using the BGPAR fit parameters at the weighted center of each of these bins in generated space. An unfolding correction is then applied which both cancels out to first order the model dependencies in the computed cross section, and conveys the statistical fluctuations of the data to the cross section tables. This correction is found by using the mapping between generated and visible space to sum up the visible space bins which have the greatest contributions to each generated bin. 
The dimuon cross section is evaluated in generated space such that:

$$
\begin{aligned}
{\left[\frac{d \sigma\left(\nu_{\mu} N \rightarrow \mu^{-} \mu^{+} X\right)}{d x d y}\right]_{(x, y, E)_{i}}=} & \frac{D_{i}}{N_{i, L O ~ f i t}^{\prime}} \int_{E_{\mu 2}>5 \mathrm{GeV}} d z d \Omega \times \\
& {\left[\frac{d \sigma\left(\nu_{\mu} N \rightarrow \mu^{-} \mu^{+} X\right)}{d x d y d z}\right]_{(x, y, E)_{i}-\text { LO fit }} }
\end{aligned}
$$

here the extracted cross section for bin $(x, y, E)_{i}$ is shown on the left, and the integral $\int_{E_{\mu 2}>5 \mathrm{GeV}} d z d \Omega$ represents the Monte Carlo integration over fragmentation $(z)$, and the semileptonic decay $(\Omega)$ with the condition that the decay muon has energy (in generated space) greater than $5 \mathrm{GeV}$. The $\frac{D_{i}}{N_{i, L O}^{\prime} \text { fit }}$ term ahead of the integral is the unfolding correction for that bin. The following describes how the unfolding term is calculated:

For neutrino and antineutrino data, the cross section table is defined in $3 E_{\nu}$, $3 y$, and $5 x$ bins in generated space. The data and MC are also divided into 11 $E_{\nu}, 9 y$, and $9 x$ bins. A mapping matrix is generated with the MC:

$$
\mathcal{M}_{i j}=\frac{\mathcal{N}_{i j}}{\mathcal{N}_{j}}
$$

where $\mathcal{N}_{j}$ is the sum of the weights of MC events in visible space bin $\left(x_{v i s}, y_{v i s}, E_{v i s}\right)_{j}$, and $\mathcal{N}_{i j}$ is the sum of the weights of events contained in $\left(x_{v i s}, y_{v i s}, E_{v i s}\right)_{j}$ and generated space bin $(x, y, E)_{i}$. In each bin the contribution due to the $\pi-K$ background in each bin is subtracted out. The data half of the unfolding correction $D_{i}$, or the number of data events corresponding to the generated bin $i$, is:

$$
D_{i}=\sum_{j \in \mathcal{C}} \mathcal{M}_{i j-\text { ordered }} \cdot D_{j}
$$


Where the sum has been limited to be only over the visible bins up to a certain coverage fraction $\mathcal{C}$. The sum is ordered from the largest elements in $\mathcal{M}_{i j}$ to the smallest until the desired coverage fraction is reached. Limiting the sum by coverage reduces the effects of smearing from fluctuations in the detector and the undetected charm decay neutrino. The coverage fraction as:

$$
\mathcal{C}=\sum_{j}^{\mathcal{N}(\mathcal{C})} \frac{\mathcal{N}_{i j}}{\mathcal{N}_{i}}
$$

If $\mathcal{C}$ reaches 1 , all visible bins are included in the sum. The denominator of the unfolding correction is also the result of a sum up to a chosen $\mathcal{C}$ :

$$
\mathcal{N}_{i}^{\prime}=\sum_{j \in \mathcal{C}} \mathcal{M}_{i j-\text { ordered }} \cdot \mathcal{N}_{j}
$$

Where like $D_{i}$ the sum is up to the same coverage fraction $\mathcal{C}$. $\mathcal{C}$ should be small enough to minimize correlations between the extracted table bins, but large enough so that there are sufficient statistics to extract the cross section.

The error on each cross section point is proportional to the number of events mapped into that bin:

$$
\delta_{i}=\frac{1}{\sqrt{\mathcal{N}_{i}}}\left[\frac{d \sigma\left(\nu_{\mu} N \rightarrow \mu^{-} \mu^{+} X\right)}{d x d y}\right]_{i}
$$

This is not the complete description of the error however. The bins in the extracted table are correlated depending on the level of coverage used in the unfolding correction. Although perfectly calculable, the covariance matrix which describes the inter-bin correlations would be difficult to work with (a $90 \times 90$ element matrix). 
To ease use of the extracted table, the effective number of degrees of freedom are calculated for each bin as a measure of its significance:

$$
D O F_{i}=\frac{\sum_{j \in \mathcal{C}} \mathcal{M}_{i j-\text { ordered }} \cdot \mathcal{N}_{j}}{\sum_{j \in \mathcal{C}} \mathcal{N}_{j}}
$$

As the coverage fraction $\mathcal{C}$ increases, $D O F_{i}$ decreases, approaching zero as $\mathcal{C} \rightarrow 1$. When performing a $\chi^{2}$ fit to the cross section, one treats the bins as statistically independent. In this case, a good fit is obtained when the $\chi^{2}$ is near $\sum_{i} D O F_{i}$ minus the number of parameters in the fit. This is effectively the same as rescaling the errors by the inter-bin correlations.

The dimuon cross section was extracted using the BGPAR fit performed in the previous section. The table values are included in appendix IV. In the following pages, plots 128 and 129 show the cross section table points for each $E_{\nu}$ and $y$ bin plotted as a function of $x$. Also shown are the effective degrees of freedom for each point in figures 130 and 131, the unfolding correction for each bin in figures 132 and 133, and the acceptance due to the $E_{\mu 2}>5 \mathrm{GeV}$ cut in figures 134, and 135 . 


\section{forward cross section vs $\mathrm{x}$}
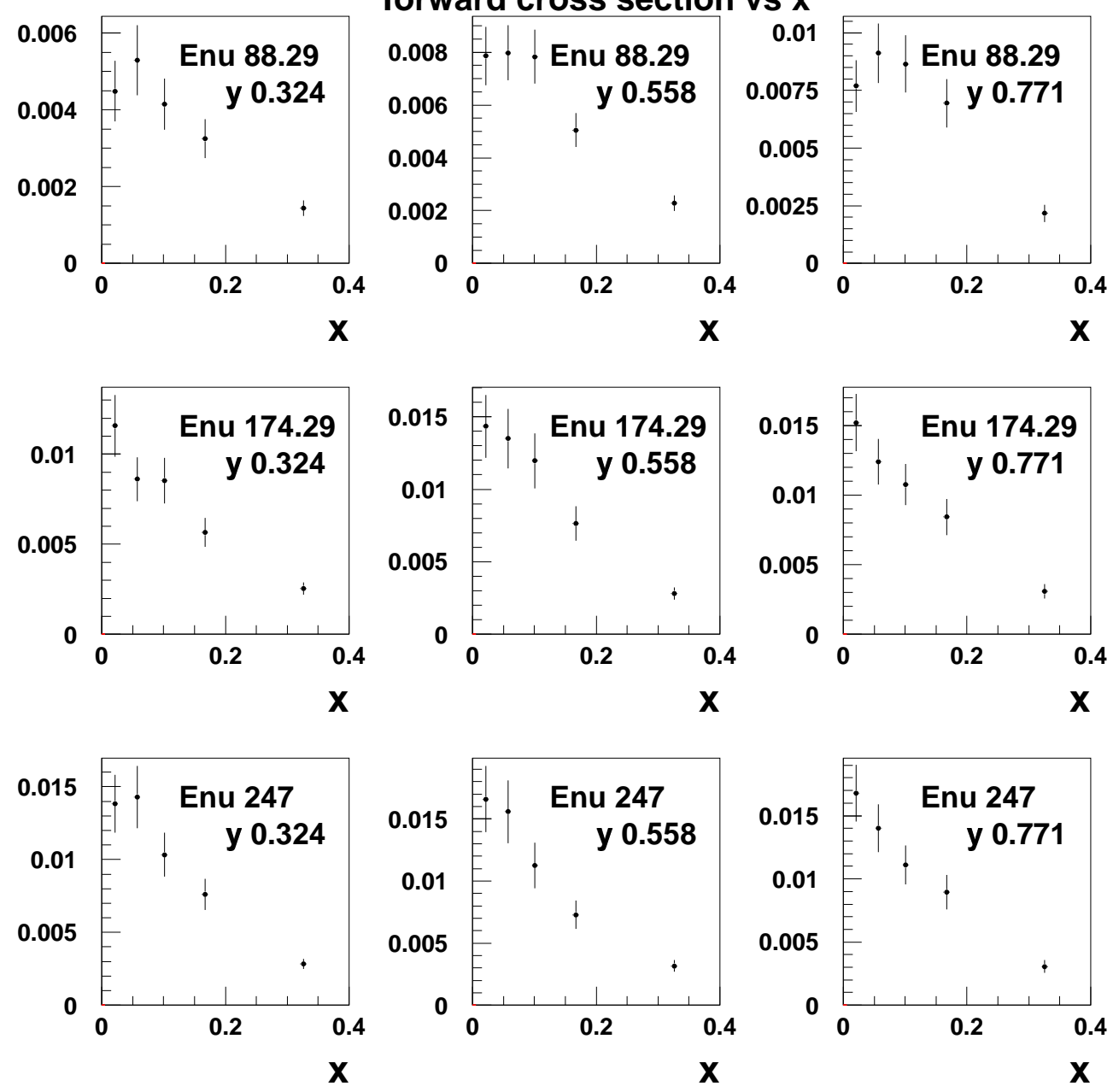

FIGURE 128. Extracted $\nu$ mode dimuon cross section table plotted vs $\mathrm{x}$ for each $E_{\nu}$ and $y$ bin. Points are shown multiplied by $\frac{\pi}{G_{F}^{2} M E_{\nu}}$. 

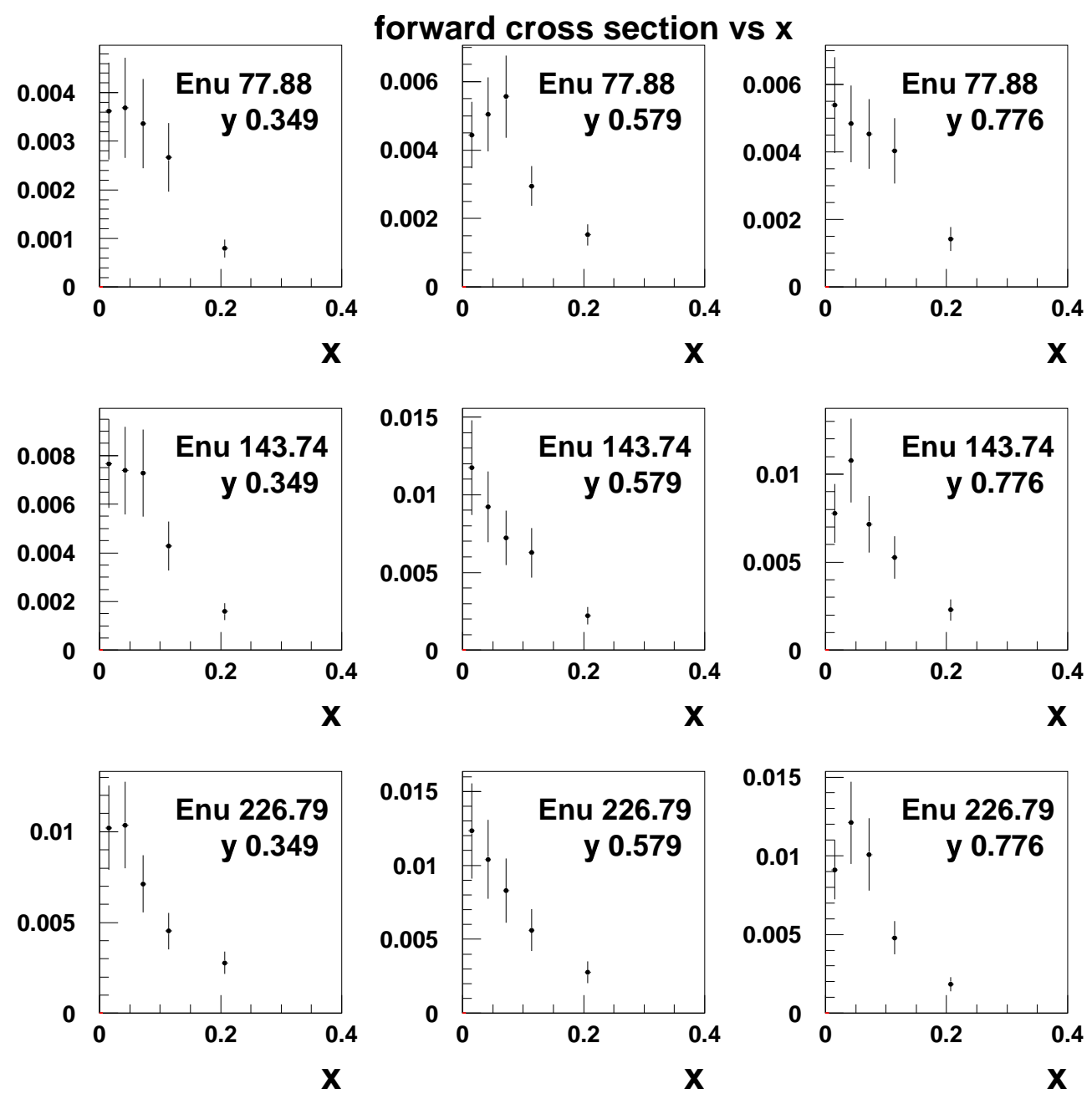

FIGURE 129. Extracted $\bar{\nu}$ mode dimuon cross section table plotted vs $\mathrm{x}$ for each $E_{\nu}$ and $y$ bin. Points are shown multiplied by $\frac{\pi}{G_{F}^{2} M E_{\nu}}$. 

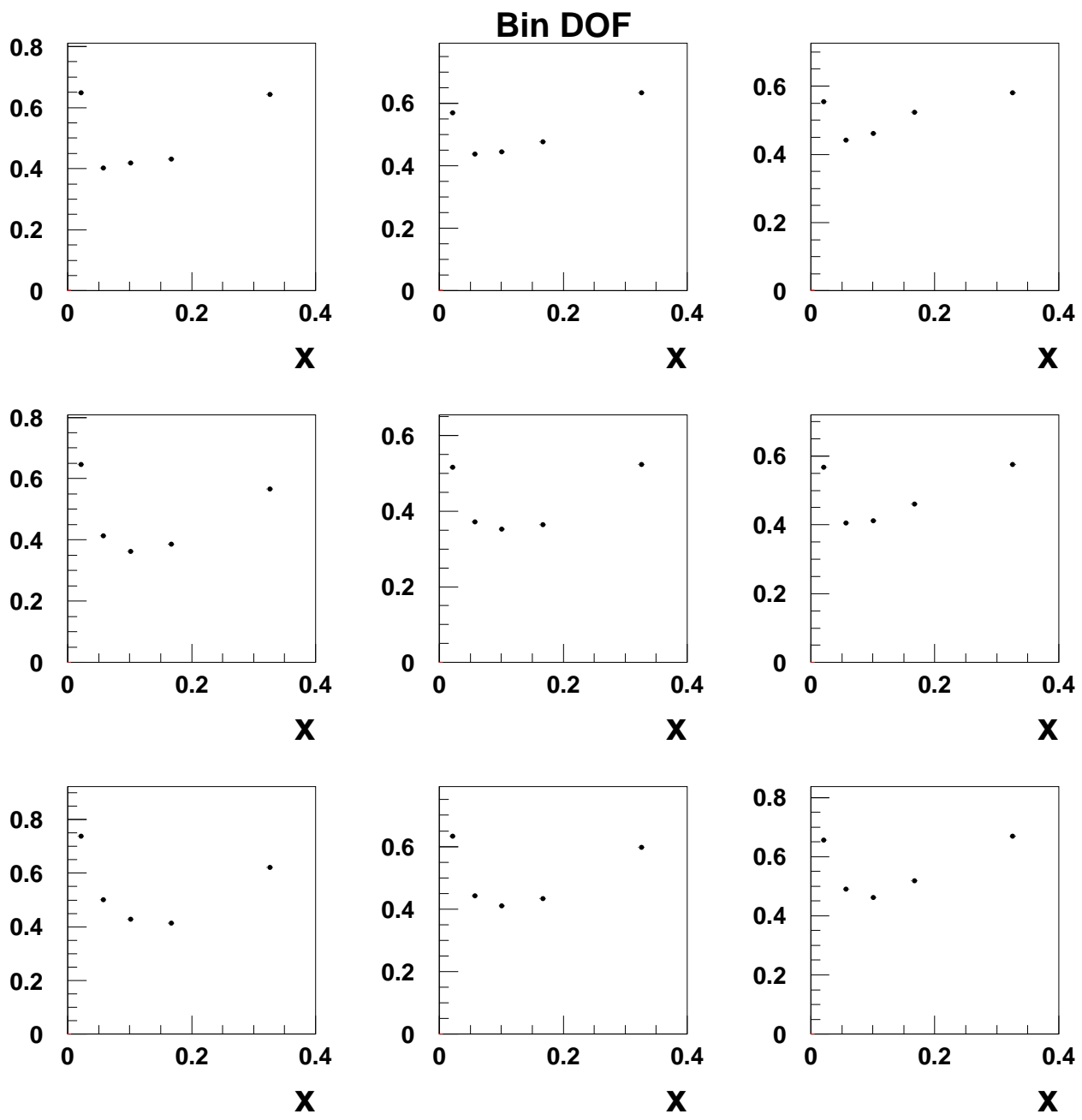

FIGURE 130. Effective degrees of freedom for $\nu$ table points plotted vs $\mathrm{x}$ for the same $E_{\nu}$ and $y$ bins shown in figure 128 

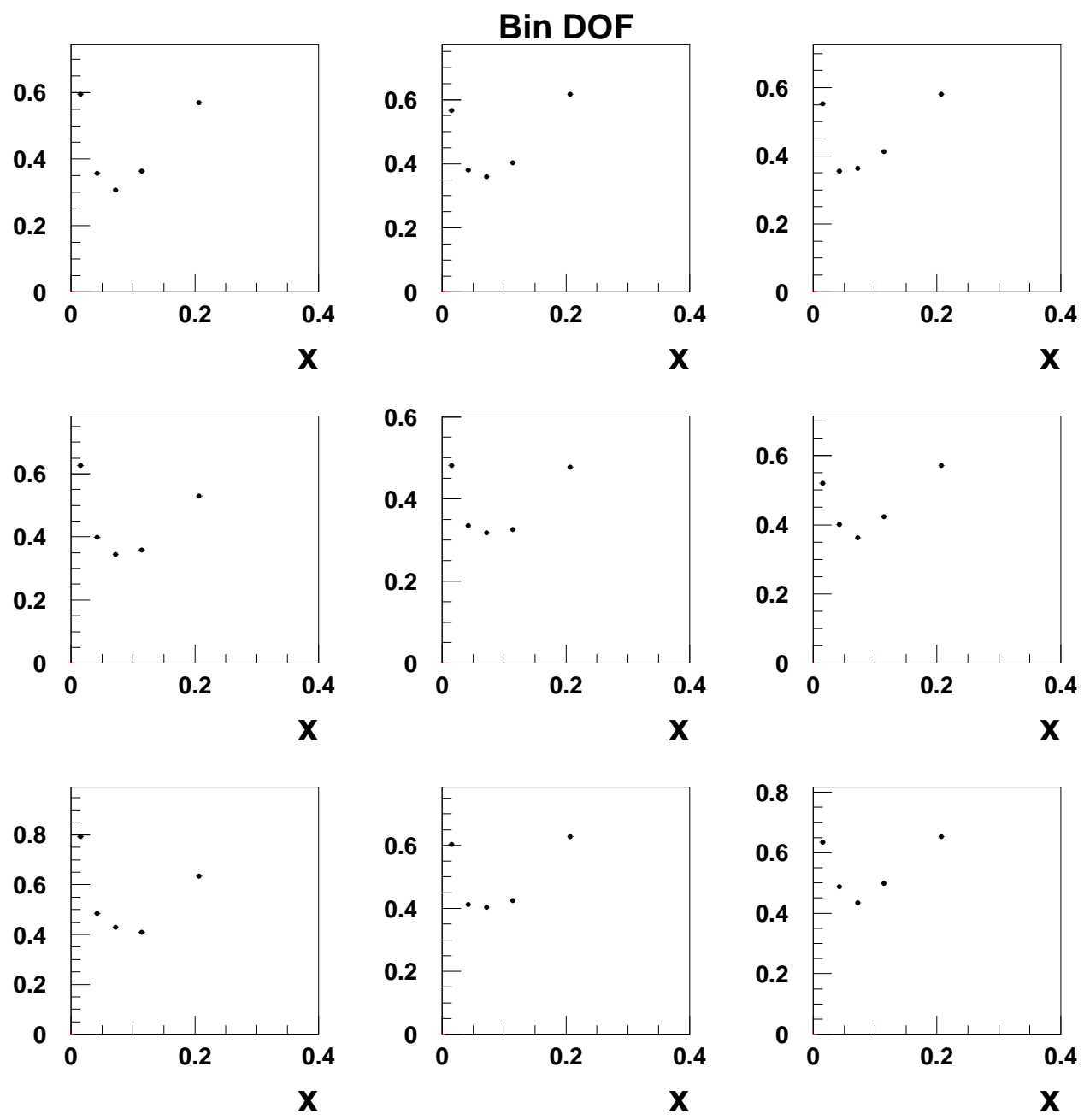

FIGURE 131. Effective degrees of freedom for $\bar{\nu}$ table points plotted vs $\mathrm{x}$ for the same $E_{\nu}$ and $y$ bins shown in figure 129 

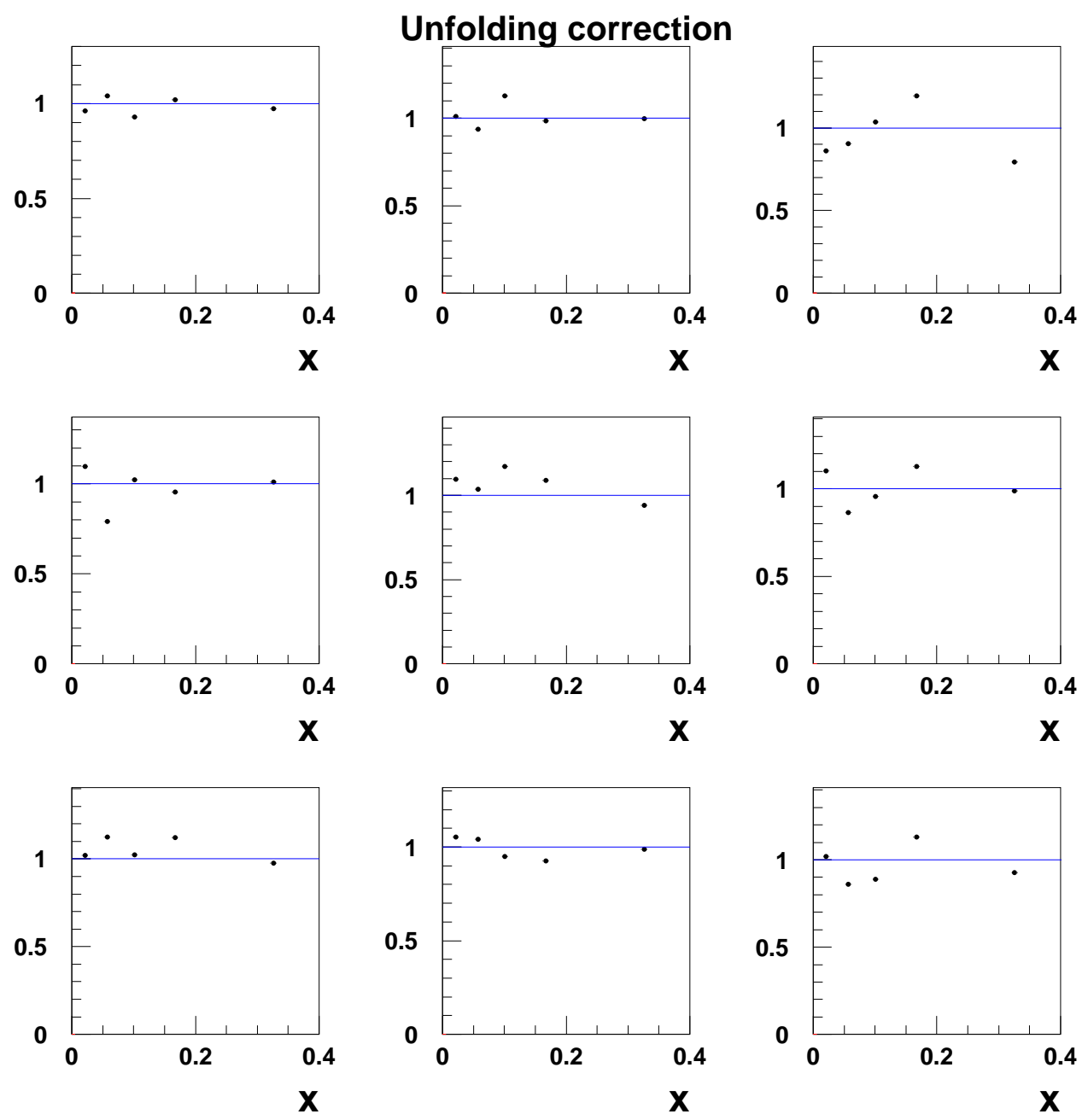

FIGURE 132. The unfolding correction for $\nu$ table points plotted vs $\mathrm{x}$ for the same $E_{\nu}$ and $y$ bins shown in figure 128 

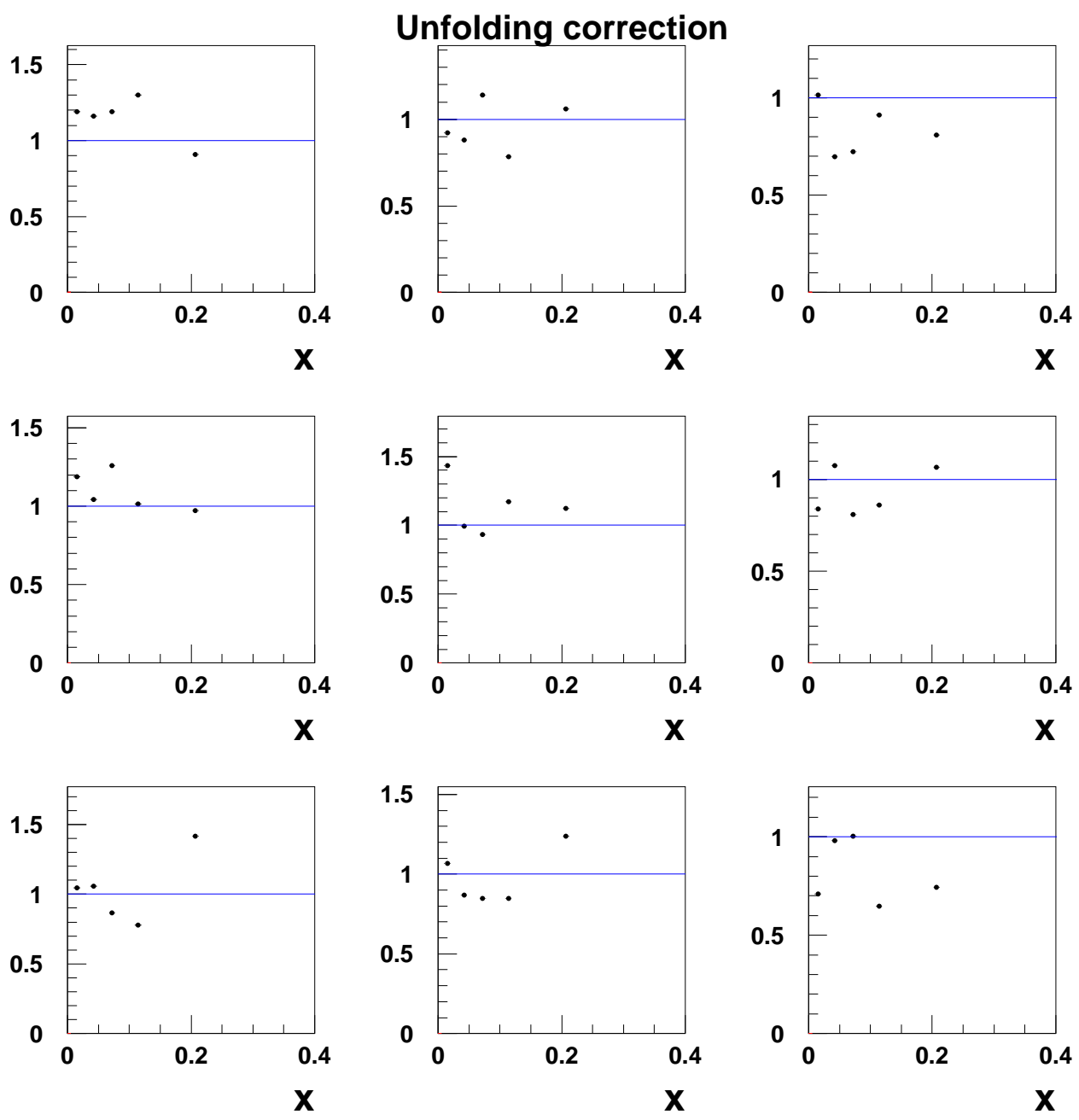

FIGURE 133. The unfolding correction for $\bar{\nu}$ table points plotted vs $\mathrm{x}$ for the same $E_{\nu}$ and $y$ bins shown in figure 129 

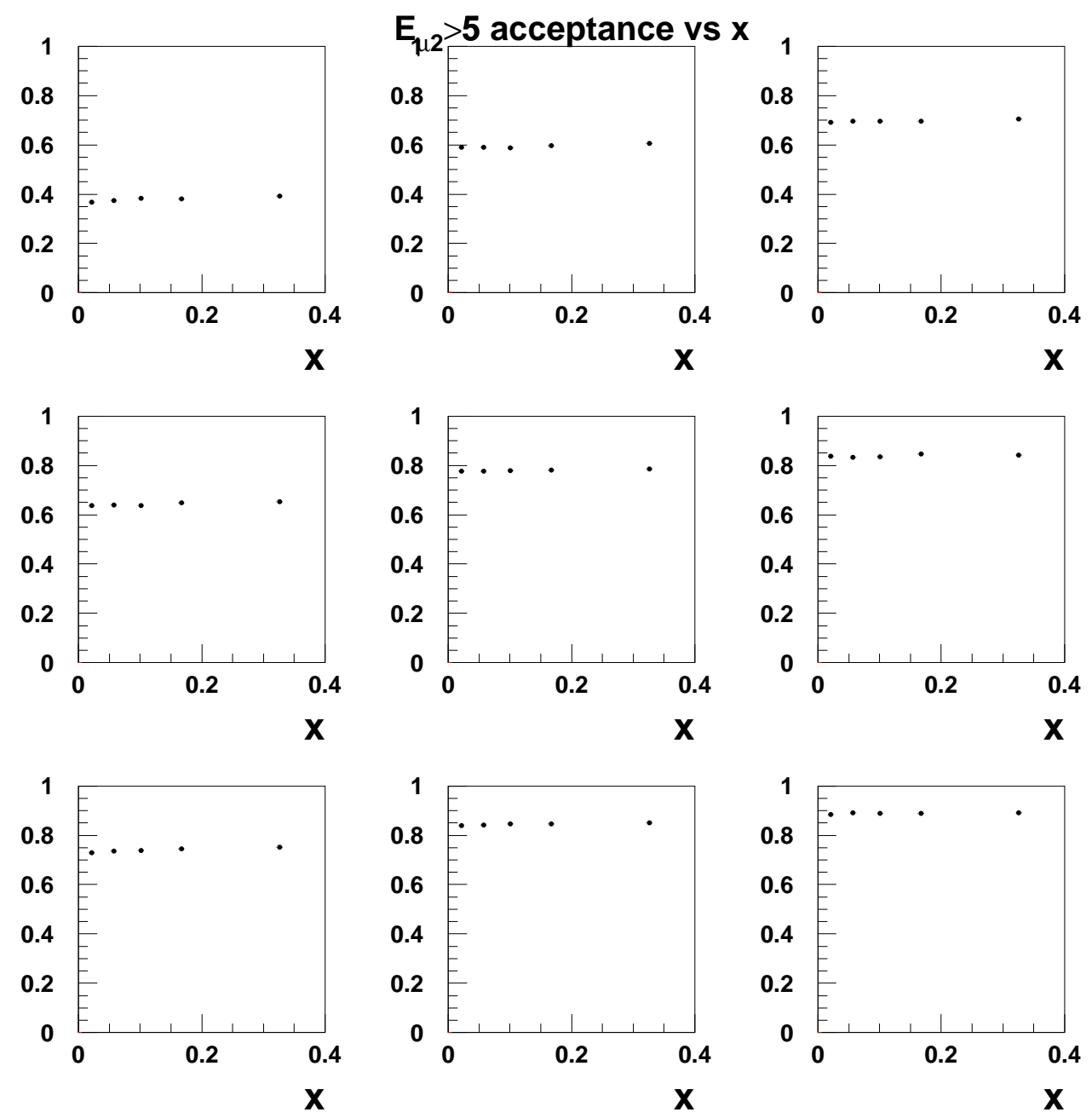

FIGURE 134. The acceptance due to the $E_{\mu 2}>5 \mathrm{GeV}$ cut for $\nu$ table points plotted vs $\mathrm{x}$ for the same $E_{\nu}$ and $y$ bins shown in figure 128 

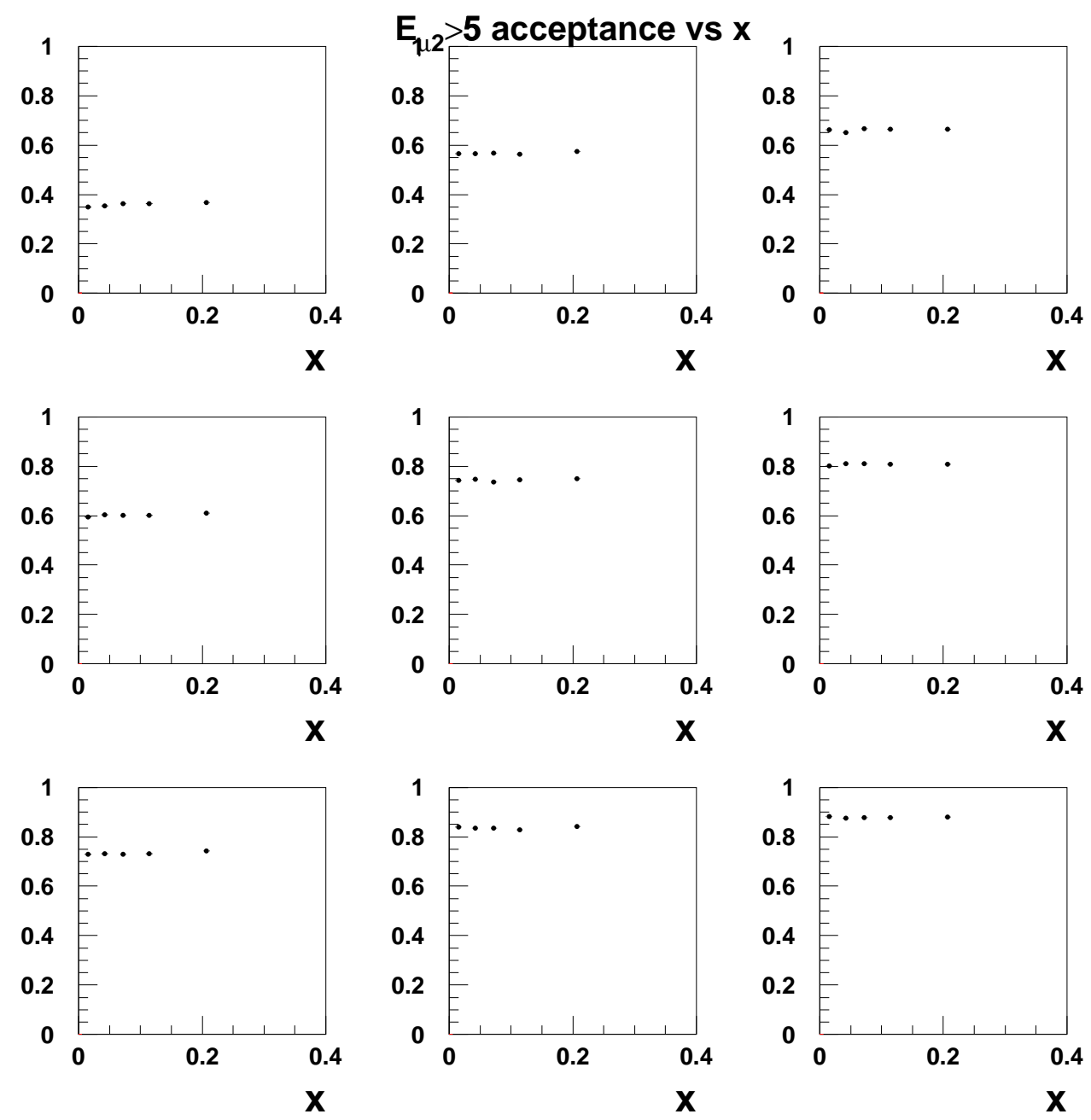

FIGURE 135. The acceptance due to the $E_{\mu 2}>5 \mathrm{GeV}$ for $\bar{\nu}$ table points plotted vs $\mathrm{x}$ for the same $E_{\nu}$ and $y$ bins shown in figure 129 


\subsubsection{Checks on the fitter and cross section}

Several checks can be performed on the fitter and cross section table to test for biases. To look for biases in the fit code itself, a series of "fake data" samples using the $\mathrm{MC}$ can be generated, , then fits performed against them, treating them as data. The resulting fit variables are compared with the ones which were used to generate the fake data to see if there is any bias in the fit procedure.

Figures 136-142 show the results of fits to 50 fake data sets generated from MC based on the BGPAR fit above. The data sets were generated by treating the MC as a parent distribution from which fake data events were selected. The same number of events as in the data were randomly pulled out of the parent MC sample from both neutrino and antineutrino modes, by selecting events whose weights passed a random throw. The usual 7 parameter fits were performed on each fake data set, with the values and statistical errors for the indicated parameters shown in each figure. The shaded band shows the central value and error of each parameter used to generate the random fake data sets, and the solid point to the right of each plot shows the average value of the 50 fits, with the error bar being from the standard deviation of those fits. The plots show there are no significant biases in the fitter itself. The RMS in the spread of the re-fit also points matches well with the statistical error on the original fit.

The table extraction procedure can be tested by extracting tables from each of these fake data sets. After fitting each data set, the dimuon cross section tables are extracted for a range of coverage fractions $\mathcal{C} . \chi^{2}$ 's from these fits are compared to the calculated DOF appropriate for that coverage. Figure 143 shows the $\chi^{2}$ values for the fits plotted on top of the curve for the total estimated DOF for 


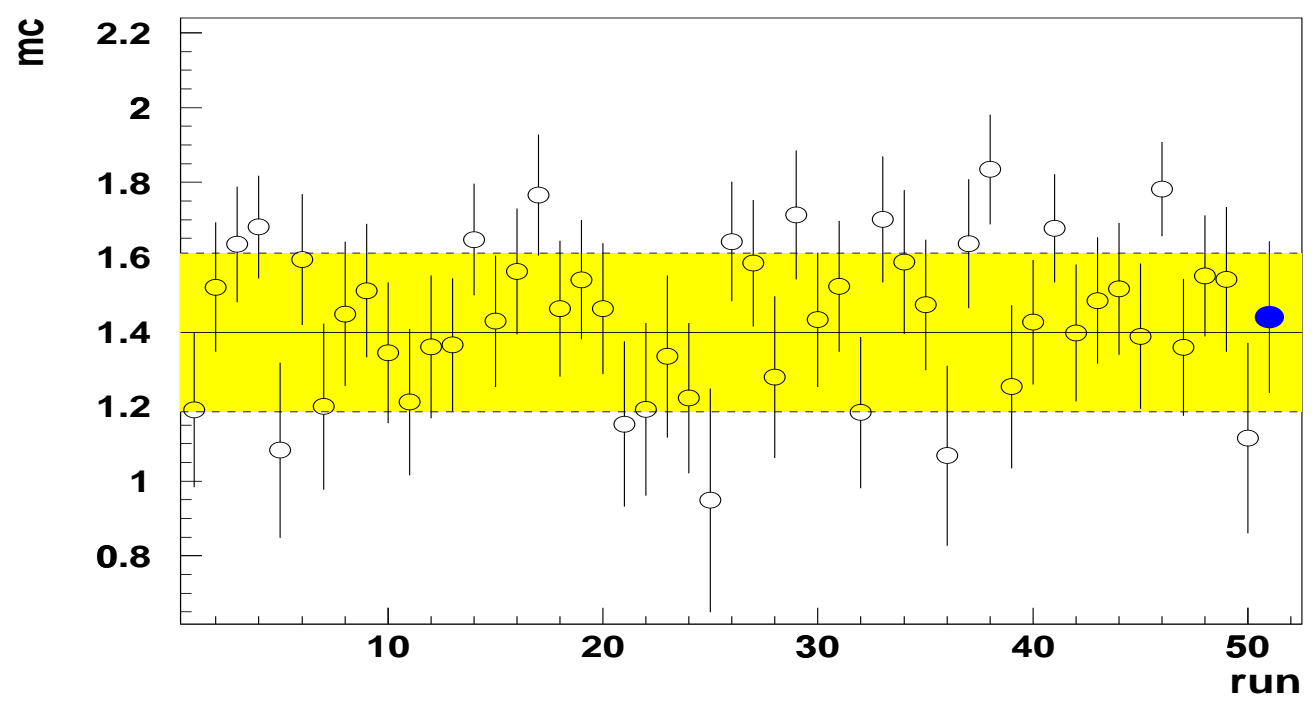

FIGURE 136. Fake data pull study $m_{c}$. Points are central values and errors for each individual fit, the shaded band is the value and error of $m_{c}$ used to generate the fake data, and the point to the right is the average of all 50 fake data fits with the error being their RMS.

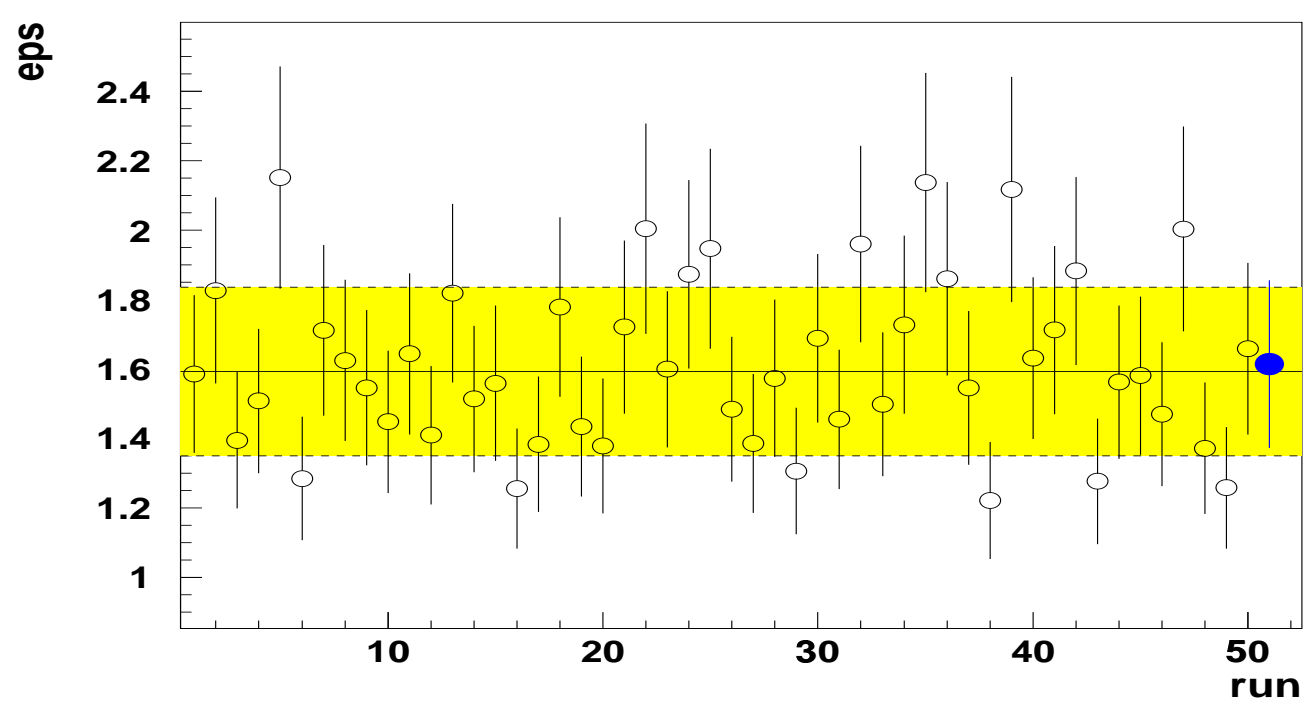

FIGURE 137. Fake data pull study $\epsilon$. Points are central values and errors for each individual fit, the shaded band is the value and error of $\epsilon$ used to generate the fake data, and the point to the right is the average of all 50 fake data fits with the error being their RMS 


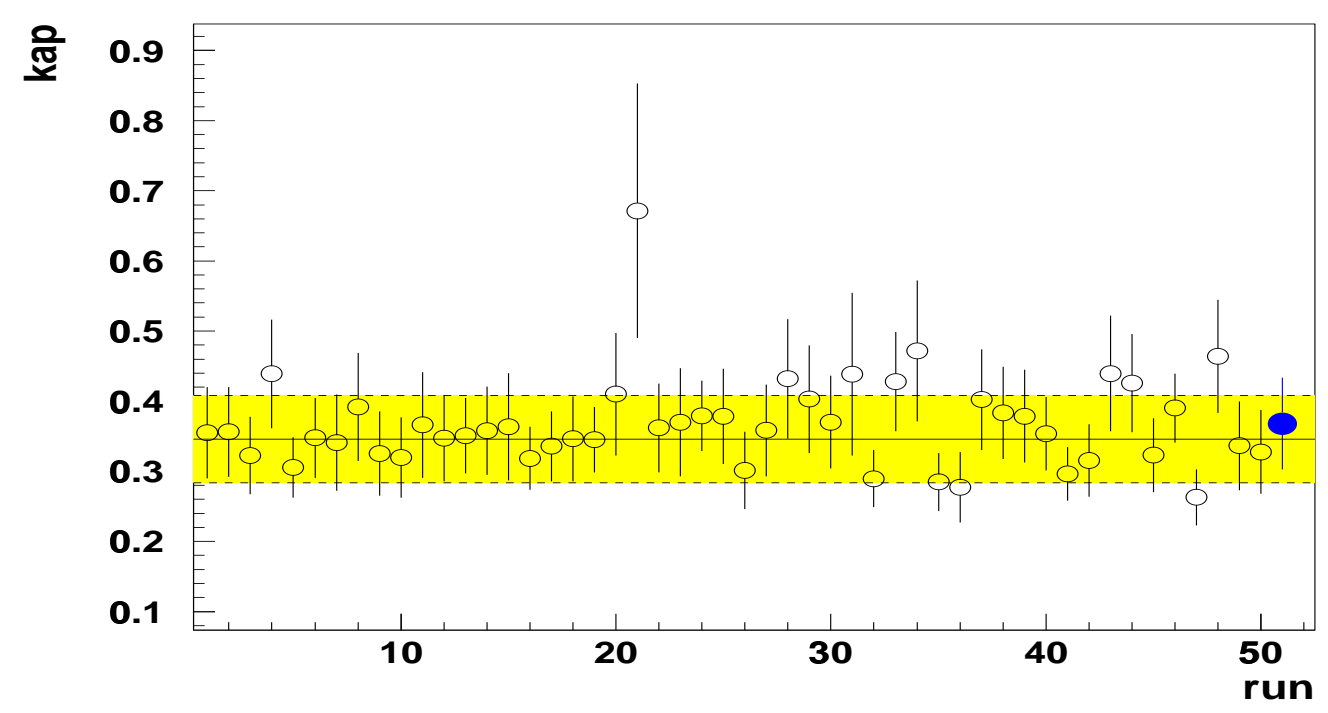

FIGURE 138. Fake data pull study $\kappa$. Points are central values and errors for each individual fit, the shaded band is the value and error of $m_{c}$ used to generate the fake data, and the point to the right is the average of all 50 fake data fits with the error being their RMS

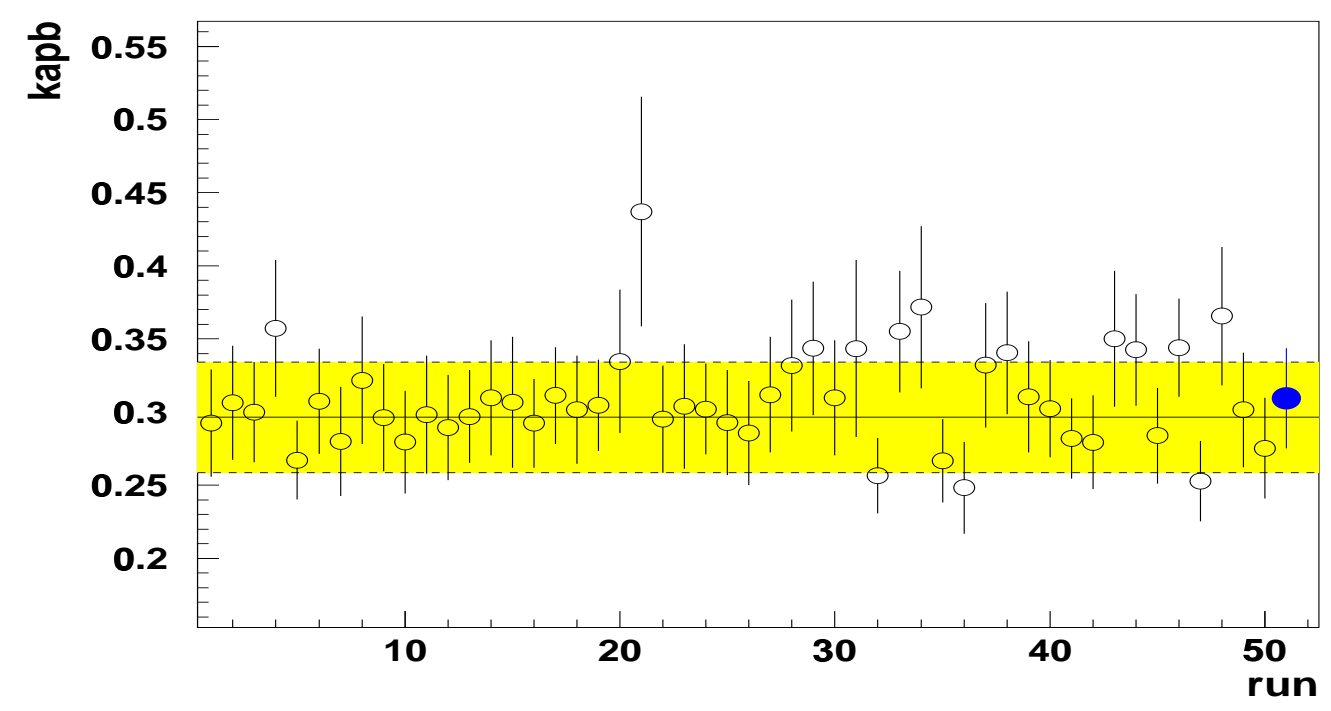

FIGURE 139. Fake data pull study $\bar{\kappa}$. Points are central values and errors for each individual fit, the shaded band is the value and error of $\bar{\kappa}$ used to generate the fake data, and the point to the right is the average of all 50 fake data fits with the error being their RMS 


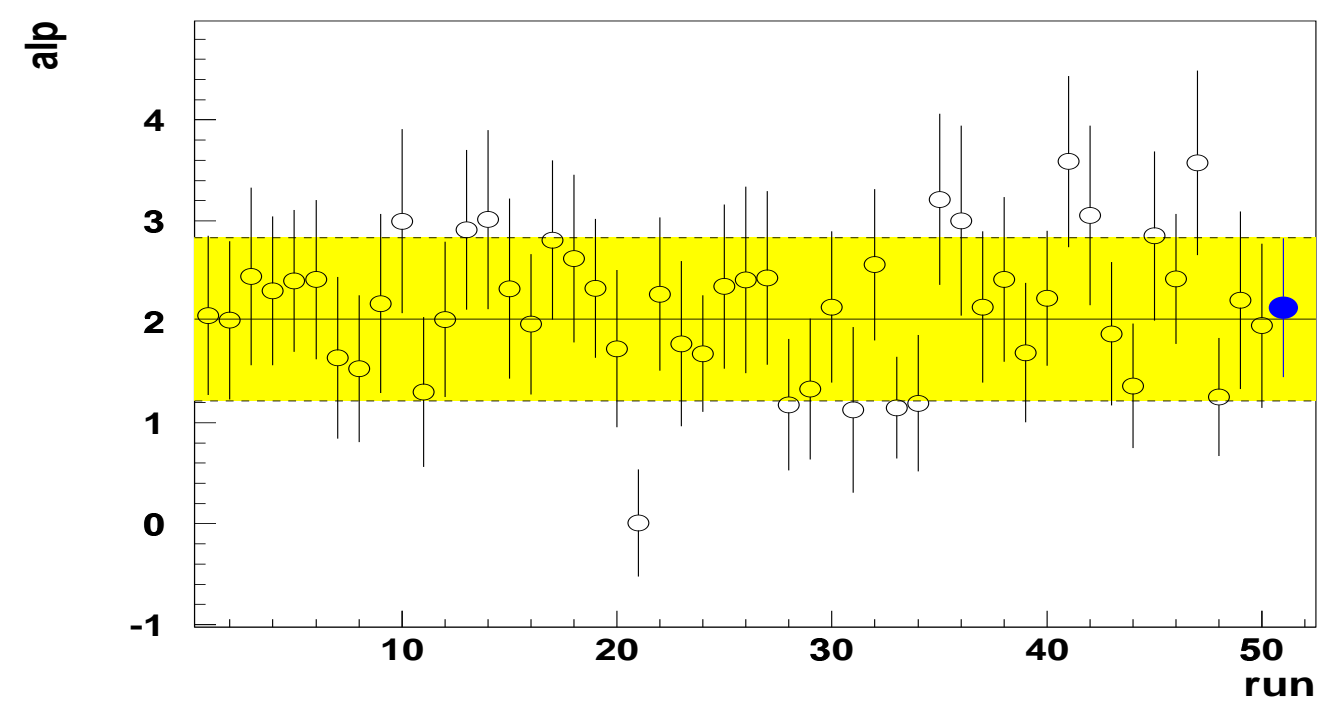

FIGURE 140. Fake data pull study $\alpha$. Points are central values and errors for each individual fit, the shaded band is the value and error of $\alpha$ used to generate the fake data, and the point to the right is the average of all 50 fake data fits with the error being their RMS

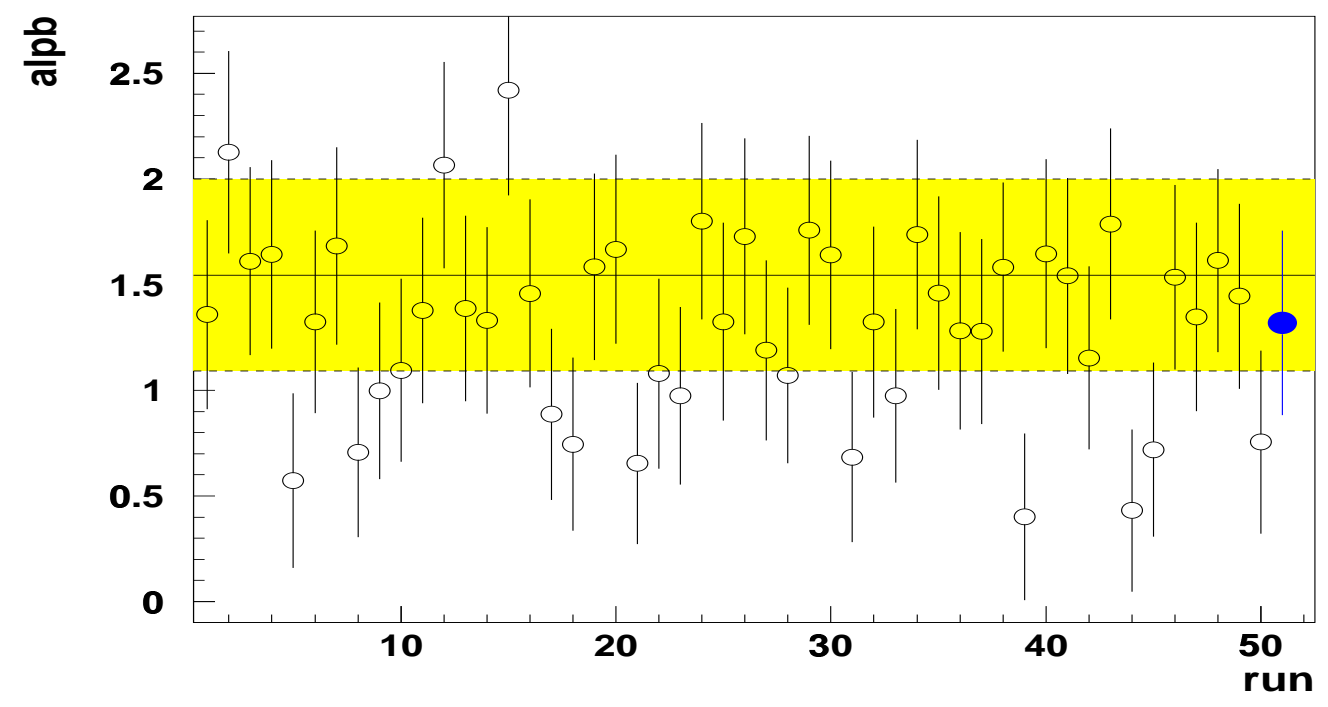

FIGURE 141. Fake data pull study $\bar{\alpha}$. Points are central values and errors for each individual fit, the shaded band is the value and error of $\bar{\alpha}$ used to generate the fake data, and the point to the right is the average of all 50 fake data fits with the error being their RMS 


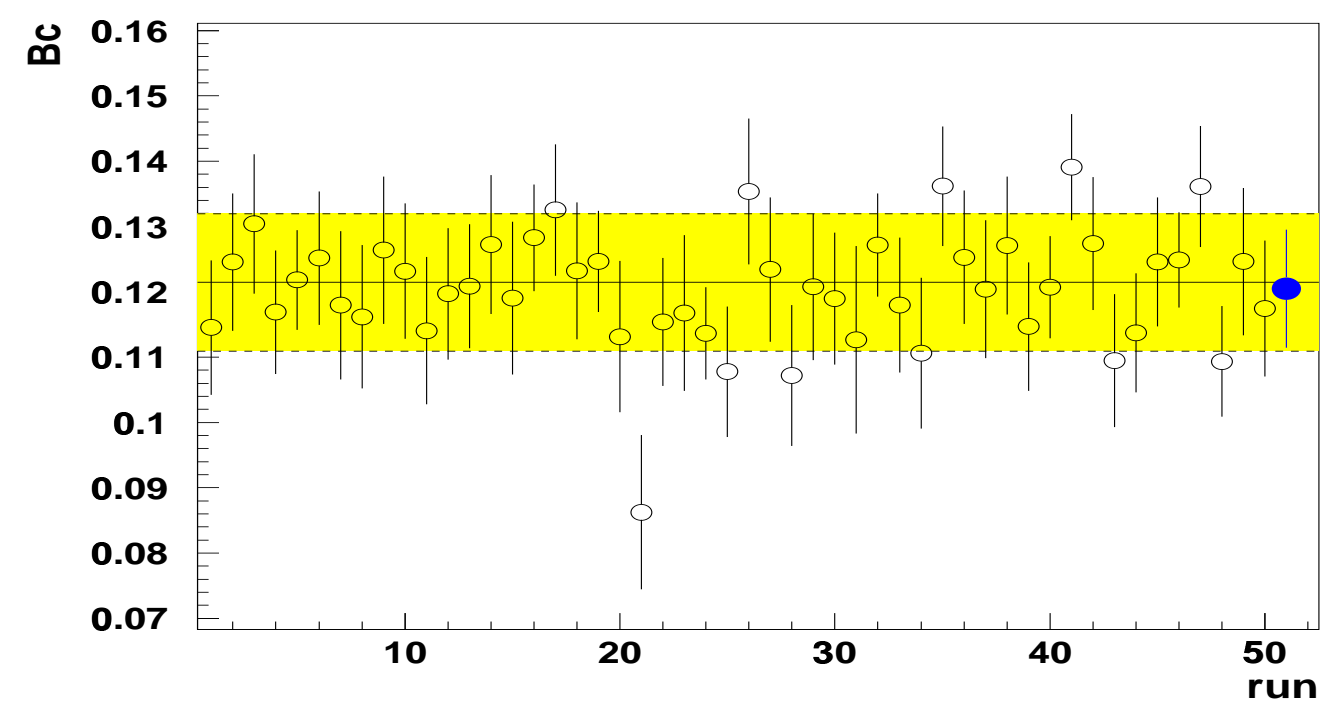

FIGURE 142. Fake data $B_{c}$ pull study. Points are central values and errors for each individual fit, the shaded band is the value and error of $B_{c}$ used to generate the fake data, and the point to the right is the average of all 50 fake data fits with the error being their RMS

the cross section table with the spread in the curve indicating the RMS from the individual fits. The band and the curve overlap within $\mathcal{C}=55 \%$ to about $95 \%$, indicating coverage fractions anywhere within that range should return the proper errors in the fit. A coverage fraction of $60 \%$ is chosen to minimize correlation between the bins, while staying in the acceptable coverage range.

Pulls or biases upon fitting the fake data tables can also be checked. Figures 144-148 show the results as a function after $\mathcal{C}$ of re-fitting the extracted cross section tables. Because fits to the cross section tables are not constrained as well as fits to the raw data, $\epsilon$, and $B_{c}$ must be held constant. The table re-fits are performed on $m_{c}, \kappa, \bar{\kappa}, \alpha$, and $\bar{\alpha}$. The figures show the average values of the fits at each coverage fraction plotted on top of the line and error band indicating the 


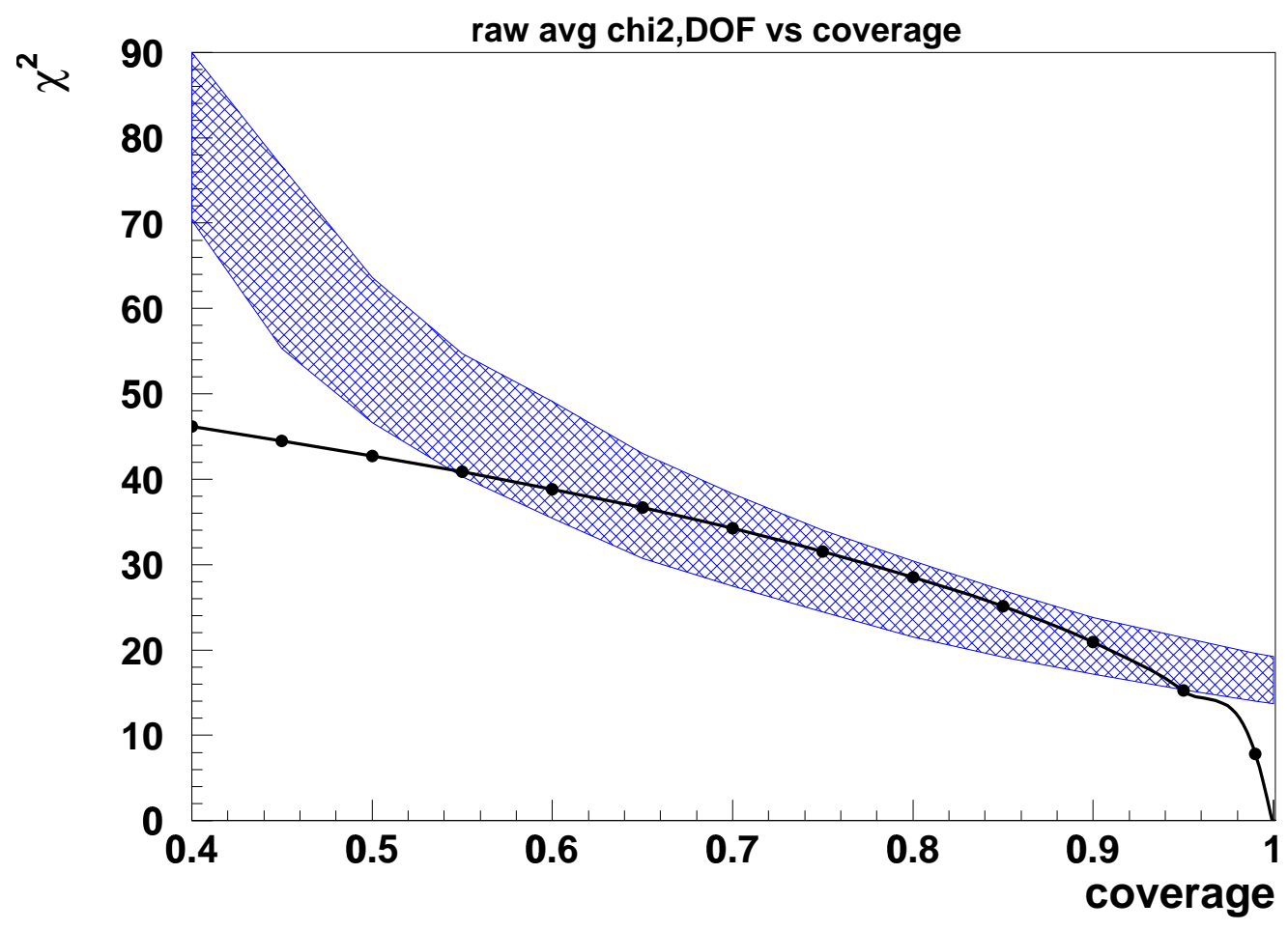

FIGURE 143. DOF vs coverage fraction $\mathcal{C}$ (line) and range of $\chi^{2}$ 's from the 50 fake data tables (band). The two overlap within the range of $\mathcal{C}=55-95 \%$, indicating coverage fractions anywhere in that range are acceptable. A coverage fraction of $60 \%$ is chosen to minimize the correlation between bins. 


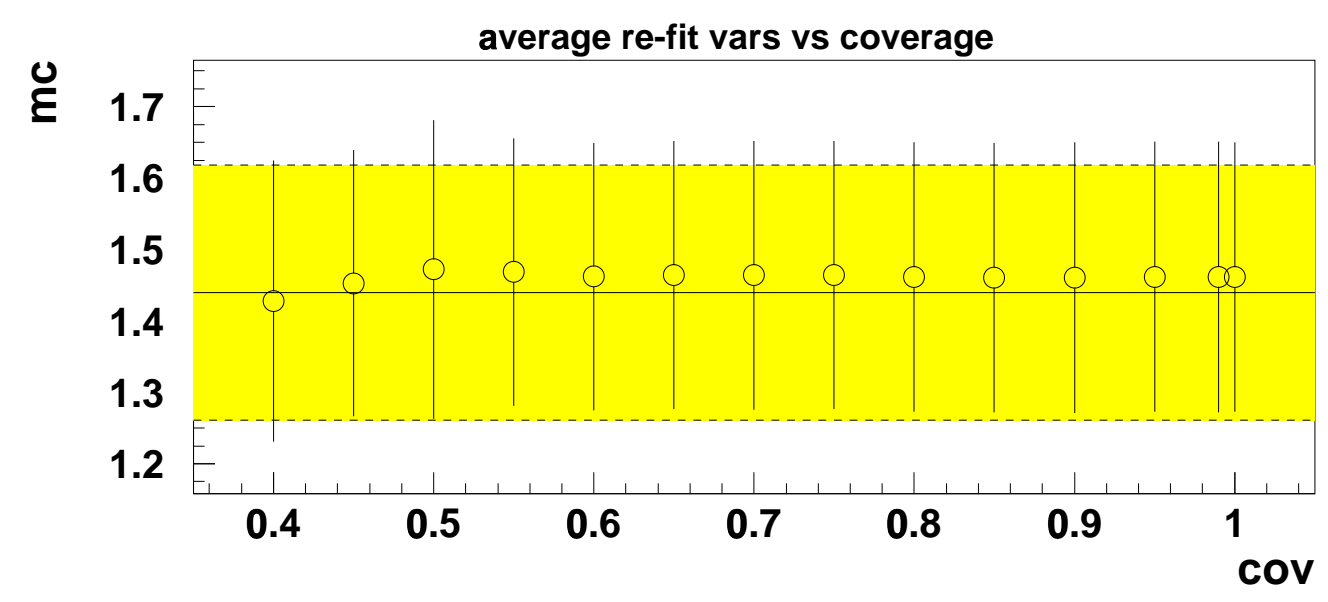

FIGURE 144. Average re-fitted $m_{c}$ vs coverage fraction. The points are the average results from the fits to the 50 extracted tables from fake data. The line and shaded band is the average value and error of the parameter used to generate the tables fit.

average parameters from which the tables were generated. All 5 re-fitted parameters are well within the error band of the generating parameters, indicating no significant bias in the table extraction procedure.

One test of model independence in the cross section table is reextracting it using a model based on an external pdf set, and comparing it to the internal BGPAR model used above. A fit was performed using the CTEQ5L pdf set [173], and a table was extracted. Figures 149 and 150 compare the two resulting tables. Very little difference is seen between the two extracted tables.

Of course extracting the cross section with different pdf sets is not a complete test of the model independence of the table extraction procedure, but its a good start. A much better test will be demonstrated in the next chapter, where the table will be re-extracted using the NLO model described there. 


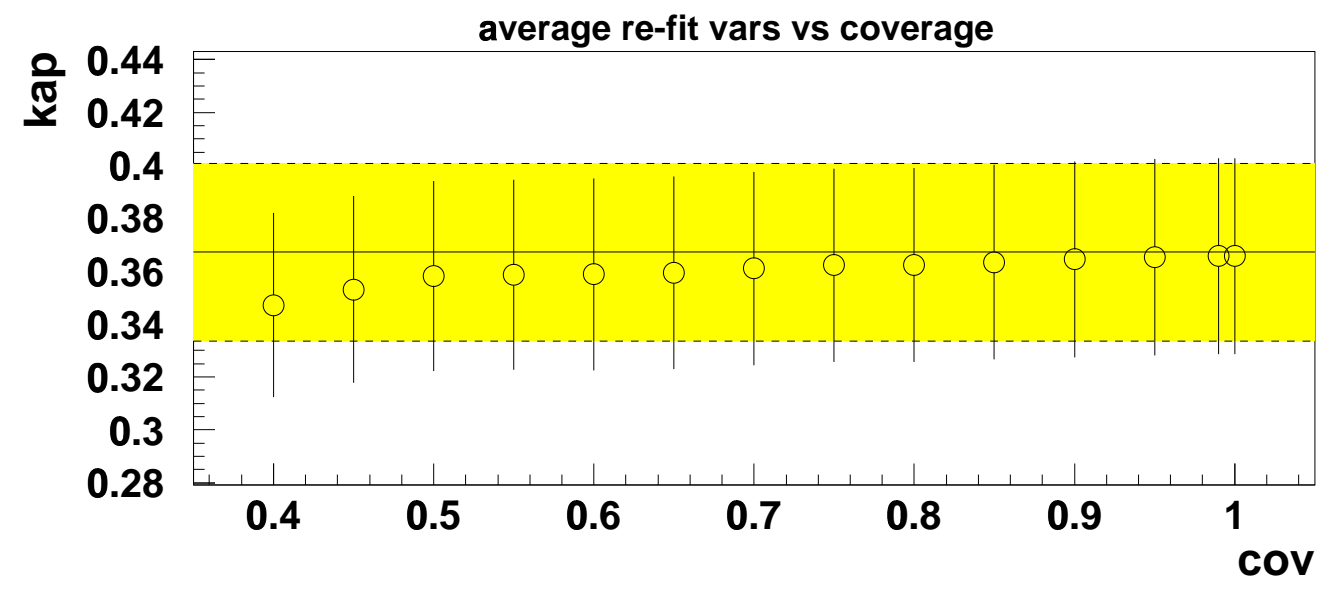

FIGURE 145. Average re-fitted $\kappa$ vs coverage fraction. The points are the average results from the fits to the 50 extracted tables from fake data. The line and shaded band is the average value and error of the parameter used to generate the tables fit.

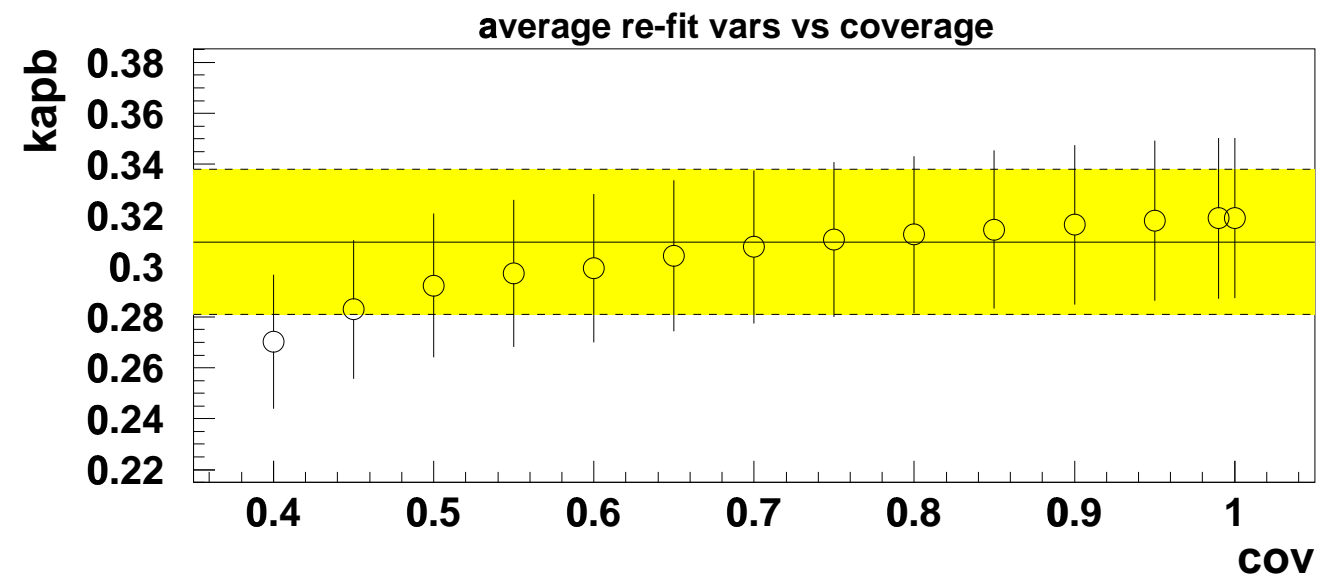

FIGURE 146. Average re-fitted $\bar{\kappa}$ vs coverage fraction. The points are the average results from the fits to the 50 extracted tables from fake data. The line and shaded band is the average value and error of the parameter used to generate the tables fit. 


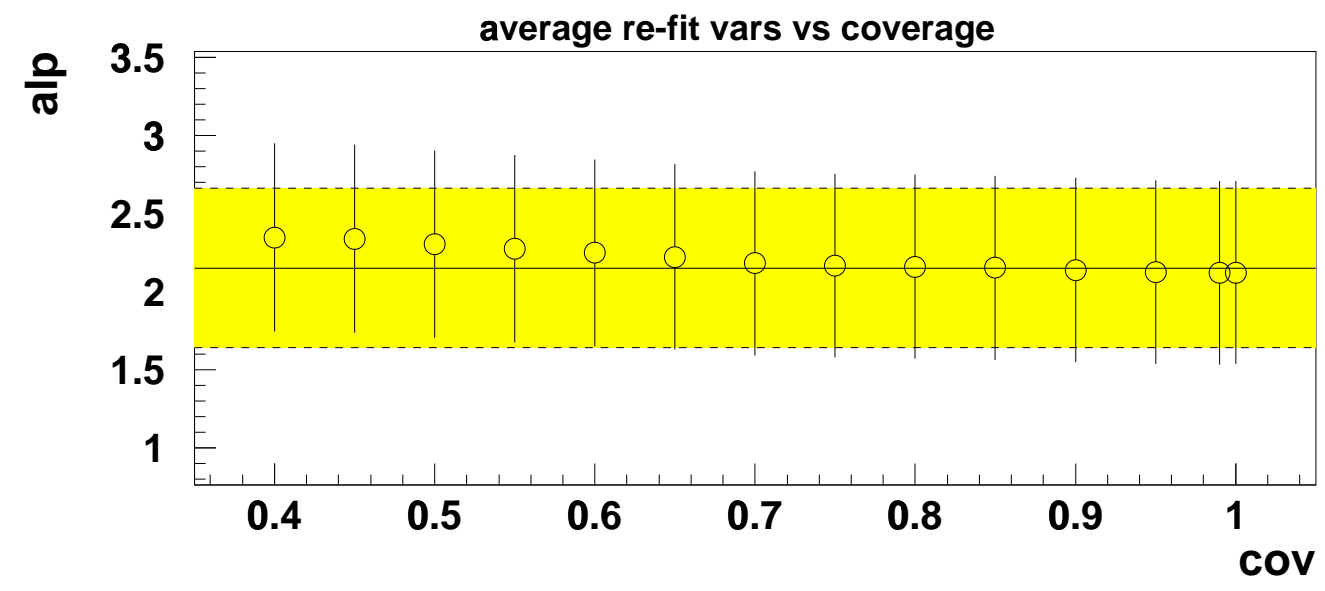

FIGURE 147. Average re-fitted $\alpha$ vs coverage fraction. The points are the average results from the fits to the 50 extracted tables from fake data. The line and shaded band is the average value and error of the parameter used to generate the tables fit.

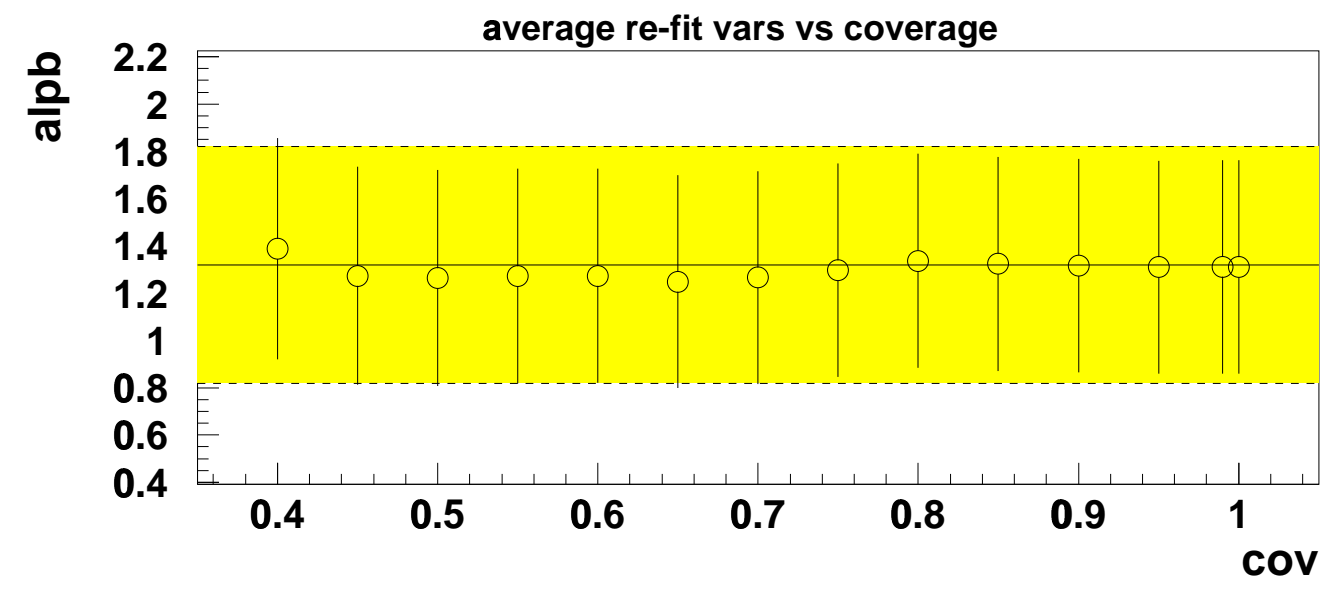

FIGURE 148. Average re-fitted $\bar{\alpha}$ vs coverage fraction. The points are the average results from the fits to the 50 extracted tables from fake data. The line and shaded band is the average value and error of the parameter used to generate the tables fit. 

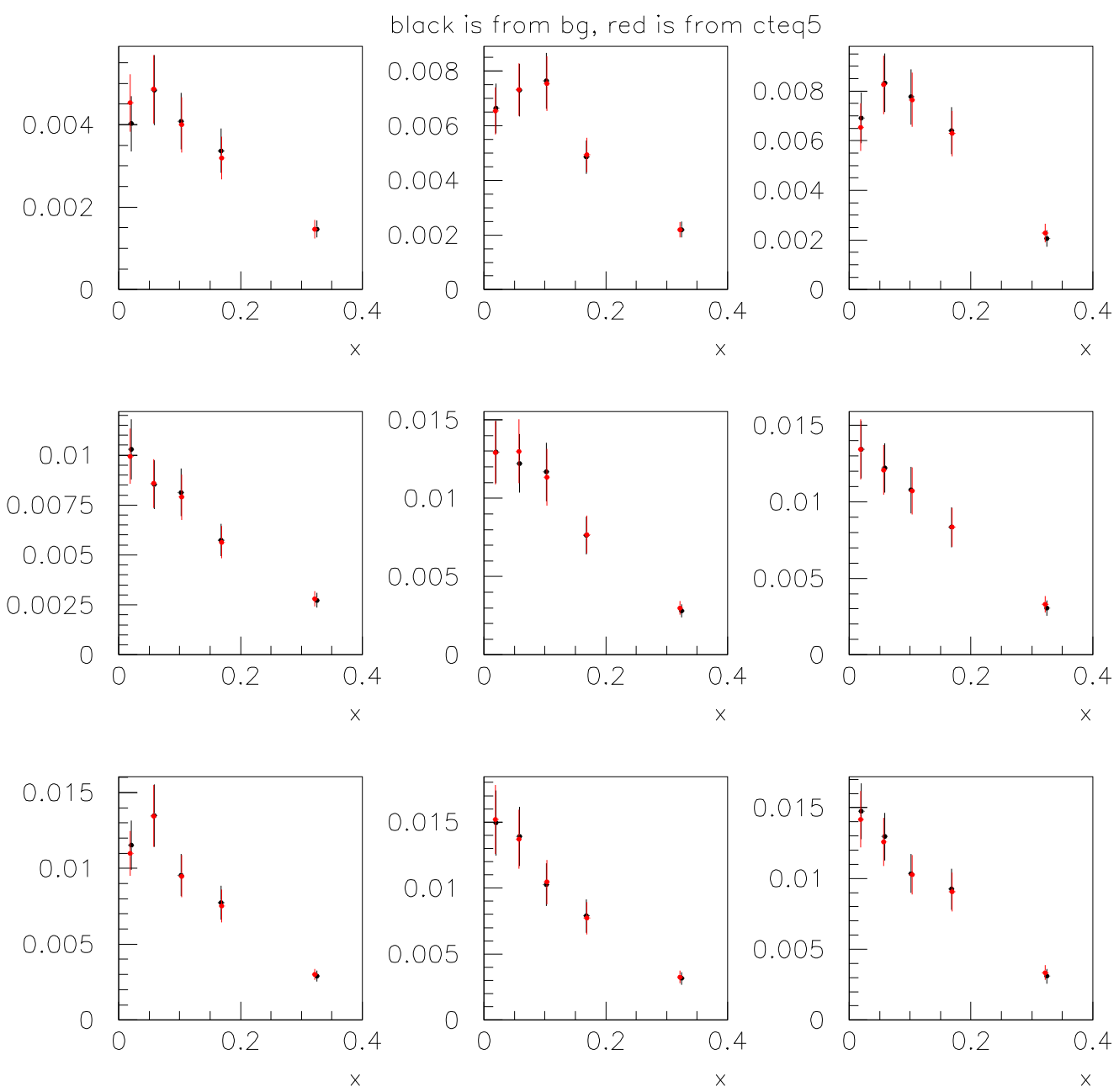

FIGURE 149. $\nu$ table extracted based on CTEQ5 pdf set and BGPAR. Points are shown multiplied by $\frac{\pi}{G_{F}^{2} M E_{\nu}}$. 

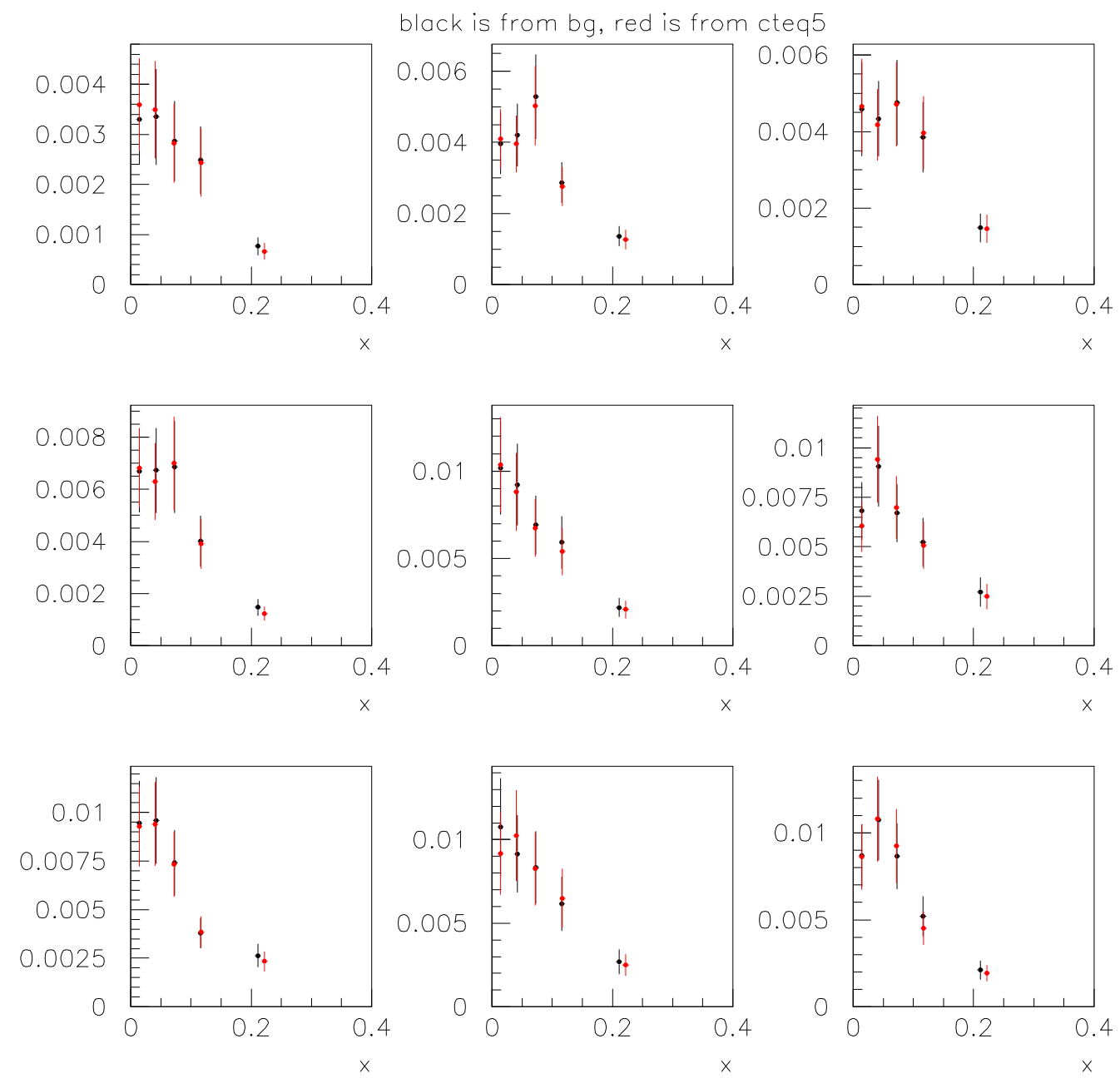

FIGURE 150. $\bar{\nu}$ table extracted based on CTEQ5 pdf set and BGPAR. Points are shown multiplied by $\frac{\pi}{G_{F}^{2} M E_{\nu}}$. 


\subsubsection{LO Fit and Table Systematic Errors}

The accuracy of the extracted cross section is dependent on the quality of fit between MC and data. Through knowledge of the [Experiment] portion of the convolution in equation 4.258, and of the $\pi-K$ background, a good fit to data constrains the cross section. The uncertainties on these pieces however translate directly to systematic uncertainties on the cross section measurement. These systematic uncertainties in the cross section are determined by re-extracting the cross section while varying those quantities upward or downward by \pm one standard deviation. The uncertainties considered are as follows:

$\pi-K$ background The uncertainty on the size of this background is $15 \%$ for neutrino data, and $21 \%$ for antineutrino data $[169,170]$. Each is taken separately and the table is extracted at the extremes for each.

$E_{\mu F F}$ scale The uncertainty on the muon momentum measurement for muons which pass through the toroid spectrometer was determined to be about $1 \%[140]$

$E_{H A D}$ scale The uncertainty on the hadronic shower energy measurement in the $\mathrm{NuTeV}$ calorimeter was measured to be about $0.5 \%[140]$

$E_{\mu 2}$ rangeout Described in the previous chapter, the uncertainty on the muon energy for muons which range out in the calorimeter was found to be $2.5 \%$.

$R_{L}$ (20\%) The uncertainty on the measurement of $R_{L}$ is $20 \%$ [114]. This has a minimal effect on the table measurement, however is still an error on the LO charm production parameters, so is evaluated. 


\begin{tabular}{|c||c|c|c|c|c|c|c||c|}
\hline & $m_{c}$ & $\epsilon$ & $\kappa$ & $\bar{\kappa}$ & $\alpha$ & $\bar{\alpha}$ & $B_{c}$ & $S^{-}$ \\
\hline Central Value & 1.39 & 1.59 & 0.346 & 0.296 & 2.03 & 1.55 & 0.1214 & 0.00128 \\
\hline Statistical Error & 0.21 & 0.24 & 0.063 & 0.038 & 0.81 & 0.45 & 0.011 & 0.00062 \\
\hline$\nu \pi-\mathrm{K}(15 \%)$ & 0.07 & 0.30 & 0.016 & 0.021 & 0.04 & 0.00 & 0.007 & 0.00016 \\
$\bar{\nu} \pi-\mathrm{K}(21 \%)$ & 0.00 & 0.08 & 0.001 & 0.014 & 0.00 & 0.25 & 0.001 & 0.00039 \\
$E_{\mu f f}$ scale (1\%) & 0.05 & 0.12 & 0.022 & 0.017 & 0.25 & 0.06 & 0.007 & 0.00050 \\
$E_{\mu 2}$ rangeout & 0.12 & 0.25 & 0.022 & 0.016 & 0.02 & 0.18 & 0.005 & 0.00014 \\
$E_{H A D}$ scale $(0.5 \%)$ & 0.01 & 0.07 & 0.009 & 0.004 & 0.06 & 0.02 & 0.001 & 0.00010 \\
$R_{L}(20 \%)$ & 0.07 & 0.02 & 0.008 & 0.005 & 0.42 & 0.26 & 0.002 & 0.00007 \\
MC statistics & 0.02 & 0.01 & 0.006 & 0.004 & 0.03 & 0.02 & 0.001 & 0.00006 \\
Flux norm & 0.00 & 0.00 & 0.000 & 0.002 & 0.00 & 0.01 & 0.000 & 0.00006 \\
\hline Total Systematics & 0.17 & 0.43 & 0.037 & 0.035 & 0.49 & 0.41 & 0.011 & 0.00068 \\
\hline
\end{tabular}

TABLE 20. BGPAR fit parameters and the itemized effects of systematics. Also shown in the far right $S^{-}$column is the integrated momentum weighted strangeantistrange asymmetry

$M C$ statistics Finite resources require that only a finite number of $\mathrm{MC}$ events may be run to do any analysis. This systematic error is estimated by dividing the MC samples used for the measurement into two parts, extracting the table with each half, then taking the difference divided by $\sqrt{2}$.

Flux normalization The relative normalization between neutrino and antineutrino fluxes was determined in the $\mathrm{NuTeV}$ inclusive cross section measurement to be $0.7 \%[154]$.

Each systematic was evaluated and the results included in table 20. Shown are the central values of the fit, statistical errors, then an itemized list of the impact of each systematic effect. Figures 151 and 152 show the dimuon cross section points, with a shaded band indicating the total systematic error superimposed on top. The systematic errors in almost all bins are smaller than the statistical errors. 
cross section vs $x$, neutrino mode
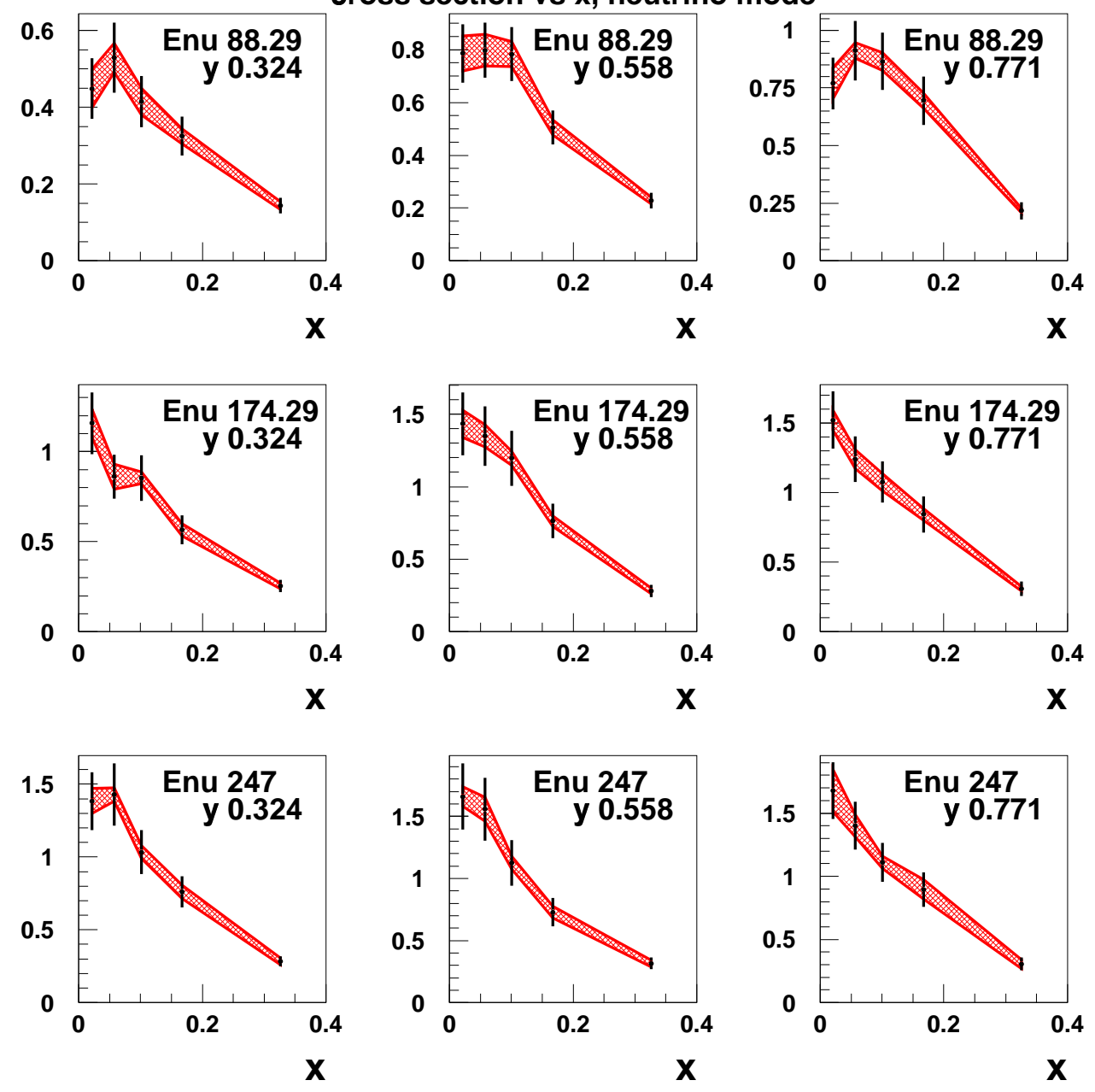

FIGURE 151. Numode Dimuon Cross Section with Systematics. Points are shown multiplied by $\frac{\pi}{G_{F}^{2} M E_{\nu}}$ 

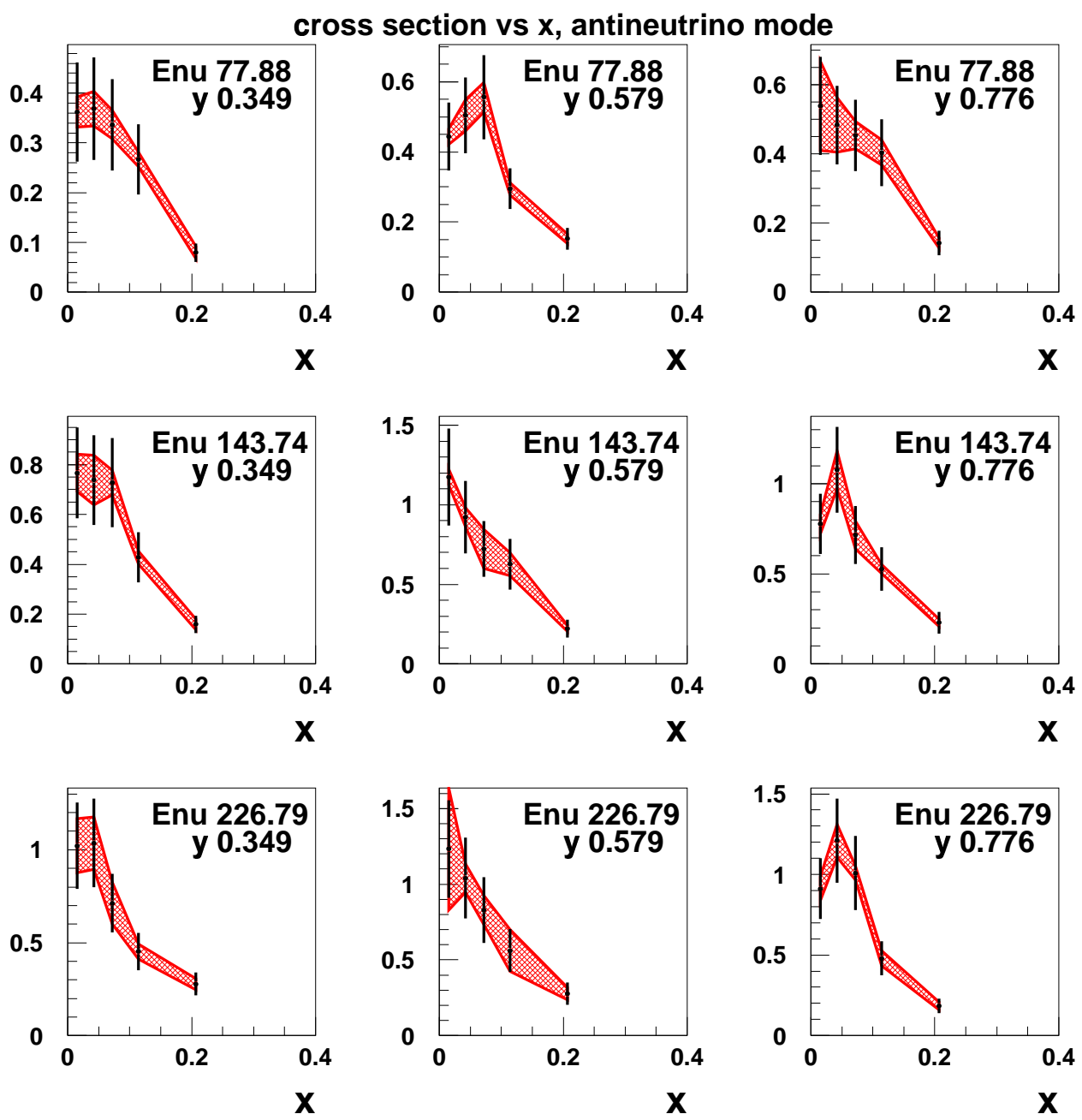

FIGURE 152. Nubarmode Dimuon Cross Section with Systematics. Points are shown multiplied by $\frac{\pi}{G_{F}^{2} M E_{\nu}}$ 


\section{CHAPTER V}

\section{NLO FITS AND PHENOMENOLOGY}

The dimuon data has been condensed into the forward dimuon cross section table, and now may be used as a tool to learn something about nucleon structure. To do this a model must be provided to work back from the observable dimuon data through the semileptonic decay and fragmentation to the production of the charmed quark. Through this model, the distribution of strange and antistrange quarks inside the nucleon can be measured.

The determination of the strange and antistrange pdfs is at some level dependent on the model chosen. Therefore, a model that as completely as possible describes the physics occurring within the nucleon will be used. This model should also be of global use. An NLO QCD (first order in $\alpha_{s}$ ) charm production cross section calculation, based on the commonly used $\overline{M S}$ factorization scheme is such a model. Moving from LO to NLO brings in the significant contribution of gluons into the model, allowing for a more physically realistic description of the kinematics and the inner-workings of the nucleon. A pdf set based on global fits to external data will also be used. An advantage of using pdfs from a global fit is that they are based on data spread over a large range of $Q^{2}$ from different processes, and so are generally applicable to other physical situations, like for example, those expected in experiments at the Large Hadron Collider. (There are disadvantages as well, namely that for $\mathrm{NuTeV}$ one must correct these proton pdfs for the nuclear environment in which the nucleons are held, but that will be addressed in detail 
shortly.) The net product of using the NLO model and external measurements is that the measurements we perform on the table should be useful outside the context of neutrino deep inelastic scattering.

The cross section table we will be performing our NLO fits on is defined as the cross section of dimuon events such that the muon from the semileptonic charm decay has energy greater than $5 \mathrm{GeV}$. In order to measure the characteristics of charm production with this cross section we must assemble the following components:

$$
\frac{d \sigma_{\text {charm }}\left(m_{c}, s, \bar{s}\right)}{d x d y} \otimes \mathcal{N}\left(A, x, Q^{2}\right) \cdot B_{c} \cdot \mathcal{A}_{\mu 2}\left(m_{c}, \epsilon\right)=\text { fit }\left.\Rightarrow \frac{d \sigma_{2 \mu}}{d x d y}\right|_{E \mu 2>5 \mathrm{GeV}}
$$

where $\left.\frac{d \sigma_{2 \mu}}{d x d y}\right|_{E \mu 2>5 G e V}$ represents the measured dimuon cross section table described in the previous chapter. The charm production cross section is embodied in $\frac{d \sigma_{\operatorname{charm}}\left(m_{c}, s, \bar{s}\right)}{d x d y}$, dependent on the charm mass, strange and antistrange seas. Convolved within the charm cross section in the form of corrections to the pdfs are nuclear corrections, $\mathcal{N}\left(A, x, Q^{2}\right)$, which correct the proton based global pdfs for nuclear effects. $B_{c}$ is the semi-muonic branching fraction, representing the average branching ratio to muons for all produced charmed mesons. $\mathcal{A}_{\mu 2}\left(m_{c}, \epsilon\right)$ represents the acceptance for the $5 \mathrm{GeV}$ cut on the charm decay muon. This acceptance correction must be determined by MC simulation at each of the table bin values, and requires an NLO cross section dependent on $x, y$, fragmentation, and the transverse momentum of the charm quark. Such a cross section code has been incorporated into the NuTeV physics Monte Carlo. 


\section{$\underline{5.1}$ NLO Enhancements to Numonte Dimuon Model}

The NLO QCD charm cross section has been discussed in section 2.5.3. The DISCO [136] cross section code has been implemented in numonte to provide the acceptance effects from fragmentation and the additional charm transverse momentum within the NLO model. DISCO is based on a calculation of the cross section within the $\overline{\mathrm{MS}}$ factorization scheme, and is differential in the variables $\xi, y$, fragmentation $z$, and charm rapidity $\eta_{c}$. In order to keep the cross section a positive definite quantity, and to smooth out collinear singularities, the cross section is integrated in bins of $z$ and $\eta_{c}$ such that:

$$
\sigma_{\mathrm{DISCO}}=\int_{z_{(i)}}^{z_{(i+1)}} \int_{\eta_{c(j)}}^{\eta_{c(j+1)}} \frac{d \sigma_{\text {charm }}}{d \xi d y d z^{\prime} d \eta_{c}^{\prime}} d z^{\prime} d \eta_{c}^{\prime}
$$

Here the $i$ 's and $j$ 's refer to the boundaries of individual bins in $z$ and rapidity.

As discussed in the previous chapter, in the LO BGPAR dimuon model, fragmentation is modeled through throwing "darts" against the fragmentation function. Using DISCO however, both $z$ and $\eta_{c}$ are thrown, then each event is weighted as:

$$
w t c c=\frac{\sigma_{\mathrm{DISCO}} \cdot B_{c}}{w t_{t o t C C}}
$$

This time generating the dimuon events exclusively as weighted Monte Carlo. This is necessary because it is computationally very expensive to find the maximum cross section value to set the upper limit on throws, and the repeatedly throw darts against the $\sigma_{\mathrm{DISCO}}$ cross section.

Constructing kinematics begins in a similar way as in the leading order case, following the outline in table 12 on page 212 up to the transition to the hadronic 
center of mass frame. At that point, code generating the NLO kinematics replaces the code in qfrag_ccfr.F. Ideally fragmentation $z$ and $\eta_{c}$ should be thrown uniformly within their kinematic limits in order to avoid biasing the Monte Carlo. This is done for $z$, but because the rapidity distribution is so sharply peaked at near collinear rapidity, the majority of the events are thrown near the collinear limit, and the weights are then adjusted for the resulting bias. This is done so that the majority of the events occur where the weights are large, to maximize the efficiency of the integration that is performed when generating a Monte Carlo sample. Rapidity is generated through the following procedure:

The bounds on rapidity are defined in the lab frame as:

$$
\begin{aligned}
\eta_{c-\min } & =\frac{1}{2} \log \left[\frac{M^{2}}{Q^{2}} x(\xi-x)\right] \\
\eta_{c-\max } & =\frac{1}{2} \log \left[\frac{M^{2}}{Q^{2}} x(1-x)\right]-\frac{1}{2} \log \left[\frac{\xi-x}{1-x}\right]
\end{aligned}
$$

with the usual definitions of Bjorken $x$, and slow rescaling variable $\xi$ (see section 2.2.1 starting on page 51). The full range of rapidity is evenly divided into $N_{\text {bins }}$ bins. It should be noted at this point that the convention here, thanks to an unfortunate $\mathrm{z}$ axis direction choice early on in calculations, is that more negative rapidity is more collinear. This is perhaps opposite of what one might be used to. However, this convention is preserved throughout the analysis. (The preference being that any confusion that occurs be on the part of the reader of this thesis rather than by the person performing the analysis.)

With the limits on rapidity defined, a throw is then performed between 0 and 1 . If this throw is greater than 0.5 , the value of rapidity will be thrown within 

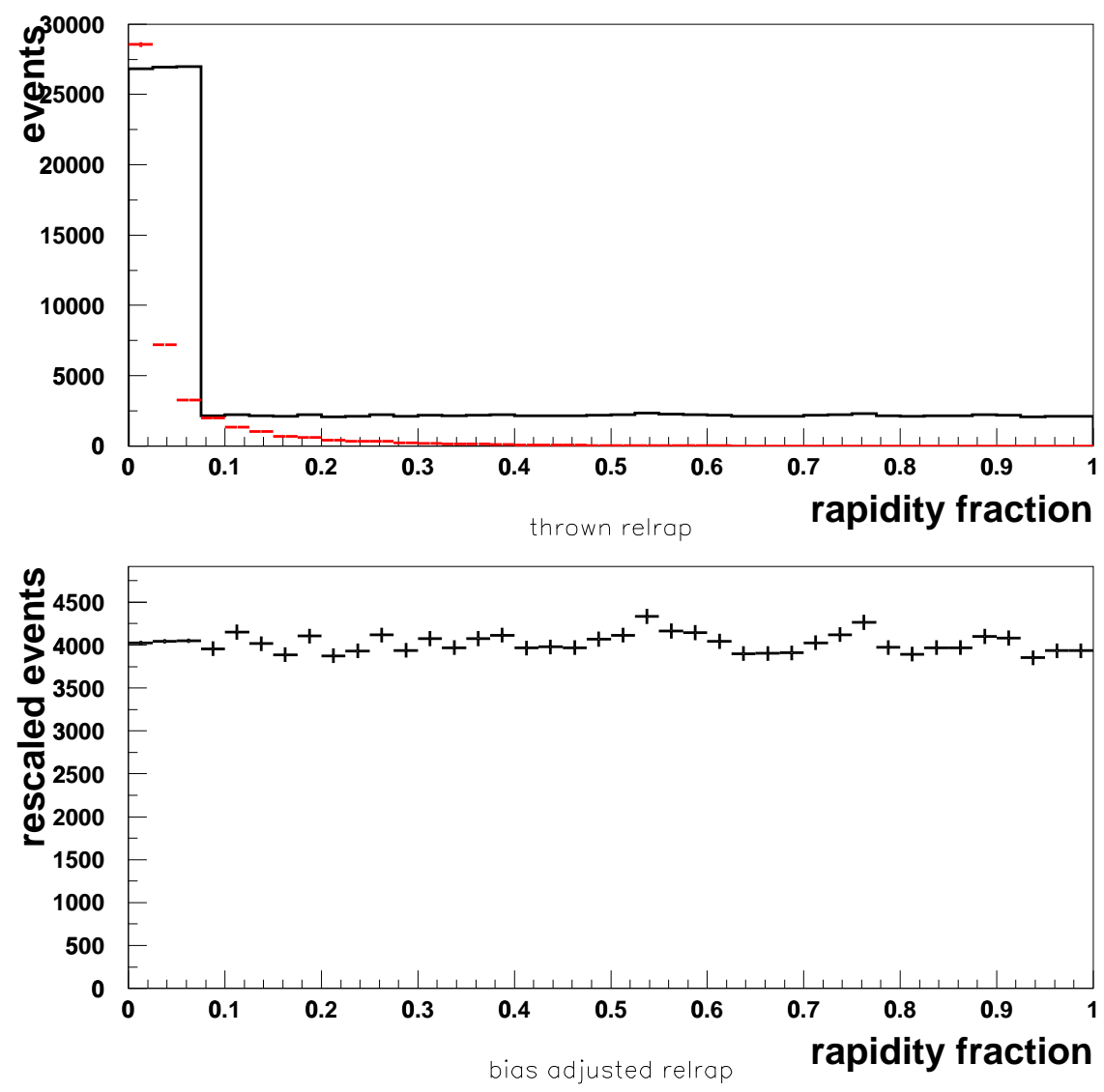

FIGURE 153. How rapidity is generated in the MC. The top plot shows the number of events thrown between the more collinear (0) and backwards going (1) rapidity. The black histogram is the raw number of events, while the superposed red histogram is the bias corrected rapidity distribution for comparison. The bottom plot shows the raw number of events in each rapidity bin, rescaled by the expressions in equations 5.271-5.272. Note that it is flat, indicating no bias, and that the errors in the collinear bins are small. 


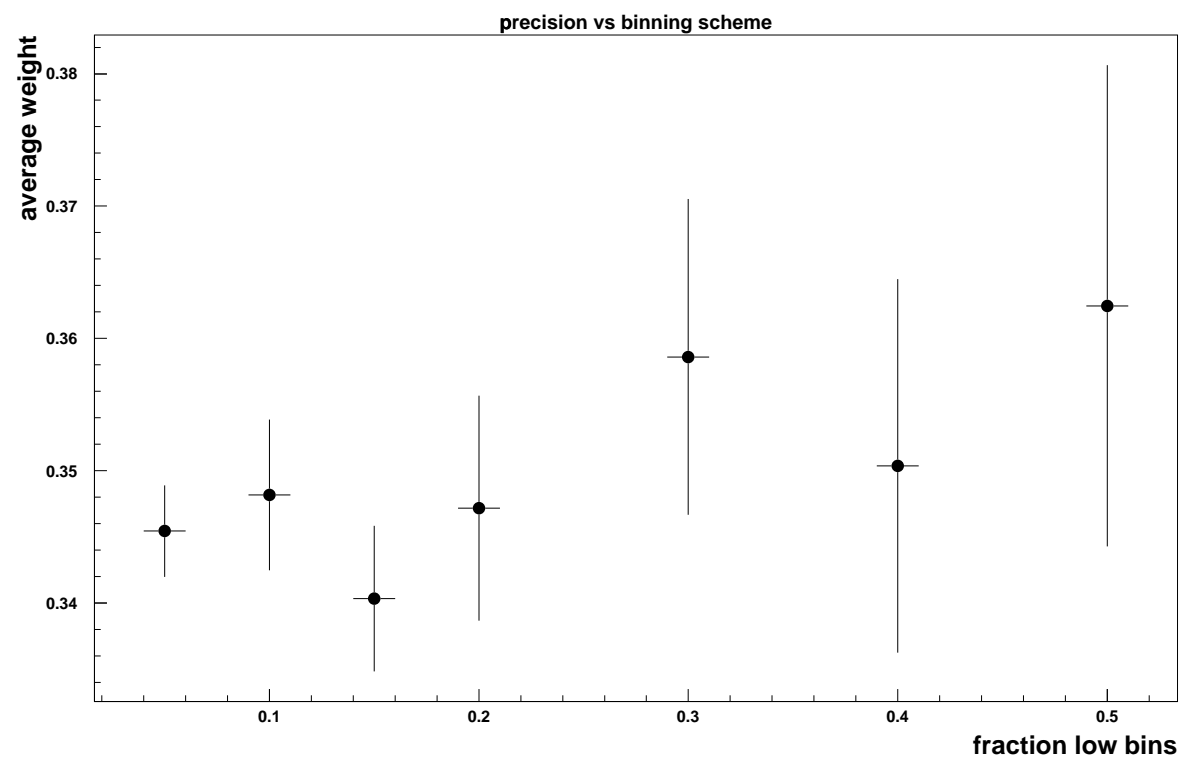

FIGURE 154. The average weight as a function of fraction of bins in the near collinear "half sample". A bin fraction of 0.05 means half the events are thrown within the lower $5 \%$ of rapidity, while 0.5 means half the events are thrown within the lower $50 \%$ of rapidity, equivalent to throwing uniformly. Note the increase in statistical error as the fraction increases.

the most collinear $5 \%$ of the rapidity bins. If there are fewer than 20 rapidity bins, the most collinear rapidity bin is chosen. If the primary throw is less than 0.5 , rapidity is thrown uniformly within the remaining $95 \%$ of the rapidity bins. An additional weight is then applied to the event weight, wtcc, to compensate for the bias introduced:

$$
\begin{aligned}
w t_{\text {col }} & =\frac{0.5 * N_{\text {bins }}}{N_{\text {lomax }}} \\
w t_{\text {off-col }} & =\frac{0.5 * N_{\text {bins }}}{N_{\text {bins }}-N_{\text {lomax }}}
\end{aligned}
$$

where $w t_{c o l}$ corresponds to the weight given to the more collinear bunch, bounded 
by bin number $N_{\text {lomax }}$, and $w t_{o f f-c o l}$ is the weight given to the remaining offcollinear bunch. Figure 153 illustrates the two groups of bins, plotted as a function of "relative rapidity", defined to be:

$$
\eta_{c-r e l}=\frac{\eta_{c}-\eta_{c-\min }}{\eta_{c-\max }-\eta_{c-\min }}
$$

for illustration. $\eta_{c-r e l}$ is zero at the collinear limit, and 1 when the charmed particle's momentum vector points opposite to the $\mathrm{W}$ boson in the W-parton CMS frame. In the figure, the top plot shows two histograms, the more blockish one being the raw numbers of events thrown for 40 rapidity bins, and the curve being the $\eta_{c-\text { rel }}$ distribution, to show where weights are largest. The bottom plot in the figure shows the raw number events after the weights from equations 5.271-5.272 are applied. The fact that this is flat shows that any bias from this throwing scheme is removed, and the benefit can be seen in the smaller error bars where the rapidity distribution is largest. Another way to see the benefit of this scheme is shown in figure 154. This shows the average event weight as a function of the width of the "collinear 50\%" bin, for MC samples generated with the same statistics. The rightmost edge of the plot, a width of $50 \%$, is equivalent to throwing rapidity uniformly throughout its range. As the width gets smaller, moving to the left, the average becomes more precise, shown by the decreasing error bars.

Once $z$ and $\eta_{c}$ are thrown, the kinematics of the charmed meson may be constructed. The rapidity, initially defined in the lab frame, is boosted to the hadronic CMS frame:

$$
\eta_{c}^{\prime}=\eta_{c} \log \left(\gamma+\sqrt{\gamma^{2}-1}\right)
$$


where $\gamma=E_{\text {had }} / \sqrt{W^{2}}$. The total momentum of the charmed meson is scaled by $z$, and then the magnitude of the charmed meson's momentum vector in the collinear direction can be written in terms of the rapidity:

$$
p_{z}=-E_{D} \frac{e^{2 \eta_{c}^{\prime}}-1}{e^{2 \eta_{c}^{\prime}}+1}
$$

The transverse component is then calculated from $p_{z}$ and the total momentum. This transverse momentum is part of what is measured in experiments like LEBCEHS and NA14/2, used earlier for parameterizing nonperturbative and higher order transverse momentum smearing. In generating NLO charm events the contribution from NLO smearing is subtracted from the nonperturbative $p_{\perp}$ smearing distribution. Once the kinematics of the charmed meson are determined, then it is decayed in the same way as in the LO case to produce an observed decay muon with the hadronic shower.

\section{$\underline{5.2 \quad \text { NLO acceptance }}$}

With the DISCO code in place in the MC, it may then be used to determine $\mathcal{A}_{\mu 2}\left(m_{c}, \epsilon\right)$. The smearing effects due to the detector environment have been corrected out of the forward cross section, so that they need not be simulated when determining the acceptance. Such smearing effects would require simulation of the full kinematic range in $E_{\nu}, x$, and $y$. Instead, only the acceptance at the centers of each $E_{\nu}, x$, and $y$ bin needs to be determined, greatly reducing the CPU time required. This acceptance, through its dependence on fragmentation and rapidity 
at NLO, depends on $\epsilon$, the fragmentation shape parameter, and the charm mass. Therefore acceptance corrections must be calculated not only for each bin point, but also for the different possible $\epsilon$ and $m_{c}$ values.

To calculate $\mathcal{A}_{\mu 2}$, a large number of charm induced dimuon events are generated with $E_{\nu}, x$, and $y$ from each of the table bin centers, and for a range values of $m_{c}$, and $\epsilon$. The acceptance is then the number of dimuon events which have a charm decay muon with energy greater than $5 \mathrm{GeV}$ divided by the total number of dimuon events run. For the purpose of fitting, a grid in $m_{c}$ and $\epsilon$ is defined, and the acceptance values for all table bins is calculated for each $m_{c}$ and $\epsilon$ point. The grid is defined with values of $m_{c}$ and $\epsilon$ close enough together so that intermediate values may be determined by linear interpolation. The values of the grid points used in this analysis were for charm mass (in GeV): $(1.10,1.25,1.40,1.55$, 1.70, 1.85), and for Collins-Spiller $\epsilon:(0.1,0.2,0.3,0.4,0.5,0.6,0.7,0.9,1.2,1.5,2.0,2.5)$. 20000 dimuon events were generated within each of $20 z$ by $40 \eta_{c}$ bins for each of the 90 cross section table bin points. This corresponds to approximately $1 \times 10^{11}$ MC dimuon events generated to produce the NLO acceptance grid.

Figures 155 and 156 show the $\sigma_{D I S C O}$ cross section, equation 5.267, and the raw acceptance convolved with it to calculate $\mathcal{A}_{\mu 2}$ for two of the dimuon cross section table bins as a function of rapidity and $z$. The cross section and acceptance histograms are multiplied together and integrated over $z$ and rapidity to obtain the acceptance correction for each table bin. In figure 155 we see the acceptance approaching zero as $z \rightarrow 0$, and as rapidity becomes more anti-collinear (with charm momentum pointing backwards in the W-parton CMS frame). At the higher energy point in figure 156, we see the off collinear component of the cross section 

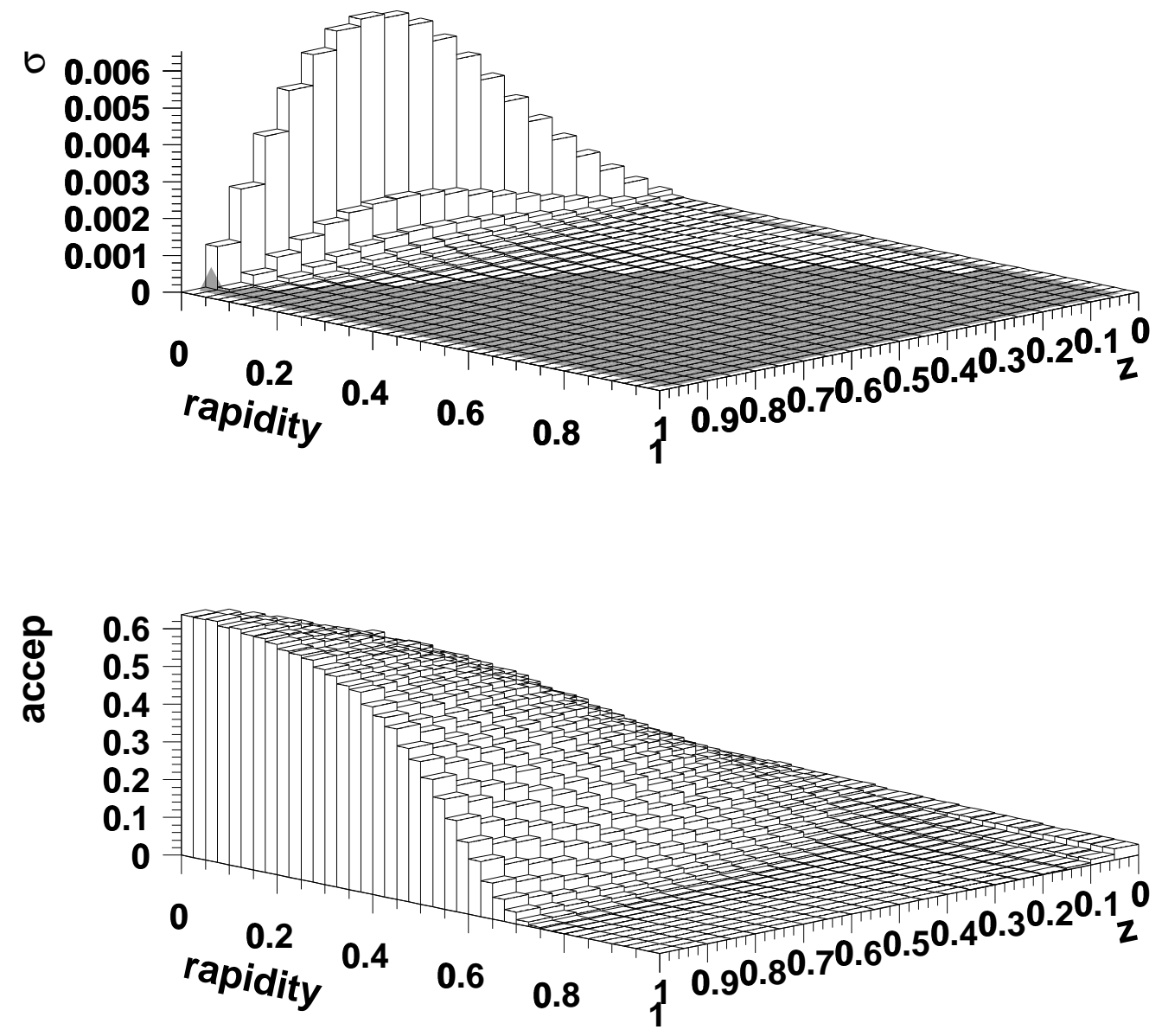

FIGURE 155. The $\sigma_{D I S C O}$ cross section (top) and $E_{\mu 2}>5 \mathrm{GeV}$ acceptance (bottom) plotted as a function of relative rapidity and z. Plots are for $\nu$ table point $\left(E_{\nu}=88.3, x=0.101, y=0.324\right)$ For relative rapidity, 0 means collinear, and 1 means anti-collinear. $z=1$ means the charmed hadron keeps all of the charmed quark's momentum. 

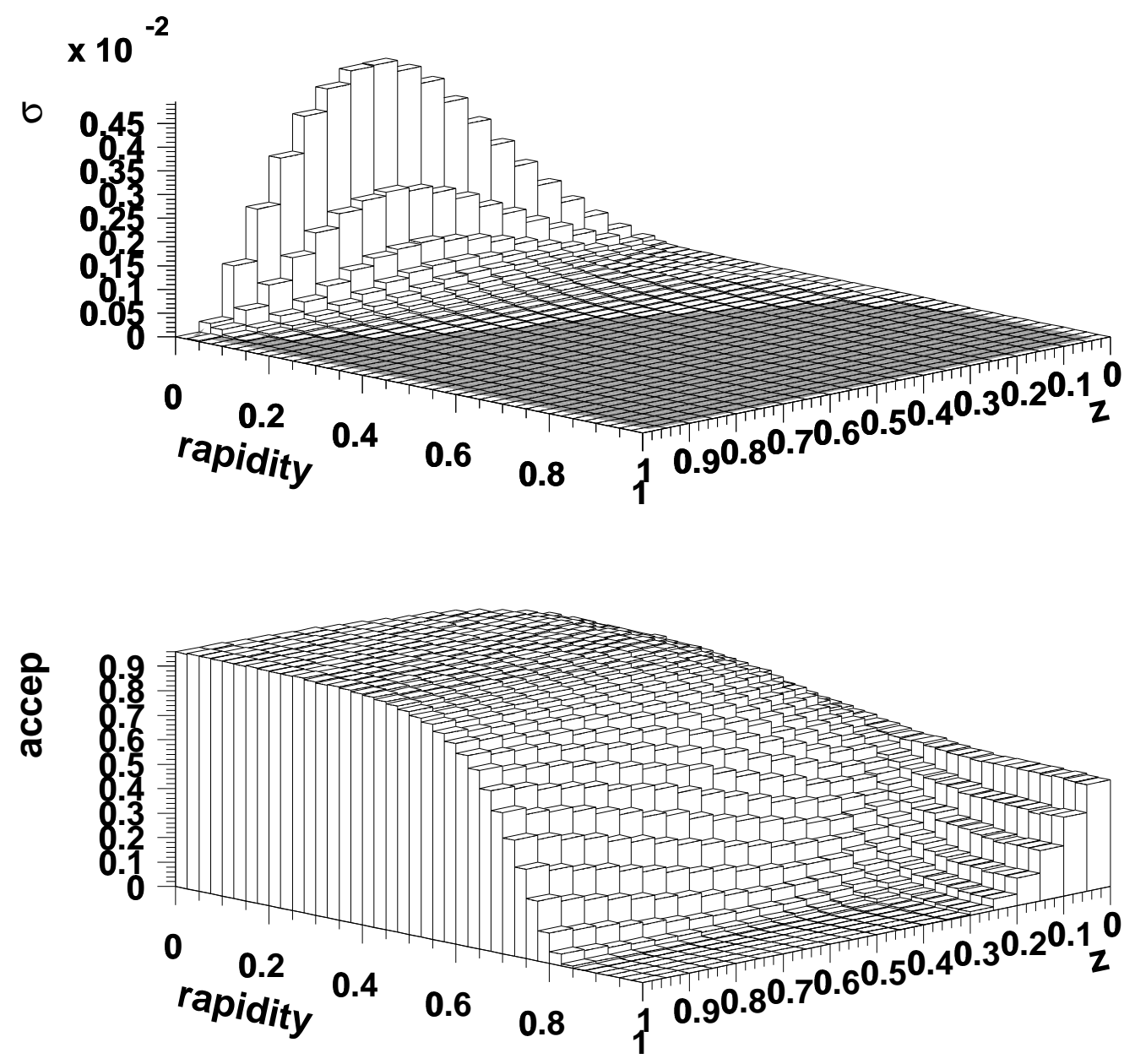

FIGURE 156. The $\sigma_{D I S C O}$ cross section (top) and $E_{\mu 2}>5 \mathrm{GeV}$ acceptance (bottom) plotted as a function of relative rapidity and $\mathrm{z}$. Plots are for $\nu$ table point $\left(E_{\nu}=247.0, x=0.101, y=0.324\right)$ For relative rapidity, 0 means collinear, and 1 means anti-collinear. $z=1$ means the charmed hadron keeps all of the charmed quark's momentum. 
(nonzero rapidity) becoming larger thanks to the higher $Q^{2}$ scale. As $Q^{2}$ decreases (or equivalently $m_{c}$ increases) there is less transverse momentum available for the charmed quark, causing the rapidity distribution to become more collinear (or another way of saying it is to become more leading-order like). This means all else being equal, the smaller the charm mass, the larger the off collinear component of the cross section, which makes acceptance smaller.

All else is not equal for the higher energy point in figure 156 though. Certainly the rapidity distribution has a larger off collinear component, but the higher energy gives better acceptance, now saturating at about 0.95 . There is also now a rise at off collinear rapidity and low $z$. Its better to think of this rise looking from low $z$ to higher $z$, i.e. as a dip as z increases, and the backwards going component at high rapidity gains strength. At this $E_{\nu}$, even a stationary $(z \rightarrow 0)$ charmed hadron in the W-parton CMS frame is boosted enough to have a significant number of decay muons pass the $E_{\mu 2}>5 \mathrm{GeV}$ cut in the Lab frame, giving the relatively high acceptance in the lowest $z$ bins. At high rapidity and finite $z$ though, the momentum vector points opposite to the boost to the lab frame, so that for $z \sim 1$ and rapidity $\sim 1$ none of the muons pass the cut.

Figure 157 shows the projection along the $z$ axis of the cross section and acceptance shown in figures 155 and 156, for the same cross section table bins. The distributions on the upper row are convolved with the acceptance curves below them in the figure to obtain the acceptance correction for each bin. Although the $z$ distributions are very similar, the acceptances as a function of $z$ at the two different $E_{\nu}$ 's are quite different. The acceptance at high $E_{\nu}$ will be higher. Similarly, figure 158 shows the $z$ acceptance distributions for the low $E_{\nu}$ table point 

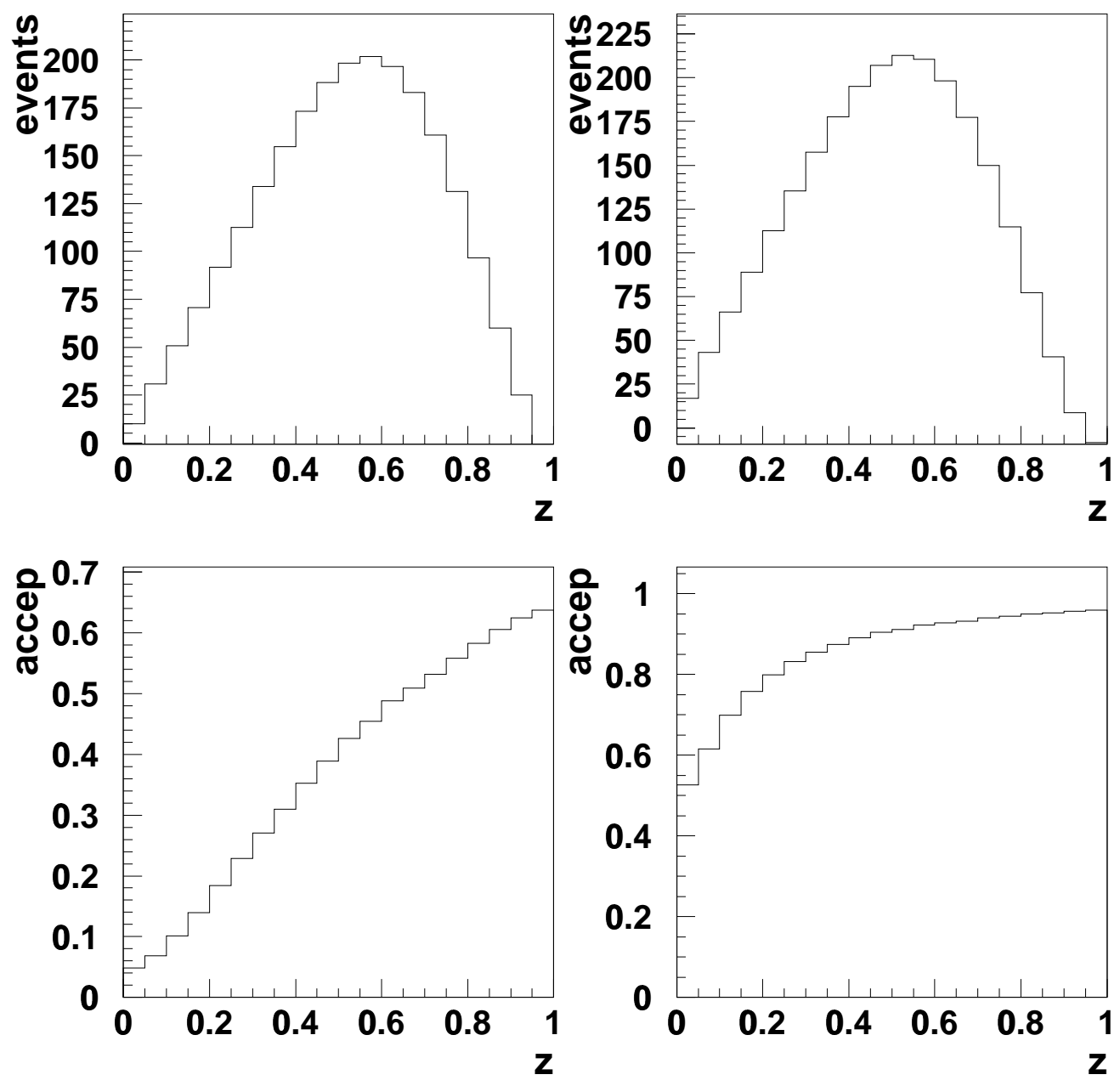

FIGURE 157. $z$ acceptance for high and low $E_{\nu}$ table bins. The top two plots show the average $z$ distribution and the bottom plots the raw acceptance for two table points: $\left(E_{\nu}=88.3, x=0.101, y=0.324\right)$ on the left, $\left(E_{\nu}=247.0, x=0.101, y=0.324\right)$ on the right.Both are plotted as a function of $z$. 

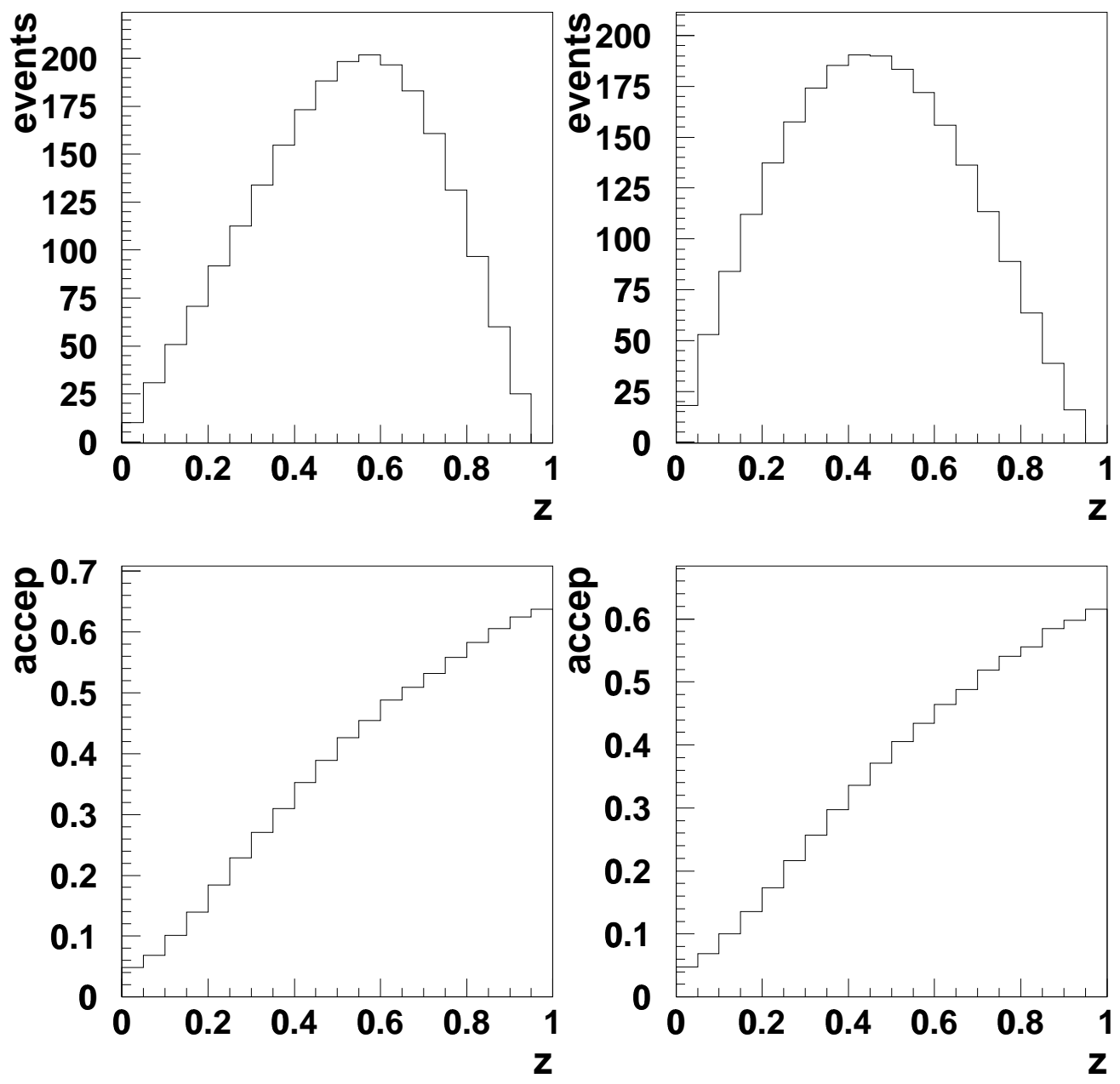

FIGURE 158. $z$ acceptance for hard and soft $\epsilon$. The top two plots show the average $z$ distribution and the bottom plots the raw acceptance for two values of $\epsilon: \epsilon$ of 0.5 on the left, and $\epsilon=1.5$ on the right. Both sets of distributions on the left and right correspond to the $\left(E_{\nu}=88.3, x=0.101, y=0.324\right) \nu$ table point, and are plotted as a function of $z$. 
with two different values of $\epsilon$, one harder (left two plots) and one softer (right two plots). Although the raw acceptances as a function of $z$ are virtually the same, the $z$ distribution of the softer $\epsilon$ has a peak at lower $z$, pulling in a greater contribution from lower $z$, and lower values of acceptance.

Figure 159 is similar to figure 157, but is a projection along the rapidity axis rather than $z$. The left and right columns again are for high and low values $E_{\nu}$. At higher $E_{\nu}$, the rapidity is slightly shifted more to off-collinear rapidity, however still strongly peaked near zero, where the raw acceptance is higher. This dominance of near collinear rapidity at $\mathrm{NuTeV} Q^{2}$ is why a leading order model is able to describe the data reasonably well. The three curves in each set of plots in figure 160 are the rapidity distributions at different charm masses. The raw acceptances for each charm mass are nearly the same, however the rapidity distribution for low charm mass pulls in a greater contribution from lower acceptance values of higher rapidity. The net result is lower overall acceptance with lower charm mass.

Figure 161 shows acceptance corrections as a function of fragmentation $\epsilon$ and charm mass for a sampling of four table points. There is a decrease with increasing (softer) $\epsilon$, and to a lesser extent, decreasing acceptance with decreasing charm mass. These points form a two dimensional grid in rapidity and $z$ which is linearly interpolated to find the acceptance correction appropriate for a particular value of $\epsilon$ and $m_{c}$.

Figures 162 and 163 compare acceptances from the DISCO-based NLO model and acceptances determined at LO with the same fragmentation $\epsilon$ and charm mass. The effect of rapidity is seen in the lower acceptance values for each table point at NLO. The relatively small difference between the leading order and NLO 

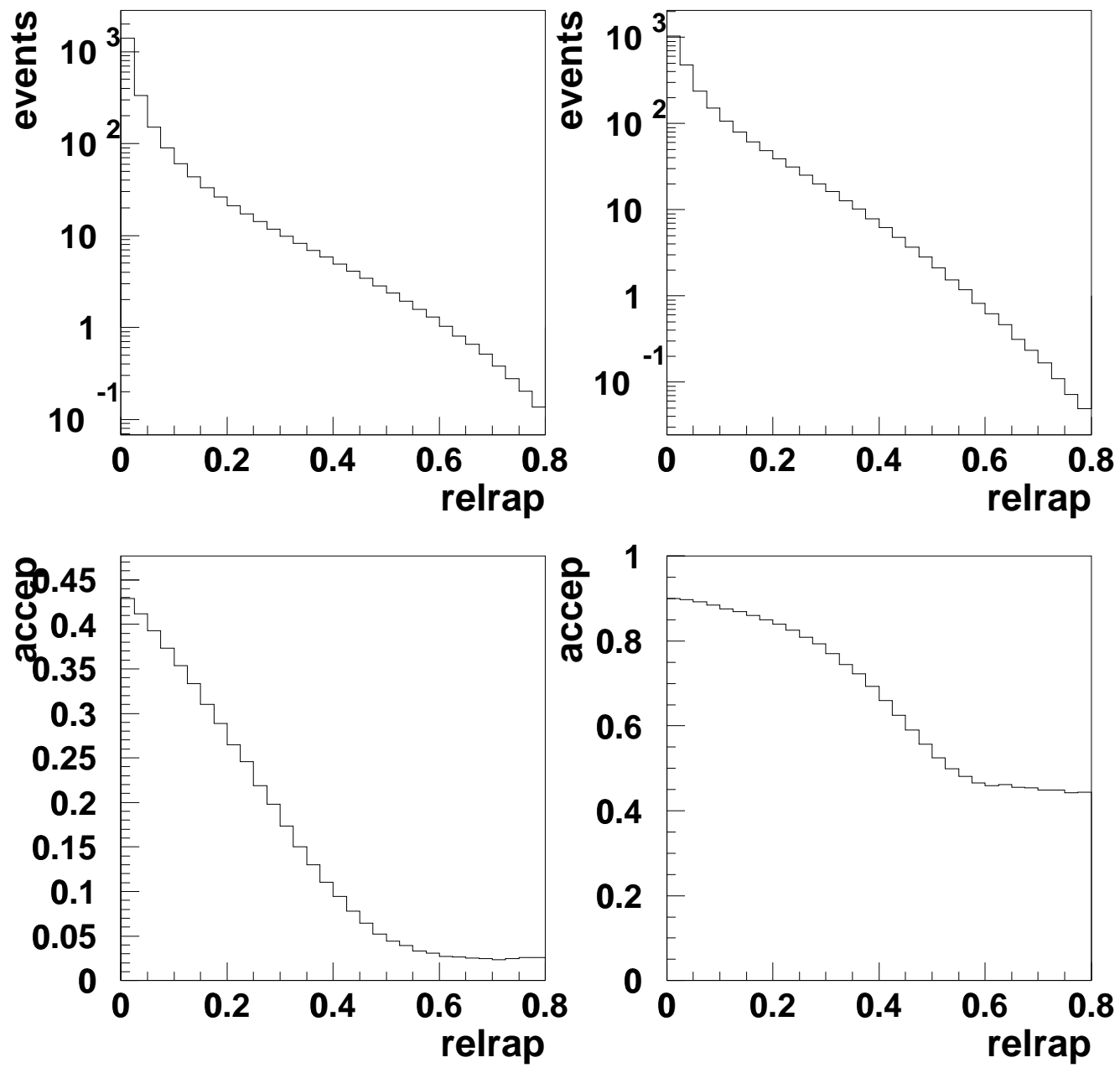

FIGURE 159. relative rapidity acceptance for high and low $E_{\nu}$ table bins. The top two plots show the average relative rapidity distribution and the bottom plots the raw acceptance for two table points: $\left(E_{\nu}=88.3, x=0.101, y=0.324\right)$ on the left, $\left(E_{\nu}=247.0, x=0.101, y=0.324\right)$ on the right.Both are plotted as a function of relative rapidity. 

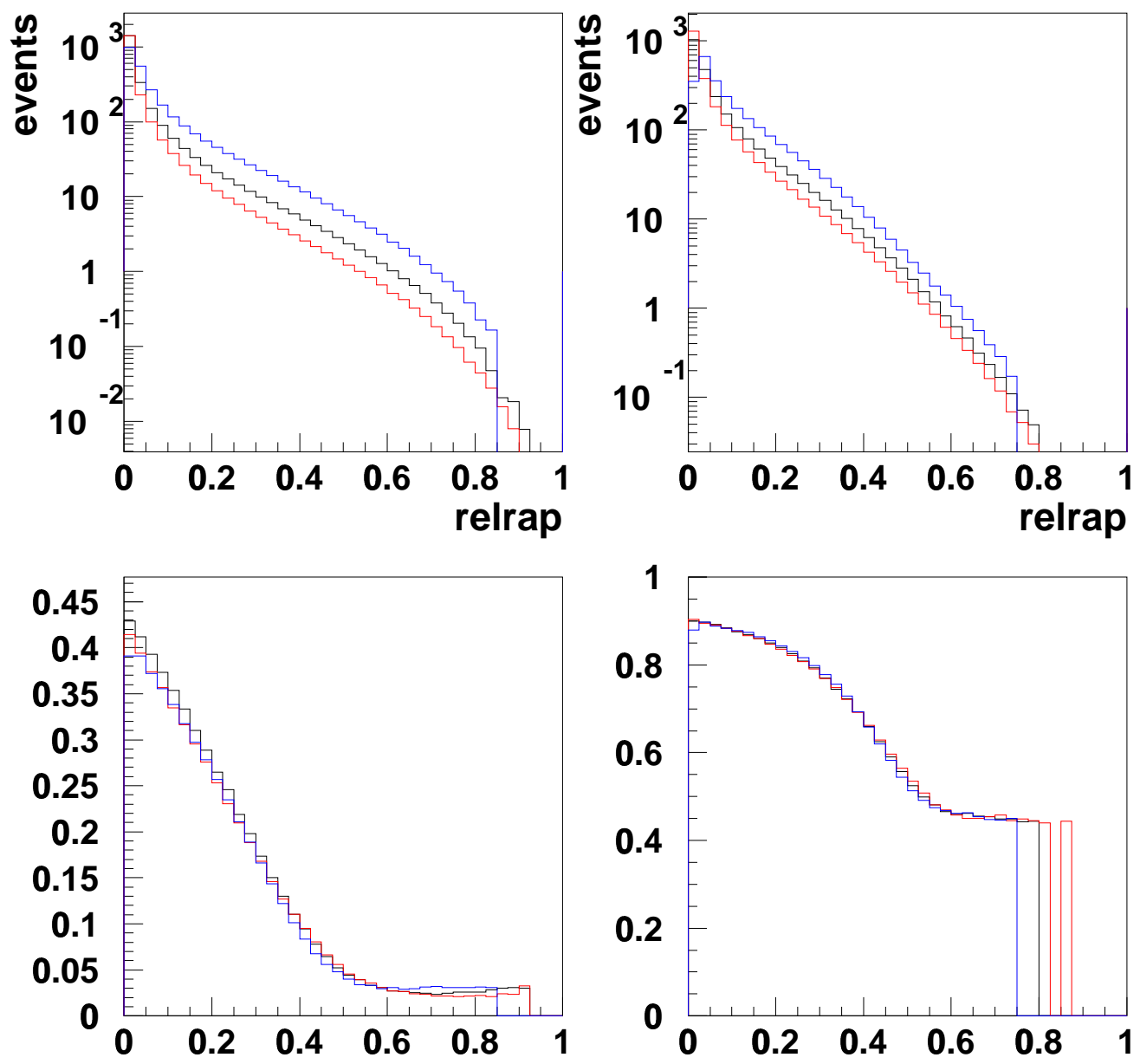

FIGURE 160. rapidity acceptance for high, low and medium $m_{c}$. Red curve corresponds to a $m_{c}$ of $1.7 \mathrm{GeV}$, Black for $m_{c}=1.25 \mathrm{GeV}$, and blue for a $m_{c}$ of 0.7 $\mathrm{GeV}$. Both sets of distributions on the left and right correspond to the $\left(E_{\nu}=88.3\right.$, $x=0.101, y=0.324) \nu$ table point 

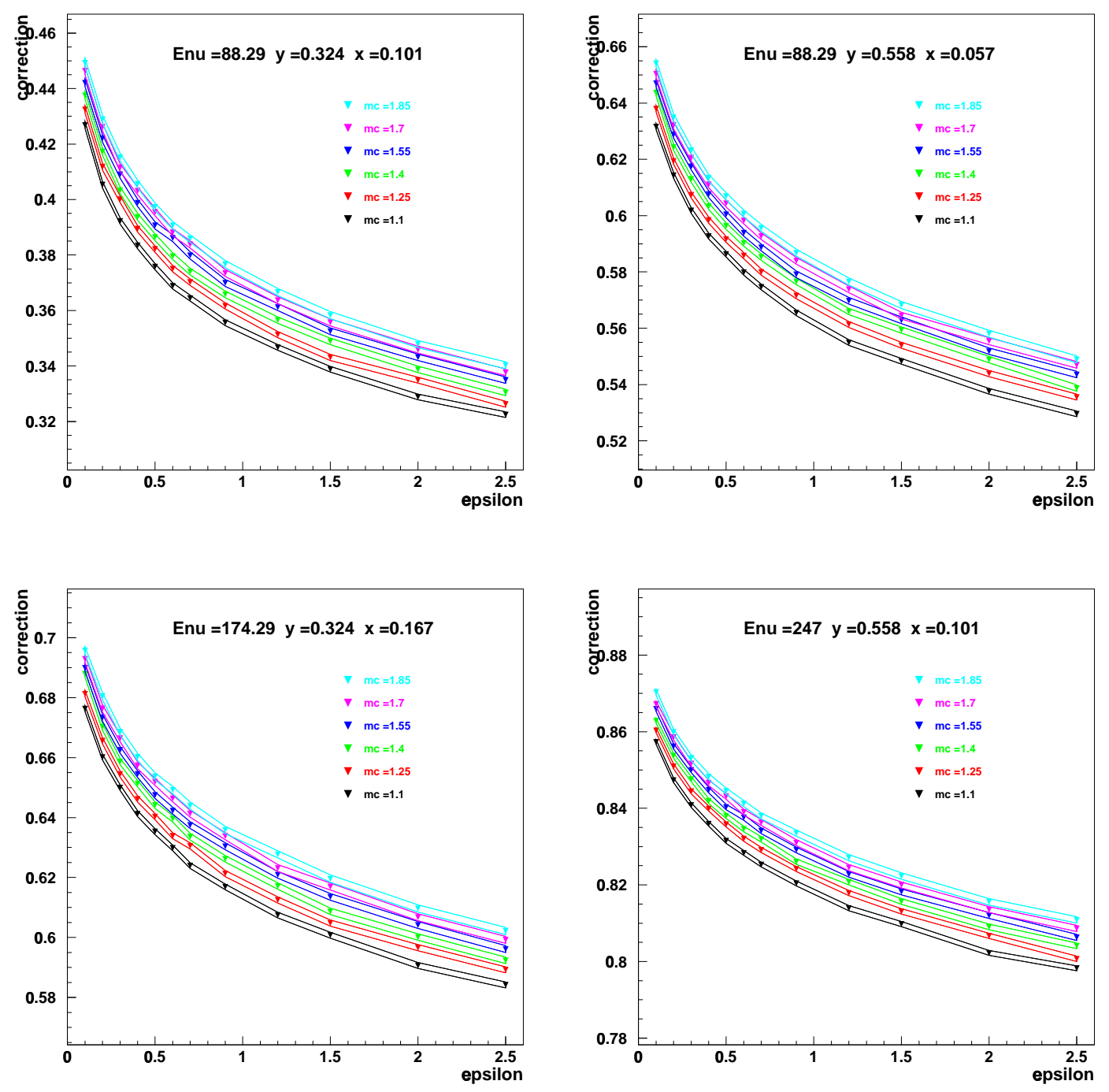

FIGURE 161. NLO acceptance corrections, plotted vs $\epsilon$ for each of the 6 charm mass points. Shown are 4 table bins spread from low to high $E_{\nu}$. There is a decrease in acceptance as $\epsilon$ increases (fragmentation becomes softer), and that acceptance slightly decreases with charm mass. 


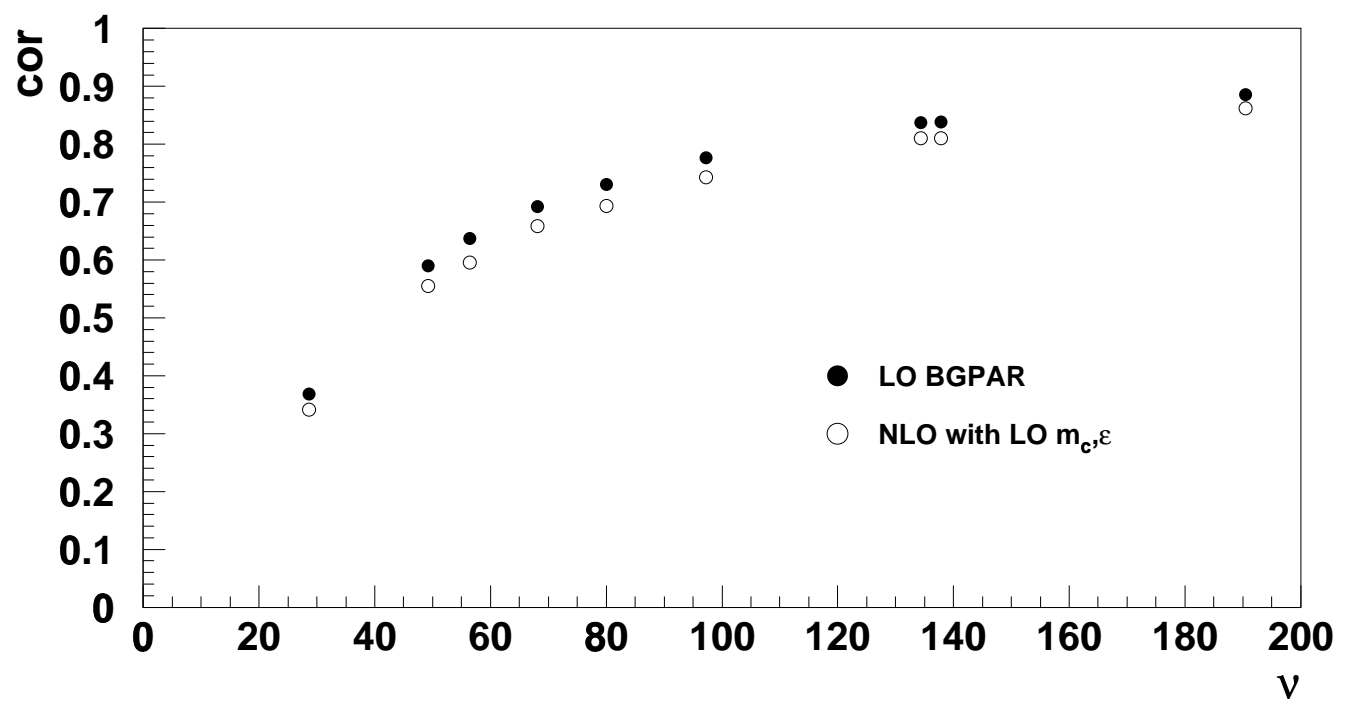

FIGURE 162. LO and NLO acceptances vs $\nu$. Plotted are $\nu$ acceptance point with $x=0.101$. Solid dots were obtained using the BGPAR LO model with the fitted parameters discussed in the previous chapter, the hollow points are with the NLO DISCO model, using those same parameters

acceptances is due to the sharply collinear distribution of the rapidity distribution at $\mathrm{NuTeV} Q^{2}$ 's. At higher values of $Q^{2}$ the rapidity distribution would be spread out over a wider range, making the LO model less appropriate for describing charm physics in, for example, environments like that of collider experiments. This is one of the reasons why it is important to perform this analysis with a model that is applicable for both situations, despite the fact that to a either LO or NLO may describe the $\mathrm{NuTeV}$ data. 


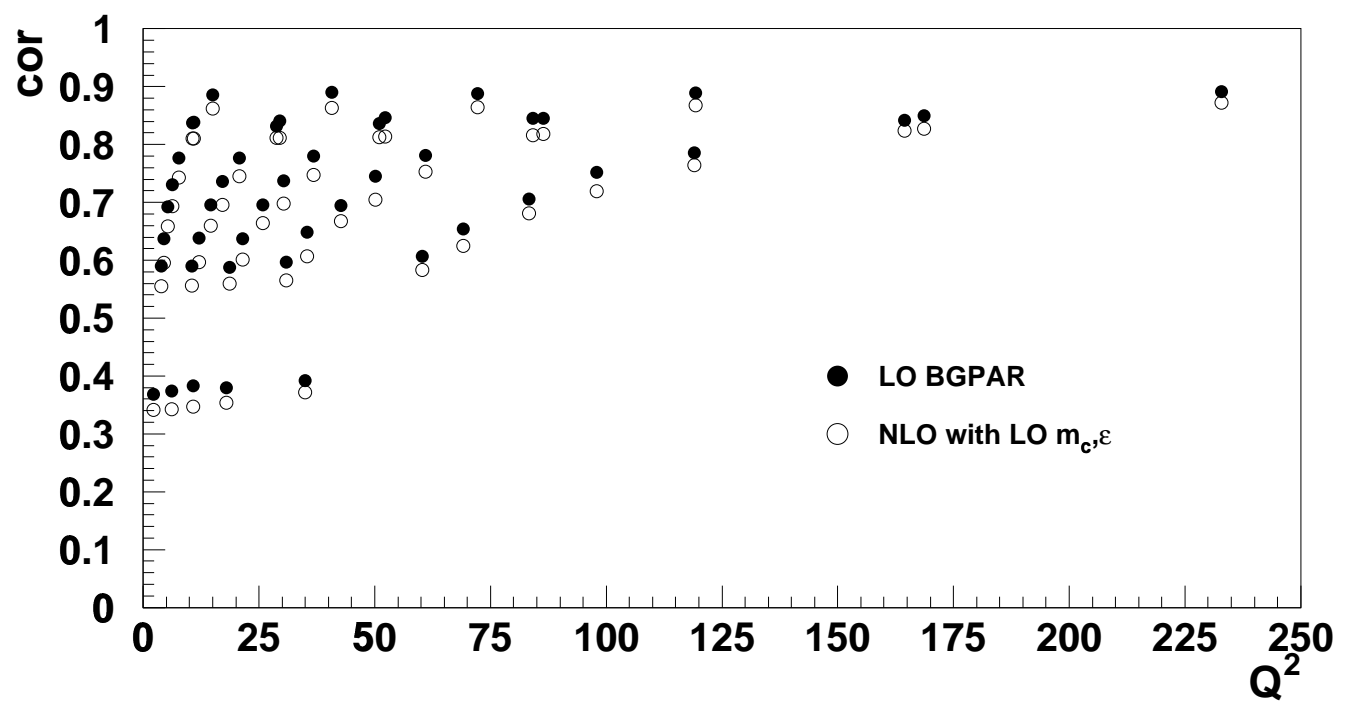

FIGURE 163. LO and NLO acceptances vs $Q^{2}$ for all $45 \nu$ table points. Solid dots were obtained using the BGPAR LO model with the fitted parameters discussed in the previous chapter, the hollow points are with the NLO DISCO model, using those same parameters 


\subsubsection{Charm Semi-muonic Branching Ratio}

The charm semi-muonic branching ratio, $B_{c}$, scales the weight given in the cross section by the average rate that each type of produced charmed meson decays semileptonically with a muon in the final state. As discussed back around page 96, the value of $B_{c}$ depends on both the semileptonic decay rate for each type of charmed meson as well as their relative production rate. The rate of production of each charmed meson is energy dependent. It is therefore important when using an external value for $B_{c}$ that it be for an energy range appropriate to $\mathrm{NuTeV}$. In our fits below, a value of $0.099 \pm 0.012$ is used, based on a re-analysis of 125 charm events measured by FNAL E531 $[164,124]$. More precise values of $B_{c}$ are available, however they include significant contributions from charmed hadrons produced at energies less than the $E_{\nu}>20 \mathrm{GeV}$ cut used in this analysis. Another emulsion based neutrino scattering experiment, CHORUS, recently performed a measurement of charm semileptonic decay events, and determined $B_{c}$ to be $0.102 \pm 0.016$ (stat) \pm 0.010 (syst) for events with $E_{\nu}>30 \mathrm{GeV}$ [174]. Figure 164 summarizes all other measurements of charm branching ratio, with the E531 number and the fit from the LO fit from the previous chapter highlighted for comparison.

\section{$\underline{5.3 \quad \text { NLO inclusive charm cross section model }}$}

After the acceptance corrections are determined, the computationally expensive DISCO cross section code is no longer needed. Only a cross section differential in $x$ and $y$ is needed when fitting the cross section tables. The integration over $z$ and $\eta_{c}$ eliminates an integral in the calculation, making the code for determining

$\frac{d \sigma_{\text {charm }}\left(m_{c}, s, \bar{s}\right)}{d x d y}$ considerably faster than DISCO. This in addition to only needing to 


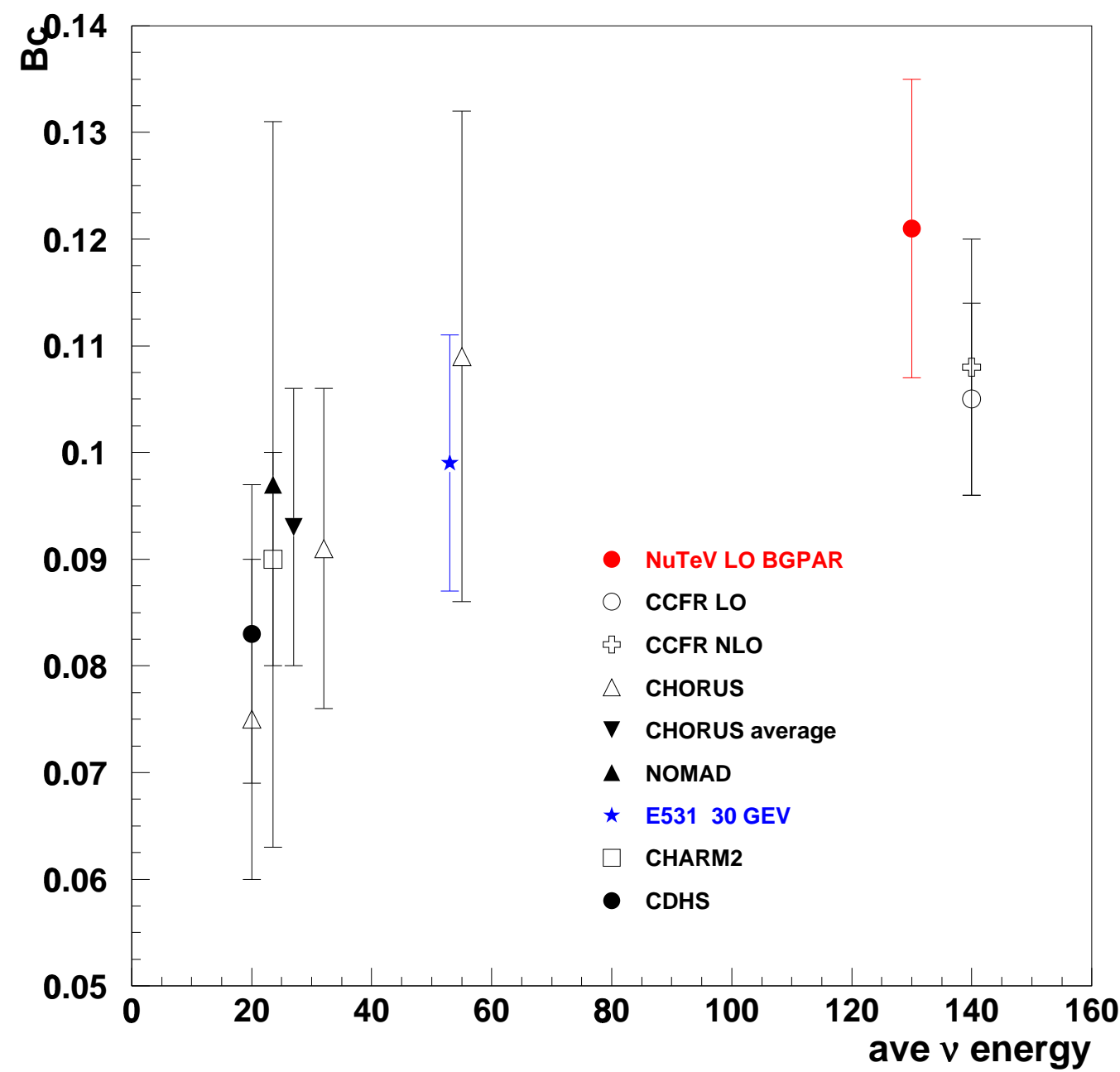

FIGURE 164. World measurements of the averaged charm semi-muonic branching ratio from neutrino DIS, including measurements from this analysis, CCFR [62, 63],CHORUS [174], NOMAD[69], E531 [164, 124], CHARM2 [74], and CDHS [58]. The E531 value, used in this analysis, is highlighted in blue. 
evaluate the cross section at the center values of each table bin makes NLO fitting to the cross section table a less painful endeavour than a fit to the raw data. Code based on the calculation in reference [133] is used to calculate the cross section in fits $[135]$

As discussed in section 2.4.2 starting back on page 88, the cross section is a sum of the charm contributing pieces of the three structure functions. These structure functions are the result of a convolution between a hard scattering term and the parton distribution functions, as described in section 2.5 on page 96. At NLO, there is a component dependent on the gluon pdf and a component dependent on the quark pdfs. The quark pdf component can be represented as a sum of all quark pdfs which can produce charm (or anticharm in the case of antineutrino scattering) multiplied by the appropriate CKM matrix elements squared. In section 2.5 it was found for an isoscalar target in neutrino scattering:

$$
\begin{aligned}
q\left(\xi, \mu^{2}\right)= & \left|V_{c d}\right|^{2}\left[d_{v}\left(\xi, \mu^{2}\right)+u_{v}\left(\xi, \mu^{2}\right)+d_{s}\left(\xi, \mu^{2}\right)+u_{s}\left(\xi, \mu^{2}\right)\right] \\
& +2\left|V_{c s}\right|^{2} s\left(\xi, \mu^{2}\right)
\end{aligned}
$$

And for antineutrinos:

$$
\bar{q}\left(\xi, \mu^{2}\right)=\left|V_{c d}\right|^{2}\left[d_{s}\left(\xi, \mu^{2}\right)+u_{s}\left(\xi, \mu^{2}\right)\right]+2\left|V_{c s}\right|^{2} \bar{s}\left(\xi, \mu^{2}\right)
$$

The NuTeV target however is not exactly an isoscalar target, which would have the same number of protons and neutrons. In this more general case then, for neutrino scattering, derived in a similar fashion as in the isoscalar case in chapter 
2, without now assuming an isoscalar target:

$$
\begin{aligned}
q\left(\xi, \mu^{2}\right)=\left|V_{c d}\right|^{2} & \frac{\left[Z d_{v}\left(\xi, \mu^{2}\right)+N u_{v}\left(\xi, \mu^{2}\right)+Z d_{s}\left(\xi, \mu^{2}\right)+N u_{s}\left(\xi, \mu^{2}\right)\right]}{A} \\
& +2\left|V_{c s}\right|^{2} s\left(\xi, \mu^{2}\right)
\end{aligned}
$$

and similarly for antineutrino mode:

$$
\bar{q}\left(\xi, \mu^{2}\right)=\left|V_{c d}\right|^{2} \frac{\left[Z d_{s}\left(\xi, \mu^{2}\right)+N u_{s}\left(\xi, \mu^{2}\right)\right]}{A}+2\left|V_{c s}\right|^{2} \bar{s}\left(\xi, \mu^{2}\right)
$$

here although not an isoscalar target, isoscalar symmetry is assumed, in that the d quark distribution in the proton is the same as the $\mathrm{u}$ distribution in the neutron. $\mathrm{Z}$ is the average atomic number of the target, $\mathrm{N}$ the number of neutrons per nucleon, and A the atomic mass. A survey of the NuTeV steel found:

$$
\begin{aligned}
& Z=23.403 \\
& N=26.215 \\
& A=49.618
\end{aligned}
$$

\subsubsection{Nuclear Corrections}

If the pdf set used in this analysis were based on iron,, as it was in the BGPAR case, adjusting up and down quark distributions according to the numbers of protons and neutrons in the target would suffice to account for the nuclear makeup of the target. Externally measured pdf sets are used here however, and these pdf sets are typically defined as the pdf distributions for the proton. 


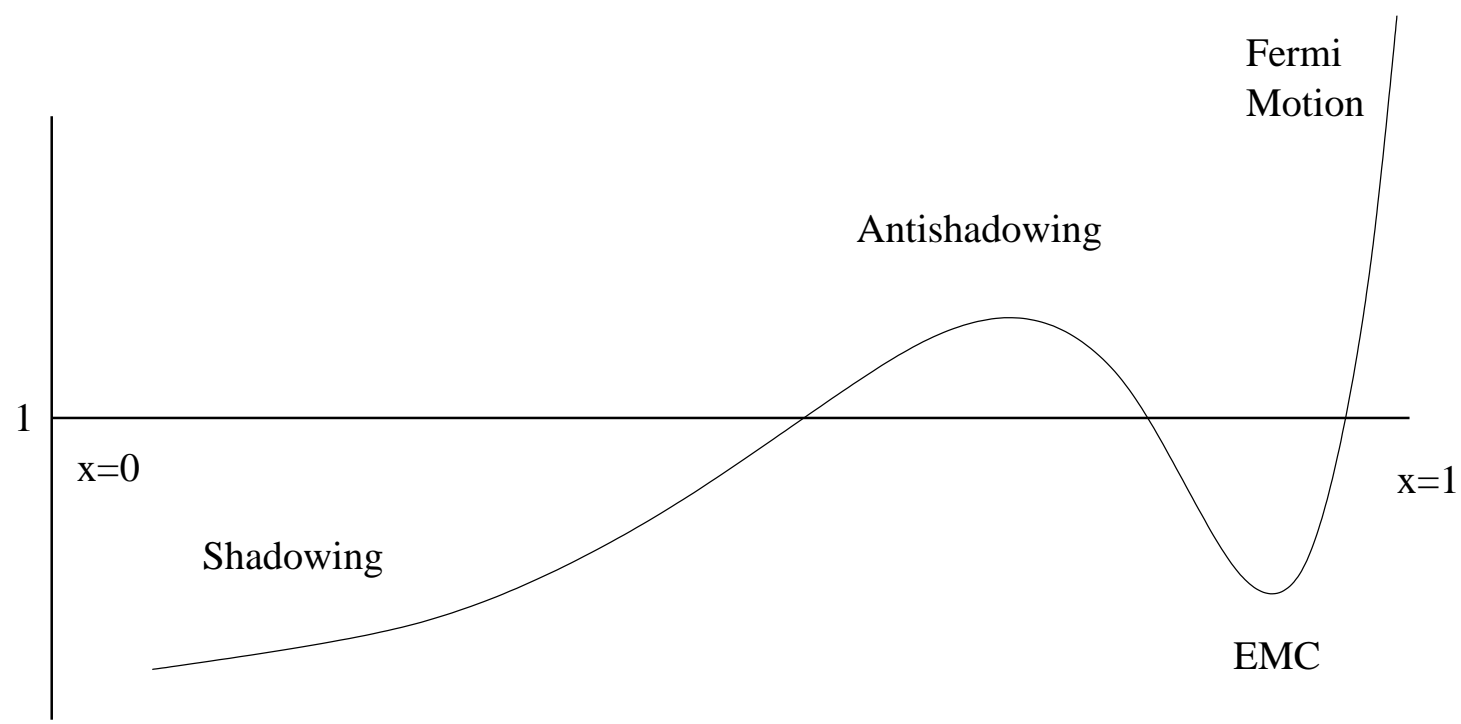

FIGURE 165. A cartoon of the the nuclear environment's effects on $F_{2}(x)$. Shown is the ratio of $F_{2}(x)$ for a multi-nucleon nucleus divided by $F_{2}(x)$ for deuterium plotted as a function of $x$. There is a suppression at low $\mathrm{x}$ due to shadowing, an enhancement at moderate $\mathrm{x}$, another suppression at moderate to high $\mathrm{x}$, followed by an enhancement at high $\mathrm{x}$ due to nucleon Fermi motion.

Scaling says that at high enough $Q^{2}$, the partons within a nucleon, and hence nucleus, should behave like free particles. For the most part this is true. One would then expect upon measuring $F_{2}^{A}(x)$ in scattering off different types of nuclei, that the ratio, $\frac{F_{2}^{A}}{F_{2}^{D}}$, would be 1 for all $x .\left(F_{2}^{D}\right.$, where "D" refers to deuterium, is typically used in the denominator because it is the simplest nucleus containing both a neutron and proton) The real situation however is sketched in figure 165 . This behavior is now well established experimentally, as discussed below, but not well understood theoretically.

At low $x$ there is a suppression of the multi-nucleon $F_{2}^{A}\left(\frac{F_{2}^{A}}{F_{2}^{D}}\right.$ below 1$)$ called the "shadowing" region. This behavior was seen from the first deep inelastic scattering experiments in the 1970's and is believed to come about when the interacting boson fluctuates into vector meson states (The model is called "vector meson 
dominance"). The intermediate vector mesons tend to interact strongly with the nucleons on the outer shell of the nucleus, which effectively "casts a shadow" on the interior nucleons, reducing the overall cross section [175].

Throughout the 1970's, and into the early 1980's, it was believed that nuclear shadowing was the only violation of scaling behavior in the structure functions, and that at higher $x$ they would behave as expected for free quarks. In 1983 however, the European Muon Collaboration (EMC) at CERN found a surprise deviation from free quark behavior when measuring the structure functions in muon scattering off hydrogen, deuterium, and iron targets [176]. When they looked at $\frac{F_{2}^{A}}{F_{2}^{D}}$, they found that instead of the flat line one would expect for free quarks, there was a downward sloping curve from moderate to high values of $x$. This suppression of the bound nucleon $F_{2}$ is now appropriately called the "EMC effect". Many models for the the EMC effect exist, usually having to do with nuclear binding effects or changes to the quark confinement behavior within the nuclear environment.

Beyond the "EMC" region, at extremely high $x$, the $F_{2}$ ratio rises significantly above 1. This rise is due to "Fermi motion", or that the nucleons are themselves moving within the nucleus. When defining Bjorken $x$ it was assumed that the nucleon was at rest, giving a maximum value for $x$ of 1 . Within the nucleus however the nucleon could have nonzero momentum, allowing $x$ (albeit infrequently) to be greater than 1. (Imagine the extreme case of the struck nucleon being slung at some high momentum towards the incoming boson, while the rest of the nucleus slightly wobbles away from it, then on top of that have the interaction happen with a quark in that nucleon with a high value of $x$. In that case it is possible to 
have a quark momentum greater than the nucleon momentum were it to be at rest relative to the nuclear center of mass.) When taking the ratio of $F_{2}$ for a large nuclear target to that of a proton, there is a component that, although small, is nonzero as $x \rightarrow 1$, divided by something approaching zero (and which is exactly zero at $x=1$ ). This causes the ratio to blow up, hence the enhancement at high $x$ [84].

The simplest way to correct for the nuclear environment's effects on the scattering cross section is to make use of the measured ratios of $F_{2}$ for the target versus that for deuterium. One could fit existing data with some kind of empirical parameterization, and use that to modify $F_{2}$ for the proton to match the $\mathrm{NuTeV}$ target. There is no evidence that $R_{L}$ is sensitive to nuclear effects [175], so for charged lepton scattering at least, the $F_{2}$ ratio could be used to correct the total cross section. There is also evidence from the BEBC experiment at CERN [177] that neutrino scattering has the same shadowing/EMC/Fermi motion behavior as charged lepton scattering. This approach has been used in the past by both the CCFR and $\mathrm{NuTeV}$ collaborations when comparing the application of external pdf sets to their measurements [153].

Recently however, several groups $[178,179,180,181]$ have taken a card from those who do global fits to determine parton distribution functions, and have produced sets of "nuclear parton distribution functions", or npdfs. The nuclear contribution to the scattering cross section results from a convolution with the bare nucleon pdfs, i.e with the structure functions looking something like:

$$
\mathcal{F}^{A}\left(x, Q^{2}\right)=\omega\left(x, Q^{2}\right) \otimes f_{q, g}\left(x, Q^{2}\right) \otimes \mathcal{N}_{q, g}\left(A, x, Q^{2}\right)
$$




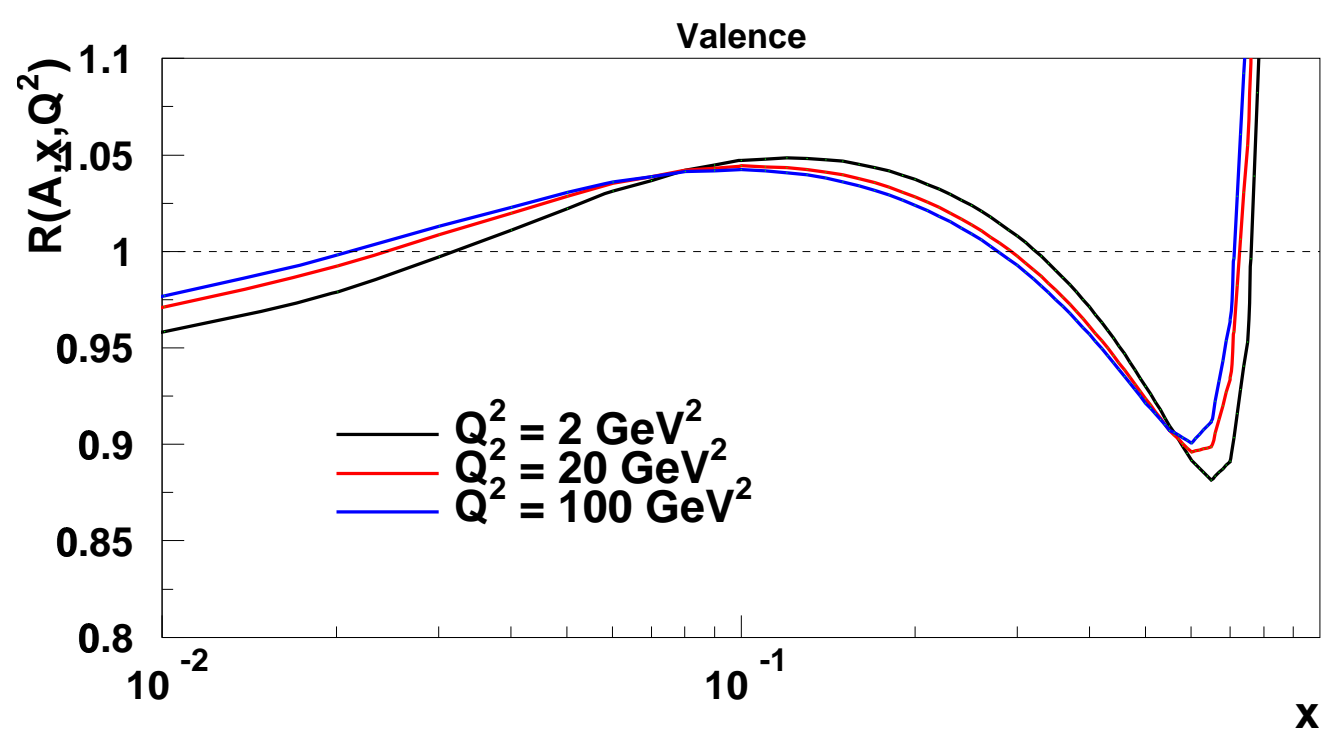

FIGURE 166. Nuclear correction to the valence quark distributions for three values of $Q^{2}$

i.e. a convolution between a hard scattering term $\omega\left(x, Q^{2}\right)$, the bare nucleon pdf $f_{q, g}\left(x, Q^{2}\right)$ and a nuclear component $\mathcal{N}_{q, g}\left(A, x, Q^{2}\right)$. What is typically done to find $\mathcal{N}_{q, g}\left(A, x, Q^{2}\right)$ (or sometimes the combination of $f_{q, g}\left(x, Q^{2}\right) \otimes \mathcal{N}_{q, g}\left(A, x, Q^{2}\right)$ ) is to assemble the world's deep inelastic scattering data off various nuclear targets, usually in the form of $\frac{F_{2}^{A}}{F_{2}^{D}}$ ratios, and to fit a parameterization of $\mathcal{N}_{q, g}\left(A, x, Q^{2}\right)$ assuming a particular pdf set.

In this analysis the npdfs from de Florian et al [179] will be used. They are the first npdfs extracted at NLO in QCD, and include both dependence on atomic mass $A$ and $Q^{2}$. The corrections are provided in the form of an interpolated table in $A, x$, and $Q^{2}$ for valence and sea quarks, and gluons of the form:

$$
R_{q, g}\left(A, x, Q^{2}\right)=\frac{f_{q, g}\left(x, Q^{2}\right) \otimes \mathcal{N}_{q, g}\left(A, x, Q^{2}\right)}{f_{q, g}\left(x, Q^{2}\right)}
$$

Figures 166-168 plot $R_{q, g}\left(A, x, Q^{2}\right)$ for valence, sea and gluon distributions for three 


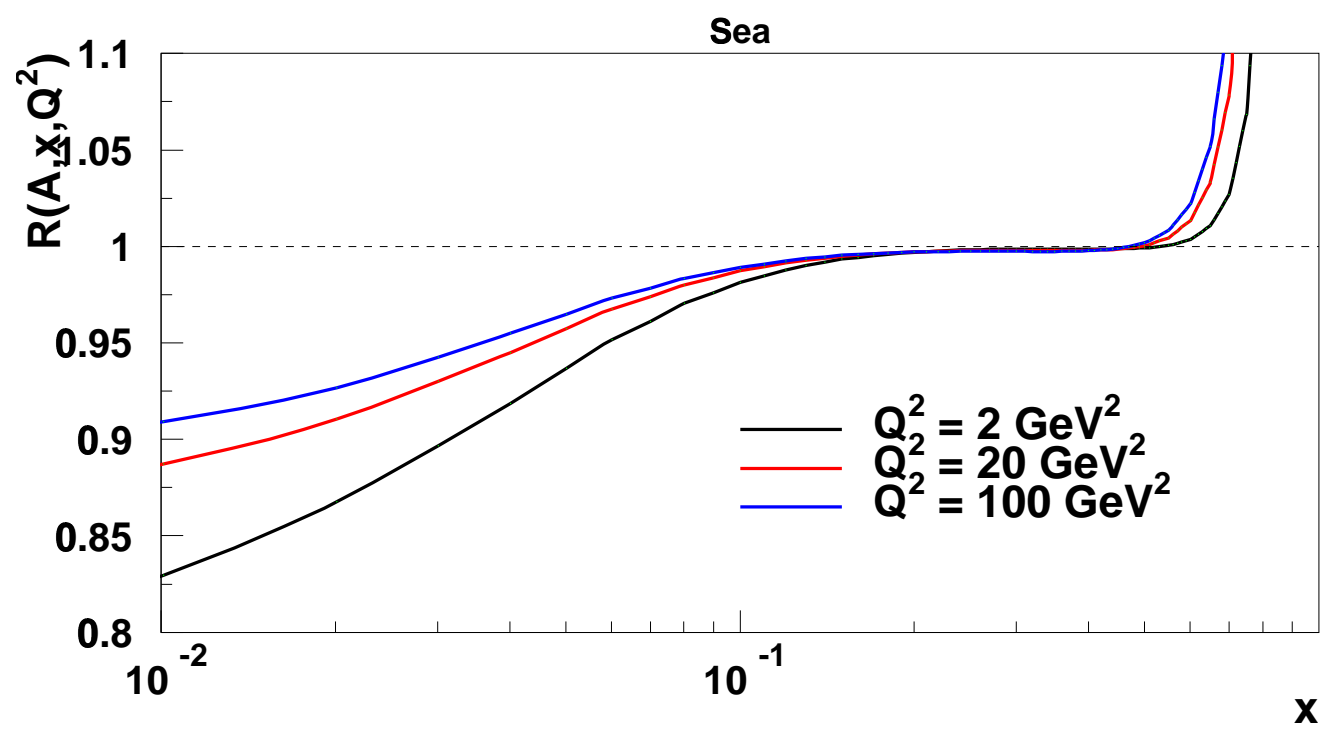

FIGURE 167. Nuclear correction to the sea quark distributions for three values of $Q^{2}$

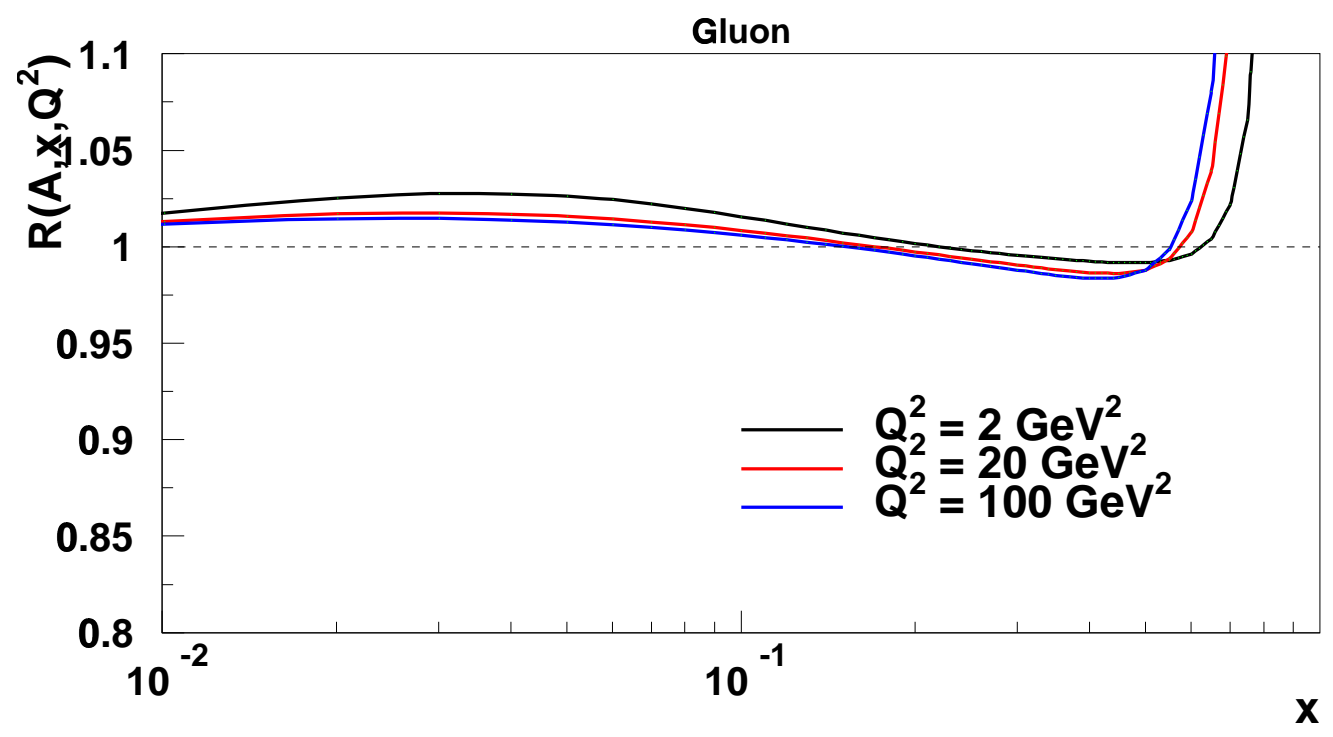

FIGURE 168. Nuclear correction to the gluon distributions for three values of $Q^{2}$ 
values of typical $\mathrm{NuTeV} Q^{2}$. As one would expect, the magnitude of the nuclear corrections begin to approach 1 as $Q^{2}$ increases. The valence quark distributions have the familiar structure seen in $\frac{F_{2}^{A}}{F_{2}^{D}}$, however the sea and gluon distributions have a different shape, most notably a virtual absence of the EMC effect. This

says that applying a simple nuclear correction along the lines of a global $\frac{F_{2}^{A}}{F_{2}^{D}}$ to both the neutrino charm cross section (stemming from $\sim 40 \%$ valence, $\sim 60 \%$ sea) and antineutrino charm cross section (100\% sea) would be a bad idea, especially when the goal is to understand the difference between the strange and antistrange distributions.

The rather busy figure 169 compares the de Florian nuclear corrections to corrections from Hirai et al $[178,180]$. The Hirai nuclear corrections are based on a leading order fit, and have slightly different assumptions regarding the EMC effect and shadowing. They also have a correction for the strange sea, though its likely not very well constrained. The Hirai npdfs will be used to get some idea of the systematic uncertainty due to the nuclear corrections.

\subsubsection{Strange Sea Modeling}

The primary goal of this analysis is to use the forward dimuon cross section to measure the strange and antistrange seas within an NLO QCD framework. Thus far the layers separating the observed dimuons from the underlying physics have been peeled back - from the acceptance corrections, charm fragmentation and decay, cross section model, nuclear effects, and now down to the parton distributions themselves. The cross section table fits will be based on the CTEQ6M pdf set [182]. It was the first of the major pdf sets to include estimates of the pdf 
uncertainties, and results from from an NLO fit to world deep inelastic scattering data over a large range of $Q^{2}$. The pdf set is treated as an external measurement of the gluon and nonstrange quark distributions.

During the fit process the strange and antistrange seas are varied. In previous dimuon measurements this has been done by defining the strange sea to be a modification of the up and down seas by a level and (sometimes) a shape parameter like:

$$
s\left(x, Q^{2}\right)=\kappa(1-x)^{\alpha}\left[\frac{\bar{u}\left(x, Q^{2}\right)+\bar{d}\left(x, Q^{2}\right)}{2}\right]
$$

where $\kappa$ scales the overall size of the sea, and $\alpha$ affects the shape of the strange sea relative to the nonstrange sea at the $Q^{2}$ of the experiment. A $\kappa$ of 1 and $\alpha$ of 0 would indicate an $\mathrm{SU}(3)$ symmetric sea, i.e. that there is no difference between the strange and nonstrange quarks in the sea.

In QCD however, the evolution of parton distribution functions is governed by the integro-differential DGLAP equation (equation 2.156 back on page 107). One cannot modify the shape of a pdf with an arbitrary function of $x$ and still expect the result to be a solution to the DGLAP equation. In other words, the values of $\kappa$ and $\alpha$ obtained by fitting with equation 5.282 would only be applicable near the $Q^{2}$ for which they were measured in, and could produce an incorrect sea much beyond that scale.

A more appropriate way to describe the strange sea would be to define the parameterization at a particular scale, $Q_{0}$, then use the DGLAP equations to evolve the pdfs to the values of $Q^{2}$ appropriate for the measurement. The strange 
sea would then become:

$$
s\left(x, Q_{0}^{2}\right)=\kappa(1-x)^{\alpha}\left[\frac{\bar{u}\left(x, Q_{0}^{2}\right)+\bar{d}\left(x, Q_{0}^{2}\right)}{2}\right]
$$

and a similar function for the antistrange sea could be defined:

$$
\bar{s}\left(x, Q_{0}^{2}\right)=\bar{\kappa}(1-x)^{\bar{\alpha}}\left[\frac{\bar{u}\left(x, Q_{0}^{2}\right)+\bar{d}\left(x, Q_{0}^{2}\right)}{2}\right]
$$

where $\bar{\kappa}$ and $\bar{\alpha}$ serve the same function as $\kappa$ and $\alpha$ in the strange sea. Technically a few rules should be followed however. The first of which is that because the proton does not have net strangeness, the integral over all $x$ of the difference between the strange and antistrange seas should be zero:

$$
\int_{0}^{1}\left[s\left(x, Q_{0}^{2}\right)-\bar{s}\left(x, Q_{0}^{2}\right)\right] d x=0
$$

This can be achieved by constraining one of the parameters such that this integral, the strange flavor sum rule, is zero. With the parameterization above, this leads to a fairly inflexible model with which to look for differences in the strange and antistrange seas. A more general parameterization, based on one used by the CTEQ group is as follows [183]:

$$
\begin{aligned}
s^{+}\left(x, Q_{0}^{2}\right) & =\kappa^{+}(1-x)^{\alpha^{+}} x^{\gamma^{+}}\left[\bar{u}\left(x, Q_{0}^{2}\right)+\bar{d}\left(x, Q_{0}^{2}\right)\right] \\
s^{-}\left(x, Q_{0}^{2}\right) & =s^{+}\left(x, Q_{0}^{2}\right) \tanh \left[\kappa^{-}(1-x)^{\alpha^{-}} x^{\gamma^{-}}\left(1-\frac{x}{x_{0}}\right)\right] \\
s & =\frac{s^{+}+s^{-}}{2} \quad \bar{s}=\frac{s^{+}-s^{-}}{2}
\end{aligned}
$$


where $\kappa^{+}, \alpha^{+}$, and $\gamma^{+}$, as well as their counterparts $\kappa^{-}, \alpha^{-}$, and $\gamma^{-}$control the size and shapes of the singlet $s^{+}\left(x, Q_{0}^{2}\right)$ and nonsinglet $s^{-}\left(x, Q_{0}^{2}\right)$ strange distributions. The strange flavor sum rule:

$$
\int_{0}^{1}\left[s\left(x, Q_{0}^{2}\right)-\bar{s}\left(x, Q_{0}^{2}\right)\right] d x=\int_{0}^{1} s^{-}\left(x, Q_{0}^{2}\right) d x=0
$$

in this parameterization is enforced for a particular $\left(\kappa^{+}, \alpha^{+}, \gamma^{+}, \kappa^{-}, \alpha^{-}, \gamma^{-}\right)$parameter set by numerically solving for the $x_{0}$ which zeroes out the integral. In code this is done through a simple search routine when the pdfs are initialized. The interval from $x=0$ to $x=1$ is divided into ten evenly spaced points where equation 5.289 is evaluated. The two points where the integral changes sign is then in turn divided in to ten more points, and the process is repeated until the desired precision is achieved. This likely sounds incredibly inefficient, however compared to the cross section calculation itself, it does not consume a significant amount of CPU time.

One last condition must be satisfied in the trip to full QCD compliance. The strange and antistrange seas are being modified within an already consistent pdf set. One of the rules that must be followed for the DGLAP equation to apply is that the total momentum distribution of the parton distributions must sum to 1:

$$
\begin{aligned}
1= & \int_{0}^{1} \sum_{\text {flavor i }}\left[x q_{i}\left(x, Q_{0}^{2}\right)+x \overline{q_{i}}\left(x, Q_{0}^{2}\right)\right] d x+\int_{0}^{1} x g\left(x, Q_{0}^{2}\right) d x \\
= & \int_{0}^{1} \sum_{\text {nonstrange i }}\left[x q_{i}\left(x, Q_{0}^{2}\right)+x \overline{q_{i}}\left(x, Q_{0}^{2}\right)\right] d x+ \\
& \int_{0}^{1} x s^{+}\left(x, Q_{0}^{2}\right) d x+\int_{0}^{1} x g\left(x, Q_{0}^{2}\right) d x
\end{aligned}
$$


where the $x s^{+}\left(x, Q_{0}^{2}\right)$ term has been pulled out of the quark momentum sum for emphasis. To conserve momentum in the fit, when $x s^{+}\left(x, Q_{0}^{2}\right)$ changes it must do so either in a way that doesn't change its integral, or the quark or gluon distributions must be changed to compensate. Constraining the $x s^{+}\left(x, Q_{0}^{2}\right)$ integral is not really desirable when trying to measure the strange sea, and the other quark sea distributions cannot be changed without external constraints. The gluon distribution however provides wiggle room here [184]. The gluon distribution is very large (about half the proton's total momentum), and the uncertainty on its measurement is also large (of order 10\%). Since any deviation in $x s^{+}\left(x, Q_{0}^{2}\right)$ during the fit is expected to be small, a small amount of momentum may safely be "stolen" from the gluon distribution to preserve momentum conservation. When establishing the pdfs for a particular set of strange parameters, both the integral of $x s^{+}\left(x, Q_{0}^{2}\right)$ and of $x g\left(x, Q_{0}^{2}\right)$ are evaluated, and the gluon distribution is rescaled by the amount required to maintain the momentum sum at 1 . In practice, in all the fits performed in this way, never more than a fraction of a percent of the gluon momentum is required to compensate for any change in the total strange and antistrange sea momenta.

Before proceeding to discussing fits and results, it should be noted that although achieving full QCD compliance is the moral and upstanding thing to do from a theoretical standpoint, it may not necessarily be the best thing to do from an experimental standpoint. Any experiment is limited in the range in $x$ that it may measure. In order to evaluate the integrals required to determine the various QCD sum rules, assumptions must be made for the behavior of the pdfs beyond the reach of the experiment. These assumptions may introduce biases in the measure- 
ment by that experiment. In the fits that follow, not only are the fits performed with the QCD compliant scheme outlined above, the ability to go back to less sophisticated schemes has been preserved for their flexibility. 


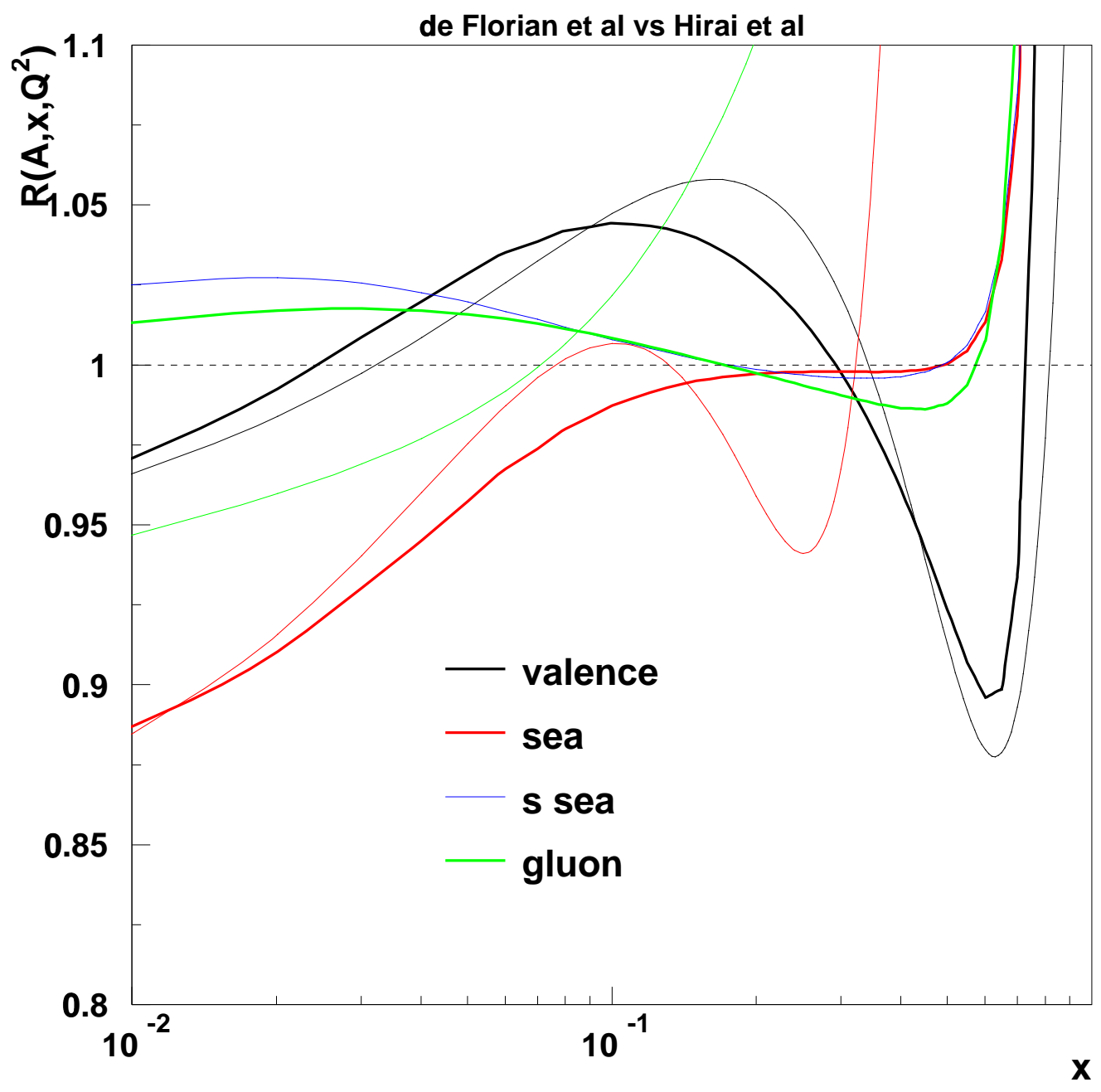

FIGURE 169. Comparison of the de Florian et al [179] nuclear corrections with the Hirai et al [180] nuclear corrections. The bold curves are from de Florian, the lighter curves are from Hirai. Hirai also includes a separate strange sea correction which roughly follows that of the gluon. 


\section{$\underline{5.4 \quad \mathrm{NLO} \text { table fits }}$}

All of the pieces required to perform fits to the dimuon cross section are now in place. Figures 170 and 171 show an example of an NLO fit to the cross section tables. Essentially all of the fits discussed below result in similar agreement with the table data points.

\subsubsection{NLO Systematic Uncertainties and Fragmentation}

Uncertainties on the table fits come from three sources. The first is due to statistics, thanks to the finite amount of data used in constructing the cross section table. The second is from systematic effects in extracting the table, such as uncertainties in energy calibrations, the $\pi-K$ background, etc, as described in section 4.5 .2 on page 296. The final source of uncertainty comes from the external ingredients in the model fitted to the table, be it from physics parameters, or parton distribution functions.

One weakness of condensing the dimuon data into the dimuon cross section tables is that some information is lost in the process. In the leading order fits, we were able to fit for the charm mass, fragmentation epsilon, strange sea parameters and the charm branching ratio simultaneously. In fitting to the cross section table, for example, charm fragmentation only comes into play via the acceptance corrections, so is not well constrained in a table fit.

External measurements are used for $m_{c}$ and $B_{c}$ in the table fits. The world average for charm mass is $1.20 \pm 0.10 \mathrm{GeV}$ [78], which is much more precise than can be achieved with fits to the $\mathrm{NuTeV}$ data alone. A fit allowing $m_{c}$ to float 

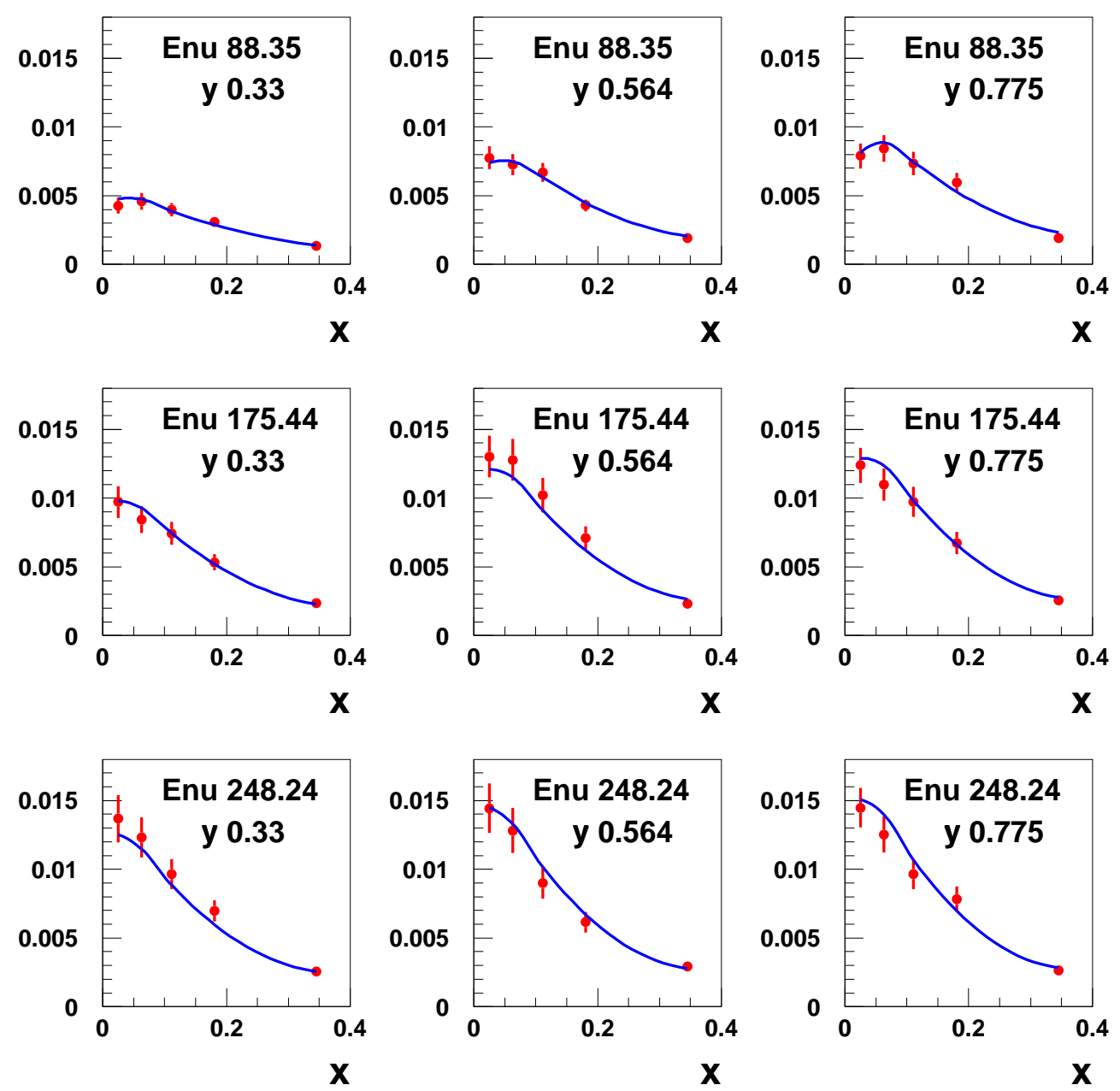

FIGURE 170. Fit of DISCO corrected $\nu$ NLO cross section to Dimuon cross section data. Points are shown multiplied by $\frac{\pi}{G_{F}^{2} M E_{\nu}}$ 

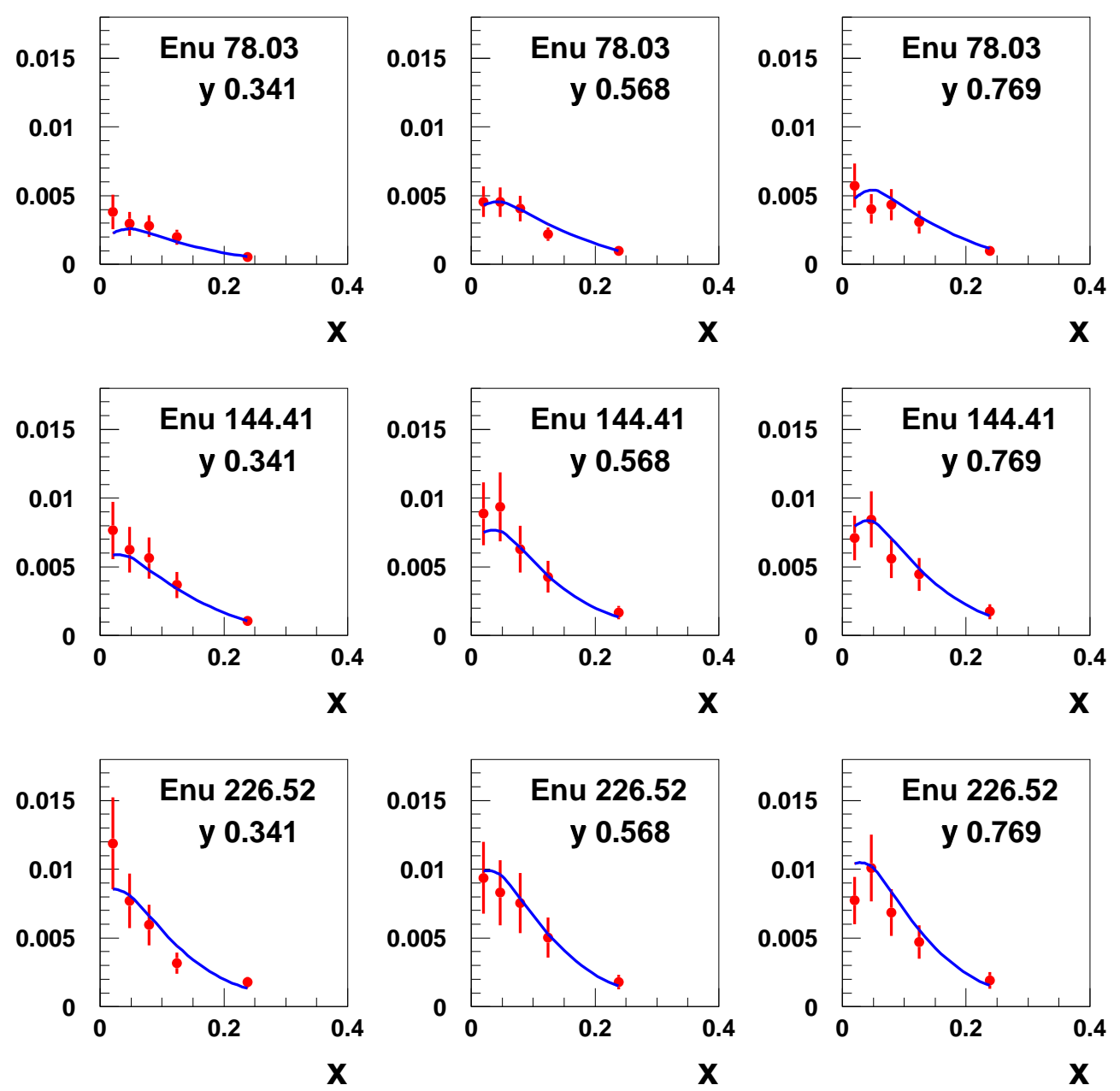

FIGURE 171. Fit of DISCO corrected $\bar{\nu}$ NLO cross section to Dimuon cross section data. Points are shown multiplied by $\frac{\pi}{G_{F}^{2} M E_{\nu}}$ 

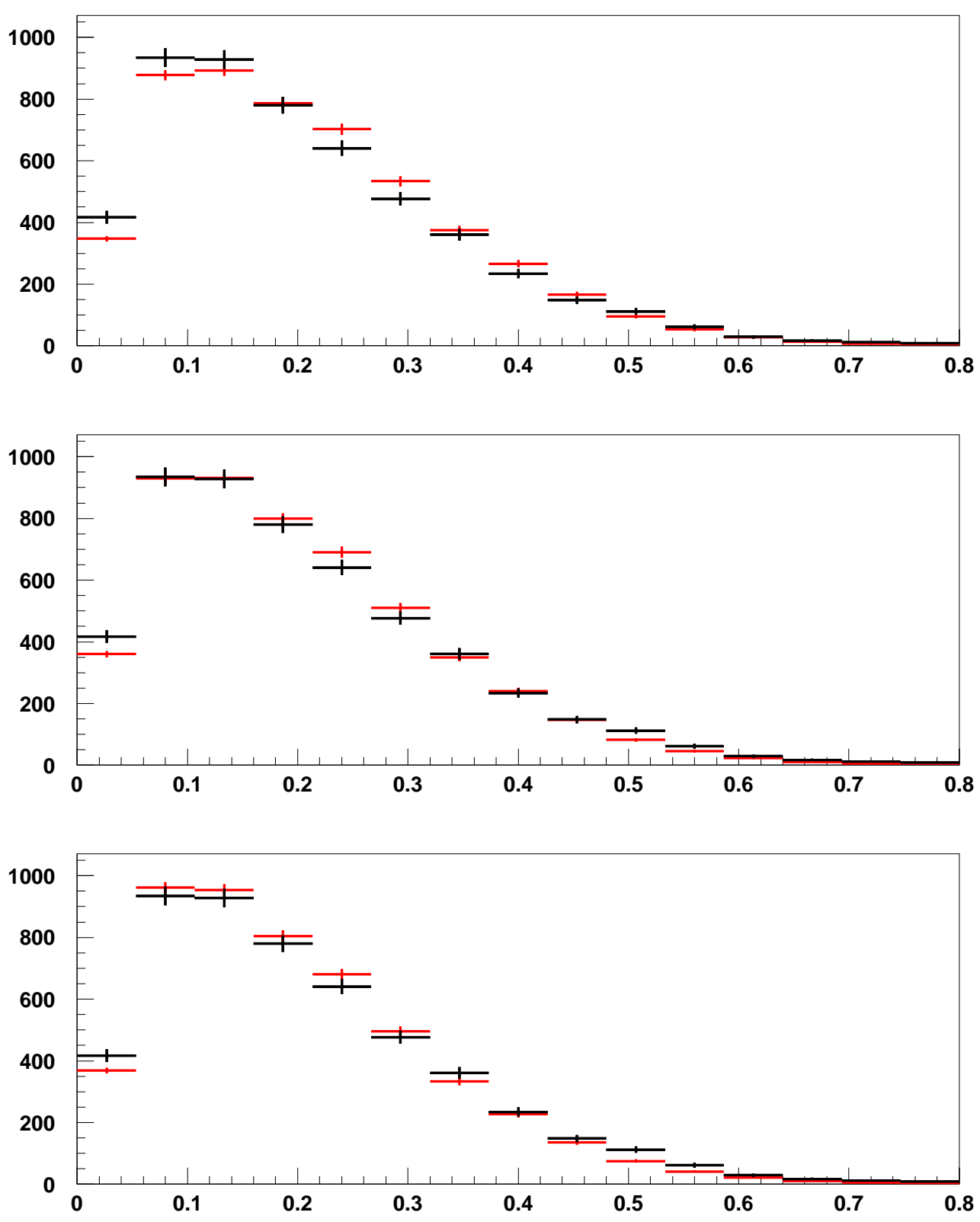

FIGURE 172. Data/MC zvis comparisons for $\epsilon=0.3$ (top),0.6 (middle), and 0.9 (bottom). MC is in red, data in black. There is an excess at higher $z_{v i s}$ with the low, harder $\epsilon$ value, moving towards an excess at low $z_{\text {vis }}$ with the softer $\epsilon=0.9$ plot. 


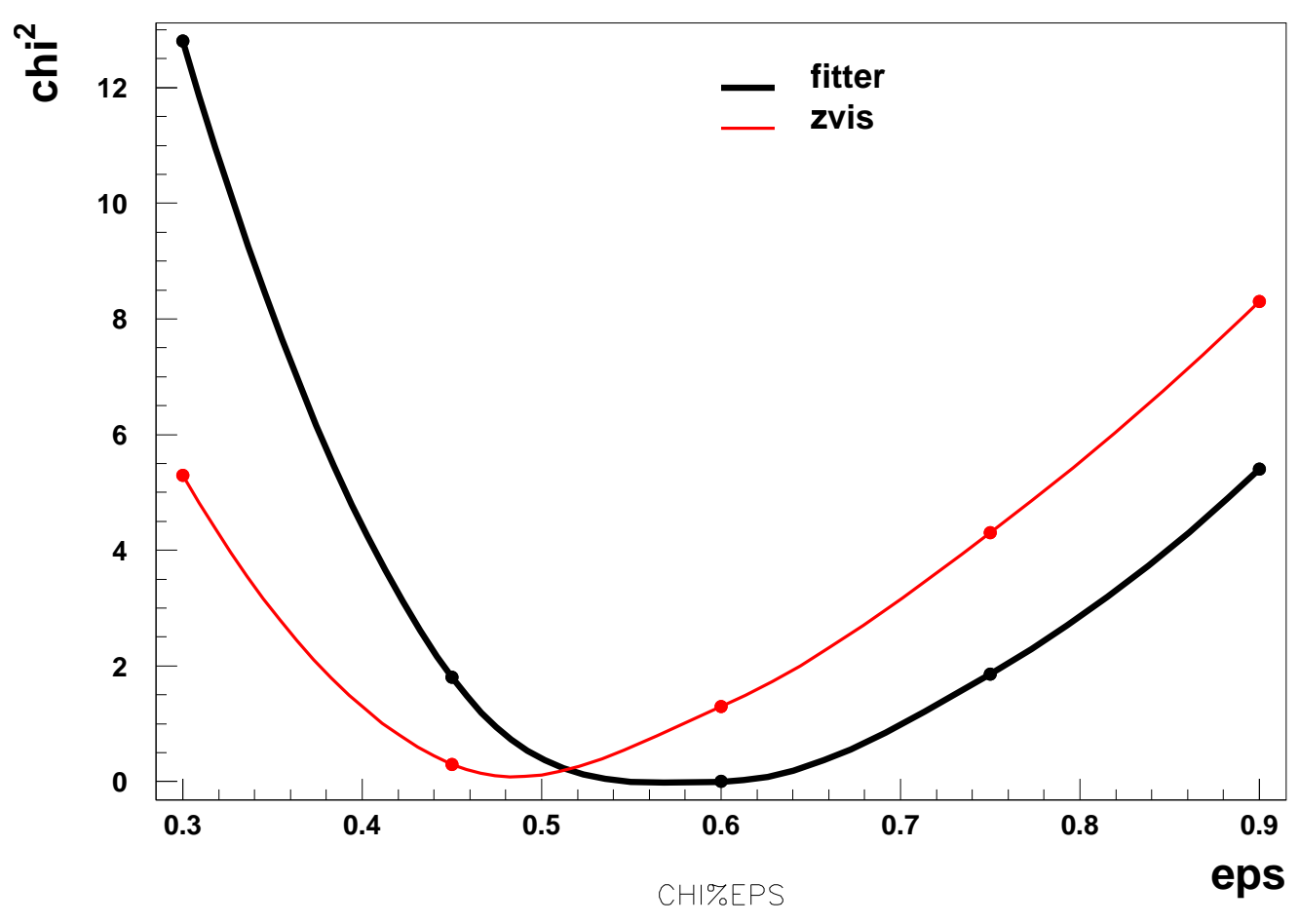

FIGURE 173. $\chi^{2}$ vs epsilon for both the $z_{v i s}$ distribution and from the data fitter. These curves are used to assign an uncertainty on $\epsilon$ ("eps" on the x axis) of 0.3 .

however, is performed below. As mentioned in the previous section a value of $B_{c}=0.099 \pm 0.012$ from a re-analysis of FNAL E531 data $[164,124]$ will be used in this analysis.

For charm fragmentation, $\epsilon$ is determined by performing a series of fits at fixed values of $\epsilon$, generating a sample of Monte-Carlo with each, and then comparing each sample to data directly. Figure 172 shows comparisons of the $3 \epsilon$ runs with data. There is a slight excess of events at high $z_{v i s}=\frac{E_{\mu 2}}{E_{H A D}+E_{\mu 2}}$ in the top most $\epsilon=0.3$ plot, while there is an excess of lower $z_{v i s}$ events at the lower $\epsilon=0.9$ plot. Figure 173 shows the results of two methods of finding an appropriate $\epsilon$ from the 


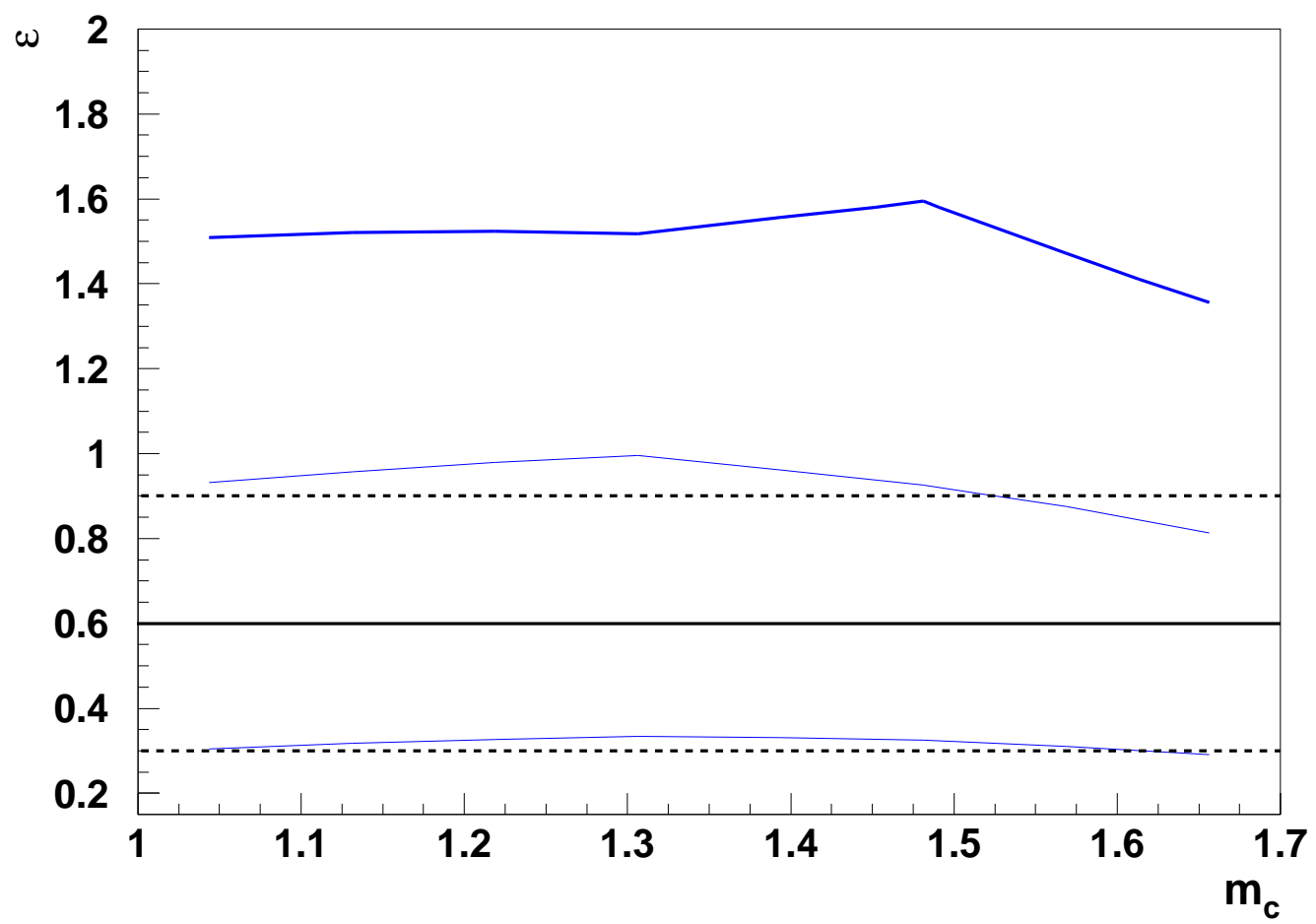

FIGURE 174. $\Delta S^{-}$vs $m_{c}$ and $\epsilon$. The horizontal line is the best fit $\epsilon$, and dotted lines the estimated uncertainty. The contours represent the shift in $S^{-}$from fits at each $\epsilon$ and $m_{c}$ value. The thin contours corresponds to a $\Delta S^{-}$of $\frac{1}{2} \sigma$ (statistical errors only), the thick contour is $1 \sigma$ from the minimum $S^{-}$. The strange asymmetry is moderately insensitive to $\epsilon$, and very insensitive to $m_{c}$.

fits. The two curves represent the $\Delta \chi^{2}$ for just the $z_{v i s}$ distributions, and the $\Delta \chi^{2}$ from the 3 dimensional grid in $E_{v i s}, y_{v i s}$, and $z_{v i s}$ used in the LO fit procedure. The two curves agree, and based on these a value of $\epsilon=0.6$ is chosen for fitting, and the uncertainty estimated to be 0.3 , based on a generous $\Delta \chi^{2}=4$, or $2 \sigma$. This uncertainty estimate becomes a systematic error on our measurement.

Figure 174, without giving away the answer, shows a contour plot of the effect on the strange asymmetry measurement of varying both $\epsilon$ and charm mass. 


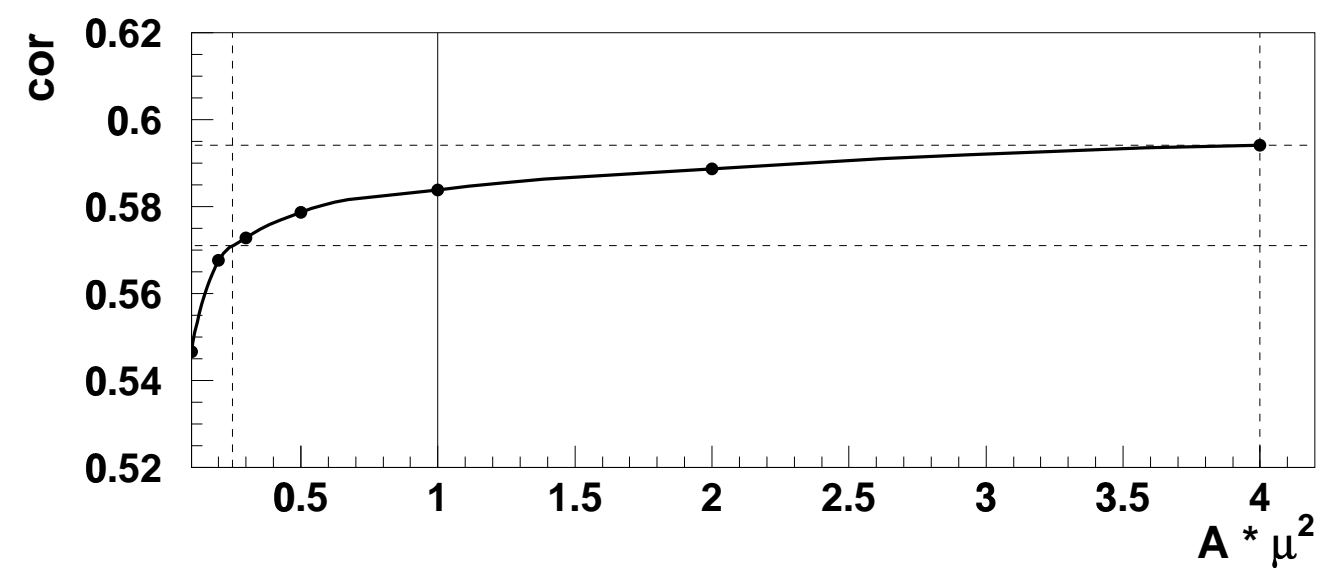

FIGURE 175. The variation of a typical acceptance point as a function of QCD scale. The $\mathrm{x}$ axis is the square of the factor that the scale is multiplied by, and the solid curve with points is the acceptance evaluated at each scale. The vertical dashed lines indicate the range of scales included in the uncertainty for that point, and the horizontal dashed lines show the uncertainty. A vertical line is drawn at 1 for reference.

A series of 88 fits were performed on a grid of 8 charm masses ranging and 11 values of $\epsilon$. The black and dotted lines indicate the central value and the assigned uncertainty for $\epsilon$, and the blue contours show the $\Delta \chi^{2}$ of 0.5 (thinner line) and 1.0 (thicker line on top). The asymmetry measurement essentially is insensitive to the charm mass, and is only slightly sensitive to $\epsilon$. Were the asymmetry measurement more sensitive to fragmentation, the value of and uncertainty on $\epsilon$ would need to be determined with more care, requiring a considerable expenditure of both CPU resources and time.

A final source of uncertainty in the NLO fit comes about by nature of the fact that the calculation necessarily must truncate the expansion in $\alpha_{s}$. This truncation results in a dependence on the QCD scale $\mu^{2}$, which is chosen here to be $Q^{2}+m_{c}^{2}$. 
Traditionally the uncertainty due to this scale choice is evaluated by looking at the dependence of observables when varying the scale $\mu$ by a factor of 2 up or down. Figure 175 shows how a typical acceptance correction point is affected by the change in scale. The acceptances change by about $2 \%$ over the factor of 2 range in scale.

In addition to the effect on the acceptances, the cross section itself is dependent on the scale. When determining the total systematic effect on the measurement due to the QCD scale, both the effect on the acceptances and on the cross section are taken into account. The scale dependence on the measurement is small, and included in the systematic tables for each fit below.

\section{$5.4 .2 \kappa, \alpha$ Approximate Evolution Fit}

Traditionally measurements of the strange sea have involved applying an external level and shape parameter to a pre-evolved nonstrange sea. Over the limited $Q^{2}$ range of a typical neutrino experiment, this is a reasonable approximation. The parameter values may only apply near the $Q^{2}$ range of the experiment measuring them however. We include this fit for two reasons: 1. It is easy to do, as it does not require formal QCD evolution code to construct the strange seas. 2. As we will see further below, the asymmetry is reasonably close to what is obtained with more formal techniques.

In this fit we define the strange and antistrange seas to be modified versions of the average between the up and down seas:

$$
s\left(x, Q^{2}\right)=\kappa(1-x)^{\alpha}\left[\frac{\bar{u}\left(x, Q^{2}\right)+\bar{d}\left(x, Q^{2}\right)}{2}\right]
$$




\begin{tabular}{|cc|}
\hline Parameter & Value \\
\hline$m_{c}$ & $1.20 \mathrm{GeV}$ \\
$\epsilon$ & 0.60 \\
$\kappa$ & $\mathbf{0 . 5 9 6} \pm \mathbf{0 . 0 2 8}$ \\
$\bar{\kappa}$ & $\mathbf{0 . 5 2 1} \pm \mathbf{0 . 0 2 6}$ \\
$\alpha$ & $\mathbf{1 . 3 4} \pm \mathbf{0 . 4 9}$ \\
$\bar{\alpha}$ & $\mathbf{1 . 5 4} \pm \mathbf{0 . 4 6}$ \\
$B_{c}$ & 0.099 \\
\hline
\end{tabular}

TABLE 21. $\kappa \alpha$ approximate evolution fit results. Bold parameters are varied in the fit. Only statistical errors are shown.

$$
\bar{s}\left(x, Q^{2}\right)=\bar{\kappa}(1-x)^{\bar{\alpha}}\left[\frac{\bar{u}\left(x, Q^{2}\right)+\bar{d}\left(x, Q^{2}\right)}{2}\right]
$$

The strange flavor sum rule, that $\int_{0}^{1} s\left(x, Q^{2}\right)-\bar{s}\left(x, Q^{2}\right) d x=0$, is also allowed to be broken. The fit results are shown in table 21 . This fit achieved a $\chi^{2}$ of 34.1 out for 39.8 degrees of freedom. The momentum weighted strange asymmetry, defined as:

$$
S^{-}\left(Q^{2}\right) \equiv \int_{0}^{1}\left[x s\left(x, Q^{2}\right)-x \bar{s}\left(x, Q^{2}\right)\right] d x=\int_{0}^{1} x s^{-}\left(x, Q^{2}\right) d x
$$

is $0.0023 \pm 0.0006$ (stat). This number has been used as a sort of shorthand to describe the overall magnitude of the asymmetry between the strange and antistrange seas $[185]$.

\subsection{3 $\kappa, \alpha$ QCD Evolution Fit}

One of the reasons why prior dimuon analyses have used a parameterization scheme as was used in the fit above, is that it does not require full blown QCD evolution code. A pdf set based on interpolated values in $\mathrm{x}$ and $Q^{2}$ could in principle be used to fit in this way. Performing a fit that evolves the pdfs properly however 


\begin{tabular}{|cc|}
\hline Parameter & Value \\
\hline$m_{c}$ & $1.20 \mathrm{GeV}$ \\
$\epsilon$ & 0.60 \\
$\kappa$ & $\mathbf{0 . 4 1 5} \pm \mathbf{0 . 0 3 1} \pm \mathbf{0 . 0 6 3}$ \\
$\bar{\kappa}$ & $\mathbf{0 . 3 3 2} \pm \mathbf{0 . 0 3 0} \pm \mathbf{0 . 0 7 2}$ \\
$\alpha$ & $\mathbf{0 . 8 7} \pm \mathbf{0 . 6 8} \pm \mathbf{1 . 4 7}$ \\
$\bar{\alpha}$ & $\mathbf{1 . 0 9} \pm \mathbf{0 . 7 1} \pm \mathbf{0 . 3 0}$ \\
$B_{c}$ & 0.099 \\
\hline
\end{tabular}

TABLE 22. $\kappa \alpha$ QCD evolution fit results. Bold parameters are varied in the fit. The first error is the statistical error, the second is the total systematic error, including both table systematics and external measurement errors.

requires numerically solving the DGLAP equations (equation 2.156 on page 107) for the different prospective strange and antistrange seas tried throughout the fit.

The CTEQ6M pdf set is accessed via the LHApdfv1 $[186,187]$ code, which serves as a wrapper for the CTEQ evolution code, EVLCTEQ. Out of the box, EVLCTEQ did not allow for the strange and antistrange seas to be different, however a version which does [188] was patched into the LHAPDF package to allow different strange and antistrange seas. The LHApdf package was also modified to allow substitution of strange and antistrange seas at initialization. LHApdf could then evolve $s(x)$ and $\bar{s}(x)$ to whatever scale the fitter required.

In this fit, the parameterization was identical to the previous fit, except now defined at the CTEQ $Q_{0}$ of $1.3 \mathrm{GeV}$, and using this hybrid LHApdf+EVLCTEQ evolution package. The nonstrange pdfs (including the gluon sea) were left unchanged from the CTEQ6M default. The starting values of the fit were usually set to the CTEQ default $(\kappa=\bar{\kappa}=0.4, \alpha=\bar{\alpha}=0)$ so that the evolution code could be checked with the standard CTEQ6M pdf set at initialization. 
Table 22 shows the results of this fit, with the parameters allowed to vary highlighted in bold. The correlation matrix for the fit is (parameters in order: $\kappa, \bar{\kappa}, \alpha, \bar{\alpha}):$

$$
\left[\begin{array}{cccc}
1.000 & 0.001 & 0.781 & 0.001 \\
0.001 & 1.000 & 0.001 & 0.806 \\
0.781 & 0.001 & 1.000 & 0.001 \\
0.001 & 0.806 & 0.001 & 1.000
\end{array}\right]
$$

The $\chi^{2}$ for this fit was 36.9 out of 39.8 effective degrees of freedom. The effect of systematic uncertainties on each of the strange sea parameters and on $S^{-}$is shown in table 23. The largest systematic is due to the charm branching ratio, which contributes roughly half the total error on the asymmetry measurement.

Figure 176 shows the strange and antistrange seas from this fit plotted on top of each other as a function of $\mathrm{x}$. As one would expect from the sign of $S^{-}$, the strange sea's momentum distribution is larger than the antistrange sea. The error bands of the two curves overlap, but correlations between the strange and antistrange sea parameters conspire to keep the difference $x s(x)-x \bar{s}(x)$ positive. The sum of the strange and antistrange seas are shown in figure 177, and the shape of the strange asymmetry is shown in figure 178. The fits prefer a positive asymmetry at low values of $x$.

\subsection{4 $s^{+}, s^{-}$QCD satisfying fit}

There are additional conditions that must be imposed on the strange and antistrange seas to perfectly satisfy the requirements of QCD. The flavor sum rule $\int_{0}^{1} s(x)-\bar{s}(x) d x$ must be zero, and the total momentum (equation 5.291) must also 


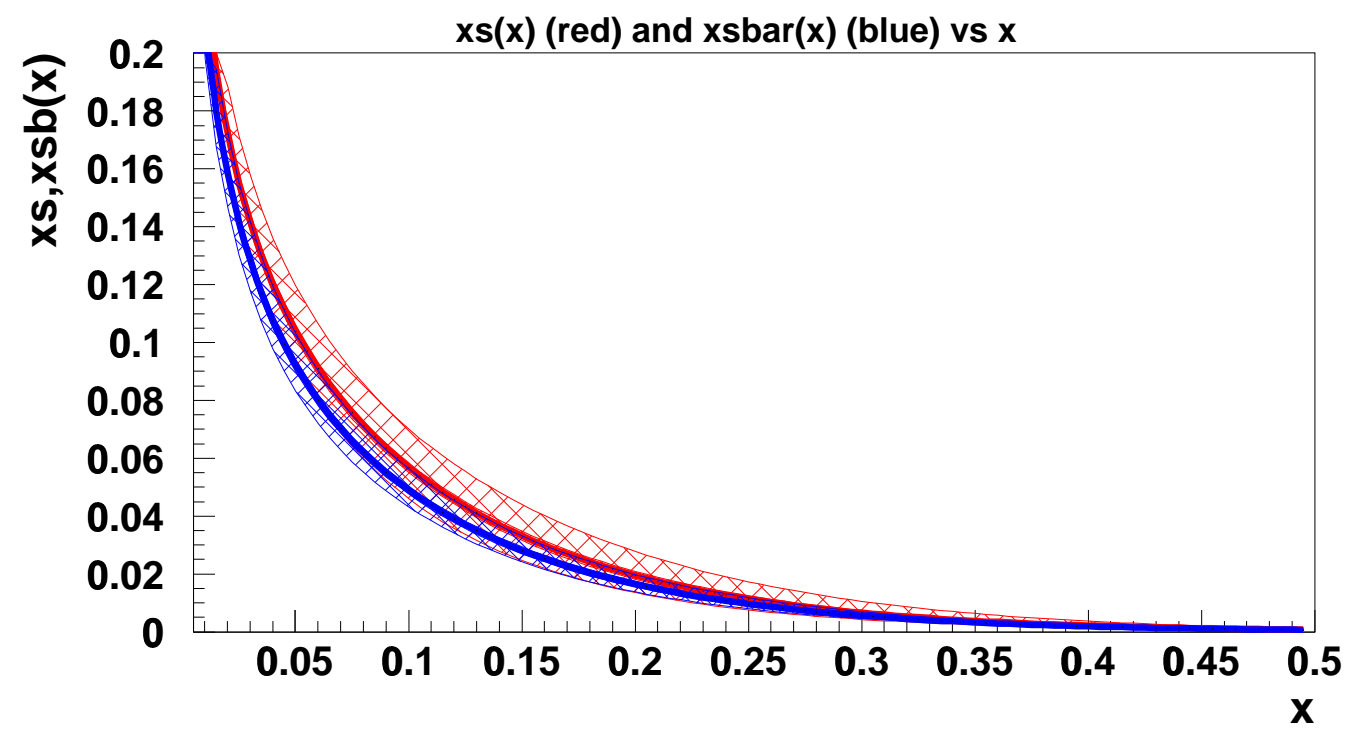

FIGURE 176. The $\kappa-\alpha$ QCD evolution fit $x s(x)$ and $x \bar{s}(x)$ vs $\mathrm{x}$. The strange sea is in red, the antistrange sea is in blue. Despite the overlap, the correlations between the two are such that the asymmetry is positive.

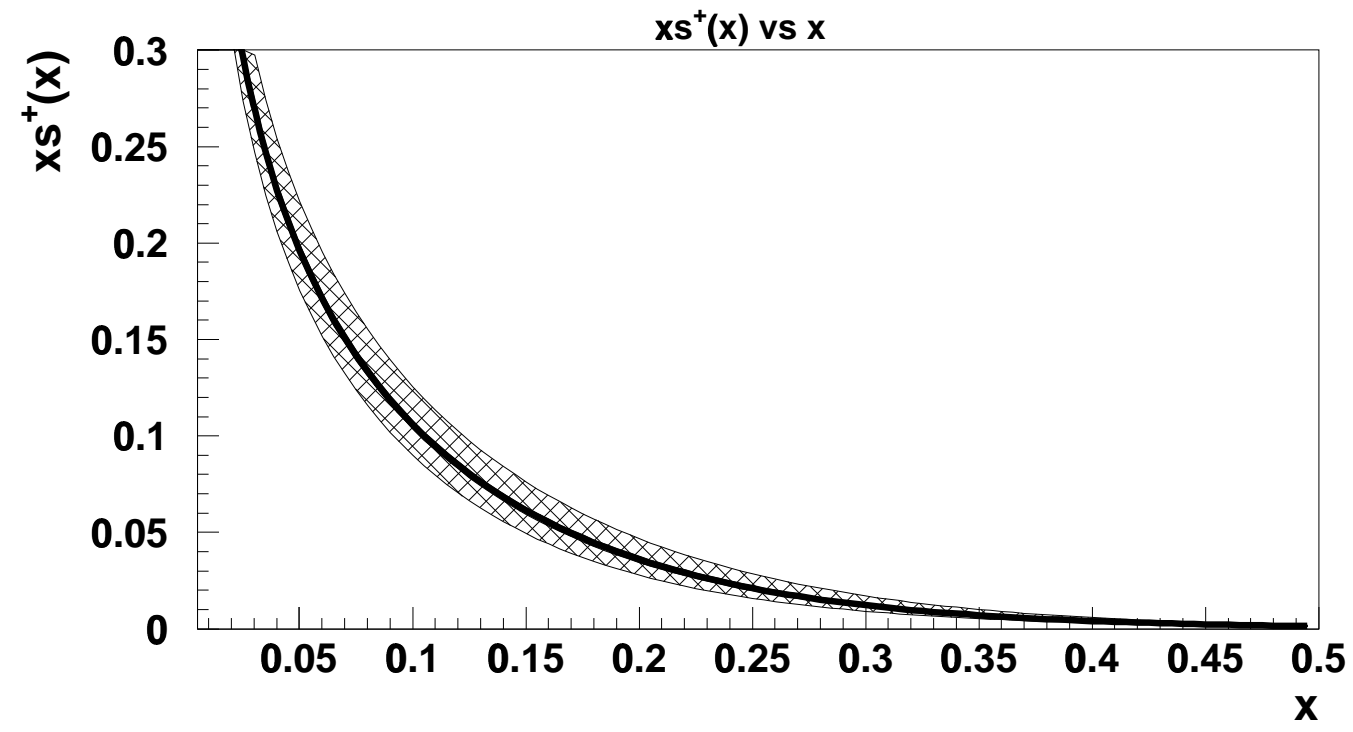

FIGURE 177 . The $\kappa$ - $\alpha$ QCD evolution fit $x s^{+}(x)$ vs $\mathrm{x}$. 


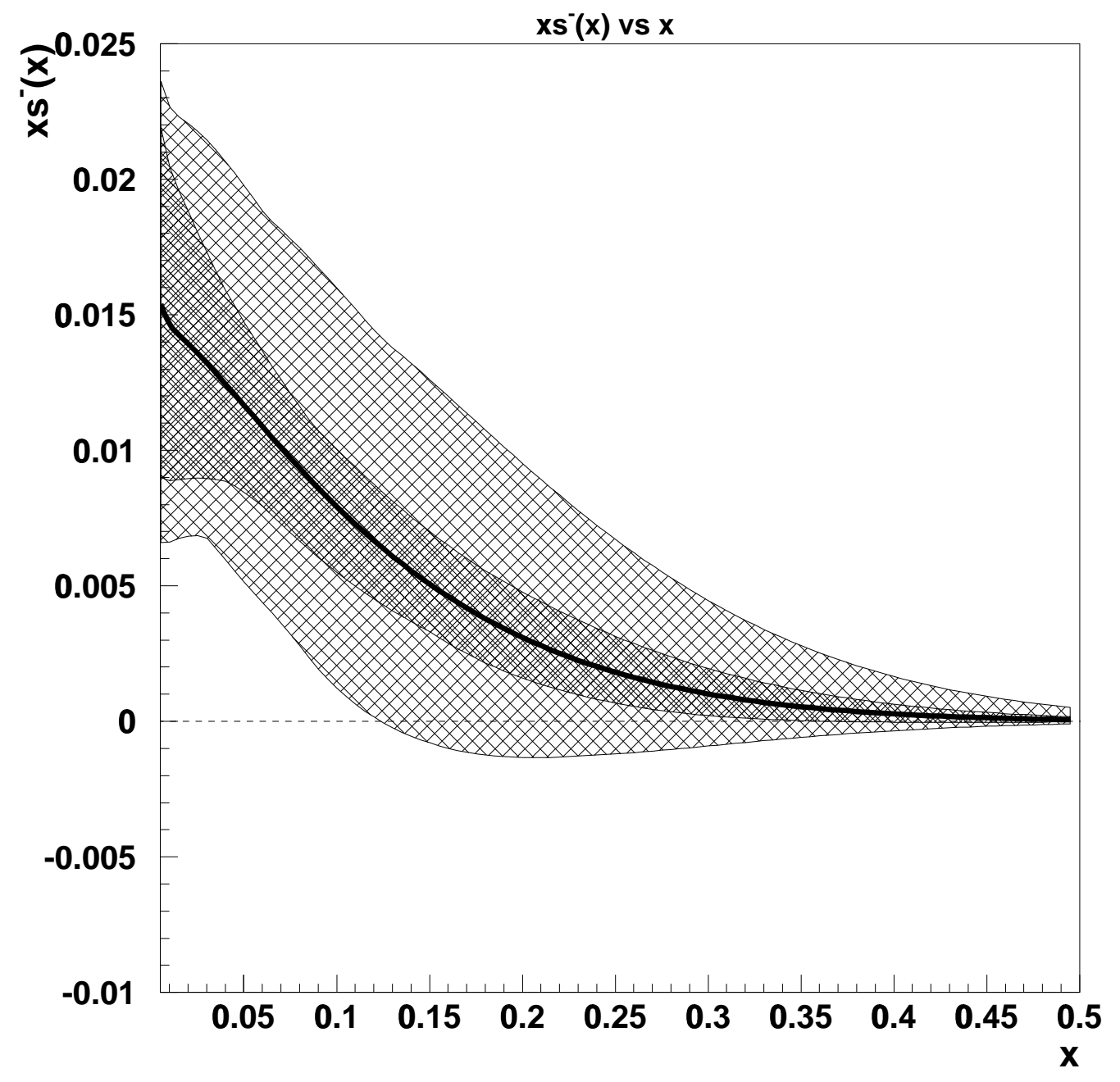

FIGURE 178. The $\kappa$ - $\alpha$ QCD evolution fit $x s^{-}(x)$ vs $\mathrm{x}$. The two shaded areas indicate the statistical errors (darkest), with the wider error band including all table systematics and external measurement uncertainties. 


\begin{tabular}{|c|cccc|c|}
\hline Description & $\kappa$ & $\bar{\kappa}$ & $\alpha$ & $\bar{\alpha}$ & $S^{-}$ \\
\hline \hline Central value & $\mathbf{0 . 4 1 5}$ & $\mathbf{0 . 3 3 2}$ & $\mathbf{0 . 8 7}$ & $\mathbf{1 . 0 9}$ & $\mathbf{0 . 0 0 1 9 5}$ \\
\hline Statistical error & $\mathbf{0 . 0 3 1}$ & $\mathbf{0 . 0 3 0}$ & $\mathbf{0 . 6 8}$ & $\mathbf{0 . 7 1}$ & $\mathbf{0 . 0 0 0 5 5}$ \\
\hline$\nu \pi-\mathrm{K}(15 \%)$ & 0.012 & 0.009 & 0.38 & 0.08 & 0.00041 \\
$\bar{\nu} \pi-\mathrm{K}(21 \%)$ & 0.006 & 0.018 & 0.05 & 0.14 & 0.00031 \\
Emuff scale $(1 \%)$ & 0.007 & 0.016 & 0.19 & 0.01 & 0.00002 \\
Had energy scale $(0.5 \%)$ & 0.008 & 0.009 & 0.15 & 0.04 & 0.00010 \\
$R_{L}(20 \%)$ & 0.011 & 0.018 & 0.06 & 0.02 & 0.00005 \\
MC statistics & 0.014 & 0.021 & 0.16 & 0.06 & 0.00000 \\
Emu2 rangeout & 0.013 & 0.021 & 0.31 & 0.06 & 0.00012 \\
Flux norm & 0.002 & 0.006 & 0.07 & 0.00 & 0.00000 \\
\hline Total Table Systematics & 0.028 & 0.044 & 0.58 & 0.18 & 0.00054 \\
\hline Charm mass & 0.015 & 0.011 & 0.07 & 0.14 & 0.00006 \\
Fragmentation $\epsilon$ & 0.009 & 0.009 & 0.25 & 0.06 & 0.00023 \\
$B_{c}$ & 0.053 & 0.055 & 1.32 & 0.19 & 0.00125 \\
\hline Total External Measurement & 0.056 & 0.057 & 1.35 & 0.24 & 0.00127 \\
\hline Total Systematics & $\mathbf{0 . 0 6 3}$ & $\mathbf{0 . 0 7 2}$ & $\mathbf{1 . 4 7}$ & $\mathbf{0 . 3 0}$ & $\mathbf{0 . 0 0 1 3 8}$ \\
\hline
\end{tabular}

TABLE 23. $\kappa \alpha$ QCD evolution fit systematics. The systematics themselves are divided into those stemming from the cross section table, and those from external measurements. The largest systematic (and largest single error) is due to the uncertainty on the charm branching ratio $B_{c}$.

be conserved. The CTEQ parameterization:

$$
\begin{aligned}
s^{+}\left(x, Q_{0}^{2}\right) & =\kappa^{+}(1-x)^{\alpha^{+}} x^{\gamma^{+}}\left[\bar{u}\left(x, Q_{0}^{2}\right)+\bar{d}\left(x, Q_{0}^{2}\right)\right] \\
s^{-}\left(x, Q_{0}^{2}\right) & =s^{+}\left(x, Q_{0}^{2}\right) \tanh \left[\kappa^{-}(1-x)^{\alpha^{-}} x^{\gamma^{-}}\left(1-\frac{x}{x_{0}}\right)\right] \\
s & =\frac{s^{+}+s^{-}}{2} \quad \bar{s}=\frac{s^{+}-s^{-}}{2}
\end{aligned}
$$

satisfies these conditions in these fits with the addition of the gluon sea rescaling scheme. The results of the fit are shown in table 24. The fit quality is good, with a $\chi^{2}$ of 38.2 for 37.8 effective degrees of freedom. The strange flavor sum rule prefers to be satisfied in these fits by producing a large negative spike at low $x$ to 


\begin{tabular}{|c|c|}
\hline Parameter & Value \\
\hline$m_{c}$ & $1.20 \mathrm{GeV}$ \\
\hline$\epsilon$ & 0.60 \\
\hline$\kappa^{+}$ & $0.551 \pm 0.126$ \\
\hline$\kappa^{-}$ & $(-0.881 \pm 0.567) \times 10^{-2}$ \\
\hline$\alpha^{+}$ & $1.11 \pm 0.69$ \\
\hline$\alpha^{-}$ & $6.31 \pm 4.06$ \\
\hline$\gamma^{+}$ & $0.072 \pm 0.064$ \\
\hline$\gamma^{-}$ & $-0.102 \pm 0.080$ \\
\hline$B_{c}$ & 0.099 \\
\hline$V_{c d}$ & 0.224 \\
\hline
\end{tabular}

TABLE 24. Sum rule satisfying fit parameters. Bold parameters are varied in the fit. Only statistical errors are shown.

compensate for a more gradual positive curve at moderate $x$. Figure 179 shows the shape of the momentum weighted strange asymmetry as a function of $x$. The crossing point for $s^{-}(x)$ prefers to be at an extremely low value of about 0.004 . This is below any of the cross section table points, so in principle the function can do whatever it pleases, and therefore does. The gluon sea only needed to be rescaled by a factor of 1.0007 (0.07\% increase), certainly well within the gluon sea errors. The correlation coefficients for the strange sea parameters are (parameters in order: $\left.\kappa^{+}, \kappa^{-}, \alpha^{+}, \alpha^{-}, \gamma^{+}, \gamma^{-}\right)$:

$$
\left[\begin{array}{cccccc}
1.000 & -0.171 & 0.889 & -0.037 & 0.975 & -0.252 \\
-0.171 & 1.000 & -0.129 & -0.220 & -0.182 & -0.767 \\
0.889 & -0.129 & 1.000 & 0.079 & 0.804 & -0.207 \\
-0.037 & -0.220 & 0.079 & 1.000 & -0.057 & -0.035 \\
0.975 & -0.182 & 0.804 & -0.057 & 1.000 & -0.264 \\
-0.252 & -0.767 & -0.207 & -0.035 & -0.264 & 1.000
\end{array}\right]
$$




\begin{tabular}{|c|c|l|}
\hline$\eta_{s}$ & $S^{-}$ & Systematic \\
\hline $\mathbf{0 . 0 6 1 2}$ & $\mathbf{0 . 0 0 1 9 6}$ & central value \\
$\mathbf{0 . 0 0 1 1}$ & $\mathbf{0 . 0 0 0 4 6}$ & statistics \\
\hline 0.0026 & 0.00034 & $\nu \pi$-K model \\
0.0019 & 0.00025 & $\bar{\nu} \pi$-K model \\
0.0020 & 0.00004 & $\mu$ spectrometer momentum scale $(1 \%)$ \\
0.0014 & 0.00008 & hadron energy scale $(0.5 \%)$ \\
0.0018 & 0.00005 & $R_{L}$ in table model $(20 \%)$ \\
0.0026 & 0.00001 & table extraction MC statistics \\
0.0030 & 0.00012 & decay $\mu$ range out energy $(2.5 \%)$ \\
0.0006 & 0.00005 & $\nu, \bar{\nu}$ relative normalization \\
0.0022 & 0.00023 & QCD scale \\
$\mathbf{0 . 0 0 6 4}$ & $\mathbf{0 . 0 0 0 5 0}$ & total systematics \\
\hline 0.0022 & 0.00002 & $\Delta m_{c}=0.10$ \\
0.0020 & 0.00021 & $\Delta \epsilon_{C-S}=0.3$ \\
0.0101 & 0.00111 & $\Delta B_{c}=0.012$ \\
0.0068 & 0.00046 & CTEQ6 PDF uncertainties \\
0.0007 & 0.00038 & Nuclear corrections \\
\hline $\mathbf{0 . 0 1 2 6}$ & $\mathbf{0 . 0 0 1 2 8}$ & total external measurement \\
\hline
\end{tabular}

TABLE 25. Contributions to the error in $S^{-}$and $\eta_{s}$ for the CTEQ parameterization fit.

The effect of the systematic uncertainties on this fit are listed in table 25 . Both the integrated asymmetry $S^{-}$, and $\eta_{s}$, the integrated ratio of the strange to nonstrange sea:

$$
\eta_{s} \equiv \frac{S^{+}}{\bar{U}+\bar{D}}
$$

where $S^{+}$is the analogue of $S^{-}: S^{+} \equiv \int_{0}^{1} x s^{+}(x) d x$ and $\bar{U}$ and $\bar{D}$ are the momentum weighted integrals of the up and down sea distributions. $\eta_{s}$ has been measured in almost every experimental dimuon analysis to date. With the usual symmetric strange and antistrange seas, $S^{+}(x)$ is typically written as $2 S(x)$. 


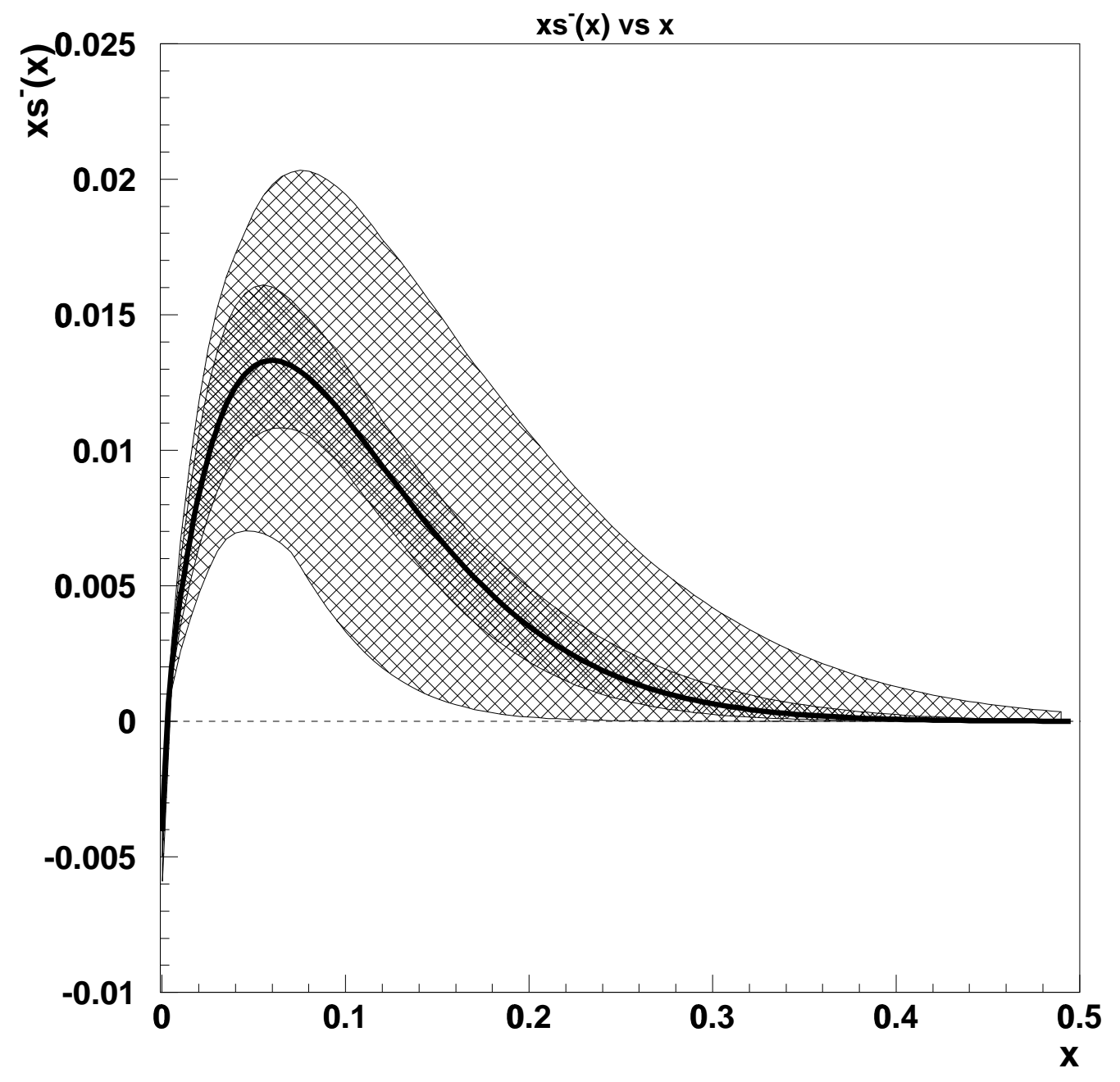

FIGURE 179. The CTEQ parameterization $x s^{-}(x)$ versus $x$. The two shaded areas indicate the statistical errors (darkest), with the wider error band including all table systematics and external measurement uncertainties. The asymmetry spikes at extremely low $x$ to satisfy the flavor sum rule. 


\begin{tabular}{|cc|}
\hline Parameter & Value \\
\hline $\mathbf{m}_{\mathbf{c}}$ & $\mathbf{1 . 4 1} \pm \mathbf{0 . 1 0} \pm \mathbf{0 . 0 8} \pm \mathbf{0 . 1 2} \mathbf{G e V}$ \\
$\epsilon$ & 0.60 \\
$\kappa$ & $\mathbf{0 . 4 3 6} \pm \mathbf{0 . 0 3 5} \pm \mathbf{0 . 0 1 7} \pm \mathbf{0 . 0 4 3}$ \\
$\bar{\kappa}$ & $\mathbf{0 . 3 4 8} \pm \mathbf{0 . 0 3 3} \pm \mathbf{0 . 0 3 6} \pm \mathbf{0 . 0 5 0}$ \\
$\alpha$ & $\mathbf{0 . 7 6} \pm \mathbf{0 . 6 7} \pm \mathbf{0 . 4 9} \pm \mathbf{1 . 1 5}$ \\
$\bar{\alpha}$ & $\mathbf{1 . 5 4} \pm \mathbf{0 . 4 6} \pm \mathbf{0 . 6 9} \pm \mathbf{0 . 1 0} \pm \mathbf{0 . 5 1}$ \\
$B_{c}$ & 0.099 \\
\hline
\end{tabular}

TABLE 26. $\kappa \alpha m_{c}$ fit systematics. The systematics themselves are divided into those stemming from the cross section table, and those from external measurements.

\section{$5.4 .5 \kappa, \alpha, m_{c}$ fit}

If an external branching ratio and fragmentation shape is assumed, the cross section table can provide enough constraint to measure the charm mass as well. At NLO, the charm mass is a more physical quantity than at LO, as the effects of the gluon in the form of rapidity are now included in the model. A variation of the $\kappa \alpha$ QCD evolution fit was tried where the charm mass was also allowed to vary in the fit. The $\kappa \alpha$ parameterization was chosen because it gives the same results as 6 parameter $s^{-} s^{+}$fit, using fewer free parameters for the strange sea. Table 26 shows the results of the fit, with the associated statistical and systematic errors. The asymmetry resulting from this fit is virtually identical to what was obtained with the fixed charm mass above, $S^{-}=0.00203 \pm 0.00057 \pm 0.00055 \pm 0.00121$. The quality of the fit was virtually identical to the fixed $m_{c}$ version, with a $\chi^{2}$ of 36.0/38.8 DOF. (It only improves slightly: The probability in this case was $40.2 \%$, while for the fixed $m_{c}$ fit it is $39.8 \%$ ). The correlation coefficients for the strange 


\begin{tabular}{|c|ccccc|c|}
\hline Description & $m_{c}(\mathrm{GeV})$ & $\kappa$ & $\bar{\kappa}$ & $\alpha$ & $\bar{\alpha}$ & $S^{-}$ \\
\hline \hline Central value & $\mathbf{1 . 4 0 9}$ & $\mathbf{0 . 4 3 6}$ & $\mathbf{0 . 3 4 8}$ & $\mathbf{0 . 7 6}$ & $\mathbf{0 . 9 0}$ & $\mathbf{0 . 0 0 2 0 3}$ \\
\hline Statistical error & $\mathbf{0 . 1 0 1}$ & $\mathbf{0 . 0 3 5}$ & $\mathbf{0 . 0 3 2}$ & $\mathbf{0 . 6 6}$ & $\mathbf{0 . 6 8}$ & $\mathbf{0 . 0 0 0 5 7}$ \\
\hline$\nu \pi-\mathrm{K}(15 \%)$ & 0.035 & 0.008 & 0.006 & 0.33 & 0.03 & 0.00040 \\
$\bar{\nu} \pi-\mathrm{K}(21 \%)$ & 0.044 & 0.002 & 0.016 & 0.01 & 0.07 & 0.00035 \\
Emuff scale $(1 \%)$ & 0.011 & 0.006 & 0.016 & 0.18 & 0.02 & 0.00001 \\
Had energy scale $(0.5 \%)$ & 0.027 & 0.005 & 0.007 & 0.12 & 0.01 & 0.00008 \\
$R_{L}(20 \%)$ & 0.038 & 0.011 & 0.018 & 0.06 & 0.02 & 0.00008 \\
Emu2 rangeout & 0.030 & 0.010 & 0.020 & 0.28 & 0.01 & 0.00010 \\
Flux norm & 0.002 & 0.002 & 0.006 & 0.07 & 0.01 & 0.00001 \\
\hline All Table Systematics & 0.079 & 0.017 & 0.036 & 0.49 & 0.10 & 0.00055 \\
\hline Fragmentation $\epsilon$ & 0.064 & 0.003 & 0.004 & 0.19 & 0.01 & 0.00021 \\
$B_{c}$ & 0.107 & 0.043 & 0.050 & 1.13 & 0.05 & 0.00119 \\
\hline All External M'mnt & 0.124 & 0.042 & 0.050 & 1.15 & 0.05 & 0.00121 \\
\hline Total Systematics & $\mathbf{0 . 1 4 7}$ & $\mathbf{0 . 0 4 6}$ & $\mathbf{0 . 0 6 1}$ & $\mathbf{1 . 2 5}$ & $\mathbf{0 . 1 1}$ & $\mathbf{0 . 0 0 1 3 3}$ \\
\hline
\end{tabular}

TABLE 27. $\kappa \alpha m_{c}$ fit systematics. The systematics themselves are divided into those stemming from the cross section table, and those from external measurements. The addition of the charm mass in the fit improves the systematics at the expense of statistical errors.

sea parameters are (parameters in order: $\left.m_{c}, \kappa, \bar{\kappa}, \alpha, \bar{\alpha}\right)$ :

$$
\left[\begin{array}{ccccc}
1.000 & 0.000 & -0.001 & 0.002 & 0.003 \\
0.000 & 1.000 & 0.001 & 0.793 & 0.001 \\
-0.001 & 0.001 & 1.000 & 0.001 & 0.817 \\
0.002 & 0.793 & 0.001 & 1.000 & 0.001 \\
0.003 & 0.001 & 0.817 & 0.001 & 1.000
\end{array}\right]
$$

As one would probably expect from looking at figure 174 the charm mass is not very strongly correlated with the strange sea parameters. Figure 180 includes the charm mass found here with the one in the LO BGPAR fit in the previous chapter, as well as with measurements from other neutrino experiments measuring dimuons. 


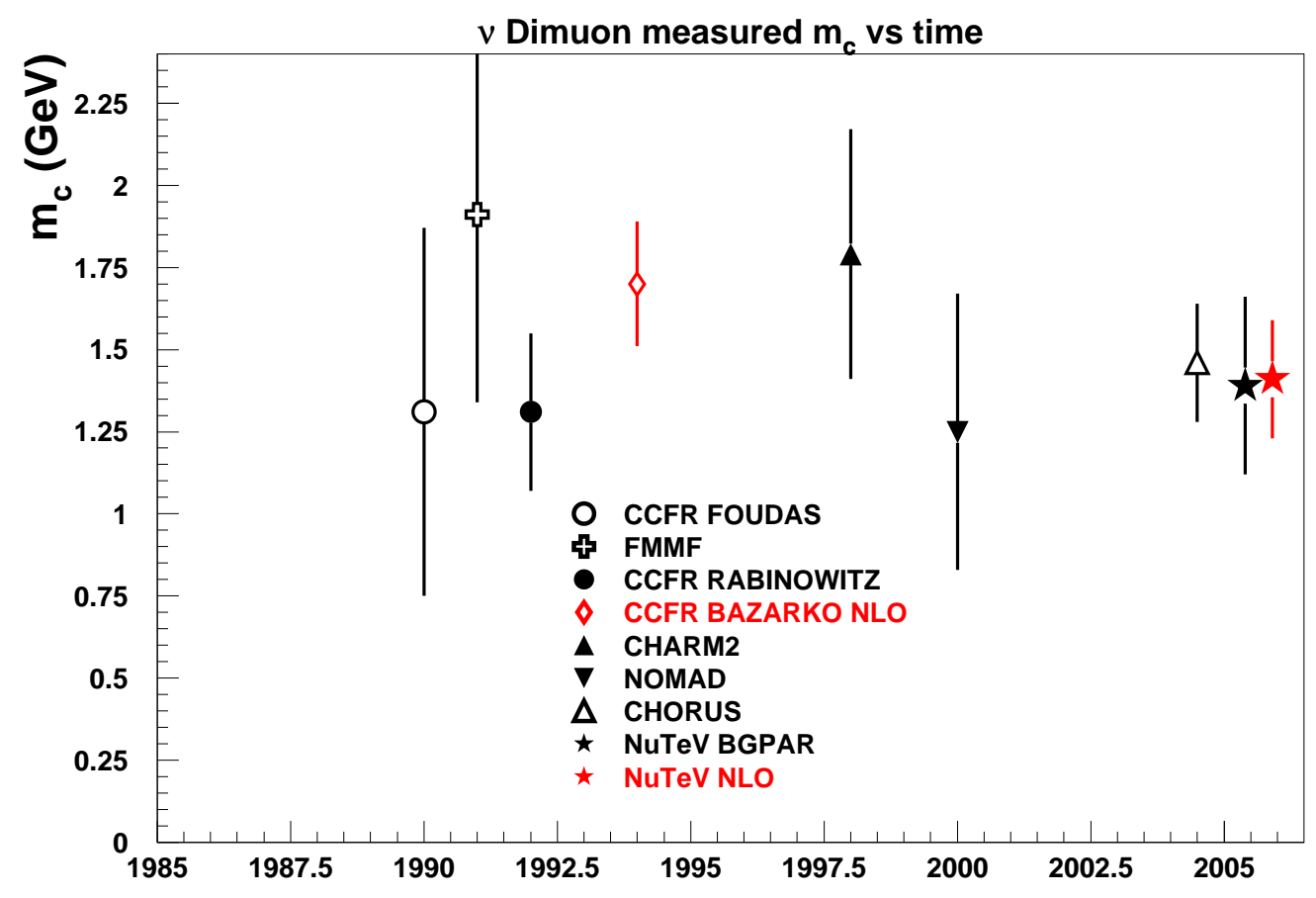

FIGURE 180. A comparison of $\nu$ dimuon charm mass measurements [61, 73, 62, $63,74,69,76]$. Measurements from this and the BGPAR analysis are starred at left, NLO measurements are in red.

\section{$\underline{5.5 \quad \mathrm{NLO} \text { MC-data comparisons }}$}

The results from the CTEQ parameterization fit above were used to generate neutrino and antineutrino dimuon $\mathrm{MC}$ to be compared to data as a check. This sample was then used to re-extract the forward dimuon cross section table as a check of the table's model dependence.

695,237 $\nu$ and 601,536 $\bar{\nu}$ numonte and $\mathrm{McNuTeV}$ events were generated, consuming a full week's CPU time from eight 1.5-2 GHz AMD processors. The results are shown in figures 181-195. The NLO MC fits the data reasonably well. 

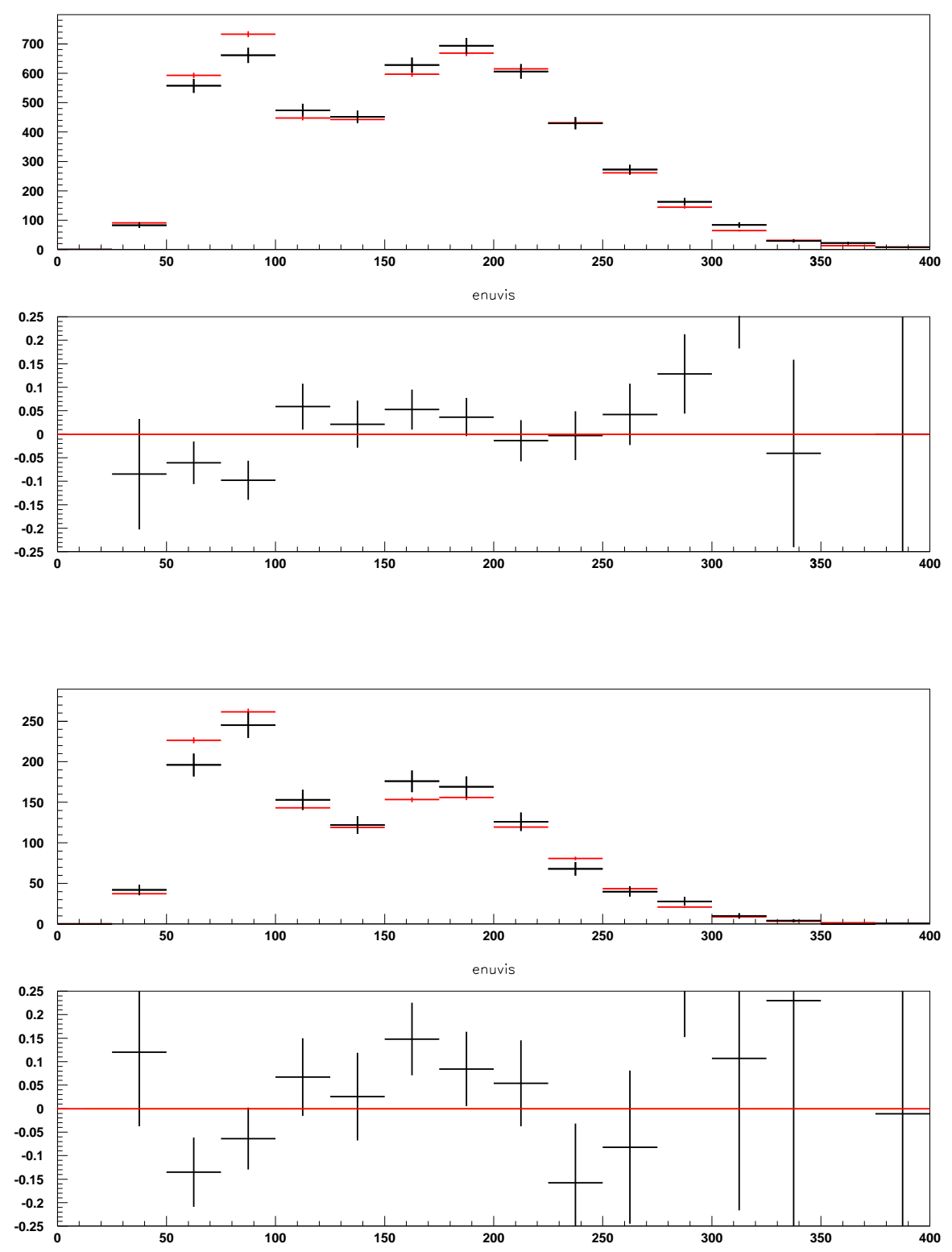

FIGURE 181. Total reconstructed neutrino energy, $E_{v i s}$, in data vs MC for the NLO dimuon sample, for both neutrino (top) and antineutrino (bottom) modes. Plotted are both absolute comparisons, with data in black, and MC in red, above ratio plots, showing data/MC-1. The $\mathrm{x}$ axis is in $\mathrm{GeV}$. 

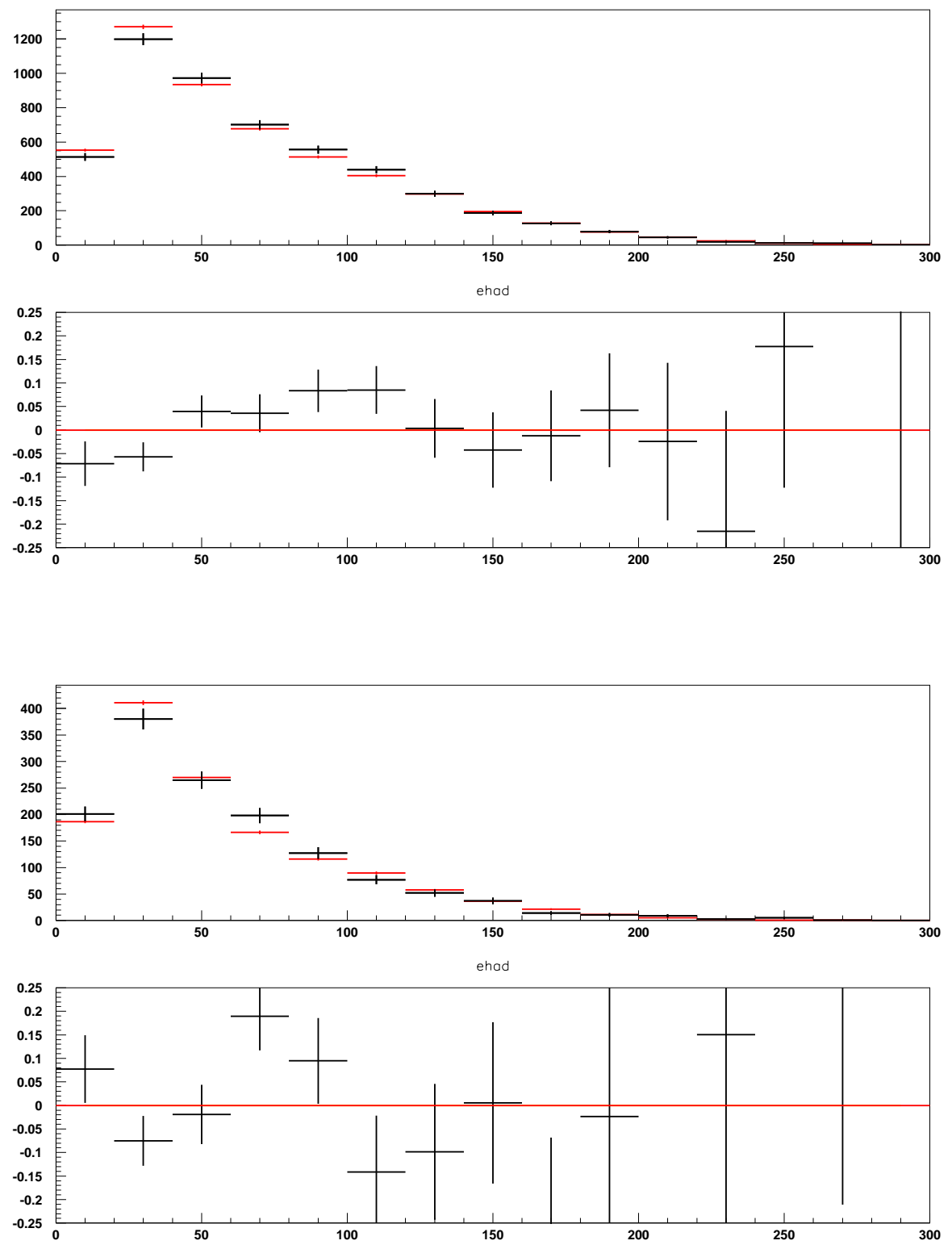

FIGURE 182. Reconstructed hadronic energy, $E_{H A D}$, in data vs MC for the NLO dimuon sample, for both neutrino (top) and antineutrino (bottom) modes. Plotted are both absolute comparisons, with data in black, and MC in red, above ratio plots, showing data/MC-1. The $\mathrm{x}$ axis is in GeV. 

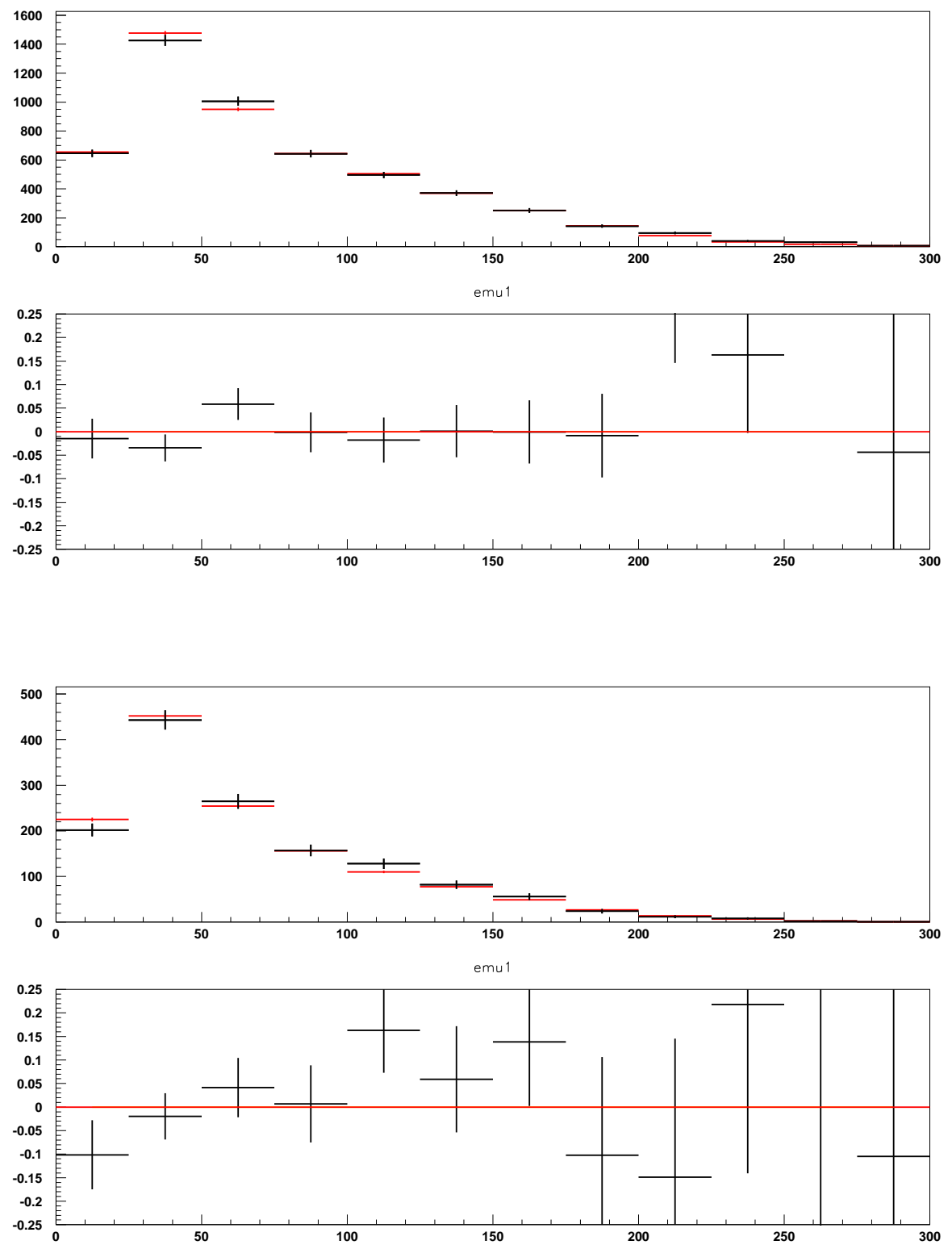

FIGURE 183. Reconstructed energy of the muon from the weak vertex, $E_{\mu 1}$, in data vs MC for the NLO dimuon sample, for both neutrino (top) and antineutrino (bottom) modes. Plotted are both absolute comparisons, with data in black, and $\mathrm{MC}$ in red, above ratio plots, showing data/MC-1. The $\mathrm{x}$ axis is in $\mathrm{GeV}$. 

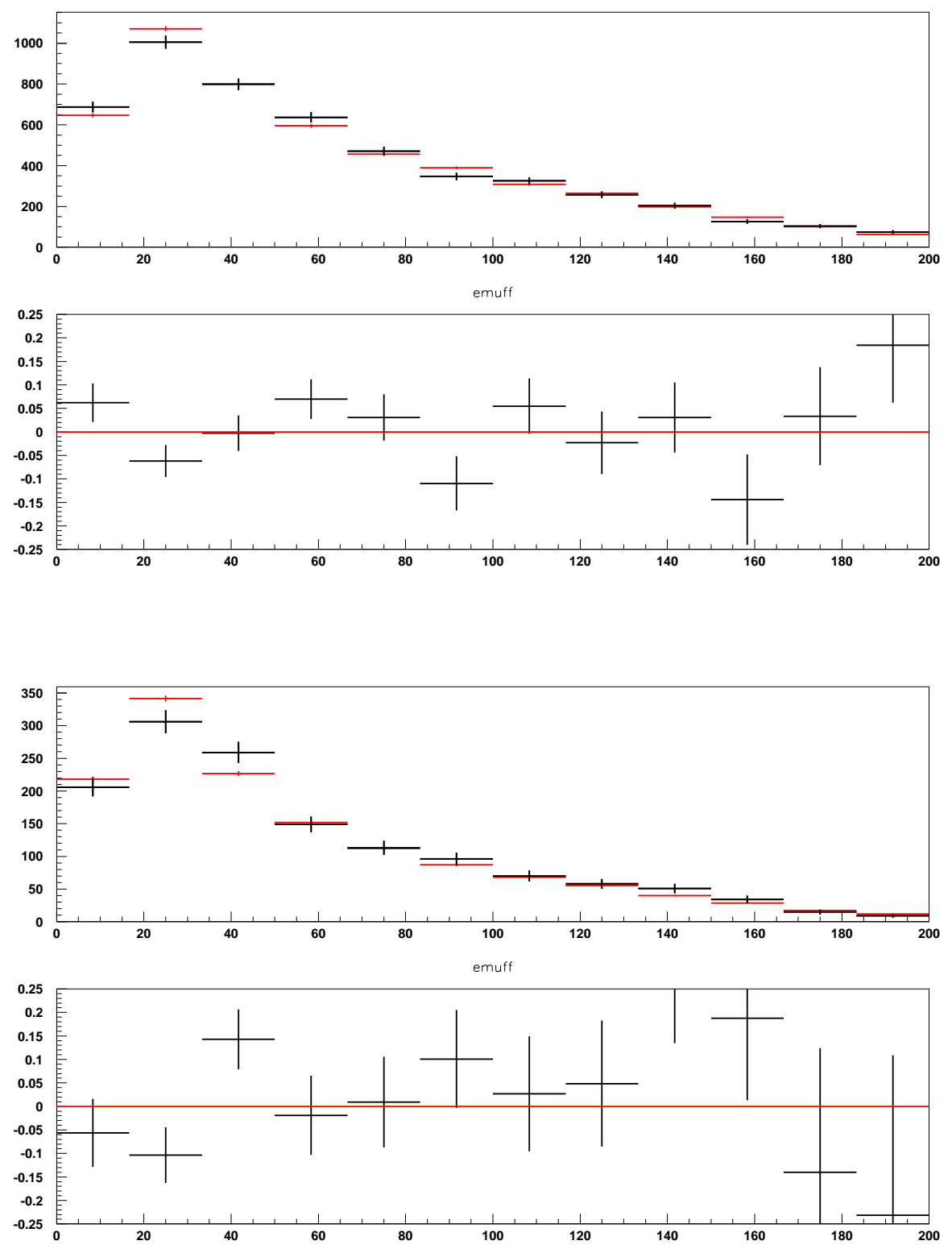

FIGURE 184. Reconstructed energy of the muon from the weak vertex, $E_{\mu F F 1}$, immediately after the calorimeter, in data vs MC for the NLO dimuon sample, for both neutrino (top) and antineutrino (bottom) modes. Plotted are both absolute comparisons, with data in black, and $\mathrm{MC}$ in red, above ratio plots, showing data/MC-1. The $\mathrm{x}$ axis is in $\mathrm{GeV}$. 

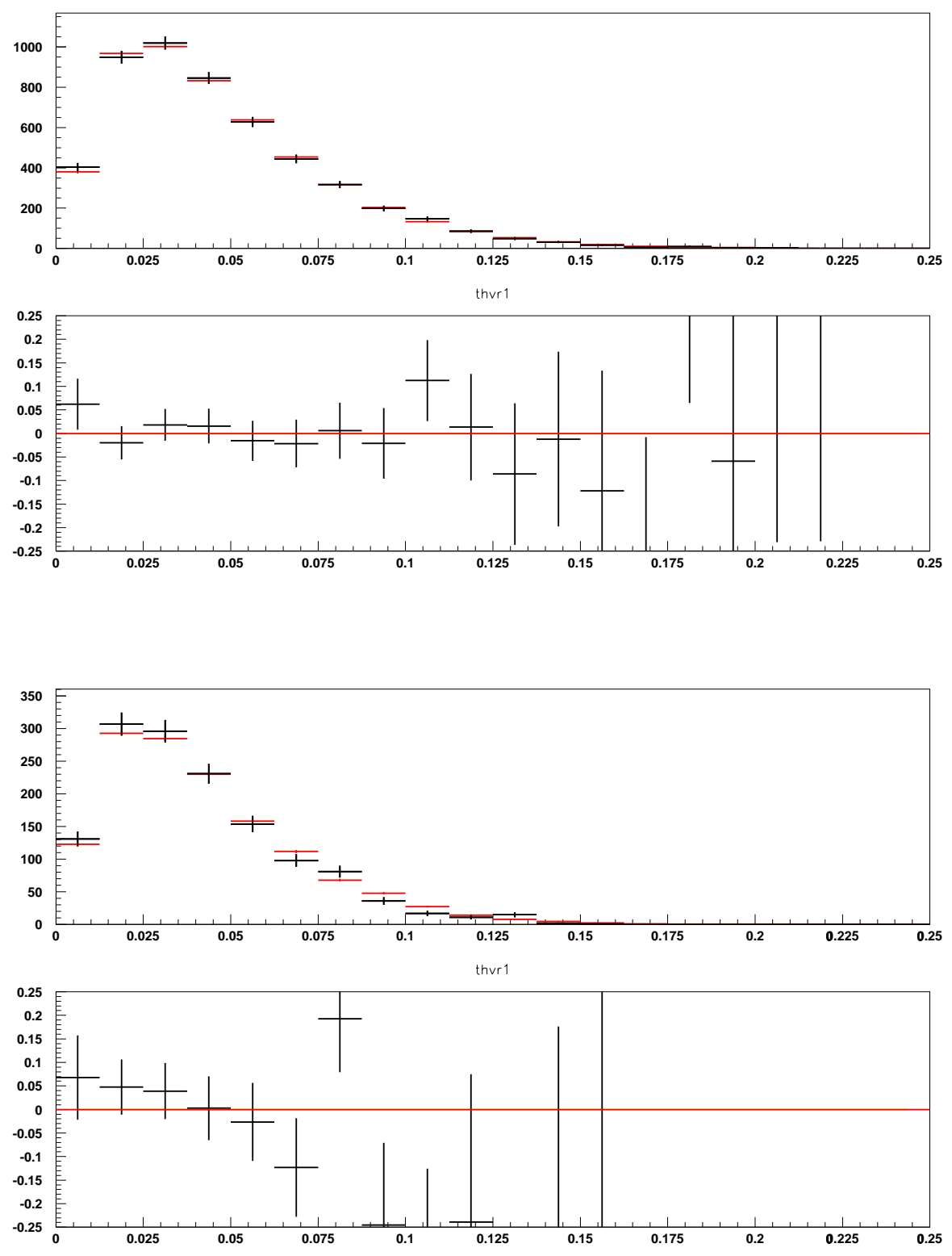

FIGURE 185. Reconstructed opening angle of the muon from the weak vertex, $\theta_{\mu 1}$, in data vs MC for the NLO dimuon sample, for both neutrino (top) and antineutrino (bottom) modes. Plotted are both absolute comparisons, with data in black, and $\mathrm{MC}$ in red, above ratio plots, showing data/MC-1. The $\mathrm{x}$ axis is in radians. 

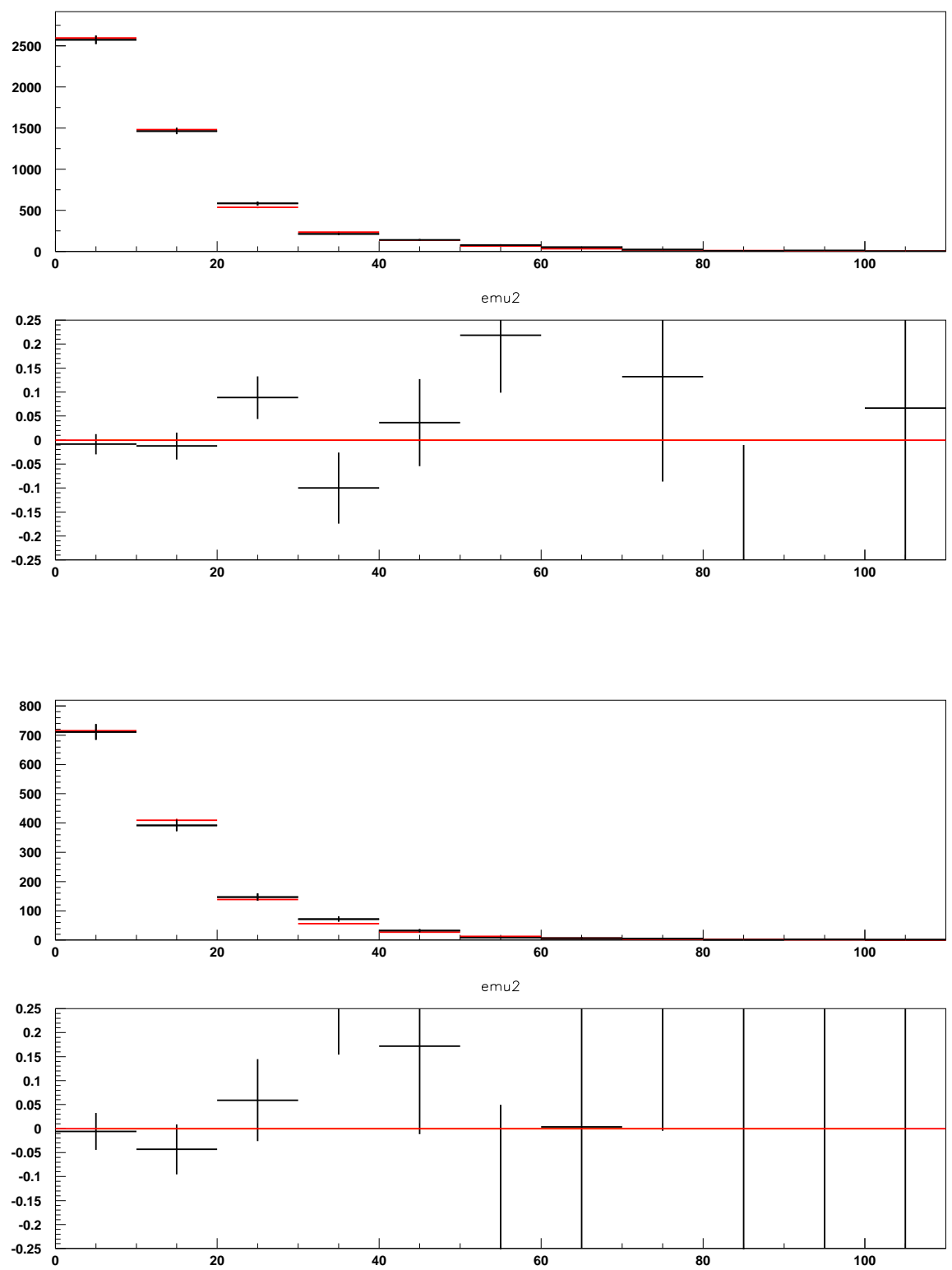

FIGURE 186. Reconstructed energy of the muon from the charm decay, $E_{m u 1}$, in data vs MC for the NLO dimuon sample, for both neutrino (top) and antineutrino (bottom) modes. Plotted are both absolute comparisons, with data in black, and $\mathrm{MC}$ in red, above ratio plots, showing data/MC-1. The $\mathrm{x}$ axis is in $\mathrm{GeV}$. 

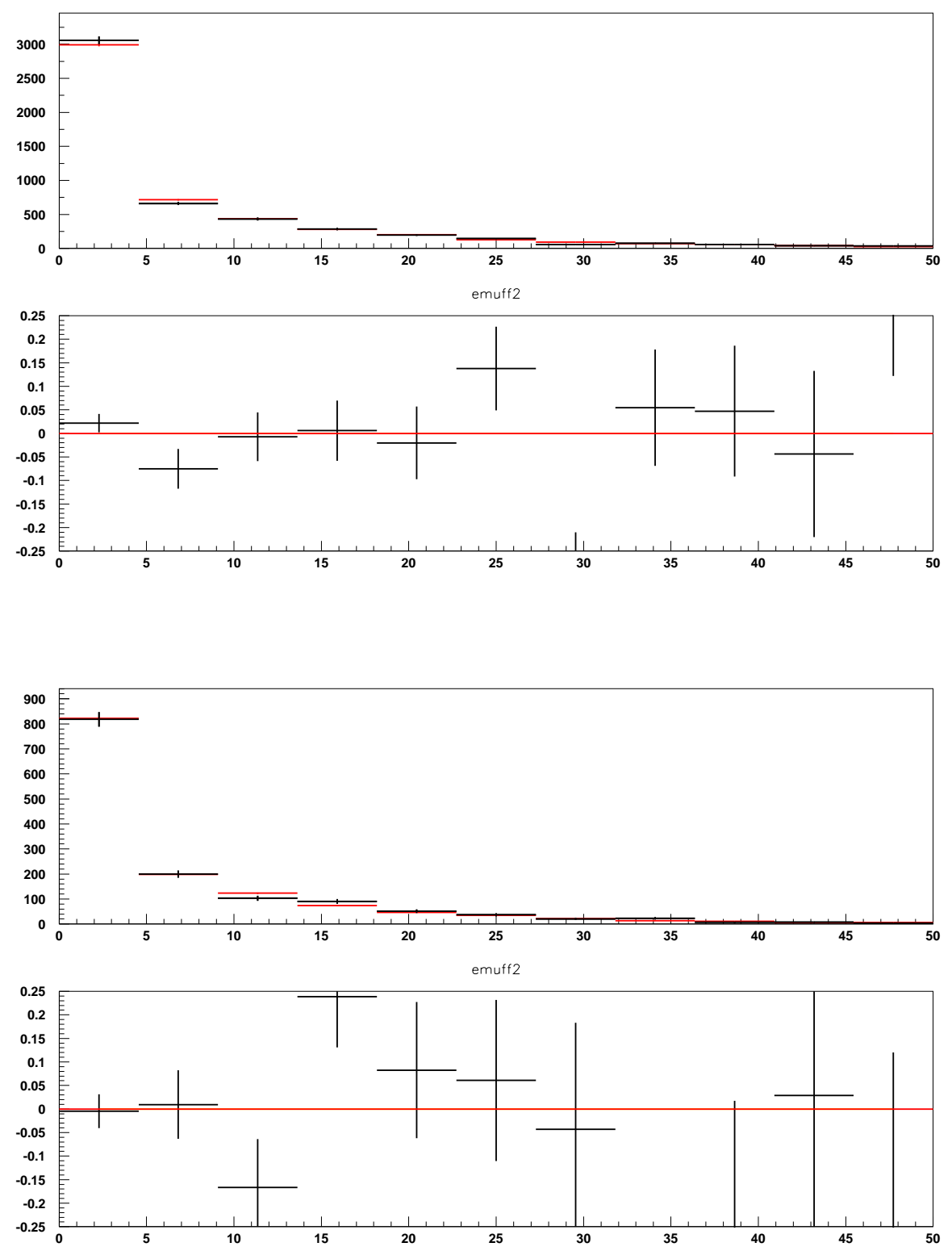

FIGURE 187. Reconstructed energy of the muon from the charm decay immediately after the calorimeter, $E_{\mu F F 2}$, in data vs MC for the NLO dimuon sample, for both neutrino (top) and antineutrino (bottom) modes. Plotted are both absolute comparisons, with data in black, and $\mathrm{MC}$ in red, above ratio plots, showing data/MC-1. The $\mathrm{x}$ axis is in $\mathrm{GeV}$. 

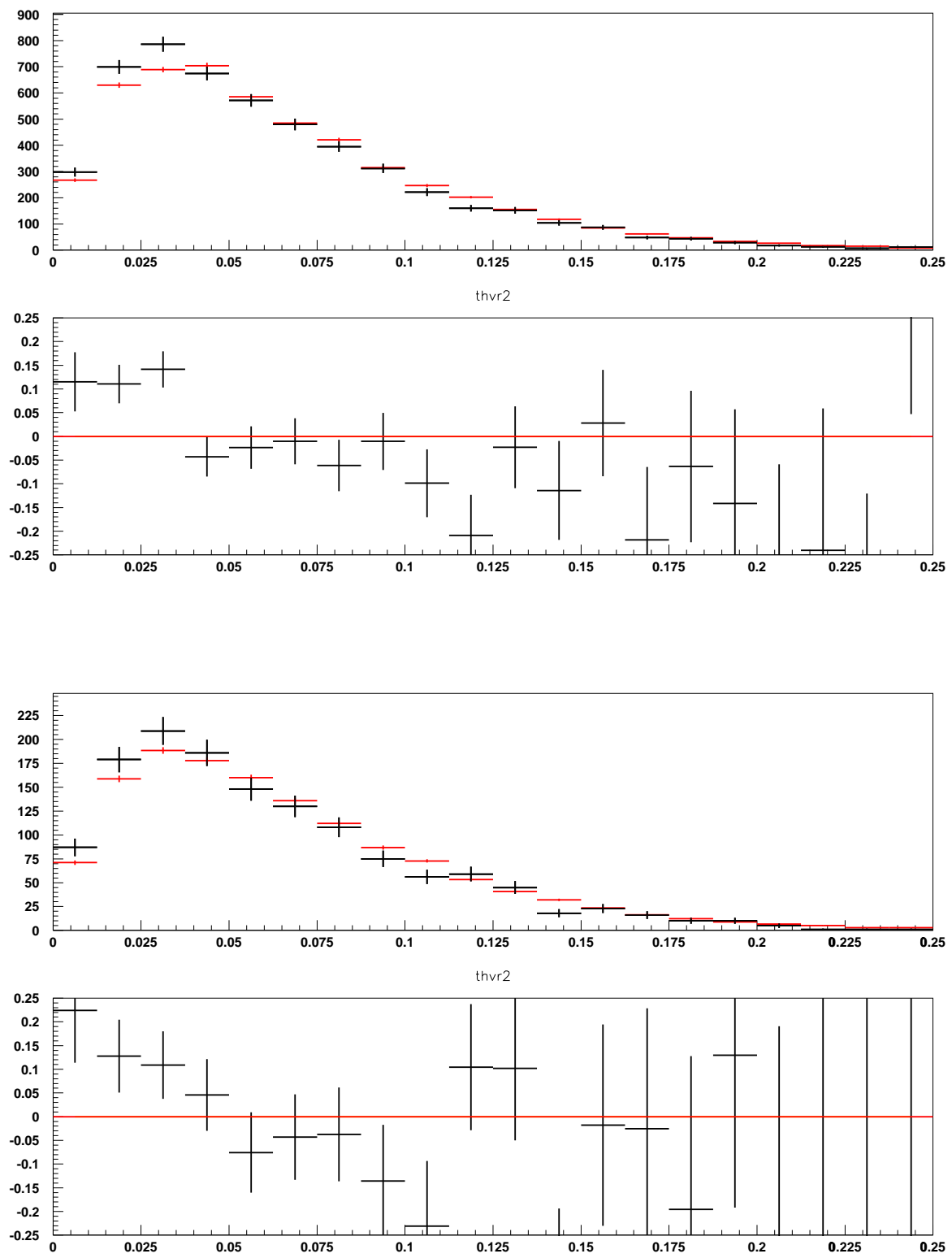

FIGURE 188. Reconstructed opening angle from the charm decay muon, $\theta_{\mu 2}$, in data vs MC for the NLO dimuon sample, for both neutrino (top) and antineutrino (bottom) modes. Plotted are both absolute comparisons, with data in black, and $\mathrm{MC}$ in red, above ratio plots, showing data/MC-1. The $\mathrm{x}$ axis is in radians. 

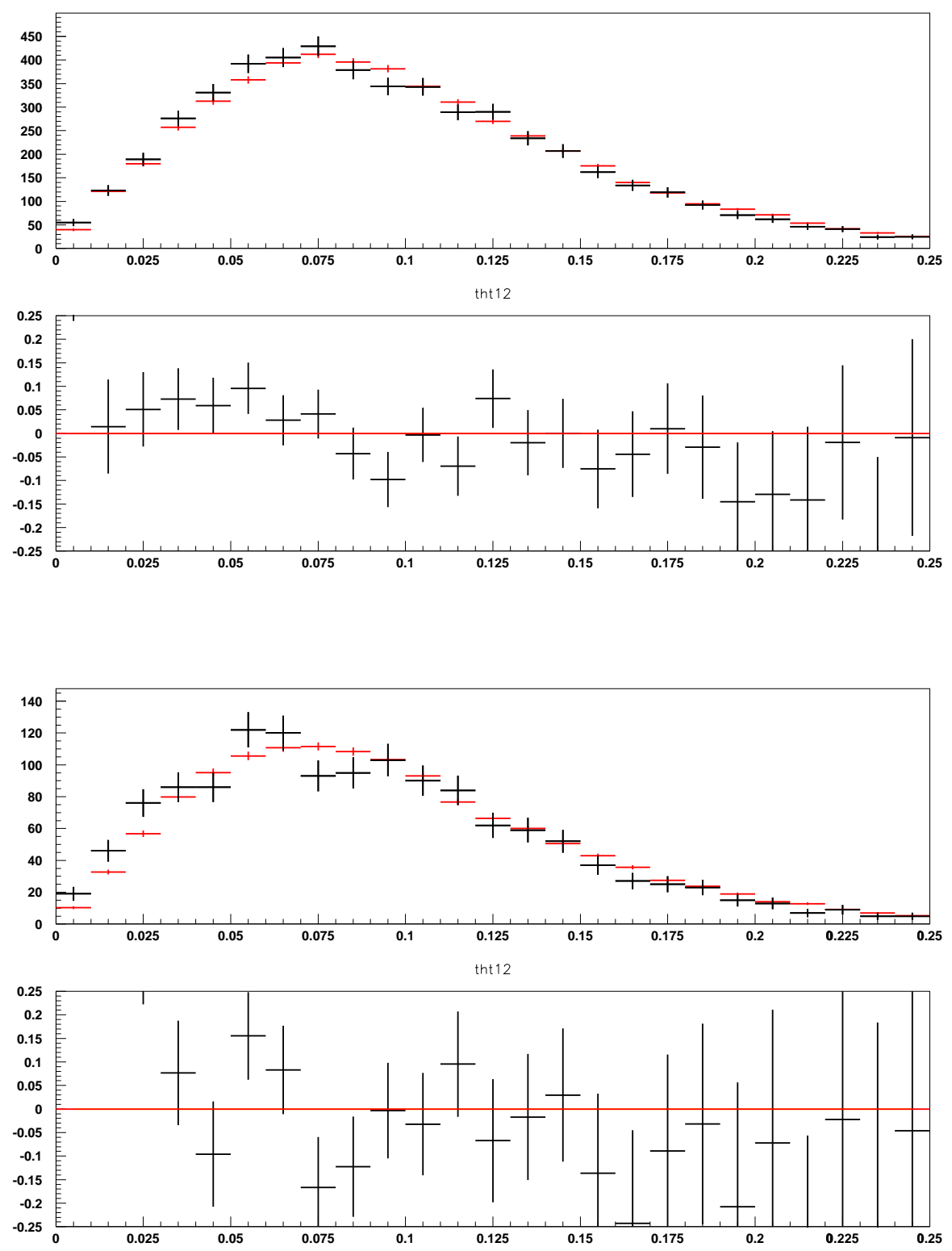

FIGURE 189. Reconstructed muon opening angle between the two muons, $\theta_{\mu 12}$, in data vs MC for the NLO dimuon sample, for both neutrino (top) and antineutrino (bottom) modes. Plotted are both absolute comparisons, with data in black, and $\mathrm{MC}$ in red, above ratio plots, showing data/MC-1. The $\mathrm{x}$ axis is in radians. 

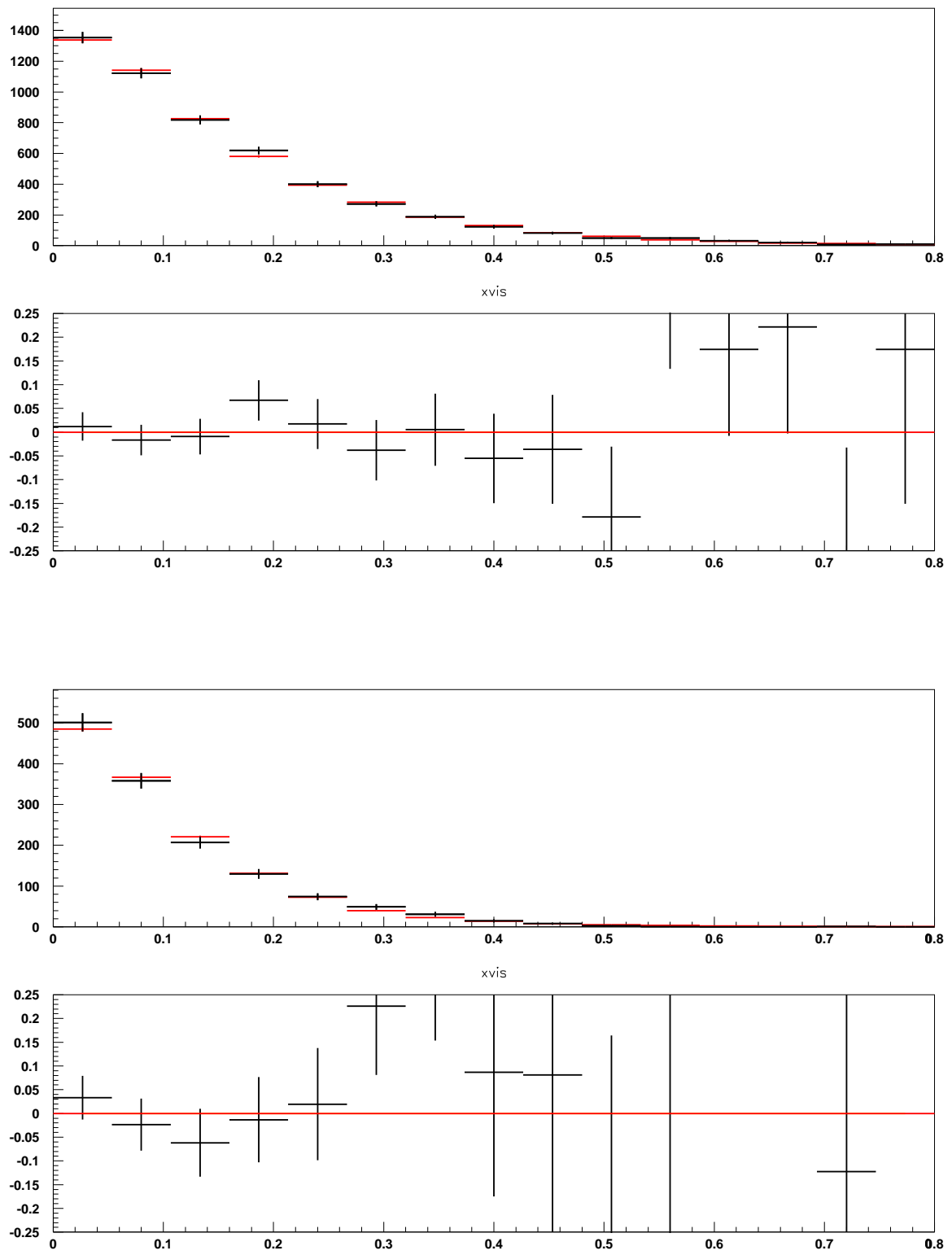

FIGURE 190. Reconstructed Bjorken $x, x_{v i s}$,in data vs MC for the NLO dimuon sample, for both neutrino (top) and antineutrino (bottom) modes. Plotted are both absolute comparisons, with data in black, and $\mathrm{MC}$ in red, above ratio plots, showing data/MC-1. 

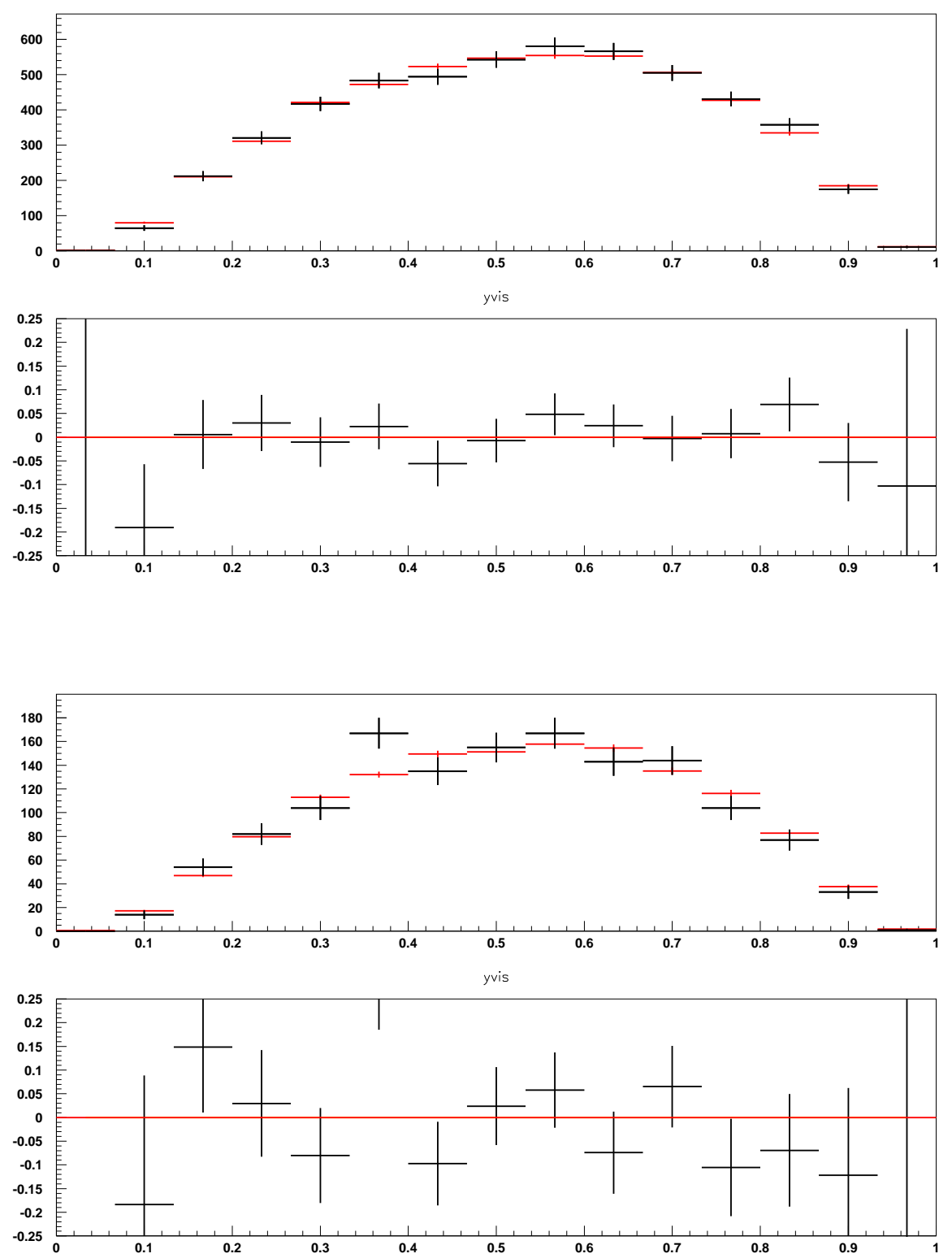

FIGURE 191. Reconstructed Inelasticity, $y, y_{v i s}$, in data vs MC for the NLO dimuon sample, for both neutrino (top) and antineutrino (bottom) modes. Plotted are both absolute comparisons, with data in black, and MC in red, above ratio plots, showing data/MC-1. 

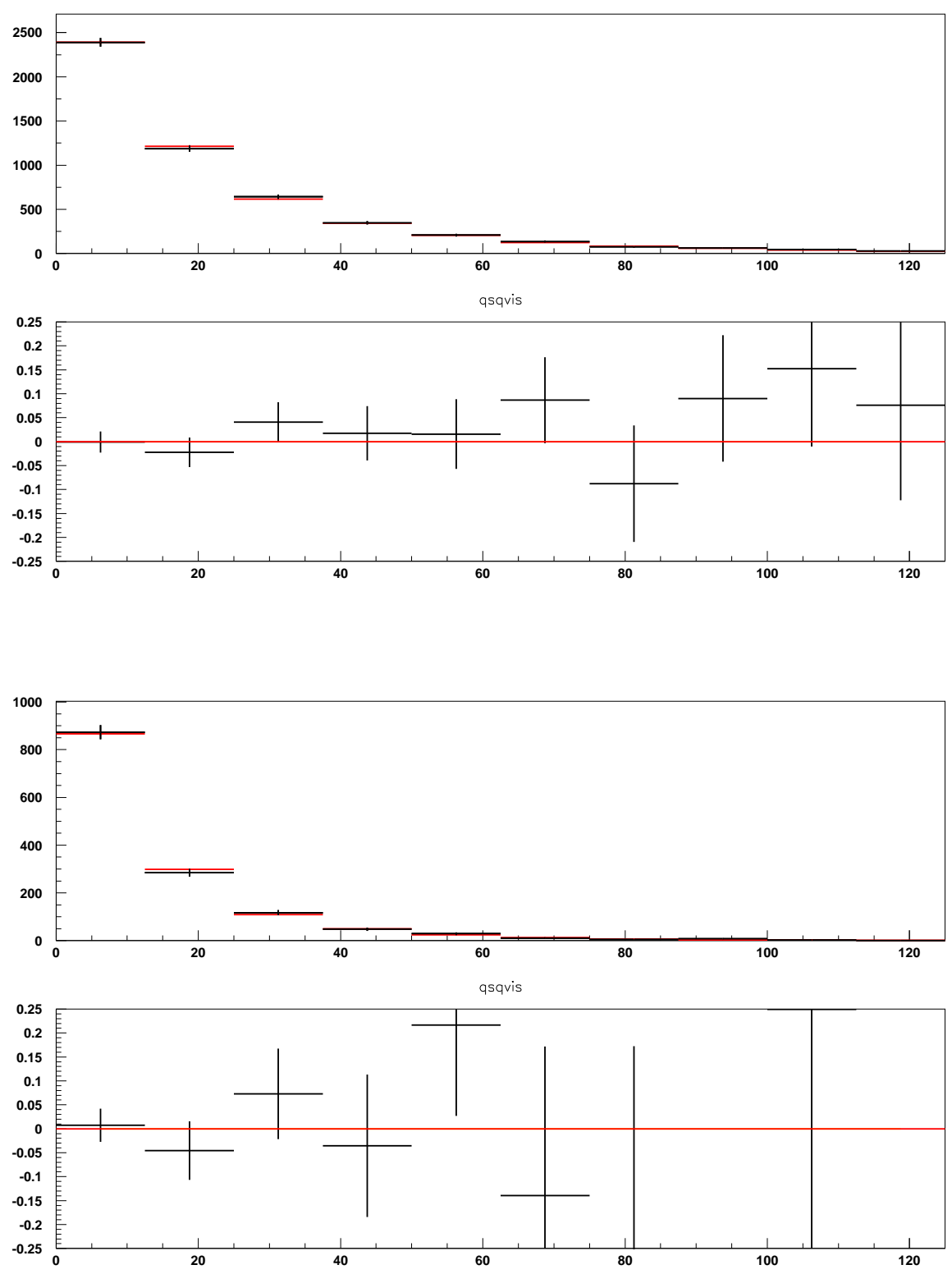

FIGURE 192. Reconstructed $Q^{2}, Q_{v i s}^{2}$, in data vs MC for the NLO dimuon sample, for both neutrino (top) and antineutrino (bottom) modes. Plotted are both absolute comparisons, with data in black, and $\mathrm{MC}$ in red, above ratio plots, showing data/MC-1. The $\mathrm{x}$ axis is in $\mathrm{GeV}^{2}$. 

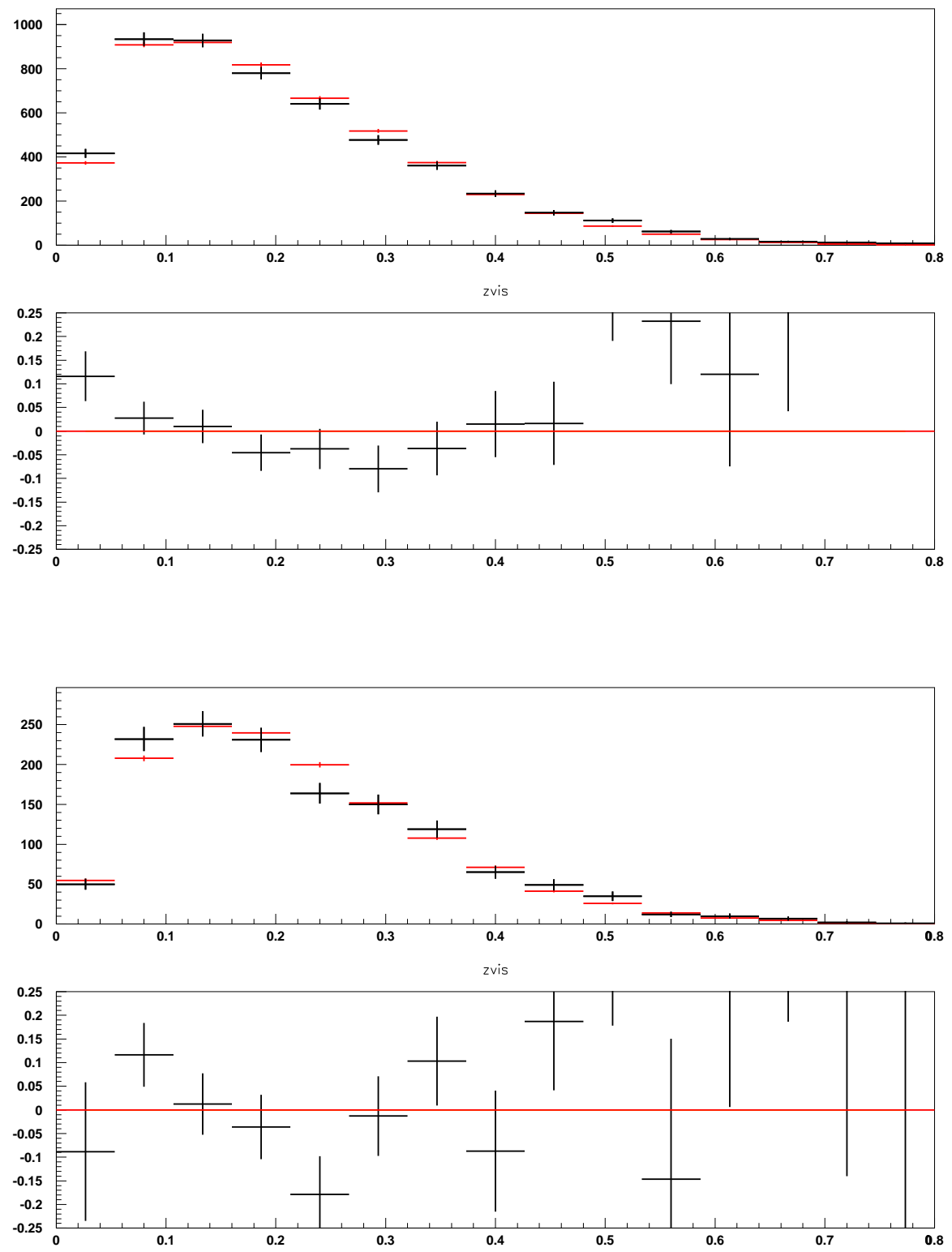

FIGURE 193. Reconstructed $z_{v i s}=\frac{E_{\mu 2}}{E_{\mu 2}+E_{H A D}}$, in data vs MC for the NLO dimuon sample, for both neutrino (top) and antineutrino (bottom) modes. Plotted are both absolute comparisons, with data in black, and $\mathrm{MC}$ in red, above ratio plots, showing data/MC-1. 

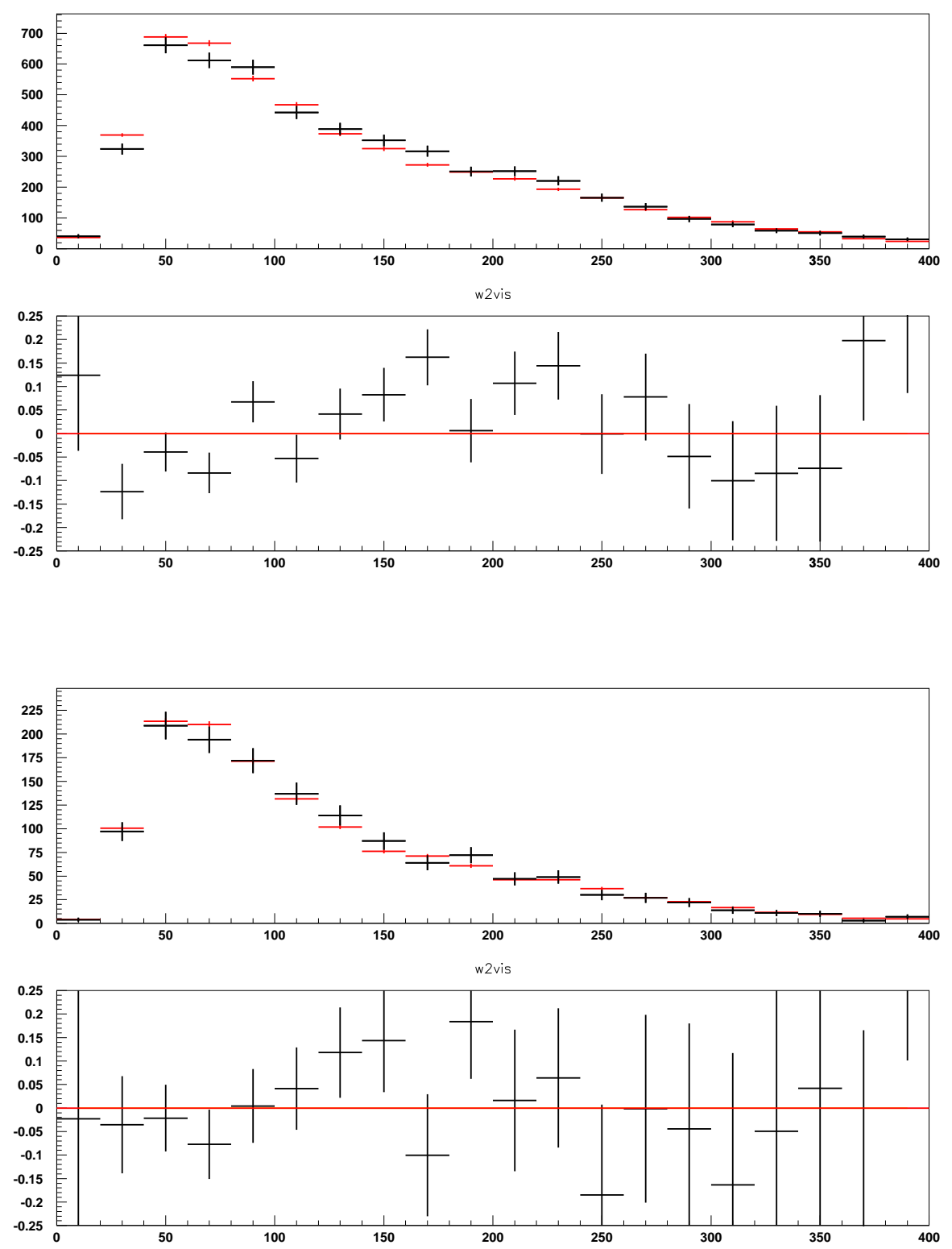

FIGURE 194. Reconstructed $W_{v i s}^{2}$, in data vs MC for the NLO dimuon sample, for both neutrino (top) and antineutrino (bottom) modes. Plotted are both absolute comparisons, with data in black, and $\mathrm{MC}$ in red, above ratio plots, showing data/MC-1. The $\mathrm{x}$ axis is in $\mathrm{GeV}^{2}$. 

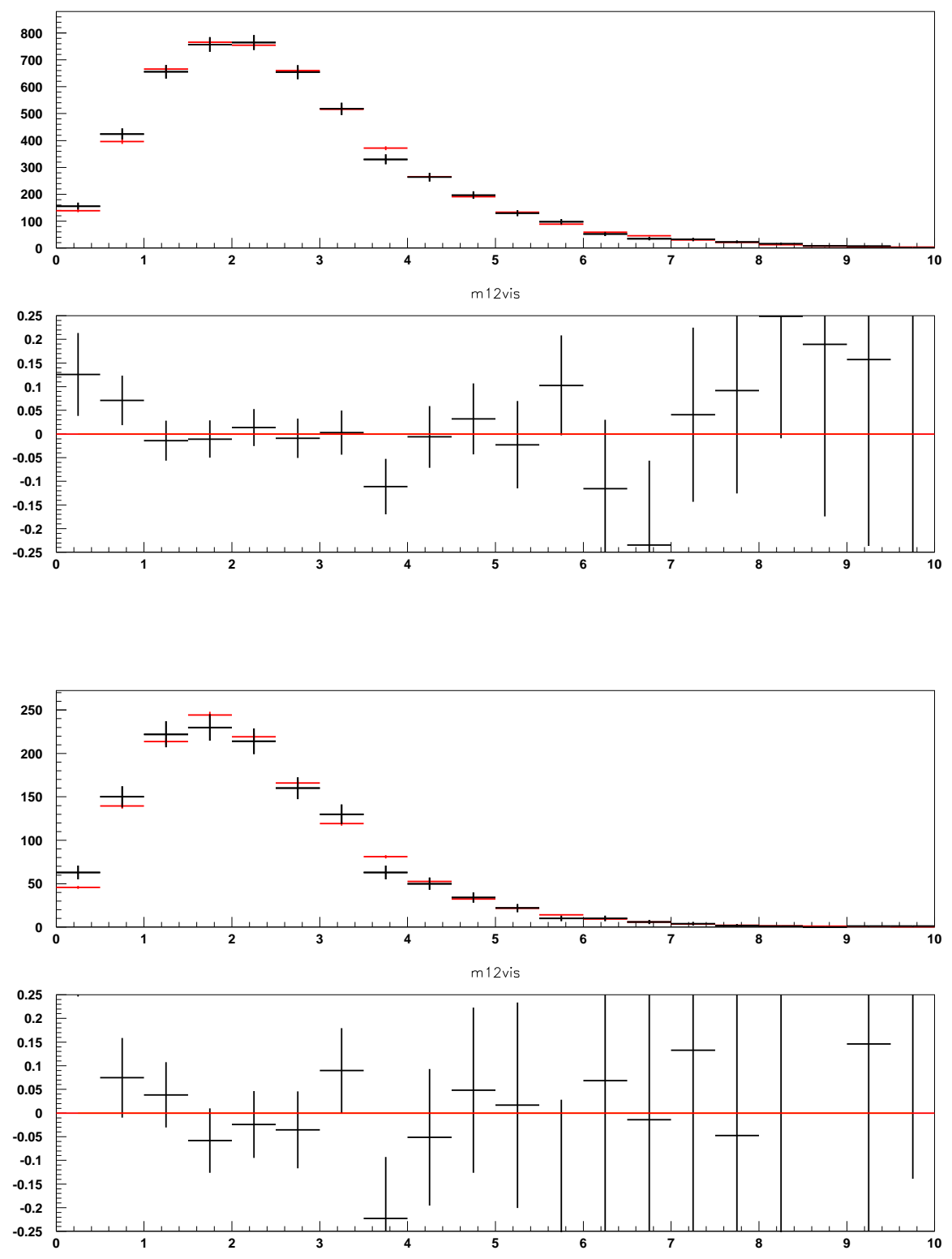

FIGURE 195. Reconstructed invariant mass between the two muons, $m_{12-v i s}$, in data vs MC for the NLO dimuon sample, for both neutrino (top) and antineutrino (bottom) modes. Plotted are both absolute comparisons, with data in black, and $\mathrm{MC}$ in red, above ratio plots, showing data/MC-1. The $\mathrm{x}$ axis is in $\mathrm{GeV}$. 


\section{$\underline{5.6}$ NLO table re-extraction}

With NLO MC that agrees with the data reasonably well, a final check remains - that the cross section table is indeed model independent. Extracting the NLO table is essentially the same as the procedure for the original table outlined in the previous chapter, except a fit to the raw data is not performed. The McNuTeV MC that was used for the NLO data/MC comparisons was fed into the NLO fitter along with the raw data, and the NLO inclusive cross section was called using the parameters from the McNuTeV run. The NLO acceptance corrections were applied from the same tables used in the NLO table fitter. The unfolding correction was determined in exactly the same way as in the LO BGPAR case, but using the NLO MC. Figures 196 and 197 show the resulting NLO extracted table in red, plotted on top of the original LO BGPAR extracted table. Figure 198 compares the strange asymmetries resulting from fits to each of the tables. The two asymmetries agree well, with the re-extracted table giving an $S^{-}$of $0.00176 \pm 0.00043$ (stat), compared to $0.00196 \pm 0.0046$ (stat) from the original table. 

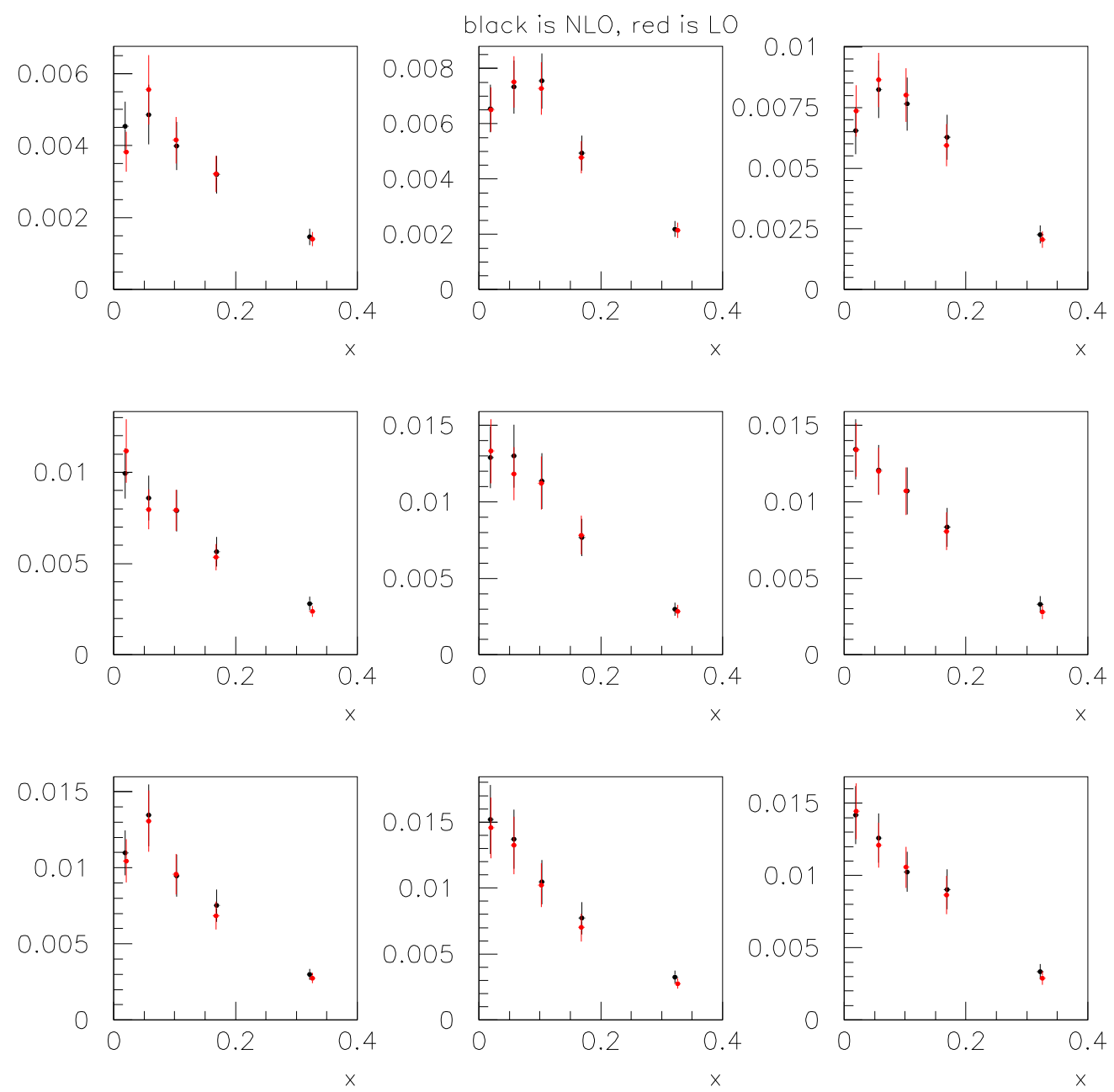

FIGURE 196. Comparison of the NLO extracted forward cross section table with the original LO extracted table ( $\nu$ mode bins). Points are shown multiplied by $\frac{\pi}{G_{F}^{2} M E_{\nu}}$ 

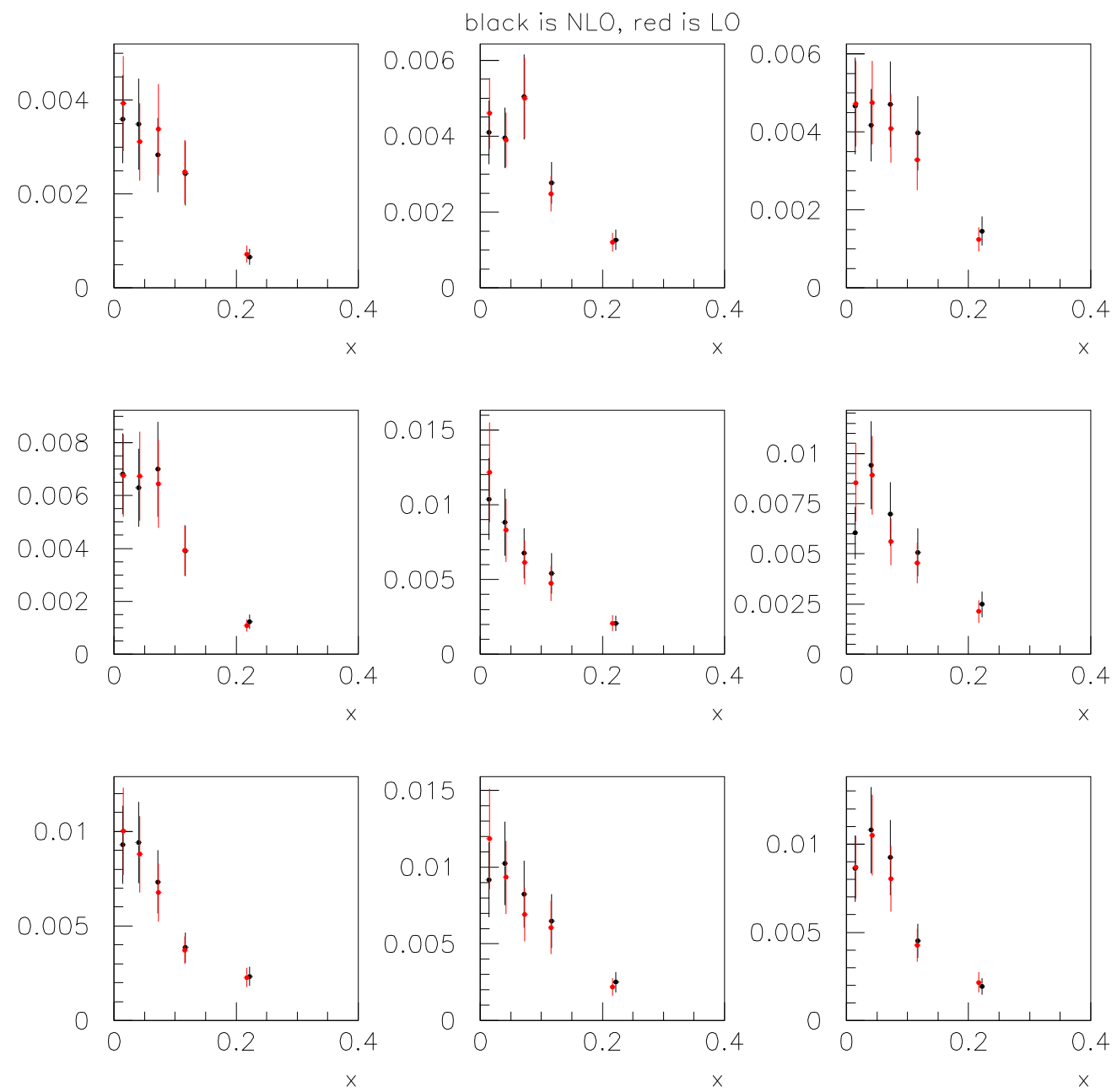

FIGURE 197. Comparison of the NLO extracted forward cross section table with the original LO extracted table ( $\bar{\nu}$ mode bins). Points are shown multiplied by $\frac{\pi}{G_{F}^{2} M E_{\nu}}$ 


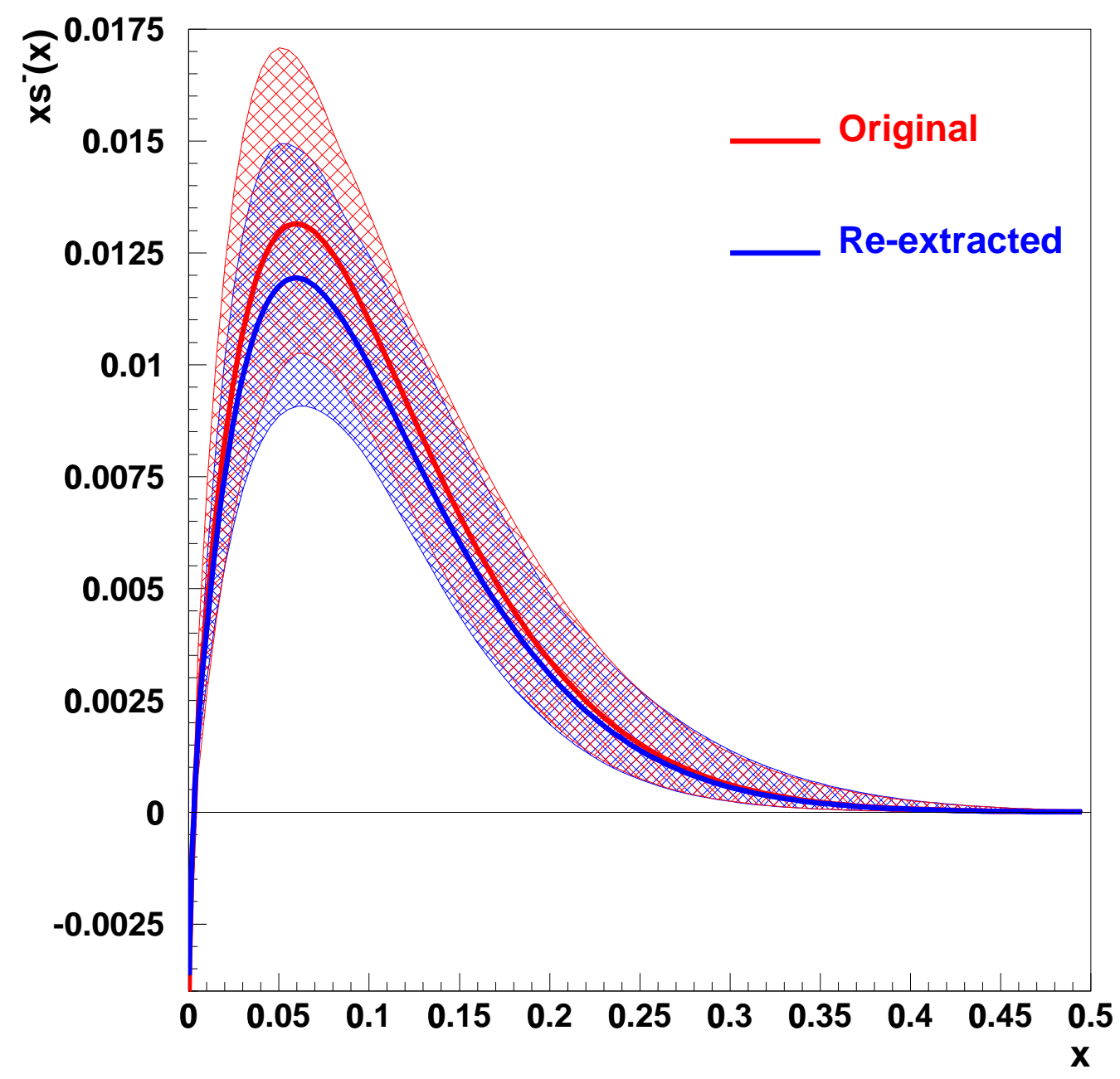

FIGURE 198. Comparison of the strange asymmetry resulting from a fit to the re-extracted table and the original asymmetry. Only statistical errors are shown. 


\section{$\underline{5.7 \text { Discussion }}$}

The strange and antistrange seas have been measured at NLO in QCD from the $\mathrm{NuTeV}$ neutrino and antineutrino dimuon data samples. This has been done using the first complete NLO description of this process. In prior measurements the strange sea has usually been assumed to be the same as the antistrange sea. Figure 199 compares $x s^{+}(x)$ from this analysis to the strange seas in three commonly used NLO pdf sets for a range in $Q^{2}$, starting at $16 \mathrm{GeV}^{2}$, close to the average $Q^{2}$ for $\mathrm{NuTeV}$. Figure 200 compares the values of $\eta_{s}$ measured over the past three decades, with the values obtained by both the LO fit in the previous chapter, and the NLO fit described above ${ }^{1}$. The sum of the strange and antistrange seas then agree well with past measurements.

If one wants to compare the difference between the strange and antistrange seas however, the field is much more limited. The two exceptions to the $s=\bar{s}$ rule were both measurements performed by the CCFR collaboration. The first, based on E-744 [61], measured the strange and antistrange seas independently, however did not do any quantitative analysis. Plots of the seas are shown in figure 14, back on page 36 . They found $x s(x)$ to be slightly larger than $x \bar{s}(x)$, however the error bars were such that the two were consistent with each other. CCFR was also limited by not having a sign selected beam, so that a transverse momentum based algorithm to choose which muon was from the leptonic vertex, and which was from

\footnotetext{
${ }^{1}$ The error on the NLO measurement is larger in part because external measurements are used for branching ratio, charm mass, etc. In the LO case, branching ratio, fragmentation $\epsilon, m_{c}$ and the overall level of the strange seas are correlated, reducing the error. This is at the expense of the branching ratio and fragmentation parameter in some sense absorbing some of the strange sea, and charm mass not really having physical meaning outside this model. The NLO error bar should be a more accurate representation of the uncertainty on the measurement.
} 

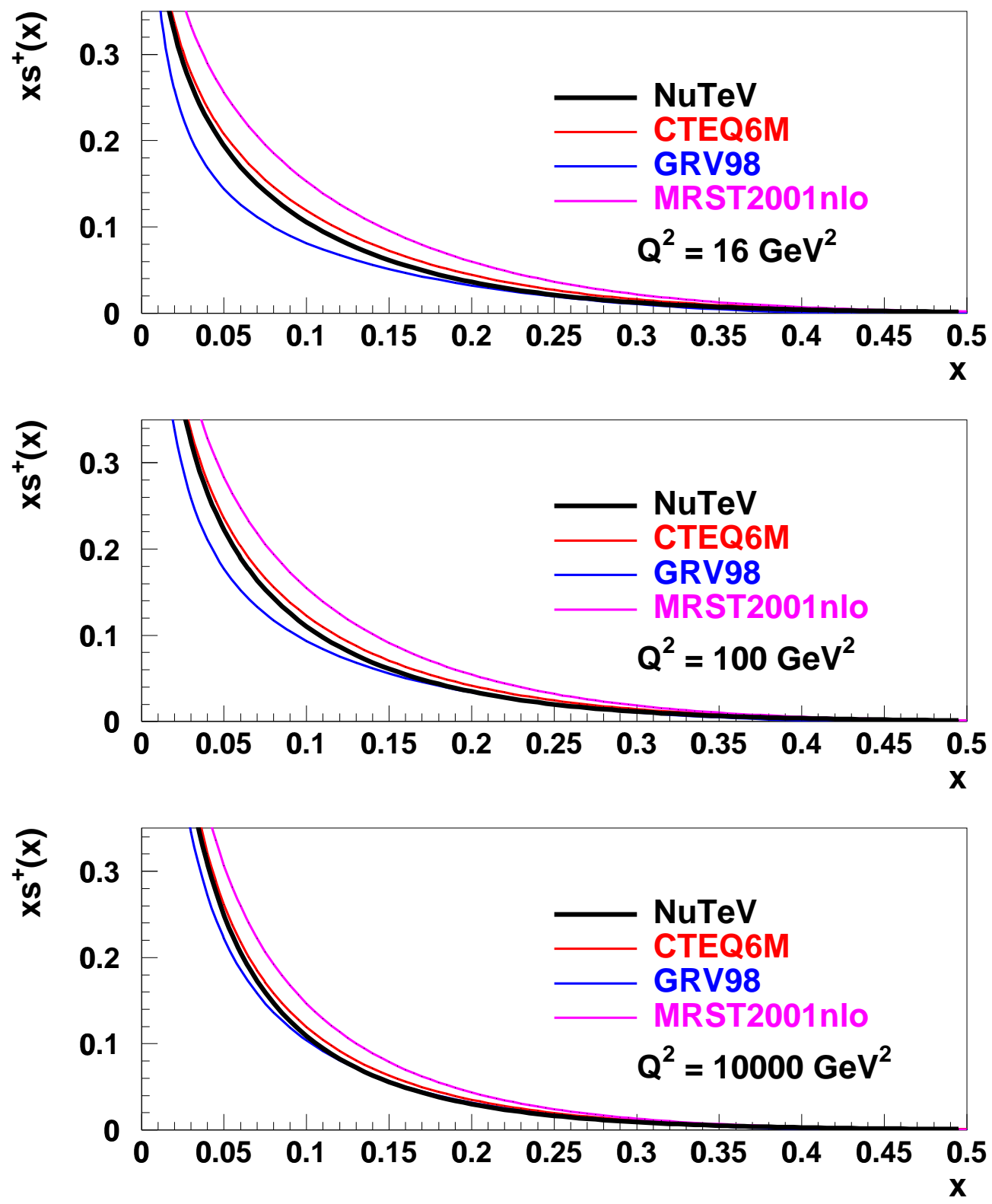

FIGURE 199. $x s^{+}(x)$ vs $x$ at $Q^{2}=16,100$, and10,000GeV ${ }^{2}$ for the CTEQ6M [182], MRST2001nlo [189], and GRV98 [190] pdf sets 


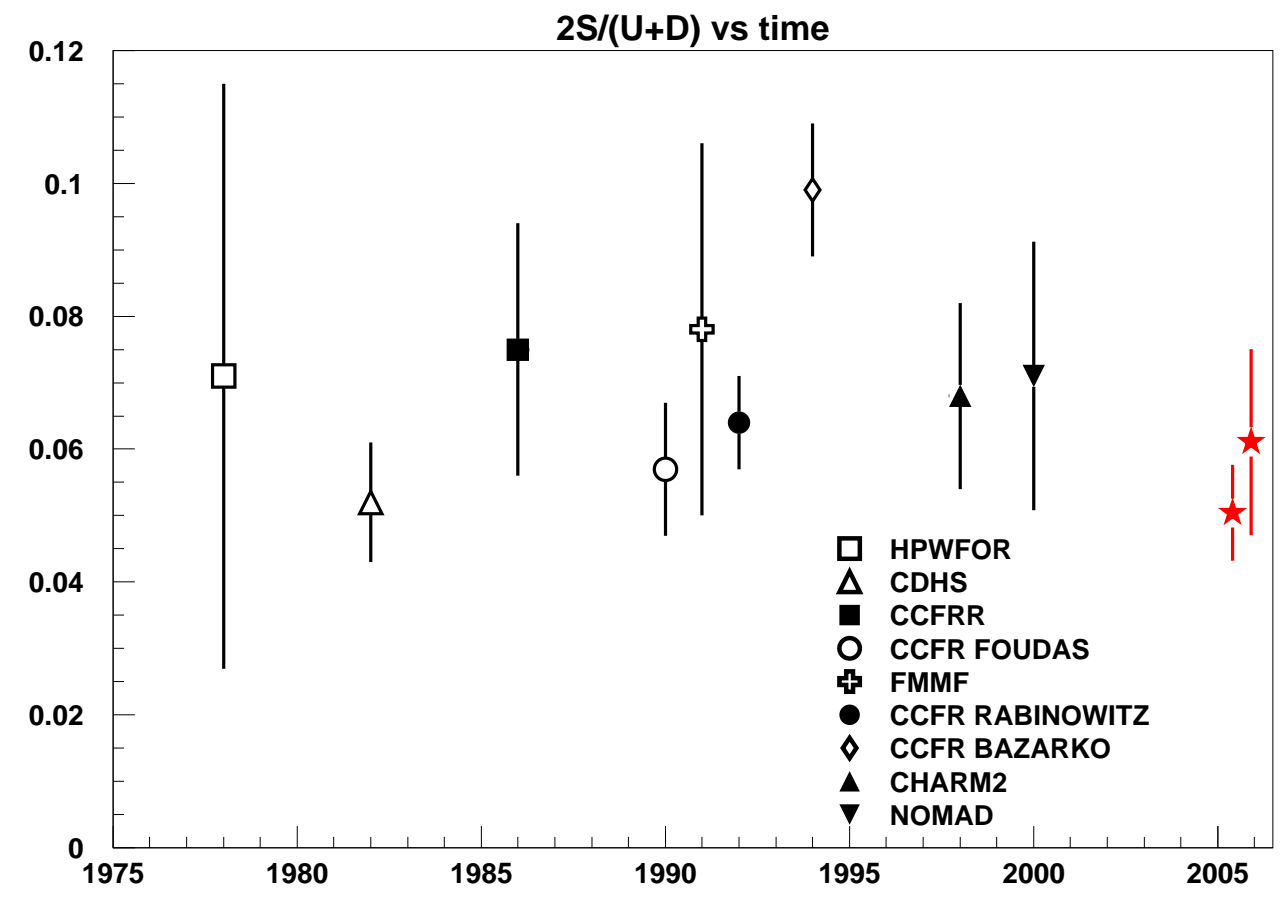

FIGURE 200. $\eta_{s}$ vs time. Other experimental values of $\eta_{s}$ dating back to the 1970's [51, 58, 71, 61, 73, 62, 63, 74, 69]. The two stars at the right of the plot are from the LO BGPAR fit in the previous chapter (left star) and the NLO analysis discussed here (right star).

charm was required. This algorithm would, for example, tend to pick the wrong muon at high $y$ or low $x$. Without knowing ahead of time whether a neutrino or antineutrino interacted, mis-assigning the muons would move neutrino generated events to the anti-neutrino side, and vice versa.

A study of the shape difference between the strange and antistrange seas was performed in the CCFR NLO analysis [63] (see pages 35 and 117). The 
parameterization however was quite limited:

$$
\begin{aligned}
& x s\left(x, Q^{2}\right)=A_{s}(1-x)^{\alpha}\left[\frac{x \bar{u}\left(x, Q^{2}\right)+x \bar{d}\left(x, Q^{2}\right)}{2}\right] \\
& x \bar{s}\left(x, Q^{2}\right)=A_{s}^{\prime}(1-x)^{\alpha^{\prime}}\left[\frac{x \bar{u}\left(x, Q^{2}\right)+x \bar{d}\left(x, Q^{2}\right)}{2}\right]
\end{aligned}
$$

where $A_{s}$ to $A_{s}^{\prime}$ were constants dependent on the overall level of the strange and antistrange seas (defined by a constant $(\kappa)$. The flavor sum rule was enforced by tying $A_{s}$ to $A_{s}^{\prime}$ such that $\int_{0}^{1} s(x)-\bar{s}(x)$ was zero. This left the only degree of freedom between the two being from the difference in $\alpha$ and $\alpha^{\prime}$.

The result was an asymmetry consistent with zero, with an $\alpha$ of $-0.78 \pm 1.30$ and an $\alpha^{\prime}$ of $-0.32 \pm 0.87$. In this case the strange sea was again larger than the antistrange sea (though not significantly), though due to the $(1-x)^{\alpha}$ factor's strength appearing at high $x$, the difference manifested at higher $x$.

The CCFR data was later re-analyzed by $\mathrm{NuTeV}[75]$ and combined with the original $\mathrm{NuTeV}$ forward dimuon cross section. The combined data was fit at LO in QCD using a more flexible parameterization like that used in section 5.4.2 [185]. The asymmetry in that case was found to be $-0.0027 \pm 0.0013$. The improved $\mathrm{NuTeV}$ table found in the previous chapter however, would certainly shift this number in a positive direction.

Aside from a possible future re-analysis of the combined $\mathrm{NuTeV}$ and CCFR $\mathrm{data}^{2}$, the best information currently available are the results described in this document. In these NLO fits to the cross section table, a positive asymmetry

\footnotetext{
${ }^{2}$ Given the mixed beam though, its unclear to what level incorporating the CCFR data would improve on this measurement
} 


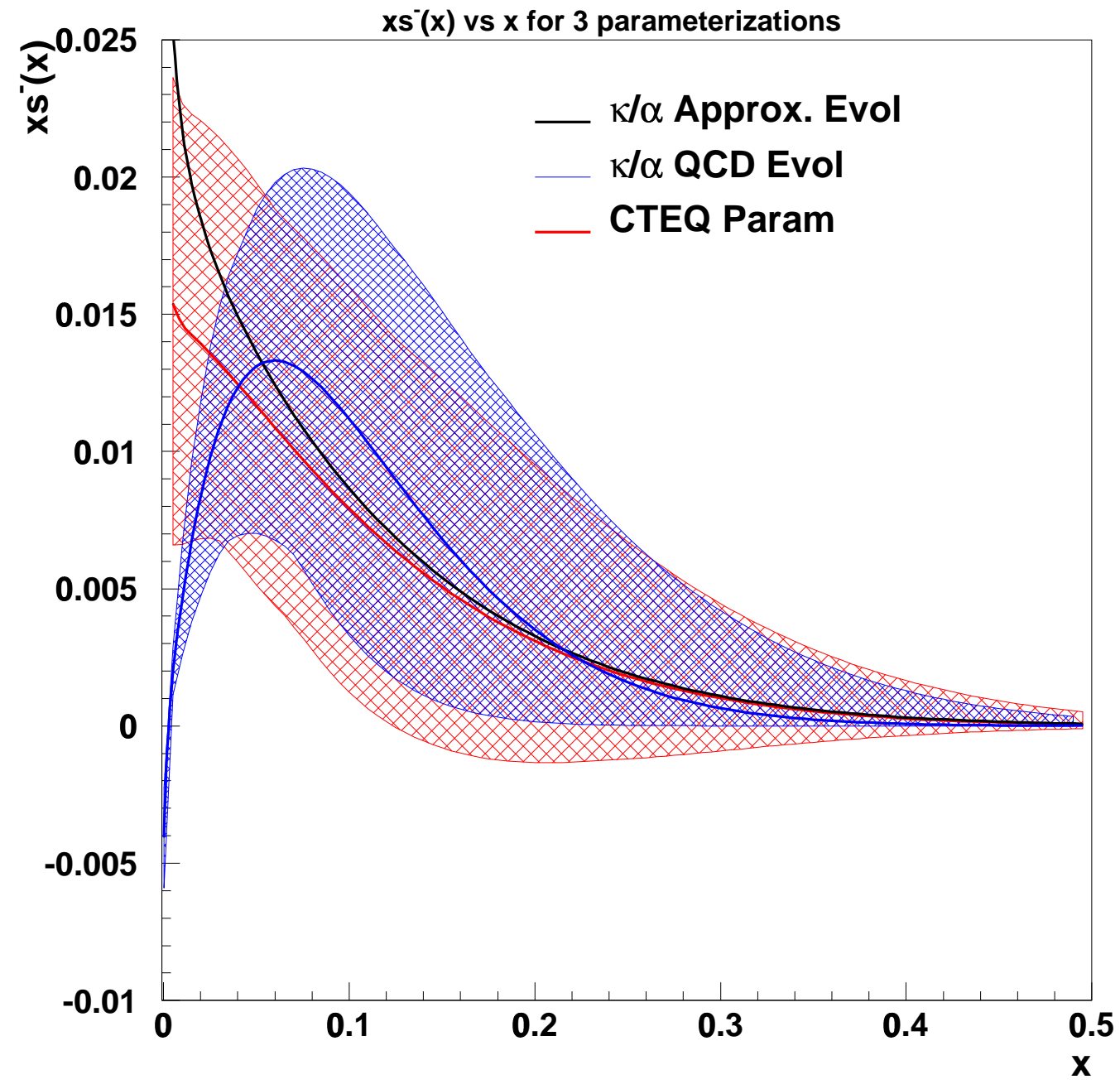

FIGURE 201. $x s^{-}(x)$ vs $x$ at $Q^{2}=16 G e V^{2}$ for the three different strange sea parameterizations tried. 
at low $x$ is preferred, no matter if the simple application of level and shape parameters to pre-evolved seas, or the more sophisticated, QCD sum rule satisfying parameterization is used. Figure 201 shows the asymmetries from each of these fits plotted on top of each other, including the total error bands. The asymmetry has been measured three different ways, and all get very similar answers. These answers can provide some constraint on theoretical models of the asymmetry.

\subsubsection{Asymmetry and crossing point}

The simplest picture of how the strange and antistrange seas come into being has strange and antistrange quarks continually pair-produced and annihilating from the gluon sea. If these $s-\bar{s}$ pairs only exist for a short time, then they will not have a chance to interact. In that case the strange and antistrange seas should be identical. If however they live long enough inside the nucleon to interact with the valence quarks, then an asymmetry can exist [191].

One possible way for this to happen is for the strange and antistrange quarks to live long enough that the nucleon fluctuates into a virtual pair of hadrons $[192,193,194]$. A proton, for example, could briefly fluctuate into a $\Lambda$ (u-d-s) and a $K^{+}(\mathrm{u} \bar{s})$. Here an asymmetry develops from the fact that the two particles have different masses and because baryon number conservation prevents the opposite, $\bar{\Lambda}, K^{-}$from happening. Because the $\Lambda$ is more massive than the $K^{+}$, it would tend to carry a larger fraction of the nucleon momentum, which would translate to a harder strange sea - a positive asymmetry.

The positive asymmetry measured here is a bit different than what the models expect. The low- $x$ spike of the CTEQ parameterization fit for example is clearly 

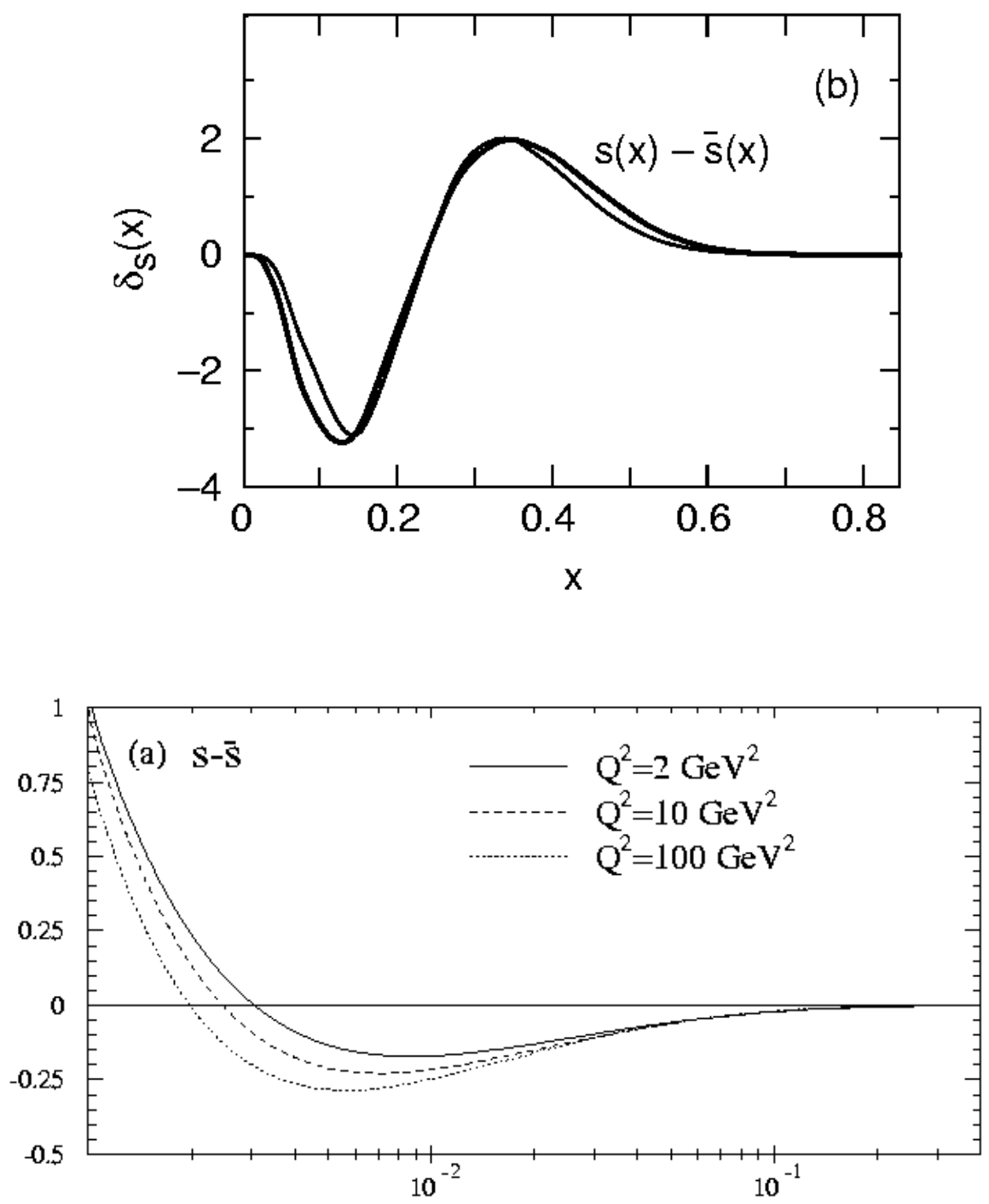

FIGURE 202. Theoretical predictions for $s(x)-\bar{s}(x)$ from references [193] (top) and [195] (bottom) 

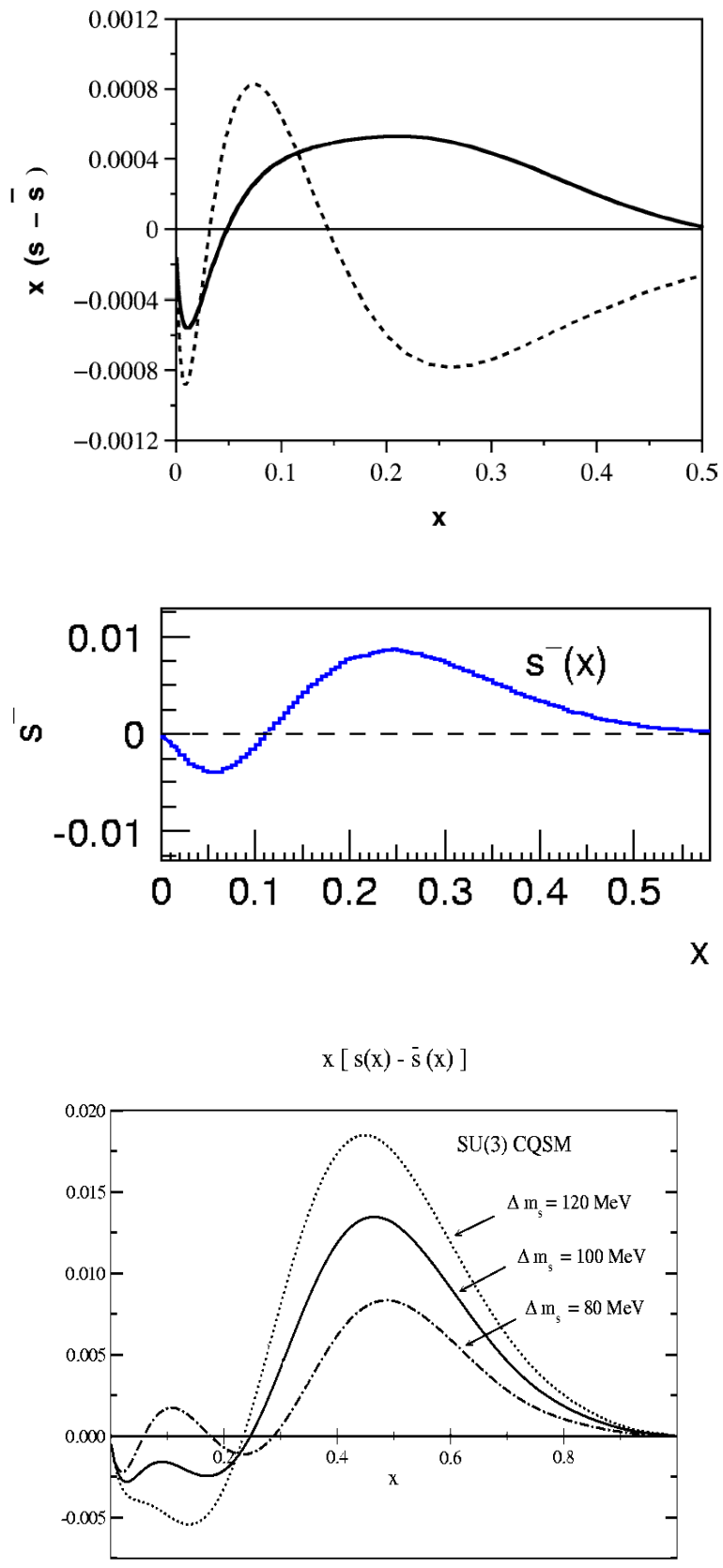

FIGURE 203. Theoretical predictions for $x s(x)-x \bar{s}(x)$ from references [196] (top), [194] (middle), and [197] (bottom) 
unphysical. This spike occurs because the fit has complete freedom to place the crossing point $x_{0}$ anywhere it wants. The crossing point however is usually constrained in the various meson fluctuation models by the difference in masses of the hadrons the nucleon is fluctuating into. The predicted crossing point values range from an $x_{0}$ around 0.05 [196] to around 0.2 [193]. Several examples of predicted asymmetries are shown in figures 202 and 203.

The dependence of the asymmetry on $x_{0}$ can be examined by performing fits with fixed values of $x_{0}$ and solving for one of the other strange sea parameters to enforce the sum rule. This is most easily achieved with $\gamma^{-}$. (It can also be achieved by solving for $\alpha^{-}$, the difference being that the lever arm is at high $x$, as opposed to $\gamma^{-}$for low $x$. Both were tried, however the " $\alpha$ " fits either failed completely, having trouble converging, or returned with zero asymmetry.) Figure 204 shows the effect of a forced crossing point on $S^{-}$and $\chi^{2}$. As $x_{0}$ increases, the asymmetry decreases at the expense of $\chi^{2}$, until it reaches zero. There the $\chi^{2}$ peaks at about 54.5 for 39.8 effective degrees of freedom. There is a small undershoot, where the asymmetry goes slightly negative, and the $\chi^{2}$ gets slightly smaller - this is due to the shape of the positive and negative bumps in the asymmetry swapping signs, with low $x$ going positive, and high $x$ negative. Figure 205 shows three of the curves at different values of $x_{0}$. Asymmetry models with crossing points at high $x_{0}$ are difficult to accommodate with the $\mathrm{NuTeV}$ data.

\section{$\underline{5.8 \text { Some Closing Remarks }}$}

Other than a few fascinating appendices, this document now (finally) comes to a close. The strange and antistrange seas have been measured, the strange 

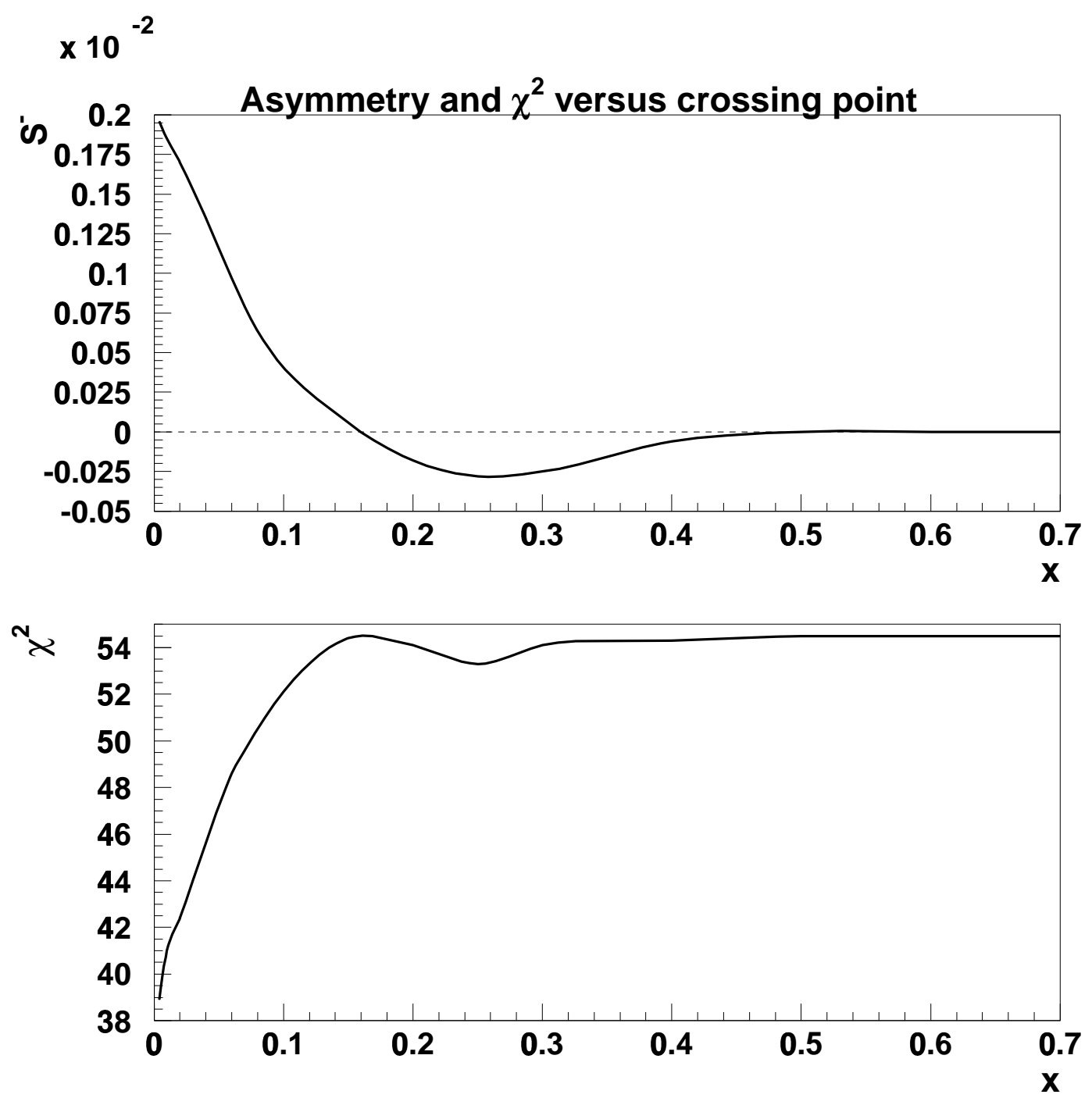

FIGURE 204. $S^{-}$and $\chi^{2}$ versus crossing point 


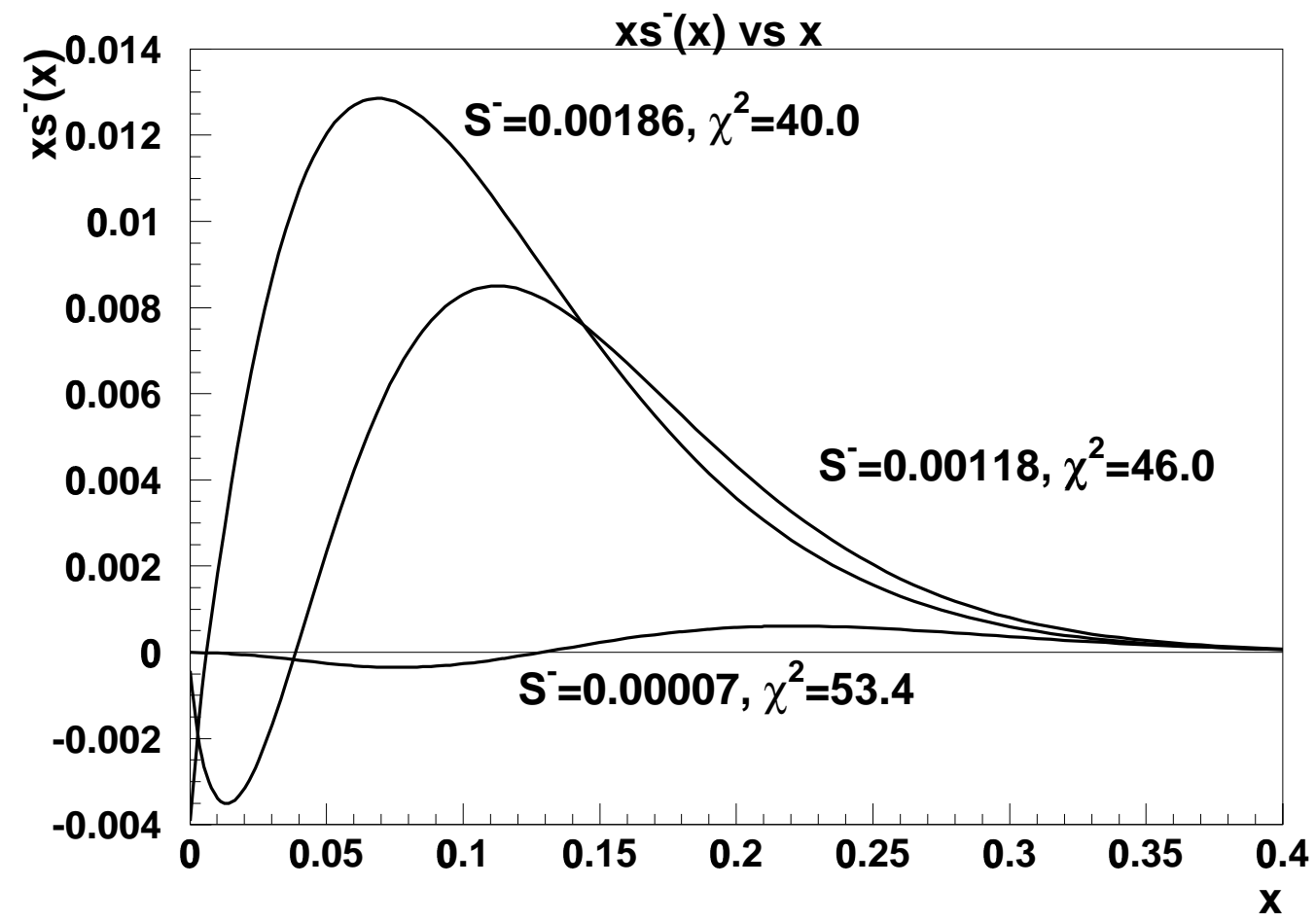

FIGURE 205. $x s^{-}$versus $x$ for $x_{0}$ of $0.01,0.05$, and $0.15 . \chi^{2}$ 's are labeled with an effective degree of freedom of 38.8 .

sea has been found to have a slightly larger momentum distribution than the antistrange sea. This can be consistent with theoretical expectations if the the model in question satisfies the flavor sum rule by crossing from $s(x)<\bar{s}(x)$ to $s(x)>\bar{s}(x)$ at relatively low $x$.

Beyond this measurement then, what can be done to improve knowledge of the strange sea and asymmetry? With the current data, certainly improving knowledge of charm semileptonic decays and charmed hadron production rates would go a long way. Figure 206 is a variation of figure 179, separating out the contribution to the error from only the charm branching ratio $B_{c}$, versus all the 
other errors. If $B_{c}$ were known very well, the error on this asymmetry measurement may be reduced by approximately half.

The other way to improve knowledge of the strange and antistrange seas would of course be be at a future $\nu-D I S$ experiment. If at some time in the distant future (and admittedly this is probably outright dreaming), the particle physics community decides to ply its talents towards building a high energy muon collider, the neutrino beams resulting from the muons decaying in the $\mu$ beamline could provide a high intensity, narrow band beam [198]. A beam like this would eliminate the need for giant coarse-grained detectors, as has been used in $\mathrm{NuTeV}$ and its predecessors. A more fine grained detector would then be able to study charm production directly through identification of the charmed hadrons themselves rather than requiring the production of an obvious decay muon. Not only would this allow better study of the strange and antistrange seas, but for detailed study of charm production and the hadronization process. Who knows, maybe this century... 


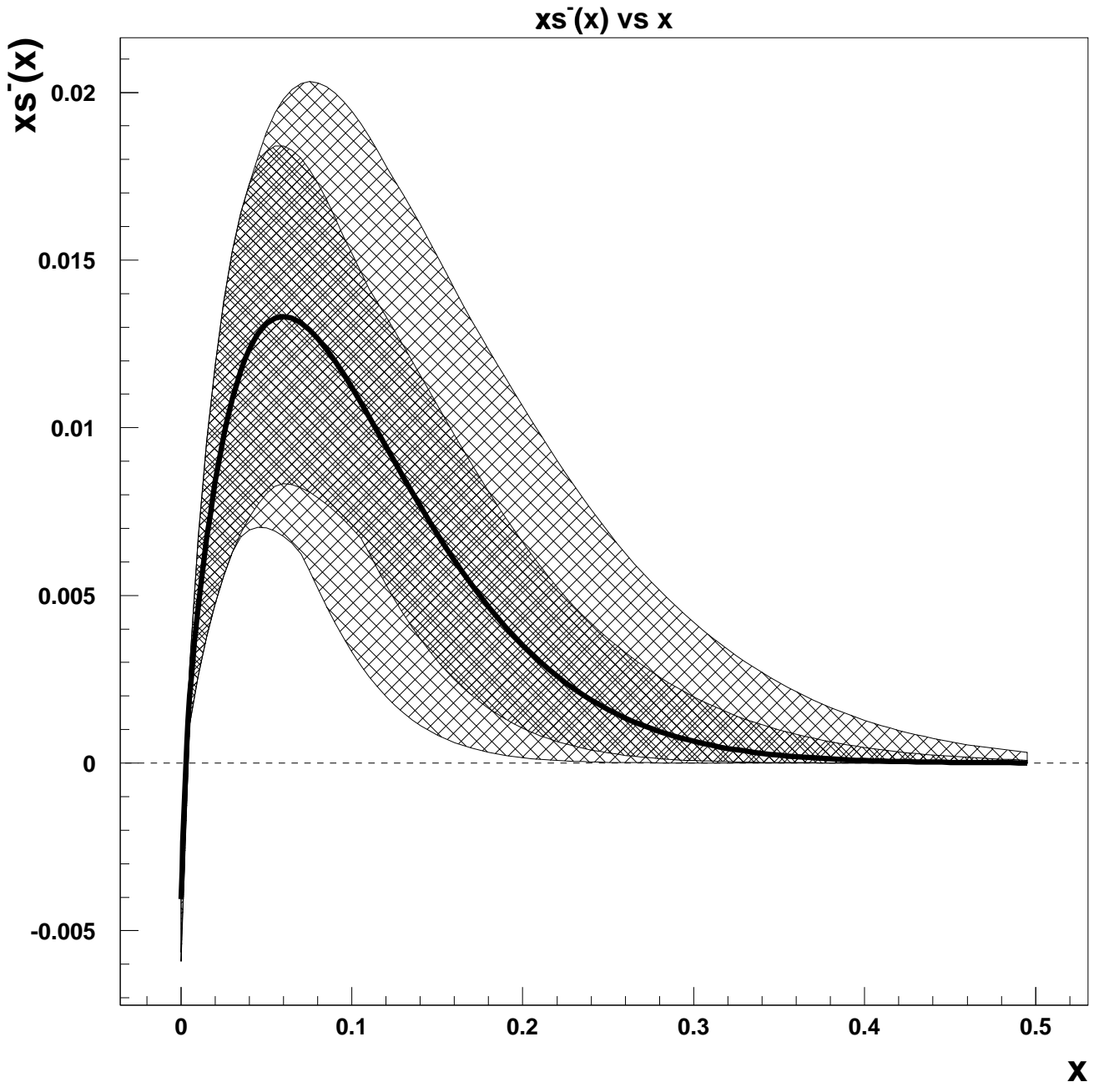

FIGURE 206. $x s^{-}(x)$ vs $x$ at $Q^{2}=16 \mathrm{GeV}^{2}$. Outer band is combined errors, inner band is without $B_{c}$ uncertainty. 


\section{APPENDIX A}

\section{THE NUTEV BEAM TOROID}

\section{$\underline{\text { A.1 Introduction }}$}

A description of the $\mathrm{NuTeV}$ beam toroid is provided here so that some form of documentation exists, were something like it to be used in a future neutrino experiment. It is was very simple device, used to measure the number of protons interacting with the primary target in addition to being an important element in NuTeV's triggering system. A photograph is shown in figure 207.

A beam toroid is a toroidal inductor coaxial with a charged particle beam to measure how many particles (how much integrated current) passed by that point during a fixed period of time. Beam toroids are part of a family of devices that are used to monitor the characteristics of particle beams without interfering with them. Some other examples include beam position monitors (BPMs), which consist of a pair of electrode plates on opposite sides of the beam, whose proximity to a beam offset induces a charge difference that can be measured to determine the position of the beam in one direction. They are usually used in pairs to get both dimensions of the transverse position of the beam. NuTeV used two pairs of BPMs to monitor the position and angle of the proton beam immediately before the primary target. Another interesting device, not used in NuTeV's case (but mentioned here to complete the resistor, capacitor, inductor set) is the resistive wall monitor. Resistive wall monitors are breaks in the metal vacuum pipe which 


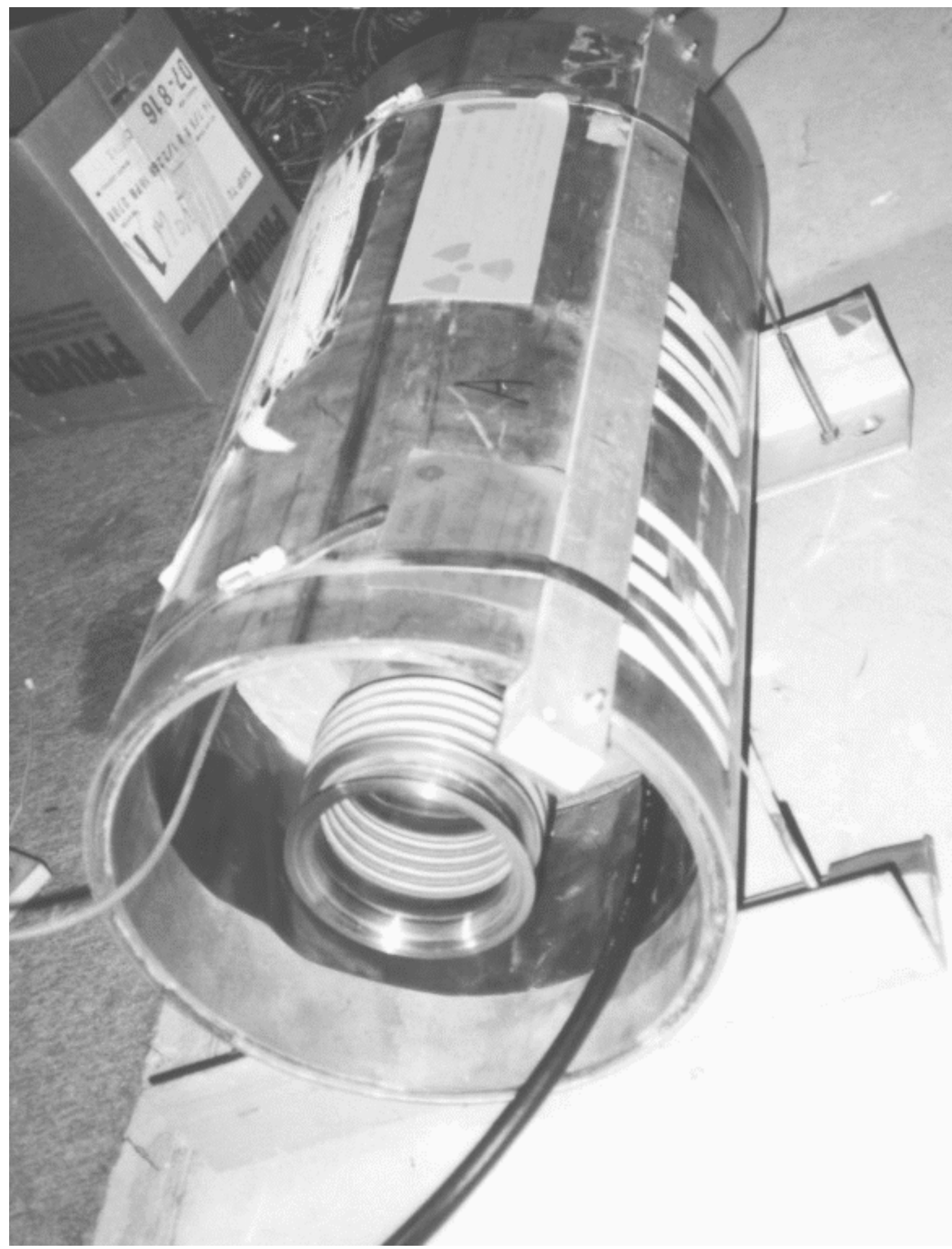

FIGURE 207. NuTeV's beam toroid before installation in the primary beam 


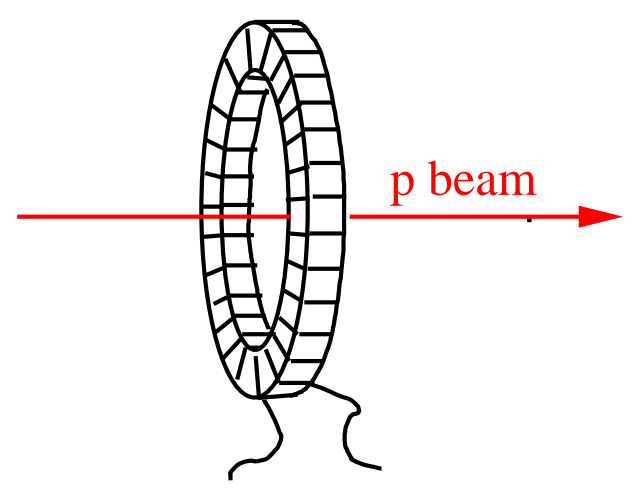

FIGURE 208. A cartoon illustrating a beam toroid

surrounds the beam, replaced with a "resistive gap". As a beam travels along the length of its vacuum pipe enclosure, an image current is induced in the pipe of opposite sign. The voltage measured across resistors bridging the gap is proportional to the image current, and therefore the beam current as well. Because they do not have the $\mathrm{AC}$ complications of toroids or BPMs, resistive wall monitors can be used to resolve the short time structure of particle beams. A disadvantage of using these devices is that the break in the beam pipe interferes with the mirror current that runs along it opposite to the beam, and the resulting RF noise can adversely affect the beam. Many more types of beam monitoring devices exist, but as a full description would more than likely be another thesis in itself, we will concentrate on just NuTeV's beam toroid.

\section{$\underline{\text { A.2 Description }}$}

A cartoon of a beam toroid is shown in figure 208. Toroids are simple devices, merely a wire wrapped around a donut shaped, highly permeable metal core, with the hole centered on a particle beam. NuTeV's toroid was based on a "supermalloy" 


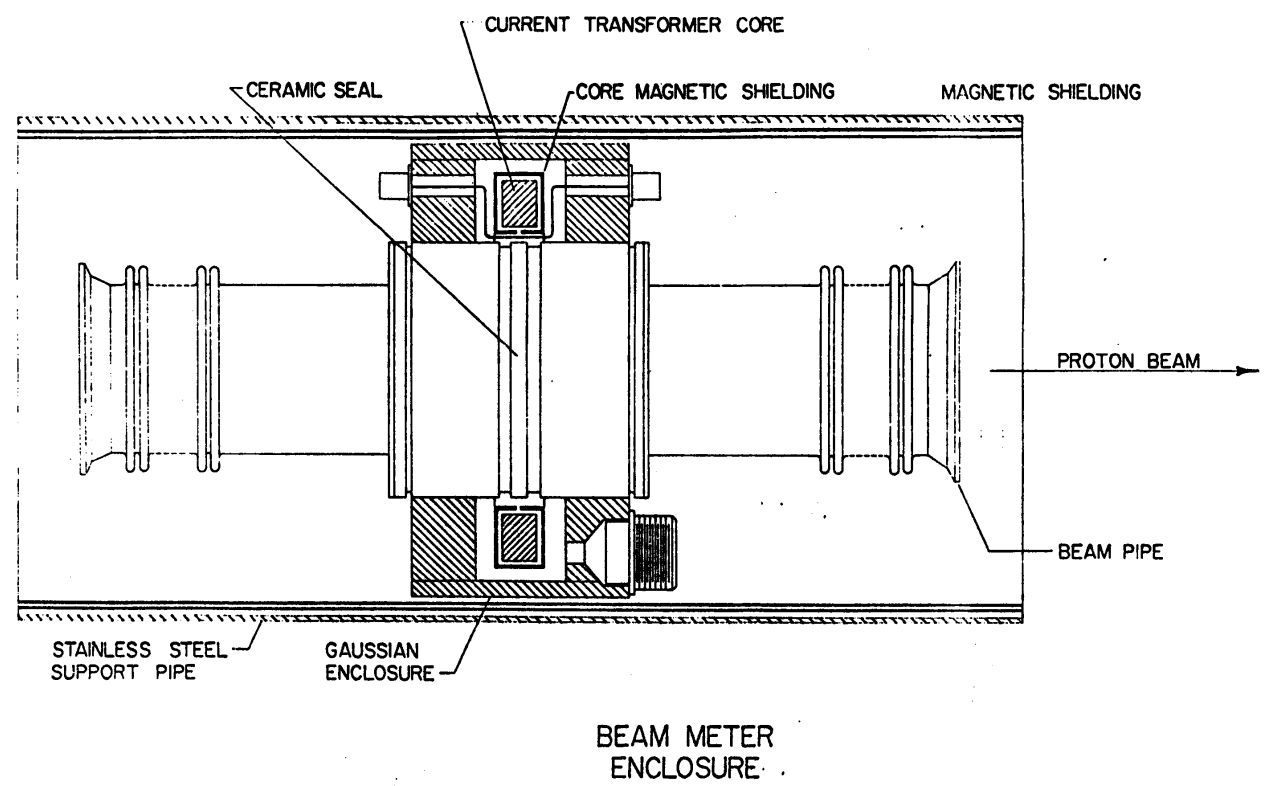

FIGURE 209. A schematic diagram of NuTeV's beam toroid

core - a Nickel, Iron, and Molybdenum alloy with very high permeability. Figure 209 shows a schematic of the actual toroid used to monitor NuTeV's beam[199]. The toroidal coil was placed coaxial with an insulating ceramic section of the beam pipe so that the vacuum surrounding the beam was preserved, but the beam's current was not shielded by the beam pipe's steel. A small wire was also placed through the coil to provide a means of calibrating the toroid with a known current.

As the particle density in the proton beam changed during a ping, a current would be induced in the wires in the toroid coil, which was then transmitted through a long coaxial cable to an amplifier and digitizer. The toroid data was used for the following purposes:

- Digitized ping waveforms were recorded via the EPICURE[145] data acquisition system, to be included along with other beam monitoring information in the $\mathrm{NuTeV}$ data. 


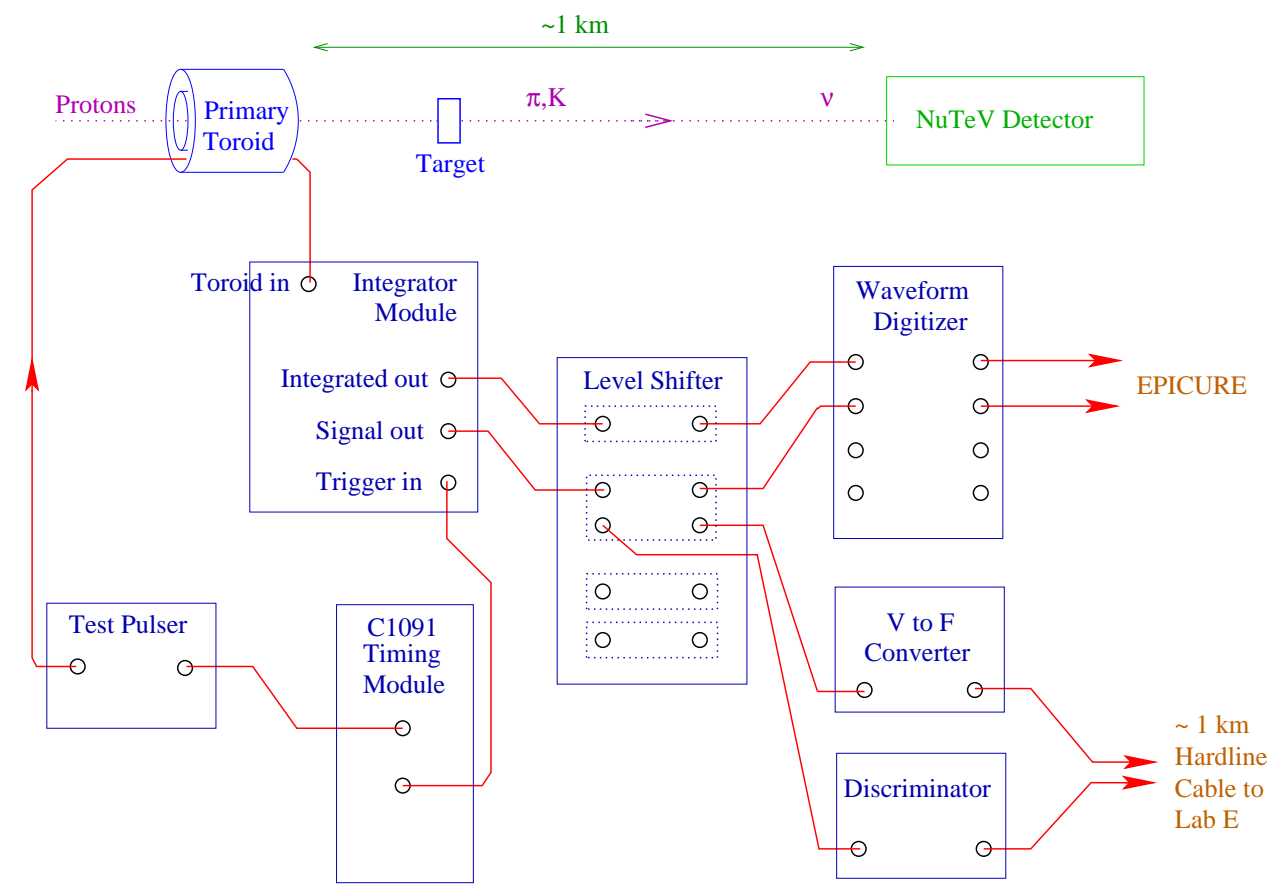

FIGURE 210. A schematic of the use of the NuTeV toroid

- A discriminated gate pulse was sent through approximately 1 kilometer of hardline cable to the NuTeV data acquisition system. This formed part of the "beam gate" that activated the detector during each ping.

- An integrated signal from the toroid was passed through a voltage to frequency ( $\mathrm{V}$ to $\mathrm{F}$ ) converter and sent down another long hardline cable to the detector control room to provide a visual measurement of the intensity of each ping for shift personnel. This data was also recorded in the $\mathrm{NuTeV}$ data.

A schematic diagram of the various paths through which signals from the toroid were processed and included in the recorded $\mathrm{NuTeV}$ data stream is shown in figure 210. 


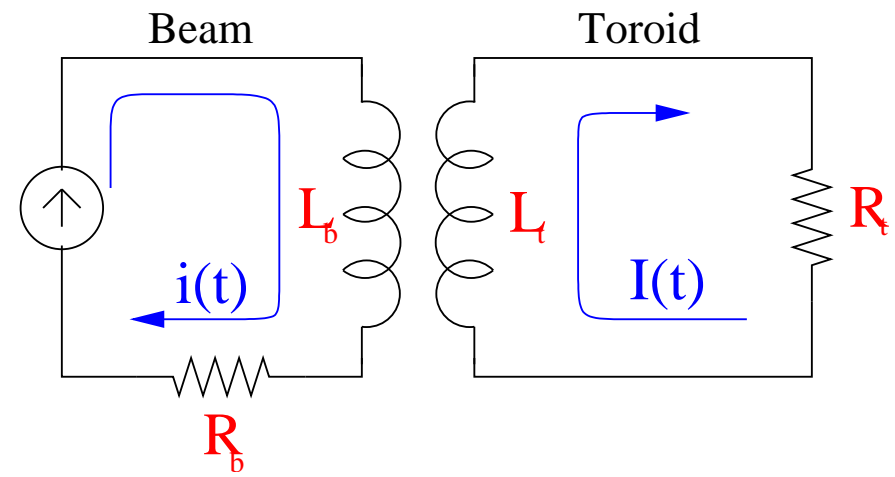

FIGURE 211. A simple equivalent circuit of the beam toroid and beam

\section{A.3 Some toroid physics and droop compensation}

A simplified equivalent circuit for the toroid is shown in figure 211. The beam (or equivalently the calibration wire looped through the toroid) is approximated by a current source and half of transformer coil $\left(L_{b}\right)$, and the toroid makes up the other half $\left(L_{t}\right)$, with the coaxial transmission line approximated by a resistor $R_{t}[200]$. To understand what kind of signal to expect from the toroid given a beam pulse $i(t)$ we start with the EMF:

$$
\begin{aligned}
\mathcal{E}_{\text {tor }} & =M \frac{d i(t)}{d t}-L_{t} \frac{d I(t)}{d t} \\
I(t) \cdot R_{t} & =M \frac{d i(t)}{d t}-L_{t} \frac{d I(t)}{d t} \\
& \vdots \\
I(t) & =e^{-\frac{R_{t}}{L_{t}} t} \frac{M}{L_{t}} \int e^{\frac{R_{t}}{L_{t}} t} \cdot\left(\frac{d i}{d t}\right) d t
\end{aligned}
$$

A relatively simple approximation for a $\mathrm{NuTeV}$ ping would be a function of the form: 


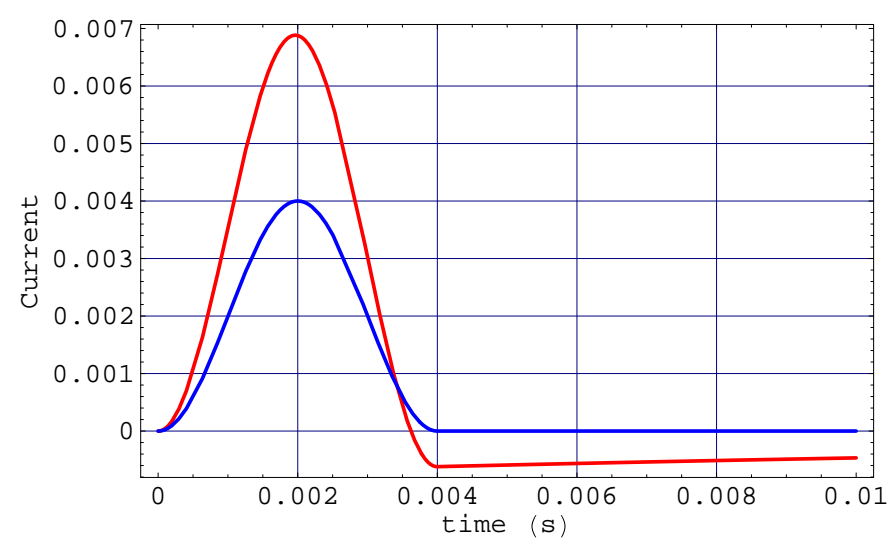

FIGURE 212. Input and output current to NuTeV toroid. The red (larger) curve is the output multiplied by 1000

$$
i(t)= \begin{cases}i_{0}[1-\cos \omega t] & 0<t<\frac{2 \pi}{\omega} \\ 0 & \text { otherwise }\end{cases}
$$

Plugging this into equation A.304, one finds that the current flowing out of the toroid would look like the following:

$$
I(t)= \begin{cases}\frac{M R_{t} i_{0} \omega}{R_{t}^{2}+L_{t}^{2} \omega^{2}} \sin \omega t+\frac{M L_{t} i_{0} \omega^{2}}{R_{t}^{2}+L_{t}^{2} \omega^{2}}\left(e^{-\frac{R t}{L_{t}} t}-\cos \omega t\right) & 0<t<\frac{2 \pi}{\omega} \\ I_{\tau} e^{-\frac{R_{t}}{L_{t}}\left(t-\frac{2 \pi}{\omega}\right)} & \text { otherwise }\end{cases}
$$

Where $I_{\tau}$ is the top half of A.306 evaluated at $t=\frac{2 \pi}{\omega}$. Figure 212 displays an input pulse of the form of A.305 with the corresponding output shape function using the following parameters: $M=19.1 \mathrm{mH}, L_{t}=1.06 \mathrm{H}, R_{t}=50 \Omega, \omega=$ $1570 \mathrm{~Hz}$, based on measurements from the actual NuTeV toroid, and an assumed ping duration of 4 milliseconds. The output pulse has a significant undershoot and exponential decay compared to the original input pulse. This undershoot is called 


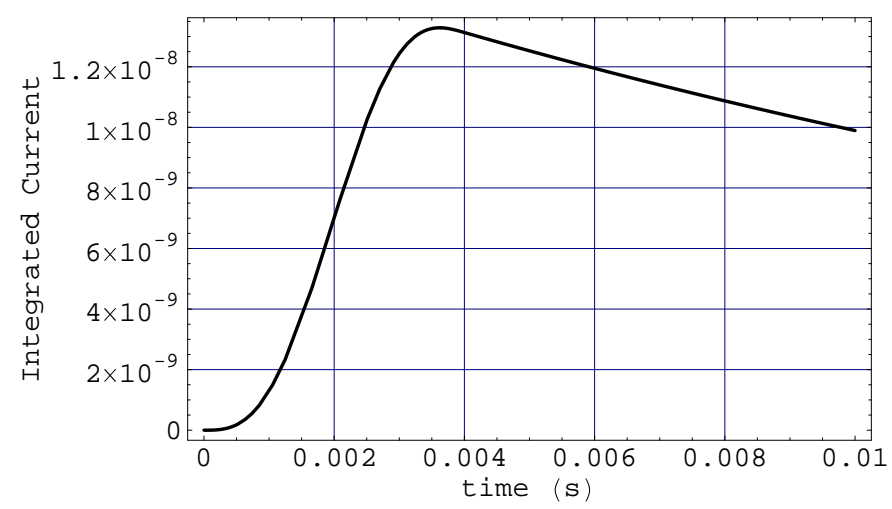

FIGURE 213. The integral of the toroid output showing decay from signal droop

"droop", and can complicate using a toroid to determine integrated charge in a ping.

Figure 213 shows the effect of droop on the integrated signal from the toroid. This decay means the integral recorded will depend on when it is recorded. This could significantly increase the uncertainty of the beam intensity calibration should the timing of the pings fluctuate. In addition to this, the analog output from the toroid was used to gate the $\mathrm{NuTeV}$ detector. The difficulty in this case is that the droop would force the pulse waveform below the gate threshold prematurely, reducing the overall active time of the detector. It is therefore important to reduce toroid droop as much as possible. 


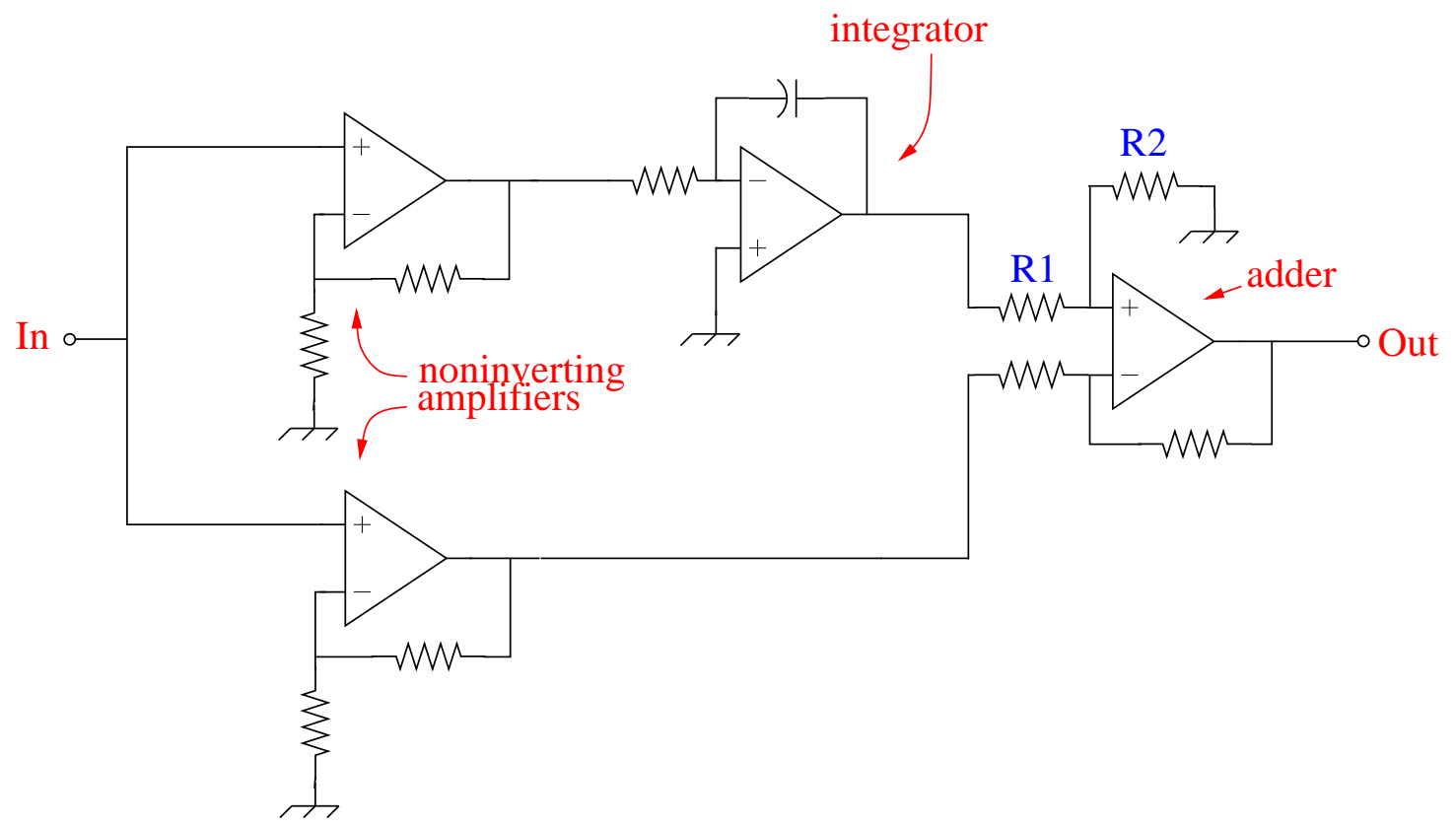

FIGURE 214. Circuit diagram of droop compensator circuit

To find a way to reduce the toroid pulse droop we must again revisit the EMF:

$$
\begin{aligned}
\mathcal{E}_{t o r} & =M \frac{d i(t)}{d t}-L_{t} \frac{d I(t)}{d t} \\
I(t) \cdot R_{t} & =M \frac{d i(t)}{d t}-L_{t} \frac{d I(t)}{d t} \\
I(t) \cdot R_{t}+L_{t} \frac{d I(t)}{d t} & =M \frac{d i(t)}{d t} \\
\frac{R_{t}}{M} \int I(t) d t+\frac{L_{t}}{M} I(t) & =i(t) \\
\frac{L_{t}}{M}\left[I(t)+\frac{R_{t}}{L_{t}} \int I(t) d t\right] & =i(t)
\end{aligned}
$$

$I(t)$ is what is measured, and to recover the shape of the original beam pulse, one needs only to add back into $I(t)$ its integral scaled by $\frac{R_{t}}{L_{t}}$. This is easy 

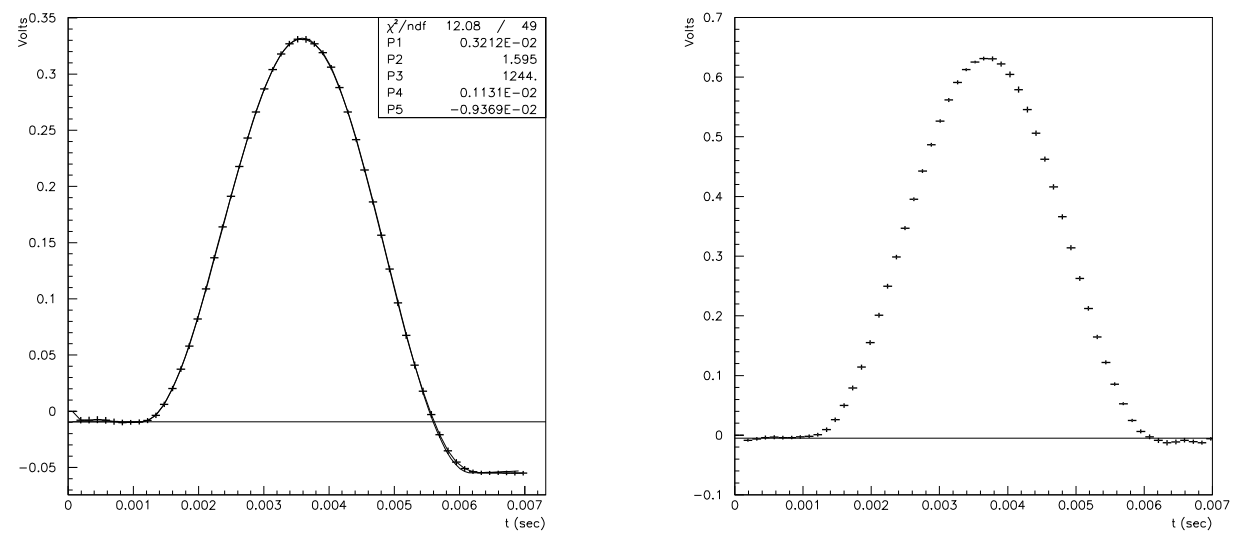

FIGURE 215. Non-droop compensated and compensated test pulse waveforms

to implement electronically with a simple integrator and adder circuit. Figure 214 shows an example of such a circuit used to cancel out the droop from the $\mathrm{NuTeV}$ toroid. The ratio of "straight" to "integrated" signal can be controlled by changing the ratio of $R 1$ and $R 2$ shown in the figure. In practice these resistors were replaced by a potentiometer which was tuned to remove the measured droop in the toroid signal.

The performance of the droop compensation electronics is shown in figures 215 and 216. During data running the raw and droop compensated signals from the toroid were digitized and recorded in the $\mathrm{NuTeV}$ data stream. At the beginning of each TeVatron cycle (at T1 +5 seconds) a test pulse with a shape similar to that in figure 212 was injected through the toroid test loop for calibration purposes. The pulse's width was tuned to be similar to the width of actual pings, as was 

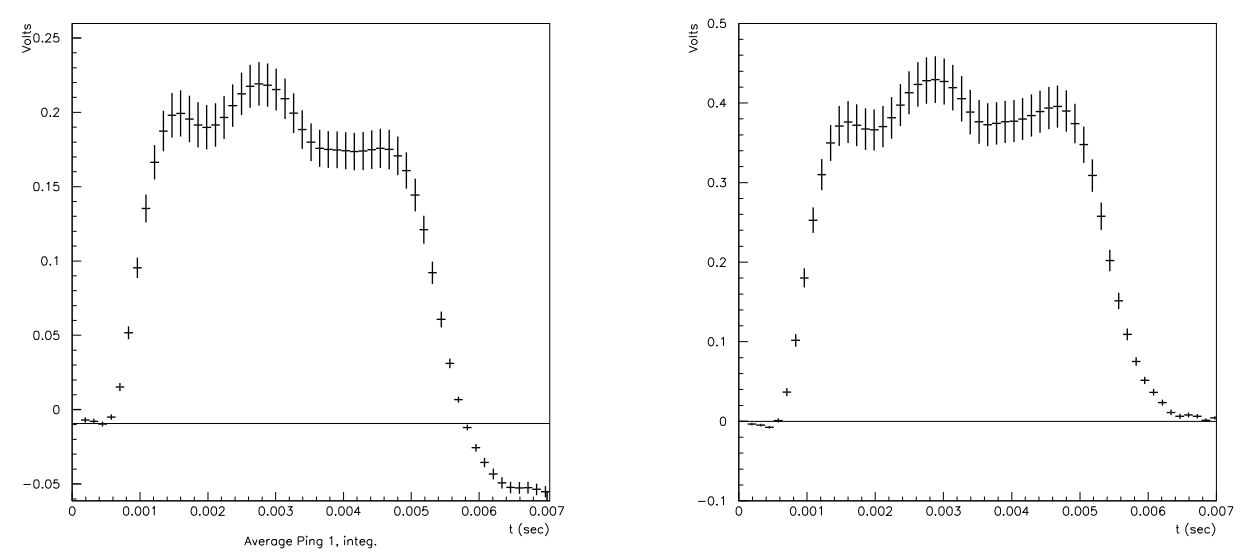

FIGURE 216. Non-droop compensated and compensated ping waveforms

the total integrated charge. Figure 215 shows both the uncompensated (left) and compensated (right) amplified outputs from the beam toroid averaged over about a dozen cycles during data running. As a check, the uncompensated curve used to measure the toroid inductance, $L$, by fitting the digitized waveform with a function of the form in equation A.306. The measured inductance of the toroid coil using this method is $1.595 \pm 0.146$, in perfect agreement with the documented value of 1.6 [199]. The effect of droop compensation on actual pings is shown in figure 216. The error bars here reflect more the fluctuation between each ping than uncertainty in the measurement. Droop compensation typically recovered approximately 400 microseconds of livetime towards the end of each ping. The recovered detector livetime is estimated to have increased NuTeV's total data sample by $2-3 \%$ over what would have been accumulated without droop compensation. 


\section{APPENDIX B}

\section{NUTEV SOFTWARE SETTINGS}

Here are settings for the different $\mathrm{NuTeV}$ software packages.

\section{B.0.1 McNuTeV Settings}

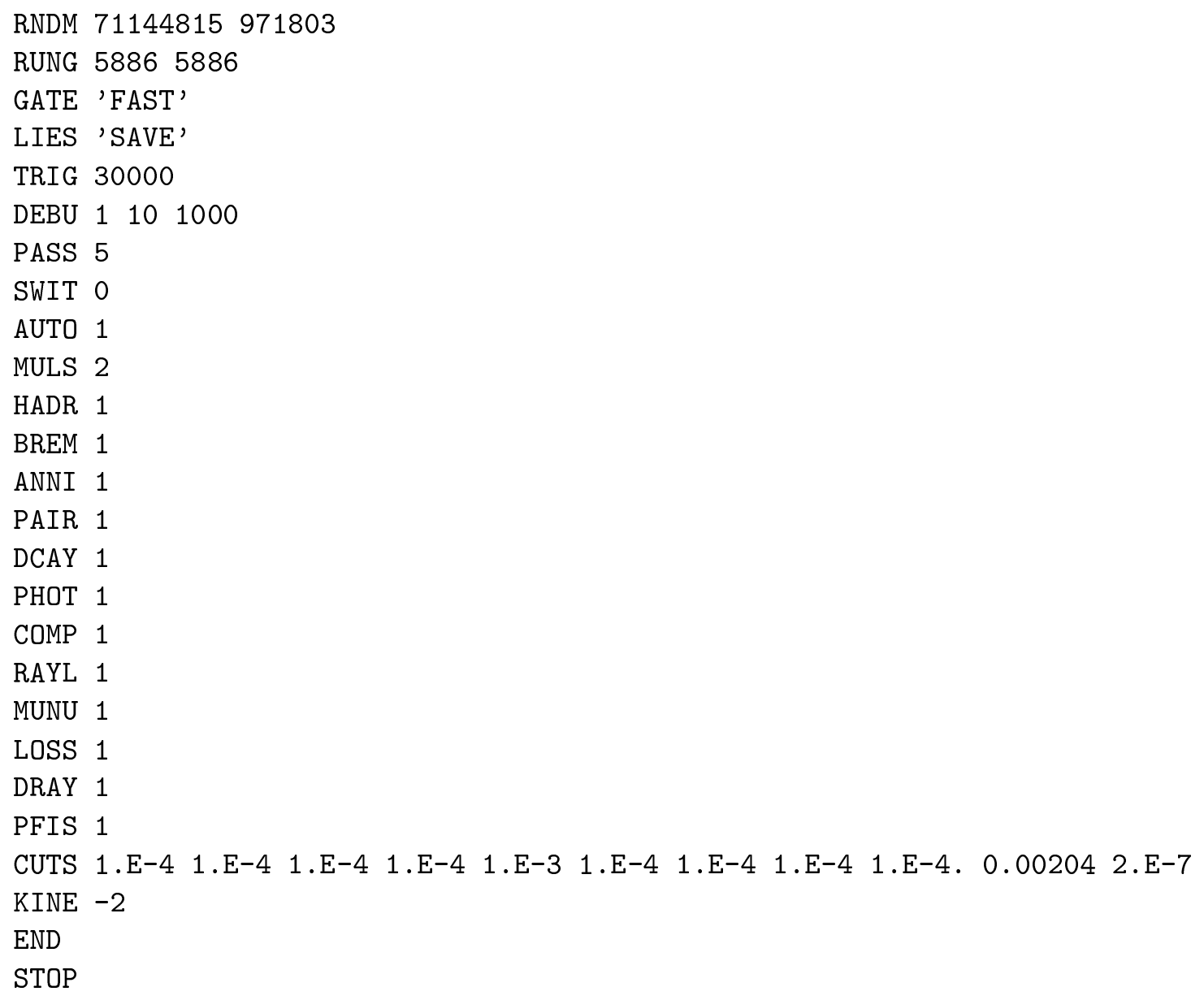




\section{APPENDIX C}

\section{RELEVANT CODE SNIPPETS}

Here we include sections of code introduced into $\mathrm{NuTeV}$ software to calculate quantities used in applying cuts, corrections, etc.

\section{C.0.2 Fixed Collins fragmentation code}

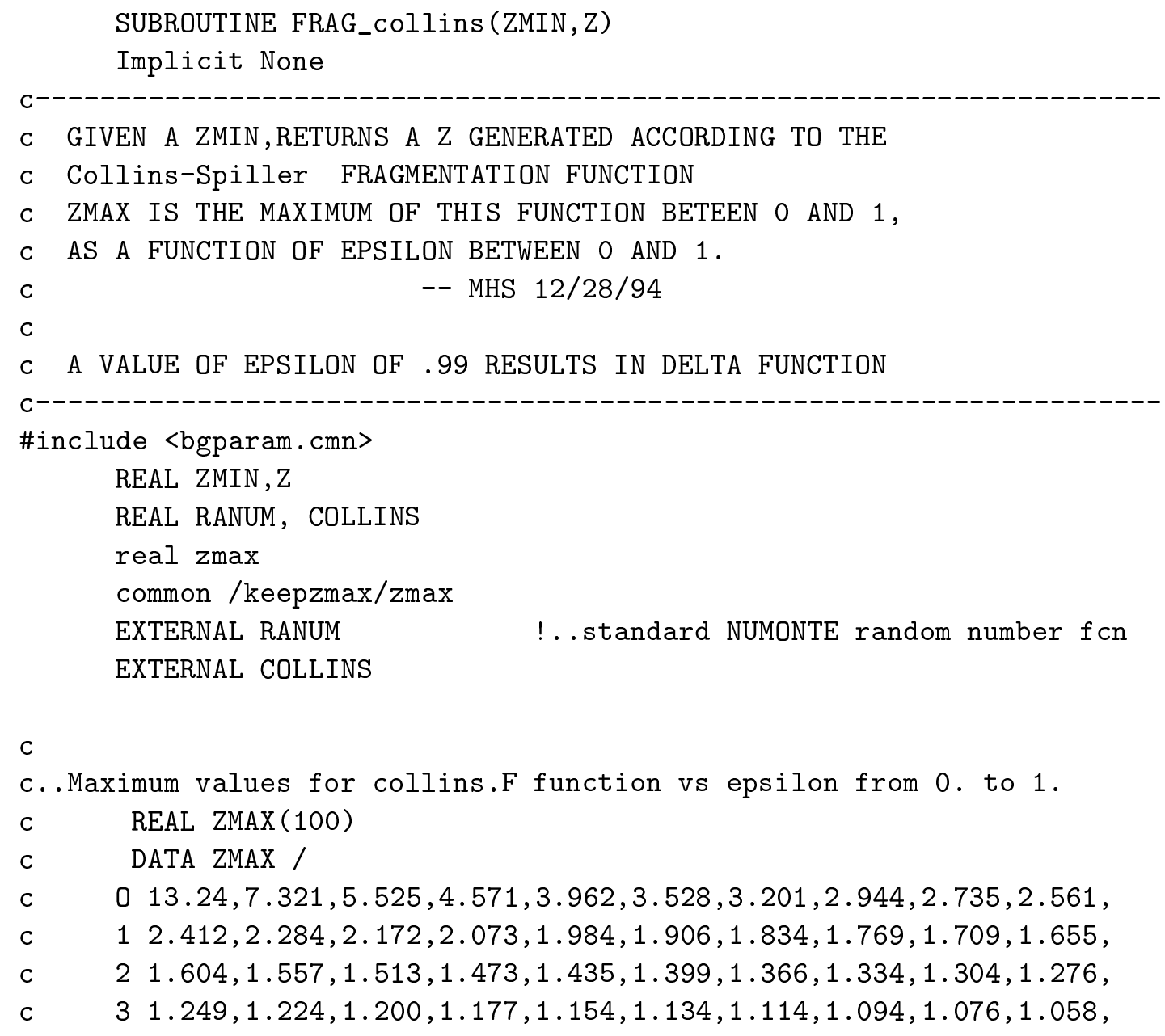


c

C

c

C

$41.041,1.025,1.009, .9939, .9794, .9652, .9515, .9385, .9258, .9135$,

$5.9014, .8899, .8788, .8680, .8574, .8471, .8371, .8276, .8182, .8090$,

$6.8000, .7913, .7830, .7748, .7667, .7589, .7512, .7437, .7365, .7294$,

c $\quad 7.7224, .7156, .7089, .7024, .6961, .6899, .6838, .6779, .6720, .6662$,

$8.6606, .6552, .6498, .6445, .6393, .6342, .6292, .6243, .6195, .6148$,

c $\quad 9.6102, .6057, .6012, .5967, .5924, .5882, .5840, .5799, .5759, .5719 /$

REAL ZF, Y, YMAX, F, ZDELTA

DATA ZDELTA / 1.0 /

INTEGER IEPS, ICNT, ICNTMX, NTRY, NTRMX

DATA ICNT, ICNTMX / 0, 5 /

PARAMETER (NTRMX $=100)$

integer iii

integer npoints

parameter (npoints $=1000)$

real point

c logical first

c save first

c data first /.false./

C

c..start of routine

c if (EPS.GE.dble(.99)) GO TO 50

C $\quad$ IEPS $=$ EPS $* 100$

if (zmax.eq.0.0) then

print *, 'first call to frag_collins'

print *,'getting maximum for future throws...'

C

first $=$.false.

$\mathrm{ymax}=0.0$

do $i i i=1, n p o i n t s$

point=1.0/iii

$y=\operatorname{collins}$ (point)

if (ymax.1t.y) ymax=y

enddo

$\mathrm{ymax}=\mathrm{ymax} * 1.01$

print $*,{ }^{\prime} \mathrm{OK}, \max$ is ', ymax

$z \max =y \max$

endif

$y \max =z \max$

NTRY $=1$

C

YMAX $=\operatorname{ZMAX}($ IEPS $) * 1.01$ 


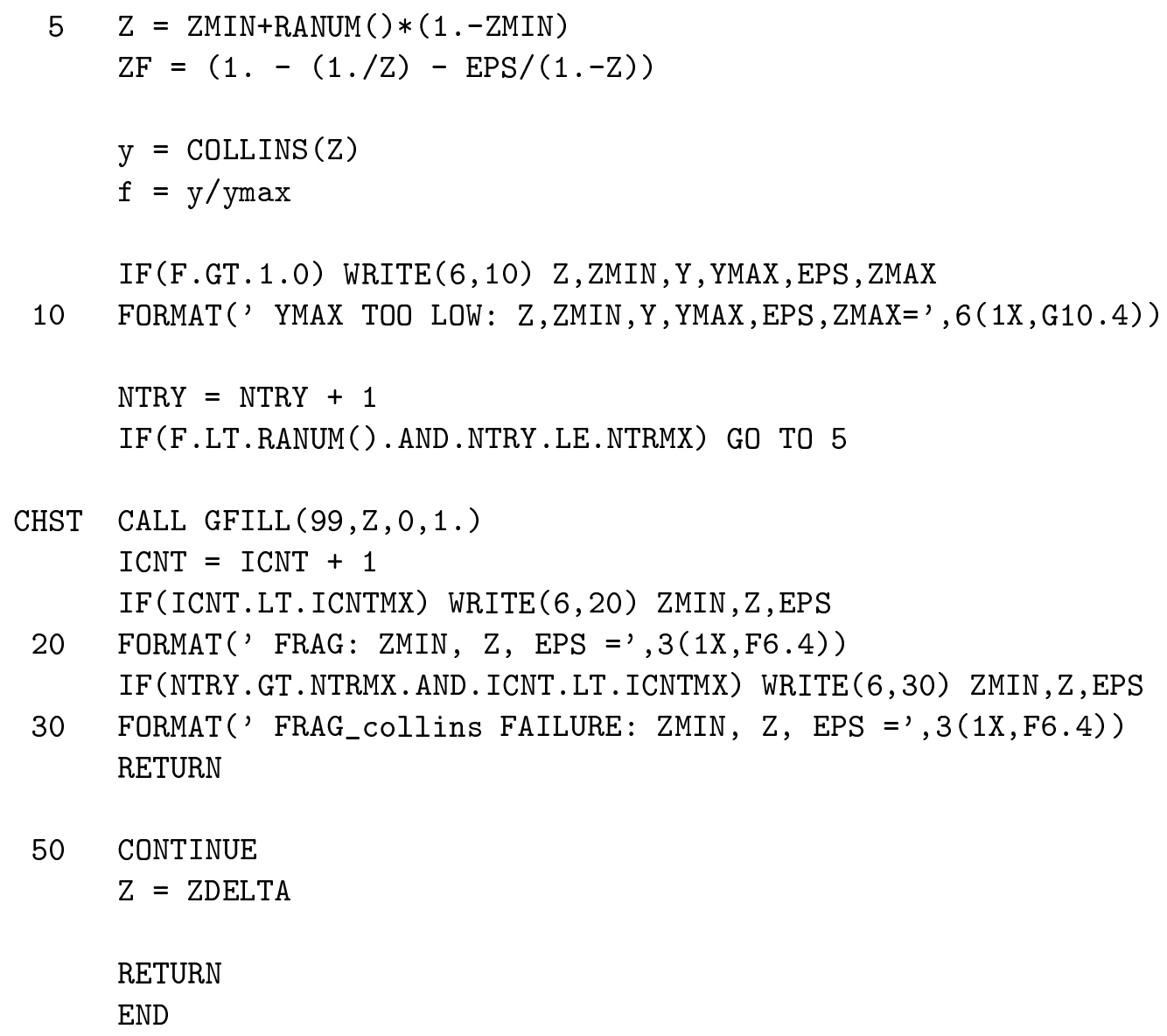

\section{C.0.3 mygap.f}

This code can be used to apply gap cuts:

real function mygap(i)

c this is just packing the links into a simple integer

c variable for each muon -- we can then do whatever

c gymnastics Re: gap cuts later on...

c $i$ is the muon (link) number

c of course since this is going into an ntuple the

c simple integer is a real just to be confusing... 


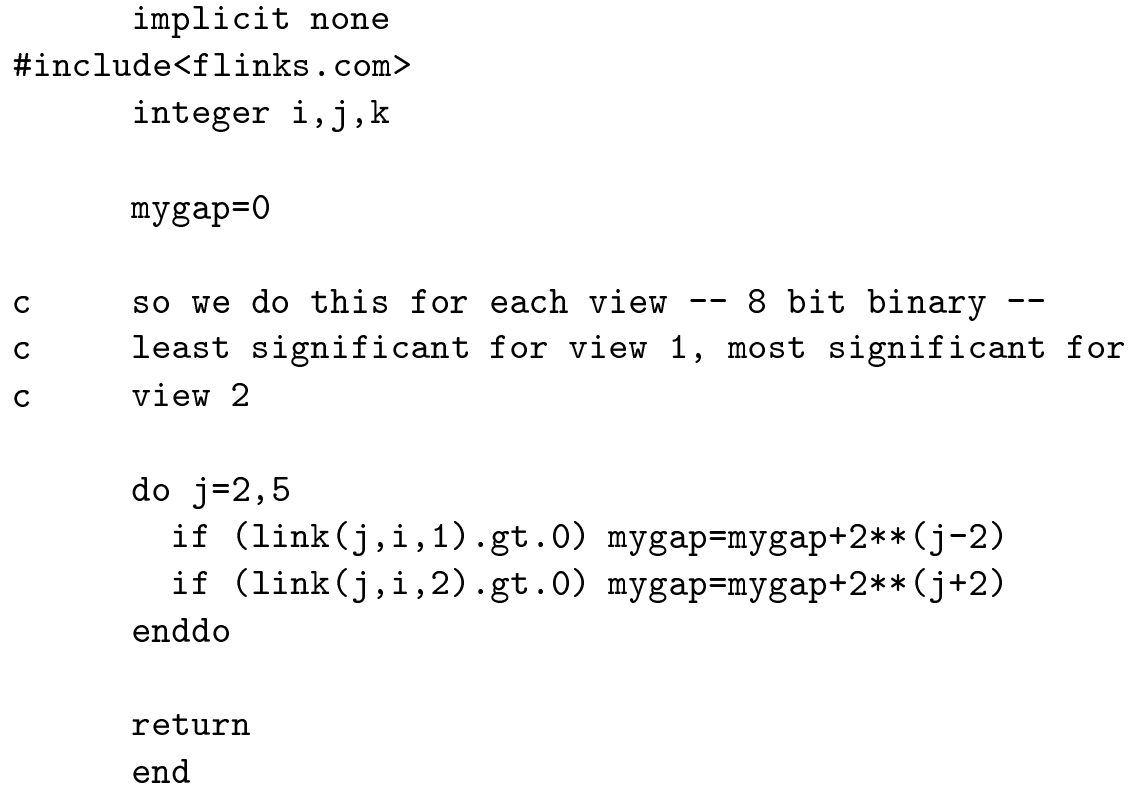

\section{C.0.4 epich,epi0}

The McNuTeV hadronic energy corrections require one know how much energy was hadronic $\left(E_{\pi^{+}}+E_{\pi^{-}}\right)$and how much was electromagnetic $\left(E_{\pi^{0}}\right)$. This code extracts that from the truth bank.

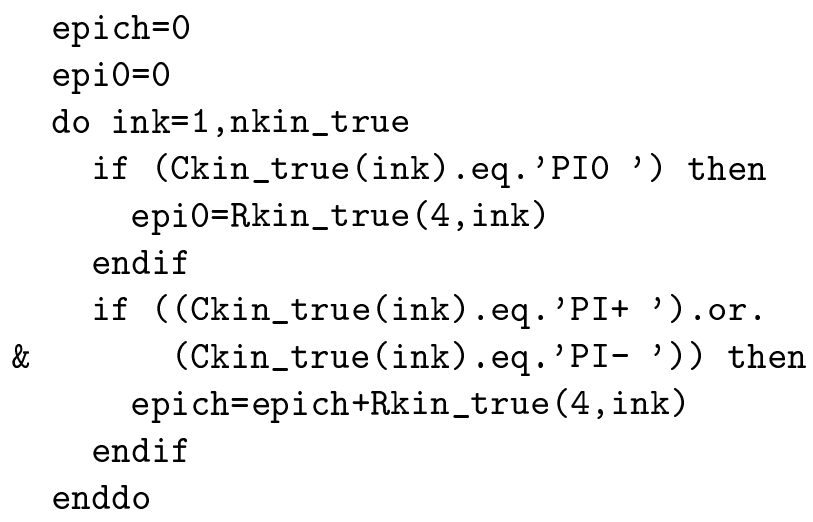




\section{C.0.5 psteel1 and 2}

The following calculates what percent of the track went through the toroid steel (psteel1 and psteel2):

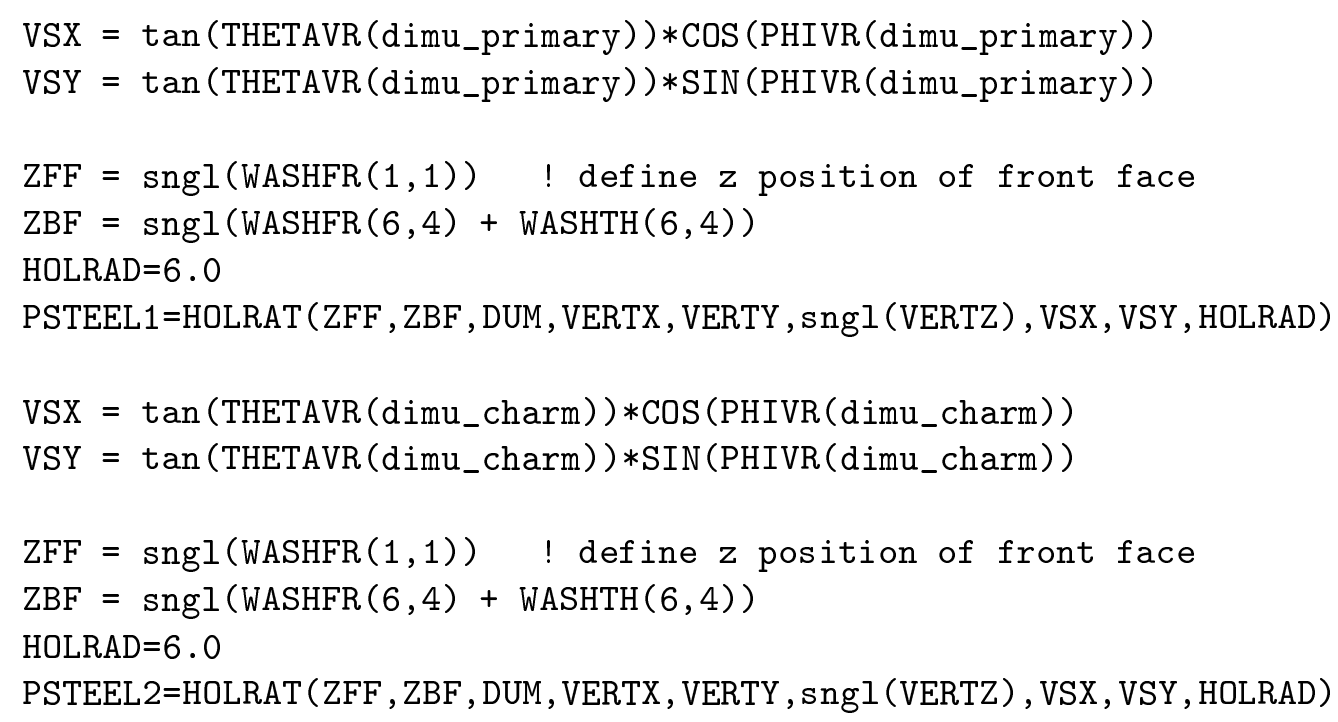

C.0.6 Bad run/event cut

c before we get going, kill bad runs! -- swiped from phdst in

c '01, updated in winter of '03.

C 


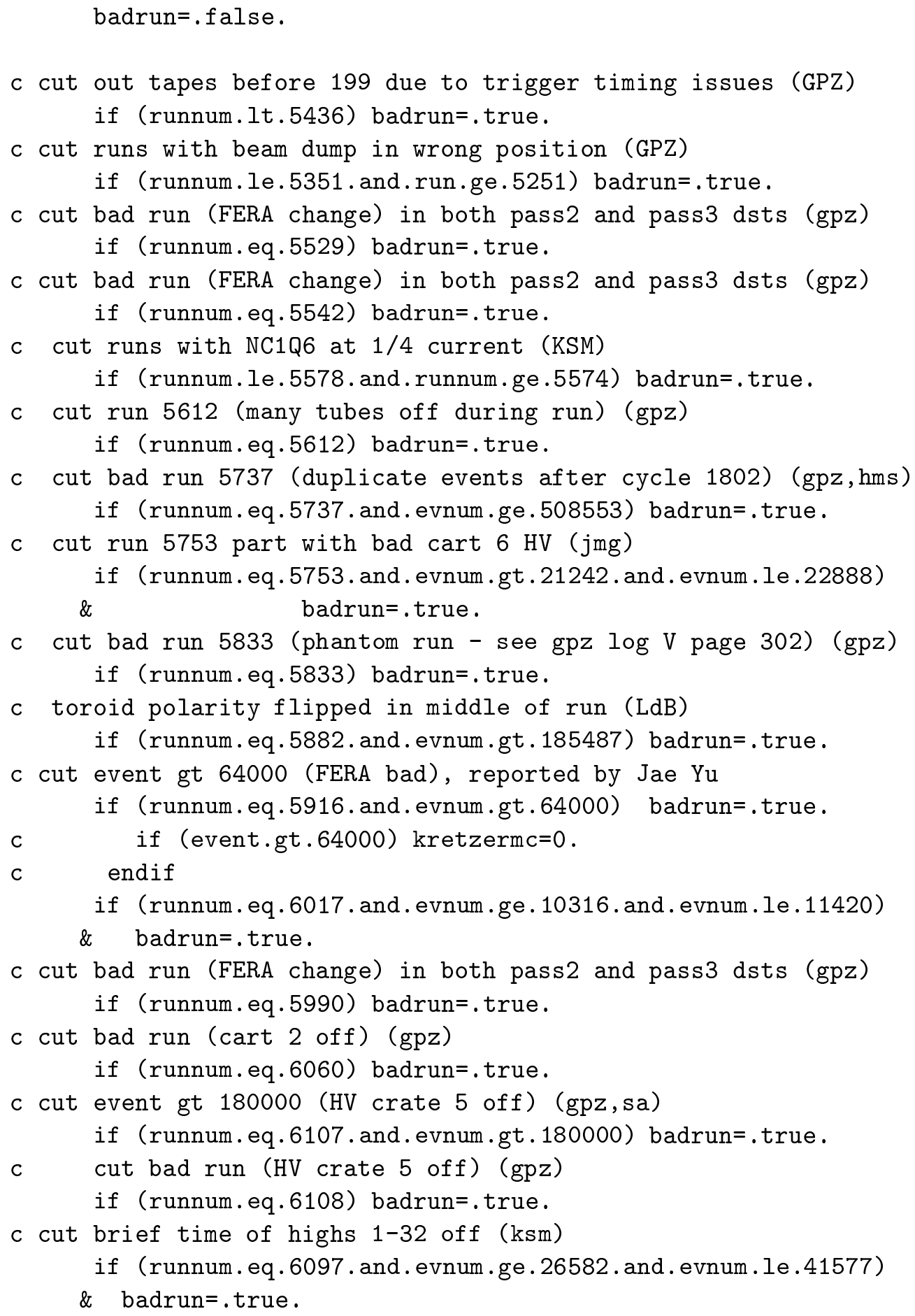




\section{C.0.7 Gap cut code}

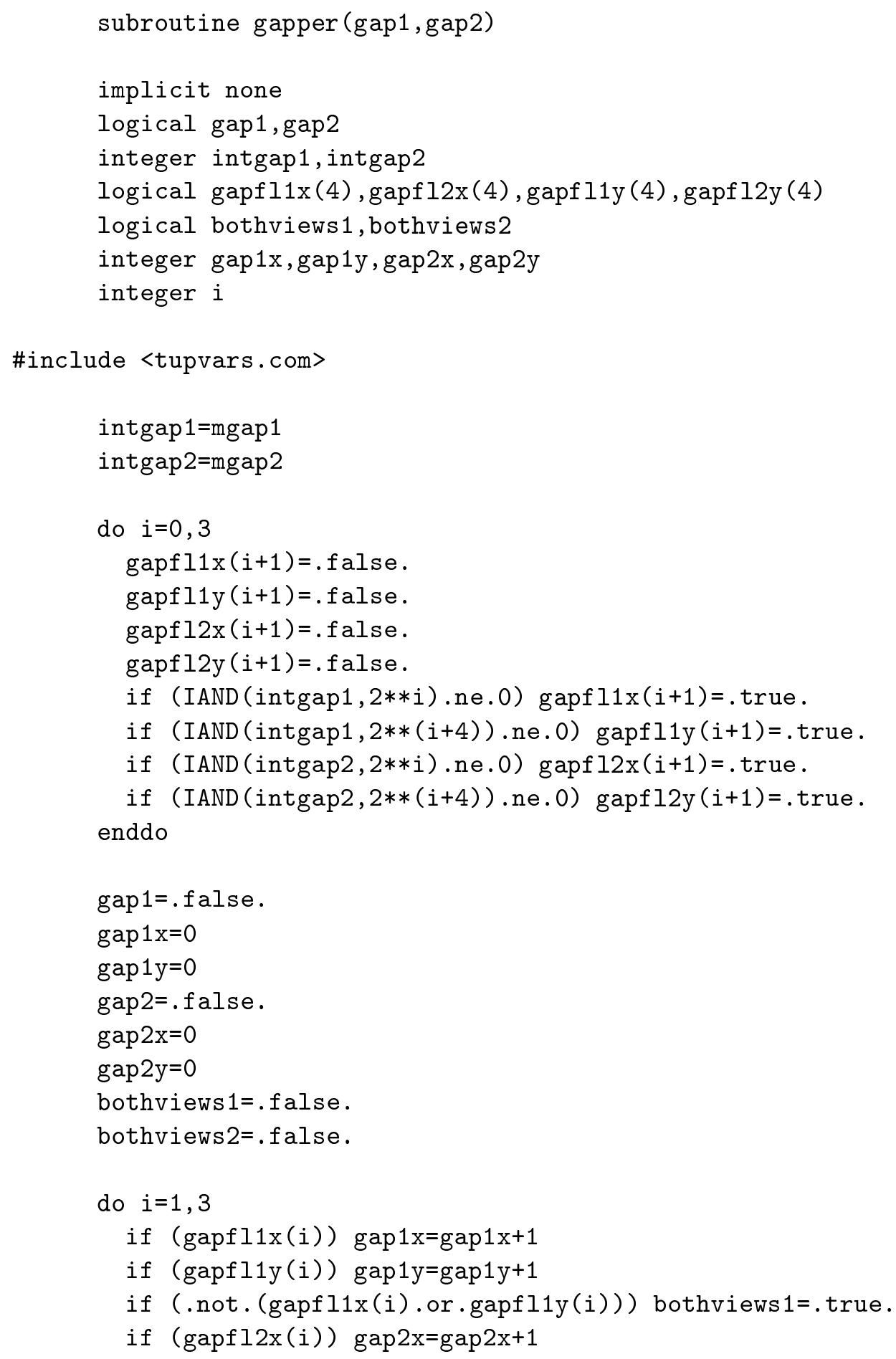




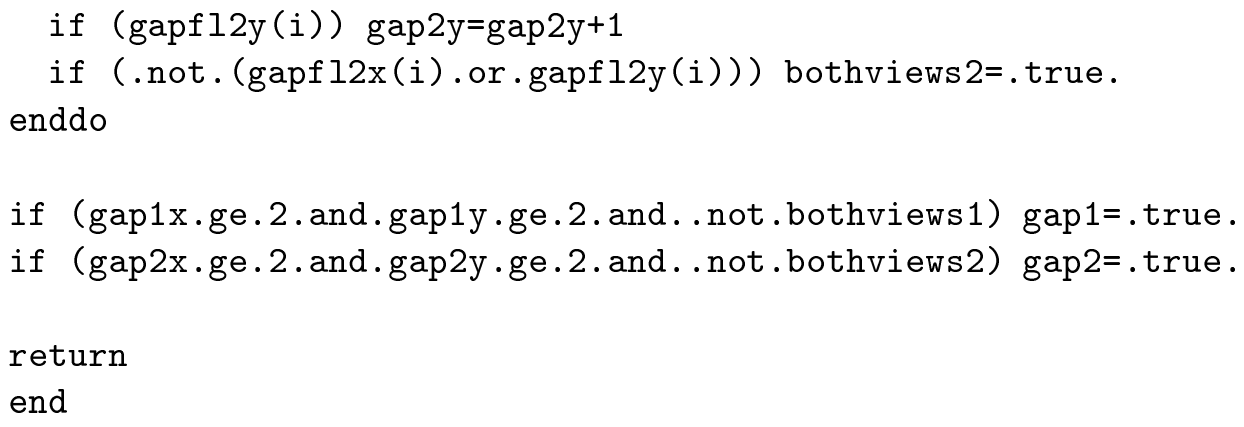

$\underline{\text { C.1 Irrelevant Code Chunks \& Snippets }}$

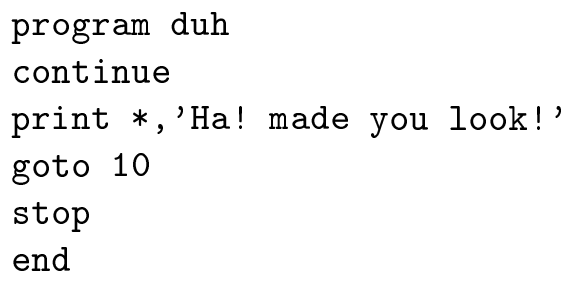




\section{APPENDIX D \\ DIMUON CROSS SECTION TABLE VALUES}

The following sets of tables list the measured dimuon cross section points described in section 4.5. Each seperate table is for a different $E_{\nu}$ bin, three for $\nu$ data, and three for $\bar{\nu}$ data. The tables list the effective degree of freedom under the $\chi^{2}$ column, the central values of $y$ and $x$, and the cross section value, with statistical and systematic errors scaled by a factor of $\frac{100 \pi}{G_{F}^{2} M E}$.

\begin{tabular}{|c|c|c|c|}
\hline \multicolumn{4}{|c|}{$E_{\nu} \sim 88.3 \mathrm{GeV}$} \\
\hline$\chi^{2}$ & $y$ & $x$ & $\frac{d \sigma\left(\nu_{\mu} N \rightarrow \mu^{-} \mu^{+} X\right)}{d x d y}$ \\
\hline 0.65 & 0.324 & 0.021 & $0.449 \pm 0.079 \pm 0.050$ \\
0.40 & 0.324 & 0.057 & $0.529 \pm 0.092 \pm 0.038$ \\
0.42 & 0.324 & 0.101 & $0.415 \pm 0.067 \pm 0.035$ \\
0.43 & 0.324 & 0.167 & $0.325 \pm 0.051 \pm 0.020$ \\
0.64 & 0.324 & 0.326 & $0.144 \pm 0.020 \pm 0.010$ \\
0.57 & 0.558 & 0.021 & $0.786 \pm 0.111 \pm 0.067$ \\
0.44 & 0.558 & 0.057 & $0.798 \pm 0.105 \pm 0.060$ \\
0.44 & 0.558 & 0.101 & $0.783 \pm 0.103 \pm 0.047$ \\
0.48 & 0.558 & 0.167 & $0.505 \pm 0.064 \pm 0.029$ \\
0.63 & 0.558 & 0.326 & $0.228 \pm 0.030 \pm 0.012$ \\
0.55 & 0.771 & 0.021 & $0.769 \pm 0.113 \pm 0.068$ \\
0.44 & 0.771 & 0.057 & $0.911 \pm 0.129 \pm 0.034$ \\
0.46 & 0.771 & 0.101 & $0.864 \pm 0.124 \pm 0.039$ \\
0.52 & 0.771 & 0.167 & $0.694 \pm 0.105 \pm 0.032$ \\
0.58 & 0.771 & 0.326 & $0.217 \pm 0.037 \pm 0.009$ \\
\hline
\end{tabular}

TABLE 28. Revised NuTeV forward differential cross-section for $\nu_{\mu} N \rightarrow \mu^{-} \mu^{+} X$. The forward cross-section requires $E_{\mu+} \geq 5 \mathrm{GeV}$, and is shown multiplied by $\frac{100 \pi}{G_{F}^{2} M E}$. The first error in the cross-section entry is statistical, the second is systematic. 


\begin{tabular}{|c|c|c|c|}
\hline \multicolumn{4}{|c|}{$E_{\nu} \sim 174.3 \mathrm{GeV}$} \\
\hline$\chi^{2}$ & $y$ & $x$ & $\frac{d \sigma\left(\nu_{\mu} N \rightarrow \mu^{-} \mu^{+} X\right)}{d x d y}$ \\
\hline 0.65 & 0.324 & 0.021 & $1.158 \pm 0.172 \pm 0.077$ \\
0.41 & 0.324 & 0.057 & $0.861 \pm 0.122 \pm 0.069$ \\
0.36 & 0.324 & 0.101 & $0.854 \pm 0.127 \pm 0.032$ \\
0.39 & 0.324 & 0.167 & $0.566 \pm 0.080 \pm 0.034$ \\
0.57 & 0.324 & 0.326 & $0.255 \pm 0.034 \pm 0.015$ \\
0.52 & 0.558 & 0.021 & $1.434 \pm 0.217 \pm 0.094$ \\
0.37 & 0.558 & 0.057 & $1.351 \pm 0.205 \pm 0.076$ \\
0.35 & 0.558 & 0.101 & $1.196 \pm 0.190 \pm 0.047$ \\
0.36 & 0.558 & 0.167 & $0.762 \pm 0.119 \pm 0.038$ \\
0.52 & 0.558 & 0.326 & $0.281 \pm 0.043 \pm 0.020$ \\
0.57 & 0.771 & 0.021 & $1.520 \pm 0.204 \pm 0.072$ \\
0.41 & 0.771 & 0.057 & $1.240 \pm 0.163 \pm 0.068$ \\
0.41 & 0.771 & 0.101 & $1.075 \pm 0.149 \pm 0.062$ \\
0.46 & 0.771 & 0.167 & $0.842 \pm 0.130 \pm 0.041$ \\
0.58 & 0.771 & 0.326 & $0.308 \pm 0.053 \pm 0.015$ \\
\hline
\end{tabular}

TABLE 29. Revised NuTeV forward differential cross-section for $\nu_{\mu} N \rightarrow \mu^{-} \mu^{+} X$. The forward cross-section requires $E_{\mu+} \geq 5 \mathrm{GeV}$, and is shown multiplied by $\frac{100 \pi}{G_{F}^{2} M E}$. The first error in the cross-section entry is statistical, the second is systematic. 


\begin{tabular}{|c|c|c|c|}
\hline \multicolumn{4}{|c|}{$E_{\nu} \sim 247.0 \mathrm{GeV}$} \\
\hline$\chi^{2}$ & $y$ & $x$ & $\frac{d \sigma\left(\nu_{\mu} N \rightarrow \mu^{-} \mu^{+} X\right)}{d x d y}$ \\
\hline 0.74 & 0.324 & 0.021 & $1.384 \pm 0.199 \pm 0.086$ \\
0.50 & 0.324 & 0.057 & $1.430 \pm 0.214 \pm 0.045$ \\
0.43 & 0.324 & 0.101 & $1.034 \pm 0.150 \pm 0.043$ \\
0.41 & 0.324 & 0.167 & $0.762 \pm 0.107 \pm 0.047$ \\
0.62 & 0.324 & 0.326 & $0.283 \pm 0.035 \pm 0.025$ \\
0.63 & 0.558 & 0.021 & $1.660 \pm 0.265 \pm 0.079$ \\
0.44 & 0.558 & 0.057 & $1.557 \pm 0.255 \pm 0.096$ \\
0.41 & 0.558 & 0.101 & $1.125 \pm 0.183 \pm 0.051$ \\
0.43 & 0.558 & 0.167 & $0.727 \pm 0.115 \pm 0.046$ \\
0.60 & 0.558 & 0.326 & $0.315 \pm 0.047 \pm 0.027$ \\
0.66 & 0.771 & 0.021 & $1.679 \pm 0.223 \pm 0.167$ \\
0.49 & 0.771 & 0.057 & $1.402 \pm 0.188 \pm 0.087$ \\
0.46 & 0.771 & 0.101 & $1.109 \pm 0.154 \pm 0.047$ \\
0.52 & 0.771 & 0.167 & $0.896 \pm 0.136 \pm 0.076$ \\
0.67 & 0.771 & 0.326 & $0.305 \pm 0.051 \pm 0.034$ \\
\hline
\end{tabular}

TABLE 30. Revised NuTeV forward differential cross-section for $\nu_{\mu} N \rightarrow \mu^{-} \mu^{+} X$. The forward cross-section requires $E_{\mu+} \geq 5 \mathrm{GeV}$, and is shown multiplied by $\frac{100 \pi}{G_{F}^{2} M E}$. The first error in the cross-section entry is statistical, the second is systematic. 


\begin{tabular}{|c|c|c|c|}
\hline \multicolumn{4}{|c|}{$E_{\nu} \sim 77.9 \mathrm{GeV}$} \\
\hline$\chi^{2}$ & $y$ & $x$ & $\frac{d \sigma\left(\bar{\nu}_{\mu} N \rightarrow \mu^{+} \mu^{-} X\right)}{d x d y}$ \\
\hline 0.59 & 0.349 & 0.015 & $0.362 \pm 0.099 \pm 0.030$ \\
0.36 & 0.349 & 0.042 & $0.369 \pm 0.103 \pm 0.034$ \\
0.31 & 0.349 & 0.072 & $0.337 \pm 0.092 \pm 0.028$ \\
0.36 & 0.349 & 0.114 & $0.267 \pm 0.071 \pm 0.015$ \\
0.57 & 0.349 & 0.207 & $0.080 \pm 0.018 \pm 0.012$ \\
0.57 & 0.579 & 0.015 & $0.444 \pm 0.097 \pm 0.023$ \\
0.38 & 0.579 & 0.042 & $0.504 \pm 0.108 \pm 0.045$ \\
0.36 & 0.579 & 0.072 & $0.556 \pm 0.120 \pm 0.042$ \\
0.40 & 0.579 & 0.114 & $0.295 \pm 0.058 \pm 0.018$ \\
0.62 & 0.579 & 0.207 & $0.153 \pm 0.031 \pm 0.014$ \\
0.55 & 0.776 & 0.015 & $0.539 \pm 0.142 \pm 0.129$ \\
0.35 & 0.776 & 0.042 & $0.484 \pm 0.114 \pm 0.079$ \\
0.36 & 0.776 & 0.072 & $0.454 \pm 0.103 \pm 0.039$ \\
0.41 & 0.776 & 0.114 & $0.404 \pm 0.097 \pm 0.036$ \\
0.58 & 0.776 & 0.207 & $0.143 \pm 0.036 \pm 0.015$ \\
\hline
\end{tabular}

TABLE 31. Revised NuTeV forward differential cross-section for $\bar{\nu}_{\mu} N \rightarrow \mu^{+} \mu^{-} X$. The forward cross-section requires $E_{\mu-} \geq 5 \mathrm{GeV}$, and is shown multiplied by $\frac{100 \pi}{G_{F}^{2} M E}$. The first error in the cross-section entry is statistical, the second is systematic. 


\begin{tabular}{|c|c|c|c|}
\hline \multicolumn{4}{|c|}{$E_{\nu} \sim 143.7 \mathrm{GeV}$} \\
\hline$\chi^{2}$ & $y$ & $x$ & $\frac{d \sigma\left(\bar{\nu}_{\mu} N \rightarrow \mu^{+} \mu^{-} X\right)}{d x d y}$ \\
\hline 0.63 & 0.349 & 0.015 & $0.767 \pm 0.183 \pm 0.075$ \\
0.40 & 0.349 & 0.042 & $0.739 \pm 0.180 \pm 0.100$ \\
0.34 & 0.349 & 0.072 & $0.728 \pm 0.179 \pm 0.050$ \\
0.36 & 0.349 & 0.114 & $0.427 \pm 0.101 \pm 0.026$ \\
0.53 & 0.349 & 0.207 & $0.159 \pm 0.035 \pm 0.022$ \\
0.48 & 0.579 & 0.015 & $1.175 \pm 0.306 \pm 0.050$ \\
0.34 & 0.579 & 0.042 & $0.923 \pm 0.227 \pm 0.059$ \\
0.32 & 0.579 & 0.072 & $0.723 \pm 0.176 \pm 0.122$ \\
0.33 & 0.579 & 0.114 & $0.626 \pm 0.159 \pm 0.072$ \\
0.48 & 0.579 & 0.207 & $0.223 \pm 0.056 \pm 0.020$ \\
0.52 & 0.776 & 0.015 & $0.777 \pm 0.168 \pm 0.054$ \\
0.40 & 0.776 & 0.042 & $1.077 \pm 0.239 \pm 0.104$ \\
0.36 & 0.776 & 0.072 & $0.716 \pm 0.160 \pm 0.079$ \\
0.42 & 0.776 & 0.114 & $0.527 \pm 0.121 \pm 0.026$ \\
0.57 & 0.776 & 0.207 & $0.229 \pm 0.060 \pm 0.020$ \\
\hline
\end{tabular}

TABLE 32. Revised NuTeV forward differential cross-section for $\bar{\nu}_{\mu} N \rightarrow \mu^{+} \mu^{-} X$. The forward cross-section requires $E_{\mu-} \geq 5 \mathrm{GeV}$, and is shown multiplied by $\frac{100 \pi}{G_{F}^{2} M E}$. The first error in the cross-section entry is statistical, the second is systematic. 


\begin{tabular}{|c|c|c|c|}
\hline \multicolumn{4}{|c|}{$E_{\nu} \sim 226.8 \mathrm{GeV}$} \\
\hline$\chi^{2}$ & $y$ & $x$ & $\frac{d \sigma\left(\bar{\nu}_{\mu} N \rightarrow \mu^{+} \mu^{-} X\right)}{d x d y}$ \\
\hline 0.79 & 0.349 & 0.015 & $1.022 \pm 0.233 \pm 0.145$ \\
0.48 & 0.349 & 0.042 & $1.037 \pm 0.238 \pm 0.141$ \\
0.43 & 0.349 & 0.072 & $0.713 \pm 0.157 \pm 0.113$ \\
0.41 & 0.349 & 0.114 & $0.452 \pm 0.100 \pm 0.042$ \\
0.64 & 0.349 & 0.207 & $0.278 \pm 0.062 \pm 0.030$ \\
0.60 & 0.579 & 0.015 & $1.233 \pm 0.323 \pm 0.405$ \\
0.41 & 0.579 & 0.042 & $1.040 \pm 0.267 \pm 0.094$ \\
0.40 & 0.579 & 0.072 & $0.829 \pm 0.216 \pm 0.095$ \\
0.42 & 0.579 & 0.114 & $0.562 \pm 0.143 \pm 0.137$ \\
0.63 & 0.579 & 0.207 & $0.276 \pm 0.074 \pm 0.040$ \\
0.63 & 0.776 & 0.015 & $0.912 \pm 0.187 \pm 0.073$ \\
0.49 & 0.776 & 0.042 & $1.210 \pm 0.262 \pm 0.102$ \\
0.43 & 0.776 & 0.072 & $1.009 \pm 0.229 \pm 0.044$ \\
0.50 & 0.776 & 0.114 & $0.479 \pm 0.107 \pm 0.048$ \\
0.65 & 0.776 & 0.207 & $0.184 \pm 0.046 \pm 0.024$ \\
\hline
\end{tabular}

TABLE 33. Revised NuTeV forward differential cross-section for $\bar{\nu}_{\mu} N \rightarrow \mu^{+} \mu^{-} X$. The forward cross-section requires $E_{\mu-} \geq 5 \mathrm{GeV}$, and is shown multiplied by $\frac{100 \pi}{G_{F}^{2} M E}$. The first error in the cross-section entry is statistical, the second is systematic. 


\section{APPENDIX E}

\section{THE NUTEV COLLABORATION}

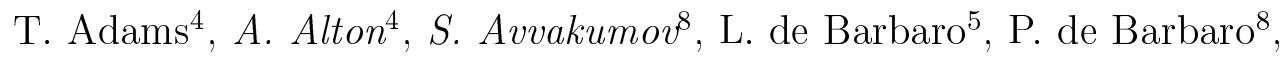

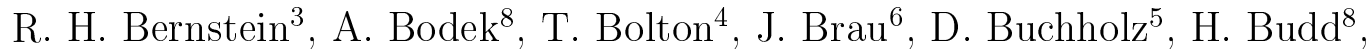

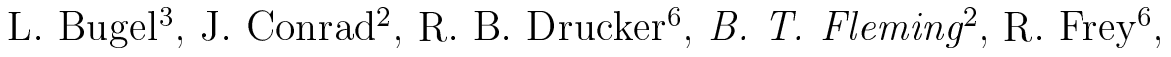

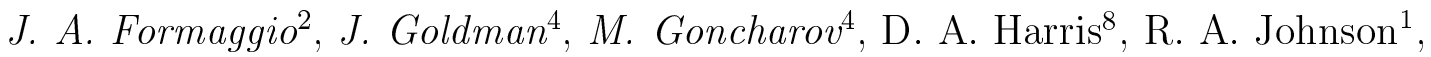

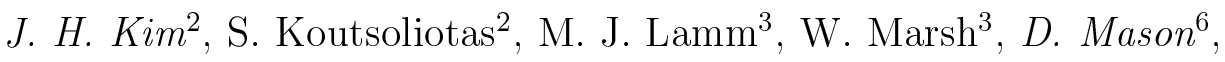
J. McDonald ${ }^{7}$, K. S. McFarland ${ }^{8,3}, C . M_{c N u l t y}{ }^{2}$, D. Naples ${ }^{7}$, P. Nienaber ${ }^{3}$,

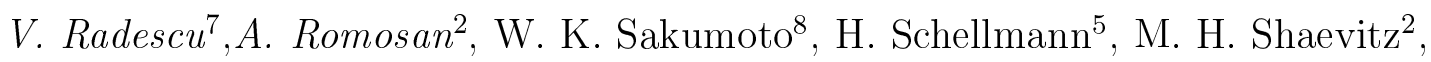
P. Spentzouris ${ }^{2}$, E. G. Stern ${ }^{2}$, N. Suwonjandee ${ }^{1}$, M. Tzanov ${ }^{7}$, M. Vakili ${ }^{1}$, A. Vaitaitis ${ }^{2}$, U. K. Yang ${ }^{8}$, J. Yu ${ }^{3}$, G. P. Zeller ${ }^{5}$, and E. D. Zimmerman ${ }^{2}$

${ }^{1}$ University of Cincinnati, Cincinnati, $\mathrm{OH}$ ${ }^{2}$ Columbia University, New York, NY

${ }^{3}$ Fermi National Acelerator Laboratory, Batavia, IL

${ }^{4}$ Kansas State University, Manhattan, KS

${ }^{5}$ Northwestern University, Evanston, IL ${ }^{6}$ University of Oregon, Eugene, OR

${ }^{7}$ University of Pittsburgh, Pittsburgh, PA

${ }^{8}$ University of Rochester, Rochester, NY 


\section{BIBLIOGRAPHY}

[1] David Alexander Mason. Measurement of the strange - antistrange asymmetry at $\mathrm{NLO}$ in QCD from $\mathrm{NuTeV}$ dimuon data. University of Oregon, FERMILAB-THESIS-2006-01. URL:http://lss.fnal.gov/archive/thesis/fermilab-thesis-2006-01.shtml.

[2] J. J. Thomson. Cathode rays. Philosophical Magazine, 44:293.

[3] E. Rutherford. The scattering of the alpha and beta rays and the structure of the atom. Proceedings of the Manchester Literary and Philosphical Society IV, 55:18-20, 1911.

[4] J. Chadwick. The existence of a neutron. Proc. Roy. Soc., A, 136:692-708, 1932.

[5] A. Einstein. On the electrodynamics of moving bodies. Annalen der Physik, 17:891, 1905.

[6] P. A. M. Dirac. The quantum theory of the electron. Proc. R. Soc. (London), A 117:610-612, 1928.

[7] P. A. M. Dirac. The quantum theory of the electron part II. Proc. R. Soc. (London), A 118:351-361, 1928.

[8] C. D. Anderson. The positive electron. Phys. Rev., 43:491-494, 1933.

[9] W. Pauli. Dear radioactive ladies and gentlemen. In N. Cooper, editor, Los Alamos Science, Celebrating the Neutrino, volume 25, page 6. Los Alamos National Laboratory, 1930.

[10] E. Fermi. Trends to a theory of beta radiation. (in Italian). Nuovo Cim., 11:1-19, 1934.

[11] S. H. Neddermeyer and C. D. Anderson. Note on the nature of cosmic ray particles. Phys. Rev., 51:884-886, 1937.

[12] R.P. Feynman. Surely You're Joking Mr. Feynman. Bantam Books, 1985.

[13] R. P. Feynman. Space-time approach to nonrelativistic quantum mechanics. Rev. Mod. Phys., 20:367-387, 1948. 
[14] C. L. Cowan, F. Reines, F. B. Harrison, E. C. Anderson, and F. N. Hayes. Large liquid scintillation detectors. Phys. Rev., 90:493-494, 1953.

[15] N. Cooper et al. The reines-cowan experiments, detecting the poltergeist. In N. Cooper, editor, Los Alamos Science, Celebrating the Neutrino, volume 25, pages 4-27. Los Alamos National Laboratory, 1997.

[16] F. Reines and C. L. Cowan. Detection of the free neutrino. Phys. Rev., 92:830-831, 1953.

[17] C. L. Cowan, F. Reines, F. B. Harrison, H. W. Kruse, and A. D. McGuire. Detection of the free neutrino: A confirmation. Science, 124:103-104, 1956.

[18] F. Reines. The detection of pauli's neutrino. In H.B. Newman and T. Ypsilantis, editors, History of the Original Ideas and Basic Discoveries in Particle Physics, pages 401-419. Plenum Press, 1996.

[19] G. Feinberg. Decays of the $\mu$ meson in the intermediate-meson theory. Phys. Rev., 110:1482-83, 1958.

[20] G. Danby et al. Observation of high-energy neutrino reactions and the existence of two kinds of neutrinos. Phys. Rev. Lett., 9:36-44, 1962.

[21] M. Schwartz. The early history of high-energy neutrino physics. In L. Hoddeson, L. Brown, M. Riordan, and M. Dresden, editors, The Rise of the Standard Model, Particle Physics in the 1960s and 1970s, page 411. Cambridge University Press, 1997.

[22] Julian S. Schwinger. A theory of the fundamental interactions. Annals Phys., 2:407-434, 1957.

[23] Abdus Salam and J. C. Ward. Weak and electromagnetic interactions. Nuovo Cim., 11:568-577, 1959.

[24] S. L. Glashow. Partial symmetries of weak interactions. Nucl. Phys., 22:579-588, 1961.

[25] J. Goldstone. Field theories with 'superconductor' solutions. Nuovo Cim., 19:154-164, 1961.

[26] Steven Weinberg. A model of leptons. Phys. Rev. Lett., 19:1264-1266, 1967.

[27] Peter W. Higgs. Broken symmetries and the masses of gauge bosons. Phys. Rev. Lett., 13:508-509, 1964. 
[28] D.J. Gross. Asymptotic freedom, confinement and QCD. In H.B. Newman and T. Ypsilantis, editors, History of the Original Ideas and Basic Discoveries in Particle Physics, pages 75-97. Plenum Press, 1996.

[29] Murray Gell-Mann. A schematic model of baryons and mesons. Phys. Lett., 8:214-215, 1964.

[30] G. Zweig. An su(3) model for strong interaction symmetry and its breaking. 2. CERN-TH-412.

[31] R. E. Millikan. On the elementary electrical charge and the avogadro constant. Physical Review, 11:109-143.

[32] S. L. Glashow, J. Iliopoulos, and L. Maiani. Weak interactions with lepton hadron symmetry. Phys. Rev., D2:1285-1292, 1970.

[33] D. Perkins. Gargamelle and the discovery of neutral currents. In L. Hoddeson, L. Brown, M. Riordan, and M. Dresden, editors, The Rise of the Standard Model, Particle Physics in the 1960s and 1970s, pages 428-446. Cambridge University Press, 1997.

[34] Gerard 't Hooft. Renormalization of massless Yang-Mills fields. Nucl. Phys., B33:173-199, 1971.

[35] F. J. Hasert et al. Observation of neutrino like interactions without muon or electron in the gargamelle neutrino experiment. Nucl. Phys., B73:1, 1974.

[36] Sheldon Lee Glashow. Charm: An invention awaits discovery. Talk given at IV International Conference on Experimental Meson Spectroscopy, Apr 26-27, 1974, Boston, Mass.

[37] J. Iliopoulos. Progress in gauge theories. Rapporteur's Talk given at 17th Int. Conf. on High Energy Physics, London, England, Jul 1-10, 1974.

[38] Burton Richter. E + e- $\rightarrow$ hadrons. Plenary Session Report at XVII International Conference on High Energy Physics, London, Eng., Jul 1-10, 1974 .

[39] Michael Riordan. The Hunting of the Quark. Simon \& Schuster, Inc., 1987.

[40] A. C. Benvenuti et al. Early observation of neutrino and antineutrino events at high energies. Phys. Rev. Lett., 30:1084-1087, 1973.

[41] A. C. Benvenuti et al. A liquid - scintillator total absorption hadron calorimeter for the study of neutrino interactions. Nucl. Instr. Meth., 125:447, 1975. 
[42] A. C. Benvenuti et al. A large area magnetic spectrometer for the study of high- energy neutrino interactions. Nucl. Instr. Meth., 125:457, 1975.

[43] C. Rubbia et al. Experimental observation of $\mu^{+} \mu^{-}$pairs produced by very high energy neutrinos. Proceedings 17h Int. Conf. on High Energy Physics, London, England, Jul 1-10, 1974.

[44] A. C. Benvenuti et al. Observation of new particle production by high-energy neutrinos and anti-neutrinos. Phys. Rev. Lett., 34:419, 1975.

[45] A. C. Benvenuti et al. Characteristics of dimuons as evidence for a new quantum number. Phys. Rev. Lett., 35:1203, 1975.

[46] Howard Georgi and Sheldon L. Glashow. Unified weak and electromagnetic interactions without neutral currents. Phys. Rev. Lett., 28:1494, 1972.

[47] B. Barish et al. Investigations of neutrino interactions with two muons in the final state. Phys. Rev. Lett., 36:939-941, 1976.

[48] C. Baltay et al. Charmed-D-meson production by neutrinos. Phys. Rev. Lett., 41:73-76, 1978.

[49] G. Goldhaber et al. Observation in e+ e- annihilation of a narrow state at $1865-\mathrm{MeV} / \mathrm{c}^{* *} 2$ decaying to $\mathrm{K}$ pi and $\mathrm{K}$ pi pi pi. Phys. Rev. Lett., $37: 255-259,1976$.

[50] A. C. Benvenuti et al. Observation of a new process with trimuon production by high energy neutrinos. Phys. Rev. Lett., 38:1110, 1977.

[51] A. C. Benvenuti et al. Rates and properties of opposite-sign dimuons from neutrinos and antineutrinos. Phys. Rev. Lett., 41:1204-1207, 1978.

[52] S. W. Herb et al. Observation of a dimuon resonance at $9.5-\mathrm{GeV}$ in $400-\mathrm{GeV}$ proton - nucleus collisions. Phys. Rev. Lett., 39:252-255, 1977.

[53] H. Deden et al. Strange particle production and charmed particle search in the gargamelle neutrino experiment. Phys. Lett., B58:361-366, 1975.

[54] N. Armenise et al. Study of neutrino induced dimuon events in gargamelle at CERN SPS. Phys. Lett., B86:115, 1979.

[55] F. Dydak. Neutrino physics. Phys. Rept., 403-404:57-67, 2004.

[56] M. Holder et al. Performance of a magnetized total absorption calorimeter between 15-GeV and 140-GeV. Nucl. Instrum. Meth., 151:69, 1978. 
[57] K. Kleinknecht. Neutrino induced trimuon and tetramuon events from the CDHS experiment. Preprint - KLEINKNECHT K (78,REC.JUL) 22p.

[58] H. Abramowicz et al. Experimental study of opposite sign dimuons produced in neutrino and anti-neutrinos interactions. Z. Phys., C15:19, 1982.

[59] M. Holder et al. Observation of a neutrino event with four energetic muons. Phys. Lett., B73:105-108, 1978.

[60] D. MacFarlane et al. Nucleon structure functions from high-energy neutrino interactions with iron and QCD results. Z. Phys., C26:1, 1984.

[61] C. Foudas et al. Neutrino production of opposite-sign dimuons at fermilab TeVatron energies. Phys. Rev. Lett., 64:1208-1211, 1990.

[62] S. A. Rabinowitz et al. Measurement of the strange sea distribution using neutrino charm production. Phys. Rev. Lett., 70:134-137, 1993.

[63] A. O. Bazarko et al. Determination of the strange quark content of the nucleon from a next-to-leading order QCD analysis of neutrino charm production. Z. Phys., C65:189-198, 1995.

[64] N. Ushida et al. Experimental details on lifetime measurements of neutrino produced charmed particles in a tagged emulsion spectrometer. Nucl. Instrum. Meth., A224:50-64, 1984.

[65] N. Ushida et al. Production characteristics of charmed particles in neutrino interactions. Phys. Lett., B206:380, 1988.

[66] K. De Winter et al. A detector for the study of neutrino - electron scattering. Nucl. Instr. Meth., A278:670, 1989.

[67] J. Altegoer et al. The NOMAD experiment at the CERN SPS. Nucl. Instrum. Meth., A404:96-128, 1998.

[68] E. Eskut et al. The CHORUS experiment to search for nu/mu -i nu/tau oscillation. Nucl. Instrum. Meth., A401:7-44, 1997.

[69] P. Astier et al. Neutrino production of opposite sign dimuons in the NOMAD experiment. Phys. Lett., B486:35-48, 2000.

[70] K. Nishikawa et al. Observation of prompt like sign dimuon production in neutrino reactions. Phys. Rev. Lett., 46:1555, 1981.

[71] K. Lang et al. Neutrino production of dimuons. Z. Phys., C33:483, 1987. 
[72] M. Jonker et al. Experimental study of opposite sign and same sign dimuon events produced in wide band neutrino and anti-neutrino beams. Phys. Lett., B107:241, 1981.

[73] B. Strongin et al. Study of opposite sign dimuon production in high-energy neutrino nucleon interactions. Phys. Rev., D43:2778-2786, 1991.

[74] P. Vilain et al. Leading-order QCD analysis of neutrino induced dimuon events. Eur. Phys. J., C11:19-34, 1999.

[75] M. Goncharov et al. Precise measurement of dimuon production cross-sections in nu/mu Fe and anti-nu/mu Fe deep inelastic scattering at the tevatron. Phys. Rev., D64:112006, 2001.

[76] A. G. Cocco. Neutrino DIS measurements in the CHORUS experiment. Prepared for 12th International Workshop on Deep Inelastic Scattering (DIS 2004), Strbske Pleso, Slovakia, 14-18 Apr 2004.

[77] L. Camilleri. Precision measurements in neutrino interactions. Nucl. Phys. Proc. Suppl., 143:129-136, 2005.

[78] S. Eidelman et al. Review of Particle Physics. Physics Letters B, 592:1+, 2004. and 2005 partial update for edition 2006.

[79] Hirotaka Sugawara, Hiroyuki Hagura, and Toshiya Sanami. Destruction of nuclear bombs using ultra-high energy neutrino beam. 2003.

[80] Y. Fukuda et al. Evidence for oscillation of atmospheric neutrinos. Phys. Rev. Lett., 81:1562-1567, 1998.

[81] Y. Ashie et al. Evidence for an oscillatory signature in atmospheric neutrino oscillation. Phys. Rev. Lett., 93:101801, 2004.

[82] T. Araki et al. Measurement of neutrino oscillation with kamland: Evidence of spectral distortion. Phys. Rev. Lett., 94:081801, 2005.

[83] D. J. Griffiths. Introduction to elementary particles. NEW YORK, USA: WILEY (1987) 392p.

[84] R. G. Roberts. The Structure of the proton: Deep inelastic scattering. Cambridge, UK: Univ. Pr. (1990) 182 p. (Cambridge monographs on mathematical physics).

[85] Janet M. Conrad, Michael H. Shaevitz, and Tim Bolton. Precision measurements with high energy neutrino beams. Rev. Mod. Phys., 70:1341-1392, 1998. 
[86] J. D. Bjorken. Asymptotic sum rules at infinite momentum. Phys. Rev., 179:1547-1553, 1969.

[87] K. Hagiwara et al. Review of particle physics. Phys. Rev., D66:010001, 2002.

[88] T. Eichten et al. Measurement of the neutrino - nucleon anti-neutrino nucleon total cross-sections. Phys. Lett., B46:274-280, 1973.

[89] A. C. Benvenuti et al. Measurements of neutrino and anti-neutrino cross-sections at high-energies. Phys. Rev. Lett., 32:125-128, 1974.

[90] J. Friedman. Deep Inelastic Scattering and the Discovery of Quarks. In L. Hoddeson, L. Brown, M. Riordan, and M. Dresden, editors, The Rise of the Standard Model, Particle Physics in the 1960s and 1970s, pages 566-588. Cambridge University Press, 1997.

[91] E. D. Bloom et al. High-energy inelastic e p scattering at 6-degrees and 10degrees. Phys. Rev. Lett., 23:930-934, 1969.

[92] Richard P. Feynman. Very high-energy collisions of hadrons. Phys. Rev. Lett., 23:1415-1417, 1969.

[93] R.P. Feynman. Photon Hadron Interactions. Benjamin, Reading MA, 1972.

[94] Raymond Brock et al. Handbook of perturbative QCD: Version 1.0. Rev. Mod. Phys., 67:157-248, 1995.

[95] J. J. J. Kokkedee and L. Van Hove. Quark model and high-energy scattering. Nuovo Cim., 42:711-716, 1966.

[96] M. Y. Han and Yoichiro Nambu. Three-triplet model with double SU(3) symmetry. Phys. Rev., 139:B1006-B1010, 1965.

[97] O. W. Greenberg. Spin and unitary spin independence in a paraquark model of baryons and mesons. Phys. Rev. Lett., 13:598-602, 1964.

[98] D.J. Gross. Asymptotic freedom and the emergence of QCD. In L. Hoddeson, L. Brown, M. Riordan, and M. Dresden, editors, The Rise of the Standard Model, Particle Physics in the 1960s and 1970s, pages 199-232. Cambridge University Press, 1997.

[99] L. Susskind. Quark confinement. In L. Hoddeson, L. Brown, M. Riordan, and M. Dresden, editors, The Rise of the Standard Model, Particle Physics in the 1960s and 1970s, page 236. Cambridge University Press, 1997. 
[100] D. J. Gross and Frank Wilczek. Asymptotically free gauge theories. 1. Phys. Rev., D8:3633-3652, 1973.

[101] H. David Politzer. Reliable perturbative results for strong interactions? Phys. Rev. Lett., 30:1346-1349, 1973.

[102] J. E. Augustin et al. Discovery of a narrow resonance in e+ e- annihilation. Phys. Rev. Lett., 33:1406-1408, 1974.

[103] J. J. Aubert et al. Experimental observation of a heavy particle J. Phys. Rev. Lett., 33:1404-1406, 1974.

[104] Jr. Callan, Curtis G. and David J. Gross. High-energy electroproduction and the constitution of the electric current. Phys. Rev. Lett., 22:156-159, 1969.

[105] D. h. Perkins. Introduction to high-energy physics. page 298. Reading, Usa: Addison-wesley ( 1982) 437p.

[106] J. J. Aubert et al. A detailed study of the nucleon structure functions in deep elastic muon scattering in iron. Nucl. Phys., B272:158, 1986.

[107] E. Oltman et al. Nucleon structure functions from high-energy neutrino interactions. FNAL-616/701 experiment. Z. Phys., C53:51-71, 1992.

[108] F. Halzen and Alan D. Martin. Quarks and leptons: An introductory course in modern particle physics. New York, Usa: Wiley ( 1984) 396p.

[109] David J. Gross and Chris H. Llewellyn Smith. High-energy neutrino nucleon scattering, current algebra and partons. Nucl. Phys., B14:337-347, 1969 .

[110] D. A. Harris et al. A measurement of alpha(s) $\left(q^{* * 2}\right)$ from the Gross-Llewellyn- Smith sum rule. 1995.

[111] Stephen L. Adler. Sum rules giving tests of local current commutation relations in high-energy neutrino reactions. Phys. Rev., 143:1144-1155, 1966.

[112] D. Allasia et al. Measurement of the neutron and proton structure functions from neutrino and anti-neutrinos scattering in deuterium. Phys. Lett., B135:231, 1984.

[113] D. Allasia et al. Measurement of the neutron and the proton F2 structure function ratio. Phys. Lett., B249:366-372, 1990. 
[114] L. W. Whitlow. Deep inelastic structure functions from electron scattering on hydrogen, deuterium, and iron at $0.6-\mathrm{GeV}^{* *} 2 i=\mathrm{q}^{* *} 2 i=30-\mathrm{GeV}^{* *} 2$. SLAC-0357.

[115] N. Cabibbo. Unitary symmetry and leptonic decays. Phys. Rev. Lett., 10:531-532, 1963.

[116] M. Kobayashi and T. Maskawa. CP violation in the renormalizable theory of weak interaction. Prog. Theor. Phys., 49:652-657, 1973.

[117] A. De Rujula, Howard Georgi, S. L. Glashow, and Helen R. Quinn. Fact and fancy in neutrino physics. Rev. Mod. Phys., 46:391-407, 1974.

[118] R. Michael Barnett. Evidence for new quarks and new currents. Phys. Rev. Lett., 36:1163-1166, 1976.

[119] R. Michael Barnett. Evidence in neutrino scattering for righthanded currents associated with heavy quarks. Phys. Rev., D14:70, 1976.

[120] R. J. N. Phillips. Slow rescaling variable for heavy quark leptoproduction. Nucl. Phys., B212:109, 1983.

[121] M. A. G. Aivazis, Frederick I. Olness, and Wu-Ki Tung. Leptoproduction of heavy quarks. 1. general formalism and kinematics of charged current and neutral current production processes. Phys. Rev., D50:3085-3101, 1994.

[122] C. Peterson, D. Schlatter, I. Schmitt, and Peter M. Zerwas. Scaling violations in inclusive e+ e- annihilation spectra. Phys. Rev., D27:105, 1983.

[123] P. D. B. Collins and T. P. Spiller. The fragmentation of heavy quarks. J. Phys., G11:1289, 1985.

[124] Andrew Orest Bazarko. Determination of the strange quark distribution from a next-to-leading order QCD analysis of neutrino and anti- neutrino production of charm. Columbia University, FERMILAB-THESIS-1994-48.

[125] J.D. Bjorken and S.D. Drell. Relativistic quantum fields. McGraw-Hill, 1965.

[126] Parvez Anandam. Factorization schemes and parton distribution functions in perturbative quantum chromodynamics. UMI-99-78244.

[127] John C. Collins, Davison E. Soper, and George Sterman. Factorization of hard processes in QCD. Adv. Ser. Direct. High Energy Phys., 5:1-91, 1988.

[128] Guido Altarelli, R. K. Ellis, and G. Martinelli. Large perturbative corrections to the drell-yan process in QCD. Nucl. Phys., B157:461, 1979. 
[129] William A. Bardeen, A. J. Buras, D. W. Duke, and T. Muta. Deep inelastic scattering beyond the leading order in asymptotically free gauge theories. Phys. Rev., D18:3998, 1978.

[130] Yuri L. Dokshitzer. Calculation of the structure functions for deep inelastic scattering and e+ e- annihilation by perturbation theory in quantum chromodynamics. (in russian). Sov. Phys. JETP, 46:641-653, 1977.

[131] V. N. Gribov and L. N. Lipatov. Deep inelastic e p scattering in perturbation theory. Sov. J. Nucl. Phys., 15:438-450, 1972.

[132] Guido Altarelli and G. Parisi. Asymptotic freedom in parton language. Nucl. Phys., B126:298, 1977.

[133] Thomas Gottschalk. Chromodynamic corrections to neutrino production of heavy quarks. Phys. Rev., D23:56, 1981.

[134] M. A. G. Aivazis, John C. Collins, Fredrick I. Olness, and Wu-Ki Tung. Leptoproduction of heavy quarks. 2. a unified QCD formulation of charged and neutral current processes from fixed target to collider energies. Phys. Rev., D50:3102-3118, 1994.

[135] M. Gluck, S. Kretzer, and E. Reya. The strange sea density and charm production in deep inelastic charged current processes. Phys. Lett., B380:171-176, 1996.

[136] S. Kretzer, D. Mason, and F. Olness. Differential distributions for NLO analyses of charged current neutrino-production of charm. Phys. Rev., D65:074010, 2002.

[137] J. Yu et al. NuTeV SSQT performance. FERMILAB-TM-2040.

[138] Robert Drucker, Richard Ford, and Gianni Tassotto. Secondary emission detectors for fixed target experiments at fermilab. To be published in the proceedings of 3rd European Workshop on Beam Diagnostics and Instrumentation for Particle Accelerators (DIPAC 97), Frascati, Italy, 12-14 Oct 1997.

[139] R. Bernstein. Sign selected quadrupole train. FERMILAB-TM-1884.

[140] D. A. Harris et al. Precision calibration of the NuTeV calorimeter. Nucl. Instrum. Meth., A447:377-415, 2000.

[141] B. Barish et al. Very large area scintillation counters for hadron calorimetry. IEEE Trans. Nucl. Sci., 25:532-536, 1978. 
[142] Geralyn P. Zeller. A precise measurement of the weak mixing angle in neutrino nucleon scattering. Northwestern University, FERMILAB-THESIS-2002-34.

[143] Arturas Genrikas Vaitaitis. Search for neutral heavy leptons in a high-energy neutrino beam. Columbia University, FERMILAB-THESIS-2000-20.

[144] T. Adams et al. Observation of an anomalous number of dimuon events in a high energy neutrino beam. Phys. Rev. Lett., 87:041801, 2001.

[145] E. Dambik, D. Kline, and R. West. The Epicure Control System. Presented at International Conference on Accelerator and Large Experimental Physics Control Systems (ICALEPCS 93), Berlin, Germany, 18-22 Oct 1993.

[146] R. Brun. ZEBRA - reference manual - RZ random access package, program library. CERN, Q100, 1991.

[147] G. Koizumi. Muon de/dx and range tables for tevatron energies: Results for some shielding materials. FERMILAB-TM-0786.

[148] Maxim T. Goncharov. Precise measurement of dimuon production cross-sections in nu/mu Fe and anti-nu/mu Fe deep inelastic scattering at the tevatron. Kansas State University, FERMILAB-THESIS-2001-14.

[149] S. Avvakumov et al. A search for $\mathrm{nu} / \mathrm{mu}-i$ nu/e and anti-nu/mu -i. anti-nu/e oscillations at NuTeV. Phys. Rev. Lett., 89:011804, 2002.

[150] G. P. Zeller et al. A precise determination of electroweak parameters in neutrino nucleon scattering. Phys. Rev. Lett., 88:091802, 2002.

[151] F. James and M. Roos. 'MINUIT' a system for function minimization and analysis of the parameter errors and correlations. Comput. Phys. Commun., 10:343-367, 1975 .

[152] R. Brun, R. Hagelberg, M. Hansroul, and J. C. Lassalle. GEANT: Simulation program for particle physics experiments. user guide and reference manual. CERN-DD-78-2-REV.

[153] William Glenn Seligman. A next-to-leading order QCD analysis of neutrino - iron structure functions at the tevatron. Columbia University, FERMILAB-THESIS-1997-21.

[154] M. Tzanov et al. Precise measurement of neutrino and anti-neutrino differential cross sections. 2005. submitted to Physical Review D.

[155] T.A. Bolton. Need for a new cross section function memo. 1994. 
[156] A. J. Buras and K. J. F. Gaemers. Simple parametrizations of parton distributions with $\mathrm{Q}^{* *} 2$ dependence given by asymptotic freedom. Nucl. Phys., B132:249, 1978.

[157] Un-Ki Yang. A measurement of differential cross sections in chargedcurrent neutrino interactions on iron and a global structure functions analysis. Rochester University, FERMILAB-THESIS-2001-09.

[158] E. A. Hawker et al. Measurement of the light antiquark flavor asymmetry in the nucleon sea. Phys. Rev. Lett., 80:3715-3718, 1998.

[159] R. S. Towell et al. Improved measurement of the anti-d/anti-u asymmetry in the nucleon sea. Phys. Rev., D64:052002, 2001.

[160] Kenneth G. Wilson. Nonlagrangian models of current algebra. Phys. Rev., 179:1499-1512, 1969.

[161] Michael E. Peskin and D. V. Schroeder. An introduction to quantum field theory. Reading, USA: Addison-Wesley (1995) 842 p.

[162] Arie Bodek, Inkyu Park, and Un-ki Yang. Improved low Q**2 model for neutrino and electron nucleon cross sections in few GeV region. Nucl. Phys. Proc. Suppl., 139:113-118, 2005.

[163] D. Yu. Bardin and V. A. Dokuchaeva. On the radiative corrections to the neutrino deep inelastic scattering. JINR-E2-86-260.

[164] Tim Bolton. Determining the CKM parameter V(cd) from nu N charm production. 1997.

[165] M. Aguilar-Benitez et al. Charm D meson production in 360-GeV/c p p interactions: Comparison with pi- p at the same energy. Phys. Lett., B123:103, 1983.

[166] M. P. Alvarez et al. Study of charm photoproduction mechanisms. Z. Phys., C60:53-62, 1993.

[167] R. Michael Barnett et al. Review of particle physics. Particle Data Group. Phys. Rev., D54:1-720, 1996. and 1997 off-year partial update for the 1998 edition available on the PDG WWW pages (URL: http://pdg.lbl.gov/).

[168] Vernon D. Barger and R. J. N. Phillips. Weak semileptonic decay distributions for new particles. Phys. Rev., D14:80, 1976.

[169] P. H. Sandler et al. Hadron shower punchthrough and muon production by hadrons of 40-GeV, 70-GeV AND 100-GeV. Phys. Rev., D42:759-770, 1990. 
[170] Pamela Helen Sandler. Neutrino production of same sign dimuons at the fermilab tevatron. RX-1381 (WISCONSIN).

[171] Torbjorn Sjostrand and Mats Bengtsson. The LUND monte carlo for jet fragmentation and e+ e- physics: JETSET version 6.3: An update. Comput. Phys. Commun., 43:367, 1987.

[172] R. Brun et al. GEANT detector description and simulation library. CERN computing document CERN CN/ASD, 1998.

[173] H. L. Lai et al. Global QCD analysis of parton structure of the nucleon: Cteq5 parton distributions. Eur. Phys. J., C12:375-392, 2000.

[174] A. Kayis-Topaksu et al. Determination of the semi-leptonic branching fraction of charm hadrons produced in neutrino charged-current interactions. Phys. Lett., B549:48-57, 2002.

[175] Michele Arneodo. Nuclear effects in structure functions. Phys. Rept., 240:301-393, 1994.

[176] J. J. Aubert et al. The ratio of the nucleon structure functions F2 (N) for iron and deuterium. Phys. Lett., B123:275, 1983.

[177] J. Guy et al. A study of the EMC effect using neutrino and anti-neutrino interactions in neon and deuterium. Z. Phys., C36:337-348, 1987.

[178] M. Hirai, S. Kumano, and M. Miyama. Determination of nuclear parton distributions. Phys. Rev., D64:034003, 2001.

[179] D. de Florian and R. Sassot. Nuclear parton distributions at next to leading order. Phys. Rev., D69:074028, 2004.

[180] M. Hirai, S. Kumano, and T. H. Nagai. Nuclear parton distribution functions and their uncertainties. Phys. Rev., C70:044905, 2004.

[181] S. A. Kulagin and R. Petti. Global study of nuclear structure functions. Nucl. Phys., A765:126-187, 2006.

[182] J. Pumplin et al. New generation of parton distributions with uncertainties from global QCD analysis. JHEP, 07:012, 2002.

[183] F. Olness et al. Neutrino dimuon production and the strangeness asymmetry of the nucleon. Eur. Phys. J., C40:145-156, 2005.

[184] D. Soper. private communication, 2005. 
[185] G. P. Zeller et al. On the effect of asymmetric strange seas and isospinviolating parton distribution functions on $\sin ^{* *} 2(\operatorname{theta}(\mathrm{w}))$ measured in the NuTeV experiment. Phys. Rev., D65:111103, 2002.

[186] W. Giele. http://pdf.fnal.gov/, 2002.

[187] M. R. Whalley, D. Bourilkov, and R. C. Group. The les houches accord PDFs (LHAPDF) and LHAGLUE. 2005.

[188] W. K. Tung. private communication, 2003.

[189] Alan D. Martin, R. G. Roberts, W. J. Stirling, and R. S. Thorne. MRST2001: Partons and alpha(s) from precise deep inelastic scattering and tevatron jet data. Eur. Phys. J., C23:73-87, 2002.

[190] M. Gluck, E. Reya, and A. Vogt. Dynamical parton distributions revisited. Eur. Phys. J., C5:461-470, 1998.

[191] M. Burkardt and Brian Warr. Chiral symmetry and the charge asymmetry of the s anti-s distribution in the proton. Phys. Rev., D45:958-964, 1992.

[192] A. I. Signal and Anthony W. Thomas. Possible strength of the nonperturbative strange sea of the nucleon. Phys. Lett., B191:205, 1987.

[193] Stanley J. Brodsky and Bo-Qiang Ma. The quark/antiquark asymmetry of the nucleon sea. Phys. Lett., B381:317-324, 1996.

[194] Johan Alwall and Gunnar Ingelman. Quark asymmetries in the proton from a model for parton densities. Phys. Rev., D71:094015, 2005.

[195] S. Catani, D. de Florian, G. Rodrigo, and W. Vogelsang. Perturbative generation of a strange-quark asymmetry in the nucleon. Prepared for 32nd International Conference on High-Energy Physics (ICHEP 04), Beijing, China, 16-22 Aug 2004.

[196] F. G. Cao and A. I. Signal. The quark-antiquark asymmetry of the strange sea of the nucleon. Phys. Lett., B559:229-234, 2003.

[197] M. Wakamatsu. On the NuTeV anomaly and the asymmetry of the strange sea in the nucleon. Phys. Rev., D71:057504, 2005.

[198] S. Geer. Neutrino beams from muon storage rings: Characteristics and physics potential. Phys. Rev., D57:6989-6997, 1998.

[199] C. R. Kerns. Radiation Hardened Proton Meter for Fermilab External Beam Lines. IEEE Trans. Nucl. Sci., 22:1104-1108, 1975. Fermi National Accelerator Laboratory, Batavia IL. 
[200] C. R. Kerns. Proton Meter for NAL External Beam Lines. IEEE Trans. Nucl. Sci., 20:204-208, 1973. National Accelerator Laboratory, Batavia IL. 
